\title{
CHARACTERIZING THE REPRODUCIBILITY OF THE PROPERTIES OF ELECTROSPUN POLY(D,L-LACTIDE-CO-GLYCOLIDE) SCAFFOLDS FOR TISSUE-ENGINEERED BLOOD VESSEL MIMICS
}

\author{
A Thesis \\ presented to \\ the Faculty of California Polytechnic State University, \\ San Luis Obispo
}

In Partial Fulfillment

of the Requirements for the Degree

Master of Science in Biomedical Engineering

by

Toni M. Pipes

June 2014 
(C) 2014

Toni M. Pipes

ALL RIGHTS RESERVED 
TITLE:

AUTHOR:

DATE SUBMITTED:

COMMITTEE CHAIR:

COMMITTEE MEMBER:

COMMITTEE MEMBER
Characterizing the Reproducibility of the Properties of Electrospun Poly(D,L-Lactide-co-Glycolide) Scaffolds for Tissue-Engineered Blood Vessel Mimics

Toni M. Pipes

June 2014

Kristen O'Halloran Cardinal, $\mathrm{PhD}$

Associate Professor of Biomedical Engineering

Dan Walsh, PhD

Professor of Biomedical Engineering

Richard Savage, $\mathrm{PhD}$

Professor of Biomedical Engineering 


\begin{abstract}
Characterizing the Reproducibility of the Properties of Electrospun Poly(D,L-Lactide-coGlycolide) Scaffolds for Tissue-Engineered Blood Vessel Mimics
\end{abstract}

Toni M. Pipes

"Blood vessel mimics" (BVMs) are tissue-engineered constructs that serve as in vitro preclinical testing models for intravascular devices. The Cal Poly Tissue Engineering lab specifically uses BVMs to test the cellular response to stent implantation. PLGA scaffolds are electrospun in-house using the current "Standard Protocol" and used as the framework for these constructs. The performance of BVMs greatly depends on material and mechanical properties of the scaffolds. It is desirable to create BVMs with reproducible properties so that they can be consistent models that ultimately generate more reliable results for intravascular device testing. Reproducibility stems from the consistency of the scaffolds. Thus, scaffolds with consistent material and mechanical properties are necessary for creating reproducible BVMs.

The aim of this thesis was to characterize the reproducibility of the electrospun PLGA scaffolds using fiber diameter measurements and compliance testing. Initial work in this investigation involved designing and testing several experimental electrospinning protocols to obtain smaller fiber diameters, which have been shown to elicit more ideal cellular responses. The most successful protocol in that regard was then analyzed for the reproducibility of fiber diameters and compared to the reproducibility of the Standard Protocol. After determining that the Standard Protocol produced scaffolds with more consistent fibers, a large-scale reproducibility study was performed using this protocol. In this expanded study, both fiber diameter and compliance were analyzed and used to characterize the scaffolds. It was established that the scaffolds demonstrated inconsistent mean fiber diameter and mean compliance. The current standard electrospinning protocol therefore does not create PLGA scaffolds with statistically reproducible properties.

Future modifications should be made to the electrospinning parameters in order to reduce variability between the scaffolds and future studies should be performed to determine the acceptable range of properties.

Keywords: electrospinning, scaffold, blood vessel mimic, tissue engineering, biomaterial, PLGA, polymer, fiber diameter, compliance, bioreactor, cultivation 


\section{ACKNOWLEDGMENTS}

First and foremost, I would like to thank Dr. Kristen O'Halloran Cardinal. Your constant support, advice, and encouragement motivated me throughout my thesis and overall graduate education. I was so blessed to have such a great advisor to guide me and make my thesis work impactful. This experience has been so challenging, exciting, and rewarding; the determination that I needed to get through this process will stay with me throughout my life. Words cannot express how grateful I am to have met and studied under you, Dr. Cardinal.

I would also like to thank my awesome lab group: Scott, Alex, Jakub, Stephan, Tanner, and the newcomers Kristen, Tristin, and Evan. Thank you all so much for giving me feedback on all of my presentations and giving me advice when needed. You are the nicest group of people I have ever had the pleasure of working with and I am so sad that I will not be a part of the group any longer. Keep up the amazing work and good luck in your future endeavors! I especially want to thank Scott and Tanner. Scott: you went above and beyond to help me whenever I needed it and definitely made this process much easier. I am incredibly grateful for everything that you did! Tanner: thank you for your hard work on improving the compliance apparatus for this thesis. You have done an amazing job!

Additionally, I want to thank my family and friends for your constant love and support throughout this experience. I especially want to give a special thanks to my mom. Thank you for being so supportive and always believing in me. I could not have done this without you. I would also like to thank my boyfriend, Michael, for always being there for me and having genuine interest in my work. Your support means the world to me.

Finally, I would like to thank my committee members, Dr. Savage and Dr. Walsh. I am so grateful to have you be a part of my culminating graduate experience. Thank you for your time and support. 


\section{TABLE OF CONTENTS}

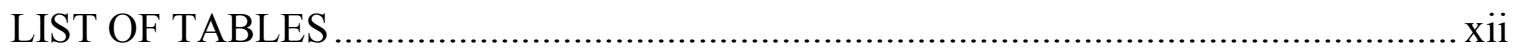

LIST OF FIGURES …..................................................................................

I. INTRODUCTION .......................................................................................... 1

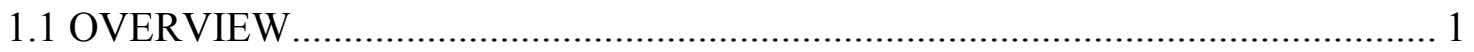

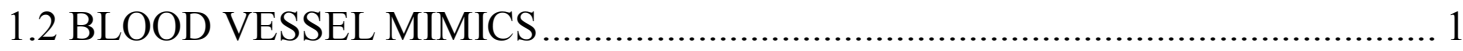

1.2.1 Blood Vessel Structure ............................................................................. 2

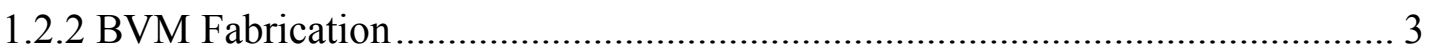

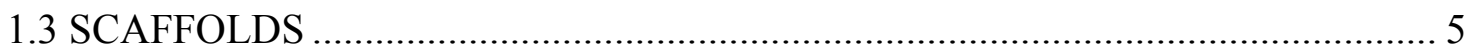

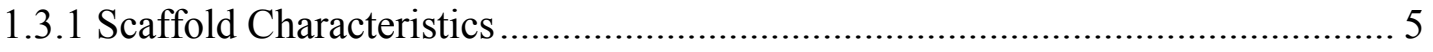

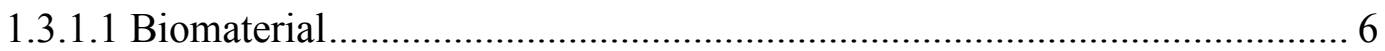

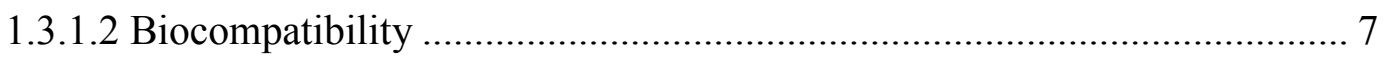

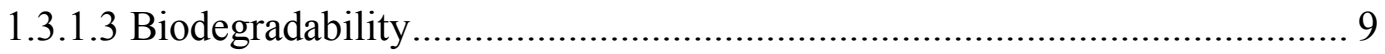

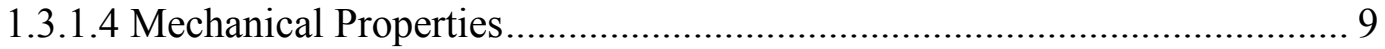

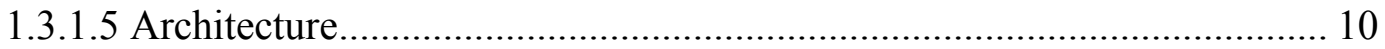

1.3.1.6 Fabrication Method ............................................................................ 10

1.3.1.6.1 Gas Foaming ....................................................................... 11

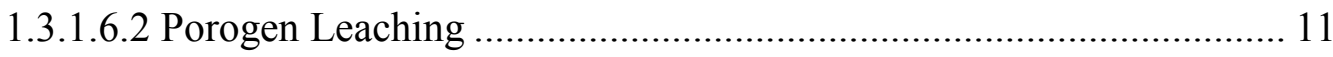

1.3.1.6.3 Thermally Induced Phase Separation......................................... 11

1.3.1.6.4 Three-dimensional Printing ........................................................ 12 
1.3.1.6.5 Selective Laser Sintering .......................................................... 12

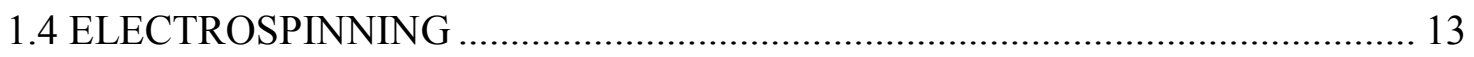

1.4.1 Electrospinning Process ............................................................................... 14

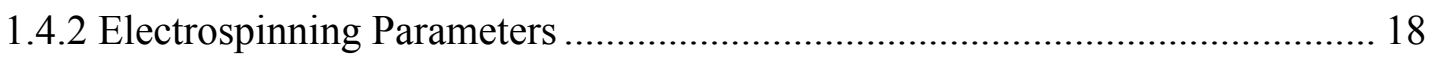

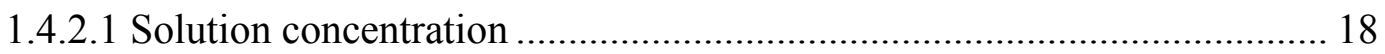

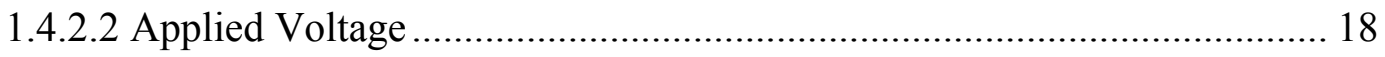

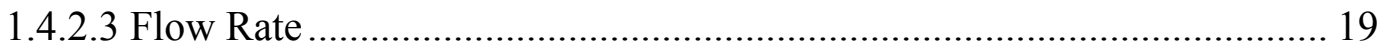

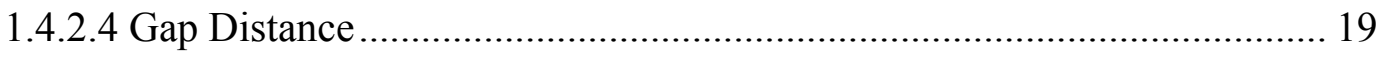

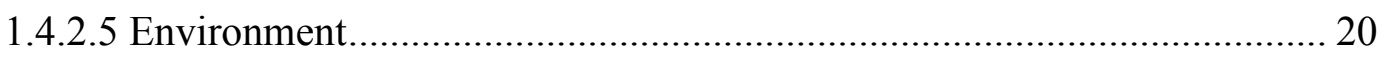

1.5 PREVIOUS ELECTROSPINNING WORK IN THE TISSUE ENGINEERING

$\mathrm{LAB}$

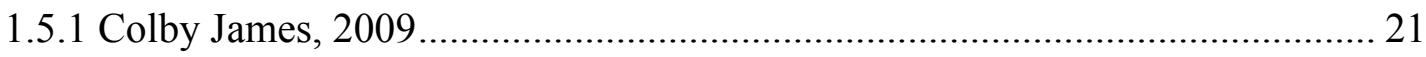

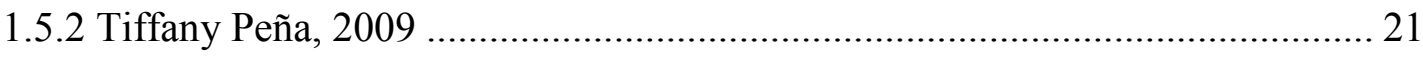

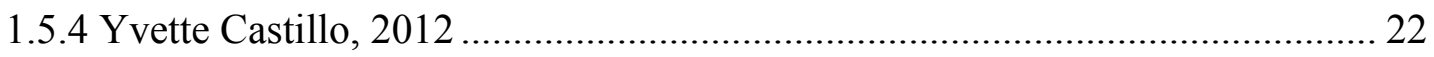

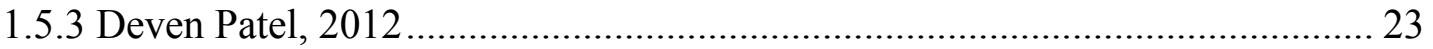

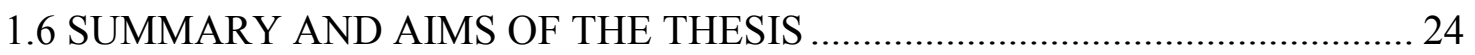

II. PRELIMINARY STUDIES TO SELECT AN ELECTROSPINNING PROTOCOL

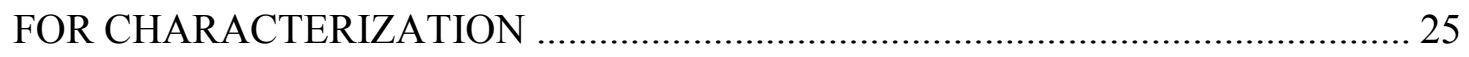

2.1 INTRODUCTION

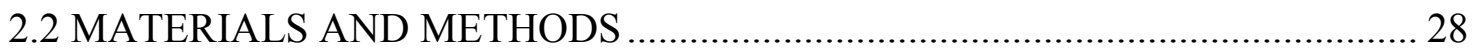




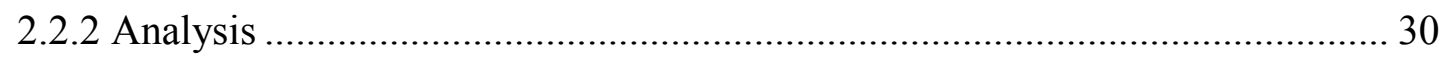

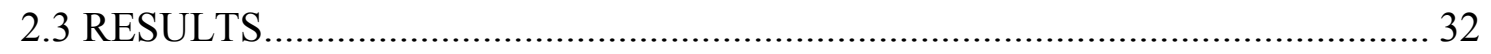

2.3.1 Spin Set 1: 5 Experimental Protocols ......................................................... 32

2.3.2 Spin Set 2: Consistency of Protocol B........................................................ 38

2.3.3 Spin Set 3: Consistency of the Standard Protocol ....................................... 44

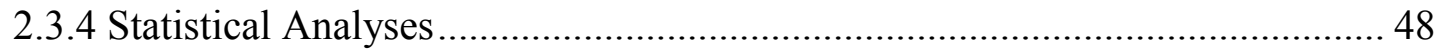

2.3.4.1 REML Variance Components Analysis .......................................... 48

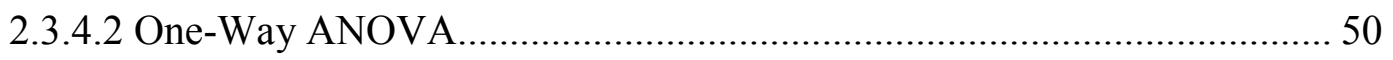

2.3.4.3 Tukey's HSD Post-Hoc Test............................................................ 51

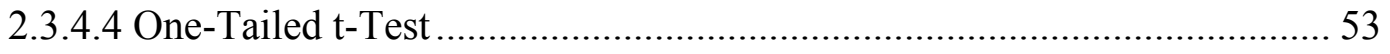

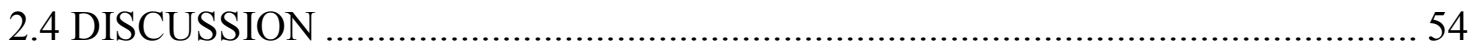

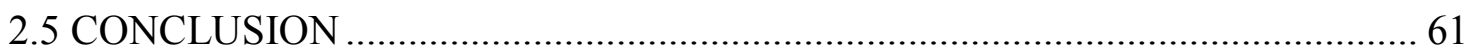

III. REPRODUCIBILITY OF THE PROPERTIES OF ELECTROSPUN PLGA

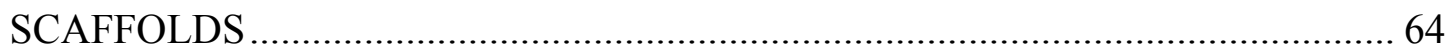

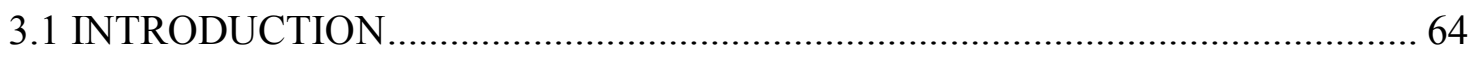

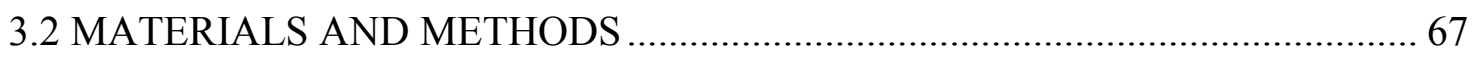

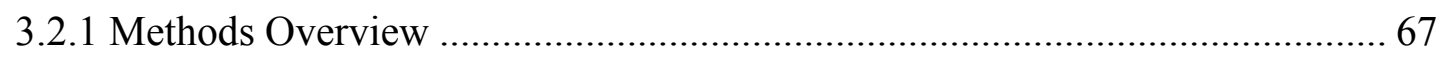

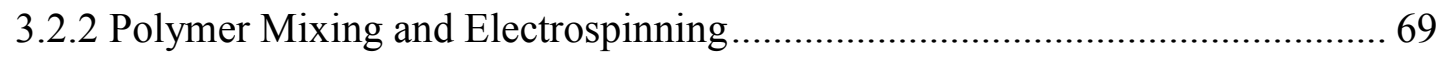

3.2.3 Fiber Diameter Measurement ............................................................ 70 


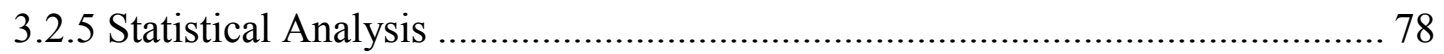

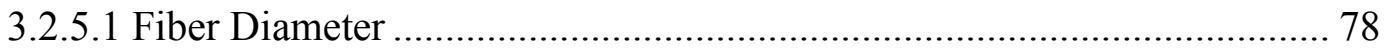

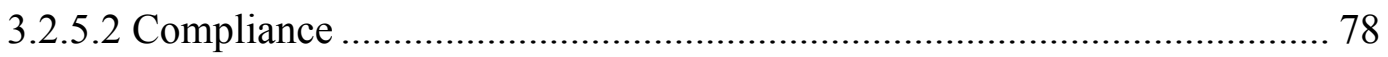

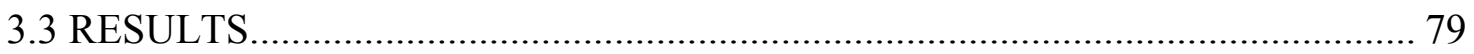

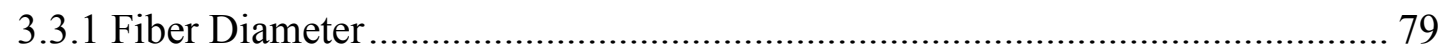

3.3.1.1 Overview of Fiber Diameter Results ............................................... 79

3.3.1.2 SEM, ImageJ, and Summary Statistics .......................................... 80

3.3.1.3 REML Variance Components Analysis ............................................. 82

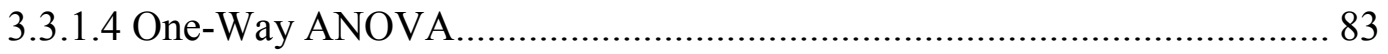

3.3.1.5 Tukey's HSD Post-Hoc Test.............................................................. 84

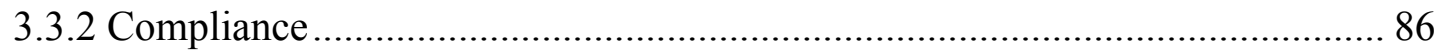

3.3.2.1 Overview of Compliance Results .................................................... 86

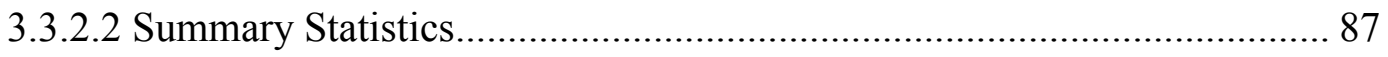

3.3.2.3 REML Variance Components Analysis............................................... 88

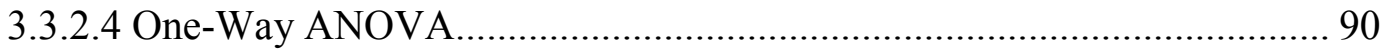

3.3.2.5 Tukey’s HSD Post-Hoc Test........................................................... 92

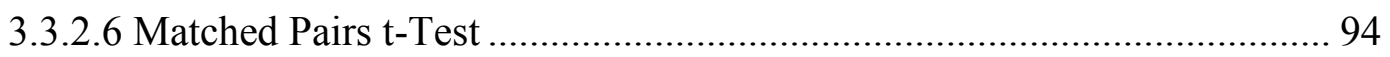

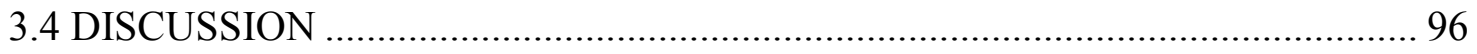

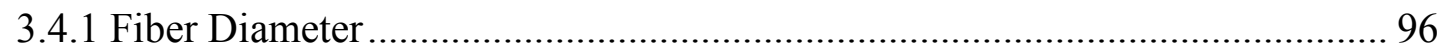




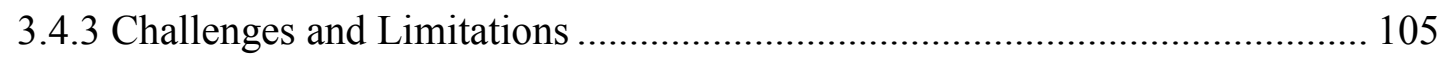

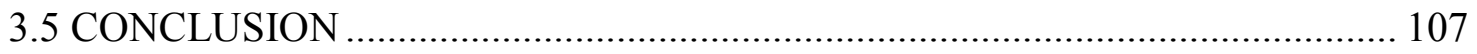

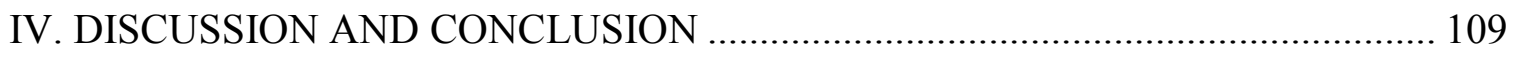

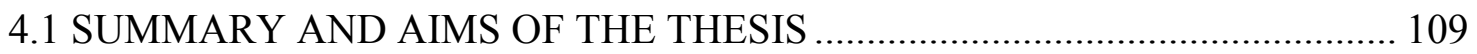

4.2 COMPARISON OF RESULTS TO LITERATURE ………................................ 111

4.3 CHALLENGES AND LIMITATIONS …………......................................... 114

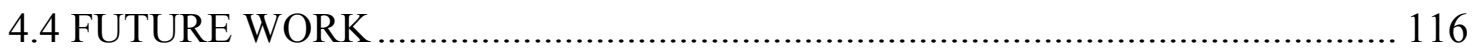

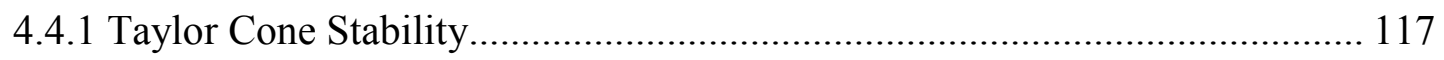

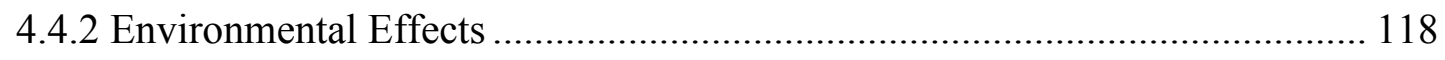

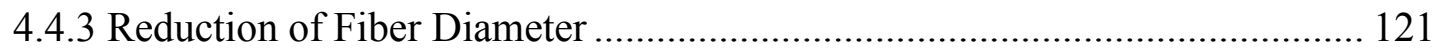

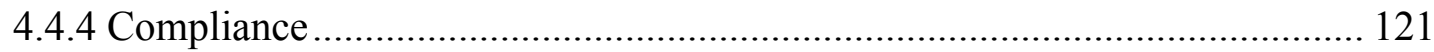

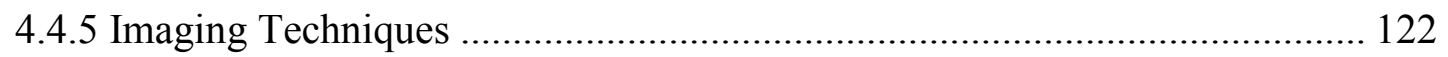

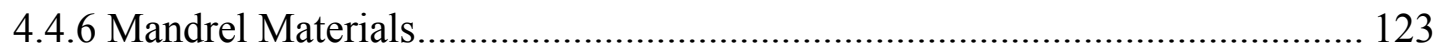

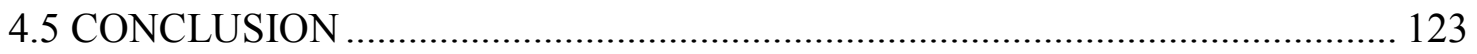

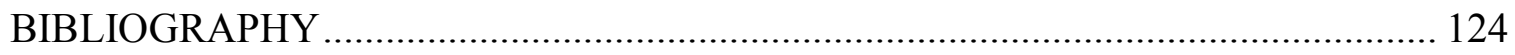

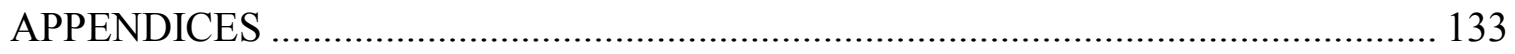

APPENDIX A: POLYMER SOLUTION MIXING PROTOCOL (SOP5310) ........... 133

APPENDIX B: STANDARD ELECTROSPINNING PROTOCOL (SOP5311)....... 134

APPENDIX C: SCANNING ELECTRON MICROSCOPY PROTOCOL................. 137 
APPENDIX D: IMAGE J PROTOCOL FOR FIBER DIAMETER

MEASUREMENT 139

APPENDIX E: STATISTICAL METHODS 146

APPENDIX F: CHAPTER II SEM AND FIBER DIAMETER RESULTS. 156

APPENDIX G: CHAPTER II JMP SOFTWARE STATISTICAL ANALYSIS 189

APPENDIX H: CHAPTER II STATISTICAL ANALYSIS WITH OUTLIERS 192 APPENDIX I: 303 SS MANDREL FABRICATION PROTOCOL 198 APPENDIX J: CHAPTER III PRELIMINARY COMPLIANCE DATA................. 200 APPENDIX K: COMPLIANCE TESTING PROTOCOL 203 APPENDIX L: SCAFFOLD CONDITIONING PROTOCOL 213 APPENDIX M: CHAPTER III FIBER DIAMETER ANALYSIS WITHOUT OUTLIERS 214

APPENDIX N: CHAPTER III SEM AND FIBER DIAMETER RESULTS. 218 APPENDIX O: CHAPTER III FIBER DIAMETER JMP SOFTWARE STATISTICAL ANALYSIS 278

APPENDIX P: CHAPTER III RAW COMPLIANCE DATA 279

APPENDIX Q: CHAPTER III COMPLIANCE JMP SOFTWARE STATISTICAL ANALYSIS 289 


\section{LIST OF TABLES}

Table I: Summary of preliminary spins.

Table II: Experimental protocols in Spin Set 1 with variations of flow rate and

voltage.

Table III: Standard Protocol electrospinning parameters. …………………………...... 29

Table IV: Parameters and results of Protocol A.............................................................. 33

Table V: Parameters and results of Protocol B. ............................................................. 34

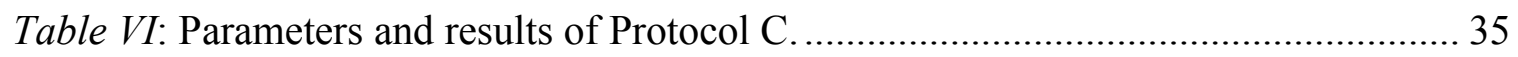

Table VII: Parameters and results of Protocol D. ........................................................... 36

Table VIII: Parameters and results of Protocol E........................................................ 37

Table IX: Mean fiber diameters and standard deviations for Spin Set 1........................ 38

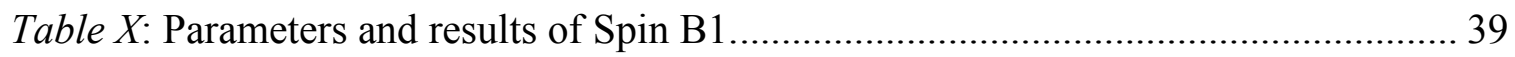

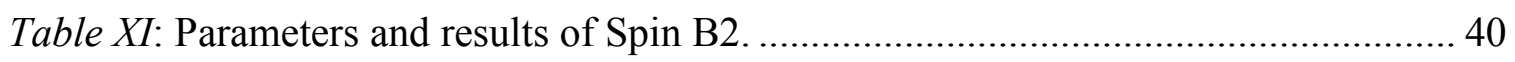

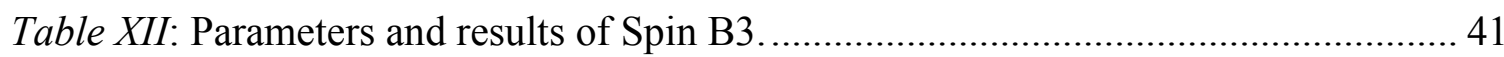

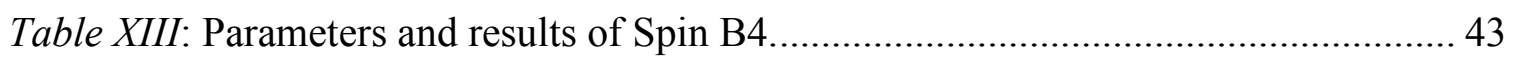

Table XIV: Mean fiber diameters and standard deviations for Spin Set 2...................... 44

Table $X V$ : Parameters and results of Spin X................................................................ 45

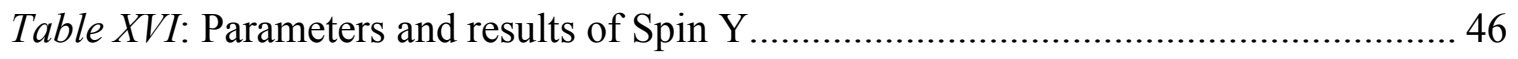

Table XVII: Parameters and results of Spin Z........................................................... 47

Table XVIII: Mean fiber diameters and standard deviations for Spin Set 3.................... 48 
Table XIX: Results of the variance components analyses for Spin Set 2 and Spin Set 3.

Table XX: Summary of the results of Spin Set 2 and Spin Set 3................................. 50

Table XXI: Overall mean fiber diameter and standard deviation for both protocols........ 53

Table XXII: Approaches for electrospinning and testing the 20 scaffolds........................ 69

Table XXIII: Mean fiber diameters and standard deviations of the 20 scaffolds.............. 82

Table XXIV: Results of the REML variance components analysis for random effects. ... 83

Table XXV: Mean compliance and standard deviations before (dry) and after (wet)

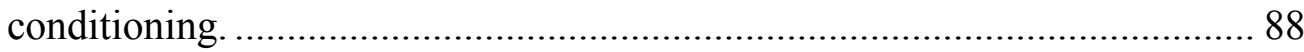

Table XXVI: Results of the REML variance components analyses for random effects.... 89

Table XXVII: Results of the matched pairs t-test for dry and wet compliance................. 95 


\section{LIST OF FIGURES}

Figure 1: Structure of the native blood vessel ........................................................ 3

Figure 2: (A) Perfusion bioreactor system used in the Cal Poly Tissue Engineering lab... 4

Figure 3: Diagram of the electrospinning setup in the Cal Poly lab. ............................. 14

Figure 4: Diagram of the electrospinning process................................................ 16

Figure 5: Onset of bending instabilities during the jet elongation stage of the

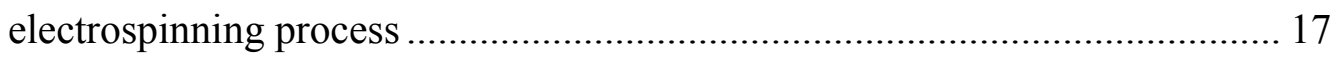

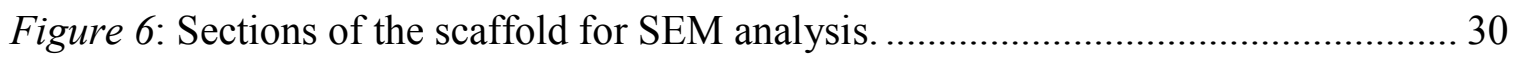

Figure 7: Sample of an SEM image with the 16-circle overlay in ImageJ..................... 31

Figure 8: SEM image of the luminal surface of Protocol A at 500x magnification;

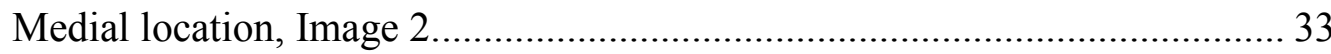

Figure 9: SEM image of the luminal surface of Protocol B at 500x magnification;

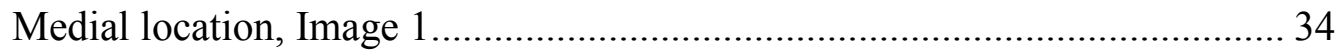

Figure 10: SEM image of the luminal surface of Protocol $\mathrm{C}$ at 500x magnification;

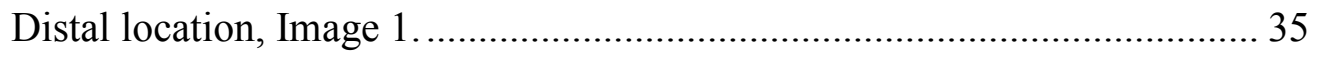

Figure 11: SEM image of the luminal surface of Protocol D at 500x magnification;

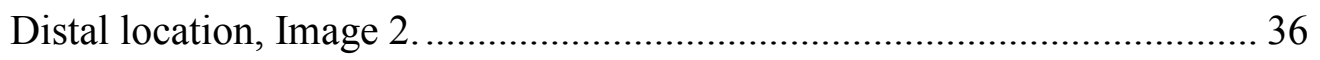

Figure 12: SEM image of the luminal surface of Protocol E at 500x magnification;

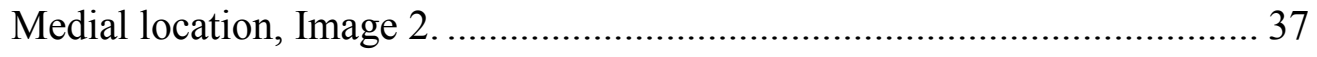

Figure 13: SEM image of the luminal surface of Spin B1 at 500x magnification;

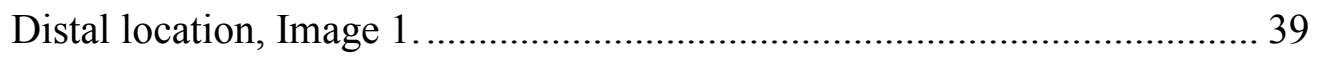

Figure 14: SEM image of the luminal surface of Spin B2 at 500x magnification;

Distal location, Image 2. 40 
Figure 15: SEM image of the middle layer of Spin B3 at 500x magnification.

Figure 16: SEM image of the luminal surface of Spin B4 at 500x magnification;

Distal location, Image 2

Figure 17: SEM image of the luminal surface of Spin $\mathrm{X}$ at 500x magnification;

Distal location, Image 45

Figure 18: SEM image of the luminal surface of Spin $\mathrm{Y}$ at 500x magnification;

Distal location, Image 1 46

Figure 19: SEM image of the luminal surface of Spin $\mathrm{Z}$ at 500x magnification;

Medial location, Image 1. 47

Figure 20: (A) Boxplot from the ANOVA results for Protocol B. 51

Figure 21: (A) Graphical results of Tukey's HSD post-hoc test for Protocol B 52

Figure 22: Boxplot of the t-test that compared the overall mean fiber diameter of

Protocol B and the Standard Protocol 54

Figure 23: (A) SEM image of the luminal surface of Spin B1 (Distal location,

Image 1) at 500x magnification. 58

Figure 24: (A) Diagram of the sectioning scheme for obtaining SEM and compliance samples from the scaffolds 71

Figure 25: SEM image with the overlay of nine circles in ImageJ. 71

Figure 26: (1) Olympus CKX41 Microscope and (2) compliance testing fixture. 72

Figure 27: (A) Dry compliance sample on the occlusion catheter centered over the $4 \mathrm{x}$ microscope objective. 73

Figure 28: (A) Scaffold sutured onto fittings......................................................... 76

Figure 29: (A) Scaffold with the flared ends removed.............................................. 77 
Figure 30: (A) SEM Image 1 for Spin 1 Distal location at 500x magnification; there was no extreme variation in fiber size 81

Figure 31: Boxplot from the ANOVA results for fiber diameter................................... 84

Figure 32: Graphical results of Tukey's HSD post-hoc test for fiber diameter. .............. 85

Figure 33: Connecting Letters Report from Tukey's HSD post-hoc test for fiber diameter. 85

Figure 34: Boxplots of the ANOVA results of the 20 scaffolds for $(A)$ dry compliance and $(B)$ wet compliance.

Figure 35: Graphical results of Tukey's HSD post-hoc test for $(A)$ dry compliance and (B) wet compliance.

Figure 36: Connecting Letters Report from Tukey's HSD post-hoc test for (A) dry compliance and $(B)$ wet compliance.

Figure 37: Matched pairs graph comparing mean dry and wet compliance of each of the 20 scaffolds. 96

Figure 38: SEM Image 2 of Spin 3 Proximal location at 500x magnification. 97

Figure 39: Results of the fixed effect tests in the REML variance components analysis.

Figure 40: Results of the REML variance components analysis showing the variability of the random effects

Figure 41: Current electrospinning setup in a fume hood. 120

Figure 42: Monitor displaying temperature $\left({ }^{\circ} \mathrm{F}\right)$ and humidity $(\%)$. 120 


\section{INTRODUCTION}

\subsection{OVERVIEW}

The focus of this thesis was to characterize the reproducibility of the properties of the electrospun poly(D,L-lactide-co-glycolide) (PLGA) scaffolds that are used to create "blood vessel mimics" (BVMs) in the Cal Poly Tissue Engineering lab. No previous work has been done to characterize these particular scaffolds in their current manufactured form, but characterization is necessary to define the consistency of material and mechanical properties of the scaffolds in order to generate reliable and reproducible constructs for BVM cultivation.

The following sections provide relevant background and research on BVMs, scaffolds, and electrospinning. Each of these topics is important to consider for scaffolds in BVM applications. The detailed electrospinning section discusses multiple parameters that can be manipulated to create the properties of the scaffolds. These details provide a basis for understanding the methods and interpreting the results of this thesis.

\subsection{BLOOD VESSEL MIMICS}

A BVM is an in vitro tissue-engineered blood vessel construct that serves as a preclinical testing environment for intravascular devices ${ }^{1-4}$. In the Cal Poly lab, BVMs are specifically created for testing the cellular response to intravascular stents. Typically, animals are used in preclinical testing of medical devices. However, there are several challenges associated with using animals, including preparation, testing execution, high costs of specific breeds, survivability during experimentation, and variability between 
animal and human results ${ }^{5,6}$. BVMs are a feasible alternative that provide a controlled testing environment with simplified human-cell based constructs ${ }^{1}$.

\subsubsection{Blood Vessel Structure}

BVMs should ideally mimic aspects of the structure of native blood vessels.

Blood vessels have three layers: intima, media, and adventitia (Figure 1). The intima is the blood-contacting surface of the vessel. It has a confluent layer of endothelial cells (ECs) that are connected to a basement membrane. The ECs create an antithrombogenic layer that prevents infection and inflammation of surrounding tissues ${ }^{7}$. It also has a role in gas and nutrient exchange. The media is the middle layer, which is composed of smooth muscle cells (SMCs) and bands of elastic tissues. The SMCs are organized concentrically around the vessel and are responsible for constriction and dilation of the vessel in response to blood pressure by signals from ECs or cytokines ${ }^{7}$. The adventitia, which provides rigidity to the vessel, is composed of an ECM of primarily collagen that contains fibroblasts and perivascular nerve cells. Internal and external elastic lamina, which provide elasticity to the structure, also separate these three layers ${ }^{7}$. 


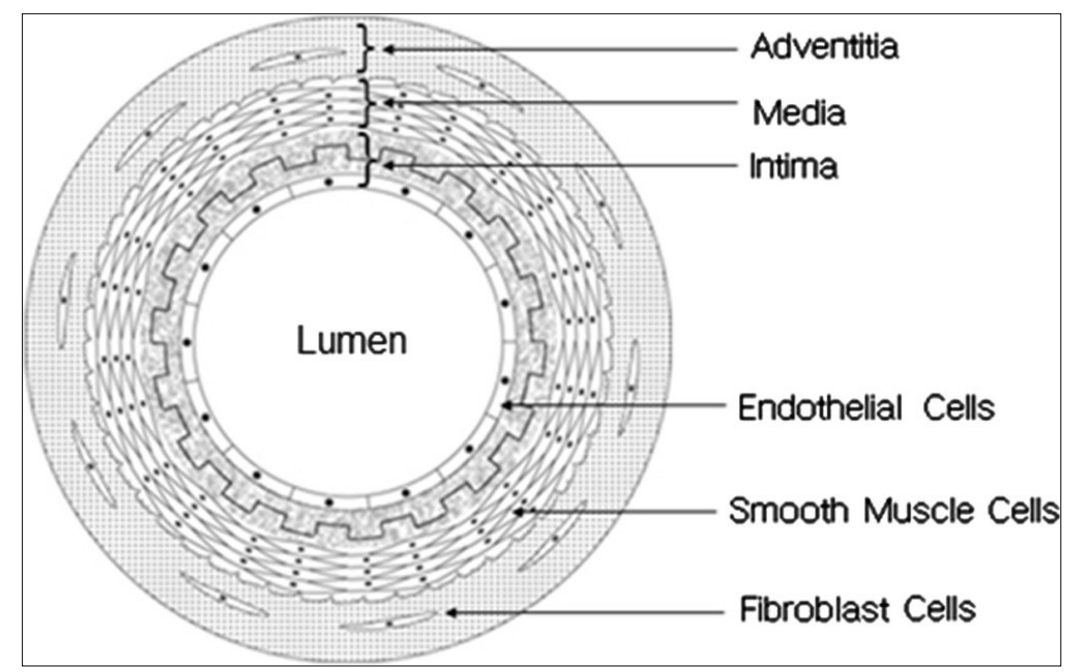

Figure 1: Structure of the native blood vessel. It consists of three layers: the intima, media, and adventitia. Each layer is composed of a cell type; ECs, SMCs, and fibroblasts are found in the intima, media, and adventitia, respectively ${ }^{7}$.

\subsubsection{BVM Fabrication}

When creating a BVM, cells and a scaffold are cultivated together in a perfusion bioreactor to form a cellular lining on the luminal surface ${ }^{1,4}$. In the Cal Poly lab, BVMs are composed of electrospun PLGA scaffolds, human umbilical vein endothelial cells (HUVECs) (Lonza, C2519A), and sometimes human umbilical artery smooth muscle cells (HUASMCs) (Lonza, CC-2579), and are cultivated within a perfusion bioreactor with three components: (1) a chamber, (2) an 8-roller peristaltic pump, and (3) a media reservoir, as shown in Figure 2(A). The cells are pressure sodded onto the lumen of the scaffold within the bioreactor chamber. The pump controls the delivery of the media throughout the BVM while the media reservoir facilitates the exchange of media. The resulting vessel has two layers; the HUVECs form the intima and the HUASMCs form the media, as shown in Figure 2(B). 

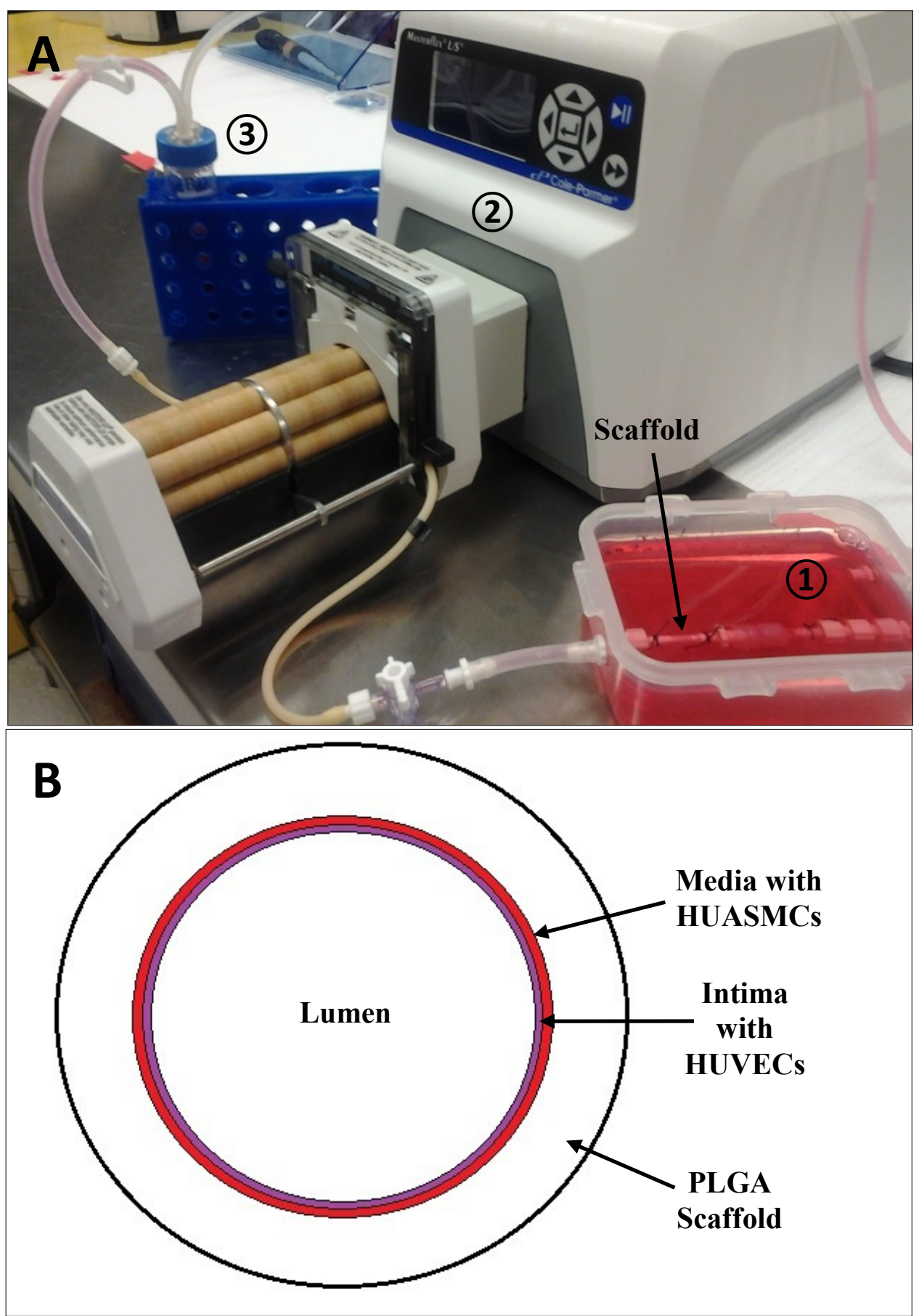

Figure 2: (A) Perfusion bioreactor system used in the Cal Poly Tissue Engineering lab. It consists of (1) a chamber that houses the vessel, (2) an 8-roller peristaltic pump, and (3) a media reservoir. (B) Resultant BVM structure. It has the intima and media layers that are formed with HUVECs and HUASMCs, respectively. 


\subsection{SCAFFOLDS}

As mentioned previously, BVM cultivation involves the combination of cells and a scaffold. Scaffolds are three-dimensional frameworks upon which tissues can be formed. Cells are seeded onto a scaffold to form a construct, which is then cultivated with the appropriate growth factors and mechanical stimuli to form a tissue. Cultivation may be carried out in vitro in bioreactors or in vivo with the natural regenerative processes of the body ${ }^{8}$. Scaffolds have three main functions in the tissue engineering process: (1) provide the overall shape of the construct, (2) facilitate the exchange of molecular and mechanical signals, and (3) support and optimize cellular functions ${ }^{9}$. The Cal Poly lab utilizes PLGA scaffolds to carry out these three functions in BVM cultivation ${ }^{10,11}$. These functions are determined by the characteristics of the scaffolds.

\subsubsection{Scaffold Characteristics}

Scaffolds mimic the role of the natural ECM, which provides structure to tissue, directs cellular functions, and provides sites for cellular adhesion ${ }^{12}$. The native ECM is composed of polysaccharides and structural proteins as well as proteoglycans within the extracellular space. This overall ECM structure facilitates tissue maintenance and remodeling in response to specific stimuli. It also directs the movement of nutrients and bioactive molecules throughout tissues ${ }^{12}$. Typically, scaffolds for tissue engineering are intended to degrade over time ${ }^{9}$. They temporarily provide mechanical support and functional guidance for cells as they attach and secrete their own ECM to eventually replace the scaffold ${ }^{8}$. 
There are several aspects to consider when creating a scaffold, including the biomaterial, biocompatibility, biodegradability, mechanical properties, architecture, and fabrication $\operatorname{method}^{8}$. Varying these parameters can produce different scaffolds for multiple applications. Each aspect is discussed in detail in the following sections.

\subsubsection{Biomaterial}

In tissue engineering, scaffolds may be composed of ceramics such as hydroxyapatite ${ }^{13}$ and tricalcium phosphate ${ }^{14}$; natural polymers such as collagen ${ }^{15}$ and chitosan $^{16}$; or synthetic polymers such as $\mathrm{PLGA}^{17}$ and $\mathrm{PCL}^{18}$. Each class of biomaterials has advantages and disadvantages, which has also led to the use of composite scaffolds. Typically, ceramic scaffolds are used in bone applications while synthetic and natural polymers are used in soft tissue applications ${ }^{8}$. Since BVMs are a soft tissue, this introduction will focus on natural and synthetic polymers that have been used as scaffolds.

Natural polymers that have commonly been used include collagen ${ }^{19}$, proteoglycans $^{20,21}$, alginate-based substrates ${ }^{22}$, and chitosan ${ }^{23,24}$. These materials are advantageous because they are biologically active and promote cell adhesion and proliferation. They are also biodegradable, which allows the host cells to replace the material with natural ECM over time. However, these materials have disadvantages. Not only is it difficult to create and reproduce homogenous structures, but they are also not ideal for applications involving great forces due to poor mechanical properties ${ }^{8,9}$. Additionally, their degradation profiles are difficult to modify ${ }^{9}$. 
Synthetic polymers that have been used include polystyrene ${ }^{25,26}$, poly(L-lactic) acid (PLLA) ${ }^{27}$, polyglycolic acid (PGA) ${ }^{28}$, and PLGA ${ }^{29,30}$. As mentioned earlier, the Cal Poly lab uses PLGA in the fabrication of BVMs, which is a copolymer of polylactic acid (PLA) and PGA. Synthetic polymers are advantageous because they are tailorable during fabrication $^{31}$. Thus, degradation rates and mechanical and biological properties can be controlled by changing the composition of the polymer. A disadvantage of synthetic polymers is reduced bioactivity, which may encumber biological integration ${ }^{8,32}$. Furthermore, some degradation by-products from hydrolysis raise concerns over biocompatibility. For example, PGA forms carbon dioxide as it is degraded, which lowers the local $\mathrm{pH}$ and may lead to cell and tissue necrosis ${ }^{33}$.

\subsubsection{Biocompatibility}

Biocompatibility is the "ability of a material to perform with an appropriate host response in a specific application ${ }^{34}$." In general, four factors are involved in defining biocompatibility: (1) toxicology, (2) extrinsic organisms, (3) mechanical effects, and (4) cell-biomaterial interaction ${ }^{34}$. Toxicology studies determine if leached substances negatively affect cells or tissues or cause adverse systemic effects ${ }^{34}$. Polymeric leached substances include additives during processing, such as unreacted monomers, oligomers, and stabilizers ${ }^{34}$. Extrinsic organisms are bacteria that may contaminate an implant. Serious biological reactions to contaminants are characterized by the presence of white blood cells in the proximity of the implanted material and leads to the formation of dense, fibrous capsules ${ }^{34}$. This response manifests in the patient as pain, redness, and heat. The third factor of biocompatibility, mechanical effects, refers to a mechanical mismatch 
between the material and tissue which may lead to undesirable host responses such as tissue irritation or damage ${ }^{34}$. Finally, cell-biomaterial interaction is the in vivo foreignbody reaction mediated by inflammatory cells. If leachables, extrinsic organisms, and mechanical effects have no part in the reaction, then all in vivo reactions to an implanted material will result in a thin fibrous capsule with mild inflammation ${ }^{34}$. The formation of this capsule isolates the material from the body, which is considered biocompatible. However, this idea of biocompatibility with inert materials is changing; now, materials may be porous instead of encapsulated to allow vascularization of the implant. They may also be bioactive by directing the incidence of specific biological processes in vivo ${ }^{34}$. In tissue engineering applications, the concept of biocompatibility of a scaffold refers to the "ability to perform as a substrate that will support the appropriate cellular activity, including the facilitation of molecular and mechanical signaling systems, in order to optimize tissue regeneration, without eliciting any undesirable local or systemic responses in the eventual host ${ }^{9}$." Scaffolds should not be immunogenic or produce an adverse inflammatory response ${ }^{35}$. Furthermore, biodegradable scaffolds should be sterilizable and their degradation products should not have cytotoxic, inflammatory, or immunogenic effects ${ }^{34}$.

As mentioned previously, some by-products of synthetic polymers have raised concerns. The PLGA used in the BVM scaffolds in the Cal Poly lab is considered biocompatible as it generally elicits a mild foreign-body response; its hydrolysis byproducts are lactic acid and glycolic acid, which cause minimal toxicity because they are used in metabolic pathways of the body ${ }^{9}$. However, this copolymer has shortcomings. First, it is hydrophobic, which limits the cell interactions with the surface ${ }^{9}$. Additionally, 
local inflammation may occur when used in living systems due to the accumulation of large amounts of acidic by-products that form as a result of its bulk degradation behavior $^{36,37}$. Despite these drawbacks, it widely used in tissue engineering ${ }^{9}$.

\subsubsection{Biodegradability}

For implantable tissue-engineered constructs, the scaffold is a temporary structure upon which cells adhere and secrete natural ECM to replace the scaffold. Thus, the scaffold should be biodegradable to a certain degree ${ }^{9}$. The type and rate of degradation depend on the particular application. Slowly degradable or nondegradable scaffolds are desirable for BVMs, since these constructs are not being used in vivo. 75:25 PLGA is currently utilized for its slow degradability ${ }^{9,10,38}$ while ePTFE was used prior to PLGA as a nondegradable scaffold ${ }^{10}$.

\subsubsection{Mechanical Properties}

The initial mechanical stiffness and strength of the scaffold should be an adequate alternate for the mechanical function of the native tissue ${ }^{9}$. In early tissue culture, the scaffold provides the mechanical integrity of the construct as the cells are attaching to and growing on the scaffold. For BVMs, the mechanical properties should be similar to those of the native blood vessel. They must withstand the stretch from blood pressure and the shear stresses from blood flow and also exhibit similar elastic modulus, ultimate stress, strain, compliance, and burst strength to the type of vessel that is being modeled, such as a coronary or peripheral artery ${ }^{7}$. For stent testing purposes in the Cal Poly lab, the 
scaffolds must be able to withstand the deployment of the stent and maintain its overall structure with the stent in place without collapsing or tearing.

\subsubsection{Architecture}

The architecture of a scaffold highly influences its performance. A tissue engineering scaffold should typically be porous with interconnected pores to allow the movement of cells and molecules throughout the material and leave sufficient space for tissue remodeling. The pores should also be large enough to allow the removal of waste and degradation products from the scaffold ${ }^{9}$. Tissue function and cell behavior are both influenced by pore size; tissue function is affected by macroscale pores (above $50 \mu \mathrm{m}$ )

while cell behavior is affected by microscale pores (below $50 \mu \mathrm{m})^{9}$. The scaffolds for the BVMs in the Cal Poly lab are highly porous and interconnected with randomly oriented microscopic fibers to allow the movement and attachment of HUVECs and HUASMCs $^{10,39}$.

\subsubsection{Fabrication Method}

There are several methods available for creating porous, three-dimensional scaffolds, including gas foaming, porogen leaching, thermally induced phase separation, three-dimensional printing, and selective laser sintering. These methods are briefly discussed in the following sections. 


\subsection{Gas Foaming}

The gas foaming technique creates porous scaffolds without the use of a solvent ${ }^{40,41}$. Instead, the polymer is pressurized with a gas, typically carbon dioxide, generating air bubbles throughout the material until saturation ${ }^{9}$. When the pressure is released, the air bubbles nucleate and grow. The resultant scaffold has interconnected pores, but a high degree of interconnectivity is not always achieved. This method may be used in conjunction with other methods, such as porogen leaching, to increase pore interconnectivity $^{42}$.

\subsection{Porogen Leaching}

Porogen leaching involves dispersing particles within a polymeric solution, fixing the structure, and removing the particles to form a porous scaffold ${ }^{9}$. A variety of materials can be used with this method to produce scaffolds ${ }^{43,44}$. The pores generated with this process are only interconnected in local regions of the scaffold. The connectivity of the pores is randomly produced with the process parameters, so the ideal interconnectivity may not be obtained every time for sufficient cellular infiltration of the scaffold $^{9}$

\subsection{Thermally Induced Phase Separation}

Thermally induced phase separation (TIPS) involves the reduction of polymer solubility ${ }^{9}$. It includes two techniques: (1) liquid-liquid phase separation and (2) solidliquid phase separation. Liquid-liquid phase separation in a polymer solution is based on the separation of regions of high and low concentration. The high concentration regions 
solidify upon cooling and the low concentration regions form pores. Solid-liquid phase separation occurs when the polymer is frozen out of solution before liquid-liquid demixing ${ }^{9}$. These techniques can be used to make various scaffold architectures and can be further molded into different shapes and sizes for multiple applications ${ }^{45,46}$.

\subsection{Three-dimensional Printing}

Three-dimensional printing (3DP) has been used to produce scaffolds by inkjet

printing a binder into specific areas of a powder ${ }^{47,48}$. A computer-aided design (CAD) model of the scaffold is used to direct the process. Multiple layers are formed by spreading a thin layer of powder over the surface of a powder bed that sits on a piston. The piston descends after each layer is printed and the layers are bound to form a 3D structure ${ }^{9}$. When the scaffold is completed, the unbound powder is removed from the pores. One disadvantage of this technique is that the material has to be in powder form; most biomaterials are not powders and require special processing 9 .

\subsection{Selective Laser Sintering}

Selective laser sintering (SLS) uses a focused laser beam to sinter specific areas of loosely compacted powder ${ }^{9}$. The powder is evenly spread into a thin layer and then raster-scanned with a laser beam. Areas of the powder that are hit with the beam are fused. Then, more layers of powder are deposited upon the previous layers and rasterscanned. The successive layers are sintered deeply so that each layer is bonded together. The material selection for SLS is limited; it is mainly used for making ceramic scaffolds, 
such as calcium phosphate-based scaffolds, for bone tissue engineering ${ }^{9}$. However, polymeric materials have also been investigated with this fabrication technique ${ }^{49,50}$.

Although all of these techniques have been successful in creating scaffolds for tissue engineering applications, electrospinning was implemented in the Cal Poly lab because it was determined to be the best fabrication method for BVM scaffolding ${ }^{39}$. Electrospinning is inexpensive, can be performed in-house, and can be used with many different materials. The fiber diameter of the scaffolds is also highly controllable and consistent through the process parameters. Furthermore, electrospun scaffolds mimic the structure of the native ECM, prevent EC infiltration into the BVM wall, and support a monolayer of ECs on the luminal surface ${ }^{39}$. Electrospinning is also the focus of this thesis; therefore additional background information and detail on this fabrication method will be provided in the following section.

\subsection{ELECTROSPINNING}

Electrospinning is an advantageous process to use for the production of scaffolds for tissue engineering in general and for BVMs specifically. As discussed earlier, the purpose of a scaffold is to mimic the natural ECM, which is a three-dimensional network composed of protein and polysaccharide fibers in the range of 50 to $500 \mathrm{~nm}^{7}$.

Electrospinning has the potential to form porous scaffolds with randomly oriented or aligned fibers smaller than $100 \mathrm{~nm}$ in diameter, which is an appropriate environment for cell adhesion, proliferation, and differentiation in comparison to the native environment $^{12,51}$. It also allows control over the composition, size, and alignment of the 
fibers through changing the parameters of the process ${ }^{52}$. This is ideal for varying the architecture and the mechanical properties of the scaffolds to suit a range of applications $^{53-55}$.

\subsubsection{Electrospinning Process}

The electrospinning process involves obtaining nano/microfibers from a polymer solution using an electrostatic force. The solution is ejected from a syringe with a pump at a slow rate toward a grounded or oppositely charged collector ${ }^{9}$. The distance between the collector and the needle tip on the syringe is called the gap distance. In the Cal Poly lab, the polymer solution is composed of PLGA and chloroform $\left(\mathrm{CHCl}_{3}\right)$ and the collector is a grounded stainless steel mandrel that translates and rotates to form a tubular scaffold with randomly aligned fibers, as shown in Figure $3^{39}$.

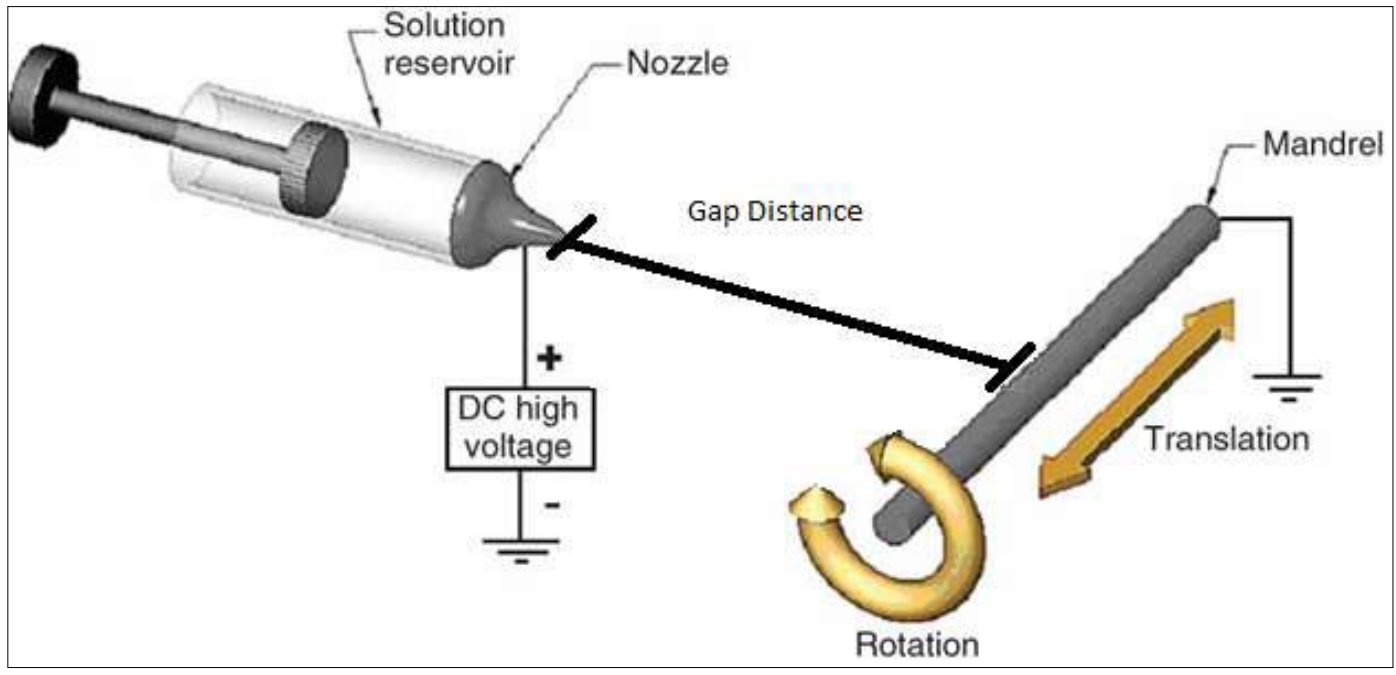

Figure 3: Diagram of the electrospinning setup in the Cal Poly lab. The grounded mandrel translates and rotates and is set at a particular gap distance away from the solution-filled syringe. Modified from J. Matthews 2001 publication $^{11,56}$. 
Electrospinning has three stages: (1) jet initiation, (2) jet elongation, and (3) jet solidification into fibers ${ }^{57}$. During the initiation stage, the solution is pumped from the syringe. Once the initial bead of solution forms at the tip of the syringe needle, a high DC voltage is applied and causes repulsive forces within the solution. The bead then forms a cone structure, called the Taylor cone, at a $49.3^{\circ}$ angle ${ }^{7,57}$. The intensity of the electric field $(V)$ reaches a critical value $\left(V_{c}\right)$, which is given by the following equation $(E q 1)$ :

$$
V_{c}^{2}=\mathbf{4}\left(\frac{H^{2}}{L^{2}}\right)\left(\frac{\ln 2 L}{R}-1.5\right)(0.117 \pi R \gamma)
$$

where $H$ is the air-gap distance $(\mathrm{cm}), L$ is the length of the capillary tube $(\mathrm{cm}), R$ is the radius of the tube $(\mathrm{cm})$, and $\gamma$ is the surface tension of the fluid $(\mathrm{dyn} / \mathrm{cm})^{57}$. Once this critical voltage is reached, the voltage is high enough to overcome the surface forces of the Taylor cone ${ }^{56}$. Consequently, a small liquid jet emerges from the cone toward the collector (Figure 4). 


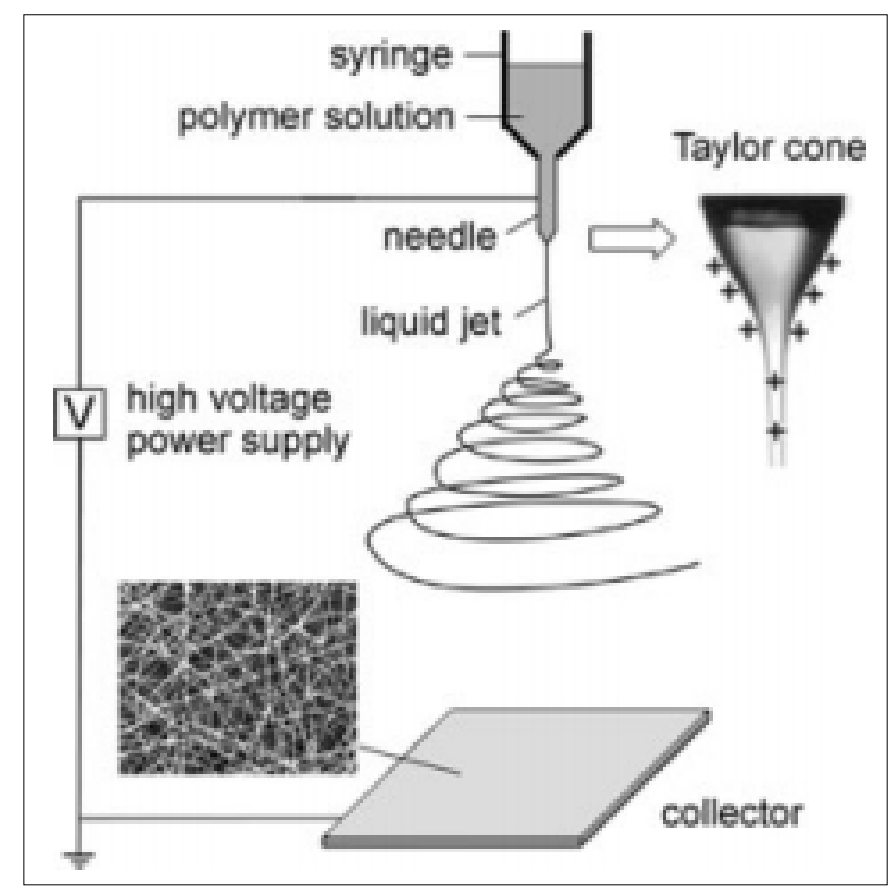

Figure 4: Diagram of the electrospinning process. The high voltage supply charges the polymer solution, forming the Taylor cone. A jet of solution emerges from the cone and travels toward the collector ${ }^{56}$.

In the elongation stage, the jet elongates and thins as it travels toward the collector $^{57}$. As it elongates, the jet experiences several forces with opposing effects which cause instabilities. Three types of instabilities exist: (1) the Rayleigh instability, which is axisymmetric to the centerline of the jet; (2) a second axisymmetric instability; and (3) the "whipping" instability, which is nonaxisymmetric ${ }^{57}$. The Rayleigh instability results from the opposing forces of electrostatic repulsion and surface tension on the surface area of the jet. Eventually, one of the forces overcomes the other due to the viscosity and surface tension of the solution. A high viscosity will cause the jet diameter to decrease until fibers form on the collector while a low viscosity breaks into droplets because it cannot resist the Rayleigh instability ${ }^{57}$. The whipping instability bends the jet and is 
involved in reducing the jet diameter from micrometers to nanometers ${ }^{57,58}$. As the jet elongates and thins, excess charge is redistributed along the jet. Due to the repulsive forces of the excess charge, the jet elongates in the axial direction. When the jet continues to elongate, it bends and forms spiraling loops with increasingly larger diameters, as shown in Figure 5 below ${ }^{59}$.

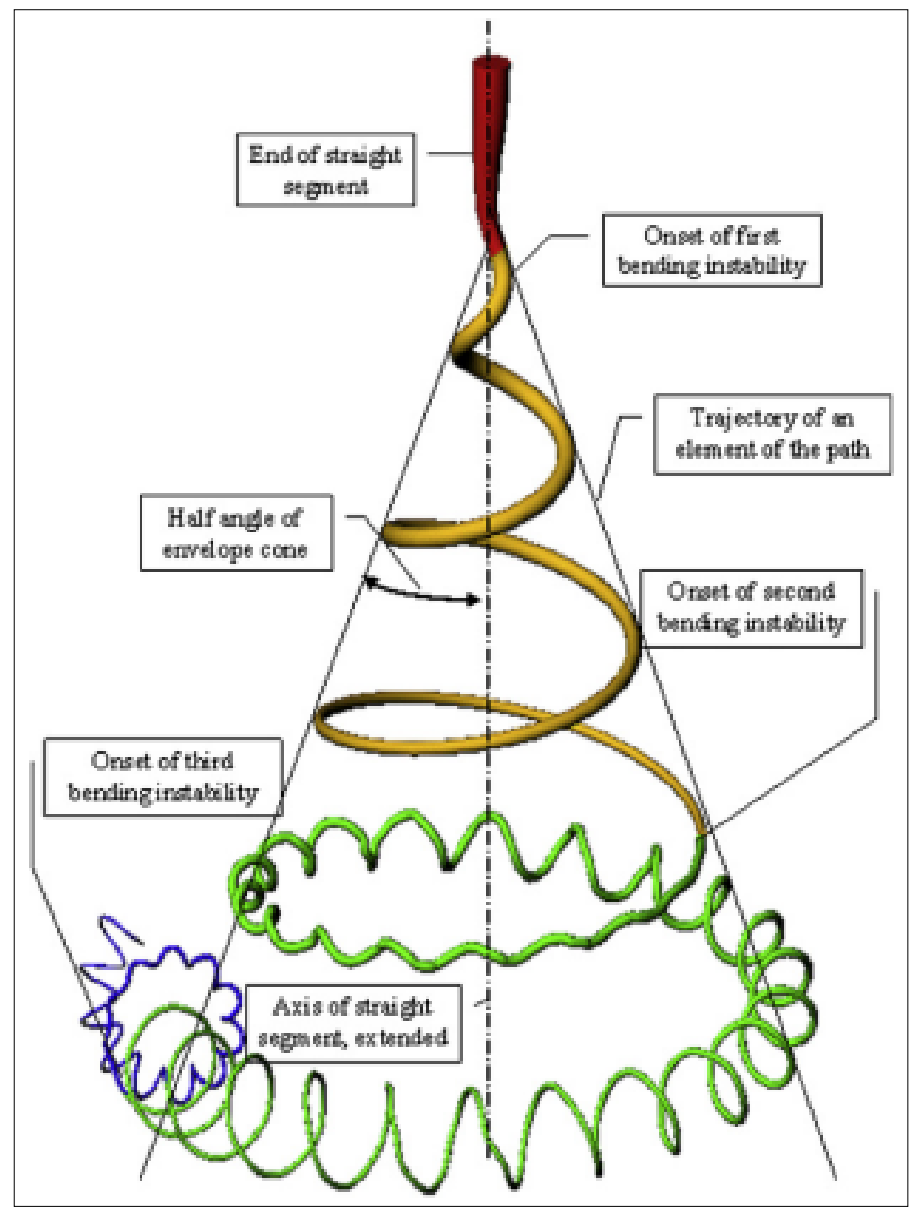

Figure 5: Onset of bending instabilities during the jet elongation stage of the electrospinning process ${ }^{59}$. 
Finally, during the solidification stage, the solvent evaporates as the jet travels toward the collector and a solid fiber is deposited ${ }^{57,59}$. The solidification rate depends on the polymer concentration, electrostatic field, and gap distance ${ }^{57}$.

\subsubsection{Electrospinning Parameters}

Several parameters can be changed to influence the characteristics of electrospun scaffolds. Fiber diameter is a common characteristic to manipulate in the electrospinning process because smaller fibers resemble the nanofibrous biological ECM, as mentioned earlier $^{12}$. Parameters that greatly influence fiber diameter include solution concentration $^{60-62}$, applied voltage ${ }^{61-63}$, flow rate $e^{60,62,63}$, gap distance ${ }^{62,63}$, and environment ${ }^{64,65}$. These are discussed in detail in the following sections.

\subsubsection{Solution concentration}

The solution concentration refers to the amount of polymer in solvent. It affects fiber formation due to the interactions of viscosity and surface tension. Low concentrations result in lower viscosities ${ }^{60}$. As a result, surface tension overcomes the bead of solution and droplets form in the scaffold. Higher concentrations create higher viscosities, which stops fiber formation. It has also been shown that increasing the concentration increases the fiber diameter and results in more uniform fibers ${ }^{60-62,66}$.

\subsubsection{Applied Voltage}

Research has indicated that increasing the voltage changes the shape of the ejection point of the fiber jet, which affects the fiber formation ${ }^{60}$. The shape of the cone is 
highly dependent on the applied voltage due to the interaction between the voltage and surface tension. Additionally, an increase of voltage results in a decrease of fiber diameter with no change in the pore size distribution ${ }^{64}$. It has been postulated that the reduction in fiber diameter can be attributed to an increased acceleration of the polymer jet which caused further charge repulsion and thinning as the jet traveled toward the collector $^{62,63,66}$. Different solutions have been shown to have the opposite effect; increasing the voltage causes an increase in fiber diameter ${ }^{57}$. It is believed that the increase in voltage increases the deposition rate because there is higher mass flow from the solution ${ }^{64}$.

\subsubsection{Flow Rate}

Flow rate and fiber diameter have been shown to be proportional because the increase in flow increases the jet velocity and mass transfer rate ${ }^{64}$. Furthermore, it has been postulated that a larger flow rate causes a larger initial bead to form, which can then be carried with the jet of solution at a faster velocity. As a result, the fibers are not dry before reaching the collecting mandrel. This also contributes to bead formation in the scaffold $^{57,62}$.

\subsubsection{Gap Distance}

The morphology of electrospun fibers is dependent on the gap distance, which is the distance between the syringe nozzle and the collector. This dependence is a consequence of evaporation rate, deposition time, and instability interval. It has been demonstrated that shorter distances result in wet fibers, bead formation, and flat fibers 
instead of round $d^{62,64}$. An increase in gap distance results in a reduction in fiber diameter and gives the solvent more time to evaporate before landing on the collecting mandrel ${ }^{63,66}$.

\subsubsection{Environment}

Humidity and temperature affect fiber morphology. It has been demonstrated with acrylic fibers that at a relative humidity of greater than $60 \%$, fibers do not dry as efficiently and consequently get tangled on the collector ${ }^{64}$. Other studies also demonstrated with certain solvents that higher humidity caused absorption of ambient water. The fibers that were collected had not completely dried, therefore resulting in larger fiber diameters ${ }^{65}$. Temperature has also been shown to affect fiber morphology; evaporation is slower at lower temperatures, which leads to the collection of wet fibers with larger diameters ${ }^{65}$.

Overall, the various parameters described above make electrospinning a versatile process that produces a range of scaffolds for tissue engineering applications. However, this versatility also poses a challenge for optimization. Due to all of the possible parameter combinations that can be made, much research is necessary to find the optimum set of parameters that can produce small, consistent fibers using a particular polymer. 


\subsection{PREVIOUS ELECTROSPINNING WORK IN THE TISSUE ENGINEERING}

\section{LAB}

\subsubsection{Colby James, 2009}

In his thesis work, Colby James implemented the in-house electrospinning apparatus that is currently used in the BVM lab ${ }^{39}$. First, multiple fabrication techniques were surveyed in order to find the most appropriate method to produce scaffolds for BVMs. Electrospinning was determined to be the most suitable technique because the structure of the scaffolds is highly controllable through process parameters and mimics the structure of the natural ECM. After building the electrospinning apparatus, trial experiments were performed with 90:10 Poly(L-Lactic-co-Caprolactone) (PLLACL) to find the optimal protocol that produced scaffolds with continuous fibers. Once this protocol was established, a consistency study was conducted on the scaffolds using fiber diameter, wall thickness, and Young's modulus of multiple spins. The following conclusions were made: the fiber diameter between scaffolds was inconsistent with fiber sizes typically between 6 and $9 \mu \mathrm{m}$, fiber diameter within scaffolds was mostly consistent, wall thickness was inconsistent between and within scaffolds, and mechanical responses were not statistically different between scaffolds.

\subsubsection{Tiffany Peña, 2009}

The ultimate aim of Tiffany Peña's thesis was to select the best polymer to use for the specific BVM application. She chose to use PLGA in the development of electrospun scaffolds for BVMs ${ }^{10}$. 75:25 PLGA was chosen based on research that indicated it was 
advantageous for vascular tissue-engineered scaffolding due to successful EC attachment, similar mechanical properties to native blood vessels, controllable degradation, and good biocompatibility. First, an optimal electrospinning protocol was developed by testing variations of flow rate, applied voltage, and solution concentration. This optimal protocol employed a voltage of $12 \mathrm{kV}$, a flow rate of $5.5 \mathrm{~mL} / \mathrm{hr}$, and a $15 \mathrm{wt} \%$ solution concentration to produced continuous, uniform fibers. The resultant fiber diameters ranged from 5 to $6 \mu \mathrm{m}$ and the scaffolds had tensile strengths that ranged from 3 to 5 $\mathrm{MPa}$, which is similar to native vessels. After establishing these characteristics, the scaffolds were then cultured up to 6 days in vitro with HUVECs. Results from fluorescent microscopy and scanning electron microscopy (SEM) analyses revealed that cells were able to penetrate the porous scaffolds beyond the luminal surface and similar cell coverage was obtained on the lumen in both proximal and distal directions to flow. These findings demonstrated that 75:25 PLGA had the potential to be used for BVM scaffolding.

\subsubsection{Yvette Castillo, 2012}

Yvette Castillo's work involved several attempts to reduce the average fiber diameter of the electrospun PLGA scaffolds due to research indicating that smaller fibers result in more successful cell adhesion ${ }^{11}$. She used a design of experiment (DOE) to determine how the electrospinning parameters interacted with each other and analyze their effect on fiber diameter. Multiple spins were performed with variations of the electrospinning parameters and mean fiber diameters were obtained for each scaffold. The parameters that were tested included solution concentration, gap distance, flow rate, 
and applied voltage. The smallest mean fiber diameter recorded was $2.74 \mu \mathrm{m}$. An equation was obtained from a regression analysis that described how all of these parameters influenced fiber diameter. The model demonstrated that decreasing the flow rate, decreasing the applied voltage, and increasing the gap distance reduced fiber diameter.

\subsubsection{Deven Patel, 2012}

Deven Patel's thesis involved the development of a PLGA scaffold for an in vitro Blood Brain Barrier (BBB) model ${ }^{67}$. His goals included upgrading the existing electrospinning system, developing an electrospinning protocol specific to BBB scaffolds, and analyzing the consistency of the resultant scaffolds by looking at fiber diameter, porosity, wall thickness, and Young's modulus. Although the BBB work is not as relevant to the current thesis, the upgrade to the equipment produced the system that is currently in use. The electrospinning equipment was optimized with a new high voltage supply, updated electrical layout and safety, and a new syringe pump and stand. The new voltage supply was capable of applying a voltage with a negative polarity, which was employed in the new BBB scaffold electrospinning protocol. The optimal protocol for the BBB work consisted of a $15 \mathrm{wt} \%$ solution, a $4.5 \mathrm{~mL} / \mathrm{hr}$ flow rate, an applied voltage of $18 \mathrm{kV}$, and a gap distance of 10 inches. The consistency studies demonstrated that the scaffolds had statistically inconsistent fiber diameter, porosity, and wall thickness, but had a consistent Young's modulus. The average fiber diameter of the scaffolds was 2.556 $\mu \mathrm{m}$, the average porosity was $70.06 \mu \mathrm{m}^{2}$, the wall thickness ranged from 0.31 to 0.54 $\mathrm{mm}$, and the average Young's modulus was 86.141 MPa. 


\subsection{SUMMARY AND AIMS OF THE THESIS}

Electrospinning has been used as an in-house process to create PLGA scaffolds for BVMs in the Cal Poly Tissue Engineering lab. BVMs are tissue-engineered in vitro models used for intravascular device testing. As discussed, scaffolds are an important aspect of a tissue-engineered construct; they provide mechanical support and guide cellular function. It is therefore important for scaffolds to be reproducible to ensure that BVMs serve as reliable models through consistent material and mechanical properties. The overall aim of this thesis was to characterize the reproducibility of the properties of electrospun PLGA scaffolds. Preliminary studies consisted of electrospinning scaffolds using several experimental protocols with varying flow rates and voltages in an attempt to reduce fiber diameter. After identifying the protocol that produced the smallest mean fiber diameter, the reproducibility of that protocol was compared to the reproducibility of the "Standard Protocol" using qualitative and statistical analyses. A large-scale study was then performed with the more reproducible protocol to analyze fiber diameter and compliance of the scaffolds. Compliance was tested before and after conditioning the scaffolds in perfusion bioreactors overnight in order to simulate more physiologic conditions and determine if the conditioning process changed the mechanical response of the scaffolds. The scaffolds were characterized using several statistical analyses that defined the capabilities of the current electrospinning process in terms of variability between scaffolds. This characterization will serve a reference for the effectiveness of future modifications that could be made to improve the electrospinning system in the Cal Poly lab. 


\section{PRELIMINARY STUDIES TO SELECT AN ELECTROSPINNING PROTOCOL FOR CHARACTERIZATION}

\subsection{INTRODUCTION}

In order to accomplish the overall goal of characterizing the reproducibility of electrospun scaffolds, the most suitable protocol had to be chosen. The first step was to design several experimental protocols in an effort to reduce fiber diameter and compare their consistency to the current "Standard Protocol." This was important due to the scaffold variability that results from modifying electrospinning protocols. This chapter had four goals: (1) select an experimental electrospinning protocol, based on several variations, that produced the smallest average fiber diameter; (2) define the scaffold variability using the chosen experimental protocol; (3) compare the result to the scaffold variability of the current Standard Protocol that is utilized in the Cal Poly lab; and (4) choose the more reproducible protocol to use for the remainder of this thesis.

The first objective in this preliminary investigation was to see if it was possible to obtain smaller fiber diameters than those generated by the prior Standard Protocol. Nanofibers are more desirable than microfibers in synthetic scaffolds because most of the tissues in the human body are inherently organized into nanoscale fibers. Thus, nanofiber scaffolds mimic the native ECM more accurately than microfibers ${ }^{68}$. Furthermore, previous studies have shown that smaller fiber diameters elicit ideal cellular responses ${ }^{68-}$

${ }^{70}$. Nanofibers provide cues that promote cell adhesion, differentiation, and production of $\mathrm{ECM}^{70}$. These events are initiated as cells interact with ECM proteins on the surface of the biomaterial through integrin adhesion receptors that control signal transduction ${ }^{71,72}$. This interaction activates signaling pathways that are involved in the regulation of 
cellular behavior, including proliferation and phenotype ${ }^{71}$. Nanofibers also promote the development of smaller focal adhesions, quicker assembly of fibronectin matrices, and more immediate cell proliferation ${ }^{70}$. These actions occur in response to the mechanical properties, similar morphology, and high porosity associated with nanofibrous scaffolds ${ }^{69}$.

Several parameters can be altered to obtain smaller fiber diameters, including flow rate, voltage, solution concentration, and gap distance. Although concentration is a strong predictor of fiber diameter, the optimal concentration of the PLGA solution was previously identified in Tiffany Peña's and Yvette Castillo's theses ${ }^{10,11}$. Yvette Castillo’s thesis also demonstrated that flow rate is the greatest predictor of PLGA fiber diameter ${ }^{11}$. Research suggests that flow rate and fiber diameter are proportional to a point; reducing the flow rate will reduce fiber diameter ${ }^{62}$. The optimal low flow rate can produce small fiber diameters with the narrowest distribution. Flow rates above and below this threshold value create a wider distribution of fiber diameter ${ }^{73}$. Another study using PLGA demonstrated that fiber diameter decreased with slower flow rates, but the distribution was more unpredictable due to the instability of the jet ${ }^{74}$. Thus, although flow rate has the potential to effectively produce smaller fibers, it is an extremely sensitive parameter; the threshold value must be identified in order to ensure homogenous distribution of fiber diameter.

Fiber diameter can also be reduced by increasing the gap distance. This inversely proportional relationship was established in previous work by Cal Poly students and is defined at an optimal distance for the current electrospinning setup ${ }^{10,11}$. Research also 
indicates that increasing the voltage can reduce fiber diameter and uniform fiber diameters are reached at higher voltages ${ }^{64,75}$.

According the DOE previously performed by Yvette Castillo, lowering the flow rate and decreasing the voltage produced smaller fiber diameters, which contradicts literature ${ }^{64,75}$. However, these experiments were performed with the old voltage supply, which had a positive polarity. In Deven Patel's thesis, a new voltage supply was adopted with a negative polarity. It was demonstrated that the use of a negative voltage produced smaller, more uniform fibers with increasing voltage ${ }^{67}$, which also supports the literature ${ }^{62}$. Furthermore, Deven Patel's thesis demonstrated that applied voltage and flow rate were the most important parameters used to control fiber morphology ${ }^{67}$. Another study also demonstrated that voltage and flow rate have a linear relationship ${ }^{76}$. It is therefore postulated that experimenting with the voltage and flow rate would greatly affect fiber diameter. Since decreasing the flow rate and increasing the voltage both reduce fiber diameter, it is believed that a specific balance exists between them to produce smaller fibers within reproducible scaffolds. Variations of both parameters were therefore tested in several experimental protocols using the values from Yvette Castillo's DOE in conjunction with a negative voltage.

Following these experiments, the experimental protocol that produced the smallest average fiber diameter was identified. The reproducibility of the experimental and standard protocols was then compared and the more reproducible protocol was selected for use in the remainder of this thesis. Reproducibility is important to define because scaffolds with consistent material and mechanical properties will result in more 
consistent BVMs. BVMs should be consistent so that they can become a reliable in vitro model for intravascular devices.

\subsection{MATERIALS AND METHODS}

Three sets of preliminary spins were performed to obtain 3-mm inner-diameter PLGA scaffolds (Table I). "Spin Set 1" consisted of five experimental protocols with varying combinations of flow rate and voltage to produce five scaffolds. All of the other

parameters followed the standard electrospinning protocol. The purpose of this first set of scaffolds was to identify the protocol that produced the smallest mean fiber diameter.

Once this protocol was identified, "Spin Set 2" was performed using that same protocol to produce three scaffolds. This second set of scaffolds was used to determine scaffold variability with smaller fiber diameters. "Spin Set 3" was performed to produce three scaffolds using the standard electrospinning protocol. The scaffold variability of the second and third sets of scaffolds was compared to identify the more reproducible process.

Table I: Summary of preliminary spins.

\begin{tabular}{|c|c|c|c|}
\hline $\begin{array}{c}\text { Spin } \\
\text { Set }\end{array}$ & Protocol & $\begin{array}{c}\text { Flow Rate } \\
(\mathbf{m L} / \mathbf{h r})\end{array}$ & $\begin{array}{c}\text { Voltage } \\
\mathbf{( k V )}\end{array}$ \\
\hline \multirow{3}{*}{$\mathbf{1}$} & Experimental A & 5.2 & -15 \\
\cline { 2 - 4 } & Experimental B & 5.2 & -18 \\
\cline { 2 - 4 } & Experimental C & 5.0 & -15 \\
\cline { 2 - 4 } & Experimental D & 5.0 & -18 \\
\cline { 2 - 4 } & Experimental E & 5.0 & -20 \\
\hline \multirow{2}{*}{2} & Experimental B & 5.2 & -18 \\
\cline { 2 - 4 } & Experimental B & 5.2 & -18 \\
\cline { 2 - 4 } & Experimental B & 5.2 & -18 \\
\hline \multirow{3}{*}{3} & Standard & 5.5 & -12 \\
\cline { 2 - 4 } & Standard & 5.5 & -12 \\
\cline { 2 - 4 } & Standard & 5.5 & -12 \\
\hline
\end{tabular}




\subsubsection{Polymer Mixing and Electrospinning}

Each spin consisted of 75:25 PLGA (Sigma-Aldrich, P1941). PLGA was mixed in $\mathrm{CHCl}_{3}$ (Acros Organics, 326820010) to make a $15 \mathrm{wt} \%$ polymer solution using the protocol outlined in Appendix A. The process resulted in a $3 \mathrm{~mL}$ solution, which is specific to $3 \mathrm{~mm}$ inner- diameter scaffolds. After mixing for 24 hours on an orbital shaker table, the solution was used in the standard electrospinning protocol that is outlined in Appendix B. All of the parameters designated in the standard protocol were held constant for each spin, excluding flow rate and voltage. Flow rate and voltage varied within Spin Set 1 and were chosen with consideration to the results from the DOE from Yvette Castillo's thesis. These varying parameters are outlined in Table II. The parameters of the standard electrospinning protocol are shown in Table III.

Table II: Experimental protocols in Spin Set 1 with variations of flow rate and voltage.

\begin{tabular}{|c|c|c|}
\hline Protocol & Flow Rate (mL/hr) & Voltage (kV) \\
\hline A & 5.2 & -15 \\
\hline B & 5.2 & -18 \\
\hline C & 5.0 & -15 \\
\hline D & 5.0 & -18 \\
\hline E & 5.0 & -20 \\
\hline
\end{tabular}

Table III: Standard Protocol electrospinning parameters.

\begin{tabular}{|l|l|}
\hline \multicolumn{1}{|c|}{ Parameter } & \multicolumn{1}{c|}{ Value } \\
\hline Flow Rate & $5.5 \mathrm{~mL} / \mathrm{hr}$ \\
\hline Voltage & $-12 \mathrm{kV}$ \\
\hline Polymer Solution & $15 \mathrm{wt} \%$ PLGA in $\mathrm{CHCl}_{3}$ \\
\hline Needle Size & 18 gauge, beveled, blunt (BD 305180) \\
\hline Gap Distance & 10 inches \\
\hline Translation Distance & $16 \mathrm{~cm}$ \\
\hline Translation Speed & 3 or $55 \mathrm{OPM}$ \\
\hline Rotation Speed & 6 or $3110 \mathrm{RPM}$ \\
\hline
\end{tabular}




\subsubsection{Analysis}

After electrospinning, the scaffolds sat on the steel mandrels in the dessicator for 48 hours. The scaffolds were then removed from the mandrels using a carbon steel razor blade. After allowing the scaffolds to sit in the dessicator for another 24 hours, they were cut into six sections. For each scaffold, three of the six sections were chosen for imaging. The designated sections are shown in the diagram in Figure 6. The sections were then cut longitudinally such that the luminal surface was exposed for viewing. SEM was used to qualitatively analyze fiber morphology. Two random images were taken in each of the three sections using a Hitachi TM-1000 tabletop microscope and the protocol outlined in Appendix $C$. Finally, ImageJ was used to measure fiber diameter using the protocol outlined in Appendix D. Briefly, an overlay of 16 circles was positioned over each SEM image to systematically select fibers (Figure 7). The fiber closest to the center of each circle was measured.

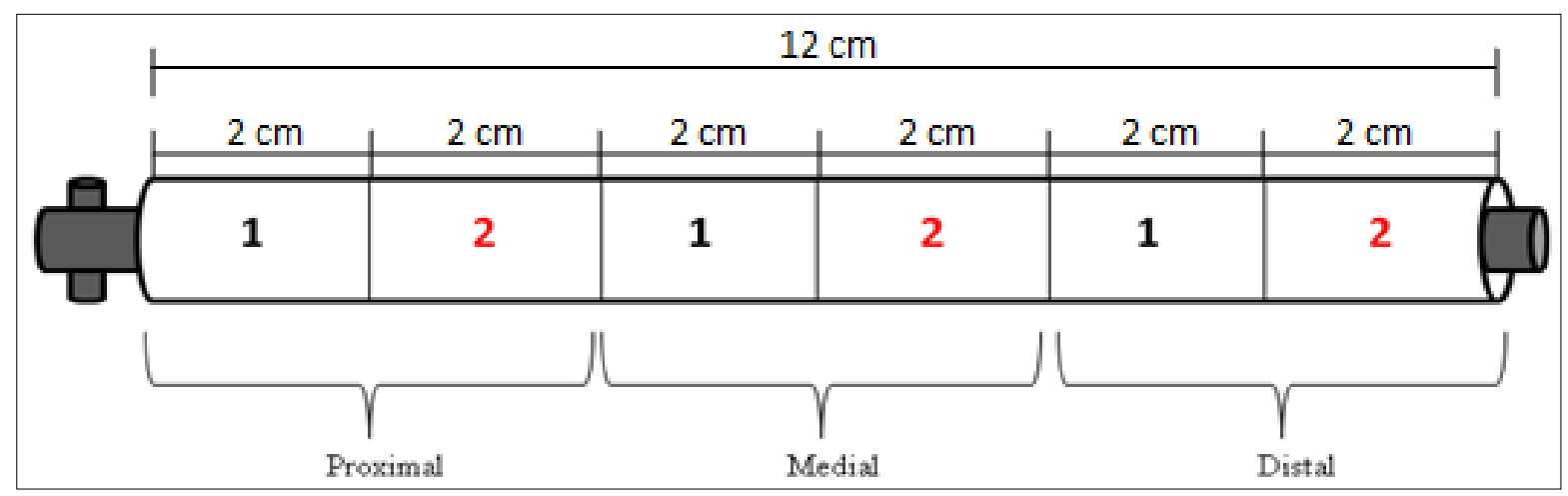

Figure 6: Sections of the scaffold for SEM analysis. 


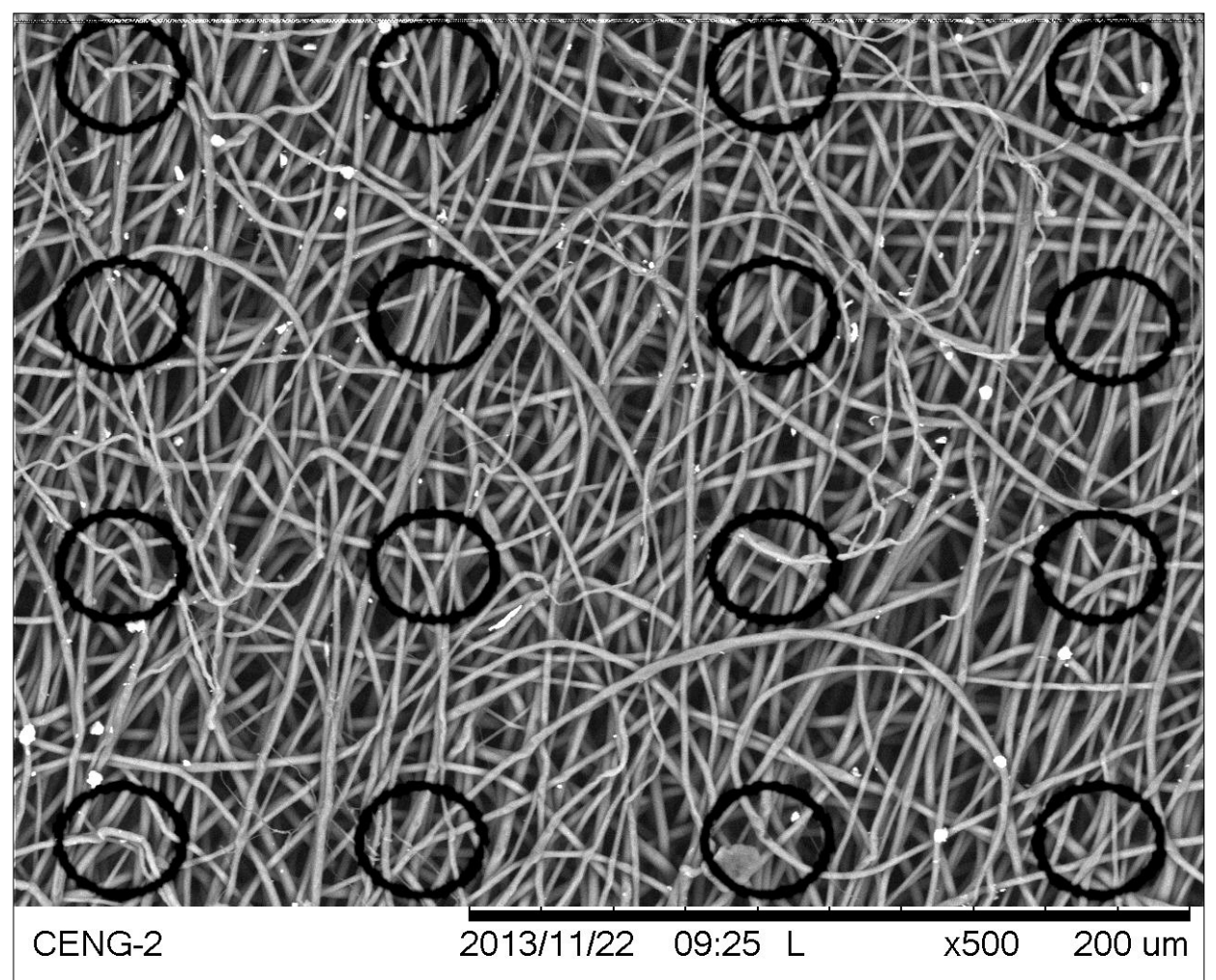

Figure 7: Sample of an SEM image with the 16-circle overlay in ImageJ. The fiber closest to the center of each circle was measured.

Using JMP software, mean fiber diameters and standard deviations were obtained for each scaffold. A Restricted Maximum Likelihood (REML) variance components analysis was performed on the fiber diameter measurements using a mixed effects General Linear Model (GLM) for Spin Set 2 and Spin Set 3 to determine the sources of variability in the scaffolds and compare the reproducibility of the experimental and standard protocols. ANOVA was also performed at a 95\% confidence level to analyze the consistency of the mean fiber diameter across scaffolds followed by a Tukey's HSD posthoc test to identify which scaffolds had statistically different mean fiber diameters. A one-tailed t-test was performed to determine if the overall mean fiber diameter was 
significantly reduced using Protocol B. The detailed procedures for these analyses are outlined in Appendix E.

\subsection{RESULTS}

\subsubsection{Spin Set 1: 5 Experimental Protocols}

The results from Spin Set 1 will be presented in the following manner: a table showing the flow rate, voltage, and the resulting mean fiber diameter (Table IV-Table VIII); observations during the process; one representative SEM image; and a description of the SEM image. Table $I X$ at the end of this section summarizes the results of the mean fiber diameters and standard deviations for the five experimental scaffolds. See Appendix $F$ for the complete set of SEM images and fiber diameter measurements. 
Table IV: Parameters and results of Protocol A.

\begin{tabular}{|l|l|}
\hline Flow Rate & $5.2 \mathrm{~mL} / \mathrm{hr}$ \\
\hline Voltage & $-15 \mathrm{kV}$ \\
\hline Mean Fiber Diameter & $2.77 \mu \mathrm{m}$ \\
\hline
\end{tabular}

Process Observations: The initial bead of solution fell off when the voltage was turned on and was replaced by the Taylor cone. The cone disappeared after a few seconds and did not form again. After closer inspection, a small cone was barely visible within the tip of the syringe. At $1.78 \mathrm{~mL}$ of solution ejected, the cone became visible and formed around the tip of the syringe. It was initially an ideal-shaped cone, but then slowly elongated and fell off. This process of cone formation, elongation, and falling occurred repeatedly for the duration of the process.

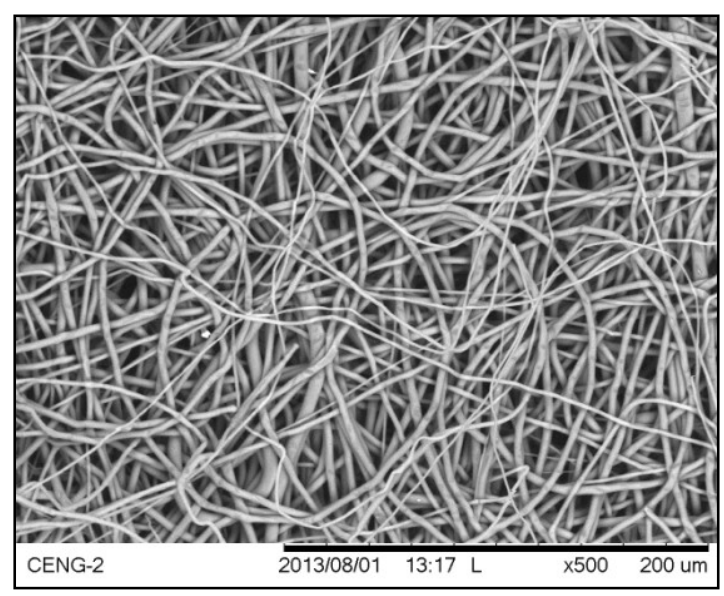

Figure 8: SEM image of the luminal surface of Protocol A at 500x magnification; Medial location, Image 2.

Image Description: The fibers noticeably varied in size. Several larger fibers were present in the layers below the surface while smaller fibers appeared on the surface. 
Table V: Parameters and results of Protocol B.

\begin{tabular}{|l|l|}
\hline Flow Rate & $5.2 \mathrm{~mL} / \mathrm{hr}$ \\
\hline Voltage & $-18 \mathrm{kV}$ \\
\hline Mean Fiber Diameter & $1.98 \mu \mathrm{m}$ \\
\hline
\end{tabular}

Process Observations: After the initial bead fell off of the needle, the Taylor cone was not visible. However, it was present within the tip of the syringe. Several extruding fibers were visible within the tip of the syringe. During the process, one thicker fiber formed and resembled a cobweb. It floated around in the gap between the end of the syringe and the mandrel. A visible, stable cone never formed during the spin.

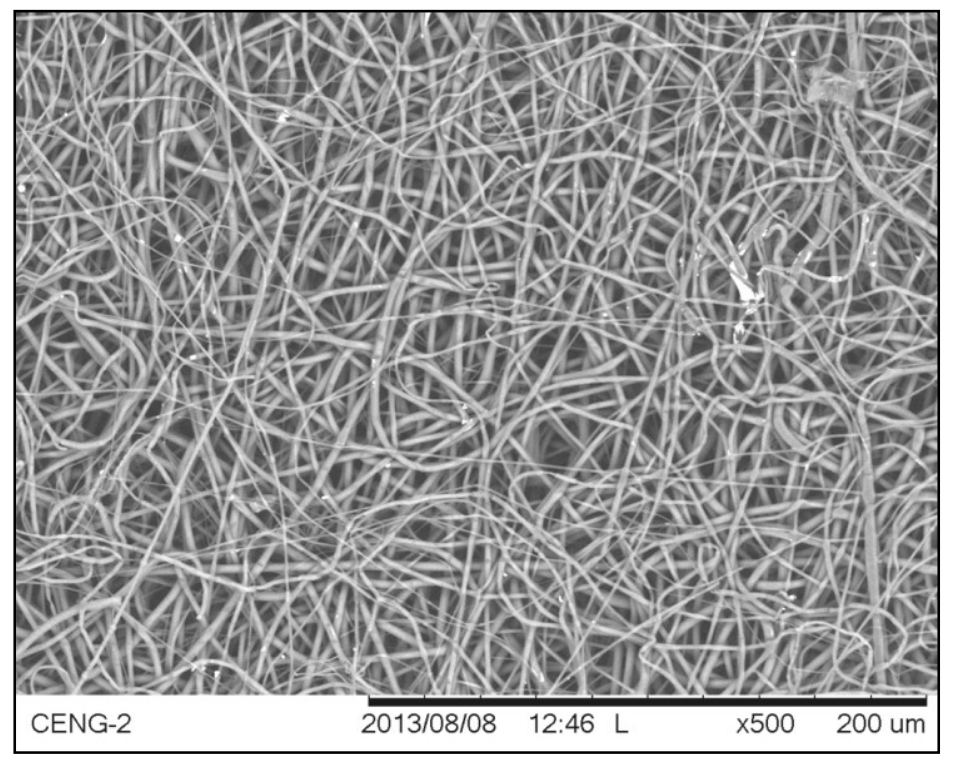

Figure 9: SEM image of the luminal surface of Protocol B at 500x magnification; Medial location, Image 1.

Image Description: Small fibers formed on the surface of this scaffold. There appeared to be less variability in fiber size. No noticeably large fibers were present. 
Table VI: Parameters and results of Protocol C.

\begin{tabular}{|l|l|}
\hline Flow Rate & $5.0 \mathrm{~mL} / \mathrm{hr}$ \\
\hline Voltage & $-15 \mathrm{kV}$ \\
\hline Mean Fiber Diameter & $2.39 \mu \mathrm{m}$ \\
\hline
\end{tabular}

Process Observations: The Taylor cone appeared around the tip of the syringe immediately after the bead fell from the needle tip. However, after a couple of minutes, the cone slowly elongated and fell. This process repeated for the duration of the spin.

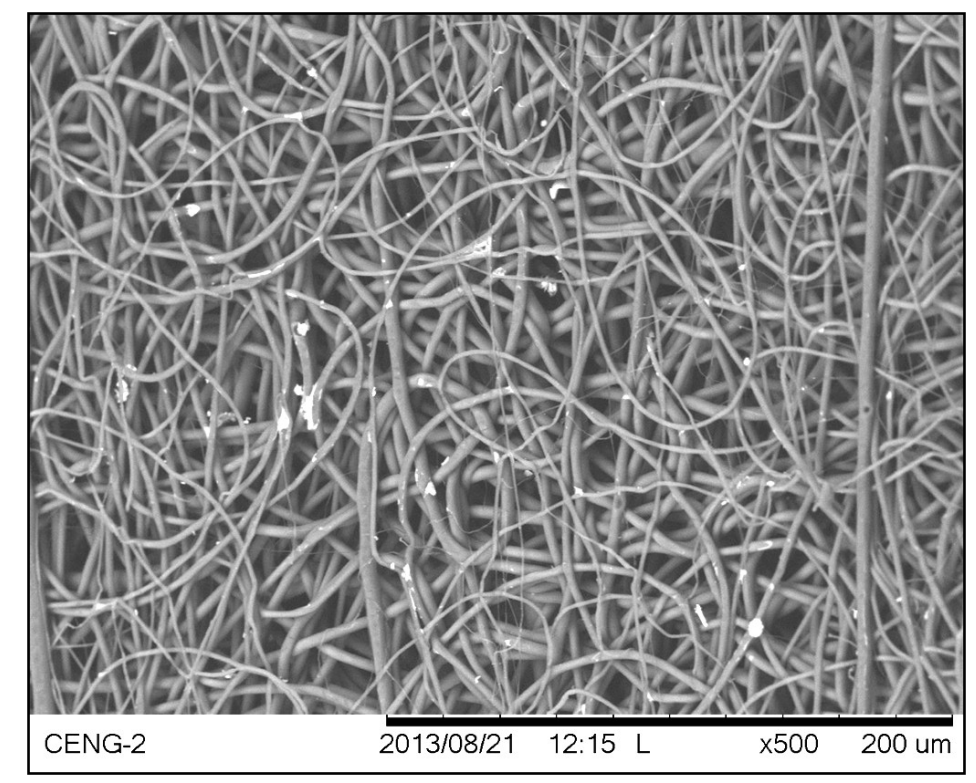

Figure 10: SEM image of the luminal surface of Protocol $\mathrm{C}$ at 500x magnification; Distal location, Image 1.

Image Description: A variety of fiber sizes were visible. The larger fibers appeared under the surface layer. Smaller fibers remained on the surface of the scaffold. Extremely small, thread-like fibers were scattered throughout. 
Table VII: Parameters and results of Protocol D.

\begin{tabular}{|l|l|}
\hline Flow Rate & $5.0 \mathrm{~mL} / \mathrm{hr}$ \\
\hline Voltage & $-18 \mathrm{kV}$ \\
\hline Mean Fiber Diameter & $3.71 \mu \mathrm{m}$ \\
\hline
\end{tabular}

Process Observations: After the initial bead fell, the Taylor cone formed within the tip of the syringe. The cone then slowly elongated and fell off of the syringe, which continuously occurred with successive cones. A cone with an ideal shape appeared at $1.02 \mathrm{~mL}$ of solution ejected, but it eventually elongated and fell. Similar cones proceeded to form, elongate, and fall throughout the spin.

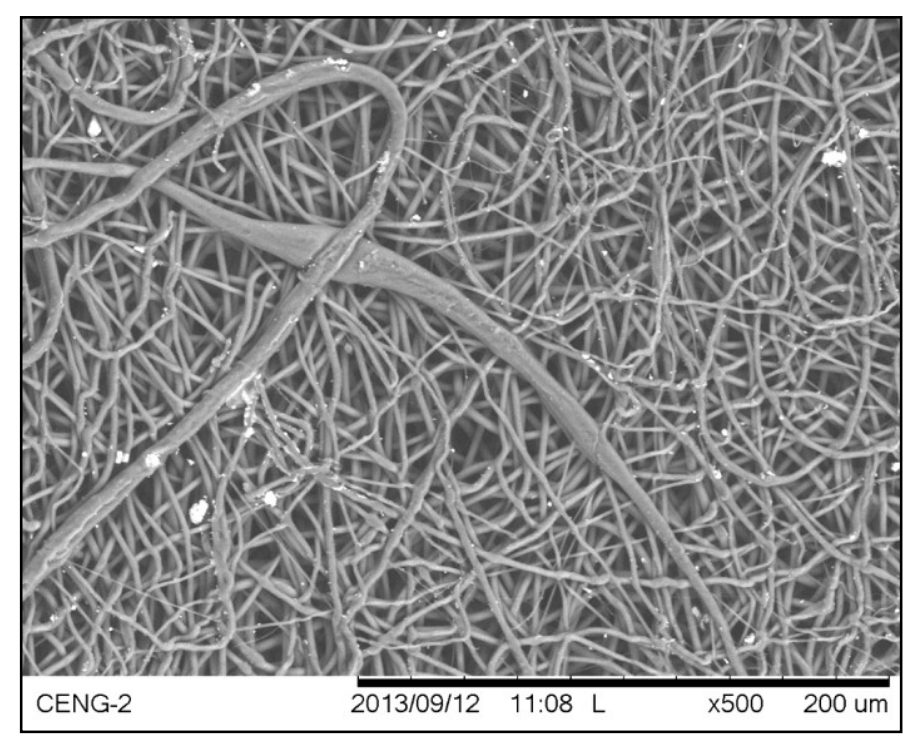

Figure 11: SEM image of the luminal surface of Protocol D at 500x magnification; Distal location, Image 2.

Image Description: Most of the fibers appeared to be small and consistent in diameter with some thread-like fibers scattered along the surface layer. However, three larger fibers were clearly visible and inconsistent with the overall scaffold. 
Table VIII: Parameters and results of Protocol E.

\begin{tabular}{|l|l|}
\hline Flow Rate & $5.0 \mathrm{~mL} / \mathrm{hr}$ \\
\hline Voltage & $-20 \mathrm{kV}$ \\
\hline Mean Fiber Diameter & $2.55 \mu \mathrm{m}$ \\
\hline
\end{tabular}

Process Observations: The initial cone was small, steady, had a well-defined shape, and was visible at the end of the syringe. However, it withdrew further into the syringe during the spin and was barely visible within the tip. The cone seemed to be steady throughout the entire spin; no elongations or falls were observed. Additionally, the humidity was notably low with this spin at $35 \%$. The humidity was in the range of $45 \%$ to $53 \%$ with the other four spins.

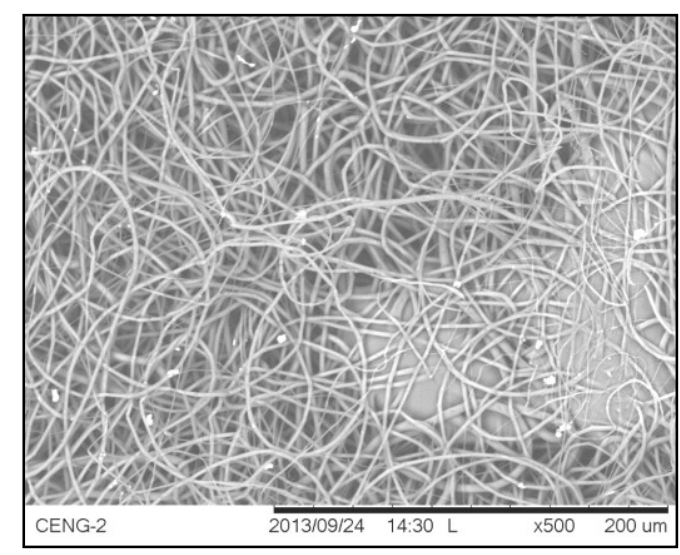

Figure 12: SEM image of the luminal surface of Protocol E at 500x magnification; Medial location, Image 2.

Image Description: There was noticeable variability among fibers in this scaffold. The surface contained small fibers while the bottom layers had increasingly larger fibers. A large clump was also present in the lower right corner underneath the large fibers, which indicated extreme inconsistency. 
Table IX summarizes the results of Spin Set 1 . The mean fiber diameter ranged from $1.98 \mu \mathrm{m}$ to $3.71 \mu \mathrm{m}$ and the standard deviation ranged from $1.11 \mu \mathrm{m}$ to $4.91 \mu \mathrm{m}$. Protocol B produced the smallest average fiber diameter.

Table IX: Mean fiber diameters and standard deviations for Spin Set 1.

\begin{tabular}{|c|c|c|}
\hline Protocol & $\begin{array}{c}\text { Mean Fiber } \\
\text { Diameter }(\boldsymbol{\mu m})\end{array}$ & $\begin{array}{c}\text { Standard } \\
\text { Deviation }(\boldsymbol{\mu m})\end{array}$ \\
\hline A & 2.77 & 1.11 \\
\hline B & 1.98 & 2.15 \\
\hline C & 2.39 & 1.25 \\
\hline D & 3.71 & 4.91 \\
\hline E & 2.55 & 3.03 \\
\hline
\end{tabular}

\subsubsection{Spin Set 2: Consistency of Protocol B}

Table IX above shows that Protocol B resulted in the smallest mean fiber diameter out of the five different protocols. In order to determine scaffold variability, three more were spun with Protocol B. Spin B3 did not have any results because the luminal surface of the scaffold stuck to the mandrel. A fourth spin (Spin B4) was performed to have data to compare from three spins. The results from the second set of spins will be presented in the following manner: a table showing the flow rate, voltage, and resulting mean fiber diameter (Table X-Table XIII); observations during the process; one representative SEM image; and a description of the SEM image. Table XIV at the end of this section summarizes the results of the mean fiber diameters and standard deviations for the three scaffolds. See Appendix $F$ for the complete set of SEM images and fiber diameter measurements. 
Table X: Parameters and results of Spin B1.

\begin{tabular}{|l|l|}
\hline Flow Rate & $5.2 \mathrm{~mL} / \mathrm{hr}$ \\
\hline Voltage & $-18 \mathrm{kV}$ \\
\hline Mean Fiber Diameter & $1.78 \mu \mathrm{m}$ \\
\hline
\end{tabular}

Process Observations: After turning on the voltage, a small cone formed within the tip of the syringe. Three thick fibers branched off of the cone and floated between the syringe and the mandrel. The cone was repeatedly pulled off of the syringe throughout the spin. The humidity was $22 \%$, which is far lower than usual.

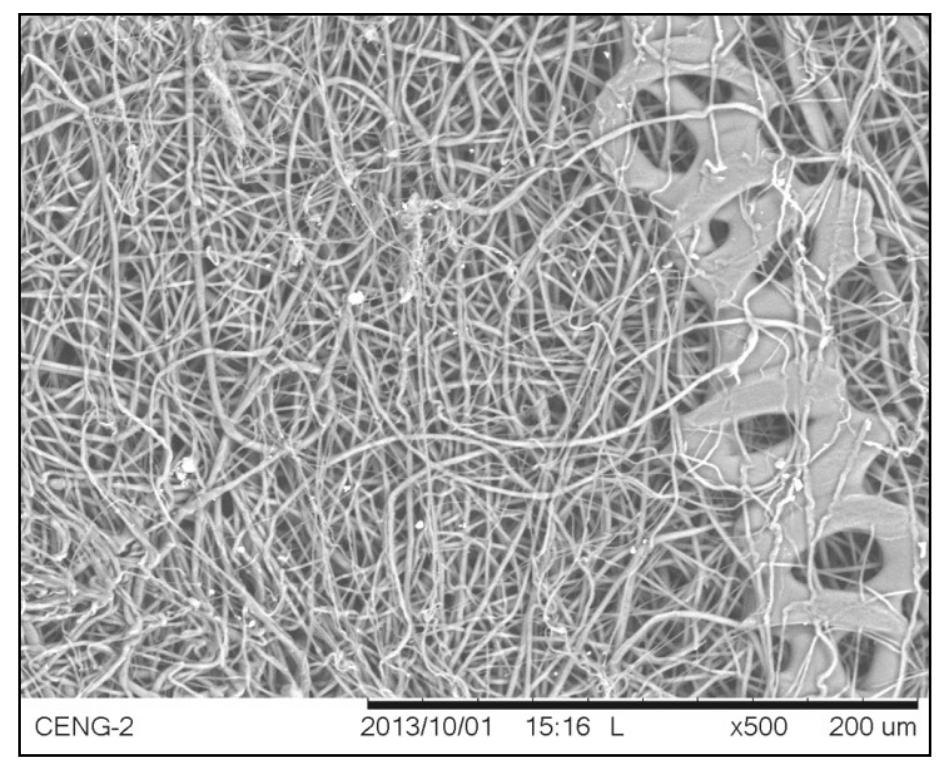

Figure 13: SEM image of the luminal surface of Spin B1 at 500x magnification; Distal location, Image 1.

Image Description: The majority of the scaffold had smaller fibers throughout with clusters of thread-like fibers on the surface. The large clump on the right was evidence of extreme inconsistency. 
Table XI: Parameters and results of Spin B2.

\begin{tabular}{|l|l|}
\hline Flow Rate & $5.2 \mathrm{~mL} / \mathrm{hr}$ \\
\hline Voltage & $-18 \mathrm{kV}$ \\
\hline Mean Fiber Diameter & $1.84 \mu \mathrm{m}$ \\
\hline
\end{tabular}

Process Observations: When the voltage was turned on, the initial bead was replaced by a Taylor cone inside of the needle tip. The cone was extremely small with multiple fibers extruding from it. Thicker fibers floated near the cone as they were pulled toward the mandrel. The same cone was present throughout the entire spin and remained steady. The humidity was also extremely low for this spin at $20 \%$.

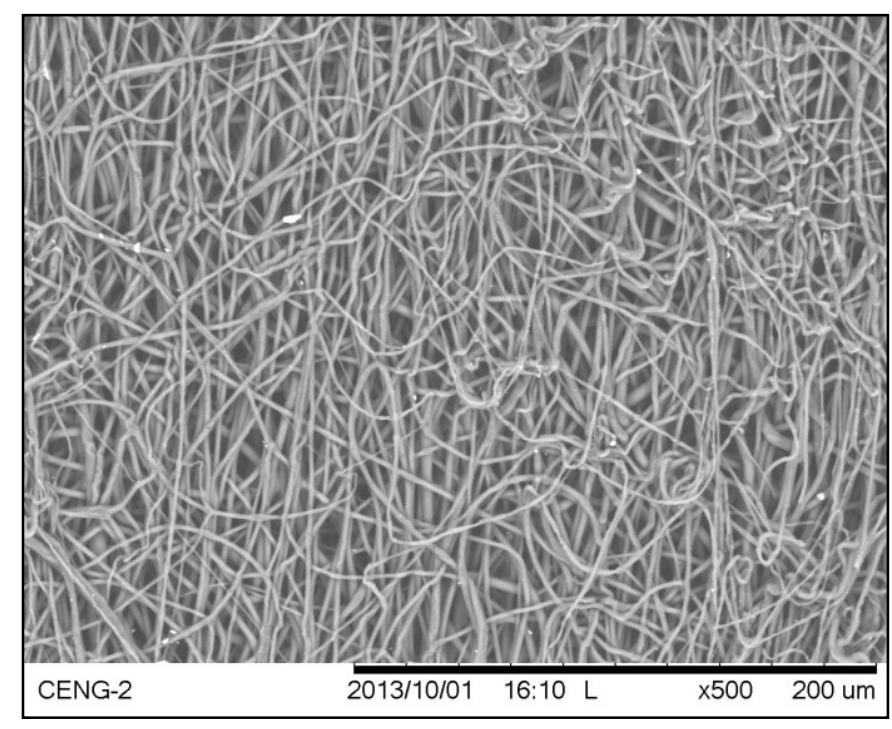

Figure 14: SEM image of the luminal surface of Spin B2 at 500x magnification; Distal location, Image 2.

Image Description: Overall, the fibers appeared to be consistently small. Upon further inspection, there appeared to be slightly larger fibers under the surface layer. However, the variability seemed to be minimal. 
Table XII: Parameters and results of Spin B3.

\begin{tabular}{|l|c|}
\hline Flow Rate & $5.2 \mathrm{~mL} / \mathrm{hr}$ \\
\hline Voltage & $-18 \mathrm{kV}$ \\
\hline Mean Fiber Diameter & - \\
\hline
\end{tabular}

Process Observations: The cone was extremely small and initially formed within the syringe tip. Thick fibers occasionally extruded from the cone in the beginning stages of the process. After this first cone fell, a steady, small cone replaced it with a steady, thin fiber extruding from it. Finally, the cone expanded at $1.91 \mathrm{~mL}$ and was pulled off of the syringe as a clump. This continued for the duration of the spin. Complications arose when taking this scaffold off of the mandrel 48 hours later. The luminal side stuck to the mandrel, which delaminated the scaffold into two layers. Only the middle layer was available for imaging. In order to be consistent, all measurements should be taken from the luminal side of the scaffolds. Consequently, no measurements were made on the images from this spin.

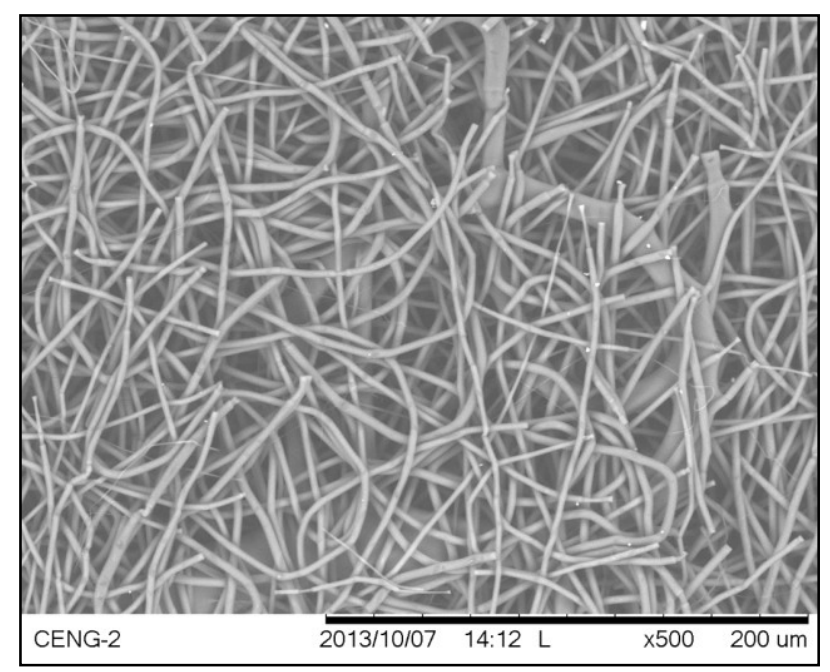

Figure 15: SEM image of the middle layer of Spin B3 at 500x magnification. 
Image Description: Most of the fibers were small and had a consistent size. There is a large, coiled fiber located under the top layer of fibers. The fibers all appeared to be broken, which is a result of the scaffold delaminating into two layers. 
Table XIII: Parameters and results of Spin B4.

\begin{tabular}{|l|l|}
\hline Flow Rate & $5.2 \mathrm{~mL} / \mathrm{hr}$ \\
\hline Voltage & $-18 \mathrm{kV}$ \\
\hline Mean Fiber Diameter & $2.21 \mu \mathrm{m}$ \\
\hline
\end{tabular}

Process Observations: The Taylor cone was small and formed within the tip of the syringe. At $1.87 \mathrm{~mL}$, the cone extended beyond the syringe tip, stretched out, and was pulled toward the mandrel in a clump. A small, steady cone replaced it for a few seconds before elongating and falling off of the needle. The elongating cone branched into two fibers before falling. This process continued for the duration of the spin.

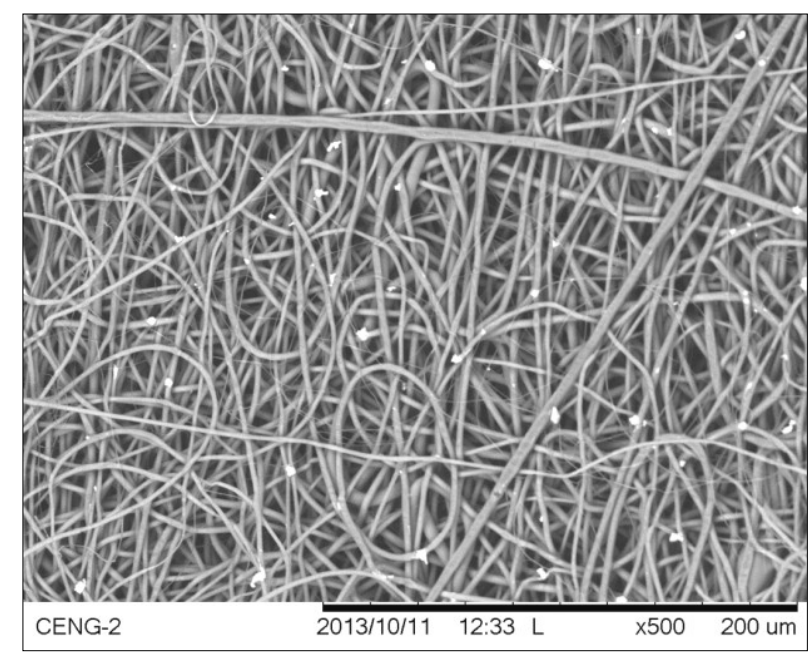

Figure 16: SEM image of the luminal surface of Spin B4 at 500x magnification; Distal location, Image 2.

Image Description: There was slight variability in fiber size. Extremely small fibers appeared on the surface layer, but slightly larger fibers were located throughout. Two large fibers were prominent on the surface. 
Table XIV summarizes the results of Spin Set 2. The mean fiber diameter ranged from $1.78 \mu \mathrm{m}$ to $2.21 \mu \mathrm{m}$ and the standard deviation ranged from $0.59 \mu \mathrm{m}$ to $0.68 \mu \mathrm{m}$.

Table XIV: Mean fiber diameters and standard deviations for Spin Set 2.

\begin{tabular}{|c|c|c|}
\hline Spin & $\begin{array}{c}\text { Mean Fiber } \\
\text { Diameter }(\boldsymbol{\mu m})\end{array}$ & $\begin{array}{c}\text { Standard } \\
\text { Deviation }(\boldsymbol{\mu m})\end{array}$ \\
\hline B1 & 1.78 & 0.68 \\
\hline B2 & 1.84 & 0.59 \\
\hline B3 & - & - \\
\hline B4 & 2.21 & 0.64 \\
\hline
\end{tabular}

\subsubsection{Spin Set 3: Consistency of the Standard Protocol}

Spin Set 3 was performed using the current Standard Protocol used in the $\mathrm{Cal}$ Poly lab. The results from the third set of spins will be presented in the following manner: a table showing the flow rate, voltage, and resulting mean fiber diameter (Table $X V$-Table XVII); observations during the process; one representative SEM image; and a description of the SEM image. Table XVIII at the end of this section summarizes the results of the mean fiber diameters and standard deviations for the three scaffolds. See Appendix $F$ for the complete set of SEM images and fiber diameter measurements. 
Table $X V$ : Parameters and results of Spin X.

\begin{tabular}{|l|l|}
\hline Flow Rate & $5.5 \mathrm{~mL} / \mathrm{hr}$ \\
\hline Voltage & $-12 \mathrm{kV}$ \\
\hline Mean Fiber Diameter & $1.92 \mu \mathrm{m}$ \\
\hline
\end{tabular}

Process Observations: After turning on the voltage, the Taylor cone formed, elongated, and fell off the tip of the needle repeatedly. In the middle of the spin, the cone finally stabilized. However, drops of solution fell from the bottom of the cone continuously as the cone maintained its shape. The syringe was not yet empty when the inputted pump volume of $3.5 \mathrm{~mL}$ was about to be reached; another $0.35 \mathrm{~mL}$ was added on the pump so that all of the solution could be used.

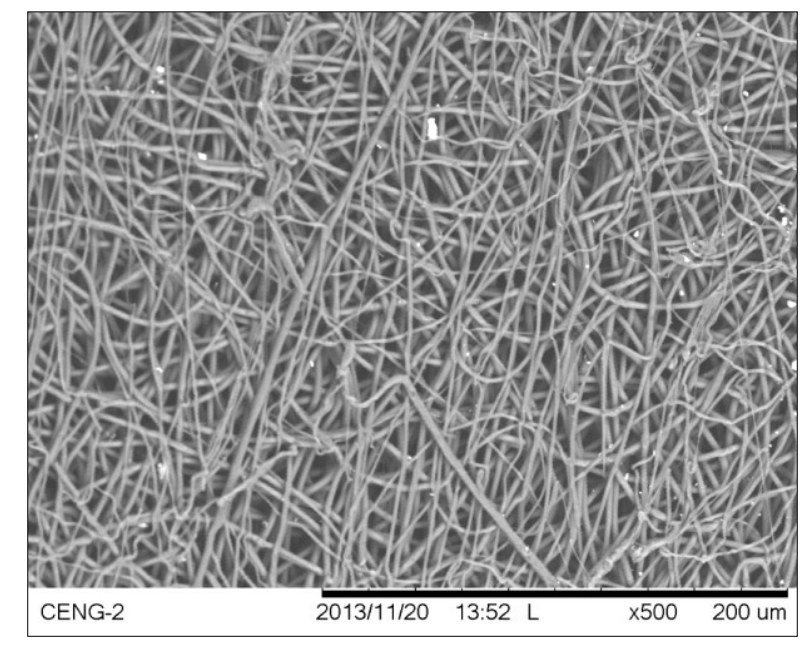

Figure 17: SEM image of the luminal surface of Spin $\mathrm{X}$ at 500x magnification; Distal location, Image 1.

Image Description: Most of the fibers appeared to have similar diameters. There were a few larger and smaller fibers present, but no obvious variations. 
Table XVI: Parameters and results of Spin Y.

\begin{tabular}{|l|l|}
\hline Flow Rate & $5.5 \mathrm{~mL} / \mathrm{hr}$ \\
\hline Voltage & $-12 \mathrm{kV}$ \\
\hline Mean Fiber Diameter & $1.94 \mu \mathrm{m}$ \\
\hline
\end{tabular}

Process Observations: After turning on the voltage, the initial cone was stable with an ideal shape. It slowly started to elongate as the process continued and eventually fell off of the needle tip. It was replaced by another stable cone. This process repeated throughout the spin.

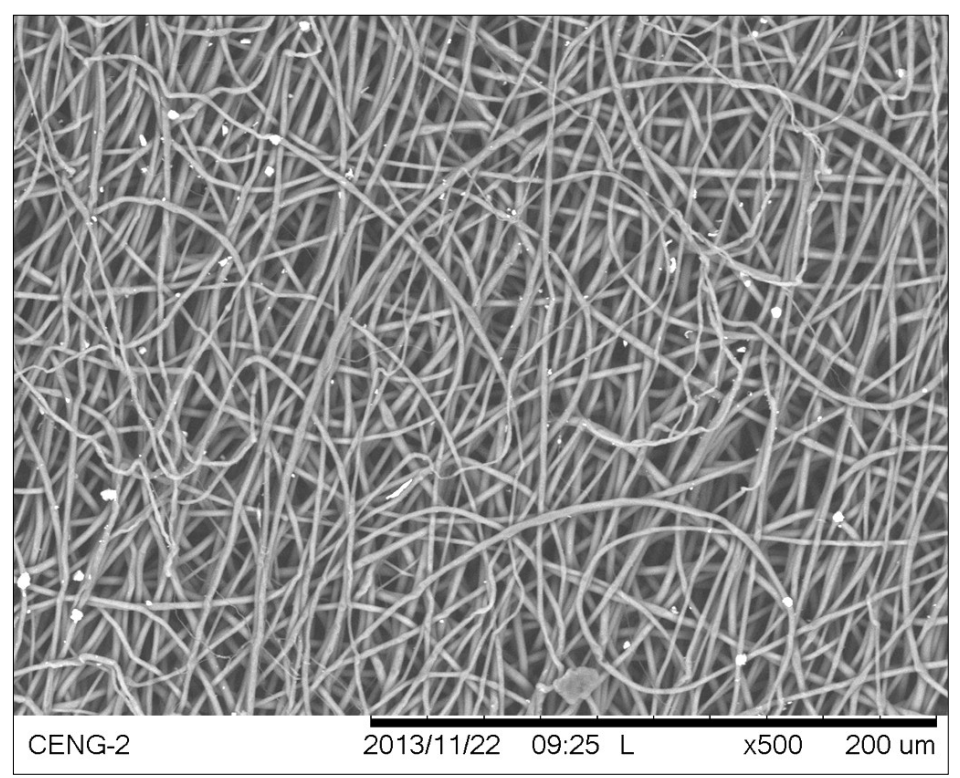

Figure 18: SEM image of the luminal surface of Spin Y at 500x magnification; Distal location, Image 1.

Image Description: The fibers appeared to have slightly variable sizes. The top layer contained a few smaller fibers while the layers underneath contained larger fibers. These variations were not extreme. 
Table XVII: Parameters and results of Spin Z.

\begin{tabular}{|l|l|}
\hline Flow Rate & $5.5 \mathrm{~mL} / \mathrm{hr}$ \\
\hline Voltage & $-12 \mathrm{kV}$ \\
\hline Mean Fiber Diameter & $2.17 \mu \mathrm{m}$ \\
\hline
\end{tabular}

Process Observations: There were small air bubbles in the solution within the syringe. After the voltage was turned on, the initial Taylor cone was small and maintained a stable shape, but solution dripped from the bottom of the cone. The cone also elongated slowly downward and fell off of the needle. It was replaced by another stable cone. This process occurred repeatedly throughout the spin.

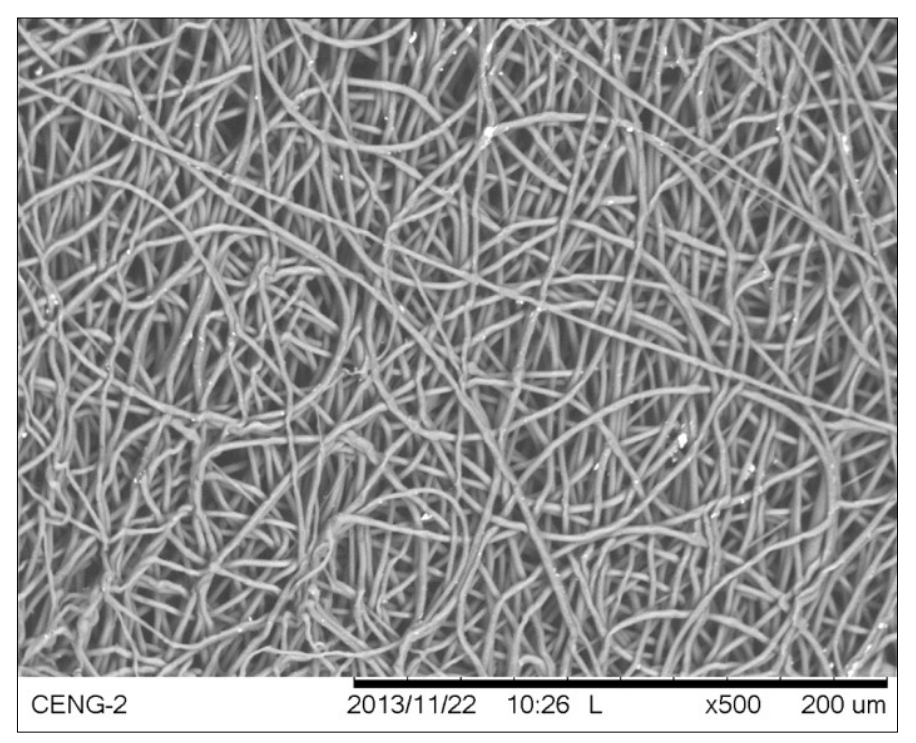

Figure 19: SEM image of the luminal surface of $\operatorname{Spin} Z$ at 500x magnification; Medial location, Image 1.

Image Description: There was not as much variability as in the two previous spins. Most of the fibers appear to have consistent diameters. No obvious variations are visible. 
Table XVIII summarizes the results of Spin Set 3. The mean fiber diameter ranged from $1.92 \mu \mathrm{m}$ to $2.17 \mu \mathrm{m}$ and the standard deviation ranged from $0.36 \mu \mathrm{m}$ to $0.49 \mu \mathrm{m}$.

Table XVIII: Mean fiber diameters and standard deviations for Spin Set 3.

\begin{tabular}{|c|c|c|}
\hline Spin & $\begin{array}{c}\text { Mean Fiber } \\
\text { Diameter }(\boldsymbol{\mu m})\end{array}$ & $\begin{array}{c}\text { Standard } \\
\text { Deviation }(\boldsymbol{\mu m})\end{array}$ \\
\hline $\mathbf{X}$ & 1.92 & 0.49 \\
\hline $\mathbf{Y}$ & 1.94 & 0.49 \\
\hline $\mathbf{Z}$ & 2.17 & 0.36 \\
\hline
\end{tabular}

\subsubsection{Statistical Analyses}

The following four analyses were performed on the data: (1) REML variance components analysis, (2) one-way ANOVA, (3) Tukey's HSD post-hoc test, and (4) onetailed t-test. The first three procedures were performed on Spin Set 2 and Spin Set 3 to analyze the consistency of the scaffolds within the experimental and standard protocols. Additionally, a one-tailed t-test was used to compare the overall mean fiber diameter from both protocols in order to determine if Protocol B yielded a statistically significant reduction in mean fiber diameter. The results of these analyses are presented in the following sections.

\subsubsection{REML Variance Components Analysis}

A REML variance components analysis using a mixed effects GLM was used to analyze the reproducibility of Protocol B and the Standard Protocol. The effects that were used in this model included images and scaffolds, which were random effects, as well as 
location, which was a fixed effect. Images were a random effect because SEM images were taken randomly. Additionally, the scaffolds were assumed to be a random sample of all of the scaffolds that could have been spun using these protocols. Location was a fixed effect because the same proximal, medial, and distal locations were analyzed for each scaffold. The variance components analysis calculated the amount of variability that was attributed to each random effect as a percentage. These percentages are shown in the columns "Between Images" and "Between Scaffolds" in Table XIX below. The remaining percentage was the residual in the column "Within Scaffold," which was the point-topoint variability within the scaffolds. When determining reproducibility, the most pertinent source of variability to analyze is the variability between scaffolds, which was $10.668 \%$ for Protocol B and $7.653 \%$ for the Standard Protocol.

Table XIX: Results of the variance components analyses for Spin Set 2 and Spin Set 3.

\begin{tabular}{|c|c|c|c|}
\hline & \multicolumn{3}{|c|}{ Variability (\%) } \\
\hline Protocol & Within Scaffold & Between Images & Between Scaffolds \\
\hline B & 86.909 & 2.423 & 10.668 \\
\hline Standard & 90.255 & 2.092 & 7.653 \\
\hline
\end{tabular}

Furthermore, the fixed effect test in this model compared the mean fiber diameters between locations on the scaffolds. The p-values for Protocol B and the Standard Protocol were 0.300 and 0.0990 , respectively, which are above the significance level of 0.05 . Thus, there were no significant differences in fiber diameter across locations. See Appendix $G$ for the JMP output of these tests. Table $X X$ summarizes the 
relevant data for Protocol B and the Standard Protocol. It includes the mean fiber diameter range, the standard deviation range, and the scaffold-to-scaffold variability.

Table XX: Summary of the results of Spin Set 2 and Spin Set 3.

\begin{tabular}{|c|c|c|c|}
\hline Protocol & $\begin{array}{c}\text { Mean Fiber Diameter } \\
\text { Range }(\boldsymbol{\mu m})\end{array}$ & $\begin{array}{c}\text { Standard Deviation } \\
\text { Range }(\boldsymbol{\mu m})\end{array}$ & $\begin{array}{c}\text { Variability Between } \\
\text { Scaffolds }(\%)\end{array}$ \\
\hline B & $1.78-2.21$ & $0.59-0.68$ & 10.668 \\
\hline Standard & $1.92-2.17$ & $0.36-0.49$ & 7.653 \\
\hline
\end{tabular}

\subsubsection{One-Way ANOVA}

After using the variance components analysis to determine the sources of variability in both electrospinning protocols, a one-way ANOVA was performed to compare the means of the three scaffolds in each protocol. While the variance components analysis identified different sources of variability and demonstrated that the scaffold-to-scaffold variability was relatively low, ANOVA determined if the low scaffold-to-scaffold variability corresponded to consistent means across scaffolds. Figure 20(A) and (B) shows boxplots from this analysis for Protocol B and the Standard Protocol, respectively. Furthermore, the p-value for Protocol B was $<0.0001$ and the pvalue for the Standard Protocol was 0.0002. These values were below the significance level of 0.05 , so the null hypothesis was rejected; both protocols had at least one scaffold with a mean fiber diameter that was significantly different than the others. The difference in means was more significant for Protocol B. See Appendix $G$ for the JMP output of this test. 


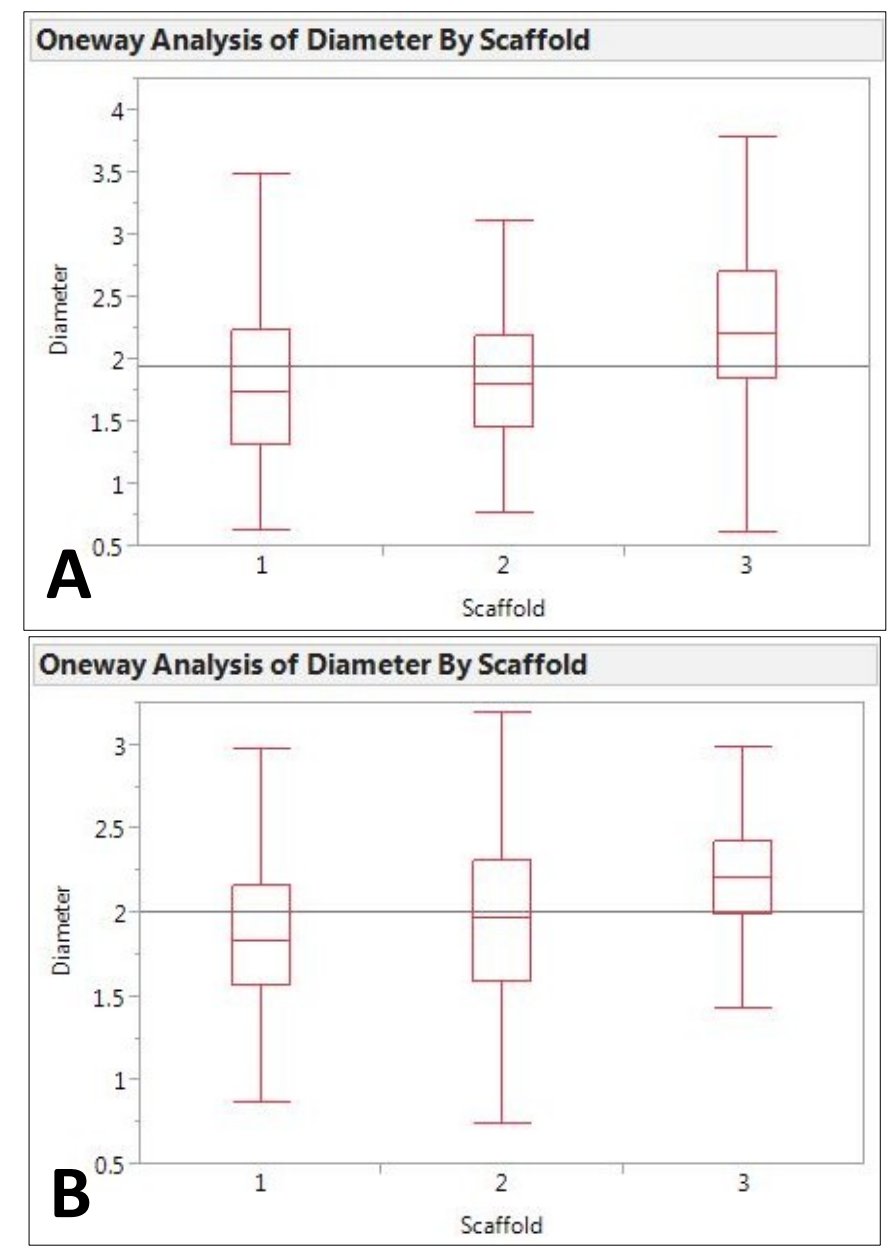

Figure 20: (A) Boxplot from the ANOVA results for Protocol B. (B) Boxplot from the ANOVA results for the Standard Protocol.

\subsubsection{Tukey's HSD Post-Hoc Test}

After running ANOVA and determining statistical significance in both protocols, Tukey's HSD post-hoc test was performed to find which scaffold had the significantly different mean fiber diameter. Figure $21(A)$ and $(B)$ shows the graphical results of this analysis for Protocol B and the Standard Protocol, respectively. The circles on the right side of the boxplots represent the comparisons that were made in the post-hoc analysis. In 
both protocols, two of the groups overlapped while the third group was clearly separated, indicating a significant difference in means.

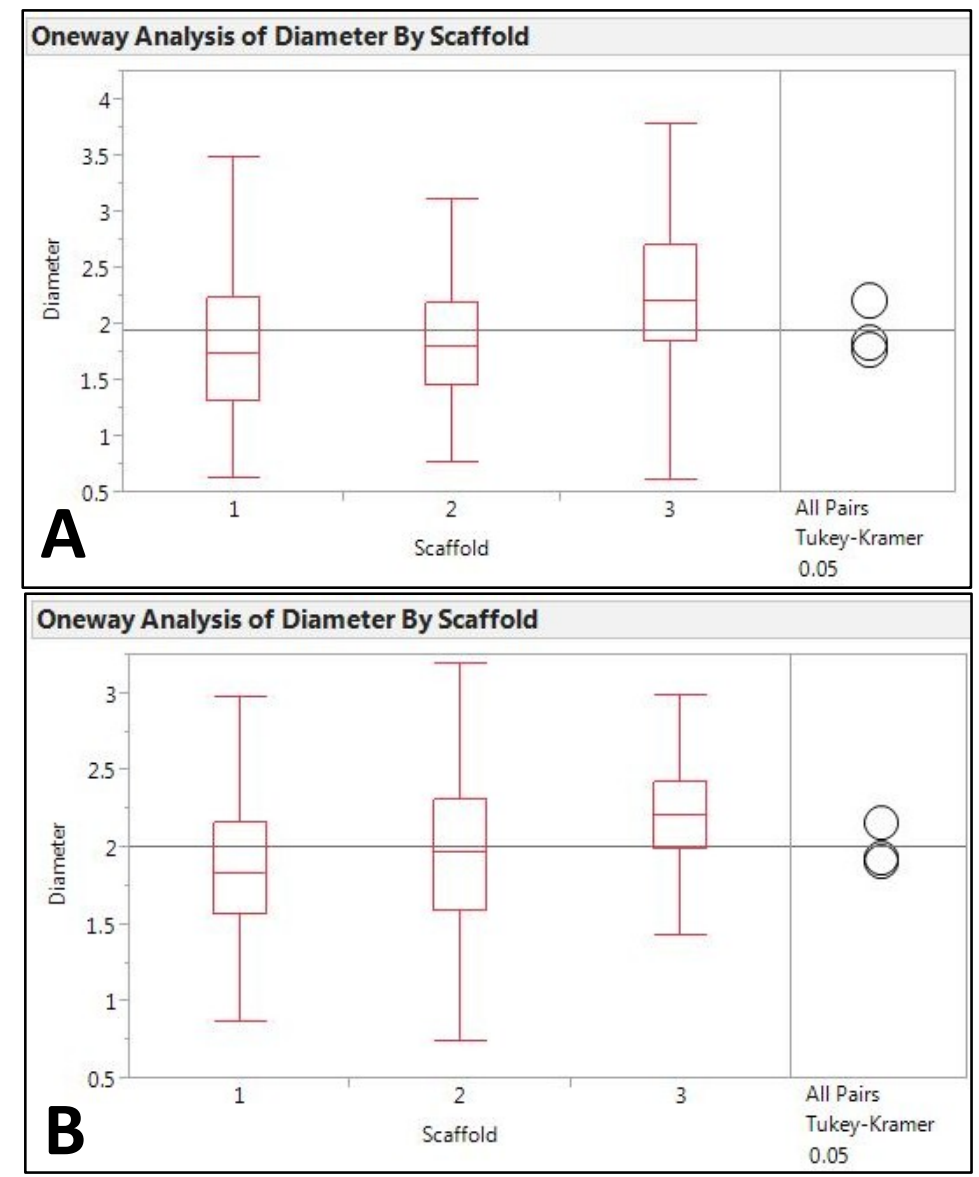

Figure 21: (A) Graphical results of Tukey's HSD post-hoc test for Protocol B. (B)

Graphical results of Tukey's HSD post-hoc test for the Standard Protocol.

Other results from Tukey's HSD test included the Connecting Letters Report and the Ordered Differences Report. The Connecting Letters Report identified the groups with significantly different mean fiber diameters and the Ordered Differences Report provided the p-values associated with each paired test. In both protocols, Scaffold 3 was significantly different from Scaffolds 1 and 2. In Protocol B, Scaffold 3 (Spin B4) had a 
significantly different mean fiber diameter from Scaffolds 1 and 2 with p-values of $<0.0001$ and 0.0002 , respectively. In the Standard Protocol, Scaffold 3 (Spin Z) also had a significantly different mean fiber diameter from Scaffolds 1 and 2 with p-values of 0.0005 and 0.0015 , respectively. The mean fiber diameter of Scaffolds 1 and 2 were not significantly different from each other in either protocol. See Appendix $G$ for the JMP output for these results.

\subsubsection{One-Tailed t-Test}

Finally, a one-tailed t-test was performed in order to determine if the mean fiber diameter was significantly reduced in Protocol B since that protocol was originally chosen for its smaller fibers. Table XXI shows the overall mean and standard deviation for each protocol.

Table XXI: Overall mean fiber diameter and standard deviation for both protocols.

\begin{tabular}{|c|c|c|}
\hline Protocol & $\begin{array}{c}\text { Mean Fiber } \\
\text { Diameter }(\boldsymbol{\mu m})\end{array}$ & $\begin{array}{c}\text { Standard } \\
\text { Deviation }(\boldsymbol{\mu m})\end{array}$ \\
\hline B & 1.95 & 0.66 \\
\hline Standard & 2.01 & 0.46 \\
\hline
\end{tabular}


Figure 22 shows a boxplot of this analysis. The p-value for the one-tailed t-test was 0.1004 , which is above the significance level of 0.05 . This failed to reject the null hypothesis; there was therefore no significant reduction in fiber diameter using Protocol B. See Appendix $G$ for the JMP output of this test.

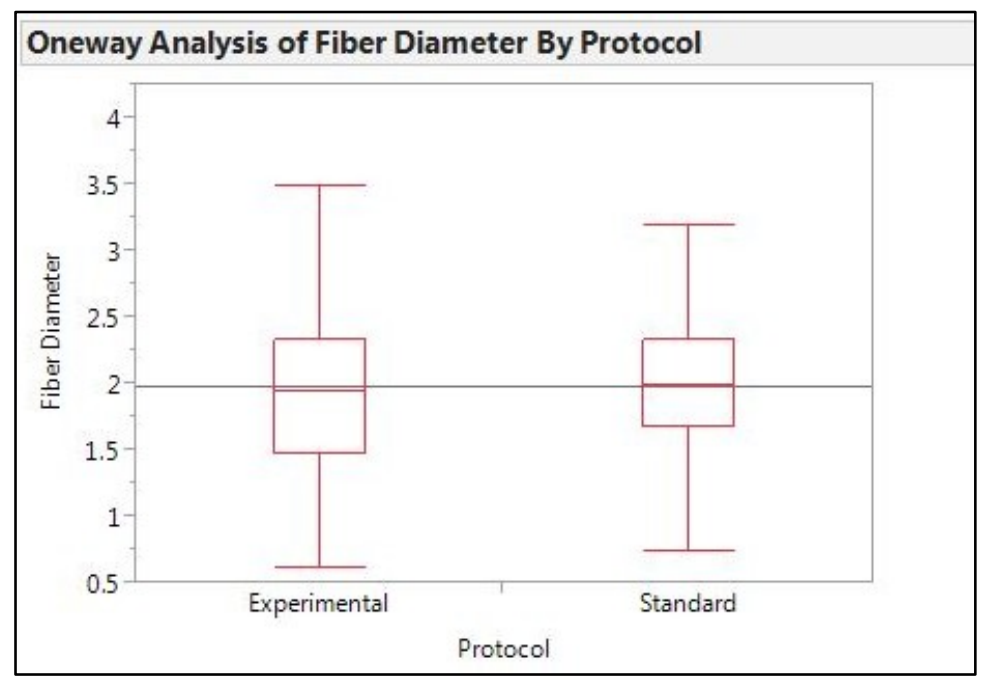

Figure 22: Boxplot of the t-test that compared the overall mean fiber diameter of Protocol B and the Standard Protocol.

\subsection{DISCUSSION}

Extreme outliers were eliminated from the data for these preliminary spins because they were highly uncharacteristic large fibers that severely skewed the results. These instabilities were not typical occurrences within the scaffolds, nor were they present in every scaffold. The same statistical analyses were performed on this preliminary data with the outliers included for comparison; see Appendix $H$ for the results. More outliers were present in the scaffolds from Protocol B than scaffolds from the Standard Protocol. Large fibers may have been a consequence of the initial instability 
of the Taylor cone. When the voltage was first applied in the electrospinning process, the initial formation of the cone was not consistently instantaneous; sometimes the bead would begin to form a cone when the voltage was applied, but was then torn off of the needle and pulled toward the mandrel along with the jet of solution that had started to extrude from the tip of the cone. A new, stable cone would then replace that initial cone. The large fibers may have formed as the bead was pulled toward the mandrel along with the jet of solution. It is postulated that the instability of the initial cone may be related to the timing involved in turning on the voltage. Instead of turning on the voltage as soon as the initial bead is visible, cone formation may be improved if a larger bead is allowed to form over the needle tip before the voltage is turned on. A larger bead may provide more resistance to the force that pulls it off as the initial surge of voltage is applied. This technique will be utilized for the duration of this thesis in an attempt to improve the stability of the Taylor cone. This modification to the standard electrospinning protocol can be found in Appendix B.

The REML variance components analysis calculated the amount of variability that each random effect had as percentage of the total variability that existed in the data. In order for a scaffold to be considered reproducible, the variability between scaffolds should be low. For Protocol B, the variability between scaffolds was $10.668 \%$ of the total variability. This indicated that there was variability between scaffolds, but it was small compared to other effects. The source of the greatest variability was the residual at $86.909 \%$, which was point-to-point variability within the scaffold. For the Standard Protocol, the variability between scaffolds was $7.653 \%$ and point-to-point variability was $90.255 \%$ of the total variability. The variability between images was low, accounting for 
approximately $2 \%$ of the overall variability in both protocols. The Standard Protocol had approximately $3 \%$ less variability between scaffolds than the experimental protocol. Therefore the scaffolds were more reproducible using the Standard Protocol. However, in order to determine if this $3 \%$ has a significant biological effect, future tests need to be performed to compare the cellular response to the scaffolds created using these two protocols.

Although the variance components analysis revealed that the variability between scaffolds was relatively low in both protocols, this corresponded to a higher amount of variability within scaffolds. High variability within scaffolds may suggest that the scaffolds are not reproducible; however, this point-to-point variability was characterized with the standard deviations for each scaffold. The overall standard deviation of Protocol B from the t-test (Table XXI) was $0.66 \mu \mathrm{m}$ while the standard deviation of the Standard Protocol was $0.46 \mu \mathrm{m}$; the Standard Protocol had a tighter range of fiber diameters than Protocol B. Following a Normal distribution, about $68 \%$ of the fibers will be within in one standard deviation away from the mean and about $95 \%$ of the fibers will be within two standard deviations away from the mean. Therefore the Standard Protocol, with an overall mean fiber diameter of $2.01 \mu \mathrm{m}$ (Table XXI), will have $95 \%$ of the fibers within the range of $1.09 \mu \mathrm{m}$ to $2.93 \mu \mathrm{m}$. If the fibers are smaller than the cells and a majority of the fibers are contained within this relatively small range, the cells will not be greatly affected. Cells can tolerate a range of fiber diameters since the fibers of the native ECM range from $50 \mathrm{~nm}$ to $500 \mathrm{~nm}^{7}$.

While nanofibers promote cell adhesion and other cellular responses ${ }^{68,70,77}$, the current electrospinning system in the Cal Poly lab is only capable of producing 
microfibers. Thus, it is important to control the variability of these fibers in order to prevent the formation of larger fibers that may interfere with ideal cellular activity. One study produced electrospun PLGA fibers in the following ranges: $0.81 \pm 0.40 \mu \mathrm{m}, 1.04 \pm$ $0.39 \mu \mathrm{m}$, and $1.11 \pm 0.58 \mu \mathrm{m}^{78}$. Although the mean fiber diameters are smaller than the ones obtained in this study, the standard deviations are comparable to those of the Standard Protocol. The electrospun microfibers within those ranges were found to influence cellular responses ${ }^{78}$. Another study that used electrospun 75:25 PLGA to coat titanium produced fibers with diameter $0.957 \pm 0.357 \mu \mathrm{m}^{79}$. Again, although the mean fiber diameter is lower than in this study, the standard deviation is comparable to the current Standard Protocol. Finally, one study that used 85:15 PLGA produced randomly aligned fibers with diameter $4.96 \pm 0.9 \mu \mathrm{m}$, which is much higher than the range in this study. Despite the higher mean and wider range of fiber diameter, the scaffolds still promoted cell proliferation and infiltration ${ }^{80}$. Clearly, there is no accepted standard for scaffold variability.

The SEM images also supported the results of the variance components analysis. Figure 23(A) shows the inconsistency of one scaffold created using Protocol B. There were clusters of extremely small, thread-like fibers as well as a large clump; these inconsistencies contributed to the higher scaffold-to-scaffold variability of $10.668 \%$. Using this protocol, the Taylor cone was usually not visible; it remained inside of the syringe tip for a majority of the spins. If it was visible, it was inconsistent and did not stabilize at any point during the process. In her thesis, Yvette Castillo discovered that the presence of a Taylor cone was influential on the structure of the scaffolds; when the cone was not visible, the scaffolds were globular instead of fibrous ${ }^{11}$. This may have 
contributed to the inconsistent fiber morphology of small, thread-like fibers and large clumps. These observations indicated that instabilities existed among the combination of parameters within the experimental protocol. Figure 23(B) shows one scaffold created with the Standard Protocol. There were no extreme variations in fiber morphology in any of the scaffolds, which was consistent with the lower scaffold-to-scaffold variability of $7.653 \%$. See Appendix $F$ for the full set of SEM images for both Protocol B and the Standard Protocol.
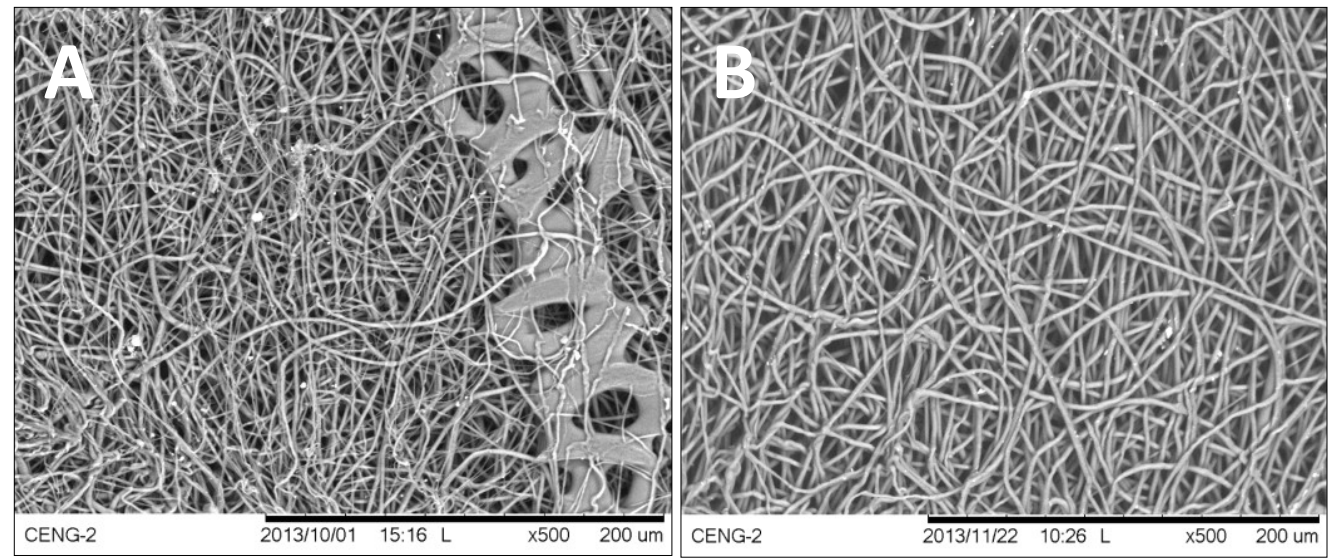

Figure 23: (A) SEM image of the luminal surface of Spin B1 (Distal location, Image 1) at 500x magnification; (B) SEM image of the luminal surface of Spin Z (Medial location, Image 1) at 500x magnification.

ANOVA was performed on both protocols in order to analyze the consistency of the mean fiber diameter between the scaffolds. Both protocols had at least one mean that was significantly different than the others. Tukey's HSD post-hoc test identified which scaffold had the significantly different mean fiber diameter; this was determined to be the third scaffold in each protocol. Although the variance components analysis revealed that 
the scaffold-to-scaffold variability was relatively low in both protocols, the variability was still large enough to produce significantly inconsistent mean fiber diameters in both protocols. However, this could be an artifact of only comparing three scaffolds in each protocol; this conclusion could change with a larger sample size.

Finally, the one-tailed t-test was used to compare the overall mean fiber diameter from both protocols. Since Protocol B was initially chosen in the first set of spins for its smaller mean fiber diameter, this test determined if Protocol B produced a significantly smaller mean fiber diameter than the Standard Protocol. The t-test resulted in a p-value above the significance level, therefore failing to reject the null hypothesis. Thus, the mean fiber diameter of Protocol B was not significantly smaller than that of the Standard Protocol. There was therefore no evidence to suggest that Protocol B was more advantageous than the Standard Protocol for the following reasons: the mean fiber diameter was not significantly reduced, the scaffold-to-scaffold variability was higher in Protocol B than in the Standard Protocol, and the SEM images of Protocol B had clumps and inferior fiber morphology. The Standard Protocol will therefore be employed for the remainder of this thesis.

Limitations of these preliminary studies included the efficiency of the REML method of statistical analysis and the accuracy of the fiber diameter measurements using ImageJ. The REML method for analyzing mixed effects GLM models provides variance components estimates that do not depend on the fixed effects. The REML method is recommended among other methods due to its ability to handle unbalanced data and provide unbiased estimates. However, a drawback of the REML method in this study is that it did not directly provide p-values for the random effects; it only calculated the 
variance components estimates. For this reason, ANOVA had to be performed to determine if the scaffolds had significantly different mean fiber diameters. Instead of having one conclusive analysis for each protocol, multiple statistical procedures had to be performed. It would have been more efficient to determine statistical significance based on the calculated REML estimates of the random effects, not on an entirely different ANOVA procedure.

Additionally, ImageJ may not have provided the most accurate fiber diameter measurements. Each time ImageJ was opened for a new session, the scale needed to be set; if the scale was not identical every session, then the subsequent measurements would also be different than the measurements made in other sessions. It was also difficult to draw perfectly perpendicular lines across the fibers, which may have contributed to slightly inaccurate fiber diameters.

Finally, temperature and humidity were recorded at the beginning of each spin. The current system does not allow for control over the environment, so these environmental levels are only monitored. Humidity varied considerably for several spins, which may have affected the variability of the scaffolds. Other studies have shown that temperature and humidity greatly affect the electrospinning process ${ }^{64,65,81}$. This will be discussed in greater detail later in this thesis.

One challenge from this preliminary study was one scaffold, Spin B3, sticking to the mandrel. The mandrels that were used for these spins had been used extensively by previous Cal Poly students. It is believed that multiple uses and cutting samples off of the mandrels with the razor blades ruined the polished surface, creating deeper scratches in the mandrels over time. Fibers may have settled within the scratches as the scaffold sat in 
the desiccator to dry, preventing a clean removal of the scaffold. In order to avoid wasting materials, a new set of mandrels were machined for use in the remainder of this thesis. The protocol for making these mandrels is outlined in Appendix I.

\subsection{CONCLUSION}

The aim of this study was to characterize the reproducibility of PLGA scaffolds using fiber diameter measurements. Four goals were used to accomplish this task: (1) select an experimental electrospinning protocol, based on several variations, that produced the smallest mean fiber diameter; (2) define the scaffold variability using the chosen experimental protocol; (3) compare the result to the scaffold variability of the current Standard Protocol that is utilized in the Cal Poly lab; and (4) choose the more reproducible protocol to use for the remainder of this thesis. Each goal is revisited below.

(1) Five experimental protocols with varying combinations of flow rate and voltage were designed to obtain a smaller mean fiber diameter than the current Standard Protocol. Flow rate and voltage did affect fiber diameter as each protocol yielded scaffolds with noticeably different qualities and mean fiber diameters. Of the five protocols, Protocol B produced scaffolds with the smallest mean fiber diameter. However, using a t-test, it was determined that this reduction was not significantly smaller than the overall average achieved using the Standard Protocol.

(2) A REML variance components analysis was used to assess the variability of the scaffolds. The variability between scaffolds in Protocol B was lower than the 
variability within scaffolds. Although the variability within scaffolds seemed high, it corresponded to a relatively small standard deviation of $0.66 \mu \mathrm{m}$. Furthermore, although the variability between scaffolds was low, ANOVA revealed that there was a significant difference in mean fiber diameter between scaffolds.

(3) The variability between scaffolds in the Standard Protocol was smaller than the scaffold-to-scaffold variability in Protocol B. Since reproducibility was defined as low scaffold-to-scaffold variability, the Standard Protocol was more reproducible than Protocol B. However, the ANOVA results demonstrated that there was still a significant difference in mean fiber diameter between the scaffolds of the Standard Protocol. However, this difference was less significant than the difference in Protocol B. The variability within scaffolds corresponded to a standard deviation of $0.46 \mu \mathrm{m}$, which was a tighter fiber diameter range than that produced with Protocol B.

(4) The variability between scaffolds was higher in Protocol B than in the Standard Protocol, but both protocols had significantly inconsistent means. Thus, a definitive conclusion could not be reached from the statistical analyses alone. This could have been due to the use of a small sample size. A more powerful conclusion was drawn from the SEM images. After qualitatively assessing the SEM images, it was determined that the Standard Protocol had fewer extreme inconsistencies and had superior fiber morphology than Protocol B. The inferior morphology of the scaffolds using Protocol B was attributed to the lack of a fully developed Taylor cone. A stable cone has been 
determined to be important for the development of fibrous scaffolds ${ }^{11}$. Thus, the Standard Protocol was selected for continued use in this thesis work.

The next chapter will expand the reproducibility study to further characterize the scaffolds created using the Standard Protocol. A large-scale study will be performed to assess fiber diameter and compliance. Compliance will be analyzed before and after conditioning the scaffolds within the flow-based bioreactors utilized in the Tissue Engineering lab. 


\section{REPRODUCIBILITY OF THE PROPERTIES OF ELECTROSPUN PLGA SCAFFOLDS}

\subsection{INTRODUCTION}

In Chapter 2, it was established that a significant reduction in fiber diameter while maintaining reproducibility was not achievable with the experimental protocol. The Standard Protocol was more reproducible with superior fiber morphology and less extreme variability present in the scaffolds. As mentioned in the previous chapters, reproducibility is important to maintain in order to create consistent BVM models for intravascular device testing; scaffolds with consistent material and mechanical properties should ultimately result in consistent BVMs. If the BVMs are reproducible, then the performance and efficiency of intravascular devices can be more consistently assessed. This chapter focused on a large-scale study of the reproducibility of PLGA scaffolds using fiber diameter and compliance to characterize the scaffolds. The goals of this chapter included the following: (1) analyze the reproducibility of fiber diameter across a larger sample size using the Standard Protocol; (2) characterize the reproducibility of scaffold compliance before and after conditioning the scaffolds overnight in perfusion bioreactors; and (3) determine if scaffold compliance significantly changed after conditioning.

The properties that were utilized in this chapter to characterize reproducibility were fiber diameter and compliance. As discussed in the previous chapter, fiber diameter influences cell behavior; smaller fibers promote more ideal cell responses, including cell adhesion, differentiation, proliferation, and production of $\mathrm{ECM}^{68,70}$. The ultimate goal is 
to obtain nanofibrous scaffolds in order to emulate the native ECM, which has a range of fibers between $50 \mathrm{~nm}$ and $500 \mathrm{~nm}^{7}$. Not only is it ideal to obtain a small mean fiber diameter, but the range of fiber diameters should also be minimized in order to prevent the formation of large fibers that may hinder cell performance. As mentioned earlier, there is no accepted standard of scaffold reproducibility. Through scaffold characterization, several published studies have established varying mean fiber diameters and ranges ${ }^{78-80}$. The scaffolds in the Cal Poly lab will therefore be characterized for the current electrospinning system.

Another important feature of scaffolds in vascular applications is compliance, which is the measure of the deformation of a vessel in response to an applied pressure. It is essential for buffering the pulsatile pressure that is generated from the left ventricle ${ }^{82}$. The proximal aorta and the major branches are the most compliant portions of the vasculature, but peripheral vessels also have storage capacity and contribute to the regulation of blood flow ${ }^{82}$. Compliance depends on geometry and mechanical properties of the vessel, which differ across vessel types, in the same vessel at different pressures, and during smooth muscle activation ${ }^{82,83}$. Thus, comparison of compliance across studies is difficult due to the lack of a standard compliance value ${ }^{82}$. However, compliance has been studied extensively through comparisons of small-diameter vascular grafts to human and animal vessels using different scaffold materials ${ }^{24,84,85}$. These studies have shown that although blood compliance is an important property to mimic, physiologic compliance is difficult to achieve with tissue-engineered constructs.

There are several different ways to define compliance. A common form of compliance $(\mathrm{mL} / \mathrm{mmHg})$ assesses the change in volume when a pressure is applied ( $E q$ 
$2)^{86}$. For small-diameter vessels, physiologic compliance $(\% / 100 \mathrm{mmHg})$ is typically calculated using $E q 3$, where $p_{1}$ is the lower pressure, $p_{2}$ is the higher pressure, $R_{i p l}$ is the radius of the vessel at the lower pressure, and $R_{i p 2}$ is the radius of the vessel at the higher pressure $^{84,87}$. This equation is used for small-diameter vessels because more reliable compliance measurements are obtained using the geometry of the vessel to measure a relative volume change ${ }^{88}$.

$$
\begin{gathered}
C=\frac{\Delta V}{\Delta P} \\
\% \text { Compliance } / 100 m m H g=\left(\frac{\frac{R_{i p 2}-R_{i p 1}}{R_{i p 1}}}{p_{2}-p_{1}}\right) * 10^{4}
\end{gathered}
$$

Compliance and viscoelasticity are desirable material properties to emulate in tissue-engineered constructs so that the mechanical performance is comparable to the native tissue ${ }^{89,90}$. This is extremely important for BVMs specifically in order to have a realistic model for testing stents and other intravascular devices. However, physiologic compliance has not been achieved in tissue-engineered constructs, though a study using poly(glycerol sebacate) (PGS) demonstrated similar compliance to a porcine carotid artery when exposed to burst pressure ${ }^{85}$. The same study also used PLGA for comparison in a variety of tests, but the scaffolds were too brittle to undergo compliance testing. This was most likely due to the fabrication methods that were used to produce the scaffolds, none of which were electrospinning ${ }^{85}$. Previous work with the compliance testing apparatus used for this thesis also revealed that PLGA was less compliant than porcine 
arteries; PLGA had a compliance of $0.5402 \%$ after being soaked in phosphate buffered saline (PBS) for 15 minutes while porcine arteries had a compliance of $2.983 \%{ }^{87}$. Even if physiologic compliance cannot currently be achieved with PLGA, it is important to produce scaffolds with consistent compliance so that BVMs exhibit similar mechanical performance.

\subsection{MATERIALS AND METHODS}

Preliminary studies on fiber diameter demonstrated that the Standard Protocol was more reproducible and had superior fiber morphology than the experimental protocol. The Standard Protocol was therefore selected for further testing in this chapter with a larger sample size.

\subsubsection{Methods Overview}

20 scaffolds were electrospun for fiber diameter and compliance studies to determine the reproducibility of the Standard Protocol. However, a total of 23 spins were performed to achieve this sample size due to complications during fabrication; this will be discussed in more detail later in this chapter. The 20-scaffold sample size was chosen because it would provide sufficient information to obtain relevant reproducibility data and could also be performed in a reasonable amount of time. Fiber diameter measurements were performed as before. Compliance testing was performed before and after conditioning the scaffolds in the perfusion bioreactors used in BVM cultivation. These "dry" and "wet" compliance tests were used to compare the reproducibility of compliance before and after conditioning, respectively, to determine if compliance was 
consistent between scaffolds, and to also determine if compliance was significantly affected by the conditioning process. Furthermore, the conditioning simulated more physiologic conditions since the scaffolds were subjected to constant fluid flow overnight.

Before beginning the expanded reproducibility study, preliminary compliance testing was performed on three 2-cm long scaffold samples in order to provide insight on the data that was to be expected using the compliance testing apparatus. Five trials were performed on each sample. It was noted that multiple trials on a sample did not have a systematic effect on the compliance reading, i.e., compliance did not increase with each trial. However, the trials did demonstrate random fluctuations of compliance within each sample. For the practicality of testing a large number of scaffolds, three trials on each sample were determined to be sufficient for the reproducibility study. See Appendix $J$ for a summary of the methods and the JMP output of this preliminary compliance data.

Electrospinning and testing were performed according to a predetermined timeline. Two scaffolds were electrospun consecutively and subjected to SEM, dry compliance testing, and wet compliance testing before the next set of scaffolds was fabricated. SEM and dry compliance testing were performed three days after electrospinning. After dry compliance testing, the scaffolds were conditioned in the bioreactors overnight for approximately 15 hours. Since each scaffold had three samples for compliance testing, six bioreactors were used at once. Wet compliance testing was then performed the following day on all six scaffold samples. However, only four scaffolds were subjected to this preliminary approach until a shortage of materials delayed the conditioning process. An adjusted approach was therefore used on the 
remaining 16 scaffolds: all of the scaffolds were electrospun and underwent SEM and dry compliance testing until conditioning materials were replenished. During this time, three scaffolds were electrospun consecutively and subjected to SEM and dry compliance testing three days after fabrication. After all SEM and dry compliance testing were completed, conditioning and wet compliance were then performed on two scaffolds at once (six bioreactors) with three days between setups. These preliminary and adjusted approaches are shown in Table XXII. The potential implications in this shift in methods will be discussed later in the chapter.

Table XXII: Approaches for electrospinning and testing the 20 scaffolds.

\begin{tabular}{|c|c|c|}
\hline $\begin{array}{c}\text { \# Scaffolds Electrospun } \\
\text { Consecutively }\end{array}$ & $\begin{array}{c}\text { Preliminary Approach } \\
\text { (4 scaffolds) }\end{array}$ & $\begin{array}{c}\text { Adjusted Approach } \\
\text { (16 scaffolds) }\end{array}$ \\
\hline SEM/Dry Compliance & 2 & 3 \\
\hline $\begin{array}{c}\text { \# Scaffolds Tested } \\
\text { Consecutively for } \\
\text { SEM/Dry Compliance }\end{array}$ & 2 & 3 \\
\hline Conditioning & 3 days after fabrication & $\begin{array}{c}\text { After all SEM/dry } \\
\text { compliance completed }\end{array}$ \\
\hline Wet Compliance & 4 days after fabrication & $\begin{array}{c}\text { After all SEM/dry } \\
\text { compliance completed }\end{array}$ \\
\hline $\begin{array}{c}\text { \# Scaffolds Tested } \\
\text { Consecutively for } \\
\text { Conditioning/Wet } \\
\text { Compliance }\end{array}$ & 2 & 2 \\
\hline
\end{tabular}

\subsubsection{Polymer Mixing and Electrospinning}

All 20 spins used $3 \mathrm{~mL}$ of a $15 \mathrm{wt} \%$ 75:25 PLGA and $\mathrm{CHCl}_{3}$ solution. The solutions were mixed using the protocol outlined in Appendix A. After mixing on an 
orbital shake table for 24 hours, the solutions were electrospun using the Standard Protocol (Appendix B). As discussed in Chapter 2, a modification was made to the protocol: a larger bead was allowed to form before turning on the voltage. This change was implemented for the spins in the present chapter. The scaffolds were then placed in the desiccator for 48 hours.

\subsubsection{Fiber Diameter Measurement}

After sitting in the desiccator for 48 hours, the scaffolds were cut into six sections and removed from the mandrels using a carbon steel razor blade. Three sections were used in SEM and three sections were used in compliance testing. Specific measurements were used to section the scaffolds. The scaffolds for compliance testing could be no shorter than $2.4 \mathrm{~cm}$ in order to fit in the bioreactors. SEM samples were $1 \mathrm{~cm}$ in length to accommodate the 2.4-cm long compliance samples. SEM and compliance samples alternated along the length of the mandrel in order to have a sample for each test from the proximal, medial, and distal locations. A diagram of the sectioning scheme is shown in Figure 24(A) and Figure 24(B) shows a sectioned scaffold. After sectioning, the scaffolds sat in the desiccator for another 24 hours. SEM samples were cut in half to expose the lumen and SEM was performed using the protocol in Appendix $C$. Three random images were obtained per section. ImageJ was later used to obtain fiber diameter measurements using the protocol outlined in Appendix D. An overlay of nine circles was placed over the SEM images to obtain nine measurements per image (Figure 25). The fiber at the center of each circle was measured. 


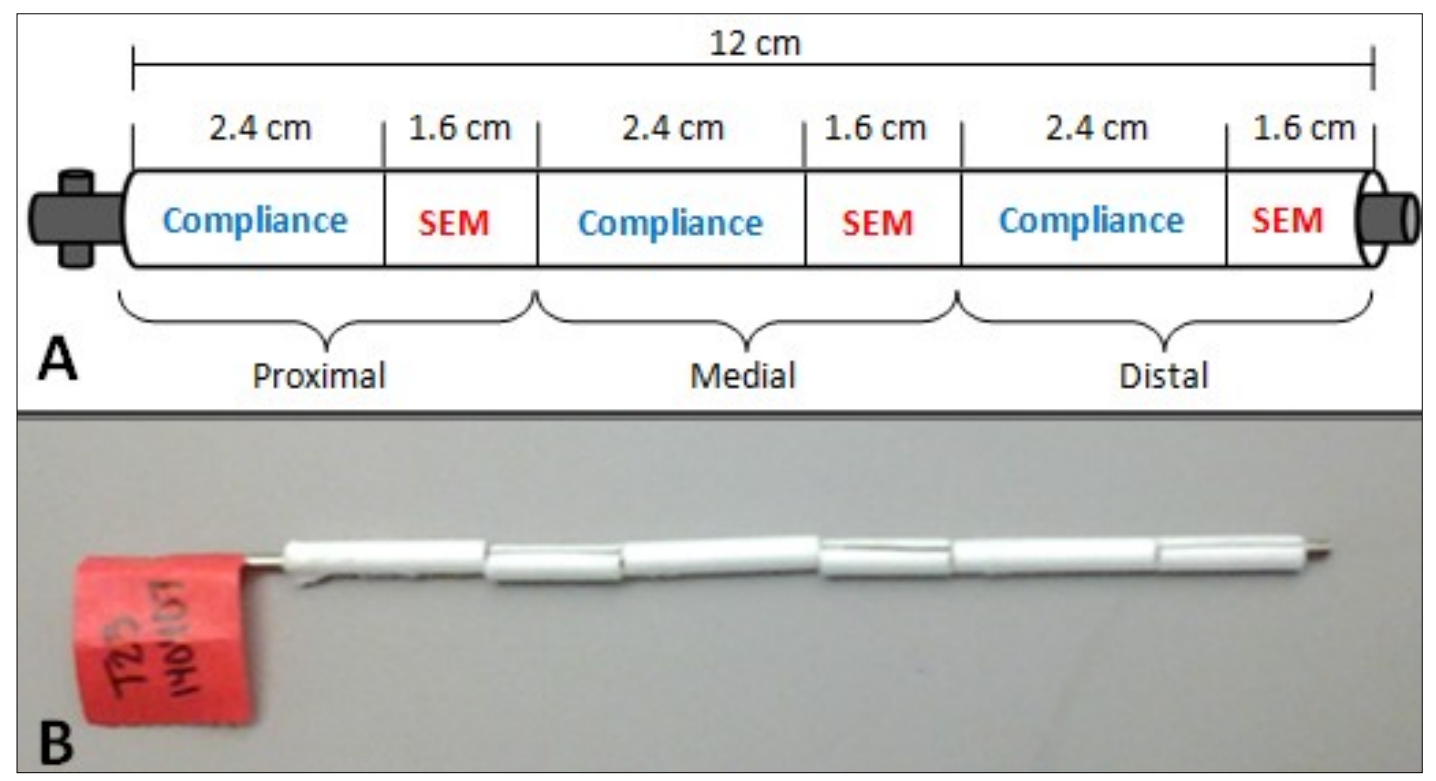

Figure 24: (A) Diagram of the sectioning scheme for obtaining SEM and compliance samples from the scaffolds. (B) Scaffold after sectioning was completed.

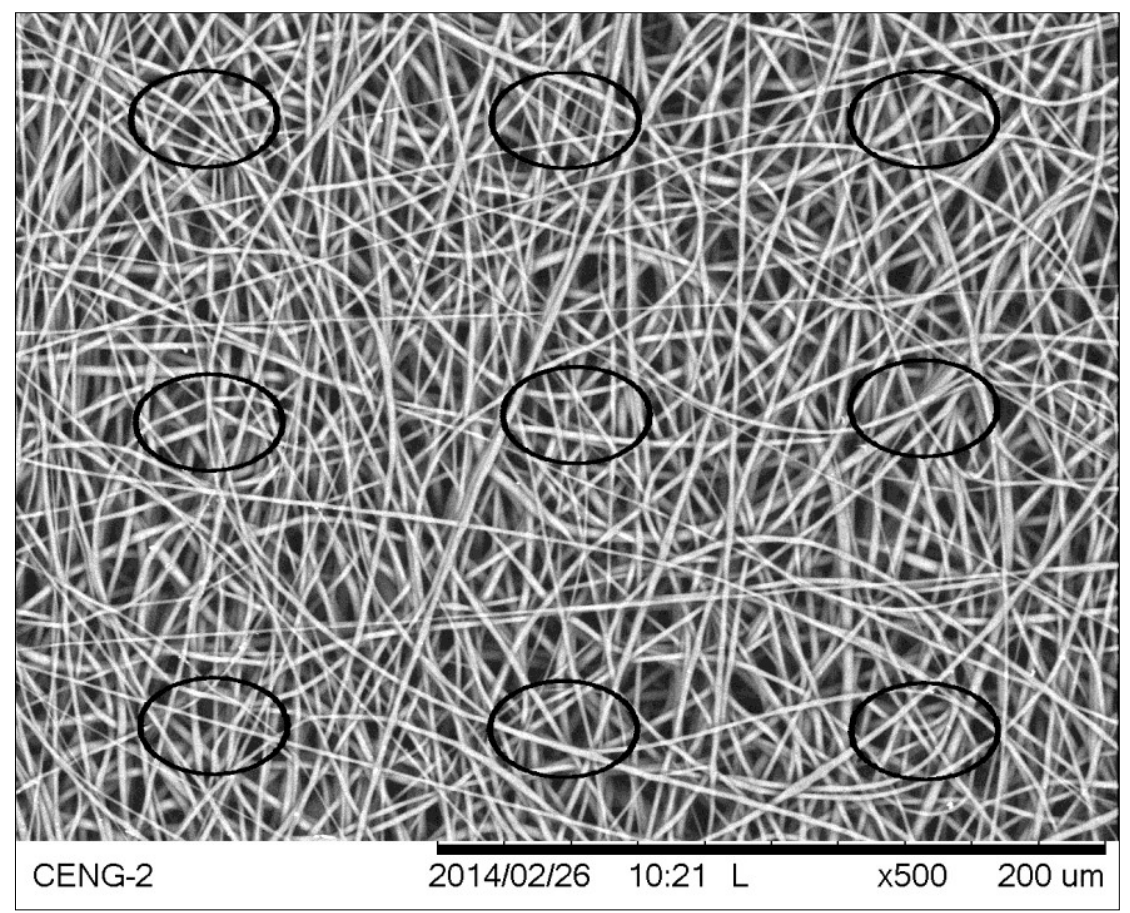

Figure 25: SEM image with the overlay of nine circles in ImageJ. The fiber closest to the center of each circle was measured. 


\subsubsection{Compliance Testing}

Immediately after SEM, compliance testing was performed on the dry samples using the Olympus CKX41 Microscope and compliance testing fixture developed inhouse, as shown in Figure 26. The compliance testing protocol is outlined in Appendix K. Briefly, the scaffolds were placed on an occlusion catheter and centered over the $4 x$ microscope objective (Figure 27(A)). MATLAB and Omega DAQ software were loaded and the scaffold was centered in the MATLAB viewing window. Pressure was applied manually using a syringe filled with $10 \mathrm{~mL}$ of deionized (DI) $\mathrm{H}_{2} \mathrm{O}$ to inflate the balloon and expand the scaffold. The process was recorded in a live video feed using MATLAB (Figure 27(B)). A video analysis was performed in MATLAB using the binary format of the video to obtain $\%$ Diameter Change $(\% \Delta D)$ as shown in Figure $27(C)$. Three trials were performed on each proximal, medial, and distal sample.

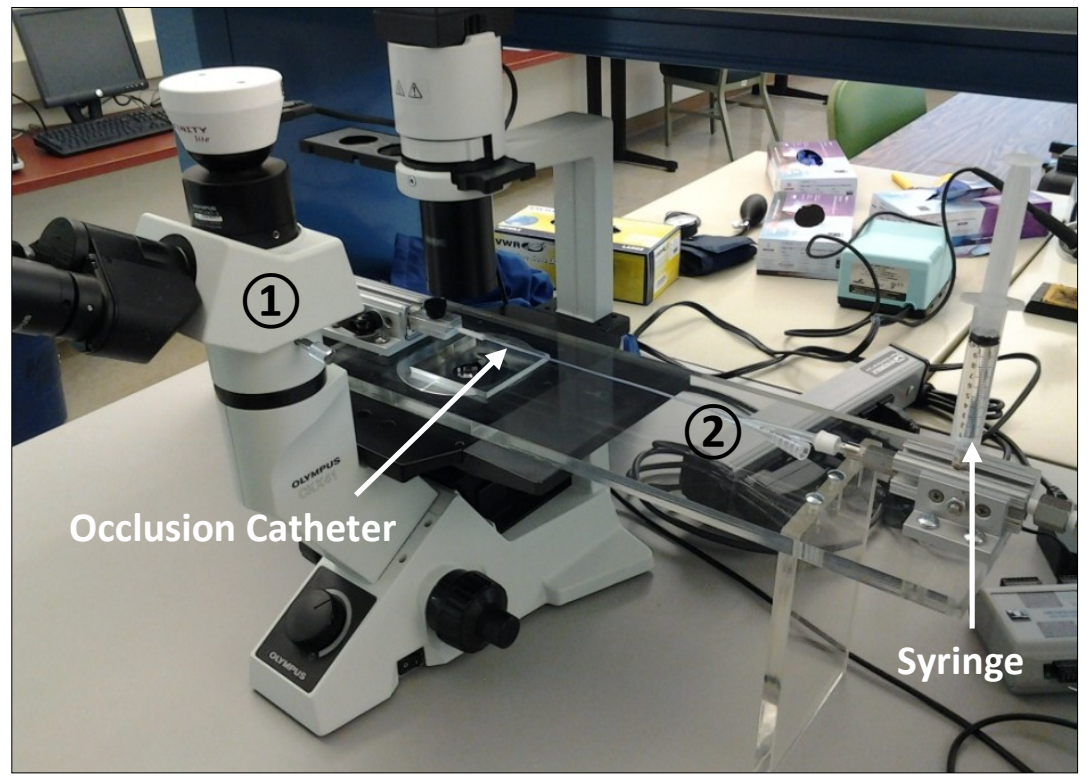

Figure 26: (1) Olympus CKX41 Microscope and (2) compliance testing fixture. 


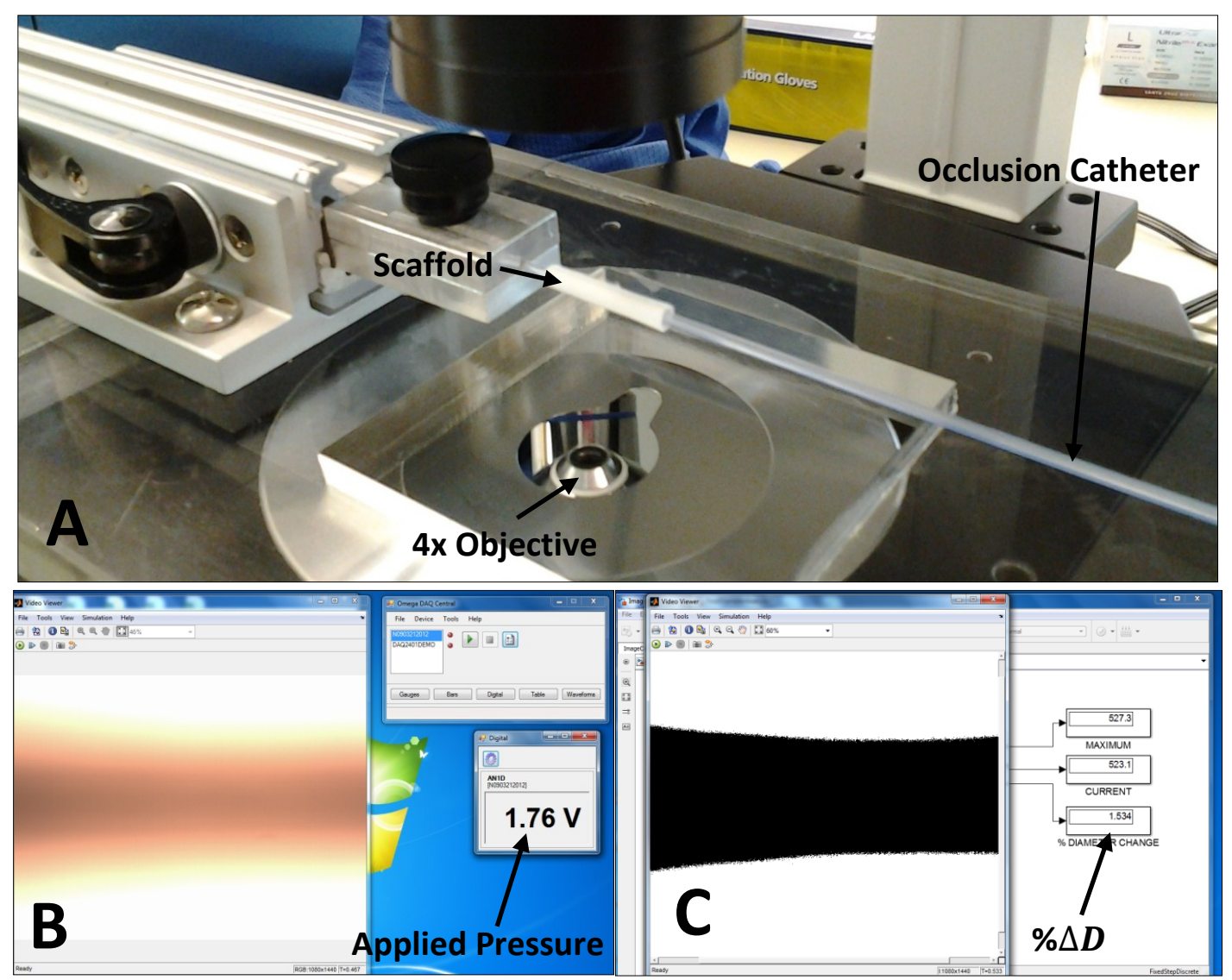

Figure 27: (A) Dry compliance sample on the occlusion catheter centered over the $4 \mathrm{x}$ microscope objective. (B) MATLAB window with the live video feed of the compliance test. (C) Video analysis using the binary format of the MATLAB video. \% Diameter Change $(\% \Delta D)$ was obtained during this analysis.

After obtaining $\% \Delta D$ from the videos, maximum and minimum voltages were obtained from Excel files of the pressures and used to calculate the change in voltage $(\Delta V)$ using $E q 4$. The change in voltage was then converted to a change in pressure $(\Delta P)$ in $\mathrm{mmHg}$ using a conversion factor provided by Omega $(E q 5)$. The original 15 psi pressure transducer in the compliance fixture was replaced by a 50 psi pressure transducer before the last dry scaffold was tested (Spin 23). A new conversion factor was 
therefore provided by Omega to convert $\Delta V$ to $\Delta P$, as shown in $E q$ 6. Compliance was then calculated using $E q 7$.

$$
\begin{gathered}
\Delta V=\operatorname{Max} V-\operatorname{Min} V \\
\Delta P=(\Delta V) *\left(3.008 \frac{p s i}{V}\right) *\left(51.7149326 \frac{\mathrm{mmHg}}{p s i}\right) \\
\Delta P=(\Delta V) *\left(10 \frac{p s i}{V}\right) *\left(51.7149326 \frac{m m H g}{p s i}\right) \\
\% \text { Compliance } / 100 \mathrm{mmHg}=\left(\frac{\% \Delta D}{\Delta P}\right) * 100
\end{gathered}
$$

The scaffolds were then prepped for conditioning. Since no cells were sodded onto the scaffolds after conditioning, non-sterile setups were performed. The conditioning protocol was therefore modified to accommodate a non-sterile setup, as shown in Appendix L. Briefly, the bioreactors were assembled and the scaffolds were mounted and sutured onto fittings (Figure 28(A)). Each bioreactor chamber was filled with $165 \mathrm{~mL}$ of bioreactor media (M199: $500 \mathrm{~mL}$, FBS: $56 \mathrm{~mL}$, HyClone ${ }^{\mathrm{TM}}$ L-Glutamine: $5 \mathrm{~mL}$, HEPES: 2.8 mL; VWR International, 12001-328; Sigma-Aldrich, F4135; Thermo Scientific, SH30034.01; Sigma-Aldrich, H0887) and each reservoir was filled with $25 \mathrm{~mL}$ of conditioning media (M199: $500 \mathrm{~mL}$, FBS: $83 \mathrm{~mL}$ ). The scaffolds were flushed luminally and transmurally with conditioning media (Figure 28(B)) and then mounted on the fittings in the bioreactor chamber (Figure 28(C)). The bioreactors were then primed by 
inverting the media reservoir until all tubing was filled with media. The tubes were clamped and the bioreactors were placed on the 8-roller pump inside the incubator (Figure 28(D)). The systems first underwent luminal flow with the distal stopcock open (Figure 28(E)) until all air was removed from the tubing. The distal stopcock was then closed (Figure 28(F)) to condition the scaffolds transmurally at $150 \mathrm{rpm}$. The systems were subjected to transmural flow overnight for 14 to 16 hours. 


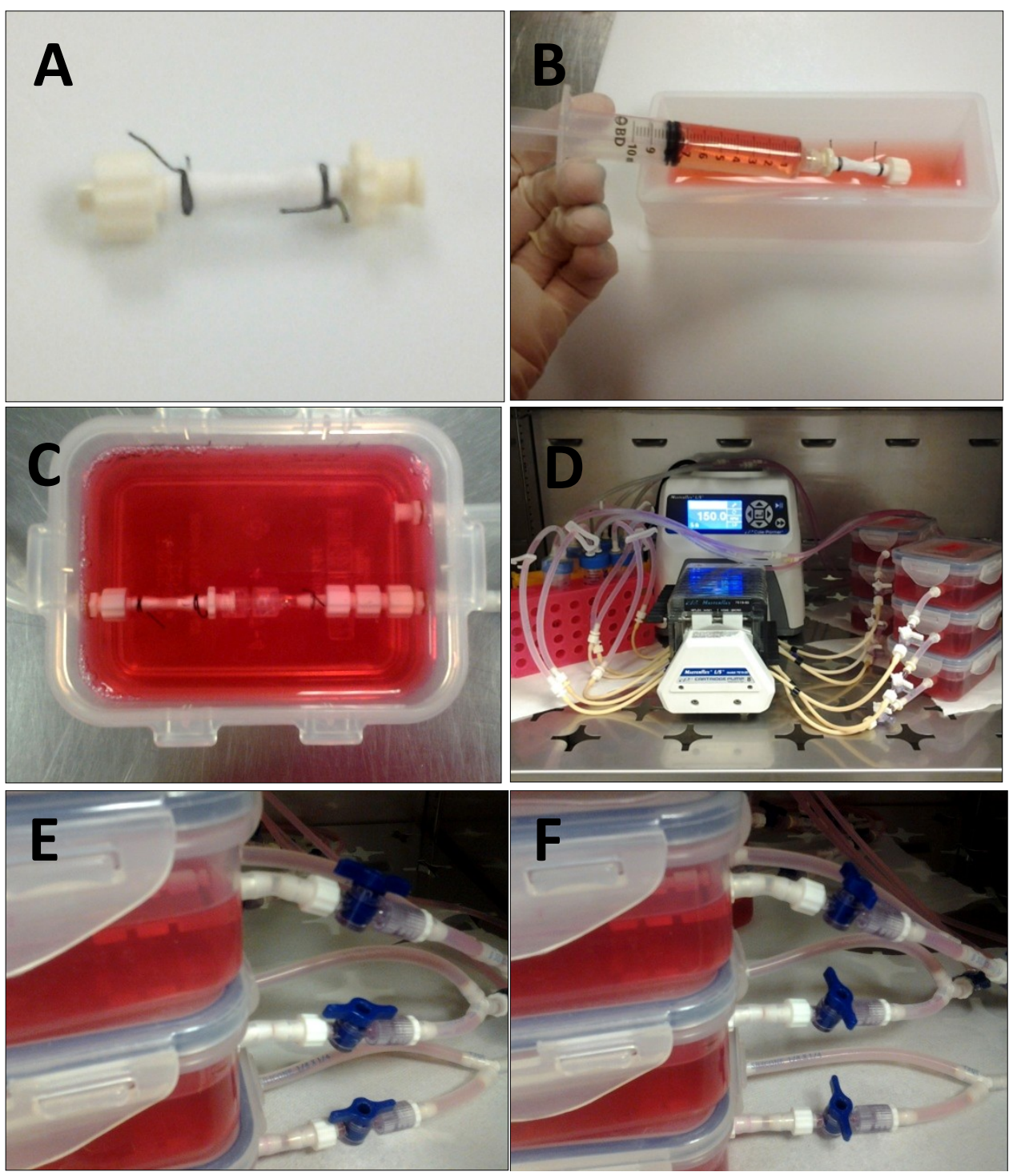

Figure 28: (A) Scaffold sutured onto fittings. (B) Scaffold flushed transluminally and transmurally with conditioning media. (C) Scaffold mounted onto fittings inside the bioreactor chamber. (D) Six bioreactor systems on the 8-roller pump inside of the incubator. (E) Transluminal flow with the distal stopcock open. $(F)$ Transmural flow with the distal stopcock closed. 
The following day, the systems were removed from the pump. The scaffolds were cut off of the fittings using a carbon steel surgical blade and placed in conicals of conditioning media. Since the ends of the scaffolds were flared from being mounted on the fittings, the ends were cut off, as shown in Figure 29(A) in order to have a uniform diameter in the MATLAB viewing window. Compliance testing was then performed on the wet samples (Figure 29(B)) using the same protocol used previously for the dry samples (Appendix $K$ ). Again, three trials were performed per proximal, medial, and distal sample. $\% \Delta D$ was recorded from the MATLAB videos. $\Delta V$ was then calculated with $E q 4$ using the Excel files of the pressures and $\Delta V$ was converted to $\Delta P$ using $E q 5$. The last four scaffolds (Spin 19, 20, 21, and 23) underwent wet compliance testing with the new 50 psi pressure transducer and therefore used the conversion factor in $E q 6$. Compliance was then calculated with $E q 7$.

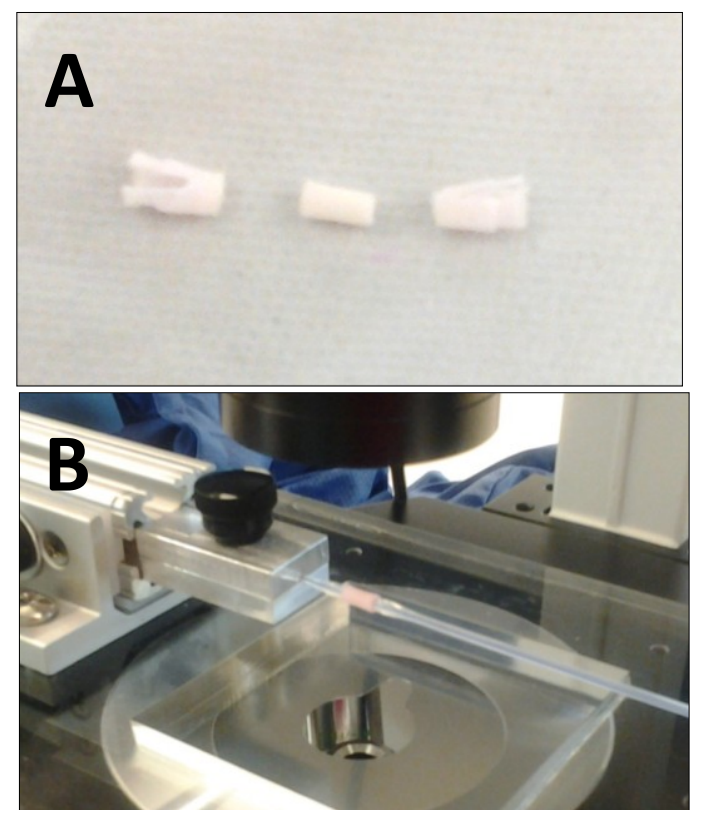

Figure 29: (A) Scaffold with the flared ends removed. (B) Wet compliance sample on the occlusion catheter centered over the $4 \mathrm{x}$ microscope objective. 


\subsubsection{Statistical Analysis}

\subsubsection{Fiber Diameter}

Mean fiber diameters and standard deviations were obtained for all 20 scaffolds using JMP software. A REML variance components analysis was performed on the fiber diameter measurements to determine the amount variability within and between scaffolds. The sources of variability were used to define the reproducibility of the Standard Protocol. ANOVA was also performed at a 95\% confidence level to determine if the scaffolds had significantly different mean fiber diameters. Tukey's HSD post-hoc test was then executed to identify which scaffolds had significantly different mean fiber diameters. The procedures for these methods are outlined in Appendix E.

\subsubsection{Compliance}

Similar statistical analyses were performed on the compliance data. Using JMP software, mean dry and wet compliance values and standard deviations were obtained for all 20 scaffolds. REML variance components analyses were completed on both sets of data to determine the sources of variability and compare the reproducibility of scaffold compliance before and after the conditioning process. ANOVA was then performed at a $95 \%$ confidence level on both data sets to determine if compliance varied significantly among scaffolds. Tukey's HSD post-hoc test was used to identify which scaffolds had significantly different compliance. Finally, a matched pairs t-test was performed to determine if the compliance of the scaffolds changed significantly after overnight conditioning. The procedures for these analyses are outlined in Appendix E. 


\subsection{RESULTS}

The results for the fiber diameter analysis and compliance testing are presented in the following sections. During the time of these experiments, two scaffolds (Spin 14 and 16) turned out thin after being electrospun. Two more spins were therefore performed (Spin 21 and 22) in order to replace those scaffolds and achieve the 20-scaffold sample size. However, one section of Spin 22 stuck to the mandrel during the removal process. This necessitated another spin (Spin 23) to be performed.

\subsubsection{Fiber Diameter}

\subsubsection{Overview of Fiber Diameter Results}

As stated in Chapter 2, the presence of larger fibers was believed to be associated with the timing of applying the voltage. During the experiments in the present chapter, the bead was allowed to form more completely before the voltage was applied in an attempt to eliminate the formation of large fibers. However, a few large fibers were still present when this technique was implemented. It is postulated that the formation of such inconsistencies may be inherent in the combination of parameters that compose the current standard electrospinning protocol. Thus, the protocol is not yet optimized to achieve consistently small fibers. Extreme outliers in these fiber diameter measurements were therefore included in this analysis since large fibers are representative of the current level of reproducibility that can be achieved. A complete fiber diameter analysis without the six outliers was also performed for comparison; see Appendix $M$ for the results. 
Images of the scaffolds were obtained using SEM and fiber diameter measurements were made with ImageJ. Summary statistics were obtained for each scaffold, including mean fiber diameters and standard deviations. The following analyses were then performed on the fiber diameter data: (1) REML variance components analysis, (2) one-way ANOVA, and (3) Tukey's HSD post-hoc test. The variance components analysis calculated the amount of variability attributed to each random effect. ANOVA determined if the mean fiber diameters were consistent among the scaffolds. Finally, Tukey's post-hoc test identified which scaffolds had significantly different mean fiber diameters.

\subsubsection{SEM, ImageJ, and Summary Statistics}

Appendix $N$ contains observations from each spin as well as the complete sets of SEM images and fiber diameter measurements. Each scaffold had nine images with nine measurements per image. Some observations common to most spins included the following: when the voltage was applied, the initial bead fell and was immediately replaced by the Taylor cone; the cone was initially stable and well-shaped; and the cone would eventually develop a repetitive cycle of elongation, falling, and replacement by a new cone. Representative SEM images are presented in Figure 30(A) and (B); Figure 30(A) shows an ideal scaffold with consistent fibers and no extreme variability while Figure $30(B)$ shows the infrequent formation of large fibers. 


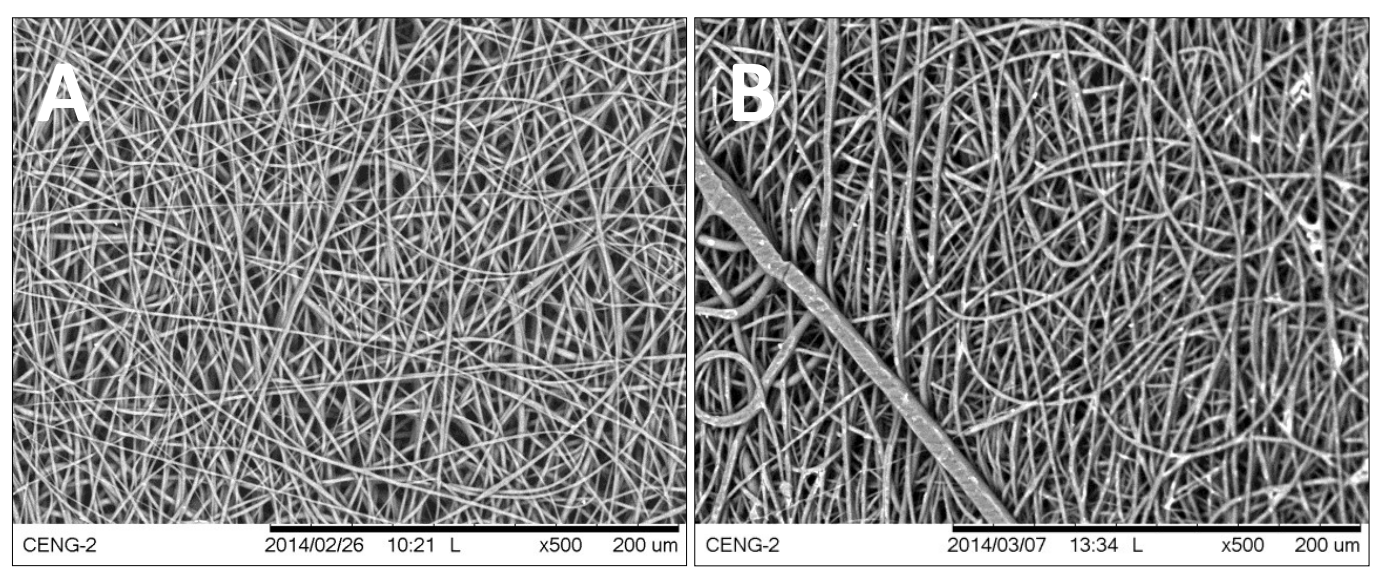

Figure 30: (A) SEM Image 1 for Spin 1 Distal location at 500x magnification; there was no extreme variation in fiber size. (B) SEM Image 1 for Spin 6 Proximal location at 500x magnification; large fibers formed.

Table XXIII contains the mean fiber diameter and standard deviation of each scaffold. The mean fiber diameter ranged from $1.71 \mu \mathrm{m}$ to $2.79 \mu \mathrm{m}$ and the standard deviation ranged from $0.37 \mu \mathrm{m}$ to $0.90 \mu \mathrm{m}$. Over all 20 scaffolds, the mean fiber diameter was $2.22 \mu \mathrm{m}$ with a standard deviation of $0.63 \mu \mathrm{m}$. 
Table XXIII: Mean fiber diameters and standard deviations of the 20 scaffolds.

\begin{tabular}{|c|c|c|}
\hline Spin & $\begin{array}{c}\text { Mean Fiber } \\
\text { Diameter }(\boldsymbol{\mu m})\end{array}$ & $\begin{array}{c}\text { Standard } \\
\text { Deviation }(\boldsymbol{\mu m})\end{array}$ \\
\hline $\mathbf{1}$ & 1.71 & 0.37 \\
\hline $\mathbf{2}$ & 2.79 & 0.51 \\
\hline $\mathbf{3}$ & 2.43 & 0.51 \\
\hline $\mathbf{4}$ & 2.18 & 0.46 \\
\hline $\mathbf{5}$ & 1.95 & 0.64 \\
\hline $\mathbf{6}$ & 2.11 & 0.57 \\
\hline $\mathbf{7}$ & 2.13 & 0.51 \\
\hline $\mathbf{8}$ & 2.07 & 0.53 \\
\hline $\mathbf{9}$ & 2.19 & 0.41 \\
\hline $\mathbf{1 0}$ & 2.58 & 0.63 \\
\hline $\mathbf{1 1}$ & 2.38 & 0.55 \\
\hline $\mathbf{1 2}$ & 2.28 & 0.64 \\
\hline $\mathbf{1 3}$ & 2.22 & 0.42 \\
\hline $\mathbf{1 5}$ & 2.41 & 0.72 \\
\hline $\mathbf{1 7}$ & 2.05 & 0.47 \\
\hline $\mathbf{1 8}$ & 2.32 & 0.89 \\
\hline $\mathbf{1 9}$ & 2.13 & 0.54 \\
\hline $\mathbf{2 0}$ & 2.45 & 0.90 \\
\hline $\mathbf{2 1}$ & 2.12 & 0.59 \\
\hline $\mathbf{2 3}$ & 1.88 & 0.53 \\
\hline Overall & 2.22 & 0.63 \\
\hline
\end{tabular}

\subsubsection{REML Variance Components Analysis}

The results of the REML variance components analysis for random effects are shown in Table XXIV. Images and scaffolds were random effects while location was a fixed effect. The variability within the scaffolds was $85.454 \%$ while the scaffold-toscaffold variability was $14.211 \%$. The variability between images was low, accounting for only $0.335 \%$ of the overall variability. As previously established, a reproducible protocol will result in low scaffold-to-scaffold variability. This data demonstrates that the variability between scaffolds was much lower compared to the variability within scaffolds. Recall that the analysis of three scaffolds from the Standard Protocol in 
Chapter 2 resulted in a scaffold-to-scaffold variability of 7.653\%; the variability in this analysis approximately doubled while the sample size was almost seven times higher. Again, the variability within scaffolds can be described by the mean fiber diameter and standard deviation; overall, this was $2.22 \mu \mathrm{m}$ and $0.63 \mu \mathrm{m}$, respectively. Thus, if $95 \%$ of the data are within two standard deviations away from the mean in a Normal distribution, then most fibers will be between $0.96 \mu \mathrm{m}$ and $3.48 \mu \mathrm{m}$.

Table XXIV: Results of the REML variance components analysis for random effects.

\begin{tabular}{|l|c|}
\hline \multicolumn{1}{|c|}{ Variability Source } & Variability (\%) \\
\hline Within Scaffold & 85.454 \\
\hline Between Images & 0.335 \\
\hline Between Scaffolds & 14.211 \\
\hline
\end{tabular}

Additionally, the fixed effect test for location compared the mean fiber diameters between the proximal, medial, and distal locations of the scaffolds. The p-value for this test was 0.0894 , which was above the significance level of 0.05 . There were therefore no significant differences in fiber diameter across locations. The JMP output for the variance components analysis and the fixed effect test are in Appendix $O$.

\subsubsection{One-Way ANOVA}

A one-way ANOVA was performed following the variance components analysis to compare the means across the scaffolds. It determined if the variability between scaffolds was associated with consistent means. A boxplot of this analysis is shown in Figure 31. Additionally, the p-value was $<0.0001$, which was below the significance level of 0.05 . The null hypothesis was therefore rejected; at least one scaffold had a mean 
fiber diameter that was significantly different than the other scaffolds. See Appendix $O$ for the JMP output of this analysis.

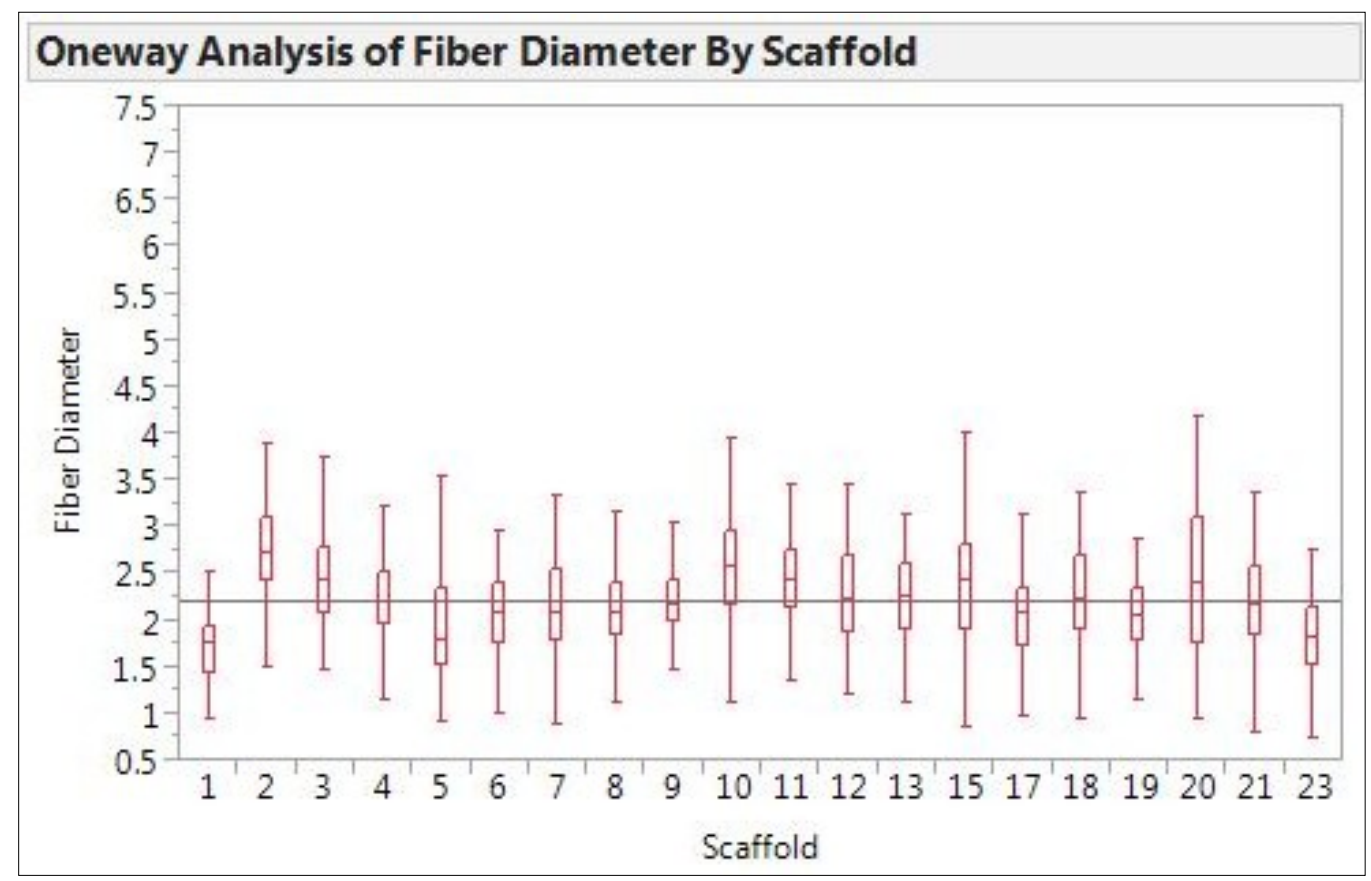

Figure 31: Boxplot from the ANOVA results for fiber diameter.

\subsubsection{Tukey's HSD Post-Hoc Test}

After determining statistical significance from the ANOVA results, Tukey's HSD post-hoc test was performed to identify which scaffold(s) had significantly different mean fiber diameter(s). Figure 32 shows the graphical results of this analysis; the circles to the right of the boxplot represent the comparisons that were made. There were multiple overlapping circles as well as distinctly separated circles. A clearer distinction was made with the Connecting Letters Report shown in Figure 33. "Level" refers to the scaffolds; scaffolds that were not connected by the same letter had significantly different mean fiber diameters. There was much overlap across the scaffolds, but it was evident that Spin 2 
and Spin 10 had significantly larger mean fiber diameters while Spin 1, Spin 23, and Spin 5 had significantly smaller mean fiber diameters.

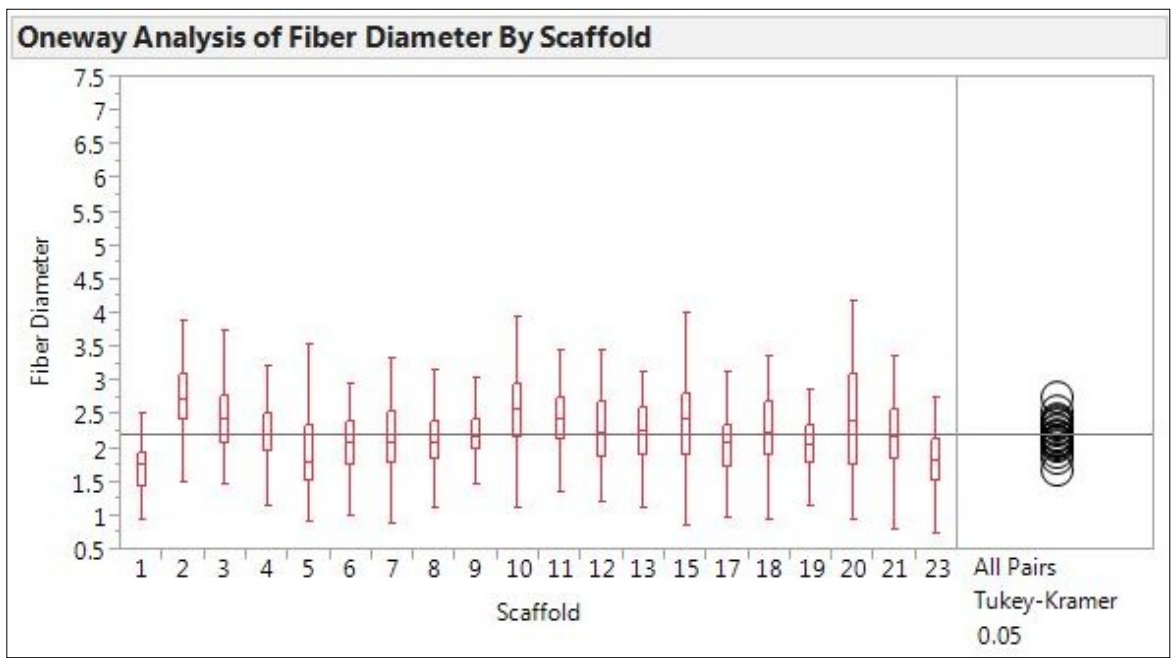

Figure 32: Graphical results of Tukey's HSD post-hoc test for fiber diameter.

\begin{tabular}{|c|c|c|}
\hline \multicolumn{3}{|c|}{ Connecting Letters Report } \\
\hline \multicolumn{2}{|c|}{ Level } & Mean \\
\hline 2 & A & 2.7864802 \\
\hline 10 & A B & 2.5779309 \\
\hline 20 & B C & 2.4497420 \\
\hline 3 & $B C D$ & 2.4280506 \\
\hline 15 & $B C D$ & 2.4101309 \\
\hline 11 & $B C D E$ & 2.3809975 \\
\hline 18 & $B C D E F$ & 2.3210074 \\
\hline 12 & $B C D E F$ & 2.2782827 \\
\hline 13 & CDEFG & 2.2223975 \\
\hline 9 & CDEF G H & 2.1894395 \\
\hline 4 & CDEFGH & 2.1812309 \\
\hline 7 & CDEF G H & 2.1339049 \\
\hline 19 & CDEFGH & 2.1250185 \\
\hline 21 & $\mathrm{CDEFGH}$ & 2.1234716 \\
\hline 6 & DEF G H & 2.1055383 \\
\hline 8 & E F G H & 2.0743556 \\
\hline 17 & F G H & 2.0459210 \\
\hline 5 & G H I & 1.9474037 \\
\hline 23 & H I & 1.8804765 \\
\hline 1 & I & 1.7085333 \\
\hline
\end{tabular}

Figure 33: Connecting Letters Report from Tukey's HSD post-hoc test for fiber diameter.

Scaffolds not connected by the same letter are significantly different. 


\subsubsection{Compliance}

\subsubsection{Overview of Compliance Results}

There were no outliers in the compliance data that needed to be removed. However, two samples could not undergo wet compliance testing. One sample (Spin 1, medial location) was pinched in the middle after overnight conditioning, which prevented insertion of the occlusion catheter. The other sample (Spin 9, distal location) had expanded during conditioning; consequently, the diameter was too large for the expansion to be captured accurately in the MATLAB viewing window. Furthermore, as stated in the Materials and Methods section, samples from the following scaffolds were tested with the new pressure transducer: dry compliance for Spin 23 and wet compliance for Spins 19, 20, 21, and 23.

Summary statistics were obtained for each scaffold, including mean compliance and standard deviations for both dry and wet samples. Then, the following analyses were performed on both sets of data: (1) REML variance components analysis, (2) one-way ANOVA, (3) Tukey's HSD post-hoc test, and (4) matched pairs t-test. The variance components analyses calculated the sources of variability in the scaffolds. ANOVAs were performed to analyze the consistency of compliance across scaffolds within the dry and wet samples. Tukey's post-hoc tests were used to determine which scaffolds had significantly different compliance. Finally, the matched pairs t-test was performed to determine if the compliance for each scaffold changed significantly after overnight conditioning. 


\subsubsection{Summary Statistics}

The raw data for both dry and wet compliance testing can be found in Appendix P. Mean compliance and standard deviations were obtained for all dry and wet scaffolds, as shown in Table $X X V$. Mean dry compliance ranged from $0.05 \% / 100 \mathrm{mmHg}$ to $0.19 \% / 100 \mathrm{mmHg}$ and the standard deviation ranged from $0.02 \% / 100 \mathrm{mmHg}$ to $0.08 \% / 100 \mathrm{mmHg}$. Mean wet compliance ranged from $0.16 \% / 100 \mathrm{mmHg}$ to $0.35 \% / 100 \mathrm{mmHg}$ and the standard deviation ranged from $0.02 \% / 100 \mathrm{mmHg}$ to $0.15 \% / 100 \mathrm{mmHg}$. The overall compliance of the dry scaffolds was $0.11 \pm$ $0.06 \% / 100 \mathrm{mmHg}$ while the overall compliance of the wet scaffolds was $0.24 \pm$ $0.09 \% / 100 \mathrm{mmHg}$. 
Table XXV: Mean compliance and standard deviations before (dry) and after (wet)

conditioning.

\begin{tabular}{|c|c|c|c|c|}
\hline Spin & $\begin{array}{c}\text { Mean Dry } \\
\text { Compliance } \\
(\% / 100 m m H g)\end{array}$ & $\begin{array}{l}\text { Dry Standard } \\
\text { Deviation } \\
(\% / 100 \mathrm{mmHg})\end{array}$ & $\begin{array}{c}\text { Mean Wet } \\
\text { Compliance } \\
(\% / 100 m m H g)\end{array}$ & $\begin{array}{l}\text { Wet Standard } \\
\text { Deviation } \\
(\% / 100 \mathrm{mmHg})\end{array}$ \\
\hline 1 & 0.06 & 0.05 & 0.17 & 0.06 \\
\hline 2 & 0.06 & 0.04 & 0.19 & 0.08 \\
\hline 3 & 0.15 & 0.05 & 0.23 & 0.09 \\
\hline 4 & 0.15 & 0.05 & 0.23 & 0.11 \\
\hline 5 & 0.10 & 0.04 & 0.20 & 0.05 \\
\hline 6 & 0.05 & 0.03 & 0.30 & 0.08 \\
\hline 7 & 0.14 & 0.06 & 0.28 & 0.15 \\
\hline 8 & 0.10 & 0.03 & 0.21 & 0.04 \\
\hline 9 & 0.14 & 0.06 & 0.19 & 0.05 \\
\hline 10 & 0.08 & 0.02 & 0.16 & 0.04 \\
\hline 11 & 0.08 & 0.04 & 0.22 & 0.03 \\
\hline 12 & 0.14 & 0.05 & 0.35 & 0.13 \\
\hline 13 & 0.19 & 0.08 & 0.20 & 0.02 \\
\hline 15 & 0.11 & 0.05 & 0.22 & 0.04 \\
\hline 17 & 0.10 & 0.07 & 0.20 & 0.04 \\
\hline 18 & 0.15 & 0.06 & 0.19 & 0.09 \\
\hline 19 & 0.16 & 0.06 & 0.30 & 0.08 \\
\hline 20 & 0.07 & 0.04 & 0.27 & 0.04 \\
\hline 21 & 0.08 & 0.05 & 0.27 & 0.03 \\
\hline 23 & 0.13 & 0.02 & 0.32 & 0.03 \\
\hline Overall & 0.11 & 0.06 & 0.24 & 0.09 \\
\hline
\end{tabular}

\subsubsection{REML Variance Components Analysis}

REML variance components analyses were performed on the dry and wet scaffolds. Scaffolds were the only random effect in the model while location was a fixed effect. Table XXVI summarizes the results. Dry compliance testing resulted in $65.546 \%$ variability within scaffolds and $34.454 \%$ variability between scaffolds; the scaffold-toscaffold variability was lower than the variability in each sample. This variability within the samples could be attributed to low repeatability of the compliance testing apparatus; the compliance readings randomly fluctuated by distinct amounts between trials on the 
same sample. Again, a reproducible process relates to low variability between scaffolds; $34.454 \%$ is relatively high, but smaller than the variability within scaffolds. Wet compliance testing resulted in $70.818 \%$ variability within scaffolds and $29.182 \%$ variability between scaffolds. Again, the scaffold-to-scaffold variability was lower than the variability within scaffolds, which is desirable for reproducibility. Furthermore, the variability between scaffolds was lower for the wet samples than the dry, which indicated that compliance was more consistent after the scaffolds were conditioned overnight.

Table XXVI: Results of the REML variance components analyses for random effects.

\begin{tabular}{|c|c|c|}
\hline & \multicolumn{2}{|c|}{ Variability (\%) } \\
\hline Compliance Test & Within Scaffold & Between Scaffolds \\
\hline Dry & 65.546 & 34.454 \\
\hline Wet & 70.818 & 29.182 \\
\hline
\end{tabular}

Finally, the fixed effect test for location determined if the compliance was consistent across locations. The p-value for dry compliance was 0.1770 while the $\mathrm{p}$-value for wet compliance was 0.7126 . Both values were above the significance level of 0.05 , failing to reject the null hypothesis. There were therefore no significant differences in compliance across locations. However, the location effect was much less significant for the wet samples than the dry samples. See Appendix $Q$ for the JMP output of the results from these analyses. 


\subsubsection{One-Way ANOVA}

One-way ANOVAs were performed on the dry and wet compliance data in order to determine if the variability between scaffolds from the variance components analyses corresponded to consistent mean compliance. Figure 34(A) and (B) shows the boxplots of these analyses for dry and wet compliance, respectively. The p-value was $<0.0001$ for dry compliance and $<0.0001$ for wet compliance, which were both below the significance level of 0.05 . Consequently, the null hypothesis was rejected; in both dry and wet scaffolds, at least one scaffold had a mean compliance that was significantly different than the other scaffolds. However, the higher F Ratio of 5.6774 for dry scaffolds indicated more significant inconsistency compared to the F Ratio of 4.6330 for wet scaffolds. See Appendix $Q$ for the JMP output of these analyses. 


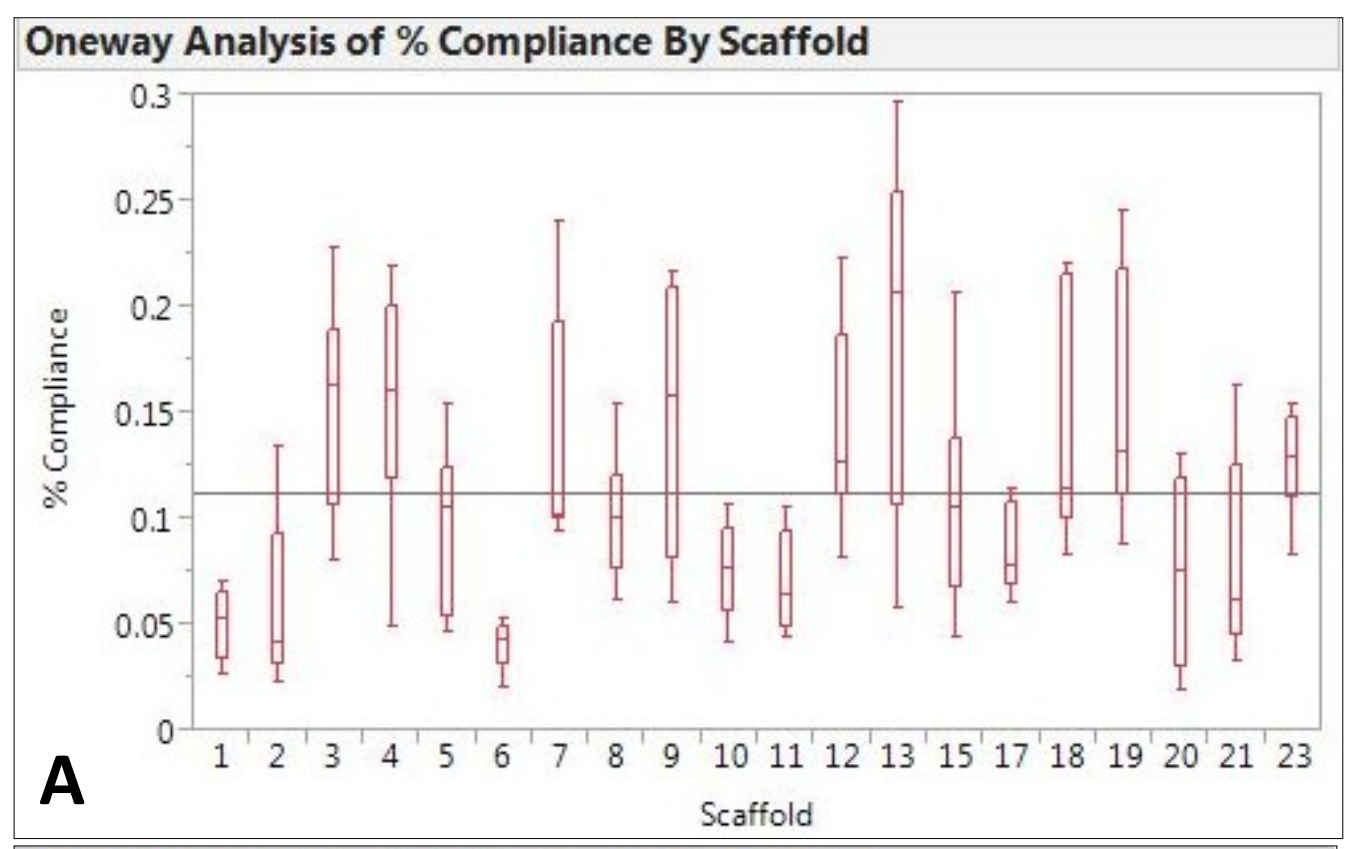

\section{Oneway Analysis of \% Compliance By Scaffold}

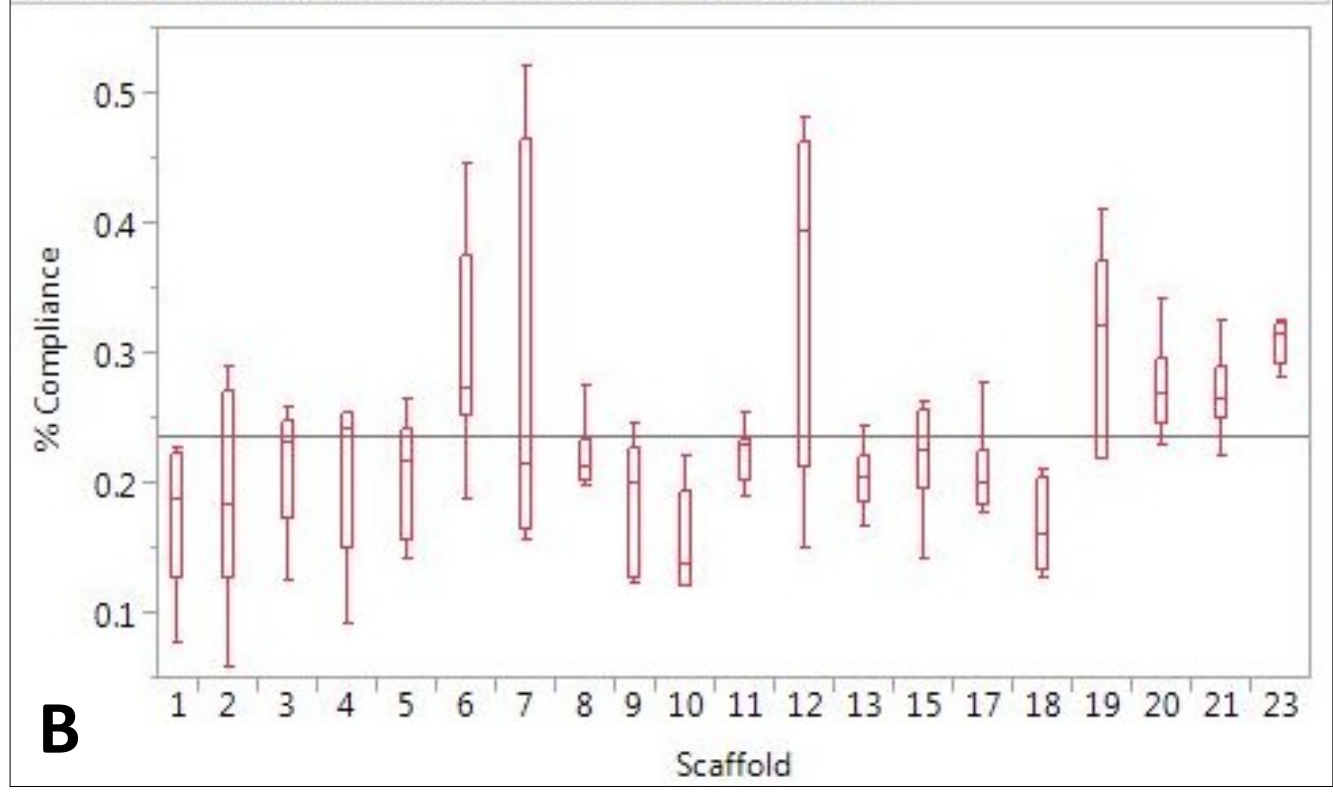

Figure 34: Boxplots of the ANOVA results of the 20 scaffolds for (A) dry compliance and $(B)$ wet compliance. 


\subsubsection{Tukey's HSD Post-Hoc Test}

After establishing that the scaffolds had significantly inconsistent mean compliance from the ANOVA results, Tukey's HSD post-hoc tests were performed to identify which scaffold(s) had significantly different mean compliance. Figure 35(A) and (B) shows the graphical results of these analyses for dry and wet scaffolds, respectively. Multiple circles overlapped in both sets of data and several circles were clearly separated. More circles seemed to overlap in a concentrated area in the wet compliance data. The Connecting Letters Reports shown in Figure 36(A) and (B) more clearly demonstrate the comparisons that were made. Again, scaffolds that were not connected by the same letter had significantly different mean fiber diameters. Fewer letters were used to connect the wet scaffolds (A-D) than the dry scaffolds (A-E); the wet scaffolds were therefore encompassed in a fewer number of significantly different groups. Furthermore, this suggests that more scaffolds had similar mean compliance when subjected to wet compliance testing rather than dry compliance testing. 


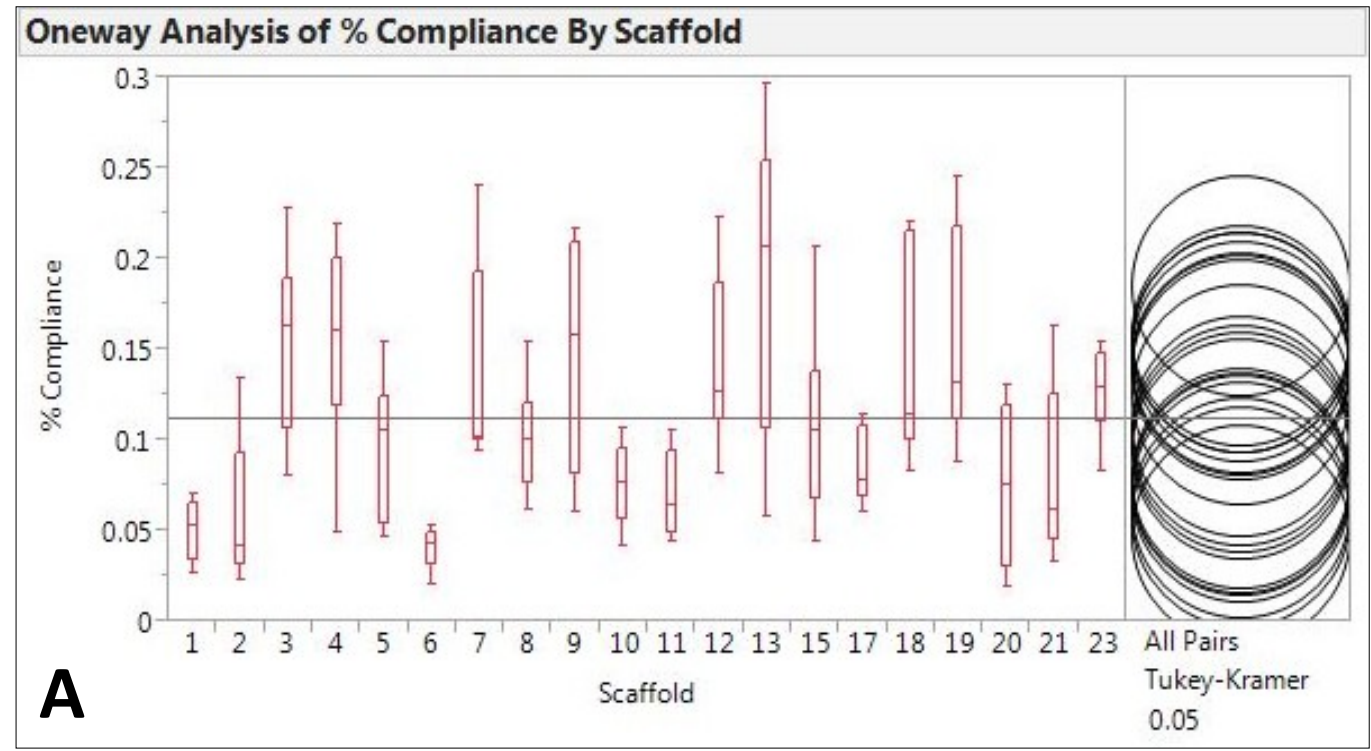

Oneway Analysis of \% Compliance By Scaffold

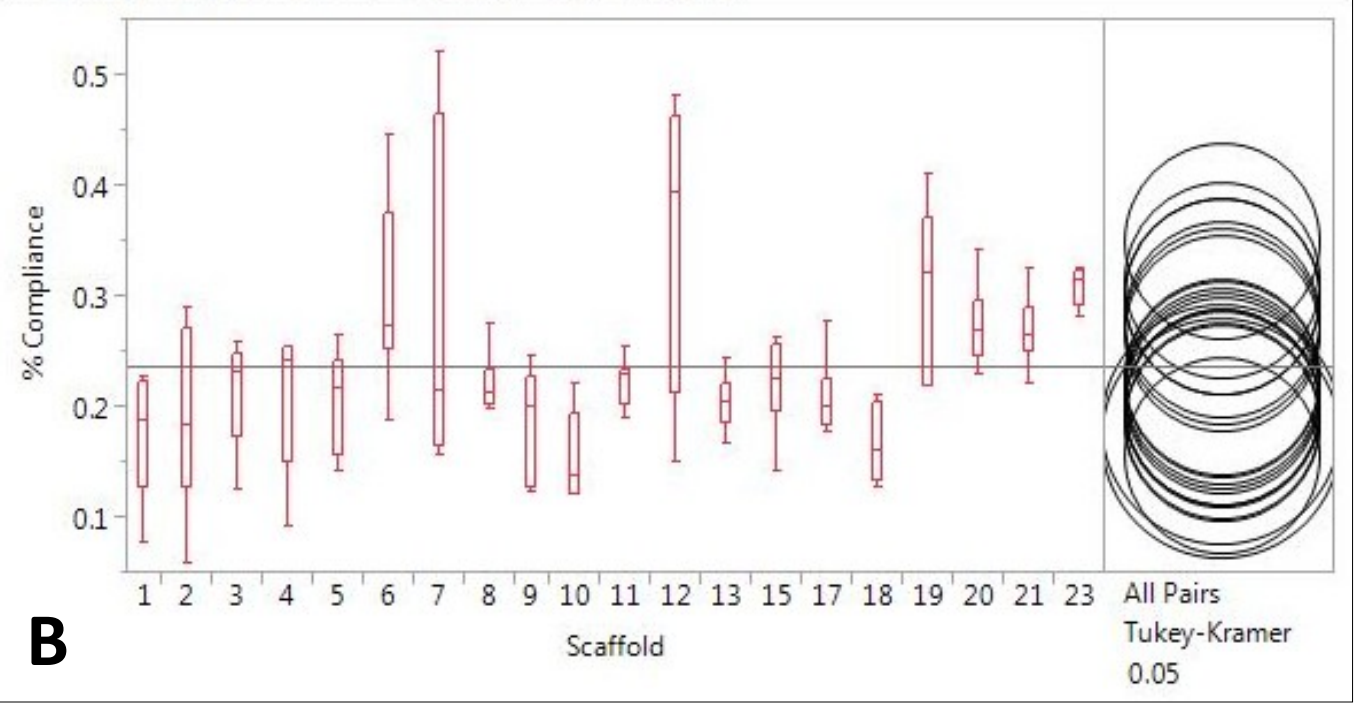

Figure 35: Graphical results of Tukey's HSD post-hoc test for $(A)$ dry compliance and (B) wet compliance. 


\begin{tabular}{|c|c|c|c|c|c|}
\hline \multicolumn{3}{|c|}{ Connecting Letters Report } & \multicolumn{3}{|c|}{ Connecting Letters Report } \\
\hline \multicolumn{2}{|c|}{ Level } & \multirow{2}{*}{$\begin{array}{r}\text { Mean } \\
0.18611831\end{array}$} & \multicolumn{2}{|c|}{ Level } & \multirow{2}{*}{$\begin{array}{r}\text { Mean } \\
0.35286950\end{array}$} \\
\hline 13 & A & & 12 & A & \\
\hline 19 & A B & 0.15876385 & 23 & A B & 0.31626743 \\
\hline 3 & $A B C$ & 0.15465173 & 6 & $A B C$ & 0.30286722 \\
\hline 4 & $A B C$ & 0.15465033 & 19 & $A B C$ & 0.30265641 \\
\hline 18 & $A B C$ & 0.15013210 & 7 & $A B C D$ & 0.28186797 \\
\hline 9 & $A B C D$ & 0.14375070 & 20 & $A B C D$ & 0.27468604 \\
\hline 12 & $A B C D$ & 0.14204092 & 21 & $A B C D$ & 0.26846310 \\
\hline 7 & A B C D & 0.13975831 & 4 & $A B C D$ & 0.22976810 \\
\hline 23 & $A B C D E$ & 0.12681566 & 3 & $B C D$ & 0.22722404 \\
\hline 15 & $A B C D E$ & 0.10847965 & 15 & $B C D$ & 0.22068075 \\
\hline 17 & $A B C D E$ & 0.10344534 & 11 & $B C D$ & 0.21673259 \\
\hline 8 & $B C D E$ & 0.10039448 & 8 & $B C D$ & 0.21256068 \\
\hline 5 & $B C D E$ & 0.09613584 & 13 & $B C D$ & 0.20184955 \\
\hline 21 & $B C D E$ & 0.08036114 & 5 & $B C D$ & 0.20140813 \\
\hline 11 & $B C D E$ & 0.07792036 & 17 & $B C D$ & 0.20079232 \\
\hline 10 & $B C D E$ & 0.07599006 & 18 & C D & 0.19033890 \\
\hline 20 & CDE & 0.07282018 & 2 & C D & 0.18738514 \\
\hline 1 & DE & 0.06369448 & 9 & $B C D$ & 0.18626834 \\
\hline 2 & $D E$ & $0.05841745 \boldsymbol{\Lambda}$ & 1 & C D & 0.17362896 \\
\hline 6 & $\mathrm{E}$ & $0.04910756 \mathrm{~A}$ & 10 & D & 0.15864212 \\
\hline
\end{tabular}

Figure 36: Connecting Letters Report from Tukey's HSD post-hoc test for (A) dry compliance and $(B)$ wet compliance.

\subsubsection{Matched Pairs t-Test}

Finally, a matched pairs t-test was performed to compare the mean compliance of each scaffold before and after overnight conditioning in the bioreactors. Table XXVII shows the results of this test. The p-value for the difference in compliance was $<0.0001$, which was below the significance level of 0.05 . The null hypothesis was rejected; the mean compliance of a scaffold before conditioning was significantly different than the mean compliance of a scaffold after conditioning. The overall mean compliance of the dry and wet scaffolds was $0.112 \% / 100 \mathrm{mmHg}$ and $0.235 \% / 100 \mathrm{mmHg}$, respectively. The mean difference between dry and wet compliance was $0.123 \% / 100 \mathrm{mmHg}$ with wet 
scaffolds being significantly more compliant than dry scaffolds. The $95 \%$ confidence interval for this difference was $(0.094,0.152)$; the mean difference between dry and wet compliance will be captured in this interval $95 \%$ of the time. Figure 37 shows a graphical depiction of these results. In the graph, the solid, horizontal, red line was the mean difference while the dotted horizontal lines represented the lower and upper bounds of the confidence interval. Since the interval did not contain zero, it followed that the difference between dry and wet compliance was significant. If the interval had contained zero, it would have followed that the difference between dry and wet compliance was zero and therefore not significant.

Table XXVII: Results of the matched pairs t-test for dry and wet compliance.

\begin{tabular}{|c|c|c|}
\hline Statistic & $\begin{array}{c}\text { Dry } \\
\text { Compliance }\end{array}$ & $\begin{array}{c}\text { Wet } \\
\text { Compliance }\end{array}$ \\
\hline $\begin{array}{c}\text { Mean Compliance } \\
\text { (\%/100mmHg) }\end{array}$ & 0.112 & 0.235 \\
\hline p-value & \multicolumn{2}{|c|}{$<0.0001$} \\
\hline $\begin{array}{c}\text { Mean Difference } \\
\text { (\%/100mmHg) }\end{array}$ & \multicolumn{2}{|c|}{0.123} \\
\hline $\begin{array}{c}\text { 95\% Confidence Interval } \\
\text { (\%/100mmHg) }\end{array}$ & \multicolumn{2}{|c|}{$(0.094,0.152)$} \\
\hline
\end{tabular}




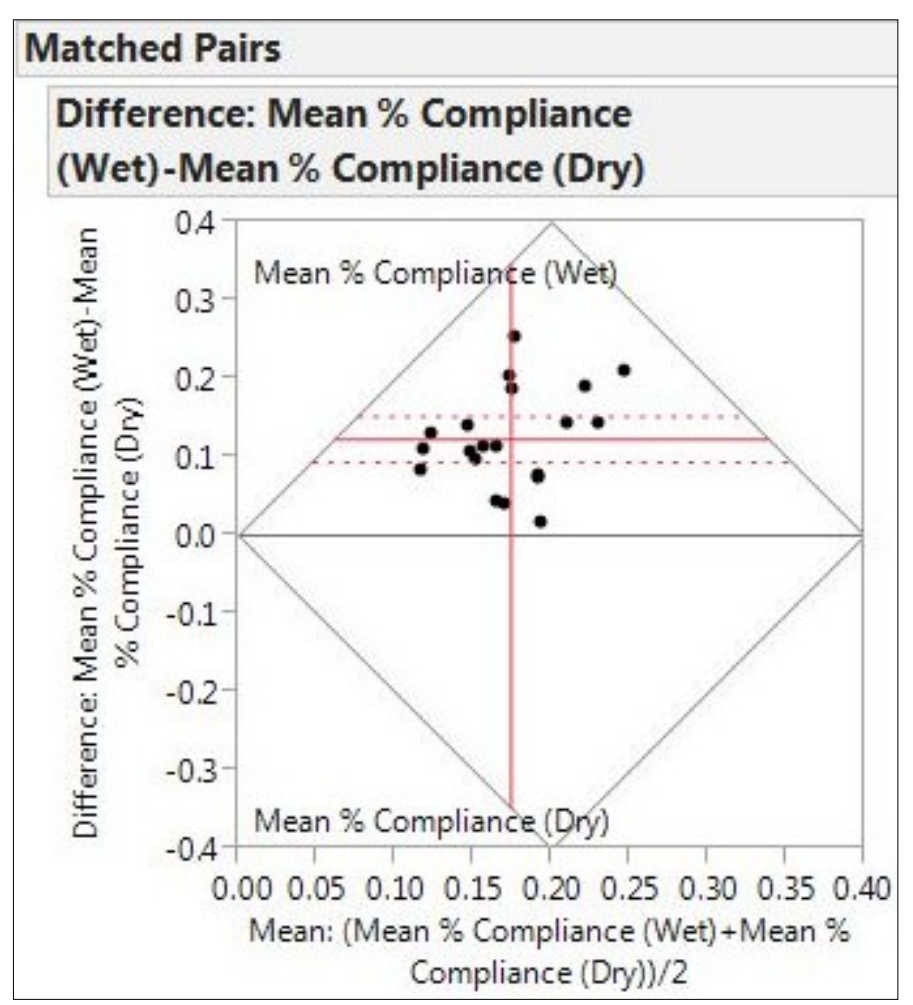

Figure 37: Matched pairs graph comparing mean dry and wet compliance of each of the 20 scaffolds. The solid, red, horizontal line represents the mean difference between dry and wet compliance samples. The dotted horizontal lines correspond to the $95 \%$ confidence interval of this difference. Since the interval did not contain zero, the means of dry and wet compliance were significantly different.

\subsection{DISCUSSION}

\subsubsection{Fiber Diameter}

Outliers were included in the fiber diameter data. There were six fiber diameter measurements that were larger than usual out of all 1,620 measurements that were made across 20 scaffolds. Some of these large fibers were flattened, as shown in Figure 38, 
which could have been a consequence of reaching the mandrel before the fibers had completely dried and solidified. The flatness of the fibers may have resulted in measurements that were larger than they would have been if the fibers were round. In Chapter 2, there were three outliers across three scaffolds from the Standard Protocol, so the ratio of outliers to the number of scaffolds decreased in this larger-scale study. Changing the timing of applying the voltage therefore appeared to reduce the incidence of larger fibers, but the problem was not completely alleviated. Furthermore, the stability of the Taylor cone slightly improved throughout most spins; the initial cone was stable, but it would eventually enter the repetitive cycle of elongation, falling, and formation of a new cone. The frequency of this cycle appeared to be slightly reduced as each cone was stable for a longer period of time before the elongation phase began. The continued inconsistency of the Taylor cone could have contributed to the presence of outliers in this study.

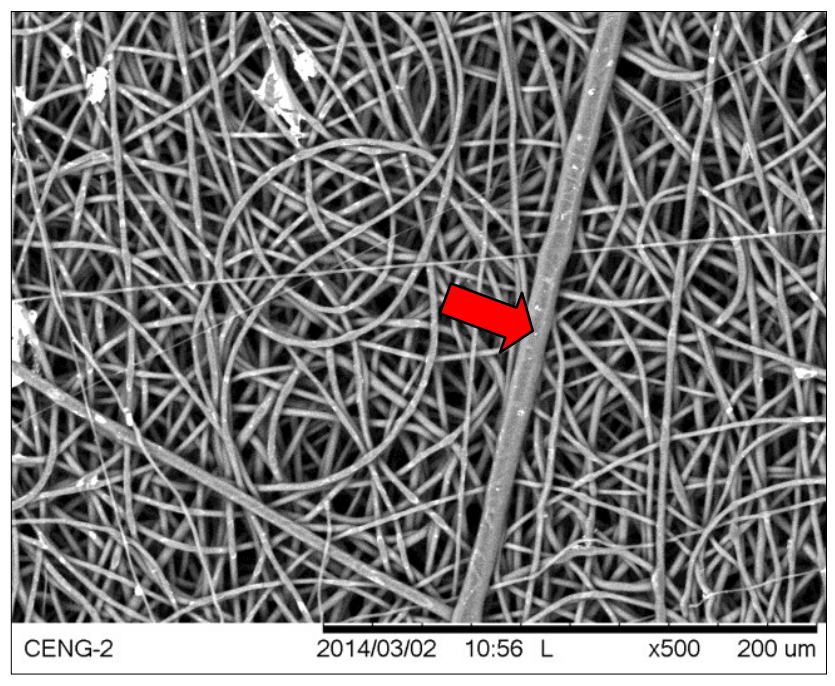

Figure 38: SEM Image 2 of Spin 3 Proximal location at 500x magnification. The large fiber, indicated by the red arrow, was flattened as a consequence of collecting on the mandrel before completely solidifying. 
It is believed that improving the consistency of the Taylor cone could greatly decrease the amount of variability in fiber diameter ${ }^{73}$. Although the timing of applying the voltage did have a slight effect on cone stability, other methods may prove to be more effective. For instance, the current set of parameters in the electrospinning protocol may not be optimal; the voltage and flow rate may need to be adjusted in order to obtain a specific balance that effectively stabilizes the Taylor cone throughout the entire electrospinning process.

The variance components analysis calculated the variability attributed to the random effects in the data, including scaffolds and images. The most important source of variability in terms of reproducibility was the variability between scaffolds, which was $14.211 \%$ of the overall variability. This was much lower compared to the variability within scaffolds at $85.454 \%$. Low scaffold-to-scaffold variability is desirable for making reproducible scaffolds; each scaffold should exhibit similar properties to any other scaffolds that are electrospun with the same process. The variability between scaffolds is higher than the $7.653 \%$ variability calculated in Chapter 2 with the Standard Protocol. However, it is important to note that the variability approximately doubled while the sample size nearly increased by sevenfold. Thus, this increase in variability between scaffolds may be inherent to the sample size; it could increase as more scaffolds are analyzed at once. Now, the reproducibility of the current Standard Protocol is characterized by a $14.211 \%$ scaffold-to-scaffold variability, which can be used as a reference for future improvements to the electrospinning system.

The residual variability within scaffolds was $85.454 \%$, which was higher than the variability between scaffolds. Again, this variability can be further characterized by the 
mean fiber diameter and standard deviation. Overall, the scaffolds exhibited a mean fiber diameter of $2.22 \mu \mathrm{m}$ and a standard deviation of $0.63 \mu \mathrm{m}$. Following a Normal distribution, $95 \%$ of the data will be within two standard deviations away from the mean. Thus, most of the fibers were within the range of $0.96 \mu \mathrm{m}$ and $3.48 \mu \mathrm{m}$, including the outliers. Three other studies using PLGA characterized scaffolds with the following mean fiber diameters and standard deviations: (1) $0.81 \pm 0.40 \mu \mathrm{m}, 1.04 \pm 0.39 \mu \mathrm{m}$, and $1.11 \pm$ $0.58 \mu \mathrm{m}$; (2) $0.957 \pm 0.357 \mu \mathrm{m}$; and (3) $4.96 \pm 0.9 \mu \mathrm{m}^{78-80}$. The overall mean and standard deviation from this thesis are larger than the statistics from the first two studies and smaller than the statistics from the third study. Implications for differences in fiber diameter size are discussed further in Chapter 4. A majority of the scaffolds from this thesis had mean diameters between $2 \mu \mathrm{m}$ and $2.5 \mu \mathrm{m}$, but three spins from this thesis, Spins 1, 5, and 23, produced scaffolds with mean fiber diameters below $2 \mu \mathrm{m}: 1.71 \pm$ $0.37 \mu \mathrm{m}, 1.95 \pm 0.64 \mu \mathrm{m}$, and $1.88 \pm 0.53 \mu \mathrm{m}$, respectively. There was no noticeable trend in humidity, temperature, or any other factor that may have contributed to this result. The standard deviations of two of these spins were also much smaller than the overall standard deviation. The ultimate goal is to obtain nanofibers in order to mimic fibers in the native ECM, which range from $50 \mathrm{~nm}$ to $500 \mathrm{~nm}^{7}$. Since the current electrospinning protocol can only produce microfibers, it is important to minimize the fiber diameter range and eliminate large fibers in order to control the variability between and within scaffolds and optimize cellular function.

While the variance components analysis calculated the amount of variability between scaffolds, ANOVA determined if the $14.211 \%$ variability corresponded to significantly different mean fiber diameters across scaffolds. ANOVA revealed with a p- 
value of $<0.0001$ that at least one scaffold had a significantly different mean fiber diameter than the others. According to Tukey's post-hoc test, there were several scaffolds that had similar means, but there were also several groups of scaffolds that had significantly different means. This was evident by the Connecting Letters Report; there were several scaffolds with similar means connected by the same letter, but nine groups of scaffolds had significantly different means from each other, as indicated by the use of nine different letters (A-I) to connect scaffolds. The $14.211 \%$ variability was large enough to produce significantly inconsistent mean fiber diameters across 20 scaffolds.

Overall, these statistics and the results from this portion of the thesis indicate that mean fiber diameter was inconsistent among scaffolds. Fiber diameter is therefore not statistically reproducible in these PLGA scaffolds with the current standard electrospinning protocol. At present, the achievable fiber diameter range is $2.22 \pm 0.63$ $\mu \mathrm{m}$. Further modifications need to be made to the electrospinning system in order to produce scaffolds with more consistent fibers. Such future work will be discussed in detail in Chapter 4.

\subsubsection{Compliance}

There were no extreme outliers in the compliance data. However, two samples could not be used in wet compliance testing: Spin 1, medial location, and Spin 9, distal location. The sample from Spin 1 had pinched in the middle during overnight conditioning, so the catheter could not be inserted through the lumen. This was due to an error when tightening the fittings. The fitting on the distal end is flexible in order to accommodate insertion of the scaffold into the bioreactor chamber. Due to its flexibility, 
this fitting was twisted too far and returned to its relaxed position overnight, twisting the scaffold in the process. The sample from Spin 9 had expanded during overnight conditioning, perhaps due to a downstream pressure buildup or a material weakness of that particular sample. Consequently, the sample was too large to capture its expansion in the MATLAB viewing window.

The variance components analysis for dry compliance testing revealed that the variability between and within scaffolds was $34.454 \%$ and $65.546 \%$, respectively. Scaffold-to-scaffold variability was lower, which is desirable for reproducibility. Overall, the mean compliance was $0.11 \% / 100 \mathrm{mmHg}$ with a standard deviation of 0.06\%/100mmHg, which encompasses a relatively small range. Wet compliance testing of the scaffolds after overnight conditioning resulted in $29.182 \%$ variability between scaffolds and $70.818 \%$ variability within scaffolds. This corresponded to an overall mean compliance of $0.24 \% / 100 \mathrm{mmHg}$ with a standard deviation of $0.09 \% / 100 \mathrm{mmHg}$. The scaffold-to-scaffold variability was again smaller than the variability within scaffolds, which is ideal for reproducibility. The variability between wet scaffolds was also less than the variability between the dry scaffolds, which indicates that scaffold compliance was more consistent across scaffolds after conditioning.

Variability within scaffolds was evident between trials on the same samples. During testing, there was no systematic increase in compliance for each successive trial, which would have suggested that PLGA was exhibiting a specific material behavior in response to compliance testing. Instead, the observed change in diameter for each trial within each sample randomly fluctuated. This random fluctuation suggests that there could have been repeatability issues with the compliance apparatus. This is highly likely 
because the student that rebuilt this apparatus is still working on improvements for repeatability and reliable compliance measurements. However, there was variability that was indicative of material-related behavior; wet scaffolds 4,7 , and 12 had noticeably larger variability than other samples, having standard deviations of $0.11 \% / 100 \mathrm{mmHg}$, $0.15 \% / 100 \mathrm{mmHg}$, and $0.13 \% / 100 \mathrm{mmHg}$, respectively. This may have been an artifact of differences in PLGA behavior developed during conditioning, especially because only three out of 20 scaffolds had such large compliance ranges. Variability within the wet scaffolds could have also been attributed to the exclusion of two samples from the analysis.

Furthermore, differences in wall thickness could have been a factor contributing to different compliance in dry and wet samples. One study showed that compliance increased as wall thickness decreased ${ }^{24}$. Previous analyses done by Colby James and Deven Patel have established that the scaffolds produced using the electrospinning system had significantly inconsistent wall thickness ${ }^{39,67}$. It is likely that the scaffolds in this study had inconsistent wall thickness which ultimately contributed to variable compliance. Furthermore, inconsistent fiber diameter may have also contributed to inconsistent compliance. It has been established in the literature that fiber diameter influences the mechanical properties of scaffolds; nanoscale variations in fiber diameter greatly impact the macroscale properties and performance of materials ${ }^{91,92}$.

ANOVA was performed to determine if the variability between scaffolds corresponded to consistent mean compliance across scaffolds. In both dry and wet scaffolds, the $\mathrm{p}$-value was $<0.0001$; at least one scaffold in each data set had a significantly different mean compliance than the others. When analyzing the F Ratios for 
both ANOVA tests, a higher F Ratio indicates more statistical significance; the F Ratio for the dry scaffolds was 5.6774 while the F Ratio was 4.6330 for wet scaffolds. Inconsistent compliance was therefore more significant in dry scaffolds than in wet scaffolds. Tukey's post-hoc tests further revealed which scaffolds had significantly different compliance. Fewer letters were used in the Connecting Letters Report for wet scaffolds (A-D) than dry scaffolds (A-E). Since scaffolds not connected by the same letter were significantly different, more wet scaffolds had similar means and fit into a fewer number of significantly different groups. This followed the lower scaffold-toscaffold variability associated with the wet samples.

Finally, a matched pairs t-test was performed to determine if the compliance changed significantly after conditioning the scaffolds overnight. Initially, it was decided to perform compliance testing before and after conditioning because the perfusion bioreactors simulate more physiologic conditions for the cultivation of blood vessel mimics through constant transmural fluid flow and induced shear stresses. It was postulated that the post-conditioned scaffolds would exhibit compliance that was more relevant physiologically. The mean difference between wet and dry compliance was $0.123 \% / 100 \mathrm{mmHg}$. The corresponding $\mathrm{p}$-value was $<0.0001$; this indicated that the difference was significant, with wet compliance significantly greater than dry compliance. One study demonstrated similar results: scaffolds that were subjected to dynamic conditioning were more compliant than those that were statically conditioned ${ }^{93}$.

There are two possible mechanisms that are believed to have contributed to the increased compliance of the scaffolds post-conditioning. First, the induced shear stresses and constant radial pressure due to transmural flow through the pores imposed constant 
mechanical forces against the walls of the scaffolds. This force could have increased the elastic capability of the scaffolds, allowing them to stretch more than they could originally. Second, the conditioning could have changed the PLGA by creating a more fibrous texture and forming nanopores on the surface of the scaffold, which were observed in a study that conditioned hydroxyapatite, chitosan, and gelatin (HCG) scaffolds for seven days ${ }^{94}$. Changing the surface features of the material may have begun to break down the walls of the scaffolds and weaken the mechanical integrity of the structures.

Other studies have defined compliance using human and animal vessels. The compliance of the human internal mammary artery has been shown to be $11.5 \pm$ $3.9 \% / 100 \mathrm{mmHg}^{84}$ while the compliance of a canine femoral artery was $10.3 \pm$ $2.3 \% / 100 \mathrm{mmHg}^{24}$. Both wet and dry compliance values from this thesis work were much less than these physiologic values. However, the standard deviations for these literature values were higher than the standard deviations calculated in this study $(0.06 \% / 100 \mathrm{mmHg}$ for dry compliance and $0.09 \% / 100 \mathrm{mmHg}$ for wet compliance).

Although physiologic compliance was not matched, the electrospun scaffolds demonstrate narrower compliance ranges, which is ideal for reproducibility. Furthermore, previous work with this compliance apparatus demonstrated that the compliance of a porcine artery, PLGA soaked in PBS, and ePTFE was $2.983 \%, 0.5402 \%$, and $0.6063 \%$, respectively ${ }^{87}$. The mean compliance value for the conditioned scaffolds is smaller than all of these values. It is known that PLGA is not a compliant material, so if physiologic compliance is desired in blood vessel mimics, a new material should be investigated for use in the future ${ }^{85,87}$. 
Overall, these results from the compliance testing portion of the thesis suggest that compliance is inconsistent between PLGA scaffolds. Therefore the scaffolds do not have statistically reproducible compliance. This was seen in both dry and wet compliance testing. The compliance ranges for dry and wet scaffolds are currently $0.11 \pm$ $0.06 \% / 100 \mathrm{mmHg}$ and $0.24 \pm 0.09 \% / 100 \mathrm{mmHg}$, respectively. Compliance did significantly increase after the scaffolds were conditioned in perfusion bioreactors overnight. However, the compliance of these scaffolds is far less than physiologic compliance.

\subsubsection{Challenges and Limitations}

Challenges were faced during scaffold fabrication. First, two scaffolds were too thin after electrospinning. During the process, the solution was dripping from the bottom of the Taylor cone, so most of the solution was not reaching the mandrel. It was not clear why this happened, but it may have been due to the environmental effects of temperature and humidity or an error in measuring the PLGA and $\mathrm{CHCl}_{3}$ during the mixing process. Furthermore, one section of a scaffold (Spin 22) stuck to the mandrel. The problem of scaffolds sticking to the mandrels was supposedly alleviated when new mandrels were machined, as discussed at the end of Chapter 2 . Since only a portion of the scaffold was stuck, the problem was most likely due to inadequate sanding and preparation of the mandrel before electrospinning.

A limitation of the fiber diameter data was the possibility of temperature and humidity having an effect on the electrospinning process. As discussed in Chapter 2, these environmental factors cannot be controlled in the current electrospinning setup; 
they are only monitored and recorded at the beginning of each spin. It has been shown in other studies that temperature and humidity affect the electrospinning process ${ }^{64,65,81}$. The environmental fluctuations therefore may have contributed to variability in fiber diameter. This will be discussed further in Chapter 4.

Additional challenges of this study occurred during compliance testing. The scaffolds were difficult to slide over the occlusion catheter because the inner diameter of the scaffolds and the diameter of the inflation zone of the catheter were similar. Consequently, some of the wet compliance samples were so tightly fitted around the catheter that it may have restricted pressurization of the catheter. Another drawback of the compliance apparatus was the repeatability of the measurements. The pressure would creep up after each trial, so the pressure difference was not consistent across all trials. It was also difficult to stop exactly at the upper voltage limit of $4.5 \mathrm{~V}$ every time with the initial 15 psi pressure transducer. With the new 50 psi pressure transducer, it was much easier to not exceed the new upper limit of $2 \mathrm{~V}$ because there was more resistance as the syringe was depressed and approached $2 \mathrm{~V}$. There did not appear to be a systematic difference in performance between the two pressure transducers; the 50 psi transducer produced larger pressures, but they were accommodated by larger diameter changes. Since the compliance calculation was a ratio of these two parameters, the resultant compliance values were comparable to those obtained with the 15 psi transducer. Additionally, the first four scaffolds were electrospun and tested according to a different timeline than the remaining 16 scaffolds. This could have caused differences in wet compliance readings since the 16 scaffolds sat in the desiccator for a few weeks before 
conditioning. However, there were no obvious differences between the results of the first four scaffolds compared to the 16 scaffolds.

Finally, in addition to switching the pressure transducers to improve repeatability, other improvements will eventually be made to the compliance apparatus. There are several parameters involved in the experimental setup that may affect the compliance results, including the tautness of the catheter when clamped into place and the lighting adjustments that are made to obtain clearer images during the live video feed. These parameters need to be tested and defined in order to ensure that compliance is tested equally across all samples.

\subsection{CONCLUSION}

The overall aim of this large-scale study was to further characterize the reproducibility of the standard electrospinning protocol using fiber diameter and compliance data. The following goals were used to accomplish this task: (1) analyze the reproducibility of fiber diameter; (2) characterize the reproducibility of scaffold compliance before and after conditioning the scaffolds overnight in perfusion bioreactors; and (3) determine if scaffold compliance significantly changed after conditioning. The goals are summarized below.

(1) 20 scaffolds were electrospun with the Standard Protocol. The overall mean fiber diameter was $2.22 \mu \mathrm{m}$ and the standard deviation was $0.63 \mu \mathrm{m}$. It was determined that the variability between scaffolds was lower than the variability within scaffolds. Lower scaffold-to-scaffold variability is desirable for reproducibility. ANOVA further 
revealed that this percentage was large enough to produce a significantly inconsistent mean fiber diameter across scaffolds.

(2) The mean compliance for scaffolds before and after conditioning was $0.11 \pm$ $0.06 \% / 100 \mathrm{mmHg}$ and $0.24 \pm 0.09 \% / 100 \mathrm{mmHg}$, respectively. The variability between scaffolds was lower than the variability within scaffolds for both dry and wet scaffolds. It was also demonstrated that the variability between scaffolds was lower in wet compliance testing than in dry compliance testing. Since the wet scaffolds had lower scaffold-toscaffold variability, compliance was more reproducible after conditioning the scaffolds in perfusion bioreactors overnight. Although the variability was lower in the wet scaffolds, ANOVA determined that the mean compliance was significantly inconsistent across scaffolds both before and after conditioning. However, compliance was more inconsistent across scaffolds before conditioning.

(3) Overnight conditioning was found to significantly increase compliance. Conditioning provided a more physiologic environment for the scaffolds by inducing shear stresses and constant radial pressure with transmural flow.

The current standard electrospinning protocol in the Cal Poly Tissue Engineering lab is characterized with these levels of variability in fiber diameter and compliance. Future research can be conducted to improve the protocol by finding the optimum set of parameters that reduce between-scaffold variability and obtain statistically consistent properties. The next chapter will discuss future work in greater detail. 


\section{DISCUSSION AND CONCLUSION}

\subsection{SUMMARY AND AIMS OF THE THESIS}

Electrospinning is an in-house fabrication technique used to create PLGA scaffolds for BVMs in the Cal Poly Tissue Engineering lab. BVMs are tissue-engineered in vitro models that are used for testing stents and other intravascular devices.

Electrospinning is an advantageous process because scaffold properties can be controlled through altering process parameters, such as flow rate, voltage, gap distance, and solution concentration. Scaffolds provide mechanical support and influence cellular function within tissue-engineered constructs. It is therefore important to establish an electrospinning process that creates scaffolds with reproducible material and mechanical properties in order to develop BVMs that serve as reliable models.

Ultimately, the aim of this thesis was to characterize the reproducibility of the properties of electrospun PLGA scaffolds. Preliminary studies involved electrospinning scaffolds using several experimental protocols with varying flow rates and voltages in an attempt to reduce fiber diameter. Smaller fibers are ideal for scaffolds because they

mimic the fibers of the native ECM and promote optimal cellular functions ${ }^{7,68,70}$. However, it was desirable to attain smaller fibers while maintaining consistent fiber diameters across scaffolds. Thus, after identifying that Protocol B produced the smallest mean fiber diameter, the reproducibility of Protocol B was compared to the reproducibility of the Standard Protocol both qualitatively and quantitatively. Reproducibility was defined with the amount of variability between scaffolds; the more reproducible protocol was identified by lower scaffold-to-scaffold variability, which was 10.668\% and 7.653\% for Protocol B and the Standard Protocol, respectively. SEM 
images further demonstrated that Protocol B produced obvious variation in fiber size and clumps while fibers from the Standard Protocol had superior morphology. Using these analyses, the Standard Protocol was determined to be more reproducible.

A large-scale study was then performed with the Standard Protocol to further characterize the reproducibility of the scaffolds with fiber diameter and compliance. Additionally, compliance was compared before and after conditioning the scaffolds in perfusion bioreactors overnight to simulate physiologic conditions of blood vessels and determine if the mechanical response of the scaffolds changed after the conditioning process. SEM and several statistical methods were performed to define reproducibility with scaffold-to-scaffold variability. The mean fiber diameter established from this study was $2.22 \pm 0.63 \mu \mathrm{m}$ while mean compliance was $0.11 \pm 0.06 \% / 100 \mathrm{mmHg}$ before conditioning and $0.24 \pm 0.09 \% / 100 \mathrm{mmHg}$ after conditioning. Fiber diameter demonstrated $14.211 \%$ variability between scaffolds while compliance demonstrated $34.454 \%$ and $29.182 \%$ variability between scaffolds before and after conditioning, respectively. Not only was compliance more consistent post-conditioning, but the scaffolds were also significantly more compliant. Although reproducibility was defined using percentages of scaffold-to-scaffold variability, it was determined that these values corresponded to significantly inconsistent mean fiber diameter and compliance across scaffolds. Now that the standard electrospinning protocol has been characterized with the current amount of variability, these values will provide references for future work to assess the efficacy of improvements to the electrospinning system. 


\subsection{COMPARISON OF RESULTS TO LITERATURE}

Previous students' theses also analyzed fiber diameter, but tested tensile strength of the PLGA scaffolds instead of compliance ${ }^{10,11,67}$. They demonstrated similar results to this thesis with fiber diameter measurements; mean fiber diameter was significantly different across scaffolds. However, mean fiber diameter was lower in this study at 2.22 $\mu \mathrm{m}$. Tiffany, Yvette, and Deven reported mean fiber diameters of 5-6 $\mu \mathrm{m}, 2.74 \mu \mathrm{m}$, and $2.556 \mu \mathrm{m}$, respectively ${ }^{10,11,67}$. Tiffany had the largest mean fiber diameter because the positive polarity voltage supply was in use at the time while Deven achieved a smaller mean diameter due to the implementation of a negative polarity voltage supply, which has been shown to produce smaller fibers upon increasing voltage ${ }^{62,64,80}$. However, Deven's electrospinning protocol was different for the BBB model, which could have contributed to a larger diameter than what was achieved in this study. The lowest mean diameter obtained in Yvette's study was larger than in this thesis because she experimented with several different parameters and therefore operated with a different electrospinning protocol. The current Standard Protocol operated with a negative voltage is the most effective in terms of achieving the smallest mean fiber diameter of the studies performed in the Cal Poly lab. However, consistency of fiber diameter across scaffolds remains an issue that needs to be improved.

The DOE from Yvette's thesis predicted that reducing the flow rate would decrease fiber diameter and Deven's thesis claimed that increasing the voltage with a negative polarity would also decrease fiber diameter ${ }^{11,67}$. These predictions are consistent with literature ${ }^{60,62,64,80}$. These trends were seen in the preliminary studies of this thesis: Protocol B had a lower flow rate and higher applied voltage and overall produced a lower 
mean fiber diameter than the Standard Protocol. However, this reduction was not significant. Additionally, the scaffolds from Protocol B had more obvious variability, poor fiber morphology, and large clumps. Further reduction to a flow rate of $5 \mathrm{~mL} / \mathrm{hr}$ and increase in voltage to $-20 \mathrm{kV}$ did not reduce fiber diameter. Instead, the scaffolds were more inconsistent with varying fiber size and the formation of large clumps. It has been demonstrated in literature that there is a threshold flow rate for fiber diameter reduction; above and below this threshold, more variation in fiber size and morphology is observed $^{73}$. Thus, the flow rates used in the preliminary studies may have been above that threshold value. Further reduction in flow rate could successfully reduce fiber diameter and produce a narrow distribution of fibers. Further experiments to test this theory could not be performed due to time constraints.

Studies in the literature have shown the following fiber diameter ranges with PLGA: $0.81 \pm 0.40 \mu \mathrm{m}, 1.04 \pm 0.39 \mu \mathrm{m}, 1.11 \pm 0.58 \mu \mathrm{m}, 0.957 \pm 0.357 \mu \mathrm{m}$, and $4.96 \pm$ $0.9 \mu \mathrm{m}^{78-80}$. Four of the five mean fiber diameters and standard deviations are lower than the mean diameter achieved in this thesis $(2.22 \pm 0.63 \mu \mathrm{m})$ while the fifth mean and standard deviation are higher. The slightly higher standard deviation from this thesis can be attributed to the formation of six large fibers that were represented as extreme outliers in the data. Differences in mean fiber diameter and variability across these studies potentially arise from differences in electrospinning parameters, including flow rate, voltage, solution concentration, and the type of solvent. For instance, one study used a $20 \% \mathrm{w} / \mathrm{v}$ PLGA solution with an applied voltage of $25 \mathrm{kV}$ and a flow rate of $2 \mathrm{~mL} / \mathrm{hr}^{78}$. Another study used a 15\% w/v PLGA solution with 1,1,1,3,3,3-hexafluoro-2-propanol (HFP) as the solvent as well as an applied voltage of $18 \mathrm{kV}$ and a flow rate of $1 \mathrm{~mL} / \mathrm{hr}^{79}$. 
The study that produced the mean diameter of $4.96 \mu \mathrm{m}$ also used chloroform as the solvent, but created a PLGA solution with a concentration of $8 \mathrm{wt} \%{ }^{80}$.

Although inconsistent mean fiber diameter was common to past theses and the present thesis, the scaffolds demonstrated consistent tensile strengths in Tiffany and Deven's theses ${ }^{10,67}$. In the present thesis, compliance was assessed and found to be significantly inconsistent. Since fiber diameter influences mechanical properties, inconsistent mechanical properties of these scaffolds may be attributed to variability in fiber diameter ${ }^{91,92}$. Additionally, a senior project that used this compliance apparatus demonstrated that PLGA scaffolds had an average compliance of $0.5402 \%$ after being soaked in PBS for 15 minutes $^{87}$. The scaffolds in this study were less compliant with a mean compliance of $0.11 \% / 100 \mathrm{mmHg}$ and $0.24 \% / 100 \mathrm{mmHg}$ before and after conditioning, respectively. The conditioned scaffolds were significantly more compliant than the dry, but still not as compliant as the scaffolds that were soaked in PBS. The study also tested a porcine artery and ePTFE for comparison, which had $2.983 \%$ and $0.6063 \%$ compliance, respectively ${ }^{87}$. PLGA had much lower compliance than both of these materials.

Other studies have also compared the compliance of polymers to native vessels $^{24,84,85}$. Physiologic compliance has been difficult to achieve in tissue-engineered constructs, though PGS was a promising polymer that demonstrated similar compliance to porcine carotid arteries at burst pressures up to $56 \mathrm{mmHg}^{85}$. The scaffolds from this thesis are far less compliant than the internal mammary artery and canine femoral artery, which were shown to have $11.5 \pm 3.9 \% / 100 \mathrm{mmHg}$ and $10.3 \pm 2.3 \% / 100 \mathrm{mmHg}$ compliance, respectively ${ }^{24,84}$. PLGA therefore does not exhibit physiologic compliance. 
The characterization that was established in this thesis is unique for the particular polymer, solvent, and electrospinning protocol currently utilized in the Cal Poly lab. Other studies using PLGA use various electrospinning parameters, including solvent, flow rate, voltage, gap distance, and solution concentration. There is also no standard level of reproducibility to which to compare these results. The current Standard Protocol is capable of producing microfibers, but nanofibers are generally better in order to mimic

the native ECM and promote optimal cellular behavior ${ }^{7,68,70}$. Furthermore, a reproducible electrospinning process is important so that scaffolds have consistent material and mechanical properties. Currently, the Standard Protocol in the Cal Poly lab produces scaffolds with neither consistent fiber diameter nor consistent compliance. Various parameters can be changed in future experiments to make improvements to this process, which are discussed in more detail later in this chapter.

\subsection{CHALLENGES AND LIMITATIONS}

A few challenges were faced during this thesis work. In the preliminary studies, an entire scaffold stuck to the mandrel. The mandrels had been in use for awhile and the surfaces were no longer in good condition, so several new mandrels were made. The new mandrels were an improvement, but toward the end of this thesis, the proximal section of a scaffold stuck to one of the mandrels. However, since only one section was affected, this occurrence was most likely due to poor sanding and preparation of the mandrel before electrospinning. The preparatory steps in the protocol are extremely important to remove the scaffolds from the mandrels effortlessly and to prevent wasting time and materials. Additionally, two other scaffolds turned out thin. The solution was dripping 
from the bottom of the cone during electrospinning, so not all of the solution was making it to the mandrel. Although the definite cause is unclear, it may have been due to inexact measurements of PLGA and $\mathrm{CHCl}_{3}$ during the mixing process that formed a less viscous solution. Another possible cause of this phenomenon could have been environmental effects; temperature and humidity have been shown to affect the electrospinning process and fiber morphology ${ }^{64,65,81}$. This issue will be discussed further in the Future Work section.

ImageJ presented another challenge. Manually measuring fiber diameters in ImageJ left much room for human error. Each time a new session began, the scale needed to be calibrated. Calibrations between sessions could have been slightly different, which would have therefore led to subsequent inaccurate measurements. Furthermore, making accurate, straight lines that were perpendicular to the edges of the fibers was challenging. If the lines were not perpendicular, the true diameter of the fiber was not measured.

There were also a few limitations in this study. The repeatability of the compliance tester may have affected the compliance data. Another student rebuilt the fixture this year and was still making improvements while these experiments were performed, which was why a new pressure transducer was implemented. Additionally, many parameters of the compliance protocol were undefined, including the tautness of the occlusion catheter and the lighting adjustments of the microscope. It was uncertain if inconsistencies in these parameters affected the change in diameter that was captured through the MATLAB video feed. Compliance may have therefore been inconsistent across scaffolds due to different catheter tautness and lighting settings. 
Time was also a limiting factor in the preliminary spins. When exploring the various experimental protocols, there was only time to test five protocols. Far more protocols could have been tested in an effort to reduce fiber diameter. If time permitted, flow rate would have been investigated further by reducing the rate in small increments while keeping other parameters constant; this could have potentially identified a

threshold flow rate, as discussed in literature ${ }^{73}$. However, a time frame had to be imposed in order to move forward into the large-scale reproducibility study, which was the focus of this thesis. Timing was also an issue to consider for the electrospinning process when reducing the flow rate in the preliminary spins. It took approximately 40 minutes to electrospin one scaffold with the Standard Protocol. Reducing the flow rate even further would increase the duration of this process, which would be extremely inefficient for producing scaffolds in a timely manner.

\subsection{FUTURE WORK}

Several issues could be the focus of future work for scaffold fabrication in the Cal Poly lab. Research could be conducted on obtaining Taylor cone stability during electrospinning, investigating environmental effects on the electrospinning process, further reducing fiber diameter, finding a more compliant material for the BVM application, using new imaging techniques, and investigating a new material to use for the mandrels. Each of these is discussed in the following sections. 


\subsubsection{Taylor Cone Stability}

It is believed that stability of the Taylor cone throughout the electrospinning process is necessary for increasing the reproducibility of these PLGA scaffolds. Another study with Nylon 6 demonstrated that a low flow rate had the following effects on the electrospinning process: stabilized the Taylor cone, formed smaller droplets, stabilized the jet, produced a narrow fiber diameter distribution, and obtained uniform nanofiber morphology $\mathrm{y}^{73}$. The presence of large fibers could be a consequence of the cone forming, elongating, and falling off of the tip of the needle repeatedly. One possible way to obtain a stable cone could be finding a way to balance the surface tension and the electrostatic forces of the polymer solution. This was attempted by changing the timing of applying the voltage; it was postulated that allowing a larger bead to form would provide more resistance to the repulsive charges, therefore preventing the cone from tearing off of the needle. The cone was stable for a slightly longer period of time, but it was never stable for the entire process. To achieve a stable cone, more research could be performed specifically on finding the balance between voltage and flow rate, which was investigated in the preliminary studies of this thesis. As mentioned before, a threshold flow rate may not have yet been reached, which could have affected the stability of the cone and subsequent variability in fiber diameter ${ }^{73}$. It could be more effective to begin with the voltage and flow rate of the standard protocol $(-12 \mathrm{kV}$ and $5.5 \mathrm{~mL} / \mathrm{hr})$ and keep the voltage constant while reducing the flow rate by increments of $0.1 \mathrm{~mL} / \mathrm{hr}$ across multiple spins in order to see if flow rate alone had an impact on improving the stability of the Taylor cone. 
Finally, environmental effects could have an impact on cone stability and subsequent variability of the scaffolds, as demonstrated in the literature ${ }^{64,65,68,81}$. An additional analysis that was performed with the preliminary data from this thesis also suggested that environment had an impact on fiber diameter. This is discussed in detail in the following section.

\subsubsection{Environmental Effects}

Humidity and temperature have been shown in other studies to affect fiber morphology in electrospun scaffolds ${ }^{64,65,81}$. One study in particular claimed that humidity has a great impact on electrospinning and that humidity below $35 \%$ is ideal for a continuous jet ${ }^{68}$. A REML variance components analysis using a mixed effects GLM was performed on the preliminary data to determine if temperature and humidity had a significant effect on fiber diameter. The variables included in this analysis were Protocol, Location, Temp, Humid, and Scaffold. All of these variables were fixed effects except Scaffold, which was a random effect. Protocol controlled for the effect of either Protocol B or the Standard Protocol on fiber diameter. Location controlled for the effect of proximal, medial, and distal locations along the scaffolds. Temp and Humid consisted of recordings that were taken at the beginning of each spin. Scaffold included three scaffolds that were created with Protocol B and three scaffolds that were made using the Standard Protocol, as seen in the preliminary studies from Chapter 2.

Figure 39 and Figure 40 below show the results of this analysis. Temperature and humidity had p-values of 0.0100 and 0.0057 , respectively (Figure 39). These values were below the significance level of 0.05 , so both had significant effects on fiber diameter. 
Additionally, the variability between scaffolds was 0\% (Figure 40). This suggests that temperature and humidity explained all of the variability that had once been attributed to scaffold-to-scaffold variability.

\begin{tabular}{|lrrrrc|}
\hline Fixed Effect Tests & & & \\
\hline Source & Nparm & DF & DFDen & F Ratio & Prob $>$ F \\
Protocol & 1 & 1 & 2 & 0.7728 & 0.4721 \\
Location & 2 & 2 & 10 & 0.4333 & 0.6600 \\
Temp & 1 & 1 & 2 & 98.6384 & $0.0100^{*}$ \\
Humid & 1 & 1 & 2 & 174.5104 & $0.0057^{*}$ \\
\hline
\end{tabular}

Figure 39: Results of the fixed effect tests in the REML variance components analysis. Temp and Humid had significant effects on fiber diameter. Protocol and Location did not significantly affect fiber diameter.

\begin{tabular}{|lrrrrrr|r|}
\hline \multicolumn{7}{|l|}{ REML Variance Component Estimates } \\
\hline \multicolumn{7}{|l}{ Var } \\
Random Effect & Var Ratio & Component & Std Error & 95\% Lower & 95\% Upper & Pct of Total \\
Scaffold[Protocol] & -0.312574 & -0.006743 & 0.003247 & -0.013107 & -0.000379 & 0.000 \\
Residual & & 0.0215731 & 0.0096478 & 0.0105321 & 0.0664407 & 100.000 \\
Total & & 0.0215731 & 0.0096478 & 0.0105321 & 0.0664407 & 100.000 \\
\hline
\end{tabular}

Figure 40: Results of the REML variance components analysis showing the variability of the random effects. There was no variability between scaffolds $(0 \%)$.

Currently, the electrospinner is set up in a fume hood (Figure 41) with a monitor that displays temperature and humidity (Figure 42). Both levels fluctuate between days, but the difference in humidity is more drastic than temperature. Temperature and humidity levels are recorded at the beginning of every spin, but they cannot be controlled with the existing equipment. According to this analysis, temperature and humidity should 
be fixed at optimum levels in the electrospinning process. Maintaining these environmental factors at constant levels could impact the reproducibility of the scaffolds by making the fibers more consistent as well as the mechanical properties. The equipment may therefore need to be relocated to a place where environment can be more easily controlled. However, before major changes are made to the current system, the accuracy of the monitor first needs to be investigated; the observed fluctuations in temperature and humidity could have been due to an inaccurate sensor.

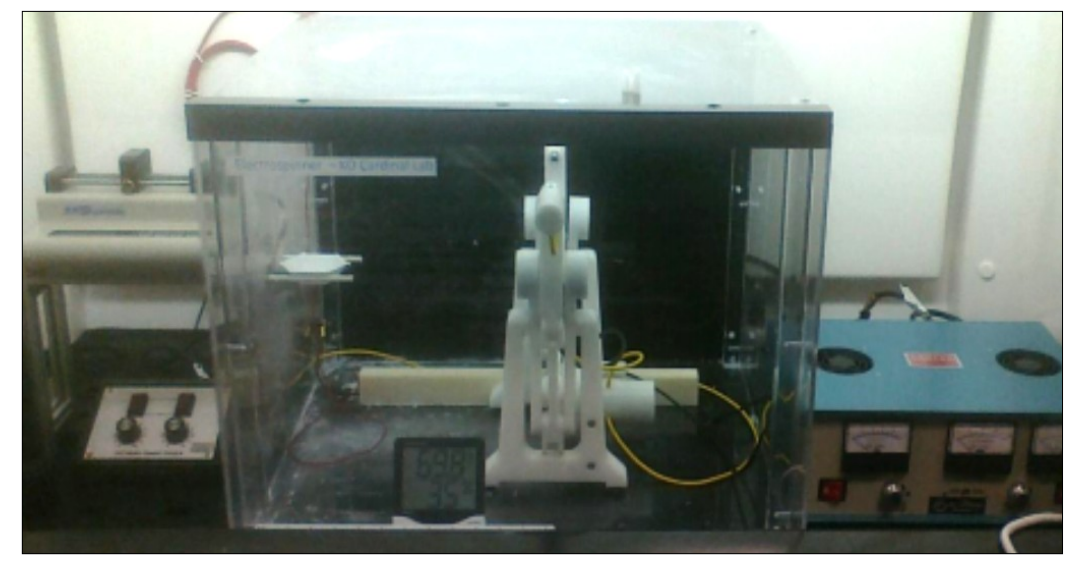

Figure 41: Current electrospinning setup in a fume hood.

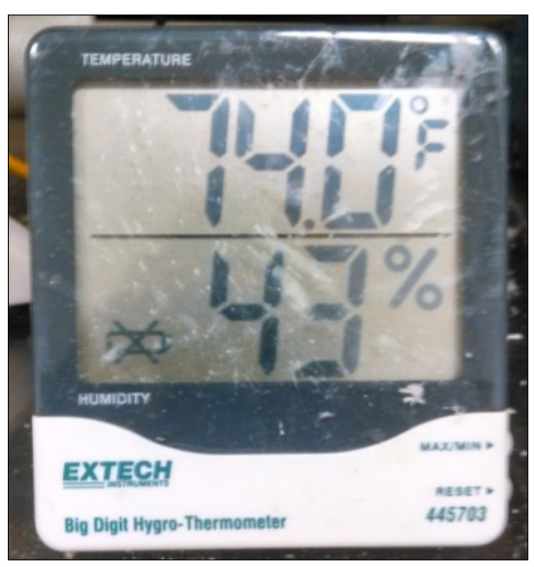

Figure 42: Monitor displaying temperature $\left({ }^{\circ} \mathrm{F}\right)$ and humidity $(\%)$. 


\subsubsection{Reduction of Fiber Diameter}

As mentioned throughout this thesis, small fiber diameters are desirable in scaffolds because they mimic fibers in the native ECM and promote ideal cellular responses, such as proliferation, differentiation, and production of $\mathrm{ECM}^{7,68,70}$. Several electrospinning parameters can be altered to reduce fiber diameter, including reducing the flow rate ${ }^{57,62}$, increasing the voltage ${ }^{62,63,66}$, decreasing the solution concentration ${ }^{61,62,66}$, and increasing the gap distance ${ }^{62,63,66}$. More experiments can be performed to obtain smaller fibers by testing each of these parameters However, other studies have also used other solvents with PLGA and obtained smaller fibers, including tetrahydrofuran (THF); $N, N$-dimethylformamide (DMF); and 1,1,1,3,3,3-hexafluoro-2-propanol (HFP) ${ }^{74,79}$. It may therefore be beneficial to switch to a different solvent to effectively reduce fiber diameter.

\subsubsection{Compliance}

In order to obtain more accurate readings with the compliance tester, experiments need to be performed to test the effects of the different parameters involved in the compliance protocol. Furthermore, if compliance is a key mechanical property to be mimicked in BVMs, a more compliant polymer should be considered for the Cal Poly lab. PLGA is known to be a less compliant material ${ }^{85,87}$. Although physiologic compliance is difficult to achieve in tissue-engineered constructs, one study demonstrated that PGS had similar compliance to a native vessel at burst pressures up to $56 \mathrm{mmHg}^{85}$. This polymer, or another similar polymer, could be considered for future use. 


\subsubsection{Imaging Techniques}

New imaging techniques could be researched for potential use in the Tissue Engineering lab. Currently, SEM is used to analyze scaffold characteristics and assess the cell coverage on BVMs. SEM is useful for surface visualization, but three-dimensional data cannot be obtained using this technique ${ }^{95}$. BVMs and other biological samples must also undergo special preparation before using SEM. Additionally, the electron beam in this technique may damage the sample and cause temporary or permanent changes in the structure $^{96}$. Other techniques that are less damaging and have the ability to provide threedimensional data should be investigated. One potential technique is optical interferometry, which uses the interference of beams of light to create three-dimensional surface topographies for analysis ${ }^{97}$. Using this technique, high resolution images can be obtained. Furthermore, biological samples are not damaged by the beams and do not require special preparation.

In addition to new imaging techniques, other areas of the scaffolds could be analyzed during characterization. For instance, images could be obtained from the abluminal surface and from different layers throughout the thickness of the scaffold. These images can then be compared to those obtained from the luminal surface, which has been the focus of characterization. By analyzing various parts of the scaffold, features such as fiber diameter can be compared throughout the whole structure. This can be useful in assessing how the consistency of the scaffolds changes throughout the electrospinning process as more fibers are deposited. 


\subsubsection{Mandrel Materials}

As discussed previously, some scaffolds stuck to the mandrels during this thesis work. These mandrels are currently made of 303 stainless steel, which accumulates scratches over multiple uses. The mandrels may not have been properly prepared before electrospinning or need to be machined and polished to remove scratches and improve the quality of the surfaces. One solution to this issue that could be employed is to sputter the mandrels with gold to create a smooth surface ${ }^{98,99}$. However, other materials altogether may be considered for the mandrels, such as Teflon or a ceramic. Materials with smooth surfaces that are resistant to scratches and wear would be more beneficial in order to ensure efficient scaffold removal and prevent wasting valuable materials.

\subsection{CONCLUSION}

In conclusion, the reproducibility of the properties of electrospun PLGA scaffolds was characterized with fiber diameter measurements and compliance testing. The consistency of these scaffolds is imperative for creating BVMs in the Cal Poly Tissue Engineering lab that can be used as reliable in vitro models for intravascular device testing. Using the current standard electrospinning protocol, it was determined that the scaffolds had inconsistent mean fiber diameter and compliance. The characterization of the existing electrospinning system can serve as a reference to determine if future modifications are significantly improving the material and mechanical properties of PLGA scaffolds. 


\section{BIBLIOGRAPHY}

1. Touroo JS. Bioengineering Human Blood Vessel Mimics for Medical Device Testing Using Serum-Free Conditions and Scaffold Variations. Tissue Eng Part C Methods. 2013;19(4):307-15.

2. Bonnema GT, Cardinal KO, Williams SK, Barton JK. An automatic algorithm for detecting stent endothelialization from volumetric optical coherence tomography datasets. Phys Med Biol. 2008;53(12):3083-98. doi:10.1088/0031-9155/53/12/001.

3. Cardinal KO, Bonnema GT, Hofer H, Barton JK, Williams SK. Tissue-engineered vascular grafts as in vitro blood vessel mimics for the evaluation of endothelialization of intravascular devices. Tissue Eng. 2006;12:3431-3438. doi:10.1089/ten.2006.12.ft-268.

4. Cardinal KOH, Williams SK. Assessment of the intimal response to a proteinmodified stent in a tissue-engineered blood vessel mimic. Tissue Eng Part A. 2009;15(12):3869-3876. doi:10.1089/ten.tea.2009.0193.

5. Virmani R, Kolodgie FD, Farb a, Lafont a. Drug eluting stents: are human and animal studies comparable? Heart. 2003;89(2):133-8. Available at:

http://www.pubmedcentral.nih.gov/articlerender.fcgi?artid=1767527\&tool=pmcen trez\&rendertype $=$ abstract.

6. Kwon JS, Park RK, Shim TJ, et al. Comparison of bare metal stent and paclitaxeleluting stent using a novel rat aorta stent model. J Vet Sci. 2011;12(2):143. doi:10.4142/jvs.2011.12.2.143.

7. Hasan A, Memic A, Annabi N, et al. Electrospun scaffolds for tissue engineering of vascular grafts. Acta Biomater. 2014;10(1):11-25. doi:10.1016/j.actbio.2013.08.022.

8. O’Brien FJ. Biomaterials \& scaffolds for tissue engineering. Mater Today. 2011;14(3):88-95. doi:10.1016/S1369-7021(11)70058-X.

9. Van Blitterswijk C, ed. Tissue Engineering. First. Academic Press; 2008.

10. Peña TR. Preparation and Characterization of Electrospun Poly(D,L-Lactide-coGlycolide) Scaffolds for Vascular Tissue Engineering and the Advancement of an In Vitro Blood Vessel Mimic. 2009;(June):1-163.

11. Castillo YS. Design of Experimentation to Systematically Determine the Interaction Between Electrospinning Variables and to Optimize the Fiber Diameter of Electrospun Poly(D,L-Lactide-co-Glycolide) Scaffolds for Tissue Engineered Constructs. 2012;(June):1-166. 
12. Araujo J, Martins A, Neves N, Reis R. Electrospun Nanostructured Scaffolds for Tissue Engineering Applications. Nanomedicine. 2007;2(6):929.

13. Will J, Melcher R, Treul C, et al. Porous ceramic bone scaffolds for vascularized bone tissue regeneration. J Mater Sci Mater Med. 2008;19(8):2781-90. doi:10.1007/s10856-007-3346-5.

14. Guo X, Zheng Q, Kulbatski I, et al. Bone regeneration with active angiogenesis by basic fibroblast growth factor gene transfected mesenchymal stem cells seeded on porous beta-TCP ceramic scaffolds. Biomed Mater. 2006;1(3):93-9. doi:10.1088/1748-6041/1/3/001.

15. Yahyouche A, Zhidao X, Triffitt JT, Czernuszka JT, Clover a JP. Improved angiogenic cell penetration in vitro and in vivo in collagen scaffolds with internal channels. J Mater Sci Mater Med. 2013;24(6):1571-80. doi:10.1007/s10856-0134912-7.

16. Zhang L, Ao Q, Wang A, et al. A sandwich tubular scaffold derived from chitosan for blood vessel tissue engineering. J Biomed Mater Res A. 2006;77(2):277-84. doi:10.1002/jbm.a.30614.

17. Yoo JJ, Bichara D a, Zhao X, Randolph M a, Gill TJ. Implant-assisted meniscal repair in vivo using a chondrocyte-seeded flexible PLGA scaffold. J Biomed Mater Res A. 2011;99(1):102-8. doi:10.1002/jbm.a.33168.

18. Kim H-J, Lee J-H, Im G-I. Chondrogenesis using mesenchymal stem cells and PCL scaffolds. J Biomed Mater Res A. 2010;92(2):659-66. doi:10.1002/jbm.a.32414.

19. Gaspar A, Moldovan L, Constantin D, Am S, Pm SB, Ic E. Collagen-based scaffolds for skin tissue engineering. 2011;4(2).

20. Yannas I V, Lee E, Orgill DP, Skrabut EM, Murphyt GF. Synthesis and characterization of a model extracellular matrix that induces partial regeneration of adult mammalian skin. 1989;86(February):933-937.

21. V BI. Biologically Active Analogues of the Extracellular Matrix : Artificial Skin and Nerves. 1990;29:20-35.

22. Ivanov R V, Lozinsky VI. Coupling of gelatin to inner surfaces of pore walls in spongy alginate-based scaffolds facilitates the adhesion, growth and differentiation of human bone marrow mesenchymal stromal cells. 2011:15291540. doi:10.1007/s10856-011-4323-6. 
23. Bakar A, Hilmi M, Halim AS, Hassan A, Lim CK, Noorsal K. In vitro characterization of a chitosan skin regenerating template as a scaffold for cells cultivation. 2013;2(1):1. doi:10.1186/2193-1801-2-79.

24. Kong X, Han B, Wang H, Li H, Xu W, Liu W. Mechanical properties of biodegradable small-diameter chitosan artificial vascular prosthesis. 2012:19381945. doi:10.1002/jbm.a.34136.

25. Baker SC, Atkin N, Gunning PA, et al. Characterisation of electrospun polystyrene scaffolds for three-dimensional in vitro biological studies. 2006;27:3136-3146. doi:10.1016/j.biomaterials.2006.01.026.

26. Bokhari M, Carnachan RJ, Cameron NR, Przyborski SA. Culture of HepG2 liver cells on three dimensional polystyrene scaffolds enhances cell structure and function during toxicological challenge. 2007;44(June):567-576. doi:10.1111/j.1469-7580.2007.00778.x.

27. Bettahalli NMS, Steg H, Wessling M, Stamatialis D. Development of poly ( 1 lactic acid ) hollow fiber membranes for artificial vasculature in tissue engineering scaffolds. J Memb Sci. 2011;371(1-2):117-126.

doi:10.1016/j.memsci.2011.01.026.

28. Wang C, Cen L, Yin S, et al. Biomaterials A small diameter elastic blood vessel wall prepared under pulsatile conditions from polyglycolic acid mesh and smooth muscle cells differentiated from adipose-derived stem cells. Biomaterials. 2010;31(4):621-630. doi:10.1016/j.biomaterials.2009.09.086.

29. Hu X, Shen H, Yang F, Bei J, Wang S. Biomaterials Preparation and cell affinity of microtubular orientation-structured PLGA ( $70 / 30$ ) blood vessel scaffold. 2008;29:3128-3136. doi:10.1016/j.biomaterials.2008.04.010.

30. In S, Yeon S, Kwan S, et al. Tissue-engineered vascular grafts composed of marine collagen and PLGA fibers using pulsatile perfusion bioreactors. 2007;28:1115-1122. doi:10.1016/j.biomaterials.2006.10.025.

31. Vacanti CA, Vacanti JP. The Science of Tissue Engineering. Orthop Clin, North Am. 2000;(31):351-355.

32. Wang H, Ji J, Zhang W, Zhang Y, Jiang J, Wu Z. Biocompatibility and bioactivity of plasma-treated biodegradable poly ( butylene succinate ). Acta Biomater. 2009;5(1):279-287. doi:10.1016/j.actbio.2008.07.017.

33. Liu H, Webster TJ. Less harmful acidic degradation of poly ( lactic- co-glycolic acid ) bone tissue engineering scaffolds through titania nanoparticle addition. 2006:541-545. 
34. Ratner BD, Hoffman AS, Schoen FJ, Lemons JE, eds. Biomaterials Science: An Introduction to Materials in Medicine. Third. Academic Press; 2013.

35. Babensee JE, McIntire L V., Mikos AG. Growth factor delivery for tissue engineering. Pharm Res. 2000;(17):497-504.

36. Bostman O, Hirvensalo E, Vainionpaa S, Al E. Ankle fractures treated using biodegradable internal fixation. Clin Orthop Relat Res. 1989;(238):892-898.

37. Fu K, Pack DW, Klibanov AM, Langer R. Visual evidence of acidic environment within degrading poly(lactic-co-glycolic acid) (PLGA) microspheres. Pharm Res. 2000;17(1):100-106.

38. Jain $\mathrm{R}$ a. The manufacturing techniques of various drug loaded biodegradable poly(lactide-co-glycolide) (PLGA) devices. Biomaterials. 2000;21(23):2475-90. Available at: http://www.ncbi.nlm.nih.gov/pubmed/11055295.

39. James CM. Assessment of Electrospinning as an In-House Fabrication Technique for Blood Vessel Mimic Cellular Scaffolding. 2009;(August).

40. Ji C, Annabi N, Hosseinkhani M, Sivaloganathan S, Dehghani F. Fabrication of poly-DL-lactide/polyethylene glycol scaffolds using the gas foaming technique. Acta Biomater. 2012;8(2):570-8. doi:10.1016/j.actbio.2011.09.028.

41. Zhou C, Ma L, Li W, Yao D. Fabrication of tissue engineering scaffolds through solid-state foaming of immiscible polymer blends. Biofabrication. 2011;3(4):045003. doi:10.1088/1758-5082/3/4/045003.

42. Harris LD, Kim BS, Mooney DJ. Open pore biodegradable matrices formed with gas foaming. J Biomed Mater Res. 1998;(42):396-402.

43. Mehraban M, Zadhoush A, Abdolkarim Hosseini Ravandi S, Bagheri R, Heidarkhan Tehrani A. Preparation of porous nanofibers from electrospun polyacrylonitrile/calcium carbonate composite nanofibers using porogen leaching technique. J Appl Polym Sci. 2013;128(2):926-933. doi:10.1002/app.38091.

44. Gong X, Tang CY, Zhang Y, Wong CT, Wu S, Liu J. Fabrication of Graded Macroporous Poly ( lactic acid ) Scaffold by a Progressive Solvent Casting / Porogen Leaching Approach. J Appl Polym Sci. 2012;125(1):571-577. doi:10.1002/app.

45. Zhao J, Han W, Tu M, Huan S, Zeng R, Wu H. Preparation and properties of biomimetic porous nano fi brous poly ( 1-lactide ) scaffold with chitosan nano fi ber network by a dual thermally induced phase separation technique. Mater Sci Eng C. 2012;32(6):1496-1502. doi:10.1016/j.msec.2012.04.031. 
46. Carrubba V La, Brucato V, Pavia FC. Morphology and thermal properties of foams prepared via thermally induced phase separation based on polylactic acid blends. J Cell Plast. 2012;48(5):399-407. doi:10.1177/0021955X12452180.

47. Lam CX., Mo X., Teoh S., Hutmacher D. Scaffold development using 3D printing with a starch-based polymer. Mater Sci Eng C. 2002;20(1-2):49-56. doi:10.1016/S0928-4931(02)00012-7.

48. Castilho M, Dias M, Gbureck U, et al. Fabrication of computationally designed scaffolds by low temperature 3D printing. Biofabrication. 2013;5(3):035012. doi:10.1088/1758-5082/5/3/035012.

49. Chen C, Shyu VB, Chen J, Lee M. Selective laser sintered poly- $\varepsilon$-caprolactone scaffold hybridized with collagen hydrogel for cartilage tissue engineering. Biofabrication. 2014;6:015004. doi:10.1088/1758-5082/6/1/015004.

50. Shuai C, Mao Z, Lu H, Nie Y, Hu H, Peng S. Fabrication of porous polyvinyl alcohol scaffold for bone tissue engineering via selective laser sintering. Biofabrication. 2013;5(1):015014. doi:10.1088/1758-5082/5/1/015014.

51. Frenot A, Chronakis IS. Polymer nanofibers assembled by electrospinning. 2003;8:64-75. doi:10.1016/S1359-0294.

52. Leach MK, Feng Z-Q, Tuck SJ, Corey JM. Electrospinning fundamentals: optimizing solution and apparatus parameters. J Vis Exp. 2011;(47):3-6. doi: $10.3791 / 2494$.

53. Kim GK, Trang H, Rainer A, Trombetta M. Electrospinning of PCL / PVP blends for tissue engineering scaffolds. 2013:1425-1442. doi:10.1007/s10856-013-48936.

54. Yoshimoto H, Shin YM, Terai H, Vacanti JP. A biodegradable nanofiber scaffold by electrospinning and its potential for bone tissue engineering. Biomaterials. 2003;24(12):2077-2082. doi:10.1016/S0142-9612(02)00635-X.

55. Vaz CM, van Tuijl S, Bouten CVC, Baaijens FPT. Design of scaffolds for blood vessel tissue engineering using a multi-layering electrospinning technique. Acta Biomater. 2005;1(5):575-82. doi:10.1016/j.actbio.2005.06.006.

56. Matthews J a, Wnek GE, Simpson DG, Bowlin GL. Electrospinning of collagen nanofibers. Biomacromolecules. 2002;3(2):232-8. Available at: http://www.ncbi.nlm.nih.gov/pubmed/11888306.

57. Garg K, Bowlin GL. Electrospinning jets and nanofibrous structures. Biomicrofluidics. 2011;5(1):13403. doi:10.1063/1.3567097. 
58. Shin YM, Hohman MM, Brenner MP, Rutledge GC. Electrospinning: A whipping fluid jet generates submicron polymer fibers. Appl Phys Lett. 2001;78(8):1149. doi:10.1063/1.1345798.

59. Reneker DH, Yarin AL. Electrospinning jets and polymer nanofibers. Polymer (Guildf). 2008;49(10):2387-2425. doi:10.1016/j.polymer.2008.02.002.

60. Zong X, Kim K, Fang D, Ran S, Hsiao BS, Chu B. Structure and process relationship of electrospun bioabsorbable nanofiber membranes. Polymer (Guildf). 2002;43(16):4403-4412. doi:10.1016/S0032-3861(02)00275-6.

61. Tan S-H, Inai R, Kotaki M, Ramakrishna S. Systematic parameter study for ultrafine fiber fabrication via electrospinning process. Polymer (Guildf). 2005;46(16):6128-6134. doi:10.1016/j.polymer.2005.05.068.

62. Tong H-W, Wang M. Electrospinning of fibrous polymer scaffolds using positive voltage or negative voltage: a comparative study. Biomed Mater. 2010;5(5):054110. doi:10.1088/1748-6041/5/5/054110.

63. Cramariuc B, Cramariuc R, Scarlet R, Manea LR, Lupu IG, Cramariuc O. Fiber diameter in electrospinning process. J Electrostat. 2013;71(3):189-198. doi:10.1016/j.elstat.2012.12.018.

64. Sharma S. Ferrolectric nanofibers : principle, processing and applications. $A d v$ Mater Lett. 2013;4(7):522-533. doi:10.5185/amlett.2012.9426.

65. Vrieze S, Camp T, Nelvig a., Hagström B, Westbroek P, Clerck K. The effect of temperature and humidity on electrospinning. J Mater Sci. 2008;44(5):1357-1362. doi:10.1007/s10853-008-3010-6.

66. Bosworth L a., Downes S. Acetone, a Sustainable Solvent for Electrospinning Poly( $\varepsilon$-Caprolactone) Fibres: Effect of Varying Parameters and Solution Concentrations on Fibre Diameter. J Polym Environ. 2012;20(3):879-886. doi:10.1007/s10924-012-0436-3.

67. Patel DC. Preparation and Characterization of Electrospun Poly(D,L-Lactide-coGlycolide) Scaffolds for Vascular Tissue Engineering and the Advancement of an In Vitro Blood Brain Barrier Model. 2012;(June):1-156.

68. Liu H, Ding X, Zhou G, Li P, Wei X, Fan Y. Electrospinning of Nanofibers for Tissue Engineering Applications. J Nanomater. 2013;2013:1-11. doi:10.1155/2013/495708.

69. Tsing P. Electrospinning Natural Polymers for Tissue Engineering Applications. $: 1-15$. 
70. Hsia, Henry C MD, Nair, Mohan R. PD, Mintz, R. Candida PD, Corbett, Siobhan A. MD. THE FIBER DIAMETER OF SYNTHETIC BIORESORBABLE EXTRACELLULAR MATRIX INFLUENCES HUMAN FIBROBLAST MORPHOLOGY AND FIBRONECTIN MATRIX ASSEMBLY. 2012;127(6):2312-2320. doi:10.1097/PRS.0b013e3182139fa4.THE.

71. Badami AS, Kreke MR, Thompson MS, Riffle JS, Goldstein AS. Effect of fiber diameter on spreading, proliferation, and differentiation of osteoblastic cells on electrospun poly(lactic acid) substrates. Biomaterials. 2006;27(4):596-606. doi:10.1016/j.biomaterials.2005.05.084.

72. Giancotti FG. Integrin Signaling. Science (80- ). 1999;285(5430):1028-1033. doi:10.1126/science.285.5430.1028.

73. Zargham S, Bazgir S, Tavakoli A, Rashidi AS, Damerchely R. The Effect of Flow Rate on Morphology and Deposition Area of Electrospun Nylon 6 Nanofiber. 2012;7(4):42-49.

74. Liu F, Guo R, Shen M, Wang S, Shi X. Effect of Processing Variables on the Morphology of Electrospun Poly[(lactic acid)- co -(glycolic acid)] Nanofibers. Macromol Mater Eng. 2009;294(10):666-672. doi:10.1002/mame.200900110.

75. Ali FAA, Haider S, Al-masry WA, Al-zeghaye Y. Fabrication of chitosan nanofibers membrane and its treatment. :1-4.

76. Smith KL, Alexander MS, Stark JPW. Voltage effects on the volumetric flow rate in cone-jet mode electrospraying. J Appl Phys. 2006;99(6):064909. doi:10.1063/1.2183351.

77. Erisken C, Zhang X, Moffat KL, Levine WN, Lu HH. Scaffold fiber diameter regulates human tendon fibroblast growth and differentiation. Tissue Eng Part A. 2013;19(3-4):519-28. doi:10.1089/ten.tea.2012.0072.

78. Mota C, Puppi D, Dinucci D, Errico C, Bártolo P, Chiellini F. Dual-Scale Polymeric Constructs as Scaffolds for Tissue Engineering. Materials (Basel). 2011;4(12):527-542. doi:10.3390/ma4030527.

79. Ravichandran $\mathrm{R}, \mathrm{Ng} \mathrm{CC}$, Liao S, et al. Biomimetic surface modification of titanium surfaces for early cell capture by advanced electrospinning. Biomed Mater. 2012;7(1):015001. doi:10.1088/1748-6041/7/1/015001.

80. Milleret V, Simona B, Neuenschwander P, Hall H. Tuning electrospinning parameters for production of 3D-fiber-fleeces with increased porosity for soft tissue engineering applications. Eur Cell Mater. 2011;21:286-303. Available at: http://www.ncbi.nlm.nih.gov/pubmed/21432783. 
81. Hardick O, Stevens B, Bracewell D. Nanofiber fabrication in a temperature and humidity controlled environment for improved fibre consistency. London

82. Mcveigh GE, Bank AJ, Cohn JN. Arterial Compliance. 1815.

83. Lee T, Kamm R. Vascular Mechanics for the Cardiologist. JACC. 1994;23(6):1289-95.

84. Konig G, Mcallister TN, Ph D, et al. Mechanical properties of completely autologous human tissue engineered blood vessels compared to human saphenous vein and mammary artery. Biomaterials. 2010;30(8):1542-1550. doi:10.1016/j.biomaterials.2008.11.011.Mechanical.

85. Crapo PM, Wang Y. Physiologic compliance in engineered small-diameter arterial constructs based on an elastomeric substrate. Biomaterials. 2010;31(7):1626-35. doi:10.1016/j.biomaterials.2009.11.035.

86. Shoukas a. a., Sagawa K. Total Systemic Vascular Compliance Measured as Incremental Volume-Pressure Ratio. Circ Res. 1971;28(2):277-289. doi:10.1161/01.RES.28.2.277.

87. Rowley AR, Tipton S, Cardinal KOH. The Construction of a Compliance Measuring Device for Vascular Scaffolds. 2012:1-34.

88. Gosling RG, Budge MM. Terminology for Describing the Elastic Behavior of Arteries. Hypertension. 2003;41(6):1180-1182. doi:10.1161/01.HYP.0000072271.36866.2A.

89. Cummings CL, Gawlitta D, Nerem RM, Stegemann JP. Properties of engineered vascular constructs made from collagen, fibrin, and collagen-fibrin mixtures. Biomaterials. 2004;25(17):3699-706. doi:10.1016/j.biomaterials.2003.10.073.

90. Zhang WJ, Liu W, Cui L, Cao Y. Tissue engineering of blood vessel. J Cell Mol Med. 2007;11(5):945-57. doi:10.1111/j.1582-4934.2007.00099.x.

91. Arinstein A, Burman M, Gendelman O, Zussman E. Effect of supramolecular structure on polymer nanofibre elasticity. Nat Nanotechnol. 2007;2(1):59-62. doi:10.1038/nnano.2006.172.

92. Camposeo A, Greenfeld I, Tantussi F, et al. Local Mechanical Properties of Electrospun Fibers Correlate to Their Internal Nanostructure. 2013.

93. Inoguchi H, Kwon IK, Inoue E, Takamizawa K, Maehara Y, Matsuda T. Mechanical responses of a compliant electrospun poly(L-lactide-co-epsiloncaprolactone) small-diameter vascular graft. Biomaterials. 2006;27(8):1470-8. doi:10.1016/j.biomaterials.2005.08.029. 
94. Sellgren KL, Ma T. Perfusion conditioning of hydroxyapatite - chitosan - gelatin scaffolds for bone tissue regeneration from human mesenchymal stem cells. 2012;(February 2011):49-59. doi:10.1002/term.

95. Denk W, Horstmann H. Serial block-face scanning electron microscopy to reconstruct three-dimensional tissue nanostructure. PLoS Biol. 2004;2(11):e329. doi:10.1371/journal.pbio.0020329.

96. Egerton RF, Li P, Malac M. Radiation damage in the TEM and SEM. Micron. 2004;35(6):399-409. doi:10.1016/j.micron.2004.02.003.

97. Kitson SC, Barnes WL, Sambles JR. e Fabrication of Submicron Hexagonal Arrays sing Multiple-Exposure Optical Interferometry. 1996;8(12):1662-1664.

98. O'Dwyer C, Gay G, Viaris de Lesegno B, Weiner J. The nature of alkanethiol selfassembled monolayer adsorption on sputtered gold substrates. Langmuir. 2004;20(19):8172-82. doi:10.1021/la049103b.

99. Chai L, Klein J. Large area, molecularly smooth $(0.2 \mathrm{~nm}$ rms $)$ gold films for surface forces and other studies. Langmuir. 2007;23(14):7777-83. doi:10.1021/la063738o. 


\section{APPENDICES}

APPENDIX A: POLYMER SOLUTION MIXING PROTOCOL (SOP5310)

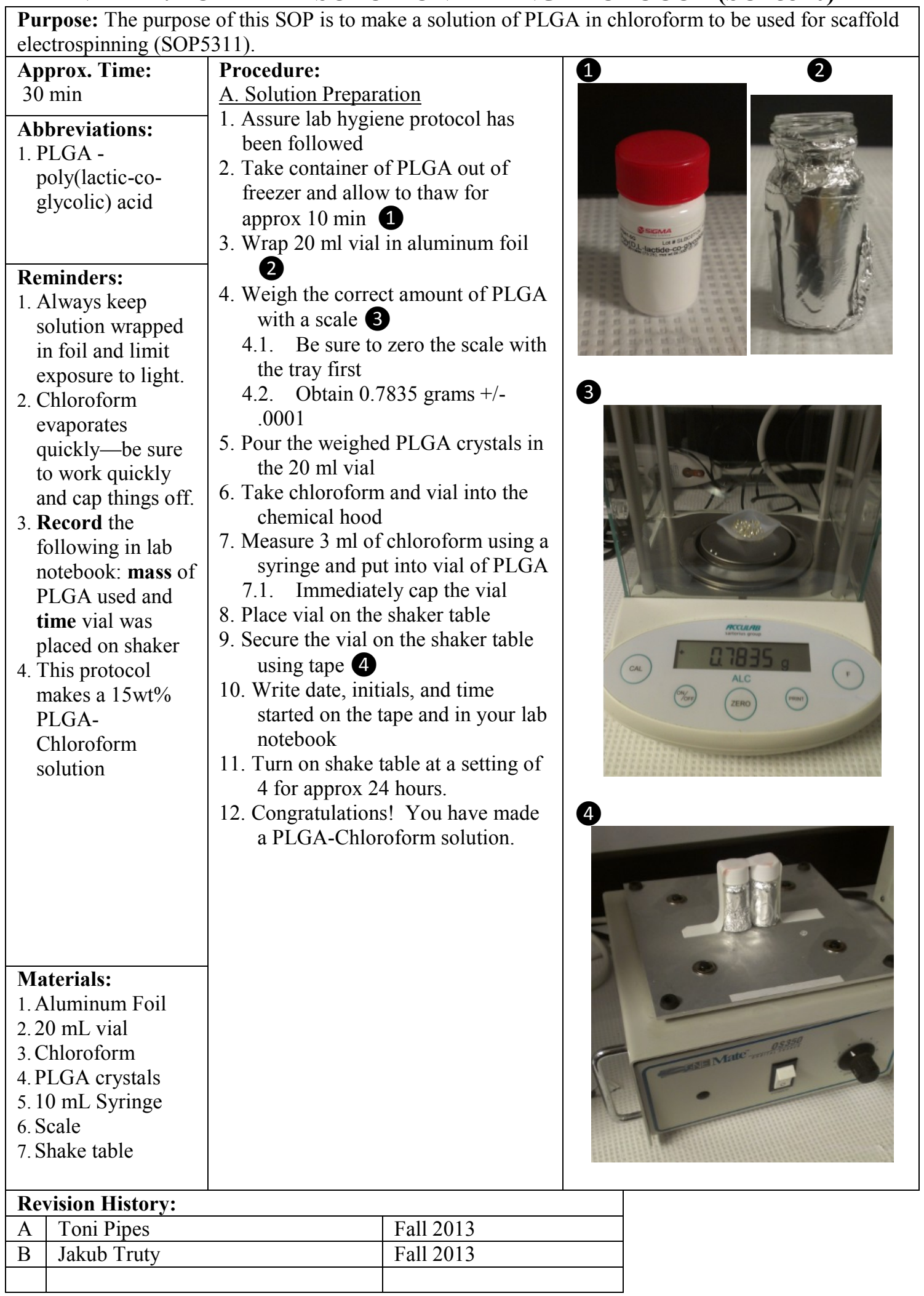


APPENDIX B: STANDARD ELECTROSPINNING PROTOCOL (SOP5311)

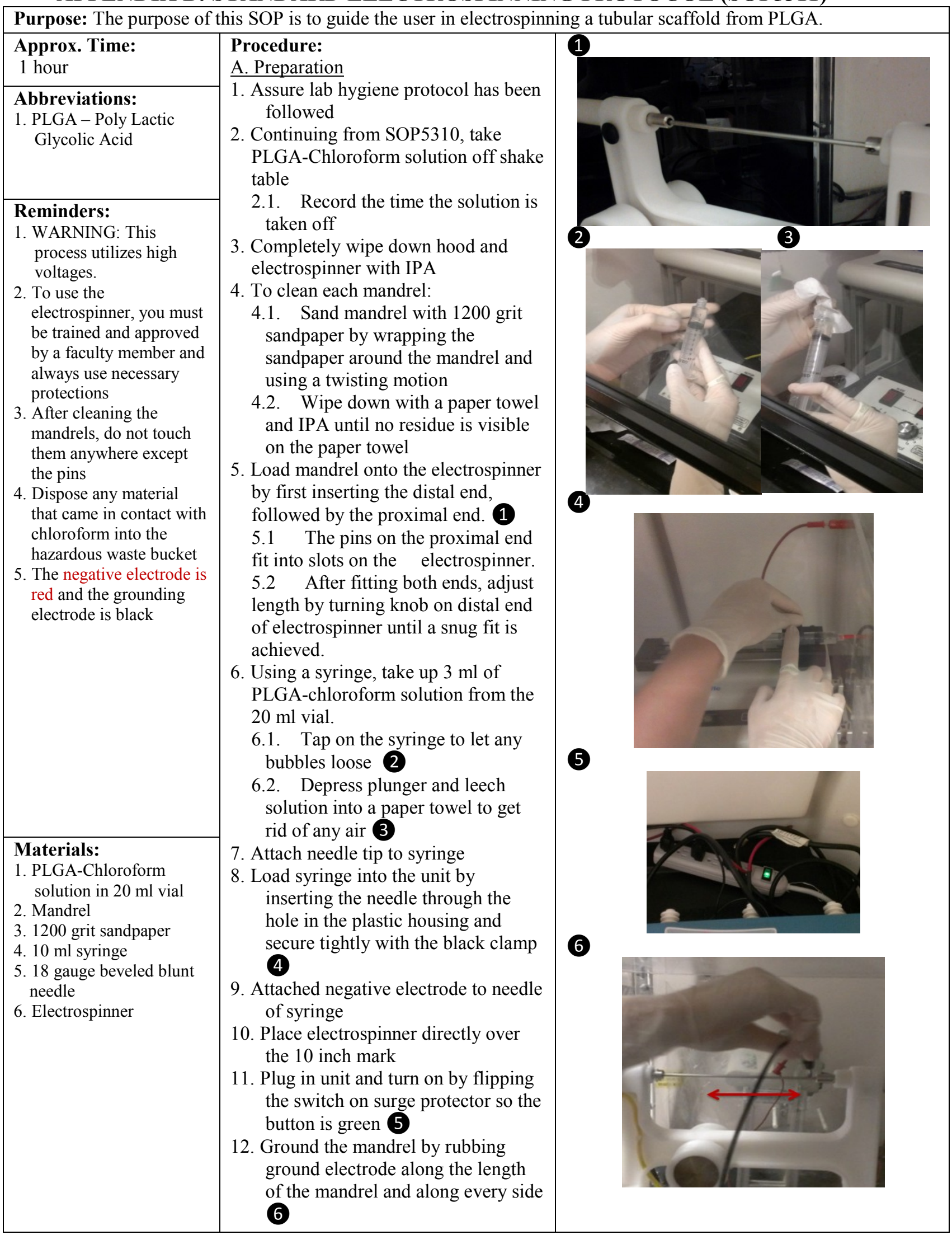




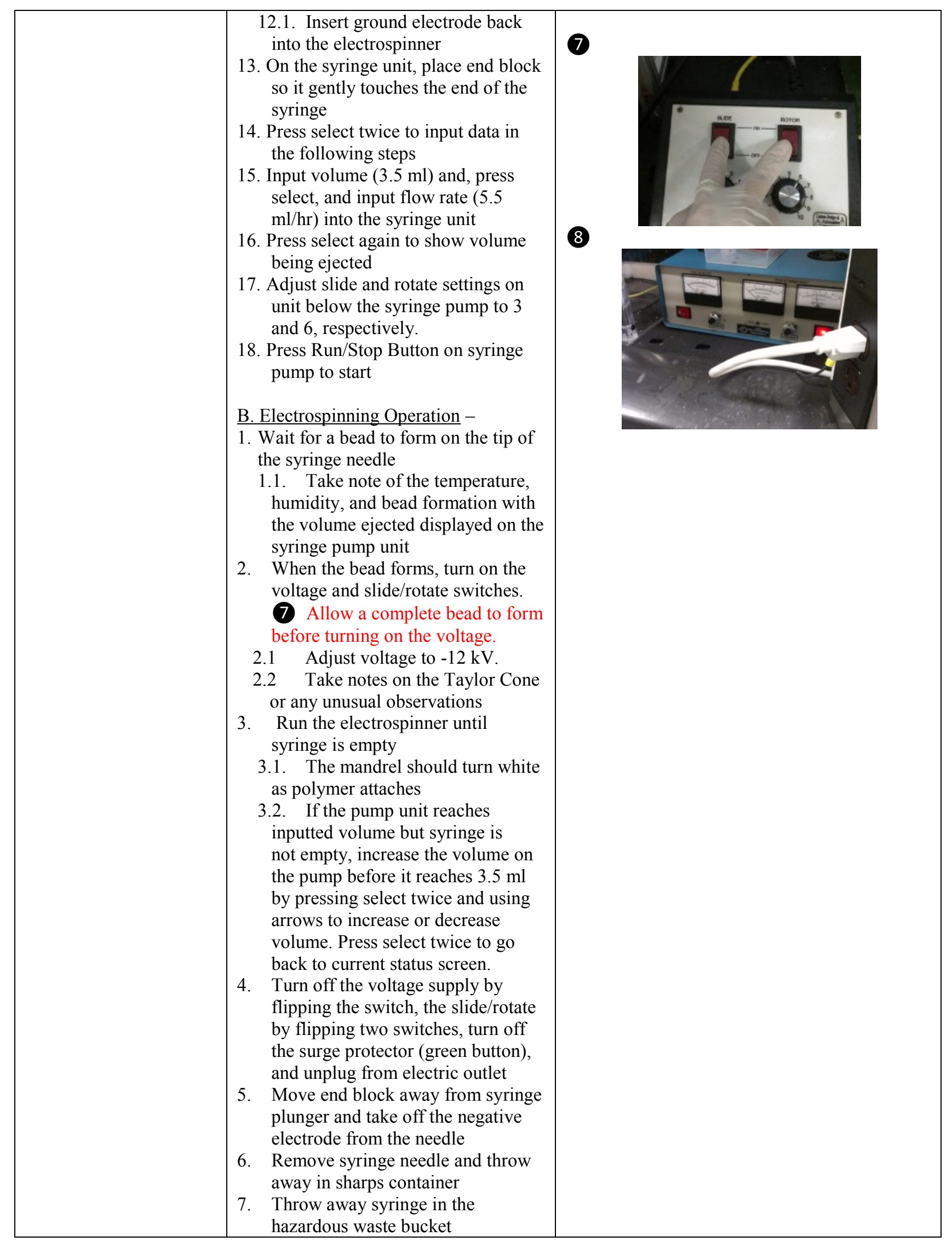




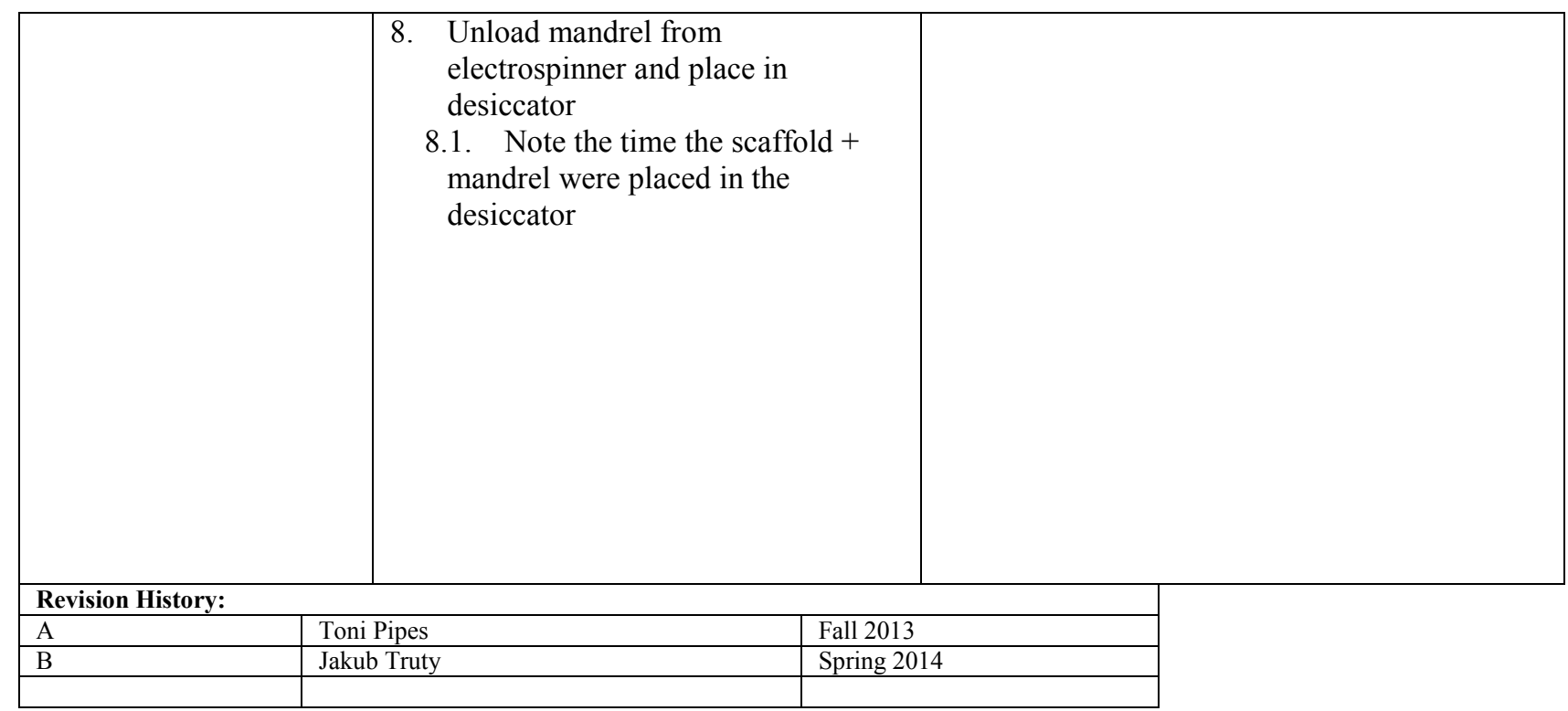

Note: A modification was made in Step 2 of the Electrospinning Operation (shown in red). This was implemented after the preliminary spins were performed in Chapter 2. 


\section{APPENDIX C: SCANNING ELECTRON MICROSCOPY PROTOCOL}
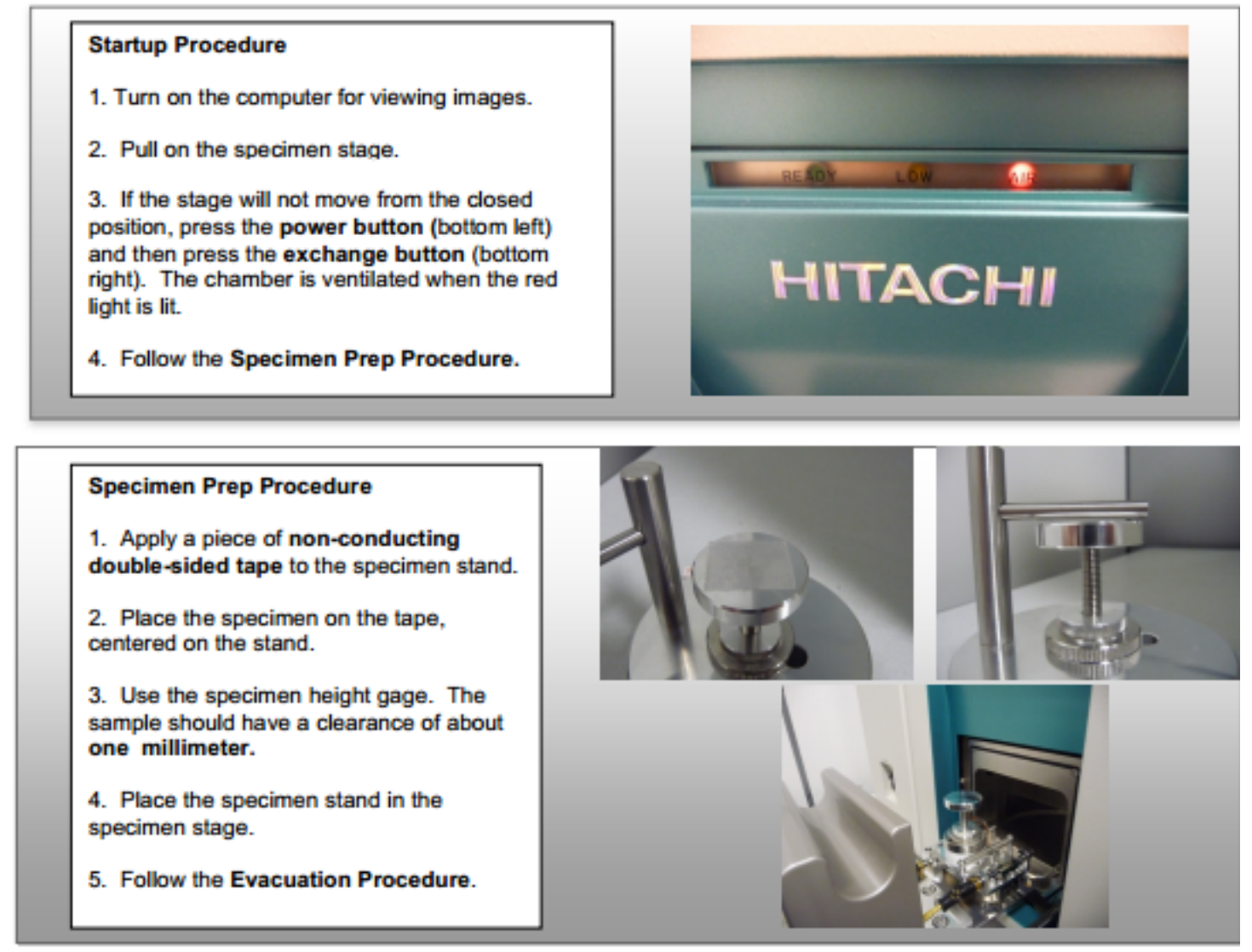

\section{Evacuation Procedure}

1. If the microscope is off, press the power button on the bottom left of the microscope

2. Check that specimen stage is completely inside the microscope.

3. Click the TM-1000 icon on the desktop.

3. Press the exchange button on the bottom right of the microscope.

4. The chamber is evacuated when the green light is lit on the top center display. The progress is also displayed in the viewing program.

5. Follow the Viewing Procedure.

\section{TM-1000}

1. Now evacuating the specimen chamber. Please wait (Code:1550)

\section{대르}

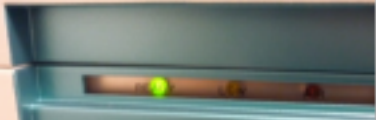

HITACHI 
Viewing Procedure

1. Press the Start Button in the viewing program.

2. To save an image: press Quick Save for low resolution or Save for high resolution.

3. When finished viewing, exit the program.

4. Press the Exchange Button. Wait for evacuation to be complete. The red light will be light.

5. Remove the specimen and close the specimen stage.

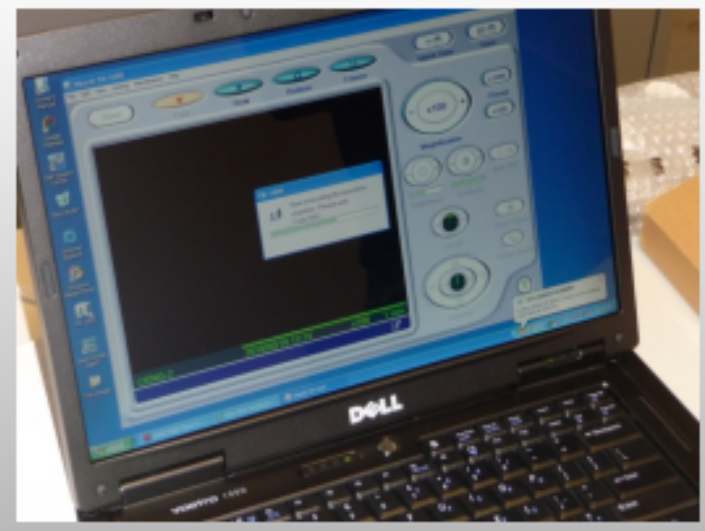

6. Press the Power Button 


\section{APPENDIX D: IMAGE J PROTOCOL FOR FIBER DIAMETER MEASUREMENT}

This protocol was taken from Deven Patel's thesis. It was originally developed by Tiffany Peña and modifications were made by Yvette Castillo. Steps in red were added to explain how to overlay a grid of circles for systematically selecting fibers.

1. Open program ImageJ. The following screen will appear.

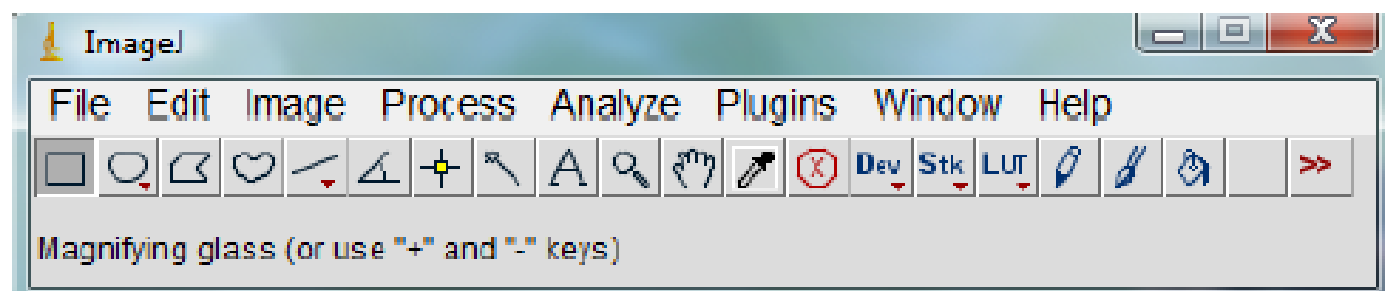

2. To place an overlay of circles, find an image of a grid of circles online $(4 \mathrm{x} 4$ and $3 \times 3$ grids were used in this thesis, as shown below). Save the image.
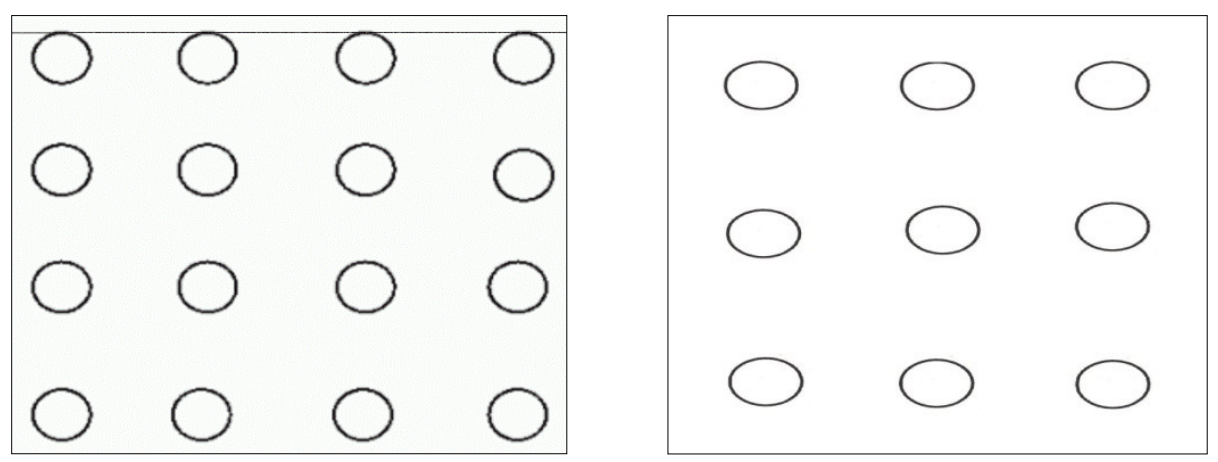

3. Select File $>$ Open. Open a saved SEM image. The image will appear in a separate screen. Then, open the saved grid image. This will also appear in a separate screen.

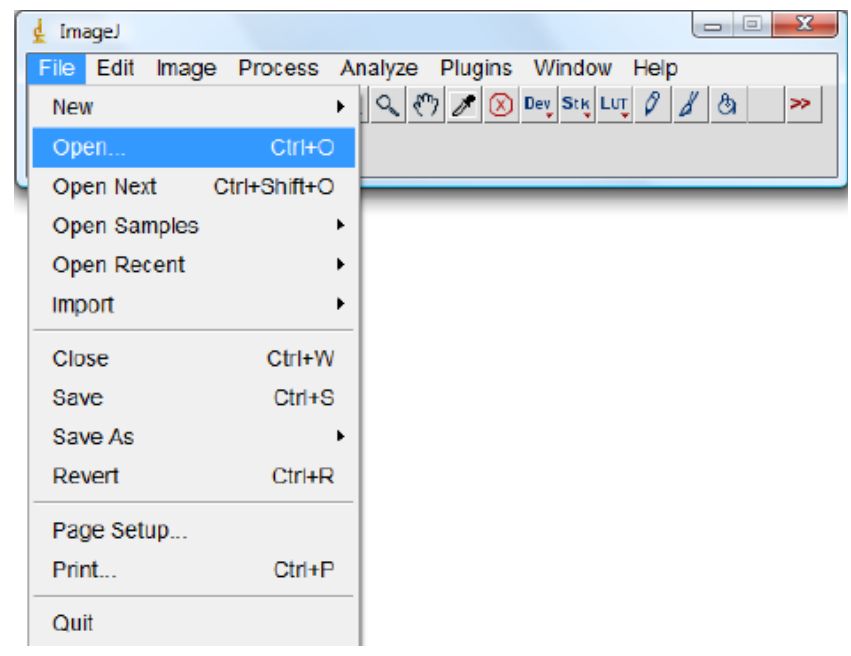


4. The grid image may need to be resized to match the SEM image. If so, click on the grid. Select Image $>$ Adjust $>$ Size.

\begin{tabular}{|l|lrl|l|l|}
\hline Image & & & \\
\hline
\end{tabular}

5. Adjust the width to 1280 pixels and the height to 1040 pixels, which are the dimensions of the SEM image. Click OK.

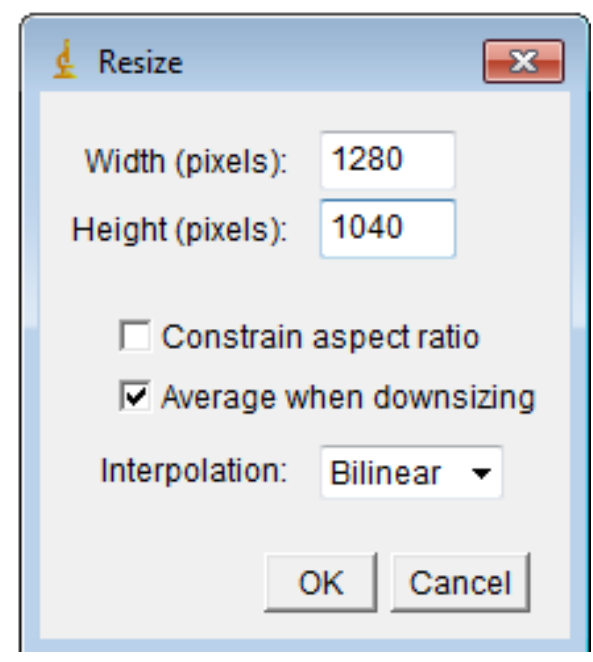


6. Click on the grid. Press $\mathrm{Ctrl}+\mathrm{C}$ to copy the image. Then, click on the SEM image. Press Ctrl $+\mathrm{V}$ to paste the grid onto the SEM image. The grid will cover the SEM image.

7. Right click the image and select Paste Control.

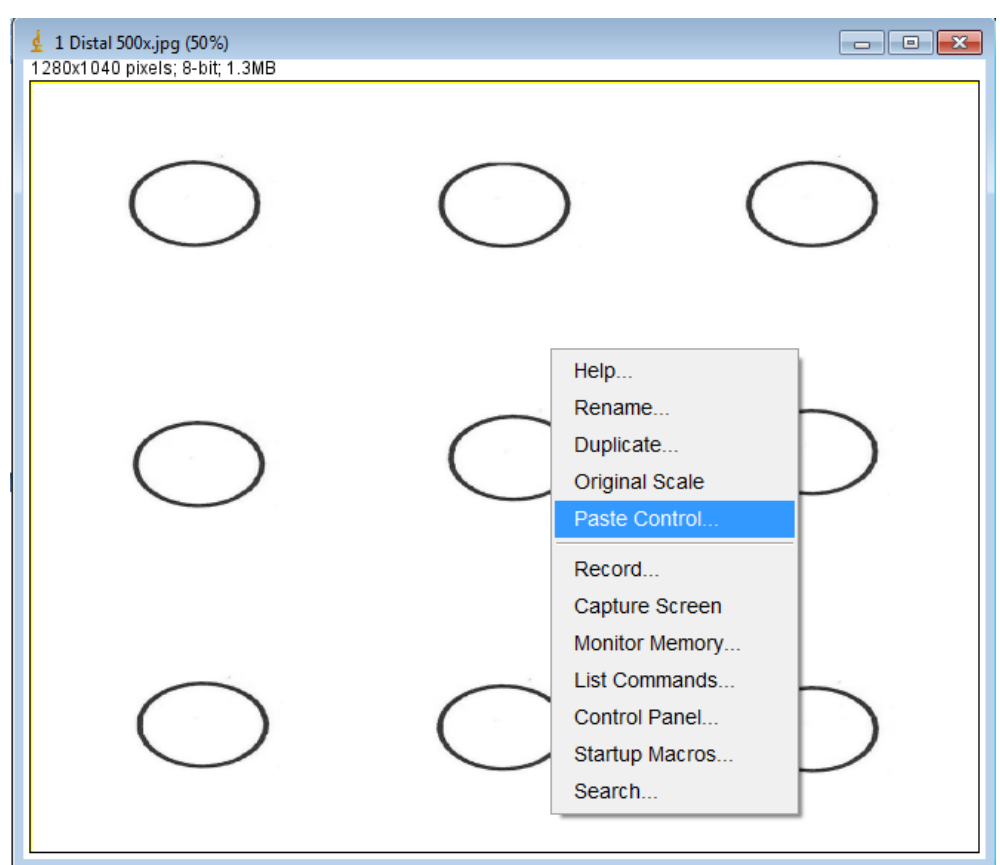

8. From the Transfer Mode drop-down menu, select Blend. Close the dialog box.

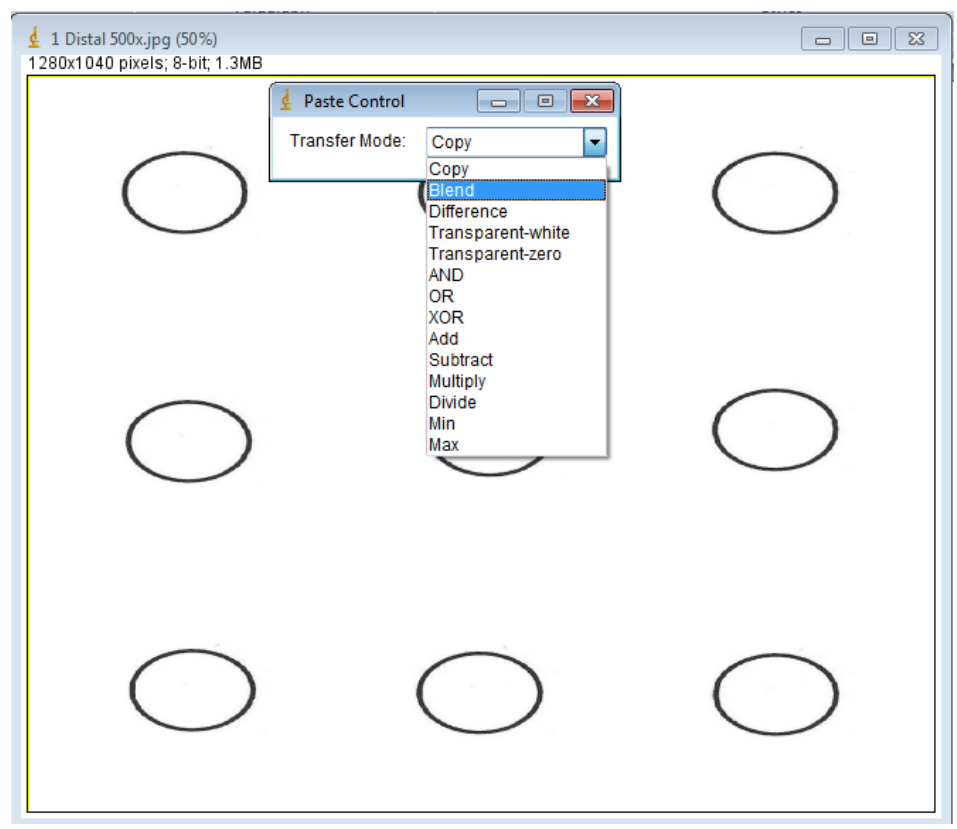


9. The white background of the grid will blend with the SEM image and the black circles will be visible, as shown below.

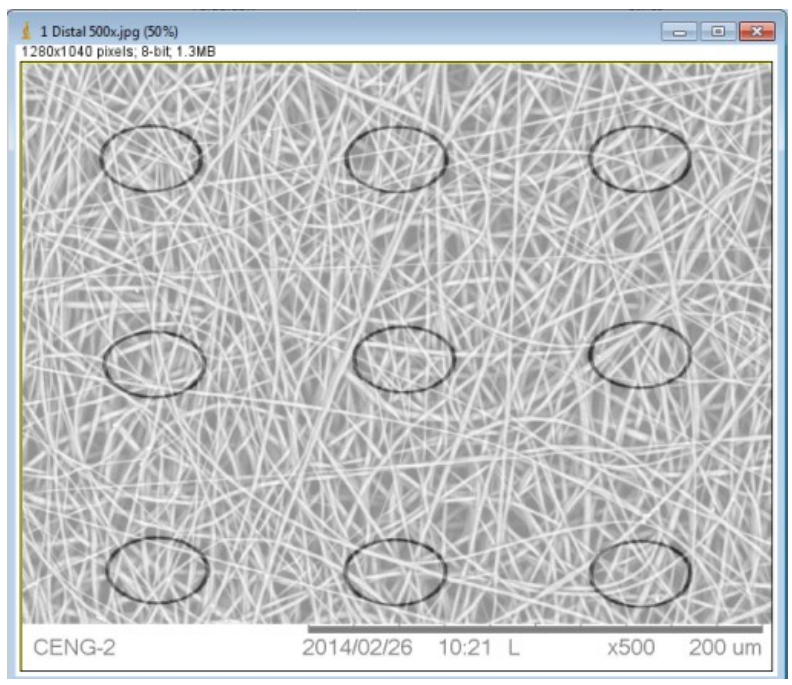

10. The brightness and contrast of the image may need to be adjusted if the fibers are not clear. If so, select Image $>$ Adjust $>$ Brightness/Contrast.

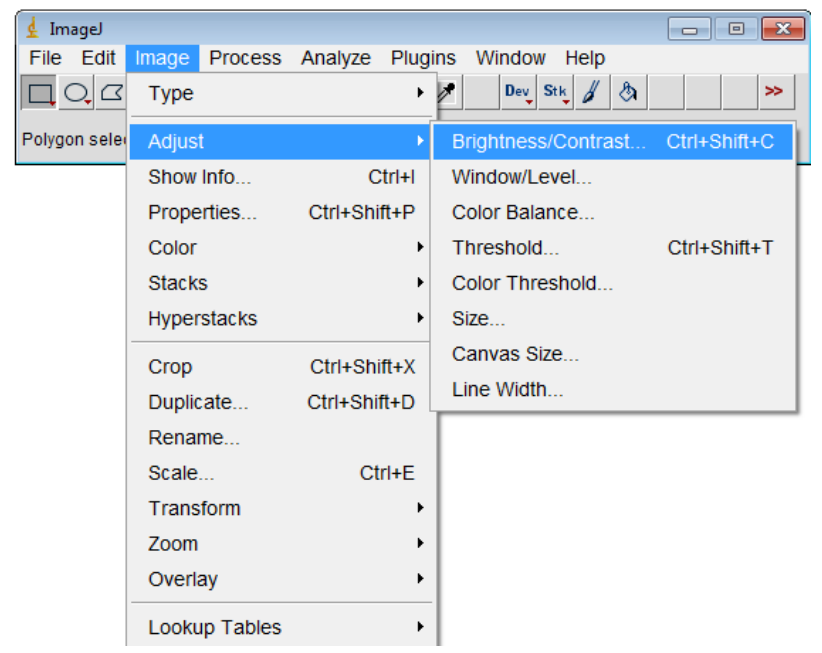


11. Click the arrows for brightness and contrast to adjust the image until the fibers are clearly visible. Click Apply and then close the dialog box.
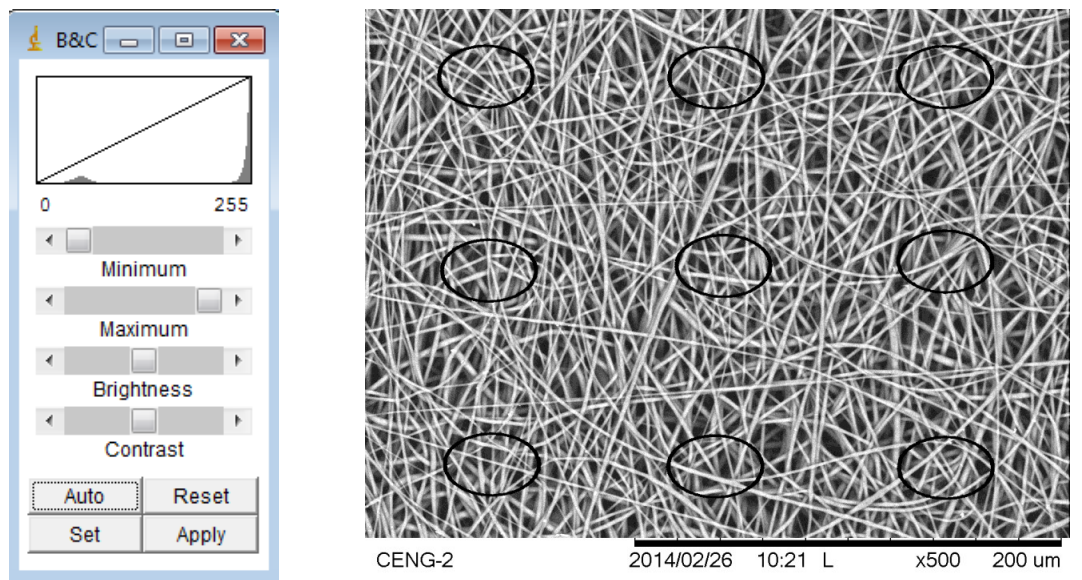

12. Select 7 and draw a line along the SEM image's scale bar. Visually ensure the line is as close to the length of the scale bar as possible as this will affect the outcome of fiber diameter measurements (See image under Step 13 for further clarification).

13. On the tool bar, select Analyze $>$ Set Scale.

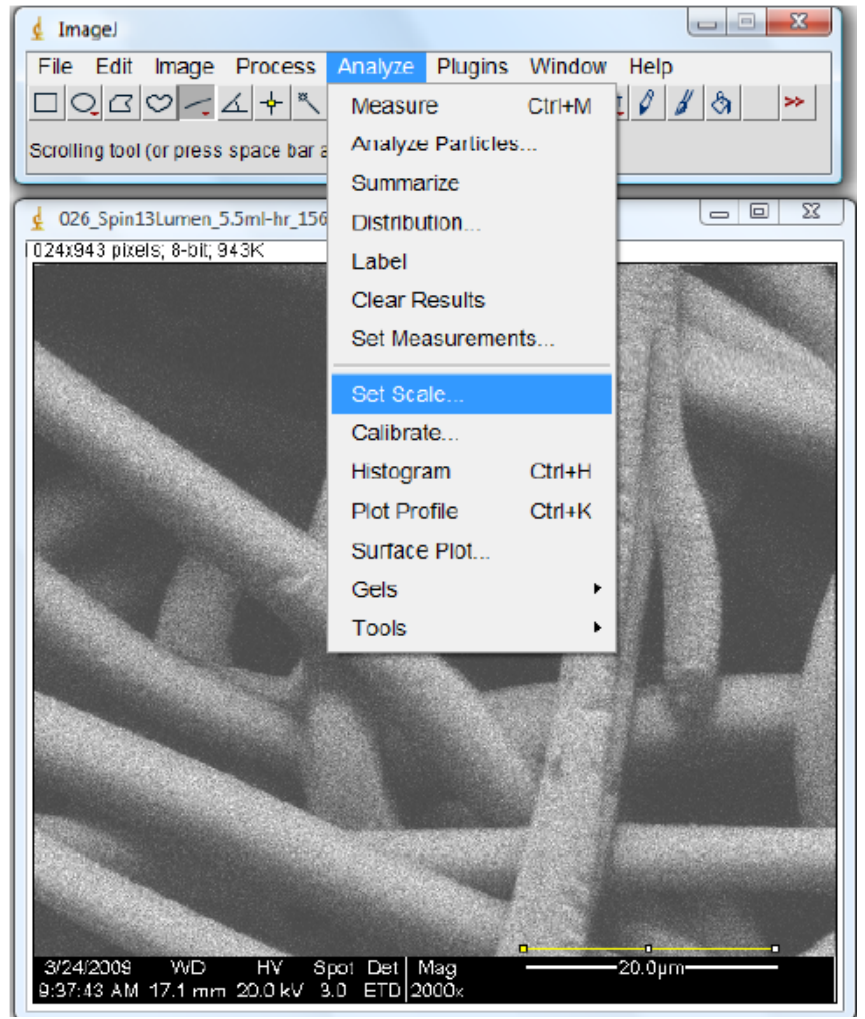


14. Insert known image scale bar distance (ex. $20 \mu \mathrm{m}$ ). Set pixel aspect ratio to 1.0 and set appropriate unit length (ex. $\mu \mathrm{m})$. Select OK. ImageJ is now calibrated to the image,

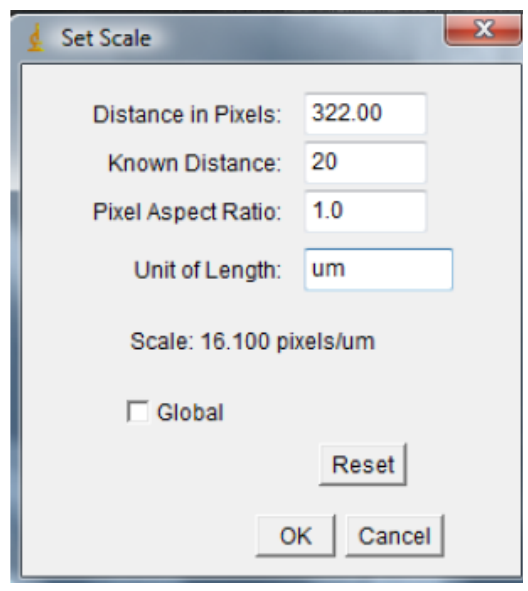

15. From the tool bar, select 7 . Draw a line across the diameter of a single fiber to be measured.

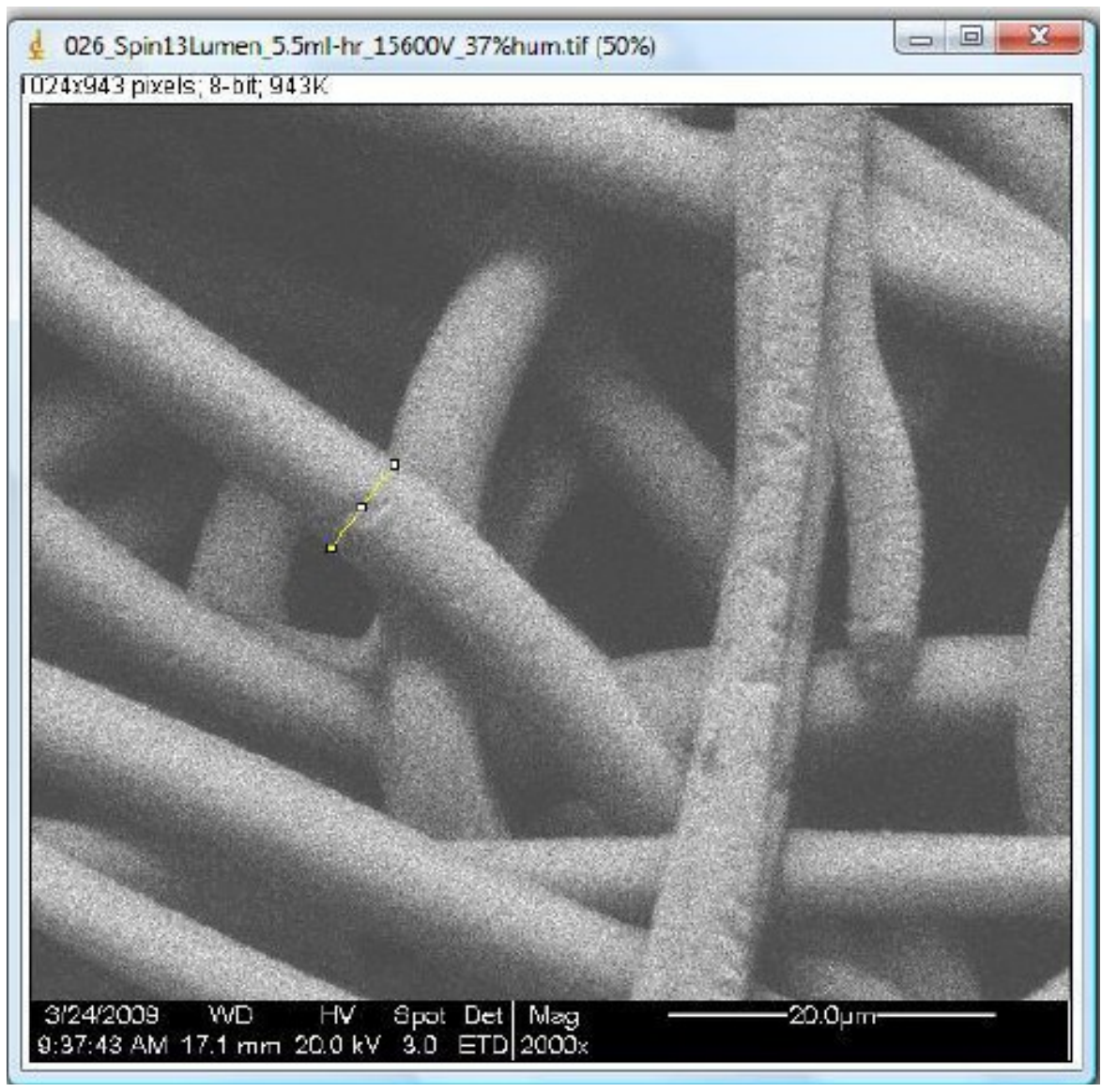


16. From the toolbar select Analyze $>$ Measure. ImageJ will open a new window reporting Results. Fiber diameter is reported as Length in the Results window in proper units (ex. $\mu \mathrm{m})$.

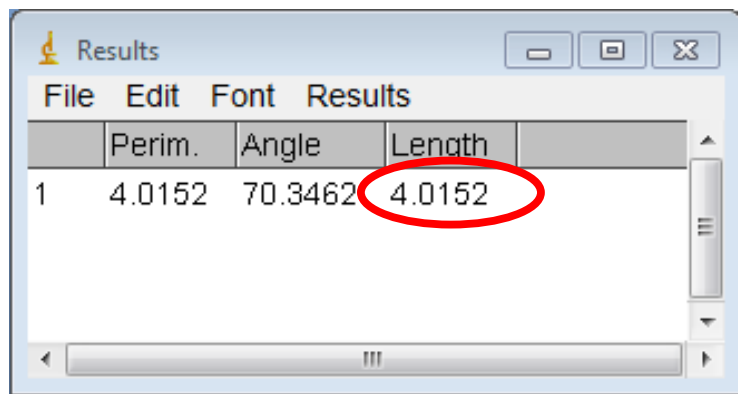

17. Leave the Measure window open and repeat Step 15 for all fiber measurements for the image.

18. Repeat for all images. NOTE: Calibration is only necessary for the first image if all images are being analyzed at the SAME magnification. Re-calibration is necessary for images taken at different magnifications. 


\section{APPENDIX E: STATISTICAL METHODS}

\section{REML Variance Components Analysis}

1. Open JMP and open the data file.

2. Select Analyze > Fit Model.

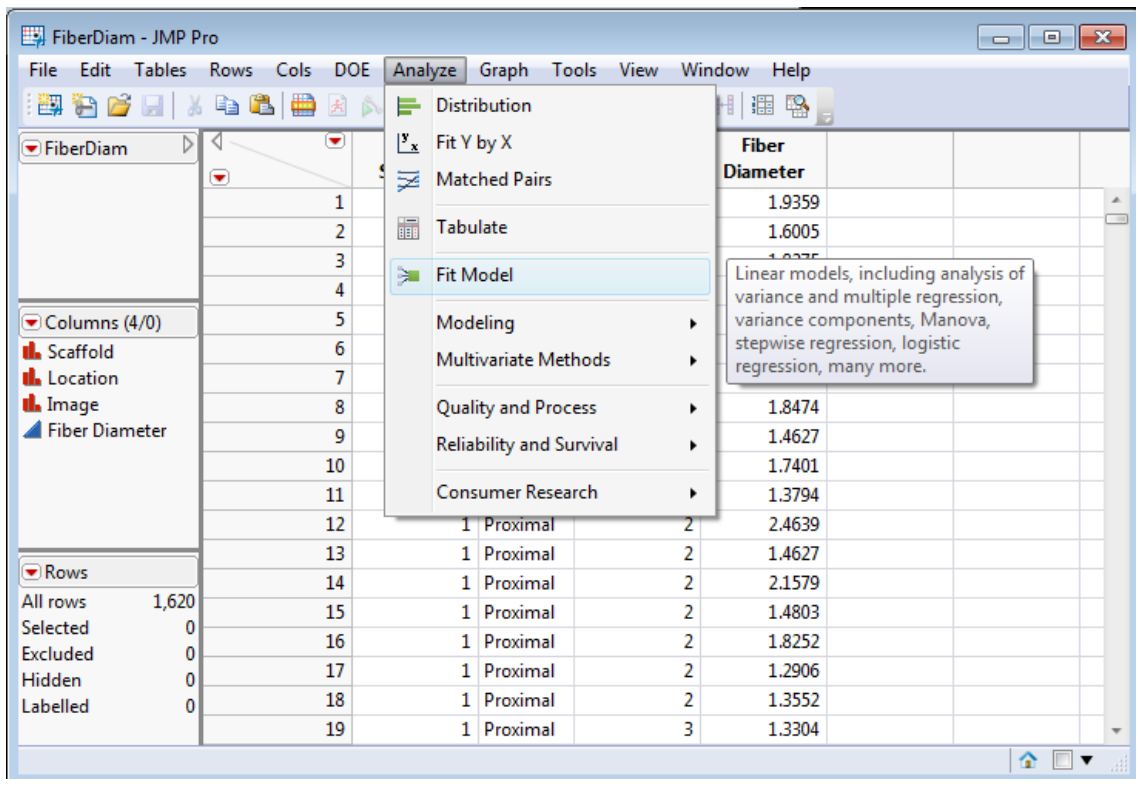

3. Place $x$ variables in the Construct Model Effects box and $y$ variables in the box next to Y under Pick Role Variables.

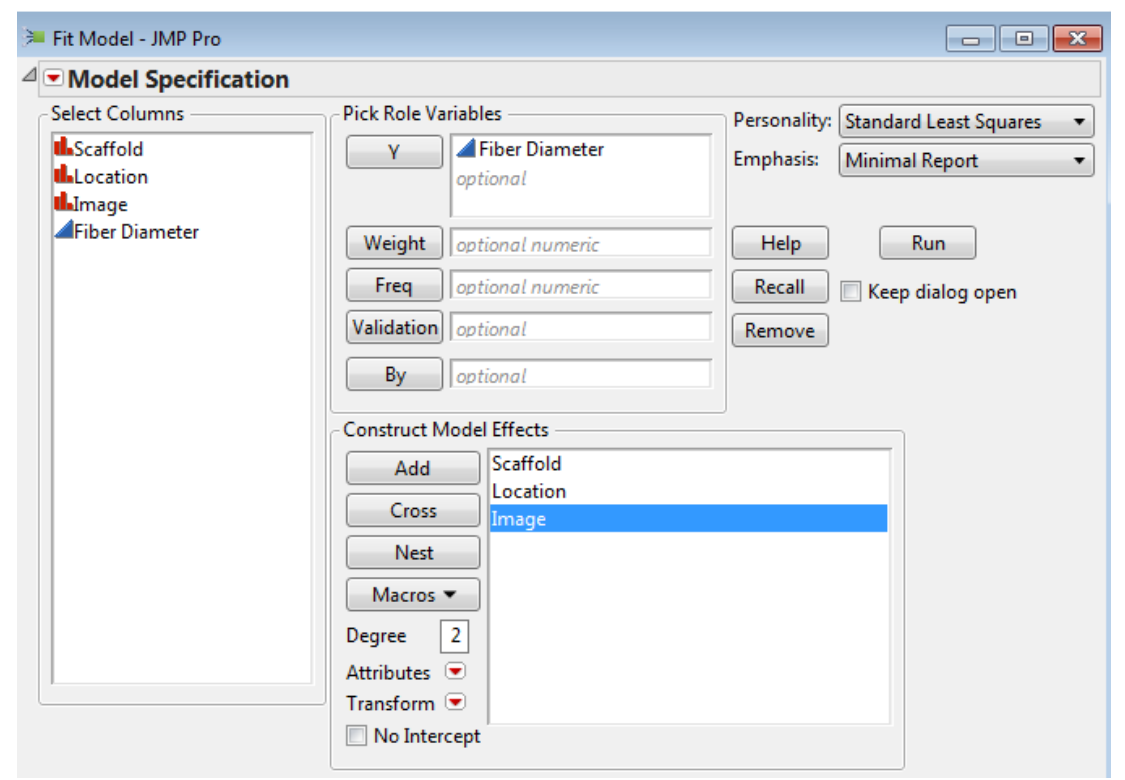


4. Change the desired $x$ variables to random effects. Click on the red arrow next to Attributes and select Random Effect. The variable will now have "\& Random" listed after it. Repeat this for all variables that need to be designated as random.

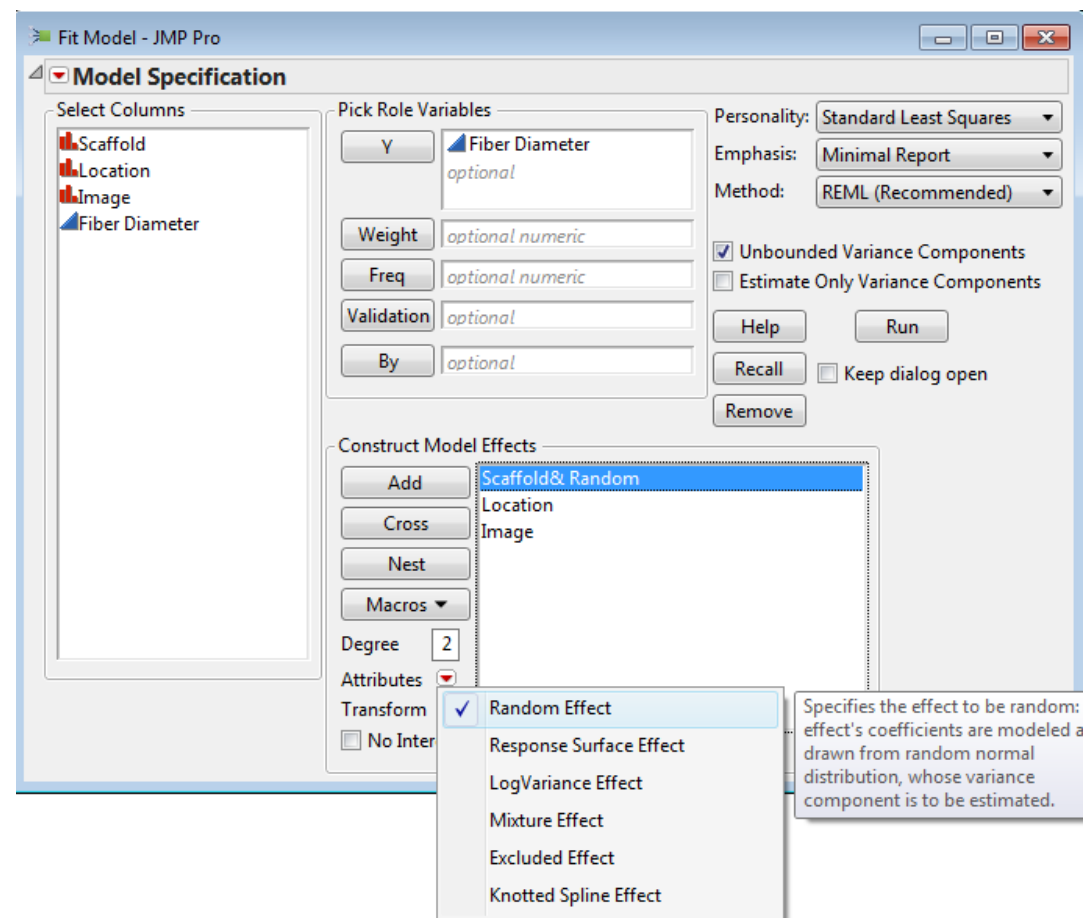

5. If a variable needs to be nested, click on the variable in the Construct Model Effects box and select the variable it is nested within in the Select Columns box. Click Nest. The variable will appear in brackets after the nested variable. Ensure that Minimal Report is selected for Emphasis and REML is selected for Method. Click Run.

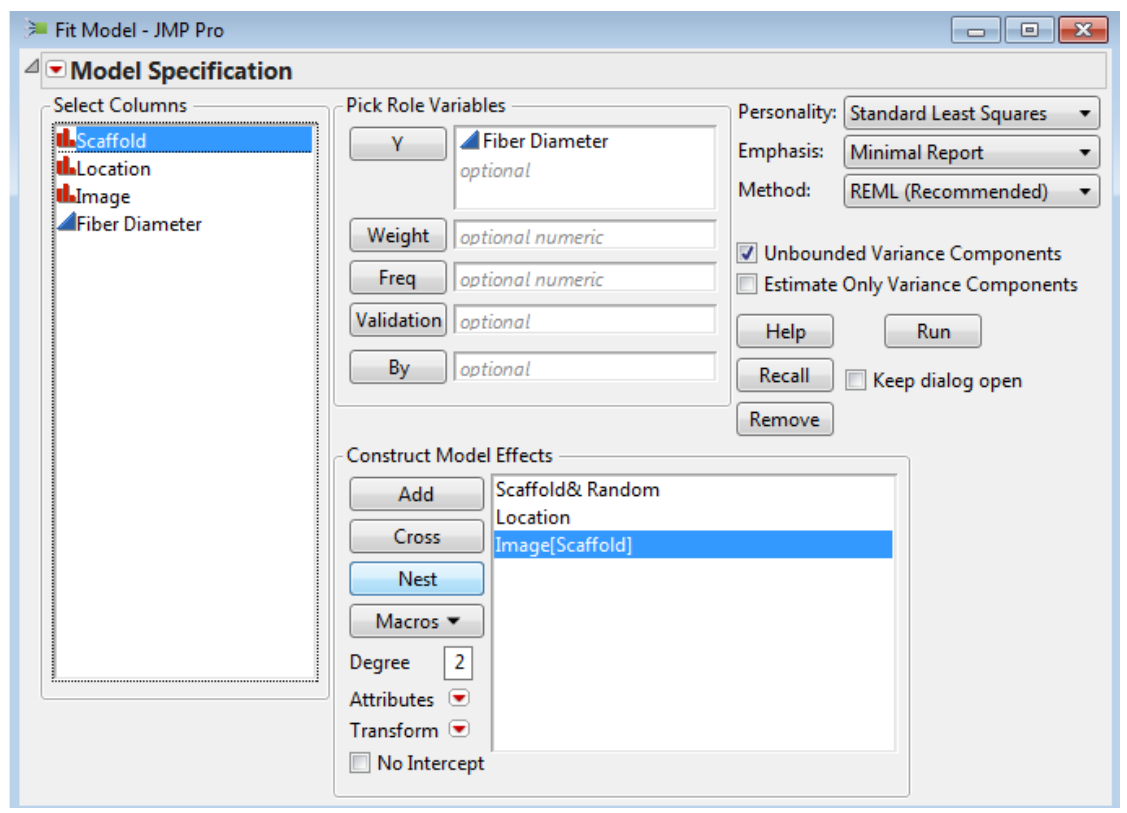


6. A new window will appear with the analysis. The results for the random effects are listed under REML Variance Components Estimates and the results for the fixed effects are listed under Fixed Effect Tests.

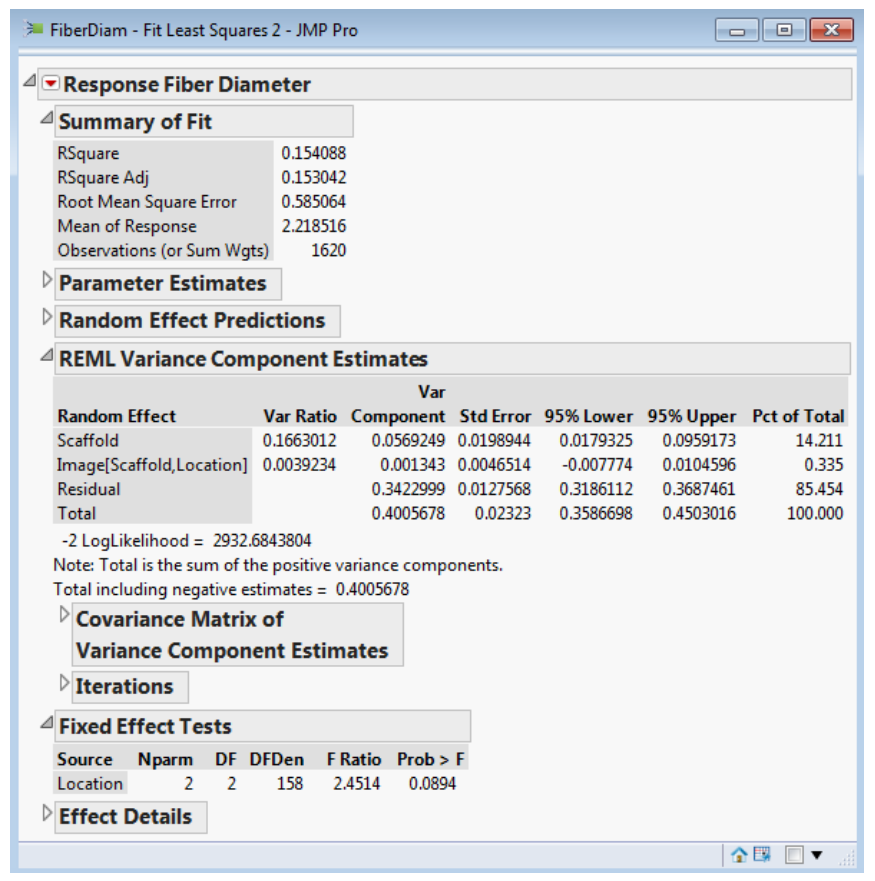

\section{One-Way ANOVA}

1. Open JMP and open the data file.

\section{Select Analyze $>$ Fit Y by X.}

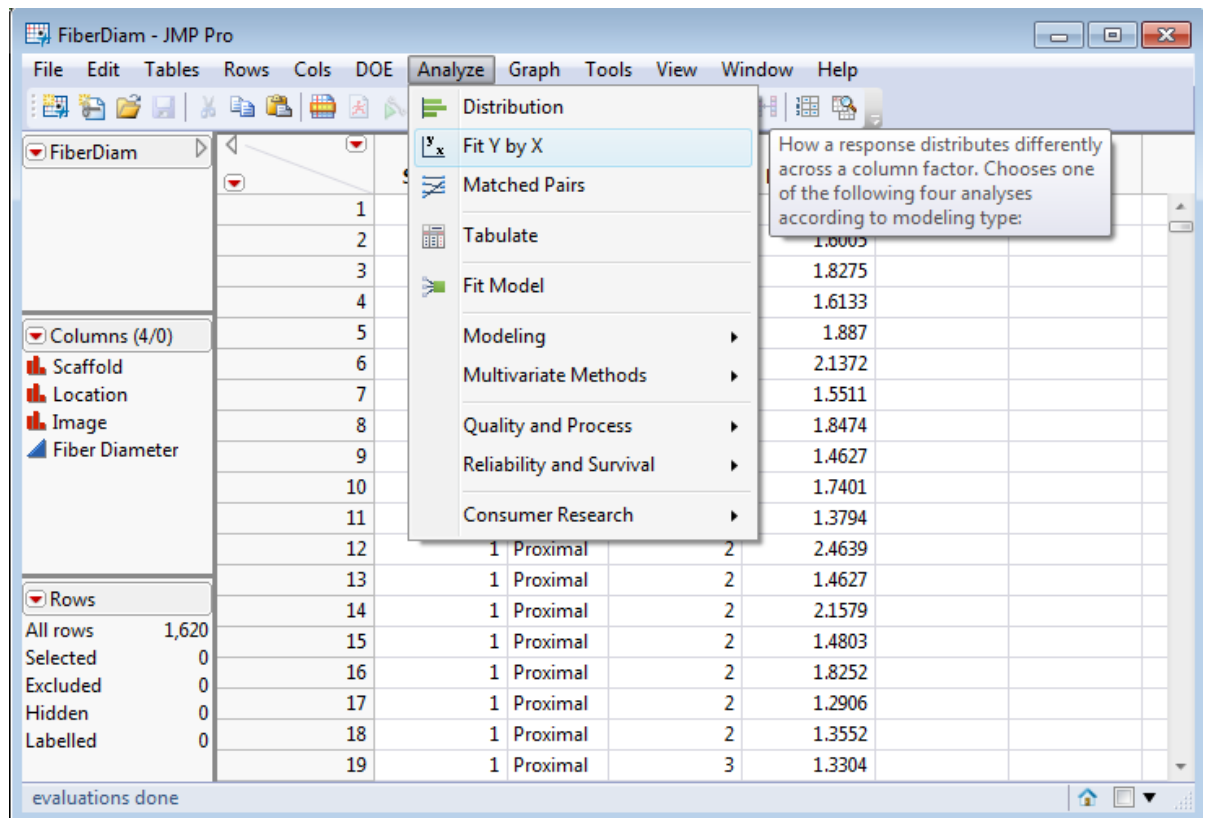


3. Place the $\mathrm{x}$ and $\mathrm{y}$ variables in the $\mathrm{X}$, Factor and $\mathrm{Y}$, Response boxes. Click OK.

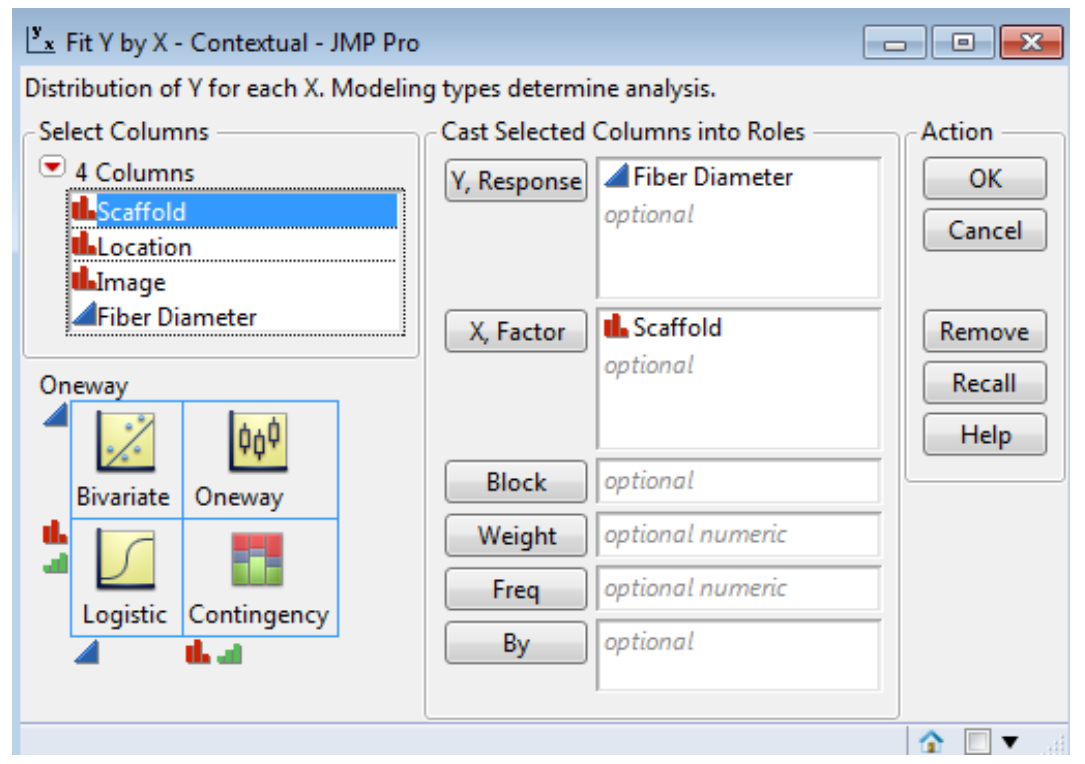

4. Click the red arrow in the top left corner. Select Means/Anova.

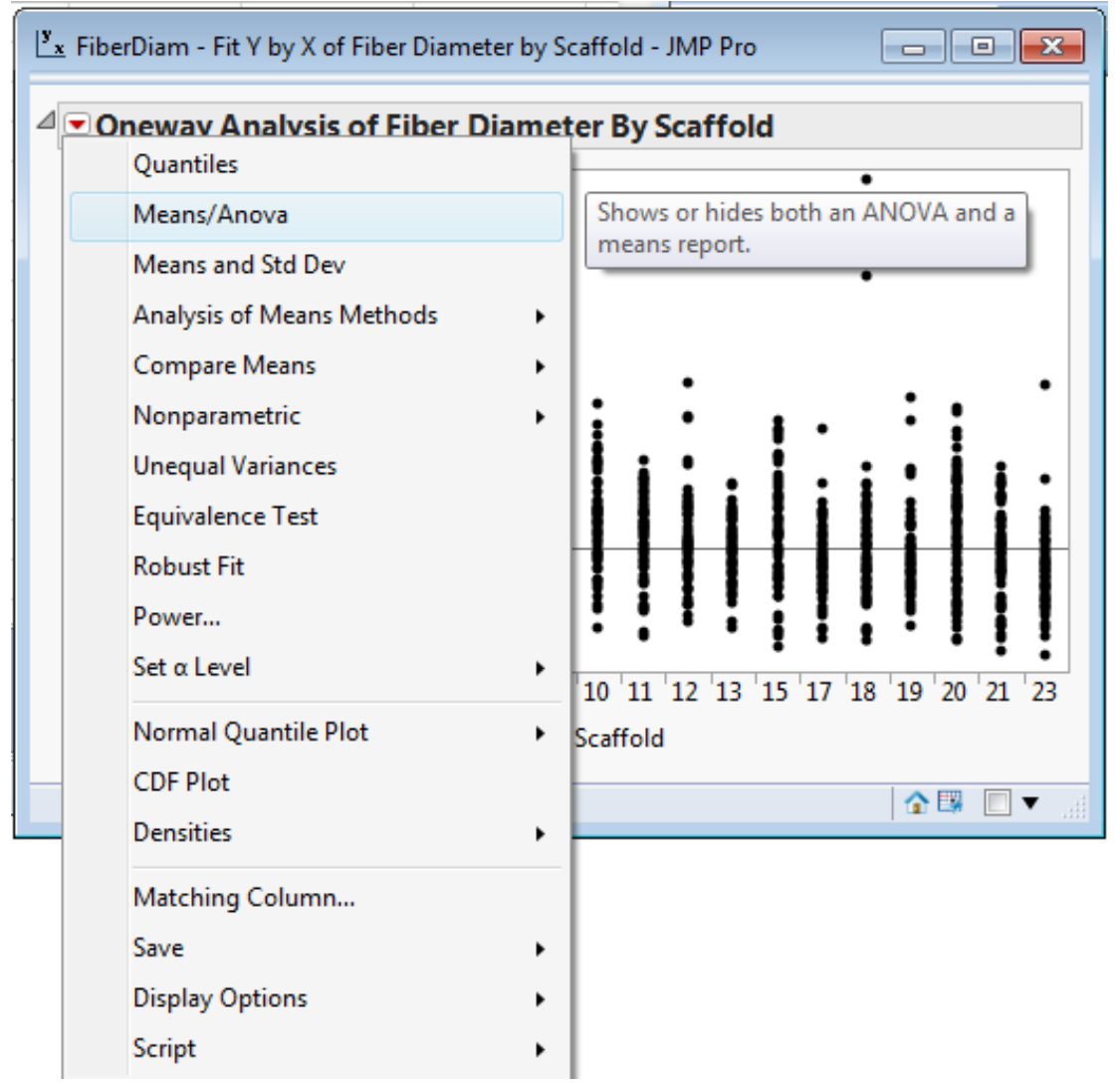


5. The ANOVA results appear under Analysis of Variance, as shown below.

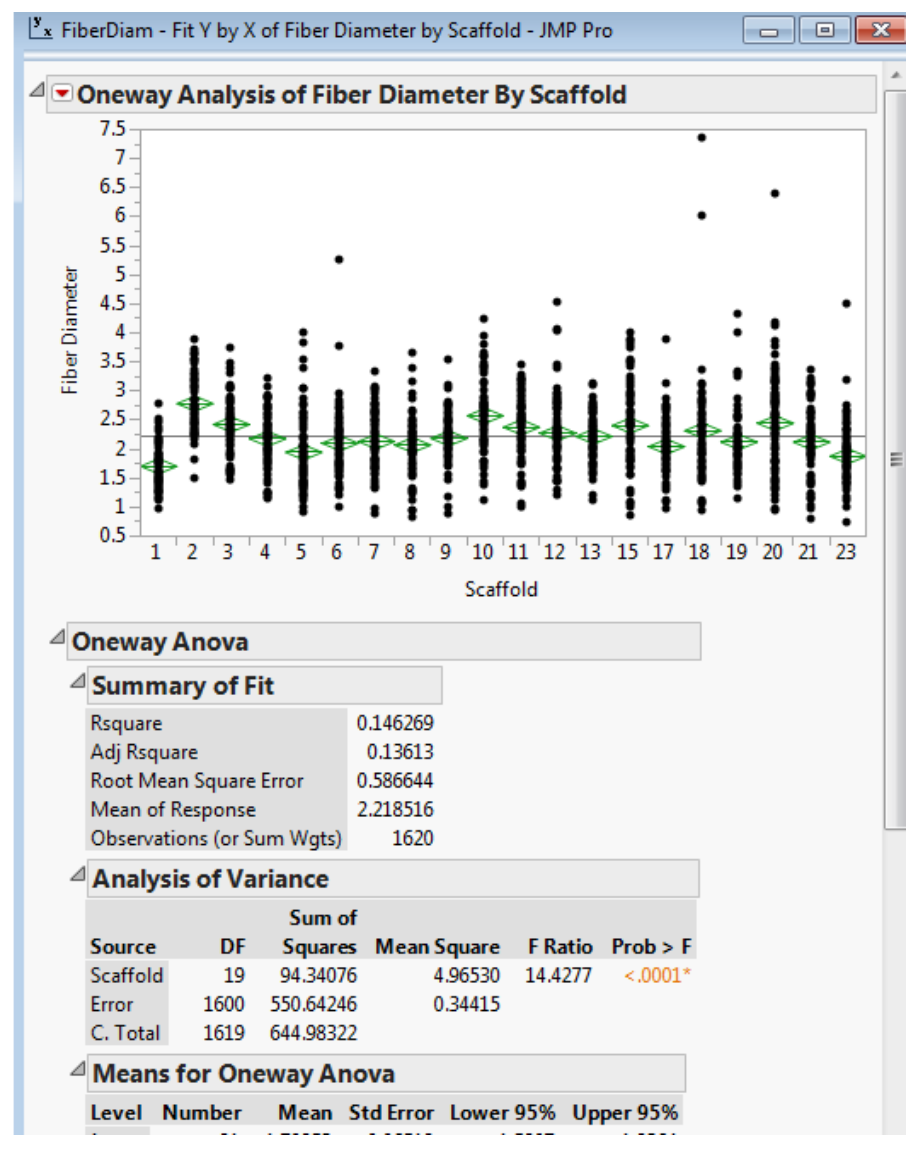

6. If needed, the graphical display can be changed. Click on the red arrow and select Display Options. Select the desired display.

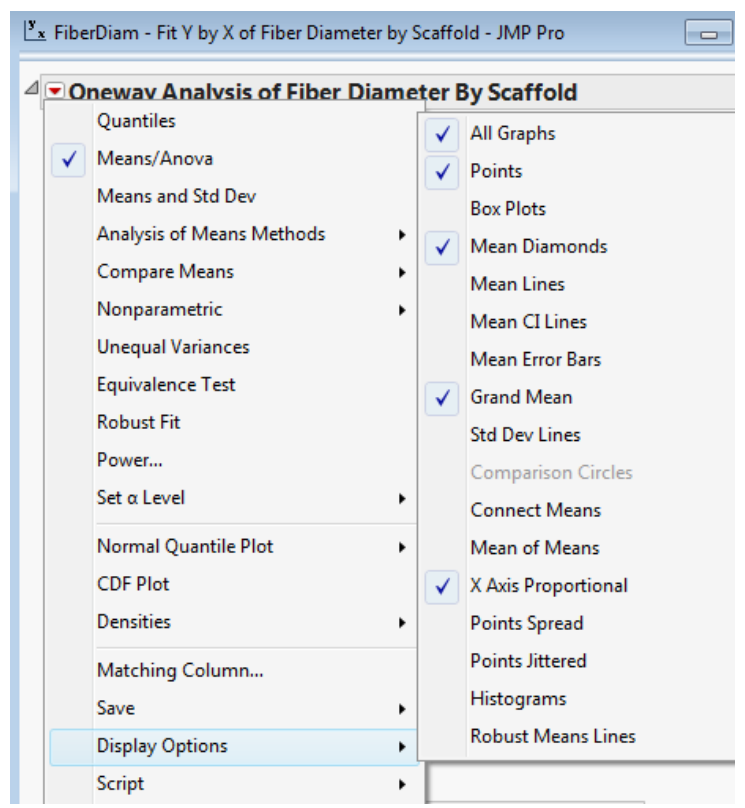




\section{Tukey's HSD Post-Hoc Test}

1. Follow the same procedure as One-Way ANOVA.

2. Click the red arrow. Select Compare Means $>$ All Pairs, Tukey HSD.

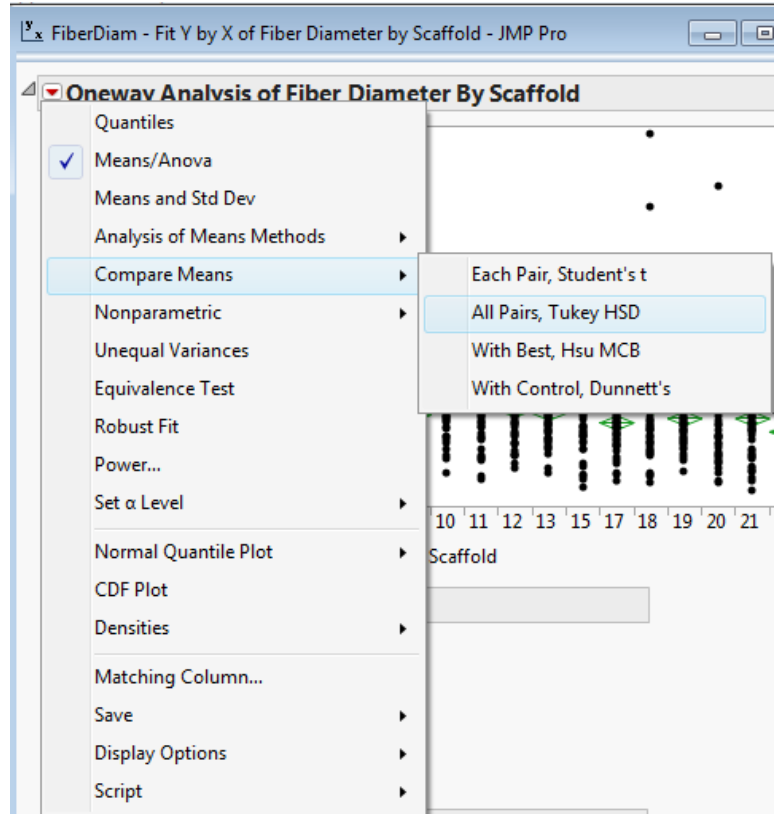

3. The results will appear after the preexisting ANOVA results, including the Connecting Letters Report and Ordered Differences Report.

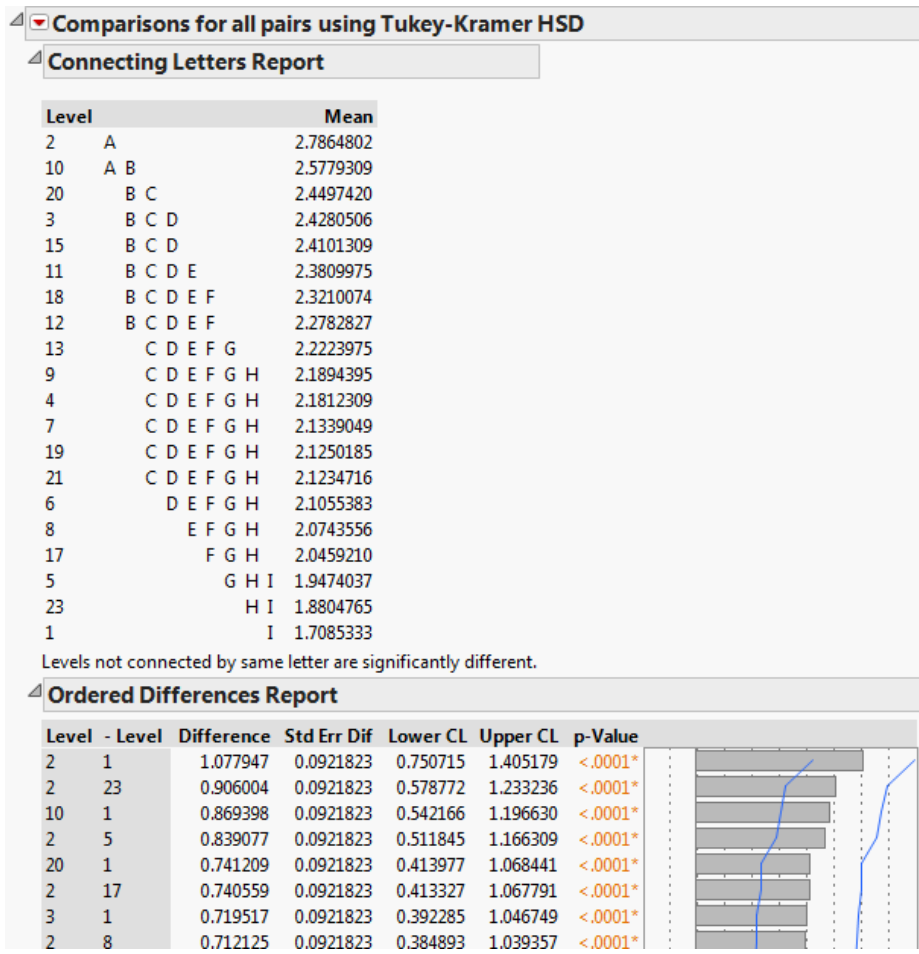




\section{One-Tailed t-Test}

1. Open JMP and open the data file.

\section{Select Analyze $>$ Fit Y by X.}

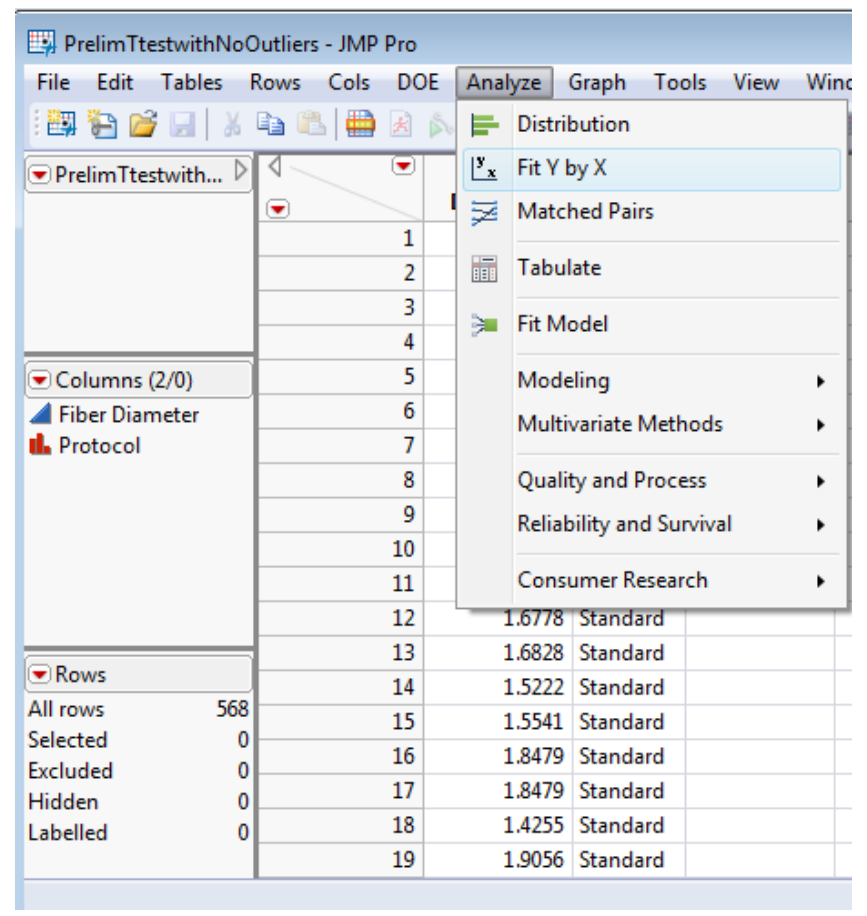

3. Place the $\mathrm{x}$ and $\mathrm{y}$ variables into the $\mathrm{X}$, Factor and $\mathrm{Y}$, Response boxes. Click OK.

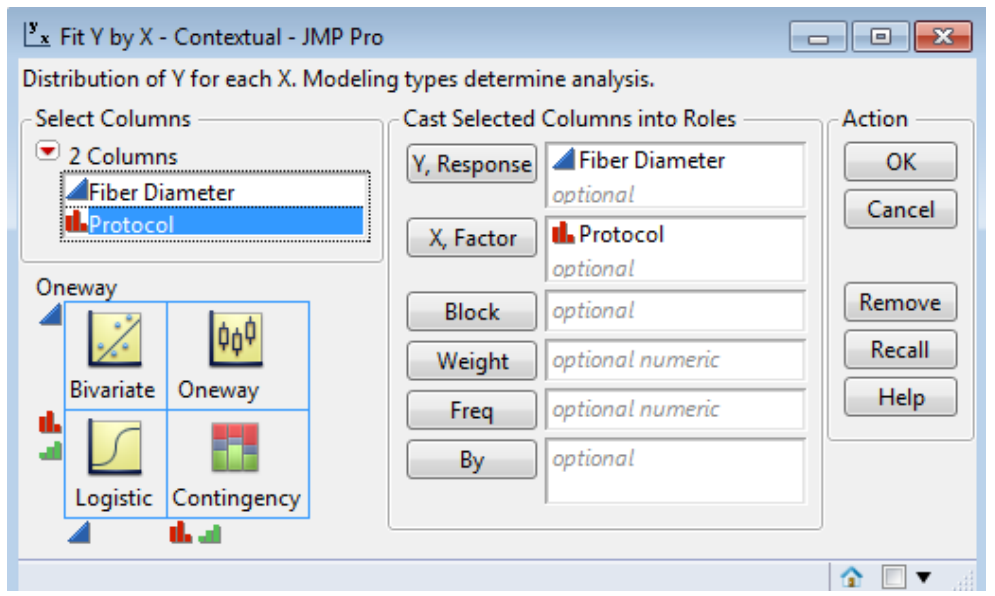


4. The results will appear in a new window. Click on the red arrow and select t Test.

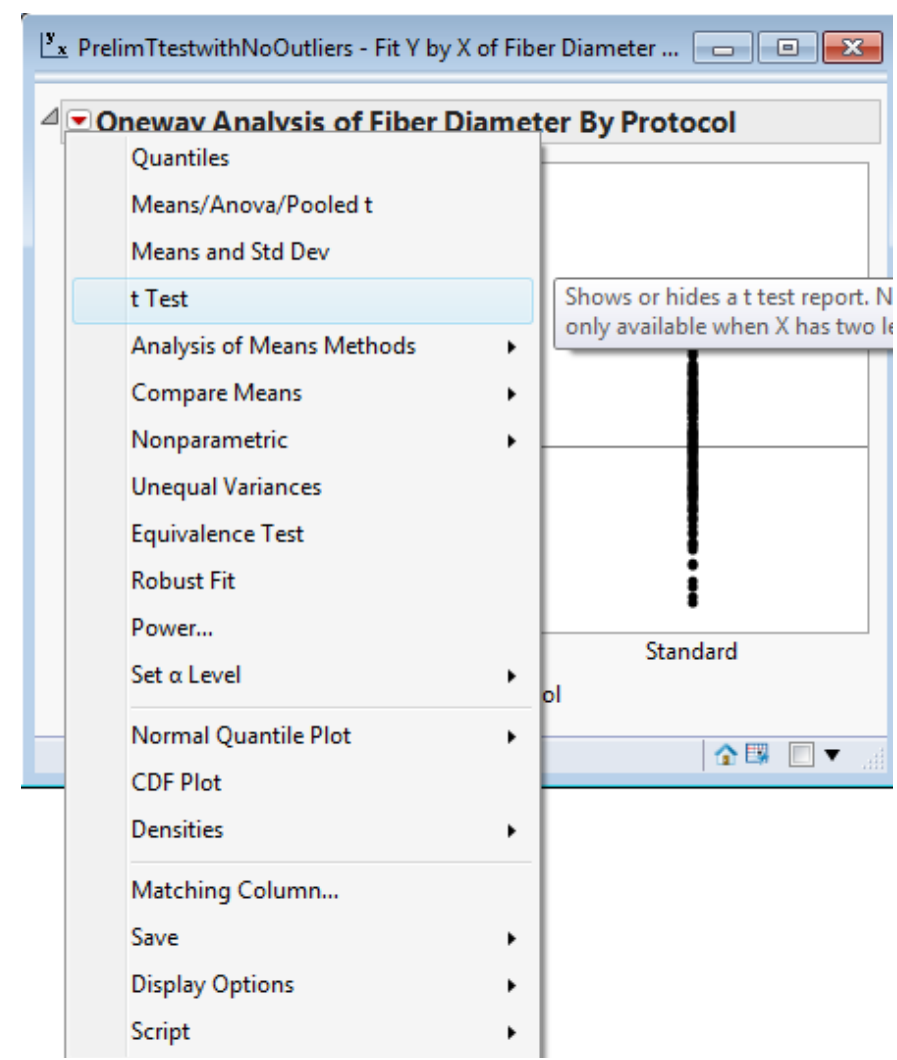

5. The results are shown below the graph. For a one-tailed t Test, look at the p-value for either Prob $>t$ or Prob $<t$, depending on the order of the variables listed directly under the t Test label (e.g. Standard-Experimental) and the alternative hypothesis.

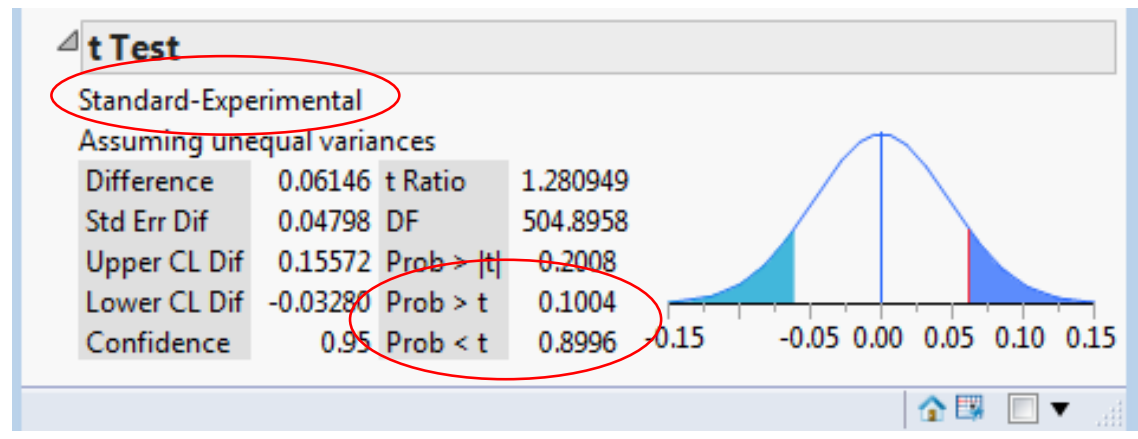




\section{Matched Pairs t-Test}

1. Open JMP and open the data file.

2. Select Analyze $>$ Matched Pairs.

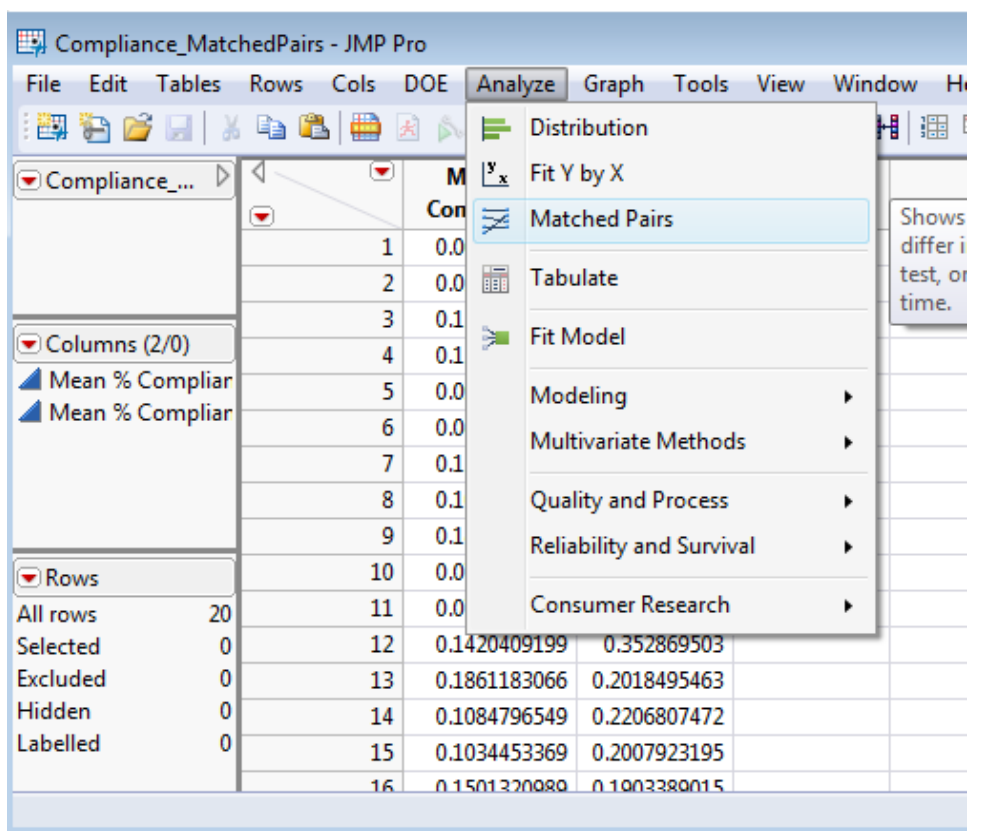

3. Place both groups into the Y, Paired Response box. Click OK.

\begin{tabular}{|c|c|c|c|}
\hline$\overline{7}$ Matched Pairs - JMP Pro & \multicolumn{3}{|c|}{$\square$ 回 $x$} \\
\hline \multicolumn{4}{|c|}{ Comparing responses in different columns } \\
\hline \multirow{7}{*}{$\begin{array}{l}\text { Select Columns } \\
2 \text { Columns } \\
\text { SMean \% Compliance (Dry) } \\
\text { LMean \% Compliance (Wet) }\end{array}$} & \multicolumn{2}{|c|}{ - Cast Selected Columns into Roles } & Action \\
\hline & \multirow[t]{2}{*}{ Y, Paired Response } & \multirow{2}{*}{$\begin{array}{l}\Delta \text { Mean \% Co...nce (Dry) } \\
\Delta \text { Mean \% Co...nce (Wet }\end{array}$} & OK \\
\hline & & & Cancel \\
\hline & $\mathrm{X}$, Grouping & optional & \\
\hline & Weight & optional numeric & Remove \\
\hline & Freq & optional numeric & Recall \\
\hline & By & optional & \\
\hline & & & 人ิ $\square \mathbf{V}$ \\
\hline
\end{tabular}


4. The results appear in a new window as a graph and corresponding statistics. Analyze the graph and look at the $p$-value for Prob $>|t|$. Means of both groups are listed as well as the mean difference between the matched pairs, as shown below.

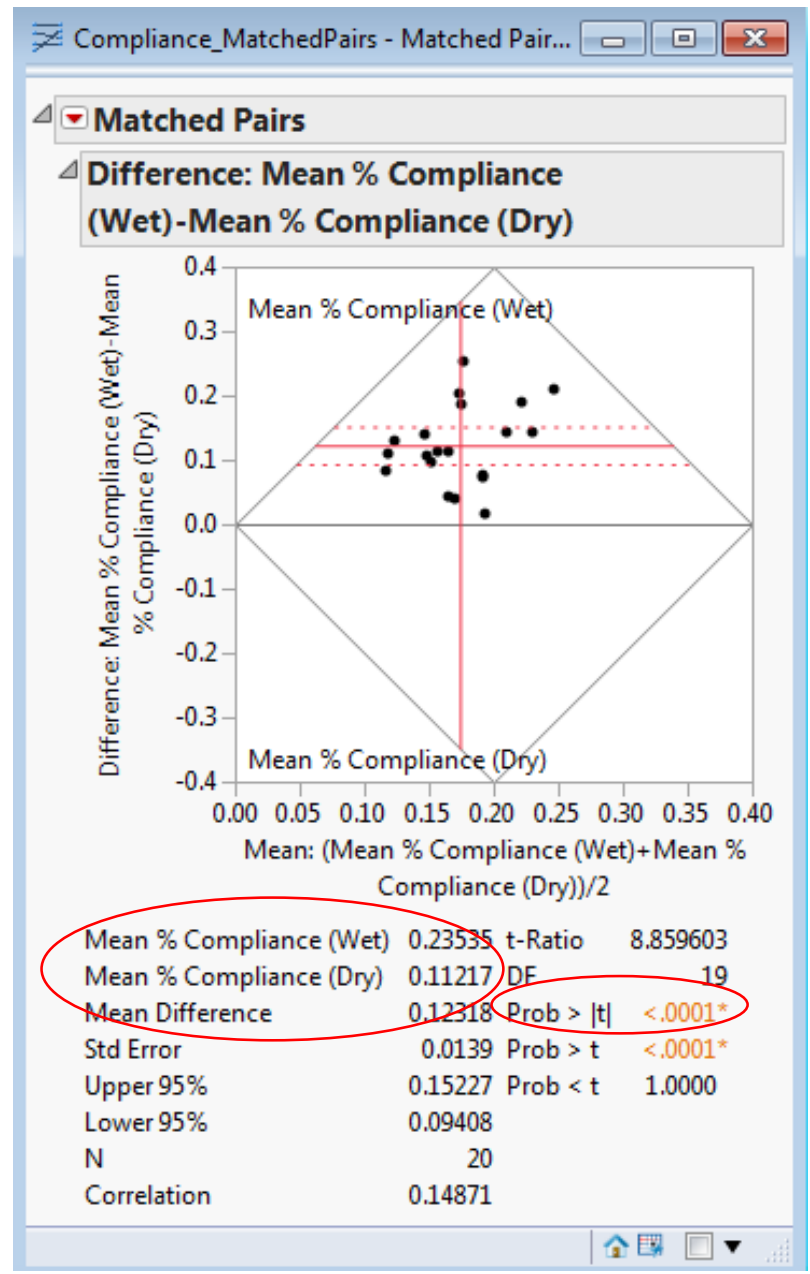




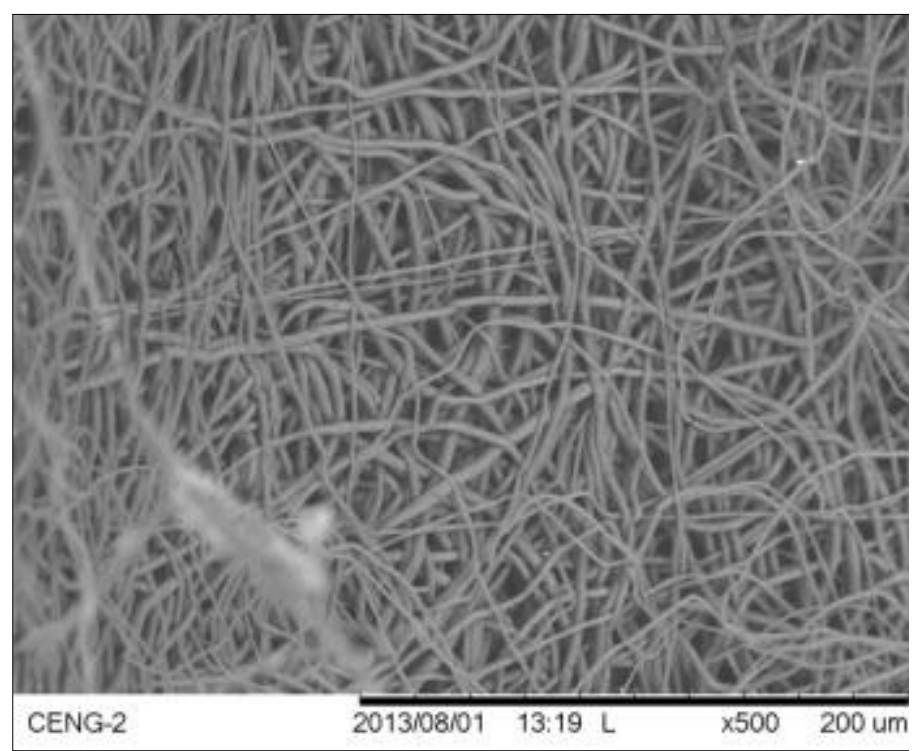

Figure F 1: SEM image of the luminal surface of

Protocol A at 500x magnification; Proximal location, Image 1.

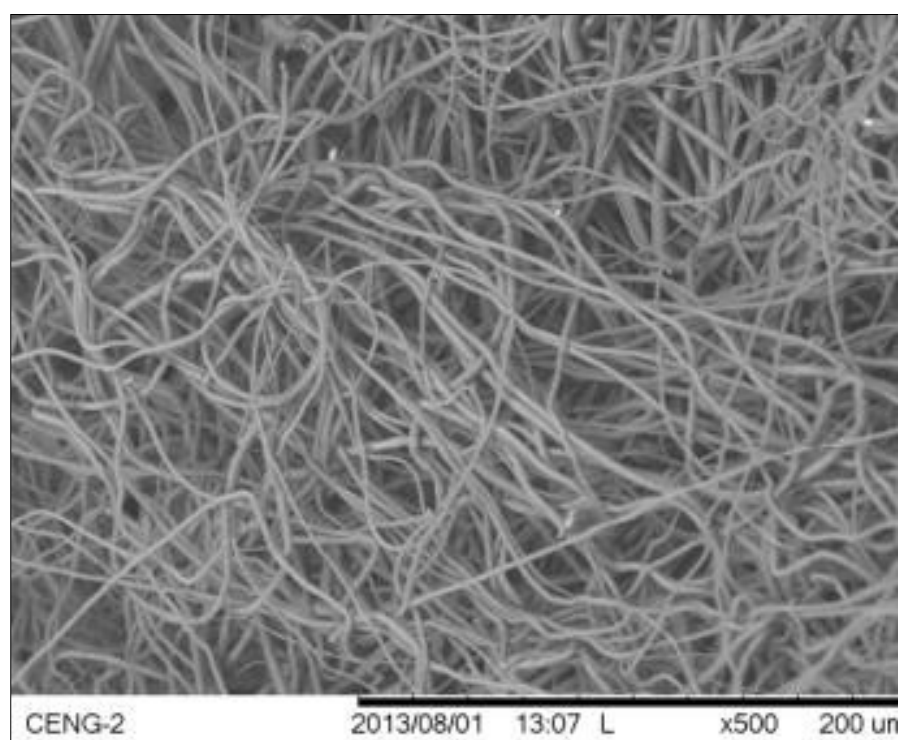

Figure F 2: SEM image of the luminal surface of Protocol A at 500x magnification; Proximal location, Image 2.

\begin{tabular}{|c|}
\hline Fiber Diameter $(\boldsymbol{\mu m})$ \\
\hline 1.3162 \\
\hline 2.0036 \\
\hline 3.4417 \\
\hline 2.2054 \\
\hline 3.5999 \\
\hline 3.8652 \\
\hline 4.13 \\
\hline 1.3106 \\
\hline 3.1402 \\
\hline 3.6505 \\
\hline 2.8511 \\
\hline 4.1481 \\
\hline 1.8479 \\
\hline 1.5511 \\
\hline 1.2971 \\
\hline 1.419 \\
\hline
\end{tabular}

\begin{tabular}{|c|}
\hline Fiber Diameter $(\boldsymbol{\mu m})$ \\
\hline 3.5697 \\
\hline 2.0686 \\
\hline 2.0078 \\
\hline 2.0811 \\
\hline 2.9431 \\
\hline 1.2046 \\
\hline 2.4556 \\
\hline 1.0405 \\
\hline 5.5927 \\
\hline 2.4352 \\
\hline 2.7378 \\
\hline 1.4715 \\
\hline 2.016 \\
\hline 1.1019 \\
\hline 0.8604 \\
\hline 1.7316 \\
\hline
\end{tabular}




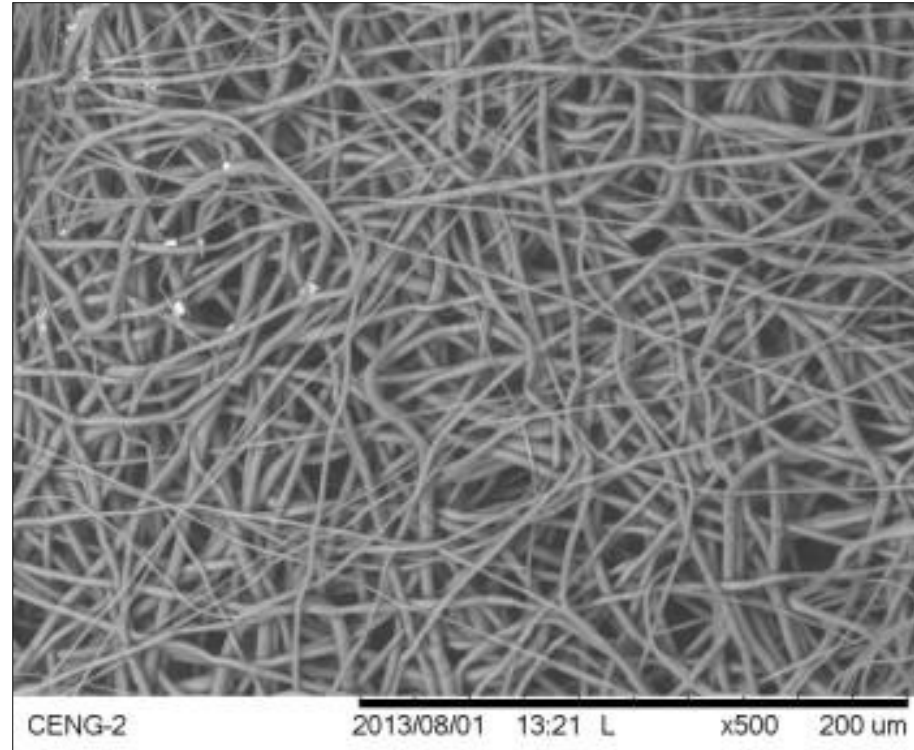

Figure F 3: SEM image of the luminal surface of

\begin{tabular}{|c|}
\hline Fiber Diameter $(\boldsymbol{\mu m})$ \\
\hline 4.0341 \\
\hline 3.3556 \\
\hline 4.9061 \\
\hline 2.9797 \\
\hline 3.1083 \\
\hline 4.4016 \\
\hline 2.065 \\
\hline 3.5109 \\
\hline 2.3231 \\
\hline 3.0378 \\
\hline 1.6828 \\
\hline 2.506 \\
\hline 1.4197 \\
\hline 1.3162 \\
\hline 4.8583 \\
\hline 1.443 \\
\hline
\end{tabular}

Protocol A at 500x magnification; Medial location, Image 1.

\section{Protocol A, Medial location, Image 2}

\begin{tabular}{|c|}
\hline Fiber Diameter $(\boldsymbol{\mu m})$ \\
\hline 3.2905 \\
\hline 3.8527 \\
\hline 3.9476 \\
\hline 2.5539 \\
\hline 1.6325 \\
\hline 1.7738 \\
\hline 6.4302 \\
\hline 3.9279 \\
\hline 2.6856 \\
\hline 1.5511 \\
\hline 1.6172 \\
\hline 4.0586 \\
\hline 3.4093 \\
\hline 1.5511 \\
\hline 2.423 \\
\hline 3.4266 \\
\hline
\end{tabular}




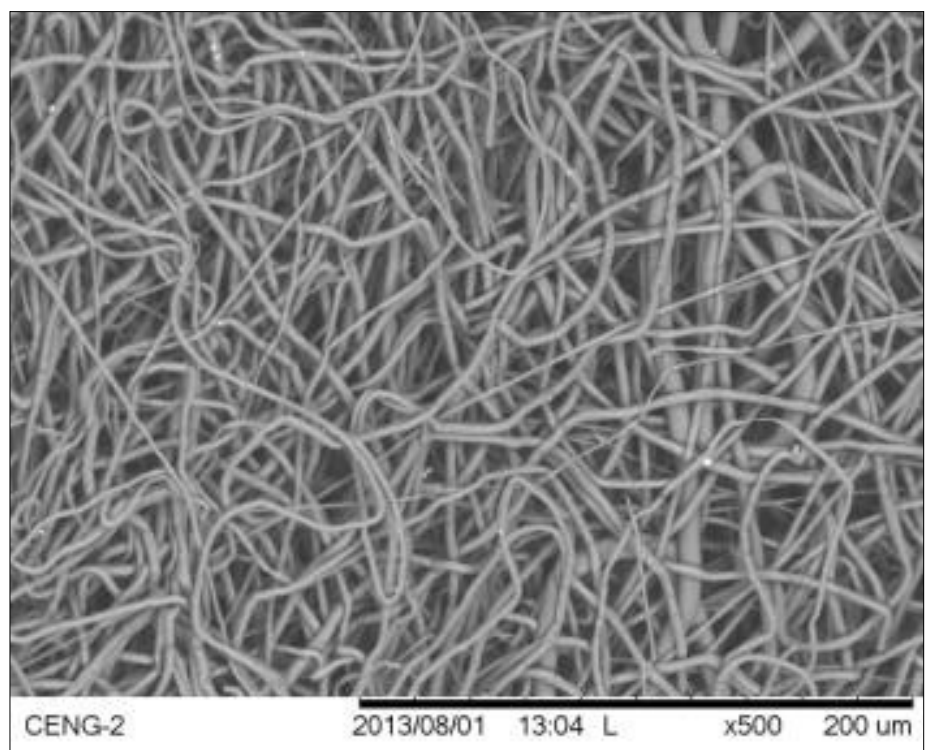

Figure F 4: SEM image of the luminal surface of

\begin{tabular}{|c|}
\hline Fiber Diameter $(\boldsymbol{\mu m})$ \\
\hline 4.7333 \\
\hline 4.2669 \\
\hline 1.5541 \\
\hline 2.9368 \\
\hline 3.3656 \\
\hline 3.6958 \\
\hline 2.5813 \\
\hline 4.3304 \\
\hline 3.1216 \\
\hline 2.7547 \\
\hline 1.4255 \\
\hline 3.3556 \\
\hline 3.042 \\
\hline 2.8859 \\
\hline 2.9719 \\
\hline 3.4631 \\
\hline
\end{tabular}

Protocol A at 500x magnification; Distal location, Image 1.

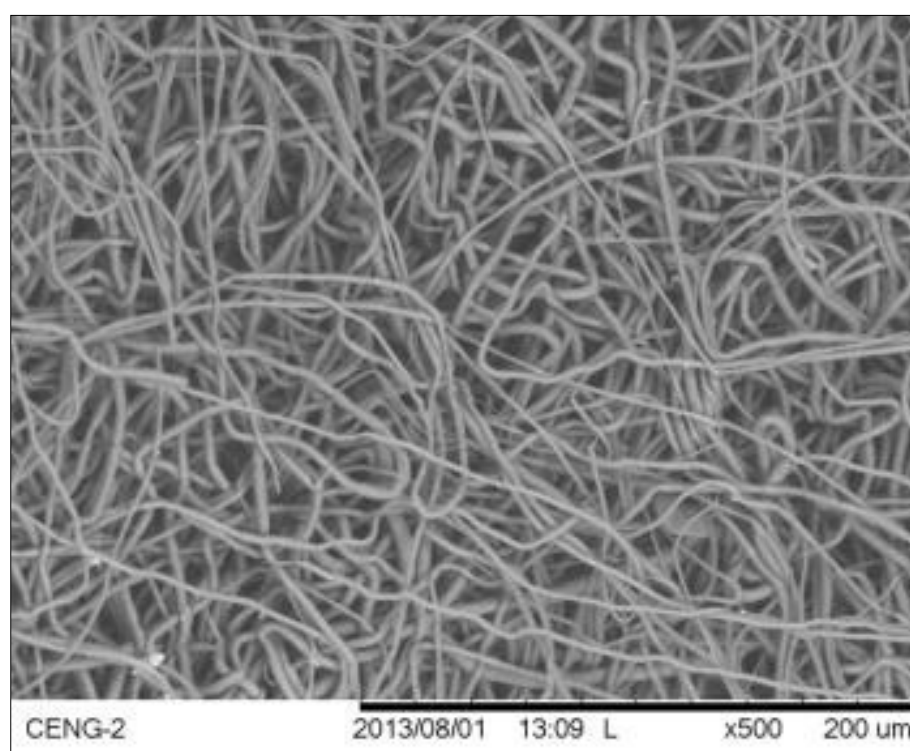

Figure F 5: SEM image of the luminal surface of Protocol A at 500x magnification; Distal location, Image 2.

\begin{tabular}{|c|}
\hline Fiber Diameter $(\boldsymbol{\mu m})$ \\
\hline 1.443 \\
\hline 1.9359 \\
\hline 2.8859 \\
\hline 1.9531 \\
\hline 3.2292 \\
\hline 3.1094 \\
\hline 1.5595 \\
\hline 3.8134 \\
\hline 3.3779 \\
\hline 3.5227 \\
\hline 2.016 \\
\hline 2.1635 \\
\hline 3.874 \\
\hline 3.2059 \\
\hline 2.3552 \\
\hline 2.6482 \\
\hline
\end{tabular}




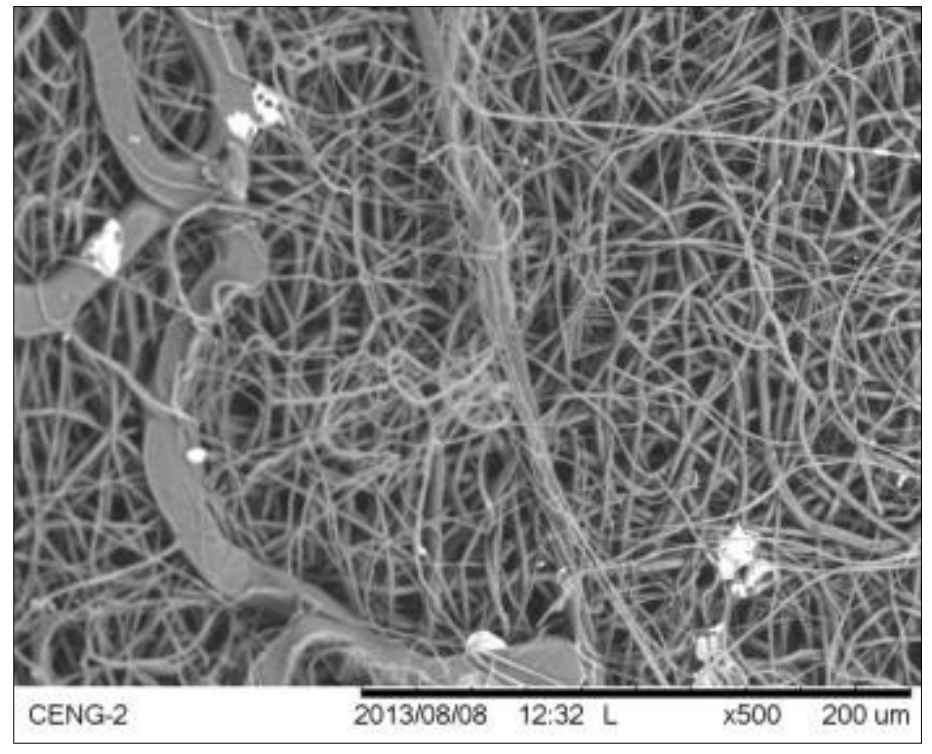

Figure F 6: SEM image of the luminal surface of

Protocol B at 500x magnification; Proximal location, Image 1.

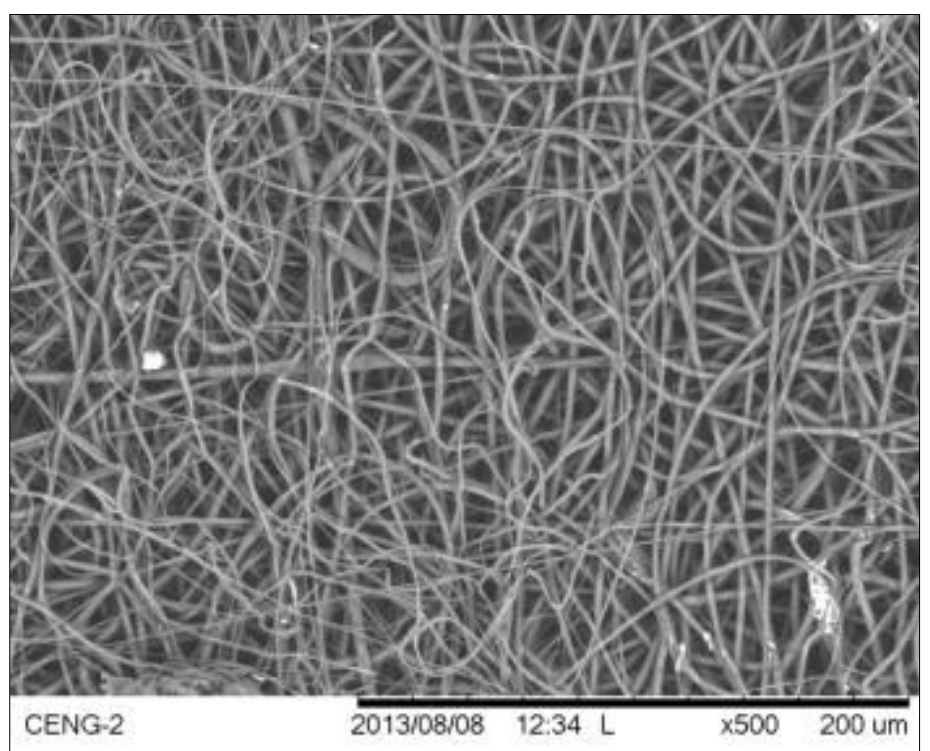

Figure $F$ 7: SEM image of the luminal surface of Protocol B at 500x magnification; Proximal location, Image 2.

\begin{tabular}{|c|}
\hline Fiber Diameter $(\boldsymbol{\mu m})$ \\
\hline 2.2371 \\
\hline 16.1878 \\
\hline 1.5051 \\
\hline 1.443 \\
\hline 1.2711 \\
\hline 1.5595 \\
\hline 1.1102 \\
\hline 7.9718 \\
\hline 1.9531 \\
\hline 1.0325 \\
\hline 2.9797 \\
\hline 0.9126 \\
\hline 1.8252 \\
\hline 1.1102 \\
\hline 2.4657 \\
\hline 1.0405 \\
\hline
\end{tabular}

\begin{tabular}{|c|}
\hline Fiber Diameter $(\boldsymbol{\mu m})$ \\
\hline 0.8775 \\
\hline 1.008 \\
\hline 2.8859 \\
\hline 1.5541 \\
\hline 1.4197 \\
\hline 5.241 \\
\hline 0.7744 \\
\hline 0.9396 \\
\hline 2.101 \\
\hline 1.6778 \\
\hline 1.6828 \\
\hline 0.6453 \\
\hline 1.9658 \\
\hline 1.1899 \\
\hline 2.8394 \\
\hline 1.5701 \\
\hline
\end{tabular}




\section{Protocol B, Medial location, Image 1}

\begin{tabular}{|c|}
\hline Fiber Diameter $(\boldsymbol{\mu m})$ \\
\hline 0.4081 \\
\hline 0.7301 \\
\hline 1.1102 \\
\hline 1.396 \\
\hline 1.9701 \\
\hline 2.5422 \\
\hline 2.0407 \\
\hline 2.6133 \\
\hline 0.8658 \\
\hline 2.3728 \\
\hline 1.396 \\
\hline 1.1544 \\
\hline 1.3604 \\
\hline 1.5701 \\
\hline 0.8163 \\
\hline 1.3288 \\
\hline
\end{tabular}

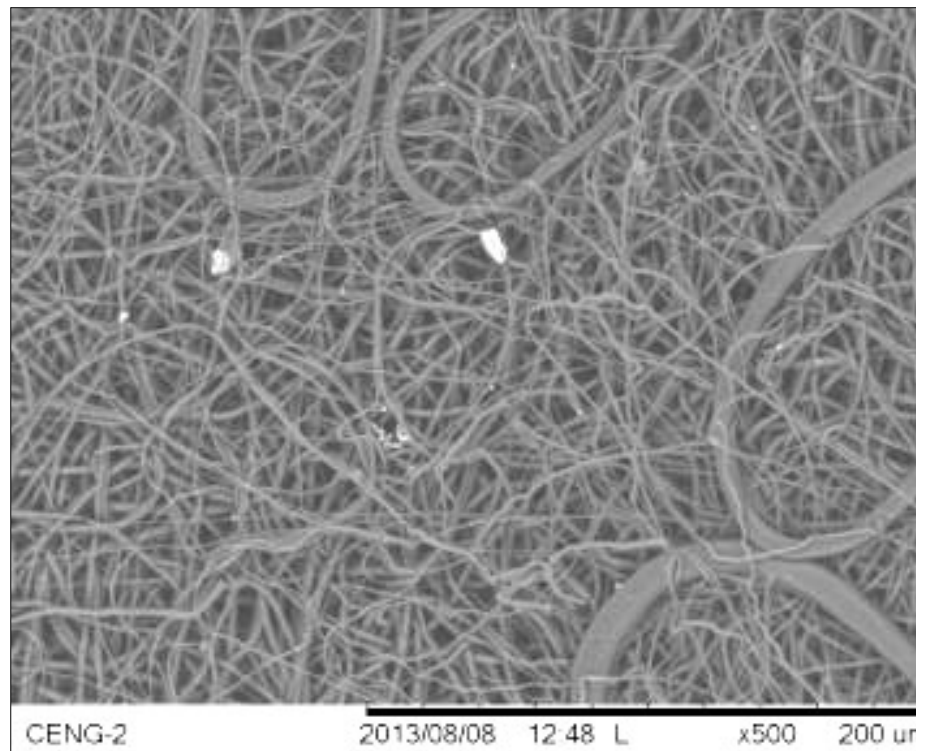

Figure $F$ 8: SEM image of the luminal surface of

\begin{tabular}{|c|}
\hline Fiber Diameter $(\boldsymbol{\mu m})$ \\
\hline 1.1899 \\
\hline 0.4302 \\
\hline 1.9621 \\
\hline 2.3247 \\
\hline 1.2971 \\
\hline 0.6453 \\
\hline 1.2906 \\
\hline 1.0325 \\
\hline 0.8264 \\
\hline 1.008 \\
\hline 1.1616 \\
\hline 13.2463 \\
\hline 0.672 \\
\hline 1.6438 \\
\hline 0.4867 \\
\hline 1.6976 \\
\hline
\end{tabular}

Protocol B at 500x magnification; Medial location, Image 2. 


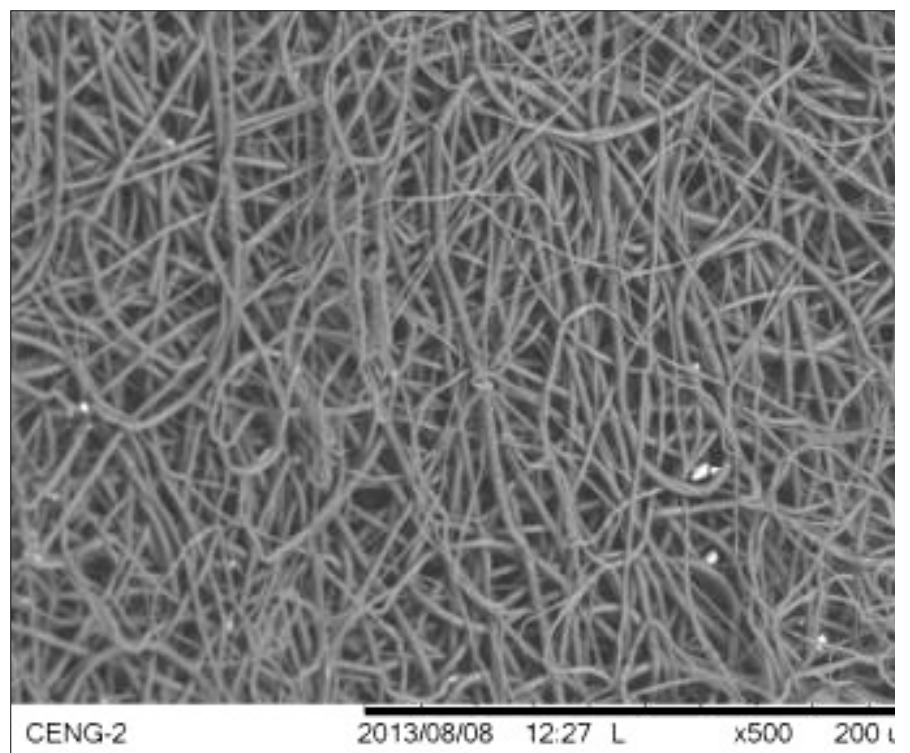

Figure F 9: SEM image of the luminal surface of Protocol B at 500x magnification; Distal location, Image 1.

\begin{tabular}{|c|}
\hline Fiber Diameter $(\boldsymbol{\mu m})$ \\
\hline 2.5941 \\
\hline 1.4803 \\
\hline 2.2371 \\
\hline 2.4639 \\
\hline 1.6172 \\
\hline 1.0884 \\
\hline 1.5488 \\
\hline 0.7526 \\
\hline 3.2292 \\
\hline 2.1941 \\
\hline 1.0071 \\
\hline 1.1899 \\
\hline 1.9359 \\
\hline 1.5541 \\
\hline 1.2244 \\
\hline 1.2906 \\
\hline
\end{tabular}

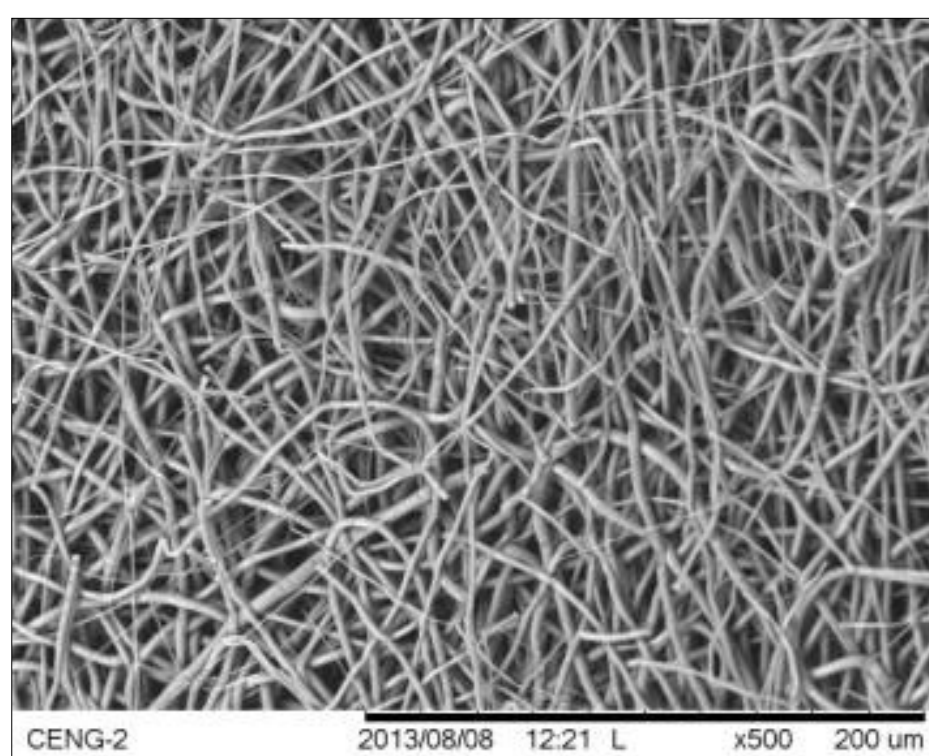

Figure F 10: SEM image of the luminal surface of

\begin{tabular}{|c|}
\hline Fiber Diameter $(\boldsymbol{\mu m})$ \\
\hline 1.4602 \\
\hline 0.7526 \\
\hline 2.1364 \\
\hline 0.6453 \\
\hline 2.1635 \\
\hline 1.6828 \\
\hline 1.0643 \\
\hline 3.3556 \\
\hline 1.5595 \\
\hline 1.3288 \\
\hline 2.101 \\
\hline 3.3056 \\
\hline 2.5845 \\
\hline 1.419 \\
\hline 2.8657 \\
\hline 4.5191 \\
\hline
\end{tabular}

Protocol B at 500x magnification; Distal location, Image 2. 


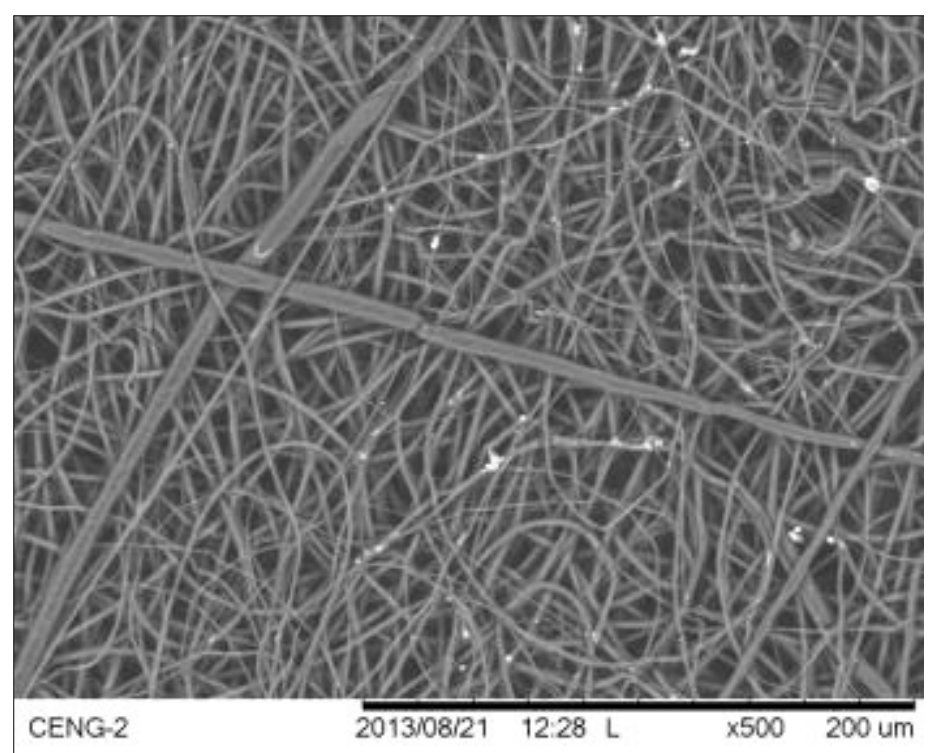

Figure F 11: SEM image of the luminal surface of Protocol $\mathrm{C}$ at 500x magnification; Proximal location, Image 1.

\begin{tabular}{|c|}
\hline Fiber Diameter $(\boldsymbol{\mu m})$ \\
\hline 2.3419 \\
\hline 7.4069 \\
\hline 0.6964 \\
\hline 2.5108 \\
\hline 2.74 \\
\hline 1.2736 \\
\hline 2.6183 \\
\hline 1.8011 \\
\hline 2.4686 \\
\hline 1.7583 \\
\hline 1.2199 \\
\hline 2.5991 \\
\hline 2.7278 \\
\hline 3.1937 \\
\hline 4.3652 \\
\hline 2.3276 \\
\hline
\end{tabular}

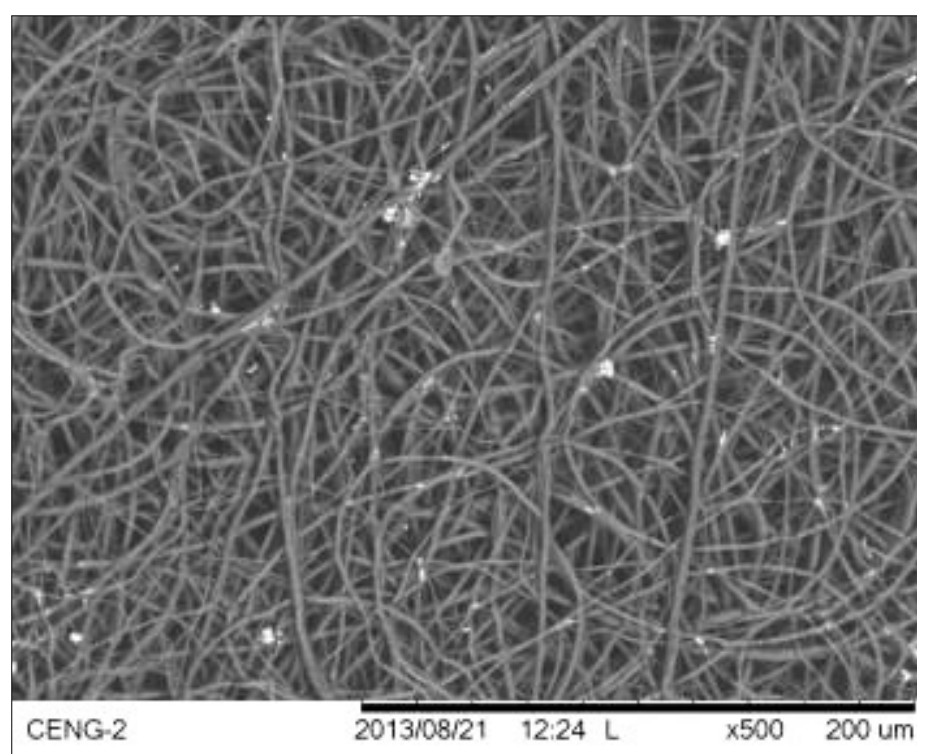

Figure F 12: SEM image of the luminal surface of

\begin{tabular}{|c|}
\hline Fiber Diameter $(\boldsymbol{\mu m})$ \\
\hline 1.5896 \\
\hline 1.0345 \\
\hline 3.4139 \\
\hline 0.9144 \\
\hline 3.016 \\
\hline 3.1303 \\
\hline 1.9696 \\
\hline 3.0737 \\
\hline 1.3466 \\
\hline 3.1701 \\
\hline 2.2097 \\
\hline 1.8514 \\
\hline 2.8712 \\
\hline 1.2267 \\
\hline 1.4283 \\
\hline 1.2931 \\
\hline
\end{tabular}

Protocol $\mathrm{C}$ at 500x magnification; Proximal location, Image 2. 


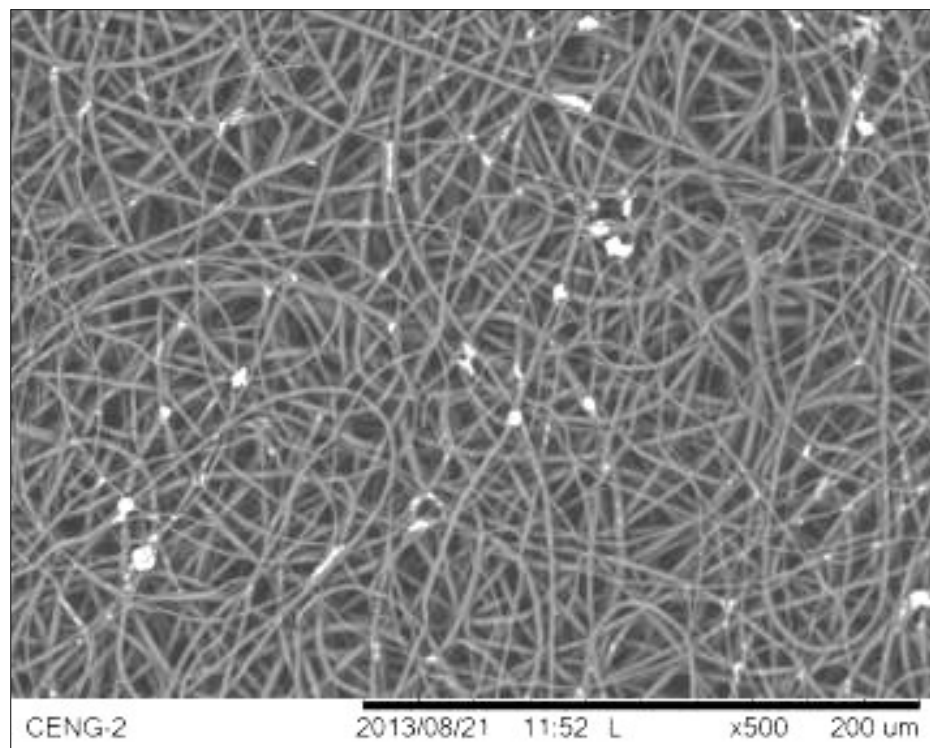

Figure F 13: SEM image of the luminal surface of

\begin{tabular}{|c|}
\hline Fiber Diameter $(\boldsymbol{\mu m})$ \\
\hline 1.4731 \\
\hline 1.0488 \\
\hline 2.0074 \\
\hline 1.3187 \\
\hline 3.0436 \\
\hline 1.104 \\
\hline 1.6265 \\
\hline 2.7643 \\
\hline 1.8966 \\
\hline 2.3003 \\
\hline 2.0107 \\
\hline 2.6374 \\
\hline 1.5731 \\
\hline 1.1638 \\
\hline 2.024 \\
\hline 1.5995 \\
\hline
\end{tabular}

Protocol $\mathrm{C}$ at 500x magnification; Medial location, Image 1.

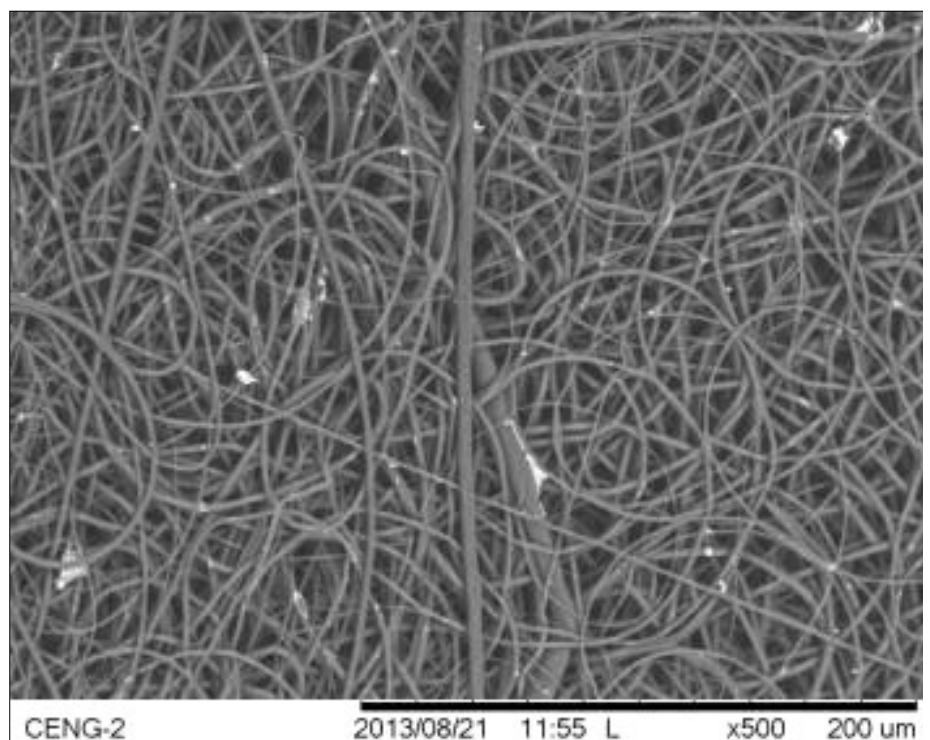

Figure F 14: SEM image of the luminal surface of Protocol $\mathrm{C}$ at 500x magnification; Medial location, Image 2.

\begin{tabular}{|c|}
\hline Fiber Diameter $(\boldsymbol{\mu m})$ \\
\hline 2.0761 \\
\hline 2.2414 \\
\hline 2.9993 \\
\hline 1.1048 \\
\hline 2.1046 \\
\hline 3.1937 \\
\hline 1.857 \\
\hline 1.9696 \\
\hline 1.2191 \\
\hline 1.9568 \\
\hline 1.6357 \\
\hline 1.4457 \\
\hline 1.7588 \\
\hline 2.2322 \\
\hline 3.2431 \\
\hline 1.7349 \\
\hline
\end{tabular}




\section{Protocol C, Distal location, Image 1}

\begin{tabular}{|c|}
\hline Fiber Diameter $(\boldsymbol{\mu m})$ \\
\hline 2.7261 \\
\hline 2.073 \\
\hline 2.2322 \\
\hline 2.4535 \\
\hline 2.3003 \\
\hline 4.7343 \\
\hline 2.9317 \\
\hline 4.0481 \\
\hline 1.7397 \\
\hline 0.5332 \\
\hline 3.678 \\
\hline 3.4914 \\
\hline 6.7241 \\
\hline 0.8178 \\
\hline 1.7349 \\
\hline 1.8103 \\
\hline
\end{tabular}

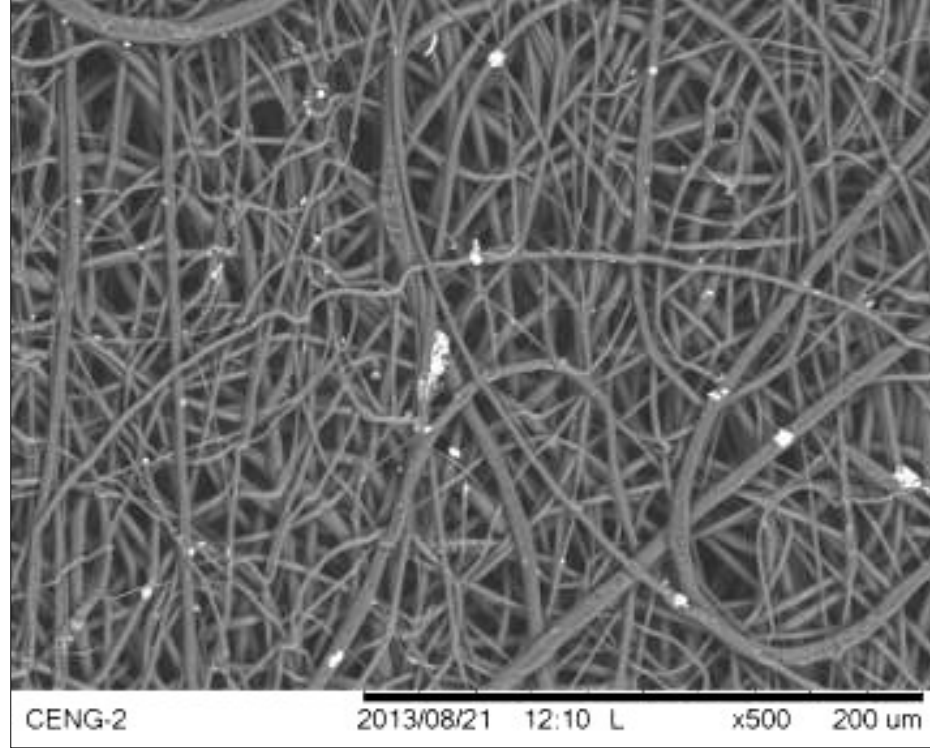

Figure F 15: SEM image of the luminal surface of Protocol $\mathrm{C}$ at 500x magnification; Distal location, Image 2.

\begin{tabular}{|c|}
\hline Fiber Diameter $(\boldsymbol{\mu m})$ \\
\hline 2.4974 \\
\hline 2.7974 \\
\hline 2.0851 \\
\hline 5.2303 \\
\hline 1.9044 \\
\hline 1.9738 \\
\hline 3.8815 \\
\hline 2.7186 \\
\hline 1.944 \\
\hline 3.1276 \\
\hline 0.6964 \\
\hline 2.1208 \\
\hline 4.8796 \\
\hline 2.4535 \\
\hline 2.073 \\
\hline 7.1857 \\
\hline
\end{tabular}




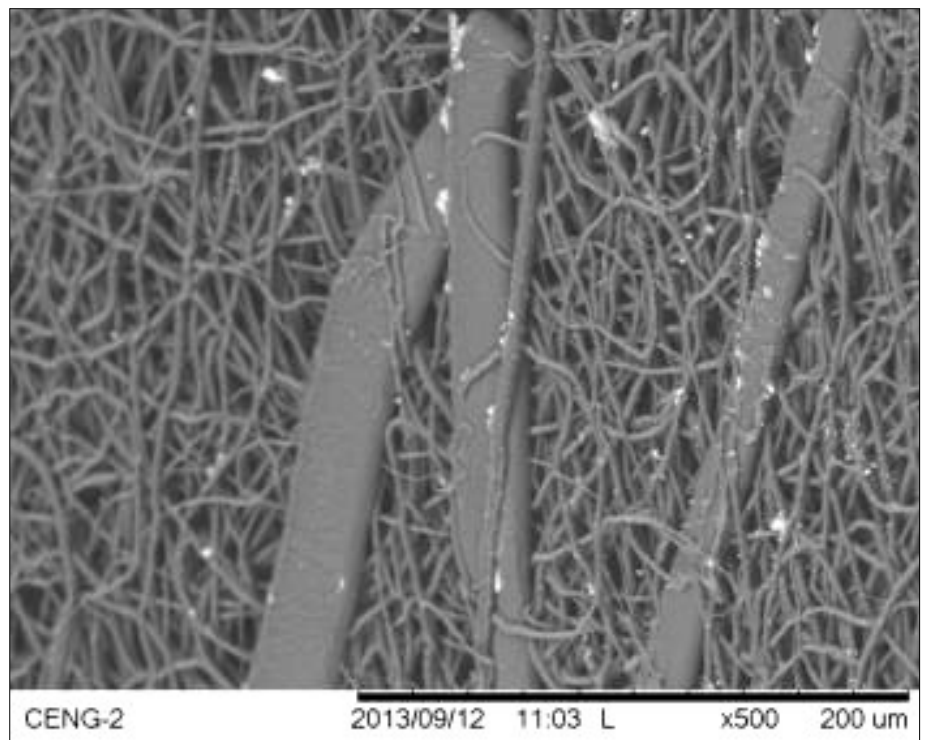

Figure F 16: SEM image of the luminal surface of Protocol D at 500x magnification; Proximal location, Image 1.

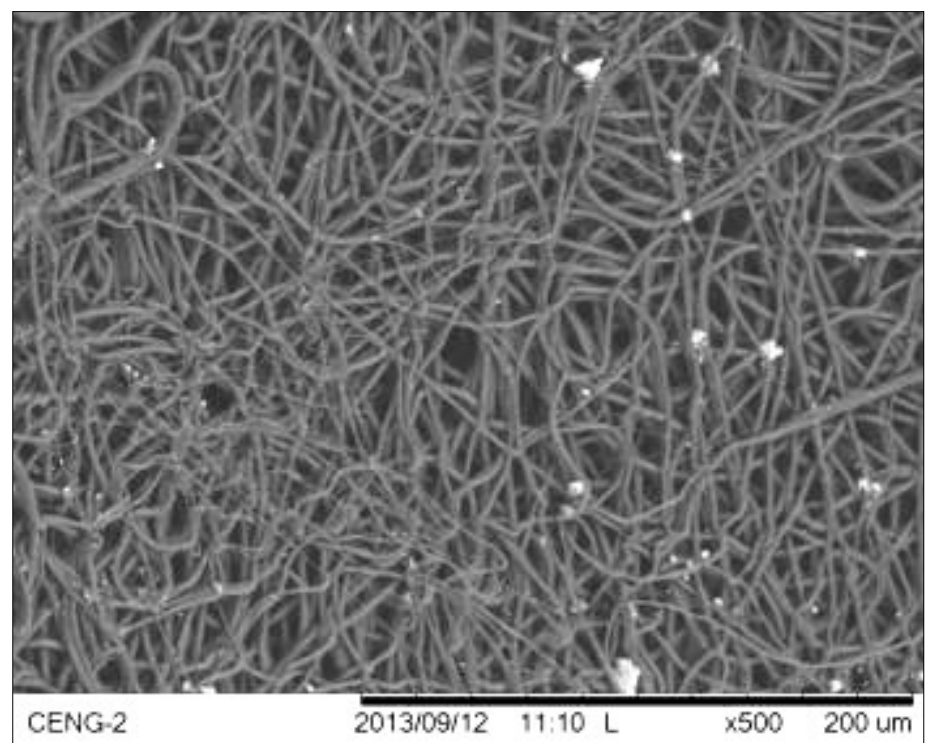

Figure F 17: SEM image of the luminal surface of Protocol D at 500x magnification; Proximal location, Image 2.

\begin{tabular}{|c|}
\hline Fiber Diameter $(\boldsymbol{\mu m})$ \\
\hline 1.8001 \\
\hline 2.248 \\
\hline 1.6203 \\
\hline 1.681 \\
\hline 1.3746 \\
\hline 1.5995 \\
\hline 31.1259 \\
\hline 33.5223 \\
\hline 1.5517 \\
\hline 2.74 \\
\hline 2.7974 \\
\hline 1.8514 \\
\hline 16.5396 \\
\hline 2.6151 \\
\hline 2.5894 \\
\hline 3.5166 \\
\hline
\end{tabular}

\begin{tabular}{|c|}
\hline Fiber Diameter $(\boldsymbol{\mu m})$ \\
\hline 2.208 \\
\hline 2.5471 \\
\hline 2.7431 \\
\hline 2.9487 \\
\hline 2.262 \\
\hline 3.8031 \\
\hline 2.0833 \\
\hline 3.2968 \\
\hline 0.8674 \\
\hline 3.3942 \\
\hline 2.6023 \\
\hline 3.5952 \\
\hline 2.7155 \\
\hline 2.7155 \\
\hline 2.7974 \\
\hline 2.4569 \\
\hline
\end{tabular}




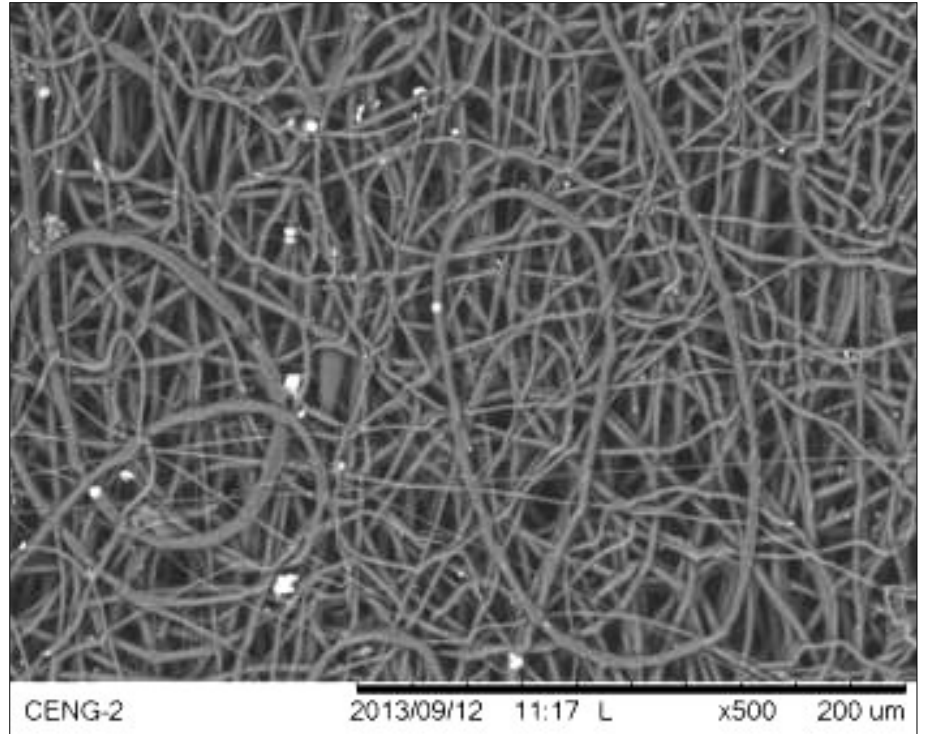

Figure F 18: SEM image of the luminal surface of Protocol D at 500x magnification; Medial location, Image 1.

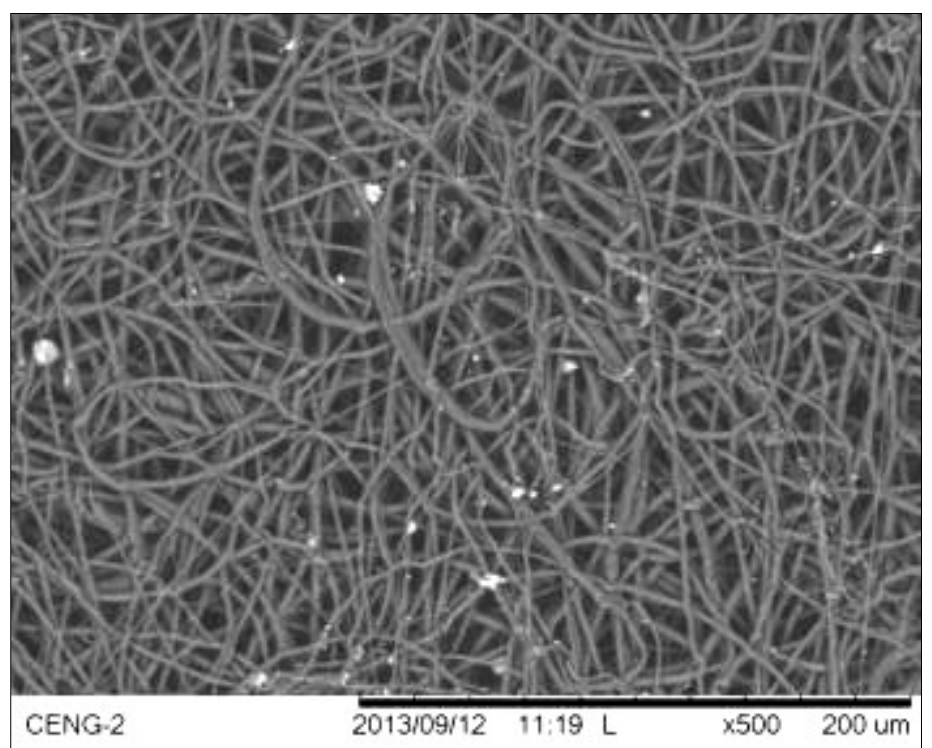

Figure F 19: SEM image of the luminal surface of Protocol D at 500x magnification; Medial location, Image 2.

\begin{tabular}{|c|}
\hline Fiber Diameter $(\boldsymbol{\mu m})$ \\
\hline 2.4398 \\
\hline 5.058 \\
\hline 1.3466 \\
\hline 2.8478 \\
\hline 4.0982 \\
\hline 2.7186 \\
\hline 1.5517 \\
\hline 3.2431 \\
\hline 2.3597 \\
\hline 3.797 \\
\hline 2.024 \\
\hline 3.994 \\
\hline 2.9487 \\
\hline 1.2736 \\
\hline 1.9738 \\
\hline 1.944 \\
\hline
\end{tabular}

Fiber Diameter $(\mu \mathrm{m})$

\begin{tabular}{|c|}
\hline 1.39 \\
\hline 1.681 \\
\hline 2.4873 \\
\hline 1.656 \\
\hline 1.9658 \\
\hline 3.6207 \\
\hline 2.105 \\
\hline 1.9092 \\
\hline 2.5765 \\
\hline 1.5784 \\
\hline 2.484 \\
\hline 2.5765 \\
\hline 4.3985 \\
\hline 2.9487 \\
\hline 2.2877 \\
\hline 2.6658 \\
\hline
\end{tabular}




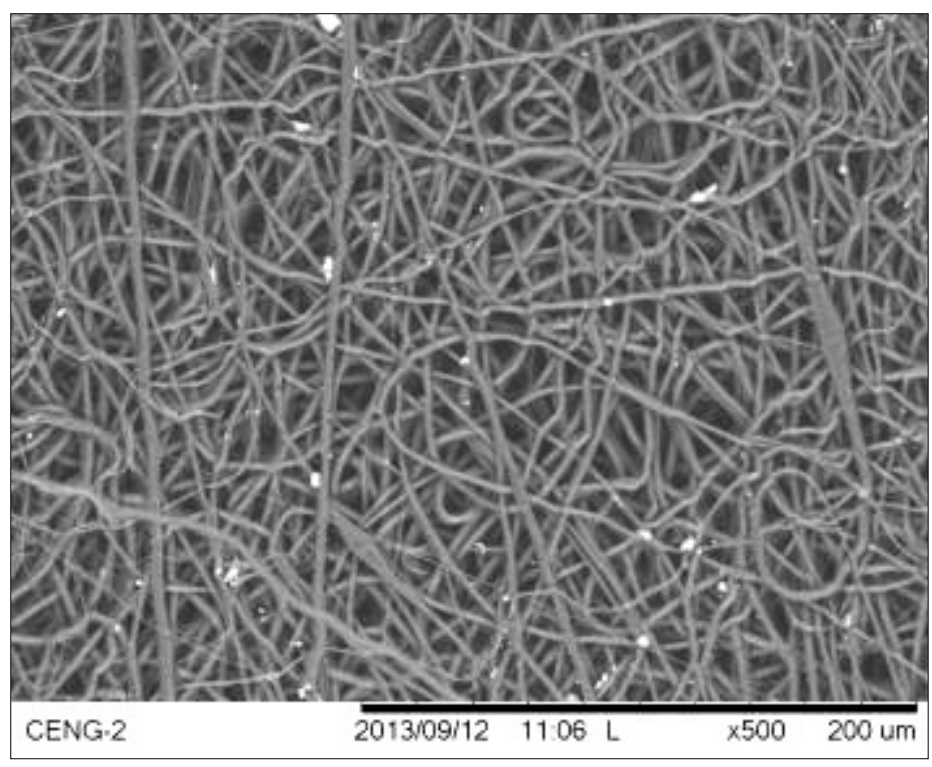

Figure F 20: SEM image of the luminal surface of

\begin{tabular}{|c|}
\hline Fiber Diameter $(\boldsymbol{\mu m})$ \\
\hline 2.5862 \\
\hline 2.4866 \\
\hline 3.3621 \\
\hline 2.6658 \\
\hline 2.4157 \\
\hline 3.8053 \\
\hline 2.9769 \\
\hline 4.9561 \\
\hline 3.2431 \\
\hline 3.372 \\
\hline 2.836 \\
\hline 3.9667 \\
\hline 2.2877 \\
\hline 1.944 \\
\hline 4.3908 \\
\hline 1.9568 \\
\hline
\end{tabular}

Protocol D at 500x magnification; Distal location, Image 1.

\section{Protocol D, Distal location, Image 2}

\begin{tabular}{|c|}
\hline Fiber Diameter $(\boldsymbol{\mu m})$ \\
\hline 1.2736 \\
\hline 1.4731 \\
\hline 3.1569 \\
\hline 2.7492 \\
\hline 8.8655 \\
\hline 18.5927 \\
\hline 3.5502 \\
\hline 2.6151 \\
\hline 3.312 \\
\hline 2.3738 \\
\hline 11.6133 \\
\hline 1.7008 \\
\hline 3.4938 \\
\hline 2.4398 \\
\hline 2.7431 \\
\hline 3.1088 \\
\hline
\end{tabular}




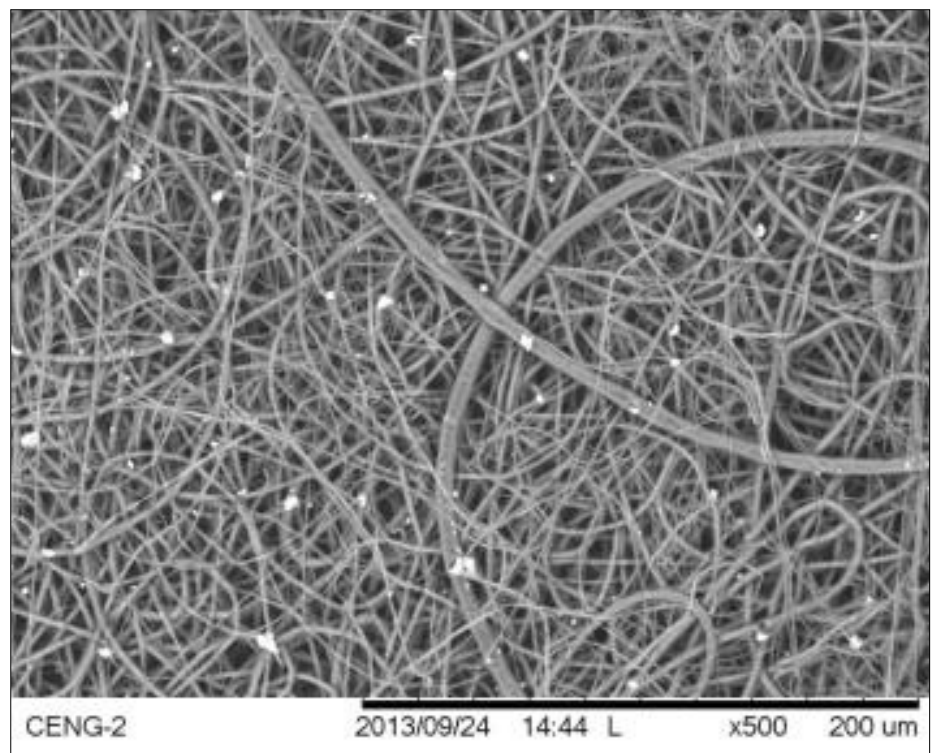

Figure F 21: SEM image of the luminal surface of Protocol E at 500x magnification; Proximal location, Image 1.

\begin{tabular}{|c|}
\hline Fiber Diameter $(\boldsymbol{\mu m})$ \\
\hline 1.4744 \\
\hline 1.35 \\
\hline 1.5517 \\
\hline 1.5135 \\
\hline 1.7349 \\
\hline 1.3045 \\
\hline 3.3371 \\
\hline 1.7252 \\
\hline 0.8534 \\
\hline 0.9753 \\
\hline 0.9052 \\
\hline 1.1922 \\
\hline 1.9846 \\
\hline 3.4698 \\
\hline 2.8478 \\
\hline 1.3927 \\
\hline
\end{tabular}

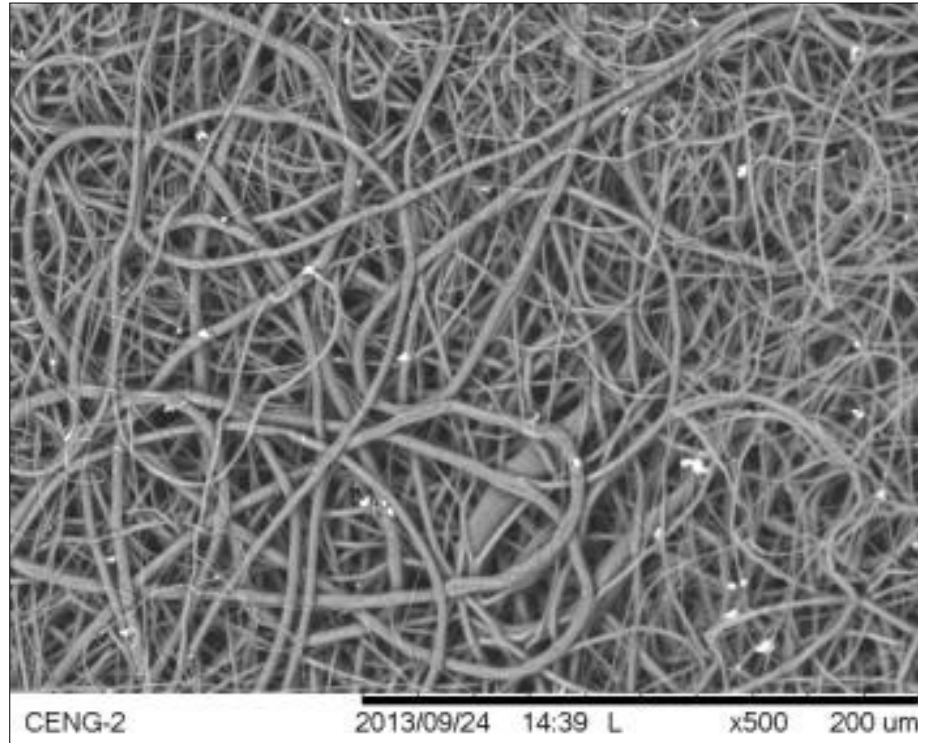

Figure $F$ 22: SEM image of the luminal surface of

\begin{tabular}{|c|}
\hline Fiber Diameter $(\boldsymbol{\mu m})$ \\
\hline 1.815 \\
\hline 1.4457 \\
\hline 2.3312 \\
\hline 1.1048 \\
\hline 0.7315 \\
\hline 3.3546 \\
\hline 4.4645 \\
\hline 4.962 \\
\hline 1.463 \\
\hline 2.2322 \\
\hline 2.2134 \\
\hline 2.0181 \\
\hline 3.634 \\
\hline 1.656 \\
\hline 1.7588 \\
\hline 3.2016 \\
\hline
\end{tabular}

Protocol E at 500x magnification; Proximal location, Image 2. 


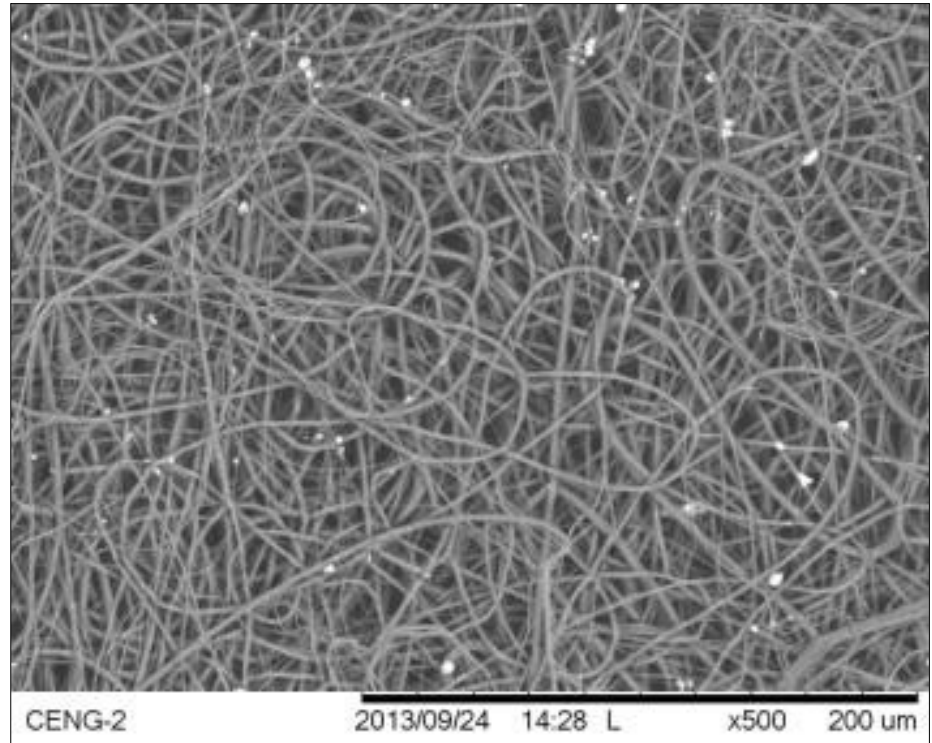

Figure F 23: SEM image of the luminal surface of Protocol E at 500x magnification; Medial location, Image 1.

\begin{tabular}{|c|}
\hline Fiber Diameter $(\boldsymbol{\mu m})$ \\
\hline 2.2677 \\
\hline 1.7008 \\
\hline 1.681 \\
\hline 1.2267 \\
\hline 2.0851 \\
\hline 1.1048 \\
\hline 2.0761 \\
\hline 1.5517 \\
\hline 0.7315 \\
\hline 2.2134 \\
\hline 1.944 \\
\hline 1.1922 \\
\hline 2.1292 \\
\hline 3.623 \\
\hline 2.069 \\
\hline 4.7424 \\
\hline
\end{tabular}

\section{Protocol E, Medial location, Image 2}

\begin{tabular}{|c|}
\hline Fiber Diameter $(\boldsymbol{\mu m})$ \\
\hline 2.1983 \\
\hline 2.248 \\
\hline 1.5613 \\
\hline 1.9658 \\
\hline 1.3466 \\
\hline 1.0904 \\
\hline 1.5517 \\
\hline 1.9696 \\
\hline 1.3187 \\
\hline 2.1945 \\
\hline 2.5667 \\
\hline 2.3597 \\
\hline 1.9397 \\
\hline 2.4295 \\
\hline 3.6437 \\
\hline 1.9223 \\
\hline
\end{tabular}




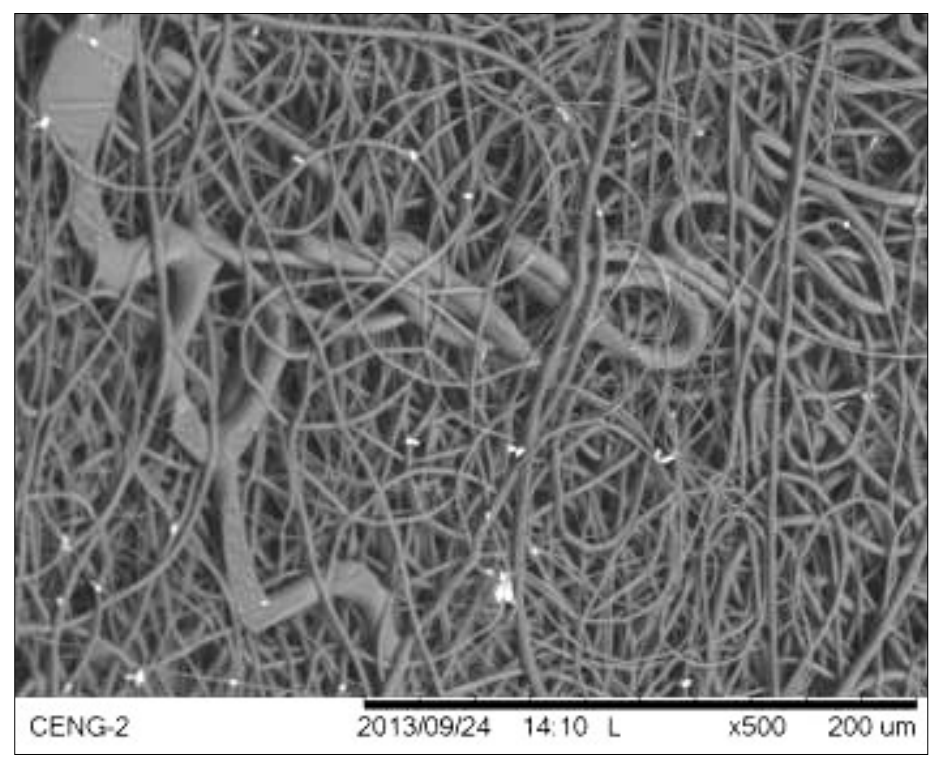

Figure F 24: SEM image of the luminal surface of Protocol E at 500x magnification; Distal location, Image 1.

\begin{tabular}{|c|}
\hline Fiber Diameter $(\boldsymbol{\mu m})$ \\
\hline 28.1943 \\
\hline 13.7124 \\
\hline 2.1326 \\
\hline 2.1809 \\
\hline 2.4398 \\
\hline 1.9568 \\
\hline 1.1638 \\
\hline 2.2914 \\
\hline 2.7914 \\
\hline 3.2431 \\
\hline 3.0188 \\
\hline 1.1566 \\
\hline 5.308 \\
\hline 3.6506 \\
\hline 3.5176 \\
\hline 1.8103 \\
\hline
\end{tabular}

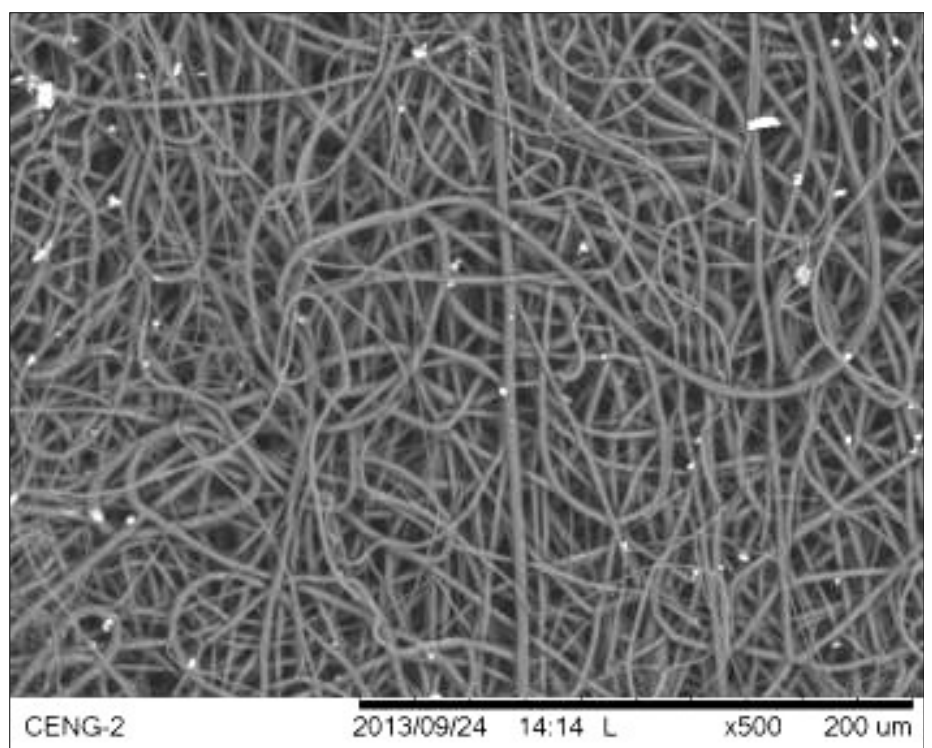

Figure $F$ 25: SEM image of the luminal surface of Protocol E at 500x magnification; Distal location, Image 2.

\begin{tabular}{|c|}
\hline Fiber Diameter $(\boldsymbol{\mu m})$ \\
\hline 1.8649 \\
\hline 2.9361 \\
\hline 1.686 \\
\hline 1.8514 \\
\hline 2.0891 \\
\hline 2.3952 \\
\hline 2.7854 \\
\hline 2.0116 \\
\hline 2.1603 \\
\hline 2.4295 \\
\hline 2.3312 \\
\hline 2.2914 \\
\hline 2.4053 \\
\hline 2.7974 \\
\hline 2.0891 \\
\hline 1.8287 \\
\hline
\end{tabular}




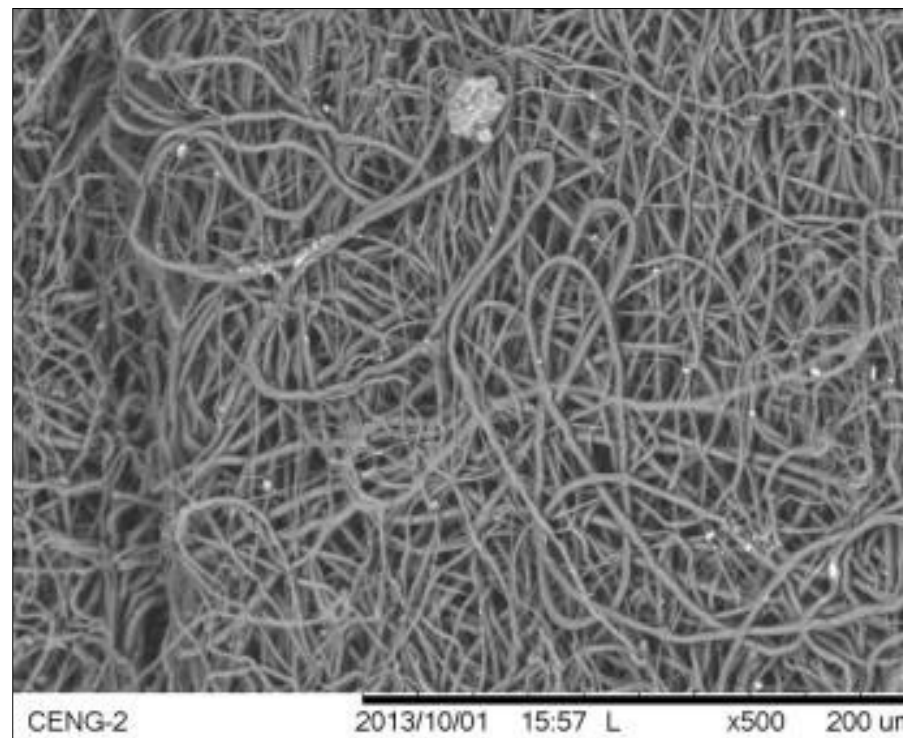

Figure F 26: SEM image of the luminal surface of Spin B1 at 500x magnification; Proximal location, Image 1.

\begin{tabular}{|c|}
\hline Fiber Diameter $(\boldsymbol{\mu m})$ \\
\hline 1.6796 \\
\hline 1.4228 \\
\hline 1.3137 \\
\hline 2.1128 \\
\hline 2.4509 \\
\hline 2.9375 \\
\hline 1.2946 \\
\hline 2.0163 \\
\hline 2.5359 \\
\hline 2.0368 \\
\hline 1.0623 \\
\hline 2.2497 \\
\hline 1.7186 \\
\hline 1.5512 \\
\hline 0.9289 \\
\hline 2.0771 \\
\hline
\end{tabular}

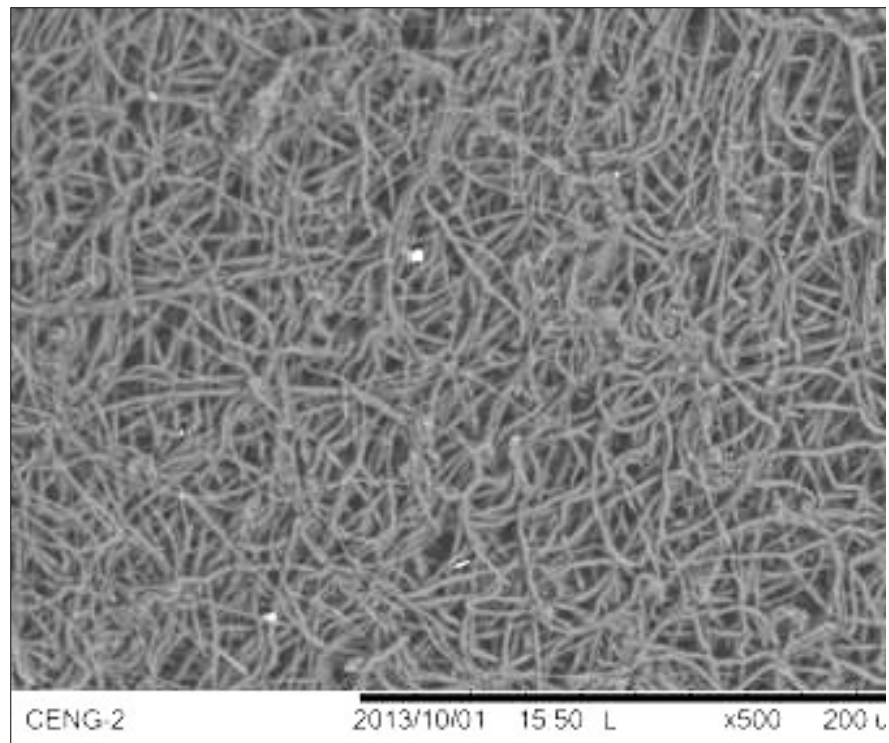

Figure F 27: SEM image of the luminal surface of Spin B1 at 500x magnification; Proximal location, Image 2.

\begin{tabular}{|c|}
\hline Fiber Diameter $(\boldsymbol{\mu m})$ \\
\hline 1.2687 \\
\hline 1.7186 \\
\hline 0.6193 \\
\hline 0.6707 \\
\hline 1.374 \\
\hline 1.6796 \\
\hline 1.8217 \\
\hline 1.9323 \\
\hline 1.6294 \\
\hline 1.093 \\
\hline 2.7387 \\
\hline 1.3693 \\
\hline 2.6052 \\
\hline 2.3648 \\
\hline 1.6396 \\
\hline 1.8034 \\
\hline
\end{tabular}




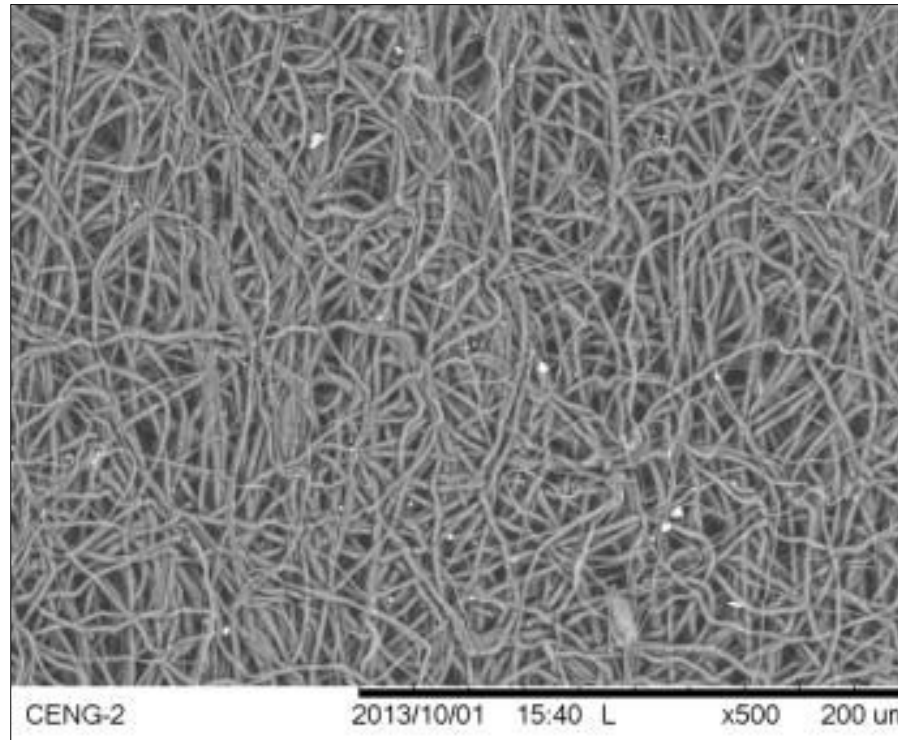

Figure F 28: SEM image of the luminal surface of Spin B1 at 500x magnification; Medial location, Image 1.

\begin{tabular}{|c|}
\hline Fiber Diameter $(\boldsymbol{\mu m})$ \\
\hline 2.205 \\
\hline 0.8248 \\
\hline 1.3137 \\
\hline 2.2237 \\
\hline 2.8369 \\
\hline 2.2534 \\
\hline 1.8217 \\
\hline 1.9705 \\
\hline 2.1245 \\
\hline 0.7681 \\
\hline 2.2534 \\
\hline 2.8252 \\
\hline 1.5671 \\
\hline 3.158 \\
\hline 2.8948 \\
\hline 1.8217 \\
\hline
\end{tabular}

\begin{tabular}{|c|}
\hline Fiber Diameter $(\boldsymbol{\mu m})$ \\
\hline 1.7186 \\
\hline 1.8756 \\
\hline 1.6141 \\
\hline 2.2295 \\
\hline 2.6525 \\
\hline 2.4202 \\
\hline 2.0811 \\
\hline 1.5458 \\
\hline 0.7337 \\
\hline 1.8217 \\
\hline 0.7777 \\
\hline 7.3437 \\
\hline 1.4085 \\
\hline 1.7521 \\
\hline 2.5308 \\
\hline 2.4778 \\
\hline
\end{tabular}

Figure F 29: SEM image of the luminal surface of Spin B1 at 500x magnification; Medial location, Image 2. 


\section{Spin B1, Distal location, Image 1}

\begin{tabular}{|c|}
\hline Fiber Diameter $(\boldsymbol{\mu m})$ \\
\hline 1.0863 \\
\hline 1.2385 \\
\hline 2.3753 \\
\hline 1.902 \\
\hline 2.0771 \\
\hline 0.7918 \\
\hline 1.8578 \\
\hline 0.807 \\
\hline 1.0305 \\
\hline 1.3693 \\
\hline 2.0368 \\
\hline 0.9222 \\
\hline 1.5512 \\
\hline 1.2946 \\
\hline 1.1876 \\
\hline 1.0341 \\
\hline
\end{tabular}

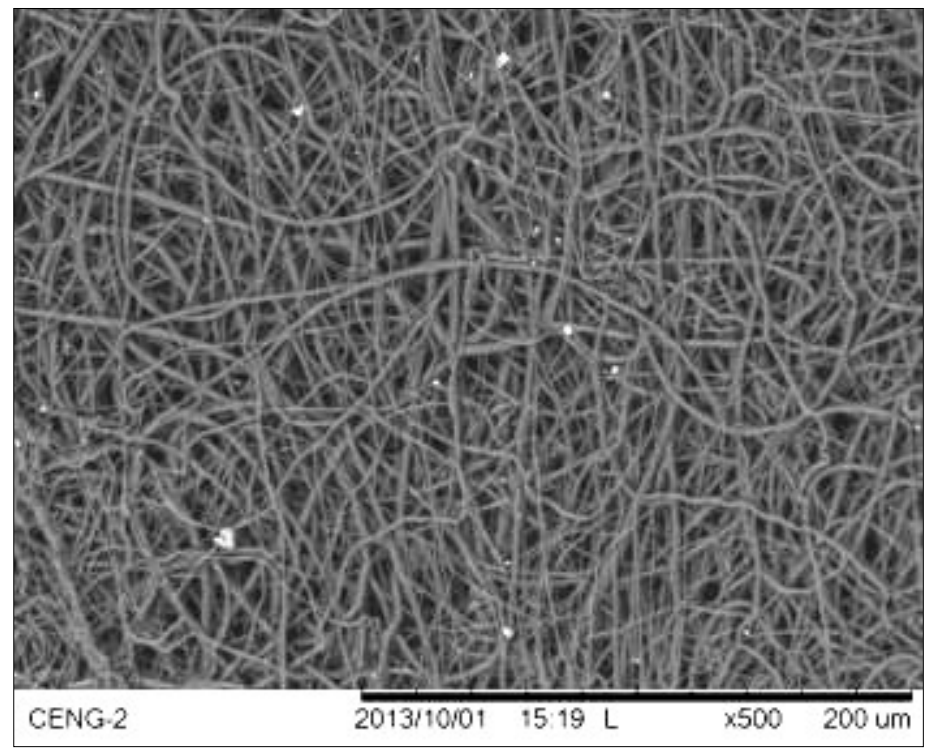

Figure F 30: SEM image of the luminal surface of Spin B1 at 500x magnification; Distal location, Image 2.

\begin{tabular}{|c|}
\hline Fiber Diameter $(\boldsymbol{\mu m})$ \\
\hline 3.8944 \\
\hline 3.4781 \\
\hline 1.7331 \\
\hline 0.6568 \\
\hline 3.0619 \\
\hline 2.7538 \\
\hline 1.7521 \\
\hline 1.4215 \\
\hline 1.3693 \\
\hline 0.9378 \\
\hline 1.3847 \\
\hline 1.1876 \\
\hline 1.398 \\
\hline 2.333 \\
\hline 1.3263 \\
\hline 1.9621 \\
\hline
\end{tabular}




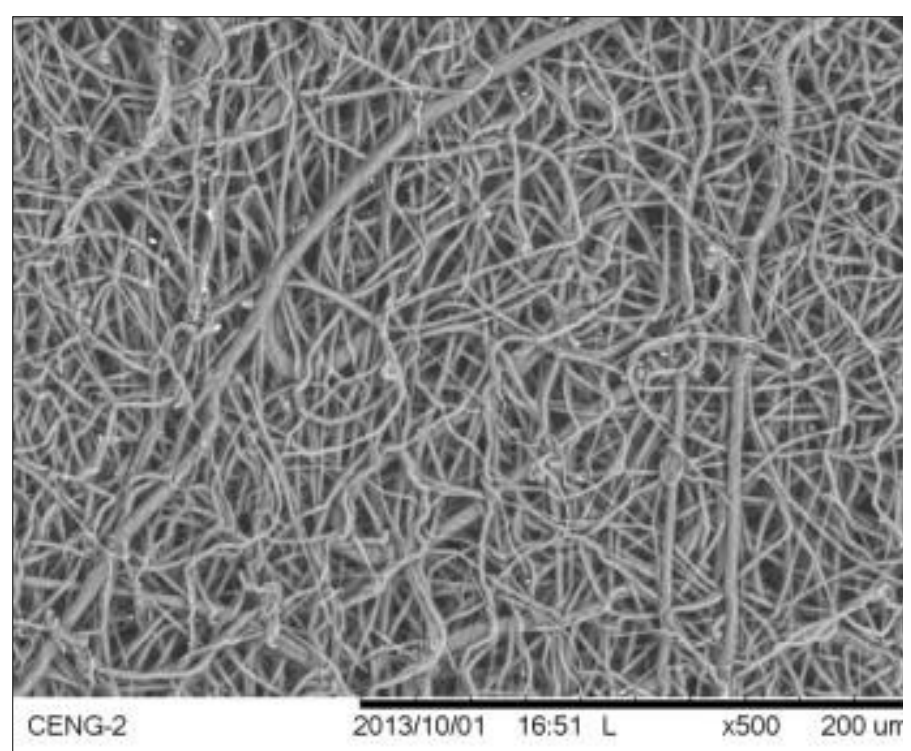

Figure F 31: SEM image of the luminal surface of Spin B2 at 500x magnification; Proximal location, Image 1.

\begin{tabular}{|c|}
\hline Fiber Diameter $(\boldsymbol{\mu m})$ \\
\hline 2.1937 \\
\hline 1.7896 \\
\hline 2.0163 \\
\hline 2.3251 \\
\hline 1.3934 \\
\hline 1.8444 \\
\hline 1.5512 \\
\hline 2.0611 \\
\hline 1.3934 \\
\hline 1.7942 \\
\hline 0.7918 \\
\hline 1.9323 \\
\hline 1.8034 \\
\hline 3.1023 \\
\hline 1.9323 \\
\hline 2.097 \\
\hline
\end{tabular}

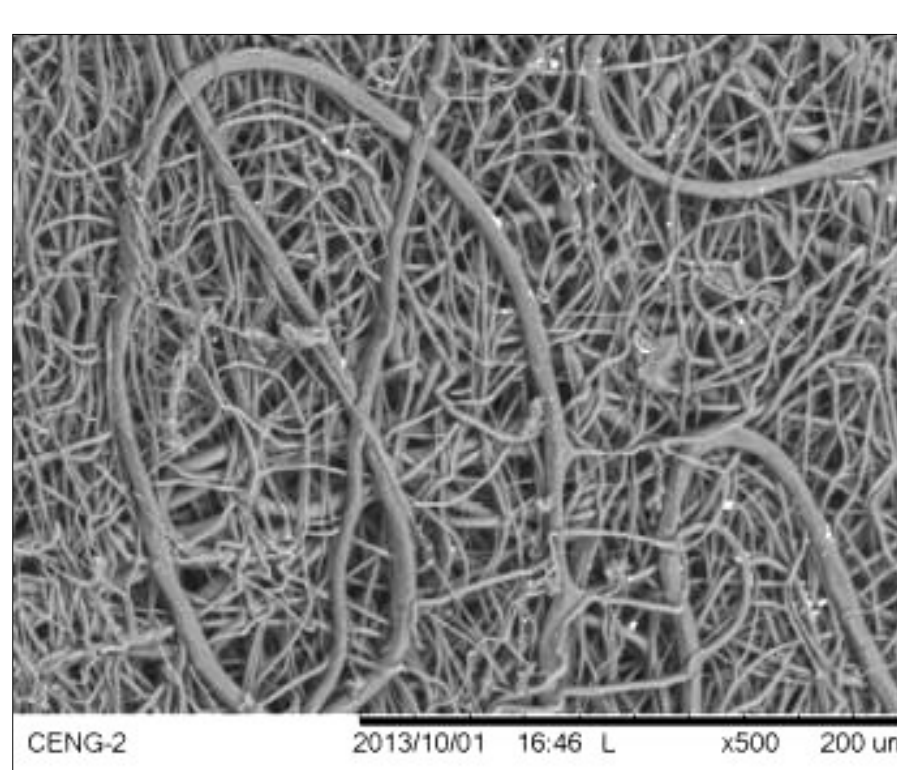

Figure F 32: SEM image of the luminal surface of

\begin{tabular}{|c|}
\hline Fiber Diameter $(\boldsymbol{\mu m})$ \\
\hline 1.5724 \\
\hline 1.6746 \\
\hline 2.097 \\
\hline 2.0163 \\
\hline 7.3346 \\
\hline 1.4574 \\
\hline 1.4228 \\
\hline 2.6805 \\
\hline 5.9256 \\
\hline 1.6497 \\
\hline 2.1899 \\
\hline 1.6294 \\
\hline 1.4402 \\
\hline 1.4687 \\
\hline 1.8756 \\
\hline 1.0623 \\
\hline
\end{tabular}

Spin B2 at 500x magnification; Proximal location, Image 2. 


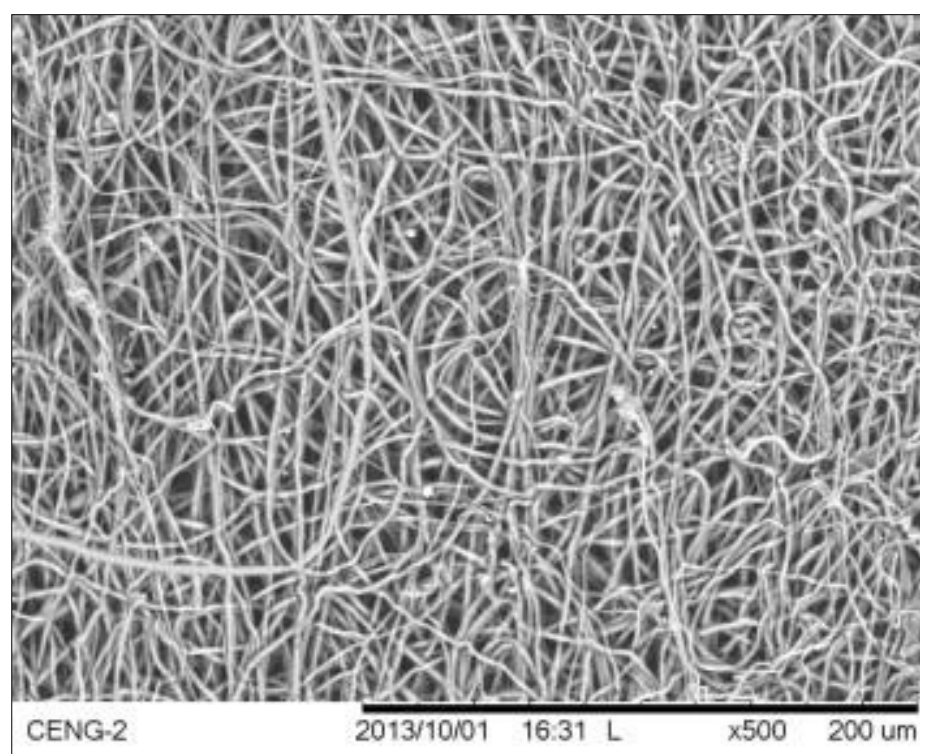

Figure F 33: SEM image of the luminal surface of Spin B2 at 500x magnification; Medial location, Image 1.

\begin{tabular}{|c|}
\hline Fiber Diameter $(\boldsymbol{\mu m})$ \\
\hline 1.7186 \\
\hline 1.5482 \\
\hline 2.0771 \\
\hline 1.4624 \\
\hline 2.205 \\
\hline 2.0122 \\
\hline 1.8217 \\
\hline 2.2737 \\
\hline 1.6746 \\
\hline 1.0061 \\
\hline 2.5012 \\
\hline 2.205 \\
\hline 2.5432 \\
\hline 1.7186 \\
\hline 0.9486 \\
\hline 2.2497 \\
\hline
\end{tabular}

\begin{tabular}{|c|}
\hline Fiber Diameter $(\boldsymbol{\mu m})$ \\
\hline 1.4228 \\
\hline 1.6396 \\
\hline 2.5763 \\
\hline 2.1899 \\
\hline 1.2687 \\
\hline 2.2497 \\
\hline 2.1594 \\
\hline 3.9954 \\
\hline 0.7729 \\
\hline 1.093 \\
\hline 2.1439 \\
\hline 1.7704 \\
\hline 1.8034 \\
\hline 2.3648 \\
\hline 1.0061 \\
\hline 2.097 \\
\hline
\end{tabular}

$\mathrm{B} 2$ at 500x magnification; Medial location, Image 2. 


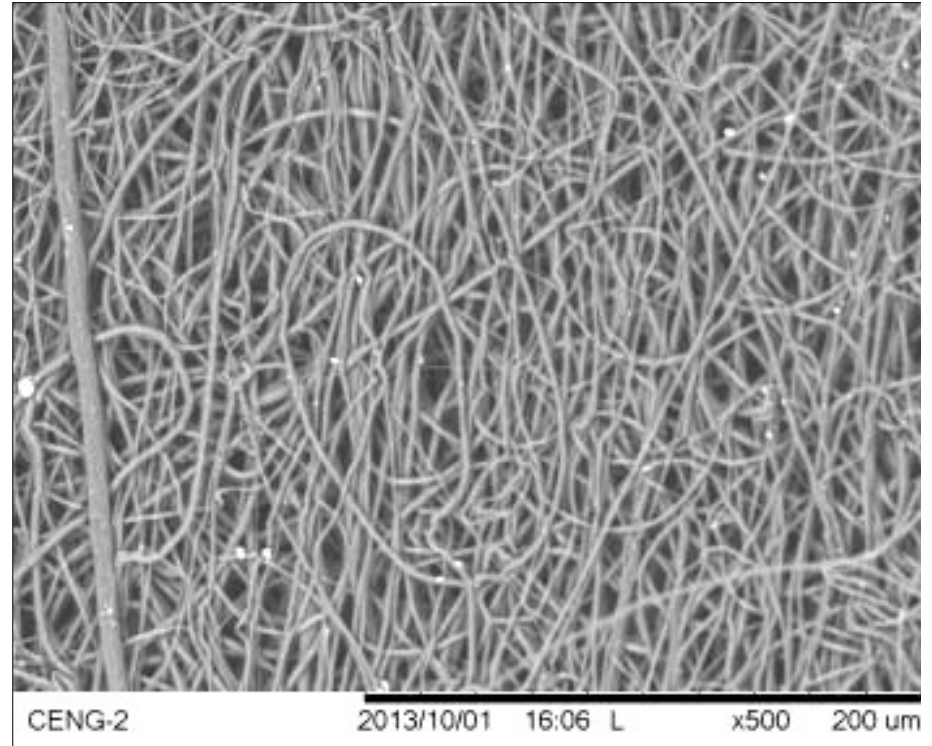

Figure $F$ 35: SEM image of the luminal surface of Spin B2 at 500x magnification; Distal location, Image 1.

\begin{tabular}{|c|}
\hline Fiber Diameter $(\boldsymbol{\mu m})$ \\
\hline 1.1876 \\
\hline 3.0725 \\
\hline 7.6176 \\
\hline 1.5565 \\
\hline 0.981 \\
\hline 2.7296 \\
\hline 1.9365 \\
\hline 2.0771 \\
\hline 2.4509 \\
\hline 1.0623 \\
\hline 2.3683 \\
\hline 1.808 \\
\hline 1.2221 \\
\hline 1.1522 \\
\hline 1.5078 \\
\hline 2.2826 \\
\hline
\end{tabular}

Spin B2, Distal location, Image 2

\begin{tabular}{|c|}
\hline Fiber Diameter $(\boldsymbol{\mu m})$ \\
\hline 1.3162 \\
\hline 2.0036 \\
\hline 3.4417 \\
\hline 2.2054 \\
\hline 3.5999 \\
\hline 3.8652 \\
\hline 4.13 \\
\hline 1.3106 \\
\hline 3.1402 \\
\hline 3.6505 \\
\hline 2.8511 \\
\hline 4.1481 \\
\hline 1.8479 \\
\hline 1.5511 \\
\hline 1.2971 \\
\hline 1.419 \\
\hline
\end{tabular}


*Spin B3 stuck to the mandrel; no data was obtained

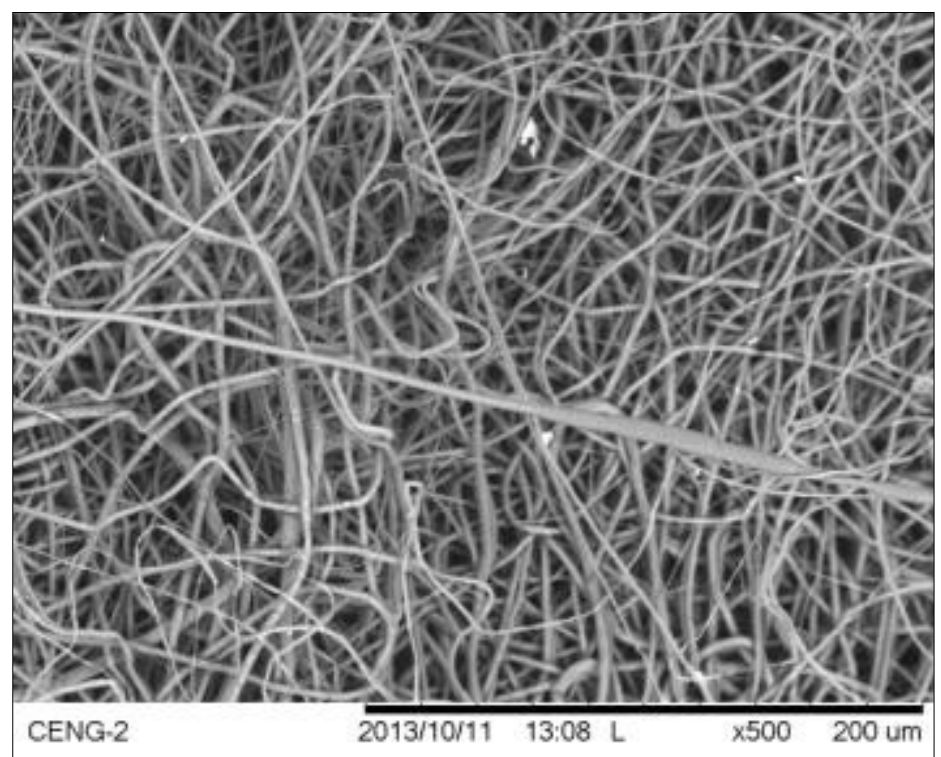

Figure F 36: SEM image of the luminal surface of Spin B4 at 500x magnification; Proximal location, Image 1.

\begin{tabular}{|c|}
\hline Fiber Diameter $(\boldsymbol{\mu m})$ \\
\hline 2.4657 \\
\hline 1.9056 \\
\hline 2.4818 \\
\hline 1.7035 \\
\hline 2.7134 \\
\hline 2.2205 \\
\hline 0.6084 \\
\hline 1.7422 \\
\hline 1.2777 \\
\hline 1.0034 \\
\hline 2.3088 \\
\hline 1.6617 \\
\hline 1.7422 \\
\hline 1.4652 \\
\hline 1.1703 \\
\hline 2.7801 \\
\hline
\end{tabular}

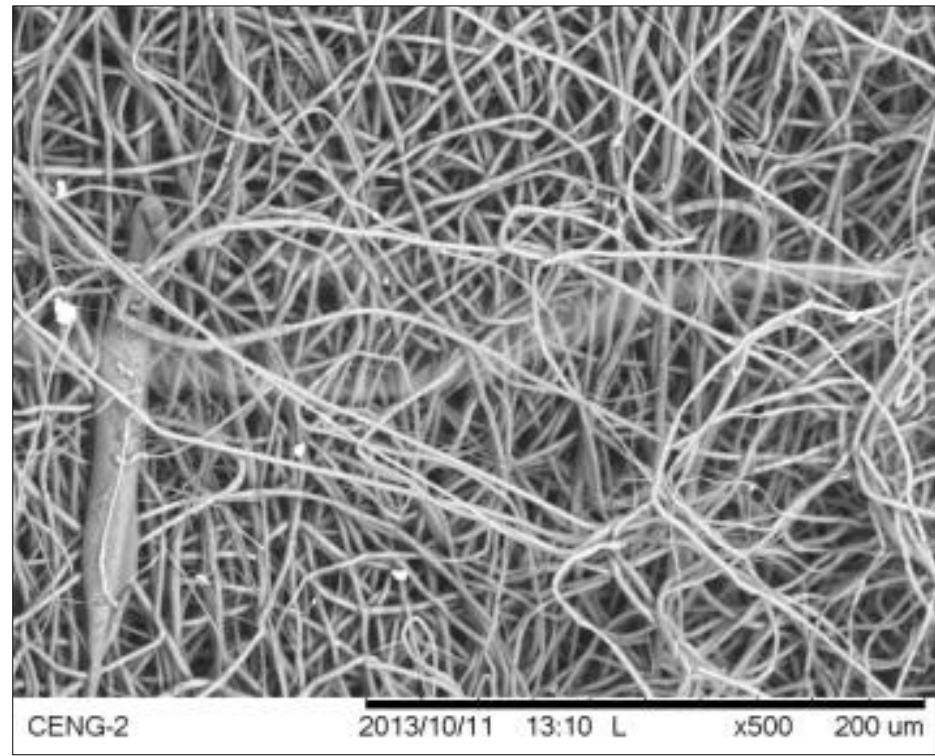

Figure F 37: SEM image of the luminal surface of Spin B4 at 500x magnification; Proximal location, Image 2.

\begin{tabular}{|c|}
\hline Fiber Diameter $(\boldsymbol{\mu m})$ \\
\hline 2.423 \\
\hline 1.8252 \\
\hline 2.8381 \\
\hline 5.7706 \\
\hline 0.7301 \\
\hline 2.5481 \\
\hline 2.0851 \\
\hline 2.6225 \\
\hline 2.2054 \\
\hline 2.8653 \\
\hline 2.1511 \\
\hline 2.0068 \\
\hline 2.7103 \\
\hline 1.9187 \\
\hline 2.4833 \\
\hline 1.0325 \\
\hline
\end{tabular}




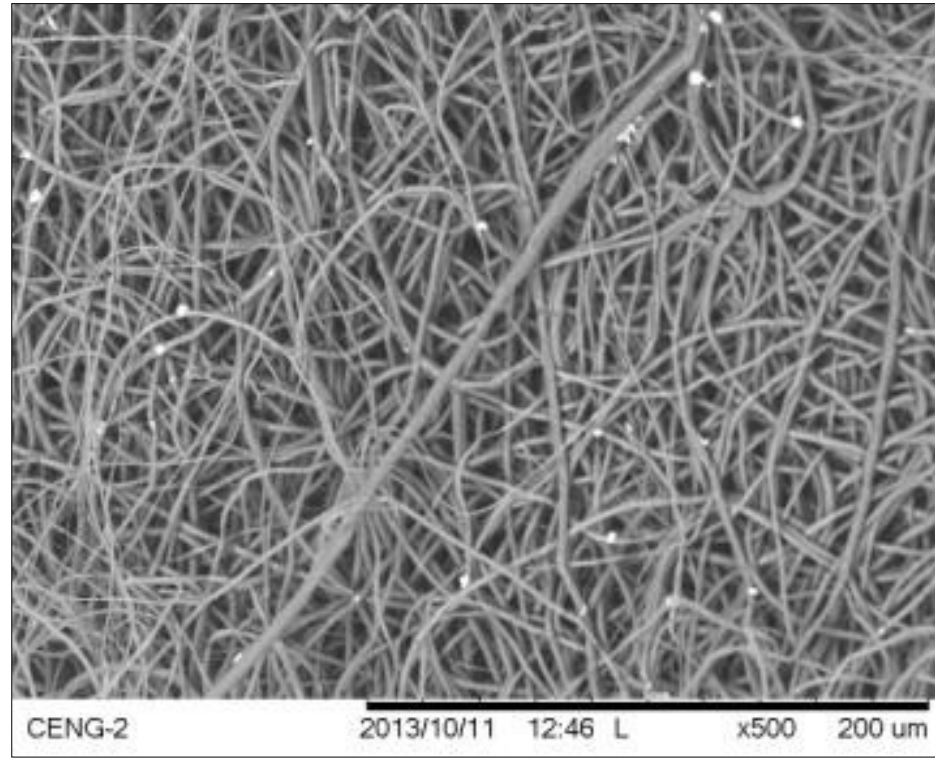

Figure F 38: SEM image of the luminal surface of Spin B4 at 500x magnification; Medial location, Image 1.

\begin{tabular}{|c|}
\hline Fiber Diameter $(\boldsymbol{\mu m})$ \\
\hline 2.1941 \\
\hline 2.7103 \\
\hline 1.4652 \\
\hline 2.2577 \\
\hline 2.6673 \\
\hline 2.2387 \\
\hline 2.0722 \\
\hline 2.2585 \\
\hline 2.5618 \\
\hline 1.7976 \\
\hline 2.2716 \\
\hline 2.0829 \\
\hline 0.8604 \\
\hline 2.9261 \\
\hline 2.101 \\
\hline 1.5051 \\
\hline
\end{tabular}

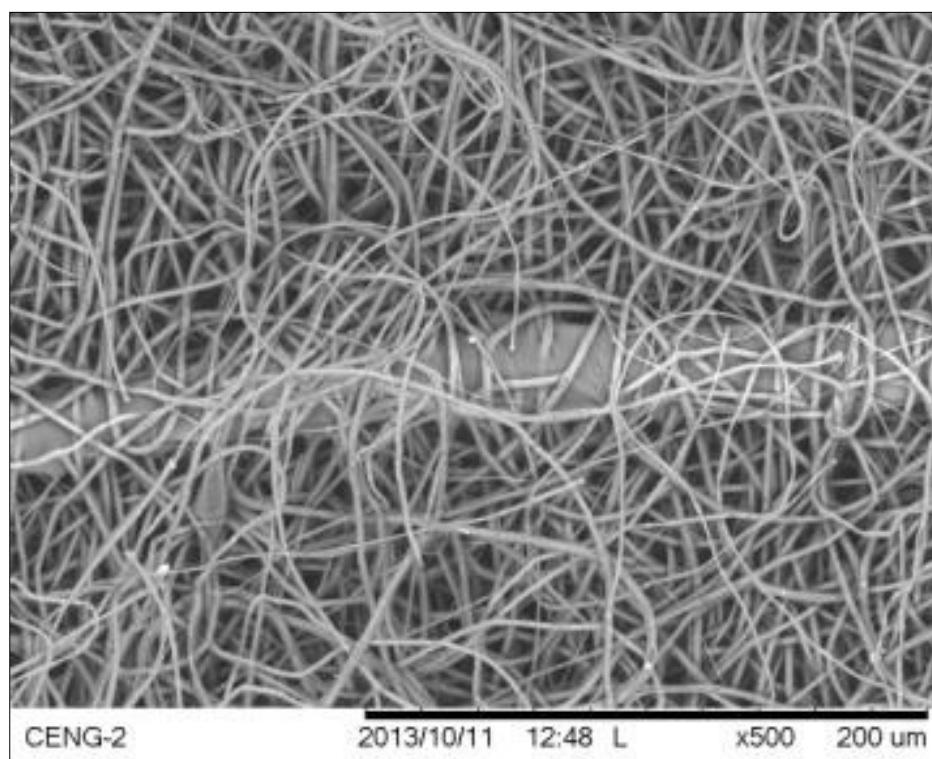

Figure F 39: SEM image of the luminal surface of Spin B4 at 500x magnification; Medial location, Image 2.

\begin{tabular}{|c|}
\hline Fiber Diameter $(\boldsymbol{\mu m})$ \\
\hline 2.8911 \\
\hline 1.5106 \\
\hline 2.6607 \\
\hline 2.6133 \\
\hline 2.7134 \\
\hline 2.7591 \\
\hline 2.0686 \\
\hline 2.0722 \\
\hline 2.291 \\
\hline 2.2038 \\
\hline 1.5222 \\
\hline 1.7422 \\
\hline 2.7004 \\
\hline 3.4158 \\
\hline 2.0452 \\
\hline 1.7738 \\
\hline
\end{tabular}




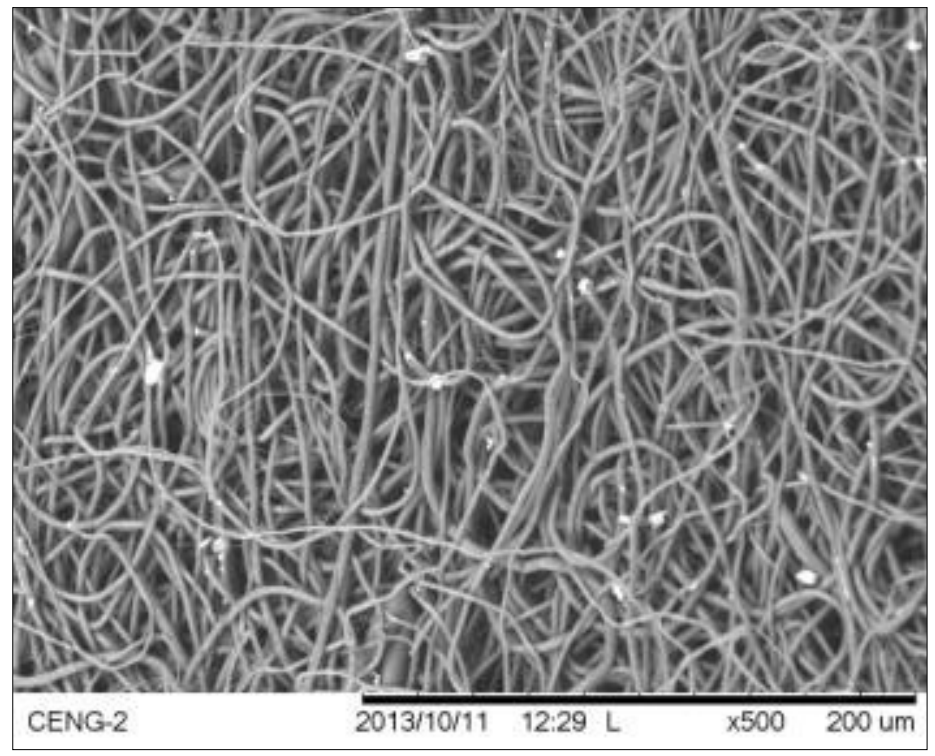

Figure F 40: SEM image of the luminal surface of Spin B4 at 500x magnification; Distal location, Image 1.

\begin{tabular}{|c|}
\hline Fiber Diameter $(\boldsymbol{\mu m})$ \\
\hline 1.9365 \\
\hline 3.2993 \\
\hline 2.7808 \\
\hline 2.2328 \\
\hline 1.7186 \\
\hline 1.8444 \\
\hline 2.6052 \\
\hline 3.7886 \\
\hline 1.0061 \\
\hline 2.0039 \\
\hline 2.5763 \\
\hline 2.9205 \\
\hline 2.429 \\
\hline 3.2204 \\
\hline 3.0155 \\
\hline 2.94 \\
\hline
\end{tabular}

Spin B4, Distal location, Image 2

\begin{tabular}{|c|}
\hline Fiber Diameter $(\boldsymbol{\mu m})$ \\
\hline 2.1861 \\
\hline 2.0039 \\
\hline 3.7796 \\
\hline 1.9365 \\
\hline 2.5308 \\
\hline 2.1439 \\
\hline 0.6193 \\
\hline 2.1594 \\
\hline 2.0485 \\
\hline 2.8602 \\
\hline 2.7748 \\
\hline 2.1895 \\
\hline 2.991 \\
\hline 2.003 \\
\hline 2.2818 \\
\hline 2.8369 \\
\hline
\end{tabular}




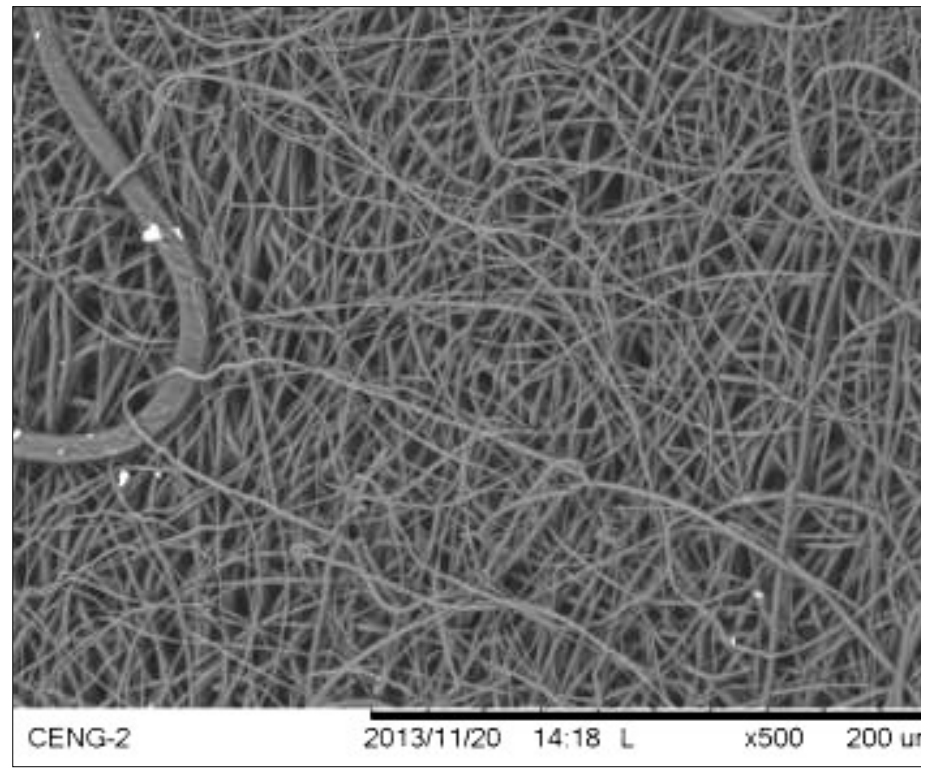

Figure F 41: SEM image of the luminal surface of Spin $\mathrm{X}$ at 500x magnification; Proximal location, Image 1.

\begin{tabular}{|c|}
\hline Fiber Diameter $(\boldsymbol{\mu m})$ \\
\hline 1.5964 \\
\hline 1.4715 \\
\hline 9.911 \\
\hline 1.6427 \\
\hline 1.7554 \\
\hline 2.101 \\
\hline 1.2906 \\
\hline 1.5541 \\
\hline 1.3475 \\
\hline 1.5541 \\
\hline 1.9743 \\
\hline 2.101 \\
\hline 1.6778 \\
\hline 1.6828 \\
\hline 1.5222 \\
\hline 1.5541 \\
\hline
\end{tabular}




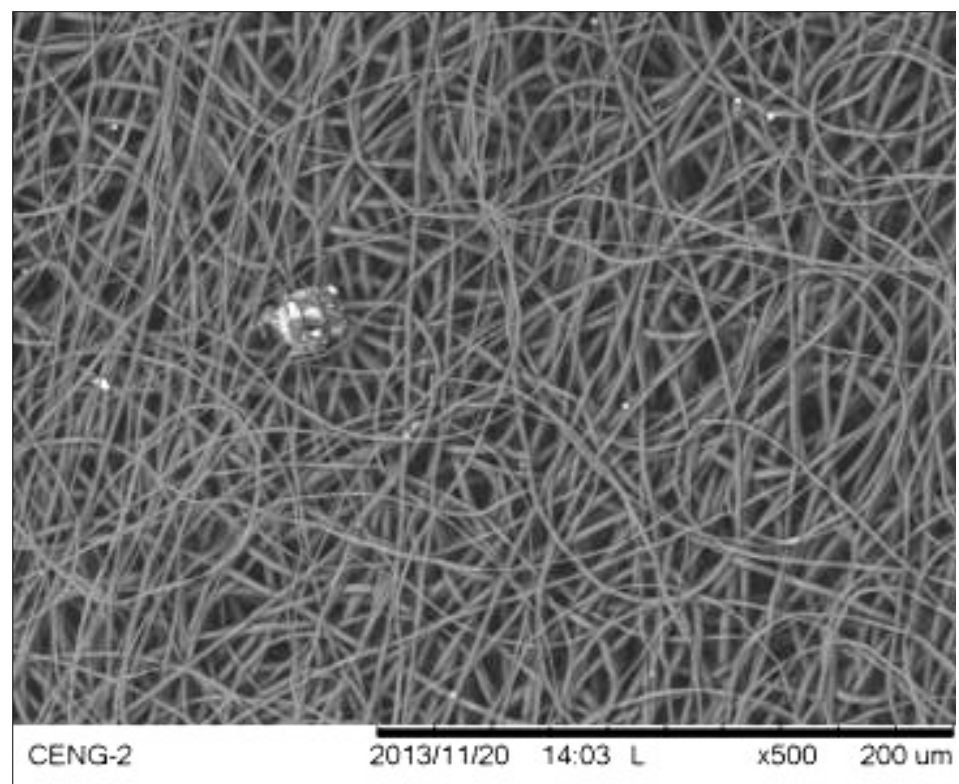

Figure F 43: SEM image of the luminal surface of Spin $\mathrm{X}$ at 500x magnification; Medial location, Image 1 .

\begin{tabular}{|c|}
\hline Fiber Diameter $(\boldsymbol{\mu m})$ \\
\hline 1.7717 \\
\hline 1.6427 \\
\hline 1.2777 \\
\hline 1.39 \\
\hline 1.2906 \\
\hline 2.8657 \\
\hline 1.8069 \\
\hline 1.6828 \\
\hline 1.9658 \\
\hline 1.9187 \\
\hline 1.9187 \\
\hline 2.3267 \\
\hline 2.4826 \\
\hline 1.9701 \\
\hline 2.8859 \\
\hline 1.7654 \\
\hline
\end{tabular}

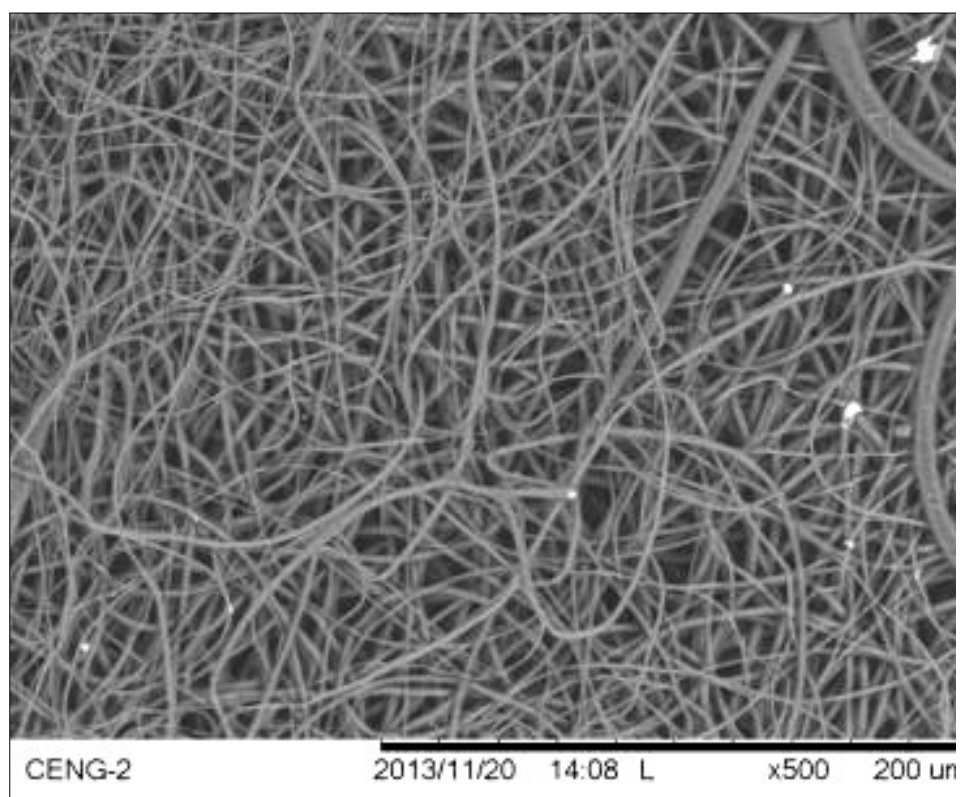

\begin{tabular}{|c|}
\hline Fiber Diameter $(\boldsymbol{\mu m})$ \\
\hline 1.5541 \\
\hline 2.9796 \\
\hline 2.759 \\
\hline 1.5754 \\
\hline 1.4715 \\
\hline 1.8614 \\
\hline 2.2979 \\
\hline 1.5051 \\
\hline 2.4007 \\
\hline 1.6617 \\
\hline 1.1899 \\
\hline 0.8658 \\
\hline 2.254 \\
\hline 2.254 \\
\hline 1.9187 \\
\hline 1.6325 \\
\hline
\end{tabular}

Figure F 44: SEM image of the luminal surface of Spin X at 500x magnification; Medial location, Image 2. 
Spin X, Distal location, Image 1

\begin{tabular}{|c|}
\hline Fiber Diameter $(\boldsymbol{\mu m})$ \\
\hline 1.9531 \\
\hline 2.9713 \\
\hline 2.9487 \\
\hline 1.2077 \\
\hline 1.8614 \\
\hline 1.8293 \\
\hline 1.8792 \\
\hline 2.0811 \\
\hline 1.5051 \\
\hline 3.1876 \\
\hline 2.016 \\
\hline 1.7364 \\
\hline 2.7134 \\
\hline 1.6528 \\
\hline 2.8188 \\
\hline 1.9359 \\
\hline
\end{tabular}

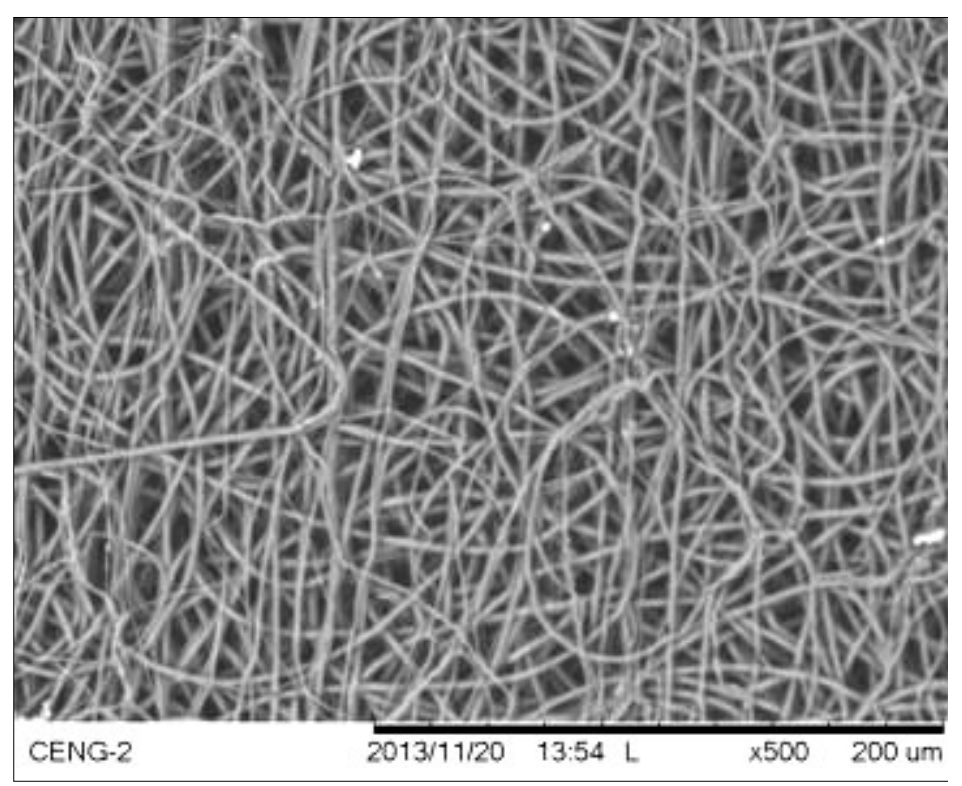

Figure F 45: SEM image of the luminal surface of Spin

\begin{tabular}{|c|}
\hline Fiber Diameter $(\boldsymbol{\mu m})$ \\
\hline 1.9359 \\
\hline 2.5973 \\
\hline 1.7294 \\
\hline 1.5541 \\
\hline 1.5583 \\
\hline 2.6324 \\
\hline 2.3552 \\
\hline 3.1082 \\
\hline 2.6101 \\
\hline 2.4926 \\
\hline 1.8929 \\
\hline 1.6828 \\
\hline 1.8252 \\
\hline 1.5701 \\
\hline 1.5583 \\
\hline 2.7439 \\
\hline
\end{tabular}

$\mathrm{X}$ at 500x magnification; Distal location, Image 2. 


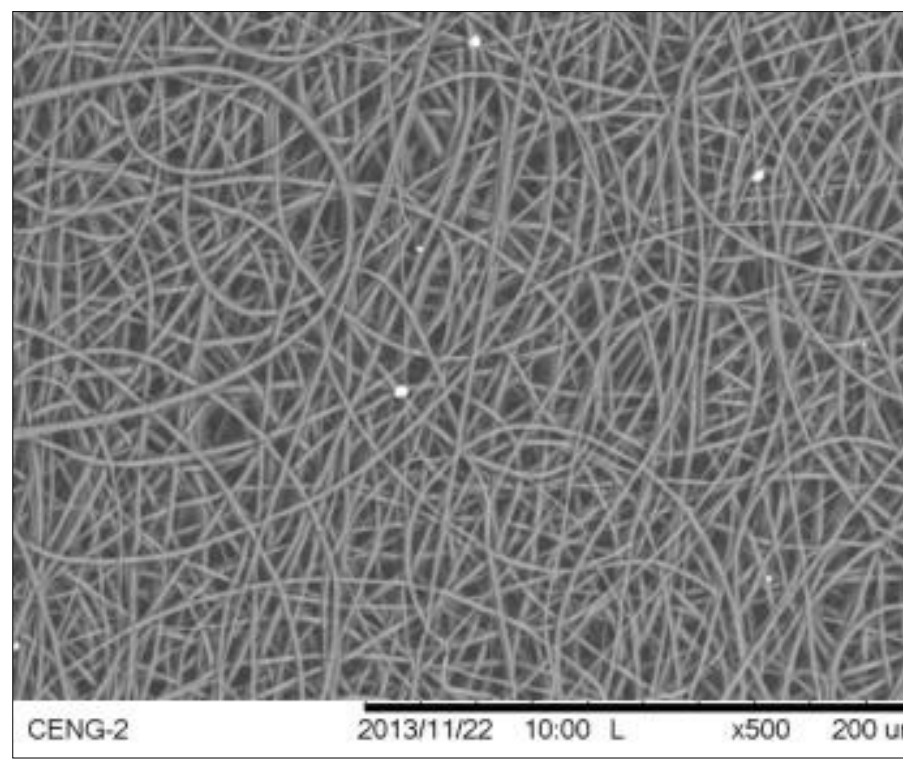

Figure F 46: SEM image of the luminal surface of Spin $\mathrm{Y}$ at 500x magnification; Proximal location, Image 1.

\begin{tabular}{|c|}
\hline Fiber Diameter $(\boldsymbol{\mu m})$ \\
\hline 1.8069 \\
\hline 1.9957 \\
\hline 2.386 \\
\hline 1.344 \\
\hline 2.151 \\
\hline 1.4242 \\
\hline 2.2054 \\
\hline 1.5488 \\
\hline 1.4627 \\
\hline 1.9621 \\
\hline 1.7316 \\
\hline 1.887 \\
\hline 1.5051 \\
\hline 1.5511 \\
\hline 1.6348 \\
\hline 2.3728 \\
\hline
\end{tabular}

\begin{tabular}{|c|}
\hline Fiber Diameter $(\boldsymbol{\mu m})$ \\
\hline 1.6528 \\
\hline 2.3267 \\
\hline 2.5669 \\
\hline 2.4107 \\
\hline 1.8414 \\
\hline 1.5595 \\
\hline 2.0013 \\
\hline 1.6528 \\
\hline 1.6074 \\
\hline 2.4473 \\
\hline 1.4627 \\
\hline 1.6172 \\
\hline 2.4249 \\
\hline 2.5408 \\
\hline 2.8757 \\
\hline 1.8089 \\
\hline
\end{tabular}

Figure F 47: SEM image of the luminal surface of Spin $Y$ at 500x magnification; Proximal location, Image 2. 


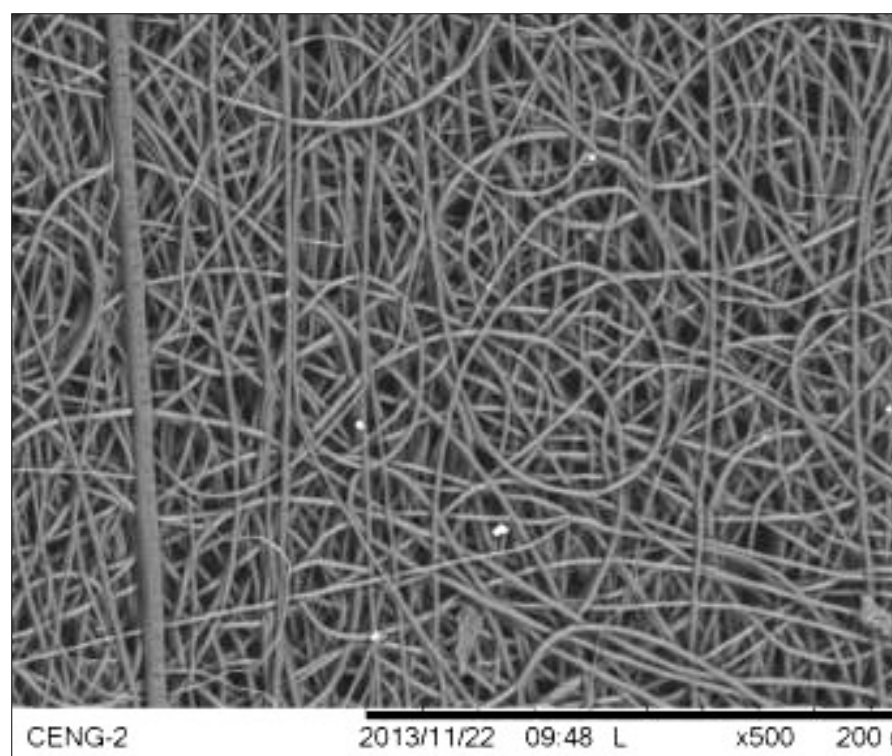

Figure F 48: SEM image of the luminal surface of Spin Y at 500x magnification; Medial location, Image 1.

\begin{tabular}{|c|}
\hline Fiber Diameter $(\boldsymbol{\mu m})$ \\
\hline 2.3267 \\
\hline 2.1168 \\
\hline 1.3874 \\
\hline 1.5488 \\
\hline 1.0467 \\
\hline 1.3475 \\
\hline 1.5865 \\
\hline 1.4112 \\
\hline 2.3406 \\
\hline 2.2092 \\
\hline 2.0668 \\
\hline 1.0467 \\
\hline 0.7791 \\
\hline 2.2437 \\
\hline 2.5085 \\
\hline 2.0142 \\
\hline
\end{tabular}

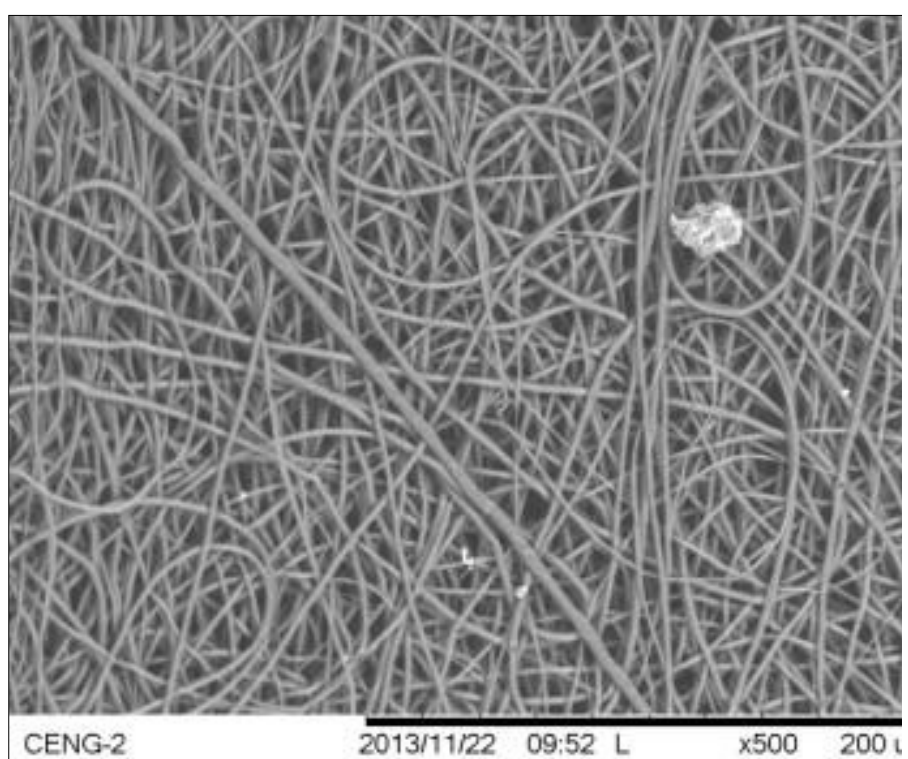

Figure F 49: SEM image of the luminal surface of

\section{Fiber Diameter $(\mu \mathrm{m})$}

\begin{tabular}{|c|}
\hline 5.0119 \\
\hline 1.4853 \\
\hline 1.6706 \\
\hline 2.1635 \\
\hline 1.9743 \\
\hline 2.0036 \\
\hline 1.8614 \\
\hline 1.7208 \\
\hline 1.7079 \\
\hline 2.9267 \\
\hline 1.5511 \\
\hline 2.065 \\
\hline 2.3152 \\
\hline 1.6528 \\
\hline 1.887 \\
\hline 2.0829 \\
\hline
\end{tabular}

Spin $Y$ at 500x magnification; Medial location, Image 2. 


\section{Spin Y, Distal location, Image 1}

\begin{tabular}{|c|}
\hline Fiber Diameter $(\boldsymbol{\mu m})$ \\
\hline 1.2077 \\
\hline 1.2244 \\
\hline 1.5865 \\
\hline 2.2092 \\
\hline 1.6828 \\
\hline 2.2054 \\
\hline 1.8089 \\
\hline 2.1936 \\
\hline 2.5059 \\
\hline 1.924 \\
\hline 3.1216 \\
\hline 2.5813 \\
\hline 2.6481 \\
\hline 2.3167 \\
\hline 1.889 \\
\hline 1.9336 \\
\hline
\end{tabular}

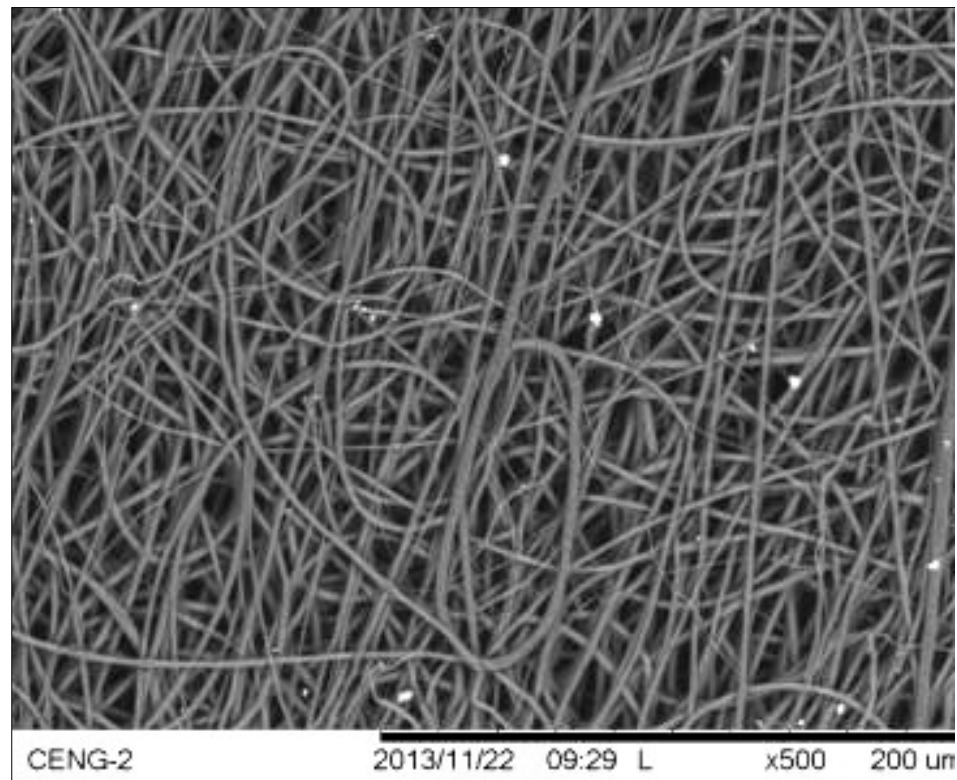

Figure F 50: SEM image of the luminal surface of Spin

\begin{tabular}{|c|}
\hline 2.2577 \\
\hline 1.9743 \\
\hline 1.0325 \\
\hline 2.5941 \\
\hline 0.7351 \\
\hline 1.5964 \\
\hline 2.423 \\
\hline 1.7144 \\
\hline 2.7861 \\
\hline 2.4926 \\
\hline 3.1928 \\
\hline 1.9658 \\
\hline 1.1576 \\
\hline 2.1936 \\
\hline 2.2387 \\
\hline 1.9621 \\
\hline
\end{tabular}
$\mathrm{Y}$ at 500x magnification; Distal location, Image 2. 


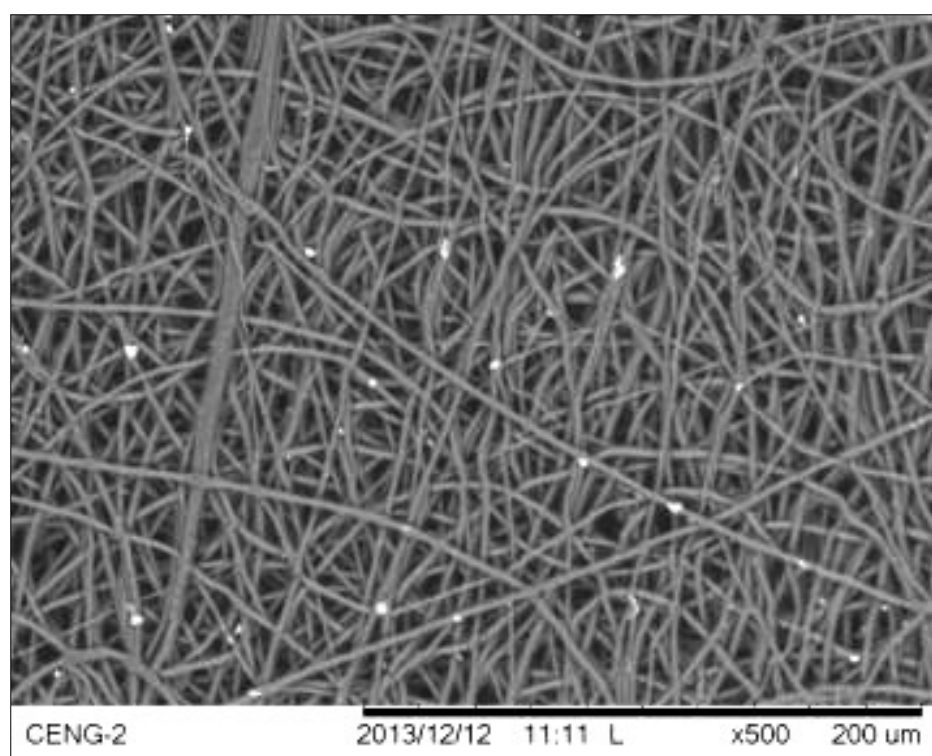

Figure F 51: SEM image of the luminal surface of Spin $\mathrm{Z}$ at 500x magnification; Proximal location, Image 1.

\begin{tabular}{|c|}
\hline Fiber Diameter $(\boldsymbol{\mu m})$ \\
\hline 2.065 \\
\hline 2.4092 \\
\hline 1.9507 \\
\hline 2.2054 \\
\hline 2.2634 \\
\hline 1.8792 \\
\hline 2.1006 \\
\hline 2.7209 \\
\hline 1.9658 \\
\hline 2.2716 \\
\hline 2.0668 \\
\hline 2.3626 \\
\hline 2.5582 \\
\hline 2.5011 \\
\hline 1.7738 \\
\hline 2.2205 \\
\hline
\end{tabular}

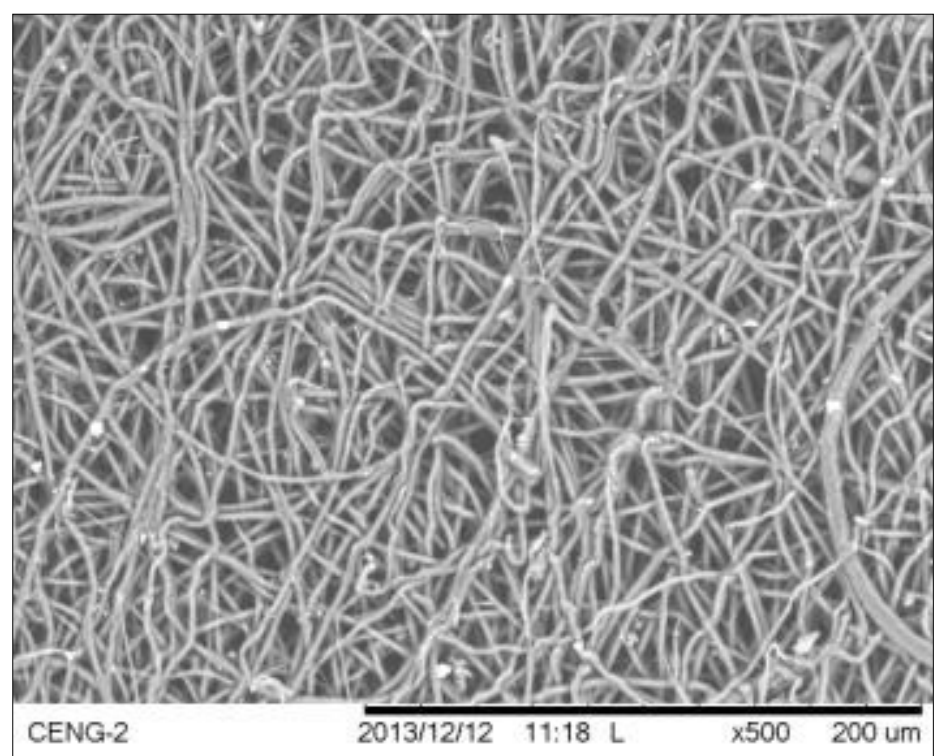

Figure F 52: SEM image of the luminal surface of Spin $\mathrm{Z}$ at 500x magnification; Proximal location, Image 2.

\begin{tabular}{|c|}
\hline Fiber Diameter $(\boldsymbol{\mu m})$ \\
\hline 2.069 \\
\hline 1.9402 \\
\hline 1.4255 \\
\hline 2.2585 \\
\hline 2.0307 \\
\hline 2.0851 \\
\hline 2.254 \\
\hline 1.2971 \\
\hline 2.2037 \\
\hline 2.2054 \\
\hline 2.2577 \\
\hline 2.3087 \\
\hline 2.3167 \\
\hline 2.5813 \\
\hline 2.4245 \\
\hline 2.6324 \\
\hline
\end{tabular}


Spin Z, Medial location, Image 1

\begin{tabular}{|c|}
\hline Fiber Diameter $(\boldsymbol{\mu m})$ \\
\hline 2.5455 \\
\hline 2.4826 \\
\hline 2.065 \\
\hline 1.7738 \\
\hline 1.2906 \\
\hline 2.0452 \\
\hline 0.9126 \\
\hline 1.8069 \\
\hline 2.101 \\
\hline 2.7134 \\
\hline 2.5539 \\
\hline 2.7004 \\
\hline 1.9621 \\
\hline 2.2862 \\
\hline 2.2732 \\
\hline 2.6169 \\
\hline
\end{tabular}

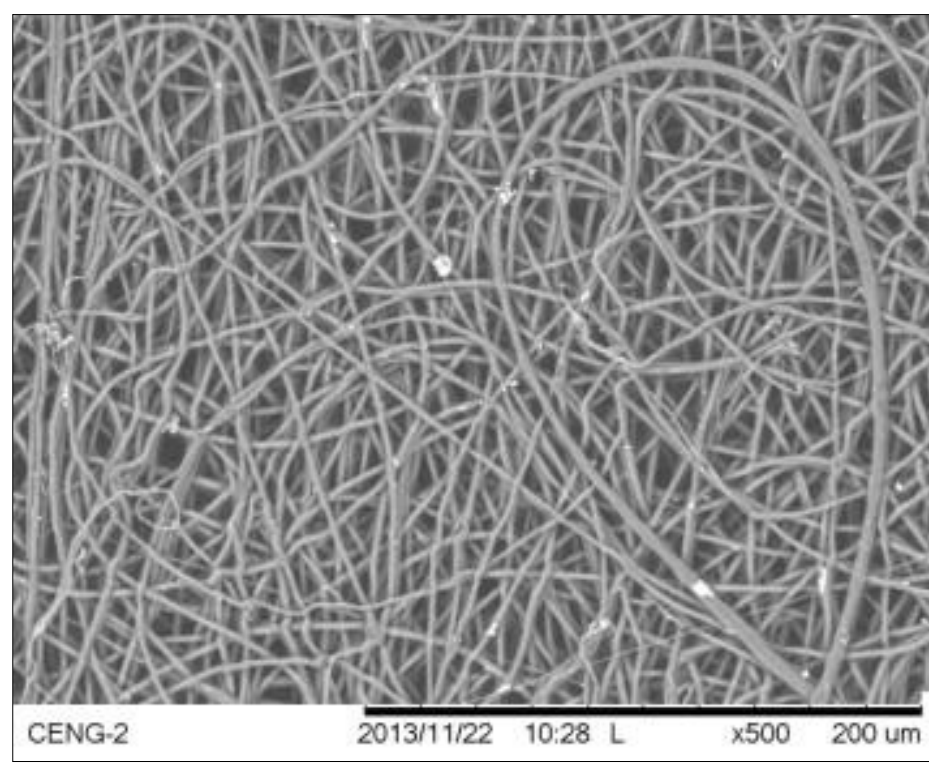

Figure F 53: SEM image of the luminal surface of Spin $Z$ at 500x magnification; Medial location, Image 2 .

\begin{tabular}{|c|}
\hline Fiber Diameter $(\boldsymbol{\mu m})$ \\
\hline 2.3798 \\
\hline 2.2634 \\
\hline 1.9412 \\
\hline 2.5669 \\
\hline 2.1635 \\
\hline 2.423 \\
\hline 2.5669 \\
\hline 2.1251 \\
\hline 1.7294 \\
\hline 2.0142 \\
\hline 2.1286 \\
\hline 2.0398 \\
\hline 2.8923 \\
\hline 5.1338 \\
\hline 1.9864 \\
\hline 2.2371 \\
\hline
\end{tabular}




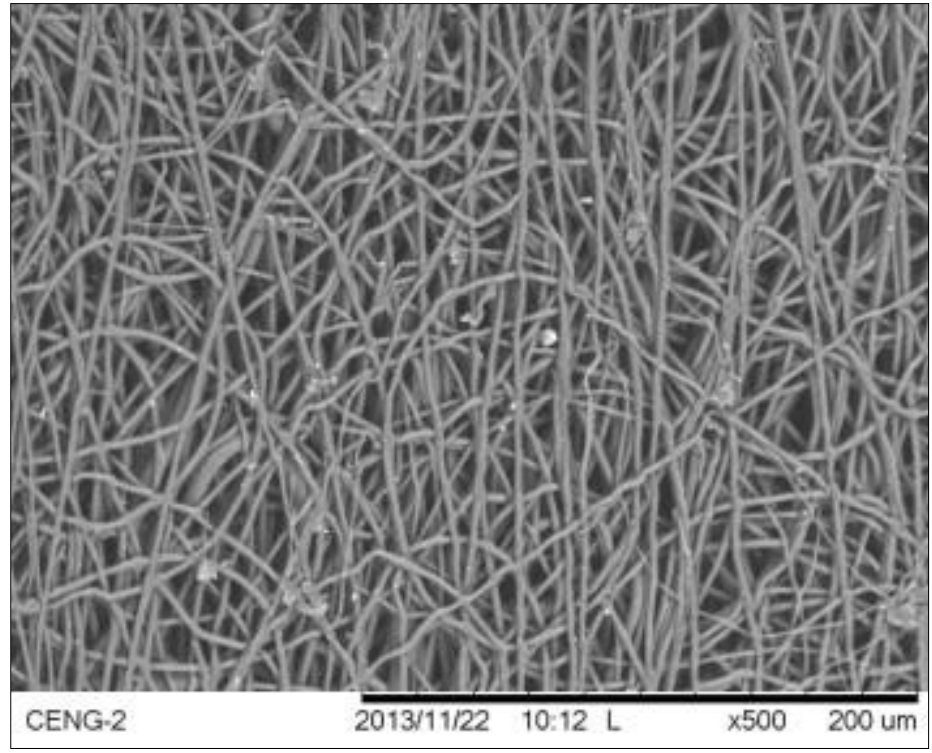

Figure F 54: SEM image of the luminal surface of Spin $\mathrm{Z}$ at 500x magnification; Distal location, Image 1.

\begin{tabular}{|c|}
\hline Fiber Diameter $(\boldsymbol{\mu m})$ \\
\hline 1.7361 \\
\hline 2.3819 \\
\hline 2.7053 \\
\hline 2.6425 \\
\hline 2.9827 \\
\hline 2.4752 \\
\hline 1.9477 \\
\hline 2.3819 \\
\hline 2.3356 \\
\hline 2.3067 \\
\hline 1.6843 \\
\hline 2.1249 \\
\hline 2.2063 \\
\hline 2.2025 \\
\hline 2.0113 \\
\hline 2.4616 \\
\hline
\end{tabular}

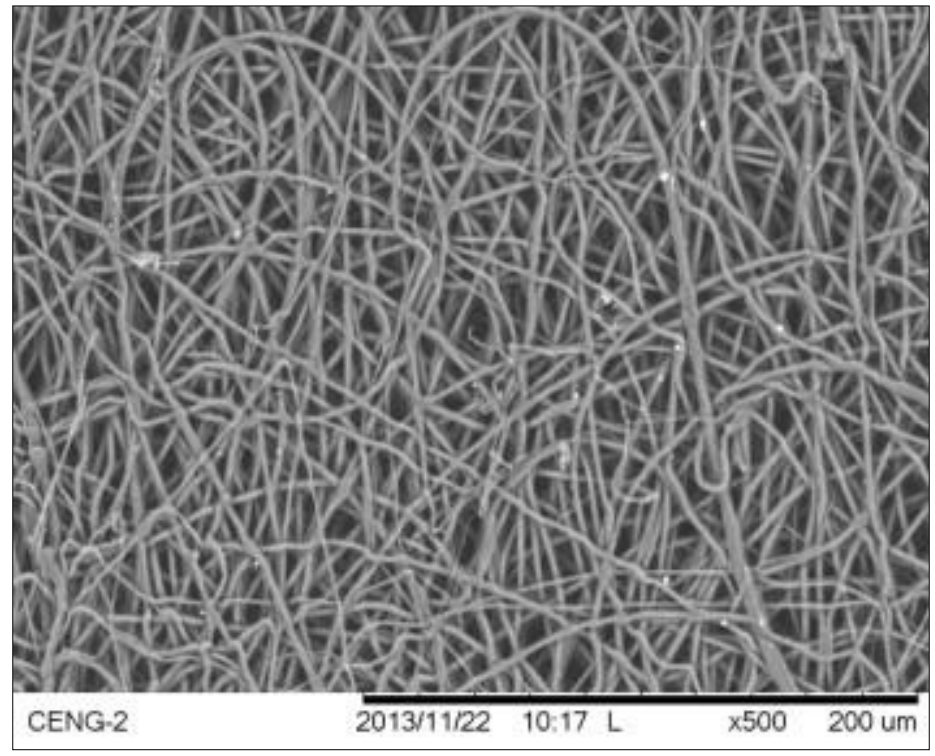

Figure F 55: SEM image of the luminal surface of Spin $\mathrm{Z}$ at 500x magnification; Distal location, Image 2.

\begin{tabular}{|c|}
\hline Fiber Diameter $(\boldsymbol{\mu m})$ \\
\hline 2.0931 \\
\hline 2.1091 \\
\hline 1.5601 \\
\hline 1.276 \\
\hline 2.4342 \\
\hline 2.0729 \\
\hline 1.6843 \\
\hline 2.0485 \\
\hline 2.2063 \\
\hline 2.2139 \\
\hline 2.4752 \\
\hline 2.2663 \\
\hline 1.6592 \\
\hline 1.8184 \\
\hline 1.9434 \\
\hline 2.4204 \\
\hline
\end{tabular}




\section{APPENDIX G: CHAPTER II JMP SOFTWARE STATISTICAL ANALYSIS}

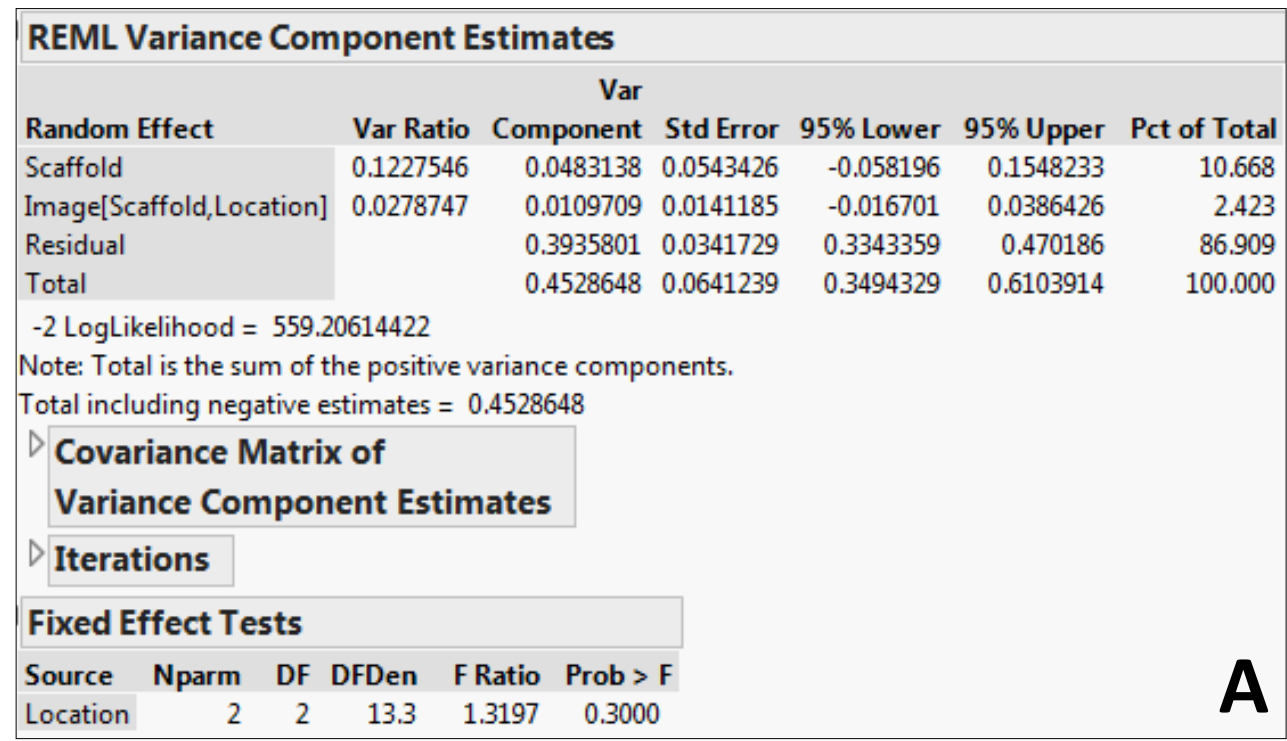

REML Variance Component Estimates

\begin{tabular}{|c|c|c|c|c|c|c|}
\hline \multirow[b]{2}{*}{ Random Effect } & \multicolumn{4}{|c|}{ Var } & \multirow[b]{2}{*}{ 95\% Upper } & \multirow[b]{2}{*}{ Pct of Total } \\
\hline & Var Ratio & Component & Std Error & 95\% Lower & & \\
\hline Scaffold & 0.0847904 & 0.0166469 & 0.019499 & -0.02157 & 0.0548642 & 7.653 \\
\hline Image[Scaffold, Location] & 0.023177 & 0.0045503 & 0.0067467 & -0.008673 & 0.0177737 & 2.092 \\
\hline Residual & & 0.1963294 & 0.0169947 & 0.1668558 & 0.2344104 & 90.255 \\
\hline Total & & 0.2175266 & 0.0257611 & 0.1747063 & 0.2783656 & 100.000 \\
\hline \multirow{3}{*}{\multicolumn{7}{|c|}{$\begin{array}{l}-2 \text { LogLikelihood }=365.60388514 \\
\text { Note: Total is the sum of the positive variance components. } \\
\text { Total including negative estimates }=0.2175266\end{array}$}} \\
\hline & & & & & & \\
\hline & & & & & & \\
\hline \multirow{2}{*}{\multicolumn{7}{|c|}{$\begin{array}{l}\text { Covariance Matrix of } \\
\text { Variance Component Estimates }\end{array}$}} \\
\hline & & & & & & \\
\hline \multicolumn{7}{|l|}{$\triangleright$ Iterations } \\
\hline \multicolumn{3}{|l|}{ Fixed Effect Tests } & & & & \\
\hline jource Nparm & DFDen & Ratio Prob & & & & \\
\hline ocation 2 & 12.95 & & & & & \\
\hline
\end{tabular}

Figure G 1: JMP output for the REML variance components analysis of (A) Protocol B and $(B)$ the Standard Protocol. 


\begin{tabular}{|lrrrrr|}
\hline \multicolumn{7}{|l|}{ Analysis of Variance } \\
Source & DF & $\begin{array}{r}\text { Sum of } \\
\text { Squares }\end{array}$ & Mean Square & F Ratio & Prob > F \\
Scaffold & 2 & 10.31719 & 5.15860 & 12.7641 & $<.0001^{*}$ \\
Error & 280 & 113.16157 & 0.40415 & & A \\
C. Total & 282 & 123.47876 & & & \\
\hline \hline Analysis of Variance & & & \\
\hline \multicolumn{7}{|c}{ Sum of } & & & \\
Source & DF & Squares & Mean Square & F Ratio & Prob > F \\
Scaffold & 2 & 3.677207 & 1.83860 & 9.0328 & $0.0002^{*}$ \\
Error & 282 & 57.400296 & 0.20355 & & B \\
C. Total & 284 & 61.077503 & & & B \\
\hline
\end{tabular}

Figure G 2: JMP output of ANOVA for (A) Protocol B and (B) the Standard Protocol.

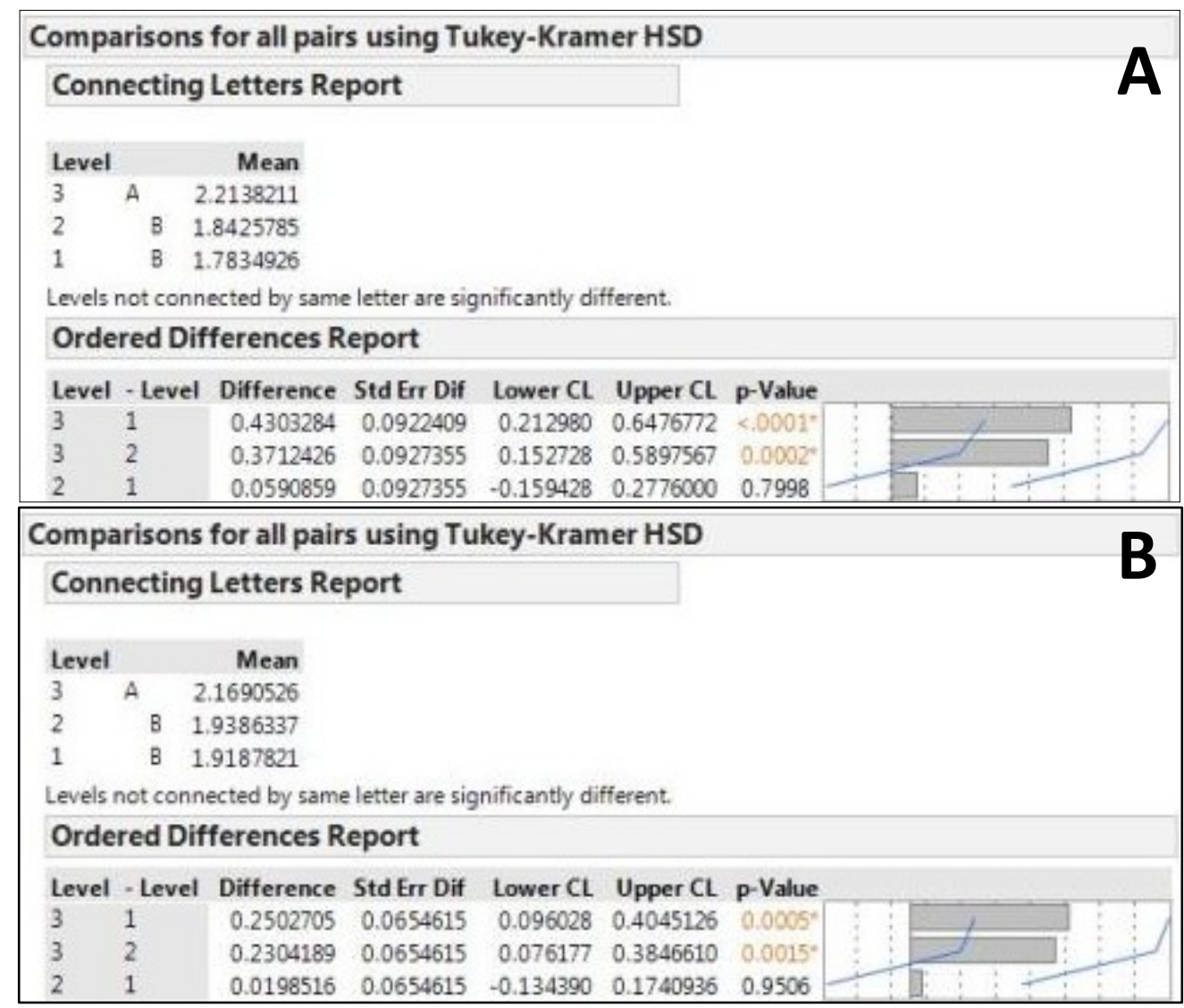

Figure G 3: JMP output of Tukey's HSD post-hoc test for $(A)$ Protocol B and $(B)$ the Standard Protocol. 


\begin{tabular}{|c|c|c|c|c|c|c|}
\hline \multicolumn{7}{|c|}{ Means and Std Deviations } \\
\hline \multirow{2}{*}{ Level } & \multirow[b]{2}{*}{ Number } & \multirow[b]{2}{*}{ Mean } & \multirow[b]{2}{*}{ Std Dev } & \multirow{2}{*}{$\begin{array}{r}\text { Std Err } \\
\text { Mean }\end{array}$} & \multirow[b]{2}{*}{ Lower $95 \%$} & \multirow[b]{2}{*}{ Upper $95 \%$} \\
\hline & & & & & & \\
\hline Experimental & 283 & 1.94737 & 0.661716 & 0.03933 & 1.8699 & 2.0248 \\
\hline Standard & 285 & 2.00882 & 0.463747 & 0.02747 & 1.9548 & 2.0629 \\
\hline \multicolumn{7}{|c|}{ t Test } \\
\hline \multicolumn{7}{|c|}{ Standard-Experimental } \\
\hline \multicolumn{7}{|c|}{ Assuming unequal variances } \\
\hline Difference & 0.06146 & t Ratio & 1.280949 & & & \\
\hline Std Err Dif & 0.04798 & DF & 504.8958 & & & \\
\hline Upper CL Dif & 0.15572 & Prob $>|t|$ & 0.2008 & & & \\
\hline Lower CL Dif & -0.03280 & Prob $>t$ & 0.1004 & & & \\
\hline Confidence & 0.95 & Prob $<\mathrm{t}$ & 0.8996 & -0.15 & -0.050 .00 & $\begin{array}{lll}0.05 & 0.10 & 0.15\end{array}$ \\
\hline
\end{tabular}

Figure G 4: JMP output for the one-tailed t-test comparing the mean fiber diameters of Protocol B and the Standard Protocol. 


\section{APPENDIX H: CHAPTER II STATISTICAL ANALYSIS WITH OUTLIERS}

Five large fibers were outliers in Protocol B while three large fibers were outliers in the Standard Protocol. These fibers were eliminated in the analysis performed in Chapter 2, but were included in the following analysis.

The same statistical procedures from Chapter 2 were performed with outliers included. Table H I and Table H II below show the mean fiber diameters and standard deviations of Protocol B and the Standard Protocol, respectively. The means did not change drastically for either protocol when outliers were included. However, the standard deviations were higher in both protocols, which increased the range of fiber diameters produced in both protocols.

Table H I: Mean fiber diameters and standard deviations for Spin Set 2.

\begin{tabular}{|c|c|c|}
\hline Spin & $\begin{array}{c}\text { Mean Fiber } \\
\text { Diameter }(\boldsymbol{\mu m})\end{array}$ & $\begin{array}{c}\text { Standard } \\
\text { Deviation }(\boldsymbol{\mu m})\end{array}$ \\
\hline B1 & 1.84 & 0.88 \\
\hline B2 & 2.00 & 1.07 \\
\hline B3 & - & - \\
\hline B4 & 2.25 & 0.73 \\
\hline
\end{tabular}

Table H II: Mean fiber diameters and standard deviations for Spin Set 3.

\begin{tabular}{|c|c|c|}
\hline Spin & $\begin{array}{c}\text { Mean Fiber } \\
\text { Diameter }(\boldsymbol{\mu m})\end{array}$ & $\begin{array}{c}\text { Standard } \\
\text { Deviation }(\boldsymbol{\mu m})\end{array}$ \\
\hline $\mathbf{X}$ & 2.00 & 0.94 \\
\hline $\mathbf{Y}$ & 1.97 & 0.58 \\
\hline $\mathbf{Z}$ & 2.20 & 0.47 \\
\hline
\end{tabular}

Figure $H 1$ on the following page shows the results for the REML variance components analyses of both protocols. The scaffold-to-scaffold variability of Protocol B was 3.614\% with outliers, which was smaller than the $10.668 \%$ variability not including the outliers. The variability between scaffolds was also reduced for the standard protocol; including outliers, the variability was $2.239 \%$ and without outliers, the variability was $7.653 \%$. However, the same conclusion was still reached when comparing Protocol B to the Standard Protocol: including outliers, the variability between scaffolds of the standard protocol (2.239\%) was smaller than the scaffold-to-scaffold variability of Protocol B $(3.614 \%)$. 


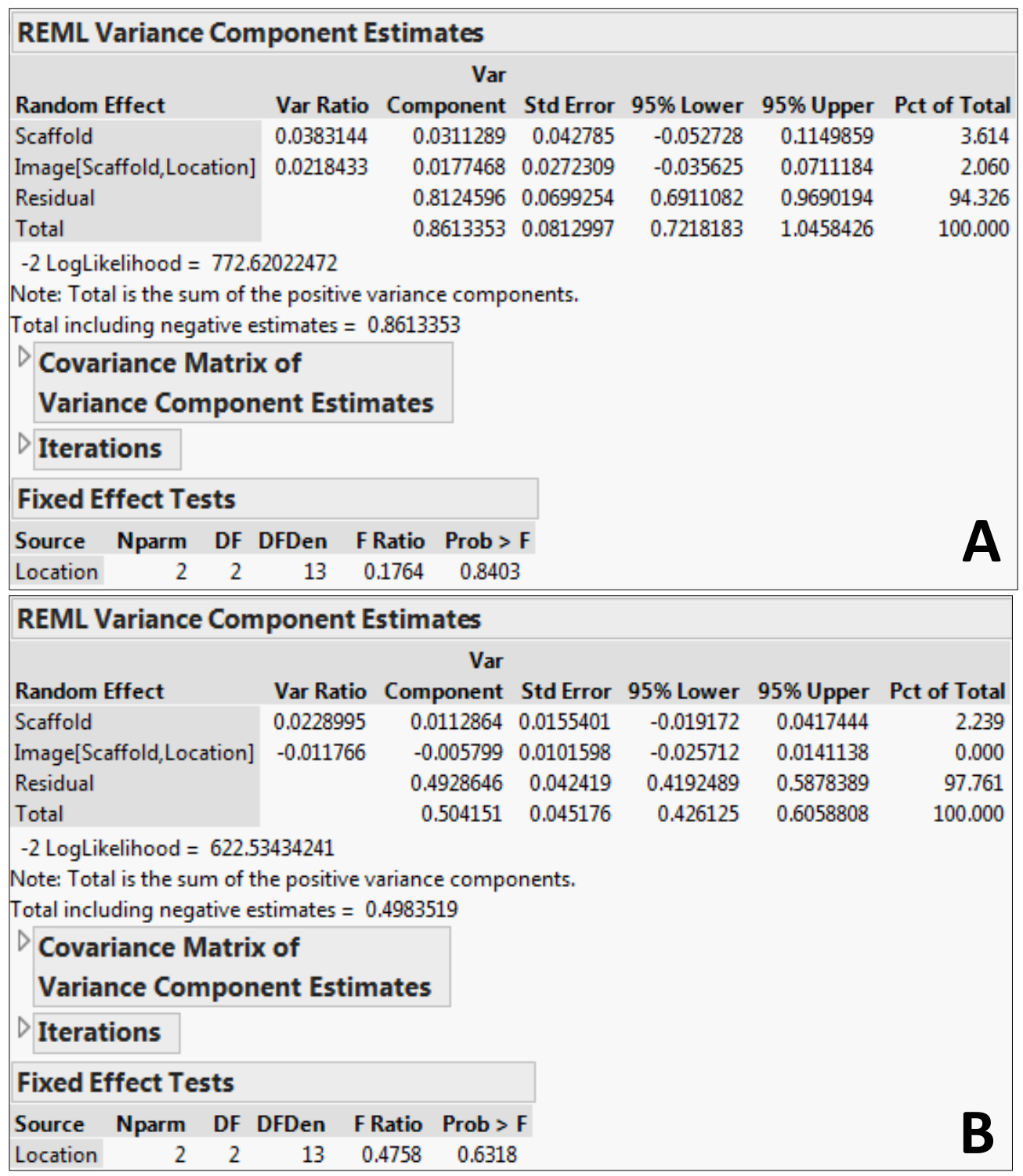

Figure H 1: JMP output of the REML variance components analysis for (A) Protocol B and $(B)$ the Standard Protocol.

Furthermore, the ANOVA results are shown in Figure $H 2$ on the next page. The p-value for Protocol B was 0.0075 while the p-value for the Standard Protocol was 0.0489. Both p-values were below the significance level of 0.05 , therefore rejecting the null hypothesis. Although the variance components analyses had low scaffold-to-scaffold variabiltiy, the variability still corresponded to significantly different means across scaffolds. Thus, the same conclusion was reached with and without outliers in the analysis. However, the p-value for the Standard Protocol was just below 0.05, indicating that the difference was less significant with outliers included. 

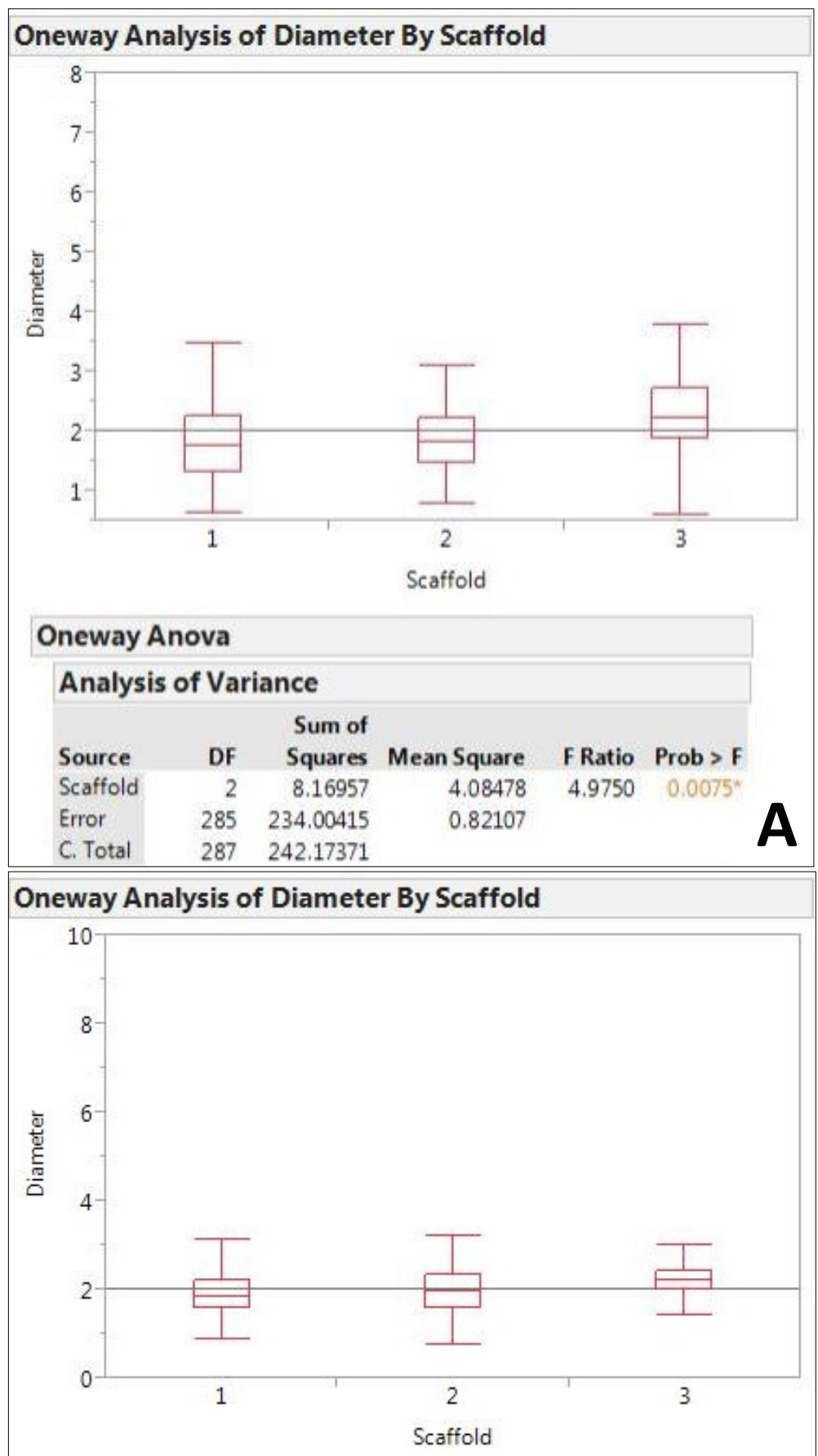

\section{Oneway Anova}

Analysis of Variance

\begin{tabular}{|c|c|c|c|c|c|}
\hline Source & DF & $\begin{array}{l}\text { Sum of } \\
\text { Squares }\end{array}$ & Mean Square & F Ratio & Prob $>$ F \\
\hline Scaffold & 2 & 2.96714 & 1.48357 & 3.0494 & $0.0489^{\star}$ \\
\hline Error & 285 & 138.65522 & 0.48651 & & \\
\hline C. Total & 287 & 141.62236 & & & \\
\hline
\end{tabular}

Figure H 2: Boxplots and JMP output of ANOVA for $(A)$ Protocol B and $(B)$ the Standard Protocol. 
Additionally, the results for Tukey's HSD tests are shown in Figure $H 3$ on the following page. For Protocol B, only Spin B4 was significantly different from Spin B1. This was different than the result without outliers, which indicated that Spin B4 was significantly different from both Spin B1 and Spin B2. The Standard Protocol also had a different result in this analysis includiing the outliers; Tukey's tests show no significant differences in any of the comparisons made between Spins X, Y, and Z, though the comparison between Spin Y and Spin Z had a p-value of 0.0606, which was close to significance. This discrepancy could be attributed to the barely significant $p$-value of 0.0489 in the ANOVA results. 


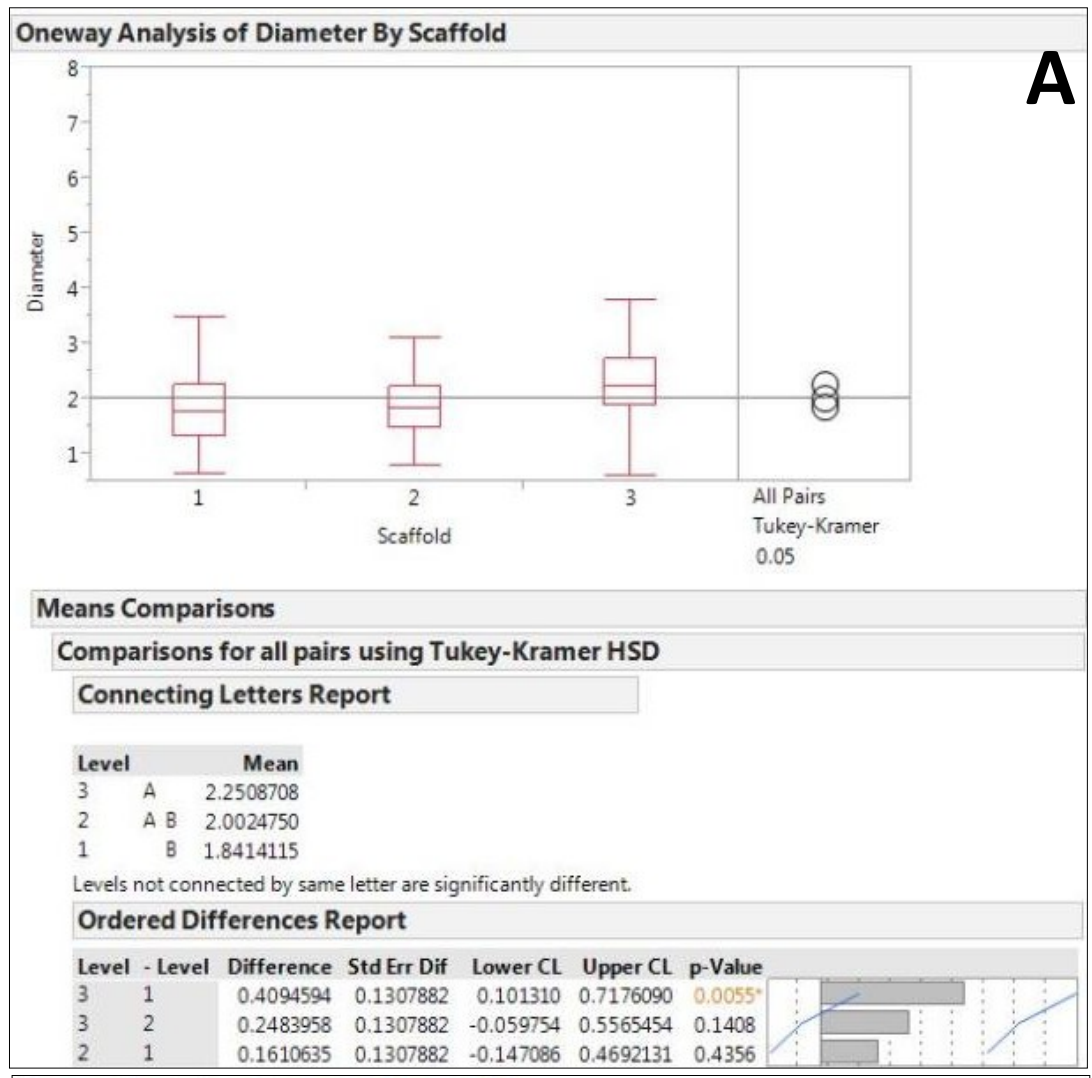

Oneway Analysis of Diameter By Scaffold

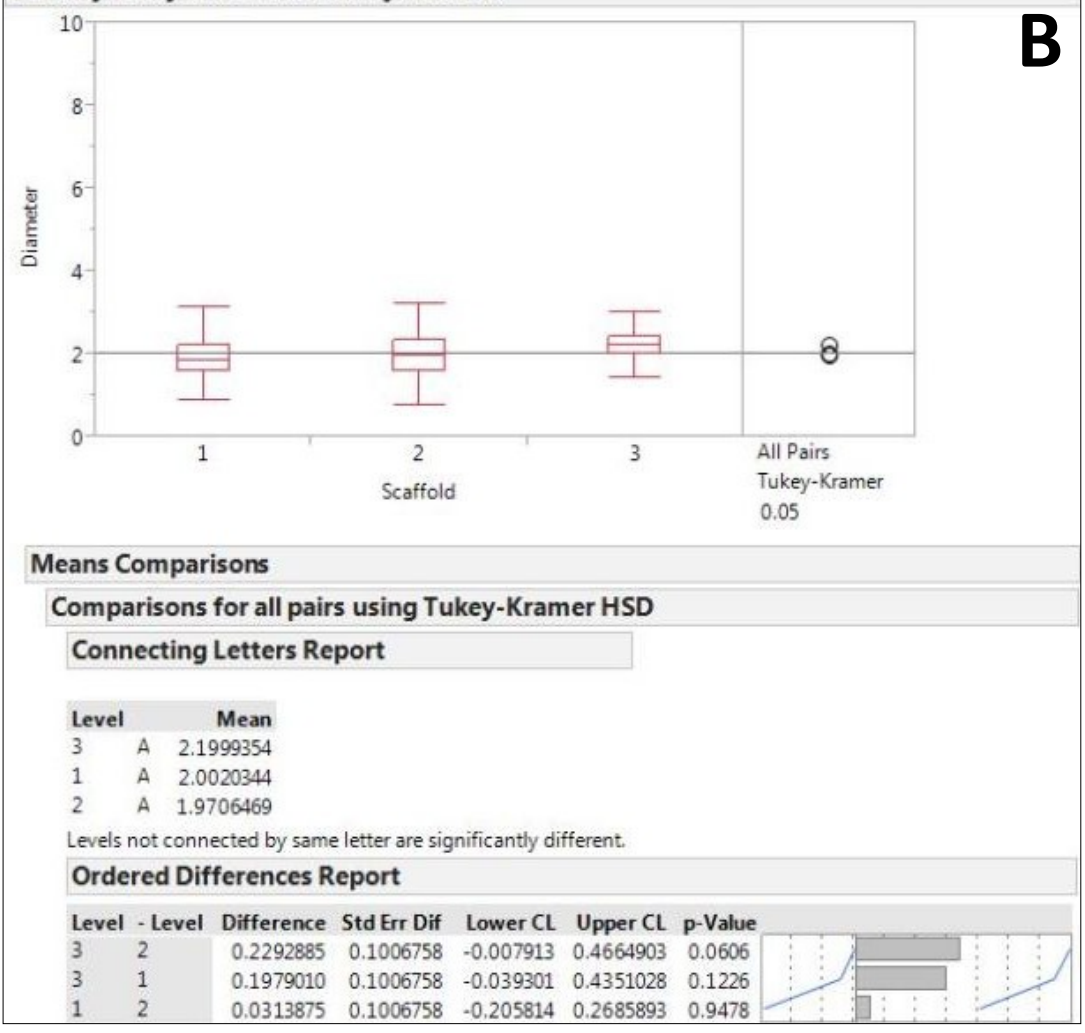

Figure $H$ 3: Boxplots and JMP output of Tukey's HSD post-hoc test for (A) Protocol B and $(B)$ the Standard Protocol. 
Finally, the results of the one-tailed t-test are shown in Figure $H 4$ below. The same conclusion was reached in this analysis with outliers: Protocol B did not significantly reduce fiber diameter. The $\mathrm{p}$-value was 0.3091 in this analysis, which was less significant than the p-value of 0.1004 achieved in the analysis excluding outliers.

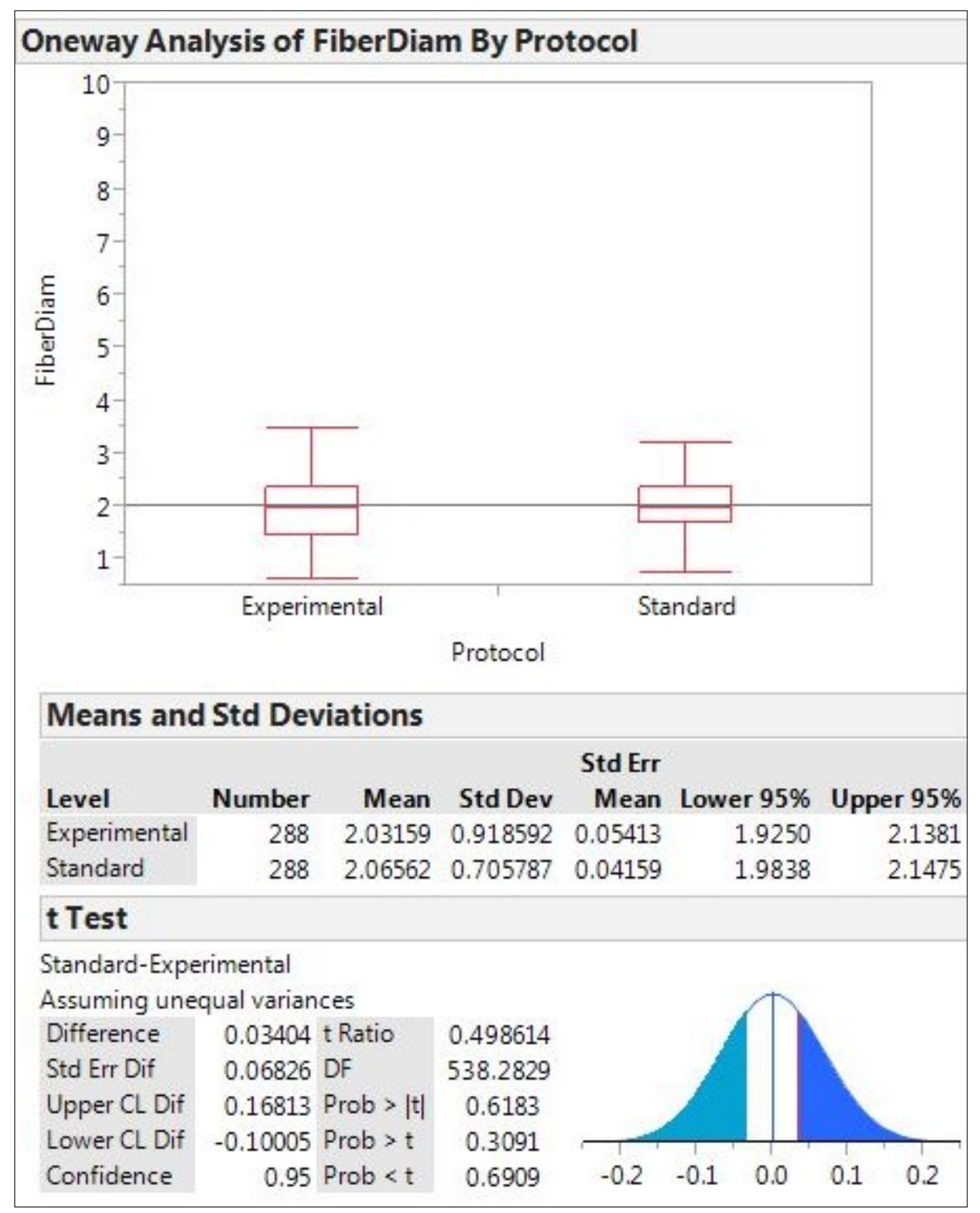

Figure H 4: Boxplot and JMP output of the one-tailed t-test comparing the mean fiber diameters of Protocol B and the Standard Protocol. 


\section{APPENDIX I: 303 SS MANDREL FABRICATION PROTOCOL}

This protocol was taken from Tiffany Peña's thesis.

\begin{tabular}{|c|c|c|c|}
\hline Materials & Vendor & Part Number & Quantity \\
\hline $\begin{array}{c}\text { 303 Stainless Steel, Unpolished } \\
\text { (diameter }=4 \mathrm{~mm}, \text { length }=1 \mathrm{~m}) \\
\text { *Precision Ground not required }\end{array}$ & McMaster Carr & $1274 \mathrm{~T} 13$ & 1 \\
\hline $\begin{array}{c}\text { Small Diameter Drill Bit } \\
\left(1 / 16^{\prime} \text { diameter, } 1 / 8^{\prime} \text { ' shank }\right)\end{array}$ & McMaster Carr & $2841 \mathrm{~A} 94$ & 1 \\
\hline $\begin{array}{c}\text { Stainless,Şteel Slotted,Şpring Pins } \\
(1 / 16 \text { diameter, } 1 / 2 \text { length })\end{array}$ & McMaster Carr & 92383A106 & $100 / \mathrm{Pkg}$ \\
\hline
\end{tabular}

**WARNING: Do not attempt to use the lathe if you have not been properly trained in all operating procedures as well as safety protocols. The lathe can cause severe injury if used improperly!

1. Read and understand all instructions before beginning fabrication.

2. If you have any questions regarding this SOP or machinery operations, contact Dr. Kristen Cardinal for assistance.

3. Cut the 1 meter stainless steel rod into $15 \mathrm{~cm}$ sections using horizontal and/or vertical band saws. There will be six $15 \mathrm{~cm}$ sections and a small scrap portion of steel left at the end. (Note: the final length of each mandrel will be $14 \mathrm{~cm}$ ).

4. Using a lathe, position the bulk of the $15 \mathrm{~cm}$ rod in the chuck, leaving only a small amount of material exposed. Face one end of each $15 \mathrm{~cm}$ rod using a carbide-tipped lathe tool. Set the speed to 1,170 RPM. Chamfer the faced edges using a fine file; there is no need to adjust the speed for this.

5. Prior to drilling the $1 / 16$ " hole, the surface of the rod must be prepared. To prevent bit- wandering and provide a better drill bit-surface contact, a file should be used to slightly grind a flat surface on the rod before drilling.

6. Before drilling the hole, provide a few drops of cutting fluid to the bit to help with lubrication. (Note: The key to drilling this size hole is to move slowly and peck the material frequently, however be careful as to not work harden the steel). Using a CNC mill, drill a $1 / 16$ " hole through diameter of each $15 \mathrm{~cm}$ rod, approximately $3 \mathrm{~mm}$ from the finished/chamfered end to the center of the hole. Set drill speed to 2,300 RPM and use the 1/16" small diameter drill bit (1/8', shank).

7. Using the lathe, face the unfinished end of each $15 \mathrm{~cm}$ rod. Set speed to 1,170 RPM. 
8. Using a \#2 drill bit on the lathe, drill a shallow hole into the faced/un-chamfered end of each $15 \mathrm{~cm}$ rod. Drill just deep enough to attach a live center. Do this for all $15 \mathrm{~cm}$ sections of steel. Set speed to 1,170 RPM.

9. Secure the $15 \mathrm{~cm}$ rod between the live center and the chuck of the lathe. Expose as much of the rod as is possible. Set speed to 510 RPM. Wet-polish the rod using 600 grit sandpaper and methanol or water. (Note: If dirty or greasy, clean rods before polishing to remove particles and to achieve a better surface finish).

10. Cut the $15 \mathrm{~cm}$ rods down to $14 \mathrm{~cm}$ ensuring to remove the face with the \#2 drill bit hole. Use a band saw to remove the bulk of the material, leaving just enough material to finish the end.

11. Using the lathe, face and chamfer the unfinished end following the same procedure as in Step 2.

12. Insert a $1 / 16$ " slotted spring pin into the $1 / 16$ " hole that was drilled in step 3 . This can be best achieved by crimping one end of the pin with pliers to get the pin partly inserted. Then using the pliers, force the pin into the center of the hole.

13. Clean mandrels thoroughly with IPA before use to ensure all oil, dirt and metal particles are removed.

14. Evaluate each mandrel manufactured by ensuring the mandrel properly fits and rotates in the electrospinner. Also, perform a test spin on each mandrel and check for any differences between polymer collection and scaffold removal compared to that of the original mandrels. The new mandrel should be nearly identical to the original. If scaffolds are difficult to remove from the mandrels, further wet polishing on the lathe may be necessary (See Step 6). 


\section{APPENDIX J: CHAPTER III PRELIMINARY COMPLIANCE DATA}

Preliminary compliance data was obtained using scrap pieces of PLGA scaffolds. The purpose of this testing was to become familiar with the compliance equipment, practice good technique, and gain perspective on the compliance behavior that was to be expected from the scaffolds. Three samples were tested using the compliance protocol outlined in Appendix $K$. Five trials were performed on each sample to see if repetitions systematically affected compliance, such as causing compliance to increase with each trial. After testing, the following statistical analyses were performed: REML variance components analysis, one-way ANOVA, and Tukey's HSD post-hoc test.

Figure $J 1$ below shows the mean compliance and standard deviations of the three scaffolds. The mean compliance ranged from $0.09 \% / 100 \mathrm{mmHg}$ to $0.25 \% / 100 \mathrm{mmHg}$ and the standard deviations ranged from $0.02 \% / 100 \mathrm{mmHg}$ to $0.03 \% / 100 \mathrm{mmHg}$.

\begin{tabular}{|c|c|c|c|c|c|c|}
\hline \multicolumn{7}{|c|}{ Means and Std Deviations } \\
\hline Level & Number & Mean & Std Dev & $\begin{array}{r}\text { Std Err } \\
\text { Mean }\end{array}$ & Lower $95 \%$ & Upper $95 \%$ \\
\hline 1 & 5 & 0.253424 & 0.027603 & 0.01234 & 0.21915 & 0.28770 \\
\hline 2 & 5 & 0.088750 & 0.030972 & 0.01385 & 0.05029 & 0.12721 \\
\hline 3 & 5 & 0.126377 & 0.015232 & 0.00681 & 0.10746 & 0.14529 \\
\hline
\end{tabular}

Figure J 1: JMP output of the mean compliance and standard deviations for preliminary compliance testing.

Figure $J 2$ shows the results for the REML variance components analysis. The scaffoldto-scaffold variability was $91.828 \%$, so a majority of the variability arose from differences between scaffolds.

\begin{tabular}{|lrrrrrr|}
\hline \multicolumn{7}{|l|}{ REML Variance Component Estimates } \\
\begin{tabular}{|l} 
Random \\
Effect
\end{tabular} & Var Ratio & Component & Std Error & $95 \%$ Lower & $95 \%$ Upper & Pct of Total \\
Scaffold & 11.236235 & 0.0073155 & 0.0074459 & -0.007278 & 0.0219091 & 91.828 \\
Residual & & 0.0006511 & 0.0002658 & 0.0003348 & 0.0017741 & 8.172 \\
Total & & 0.0079665 & 0.0074487 & 0.0022867 & 0.2118209 & 100.000 \\
\hline
\end{tabular}

Figure J 2: JMP output for a REML variance components analysis of the preliminary compliance testing. 
ANOVA results are shown in Figure $J 3$ below. The p-value was $<0.0001$, which was below the significance level of 0.05 . This matches the high between-scaffold variability that was observed in the REML variance components analysis.

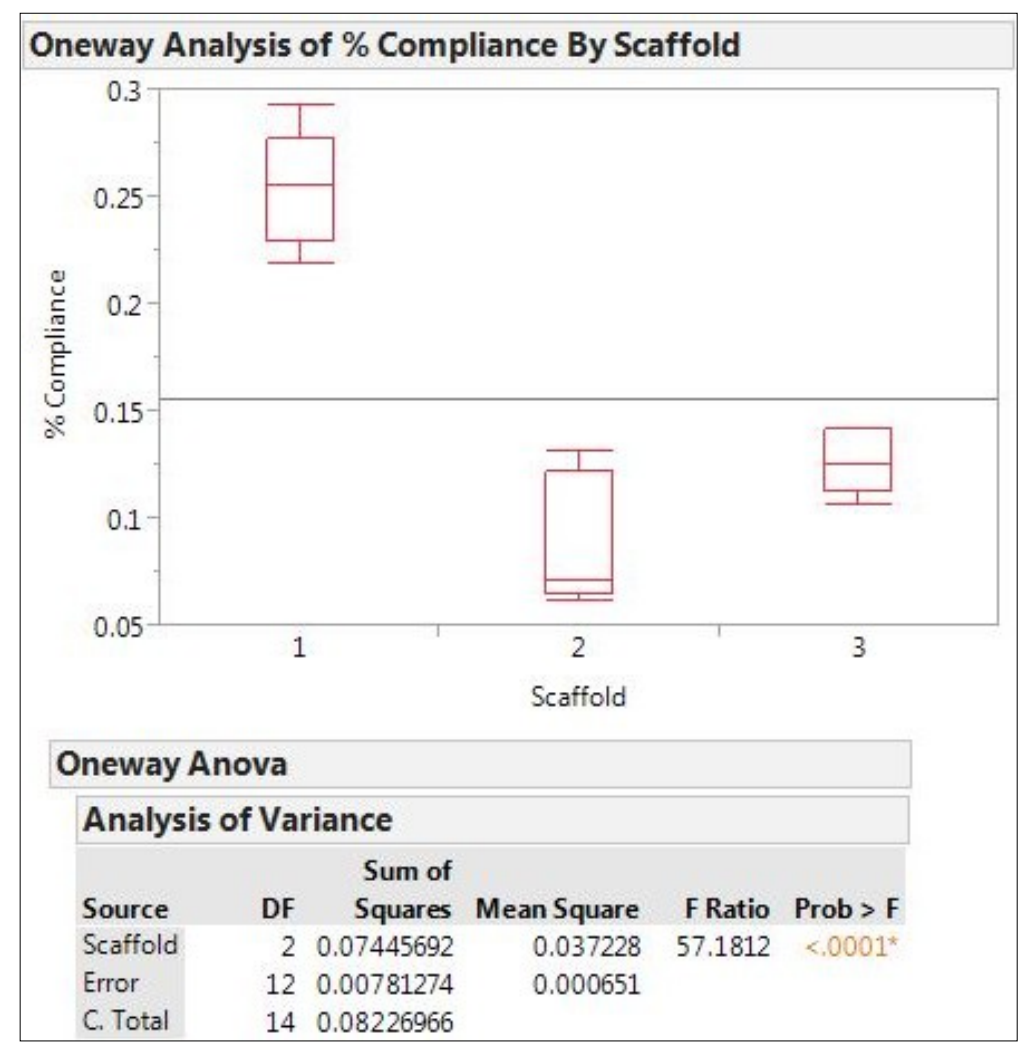

Figure J 3: Boxplot and JMP output of ANOVA for preliminary compliance testing.

The results of Tukey's HSD test are shown in Figure $J 4$ on the following page. It was evident that Scaffold 1 was significantly more compliant than Scaffolds 2 and 3. Scaffolds 2 and 3 did not have significantly different compliance. 


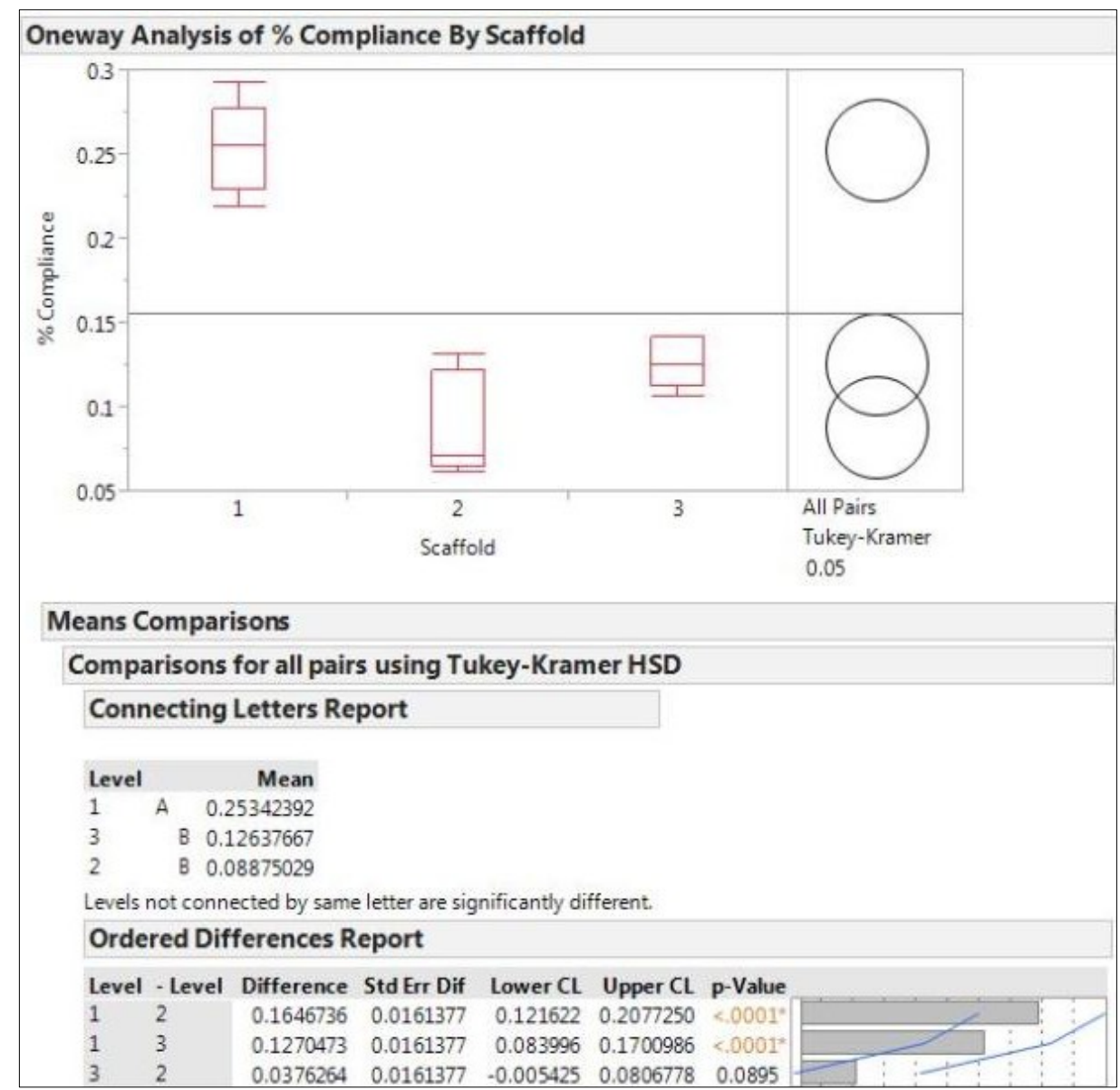

Figure J 4: Boxplot and JMP output of Tukey's HSD test for preliminary compliance testing.

Finally, the data for all trials (Table $J I$ ) did not reveal any systematic patterns in compliance; specifically, multiple trials on one sample did not cause the compliance to increase with each trial. Thus, multiple trials were acceptable to perform. However, since 20 scaffolds were going to be tested in the reproducibility study with three compliance samples per scaffold, it was determined that performing three trials per sample was sufficient.

Table J I: Compliance data of all trials performed on the three scaffolds.

\begin{tabular}{|c|c|c|c|c|c|}
\hline Scaffold & $\begin{array}{c}\text { Compliance } \\
(\% / 100 \mathrm{mmHg})\end{array}$ & Scaffold & $\begin{array}{l}\text { Compliance } \\
(\% / 100 \mathrm{mmHg})\end{array}$ & Scaffold & $\begin{array}{c}\text { Compliance } \\
(\% / 100 \mathrm{mmHg})\end{array}$ \\
\hline 1 & 0.260792837 & 2 & 0.061121827 & 3 & 0.124652755 \\
\hline 1 & 0.218426251 & 2 & 0.06885631 & 3 & 0.11773215 \\
\hline 1 & 0.292986671 & 2 & 0.070321688 & 3 & 0.141309057 \\
\hline 1 & 0.239330916 & 2 & 0.130770476 & 3 & 0.10653158 \\
\hline 1 & 0.255582911 & 2 & 0.112681163 & 3 & 0.141657786 \\
\hline
\end{tabular}




\section{APPENDIX K: COMPLIANCE TESTING PROTOCOL}

\section{METHODS}

PROTOCOL PART A: SET-UP

1. Identify the Olympus CKX41 microscope and compliance tester fixture. Make sure everything is plugged in to the power strip. (Figure K 1 ).

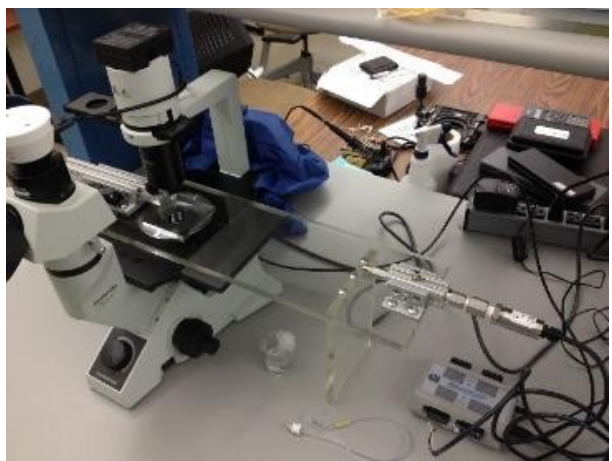

Figure K 1: The Olympus CKX41 Microscope and compliance testing fixture.

2. Turn the microscope on. Verify that the microscope and Omega DAQ Board are plugged into the laptop via the USB ports.

3. Verify that the transducer wires are connected as shown in Figure $K 2$.

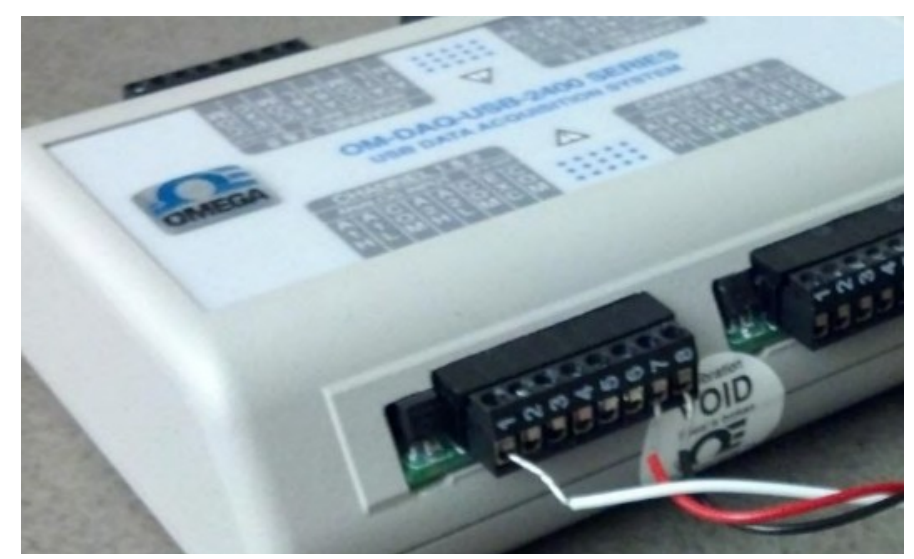

Figure K 2: DAQ wires. The proper DAQ connections.

4. Push the condenser all of the way to the left (1) and move the aperture knob (2) to the left so the light is brightest (Figure $K 3$ ). Turn the microscope objective to $4 \mathrm{x}$. 


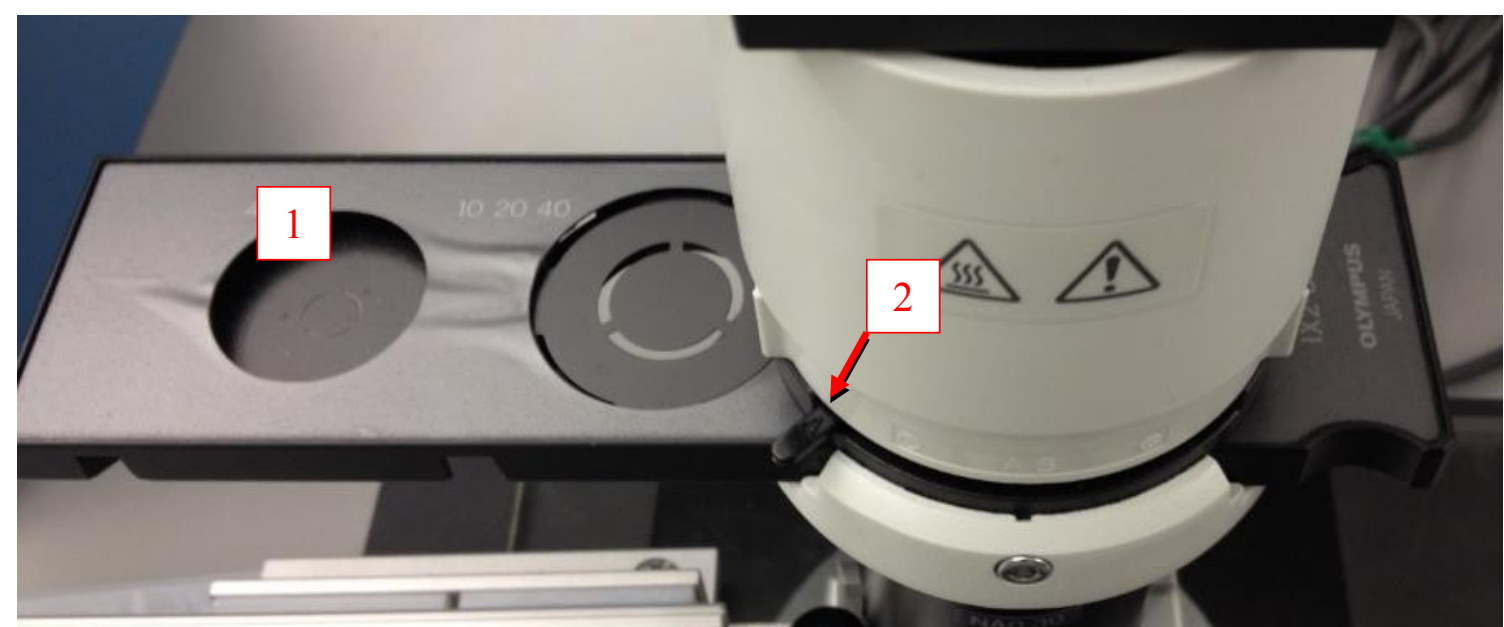

Figure K 3: Light condenser. Image of shutter and filter in proper placement.

5. Connect the occlusion catheter to the male luer fitting as seen in Figure $K 4$.

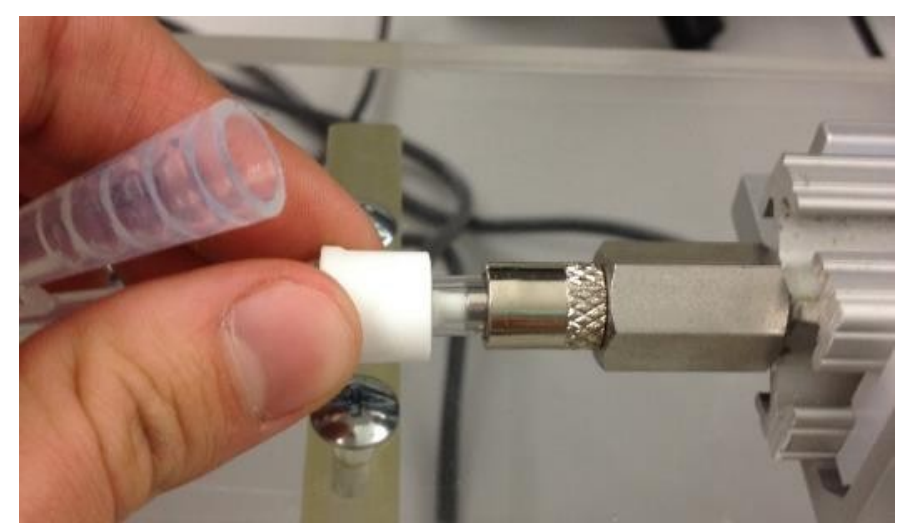

Figure K 4: Catheter connection. Attach the catheter to the male luer.

6. Fill the $10 \mathrm{~mL}$ syringe with DI water and screw it into the female luer fitting, as seen in Figure K 5 .

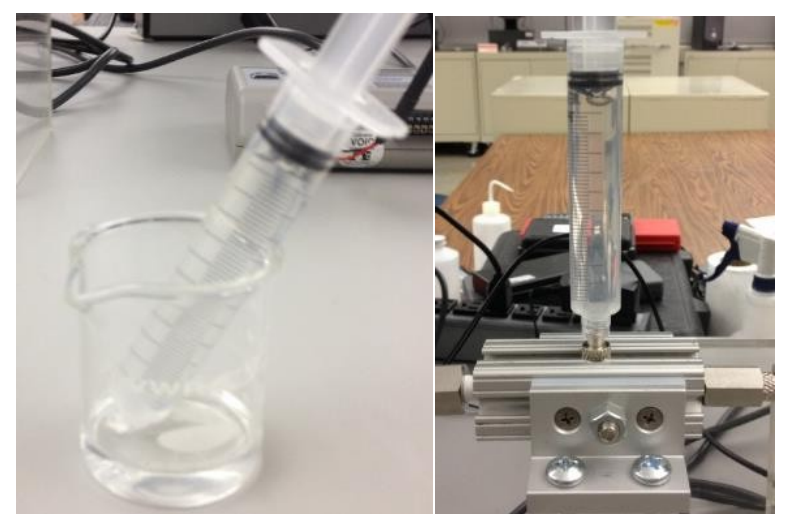

Figure K 5: Syringe connection. Image of fluid-filled syringe screwed into the female luer fitting. 
7. Cut one $\sim 2 \mathrm{~cm}$-length tubing sample for each material (you only need one sample for each). The sample length should be just long enough to cover the inflation zone; not any longer (see below).

8. Load the first tubing sample onto the catheter, making sure it is covering the inflation zone (Figure $K$ 6).

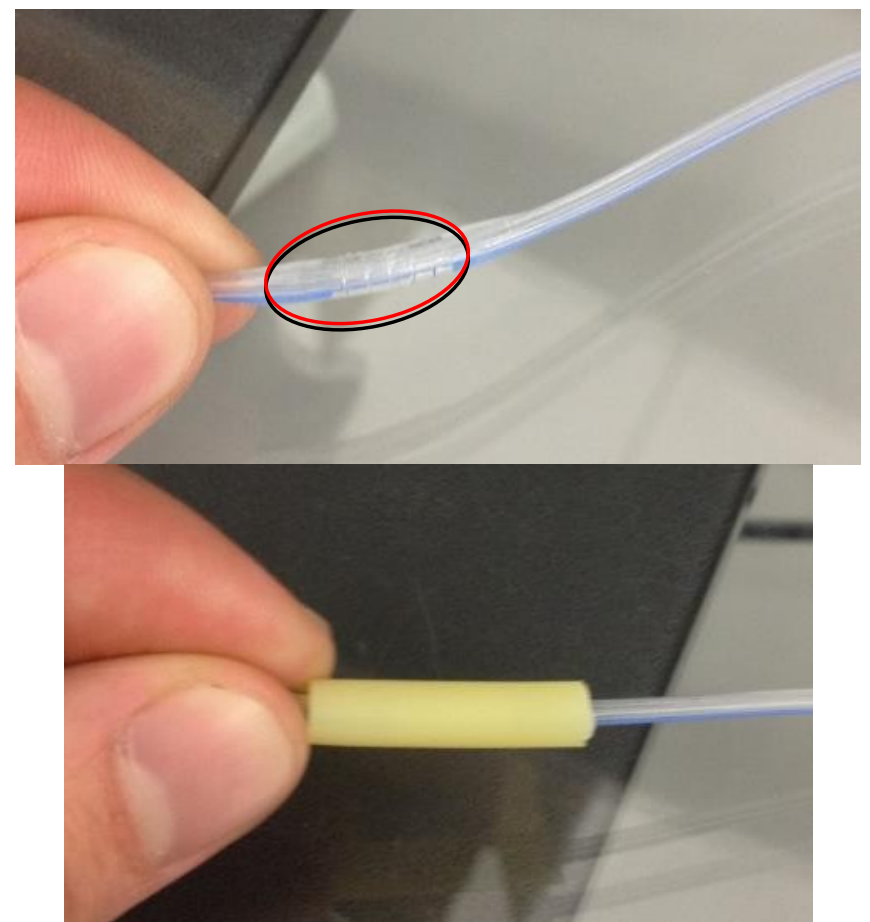

Figure K 6: Image of balloon region (left) and the tubing sample loaded to cover the balloon region (right).

9. Place the tip of the catheter into the clamp just until the hole in the catheter is covered. Tighten the thumb screw all the way (Figure $K 7$ ).

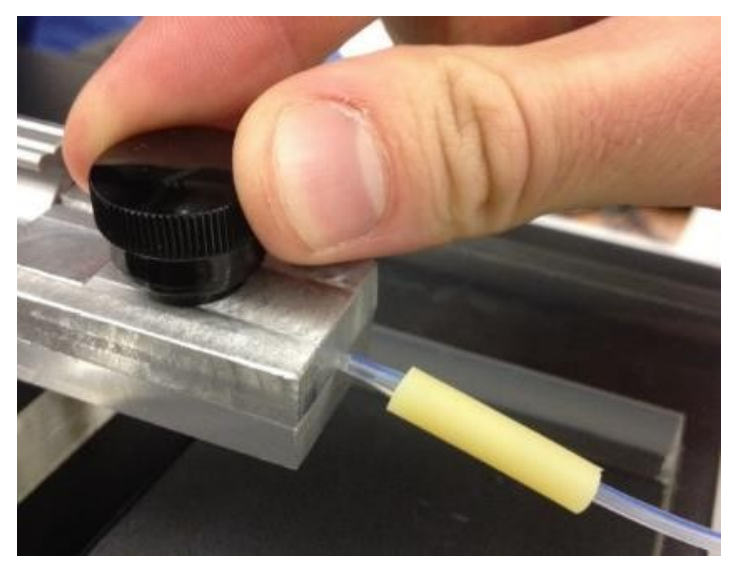

Figure K 7: Placement of the catheter in the clamp. 
10. Slide the clamp back so that the black line is aligned with the edge of the platform (Figure $K 8$ ).

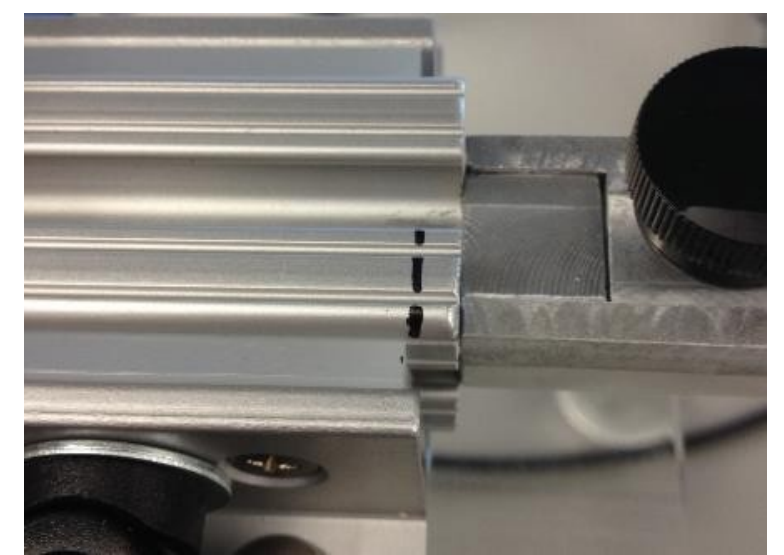

Figure $K 8$ : Location of slider when pulled back.

11. Position the fixture so that the tubing sample is roughly centered over the objective lens.

\section{PROTOCOL PART B: SOFTWARE}

1. Open up the "DAQ Central" software package by clicking on the icon seen in Figure K 9 .

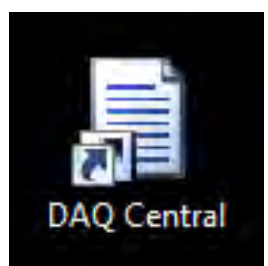

Figure K 9: DAQ Icon. An image of the icon used to open up the DAQ program.

2. From the DAQ main window (Figure K 10), click "Devices" and then "Detect Devices". The device "N0903212012" should appear in the window: this is the pressure transducer.

NOTE: If the device does not appear, ensure that the green power LED is lit up on the DAQ unit, that the USB cable is plugged in, and that the wires are correctly hooked up per Part A. 


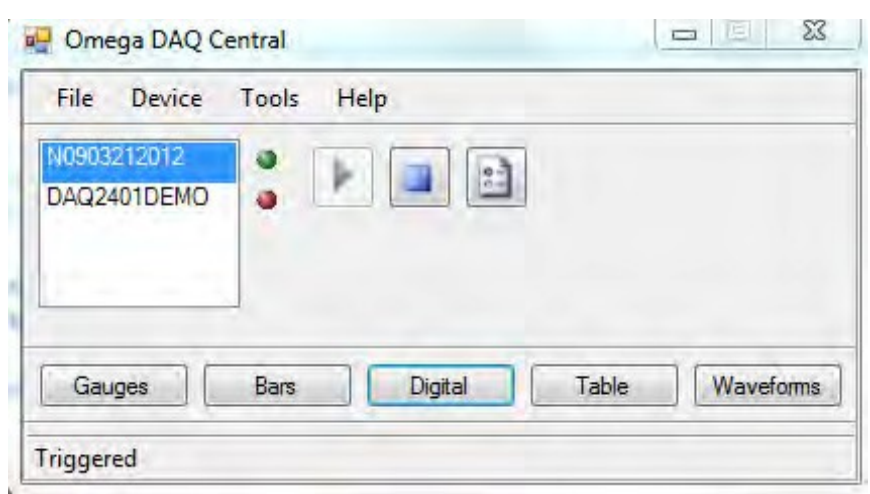

Figure K 10: DAQ Program. The pressure transducer can be seen highlighted in blue.

3. Click "Devices" and then "Configuration". From the menu seen in Figure K 11, make sure that the AN1D channel is set to "ON", "Differential", and "+/- 5V". Also make sure the Scan Rate is set to $100 \mathrm{~Hz}$.

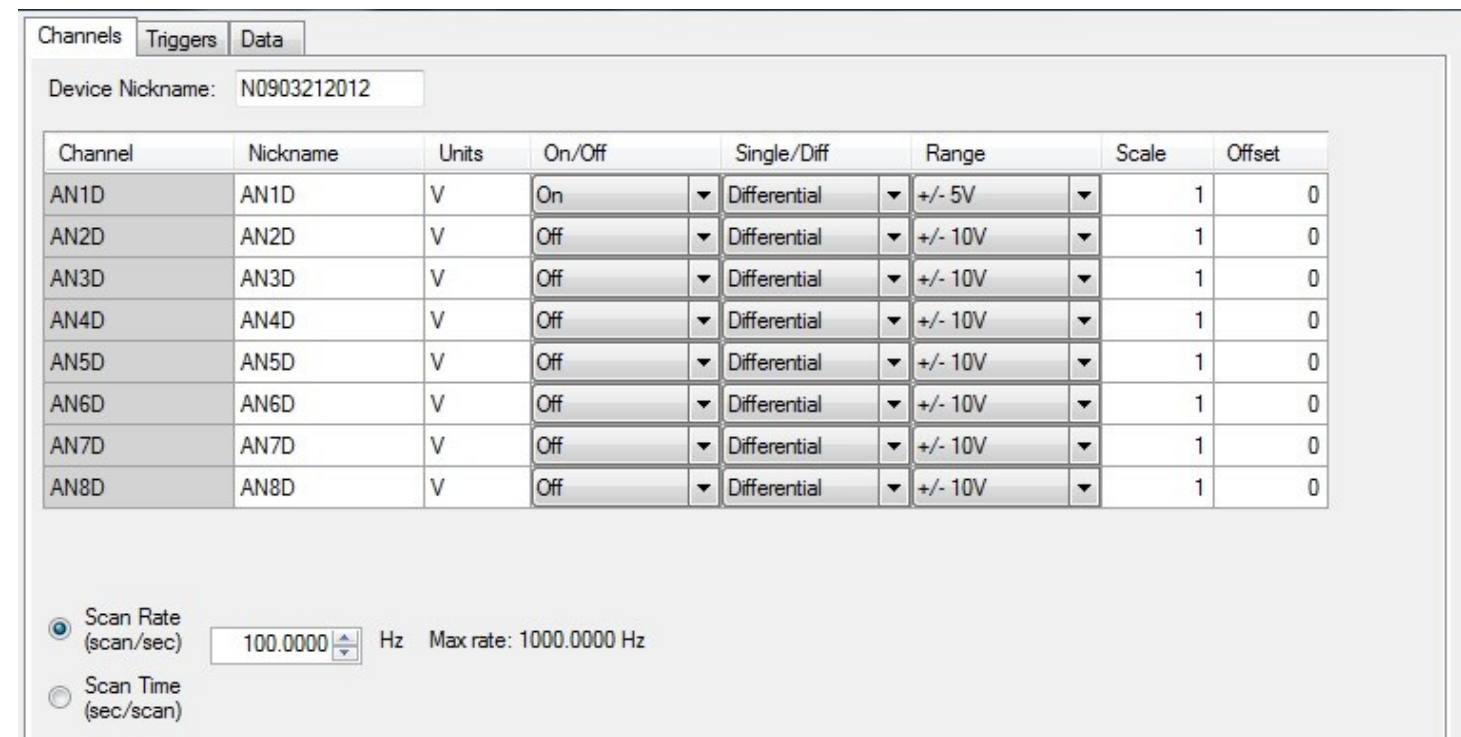

Figure K 11: DAQ Configuration. The correct configuration for the pressure transducer.

4. From the same window, click the "Data" tab. As seen in Figure $K 12$, set up the correct parameters. For "File", create a unique file name in the "Pressure Files" folder on the desktop where you can easily access the Excel file that will be created.

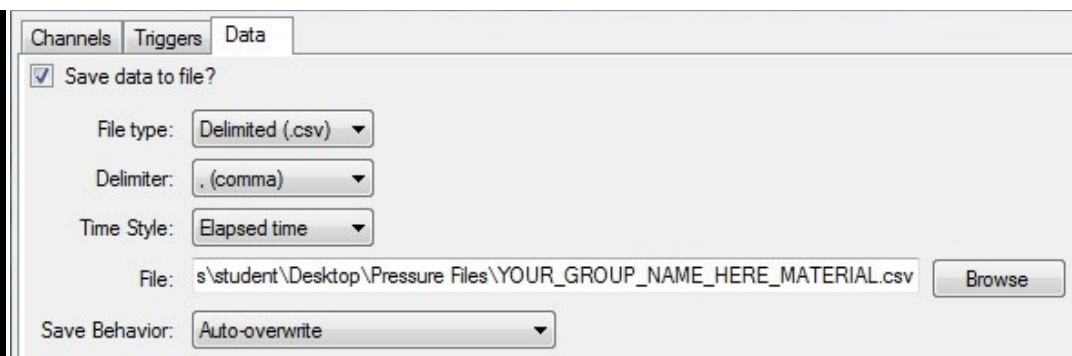

Figure K 12: DAQ Data settings. An image of the correct data parameters. 
5. From the main DAQ menu, hit the "play" button as seen in Figure $K 10$. Then hit the "Digital" button. The image seen in Figure K 13 should appear. This displays the voltage that the transducer is reading. "Prime" the system by applying a bit of pressure to the system (about $0.5 \mathrm{~V}$ ).

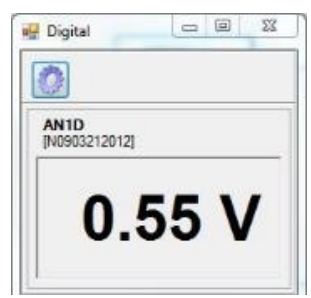

Figure K 13: The DAQ Voltage Display.

6. Hit the "stop" button. You are now ready to prepare the MATLAB programs.

7. Open up the MATLAB software suite. From the drop-down menu, expand the folder "Compliance Matlab Files" on the desktop and choose "ImageCapture.mdl," as seen in Figure K 14.

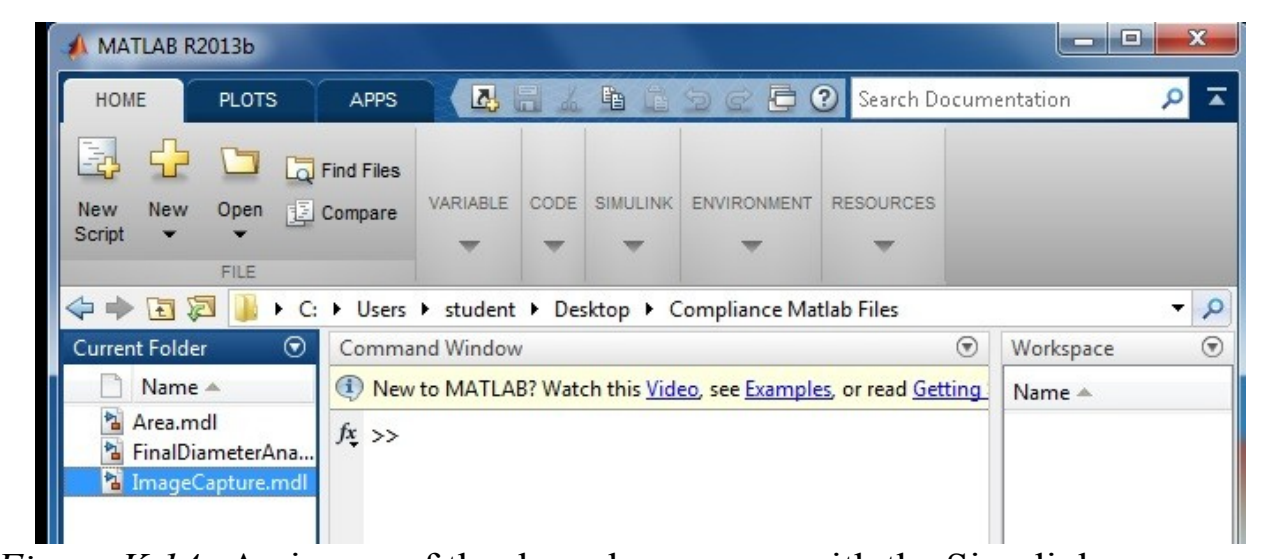

Figure K 14: An image of the drop-down menu with the Simulink programs.

8. From the Image Capture GUI (Figure K 15) open the "From Video Device" block and ensure that the Microscope is chosen. If the Infinity microscope is not chosen, try plugging the microscope USB back into the computer, make sure there is power, that the scope is plugged in, and that the microscope button is in the "on" position.

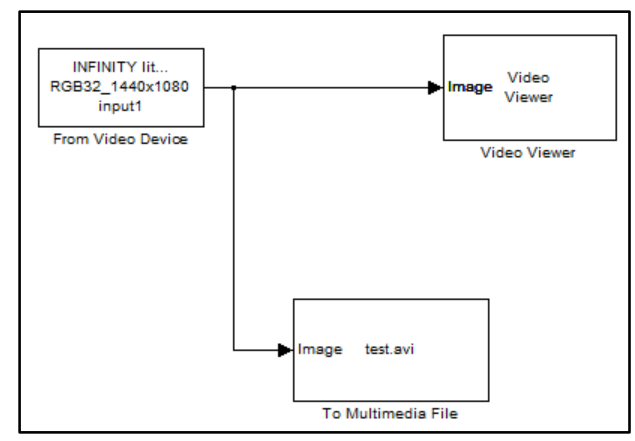

Figure K 15: The Image Capture simulink graphical user interface (GUI). 
9. Open up the "To Multimedia File" block. For "File name", create a unique file name in the "Video Files" folder on the desktop where you can easily access the video file that will be created (Figure K 16).

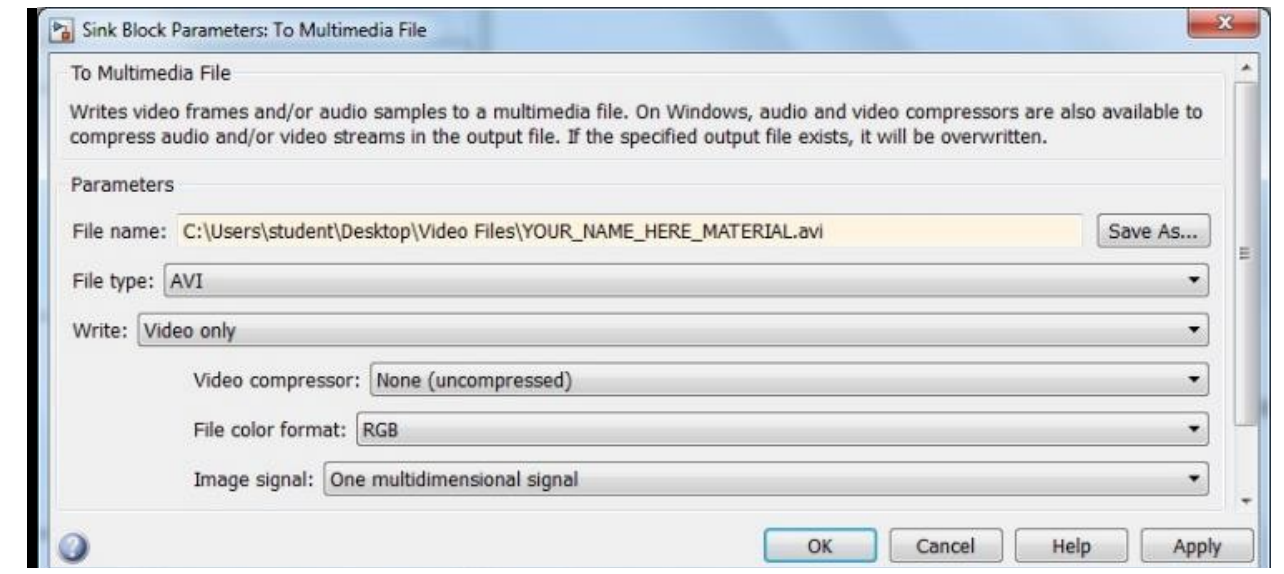

Figure K 16: Saving the video. An image of the "To Multimedia File" menu.

10. Once a location for the file has been saved, click "OK". Hit the "play" button located in the Simulink toolbar (towards the top of the program), as seen in Figure K 17.

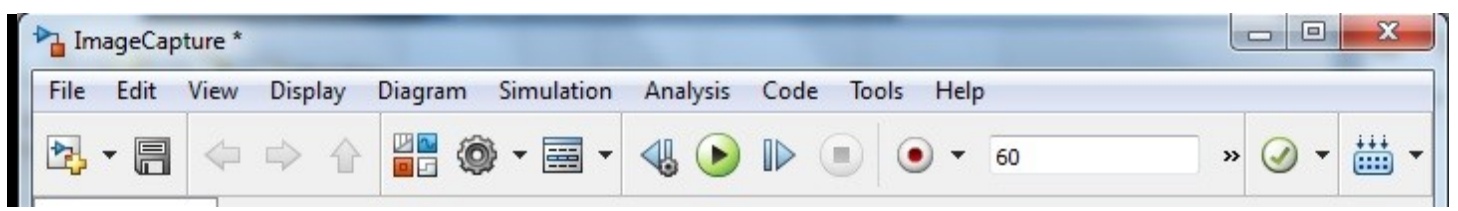

Figure K 17: Starting the Program. The play button will begin video capture.

11. A "video viewer" window will pop up. Use this live video feed to position the scaffold in the center of the screen. Nudge the stage back and forth until you have properly placed the scaffold. Note: The video feed will lag behind your motions, so be slow, and wait for the video to "catch up" with your motions. This will make placement easier. Try to make the sample as horizontal as possible.

12. It may be necessary to adjust the lighting and focus in order to create an image like the one shown below in Figure K 18. Try to eliminate all fuzziness around the edge as possible. 


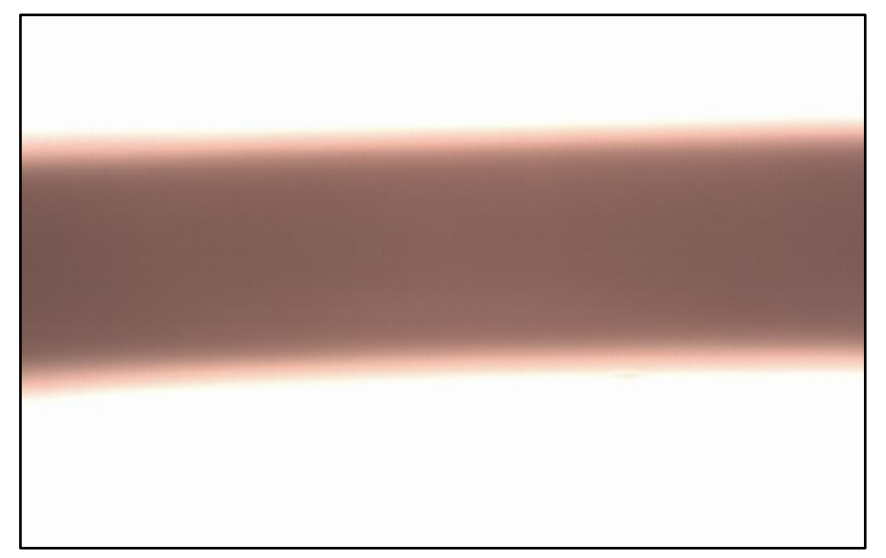

Figure K 18: An example of an acceptable image of the tubing.

13. Once the scaffold has been centered, hit the "stop" button to end the Image Capture program.

14. Now you are ready to capture the video of the pressure application. First, apply a little bit of pressure on the syringe and hold it steady. In quick succession, hit the "play" button for the DAQ pressure sensor (Step 5), then hit the "play" button on the Image Capture GUI (step 11). Begin applying pressure once the video feed is displayed.

15. Apply pressure slowly, and consistently to avoid spiking the pressure reading, but apply with urgency. Bring the voltage to around $2 \mathrm{~V}$ and hold it there for a couple of seconds. Relieve the pressure then hit the "stop" button for both the video and pressure readings. Take note of the $\mathrm{T}$-value in the bottom right corner of the video viewer window when you relieve the pressure (Figure K 19). Note: the smaller the T-value the better.

Figure K 19: The T-value to observe when the pressure is relieved.

16. Return to the main MATLAB window and choose "FinalDiameterAnalysis.mdl" from the "Compliance Matlab Files" folder.

17. From the main Final Diameter Analysis GUI, open the "From Multimedia File" block, as seen in Figure $K 20$.

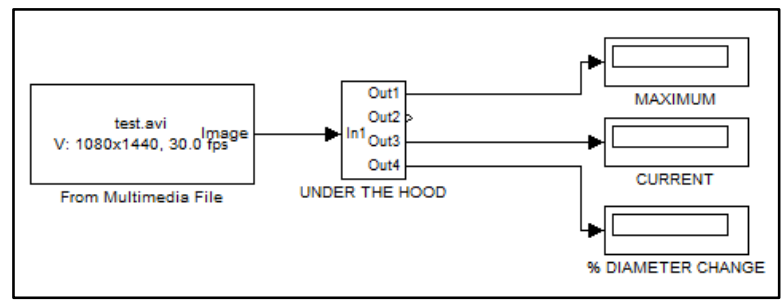

Figure K 20: Diameter Analysis GUI. An image of the GUI of the diameter Simulink program. 
18. A dialog box will appear (Figure K 21). Make sure the video you load is the video you named in Step 10. Click "OK" when finished.

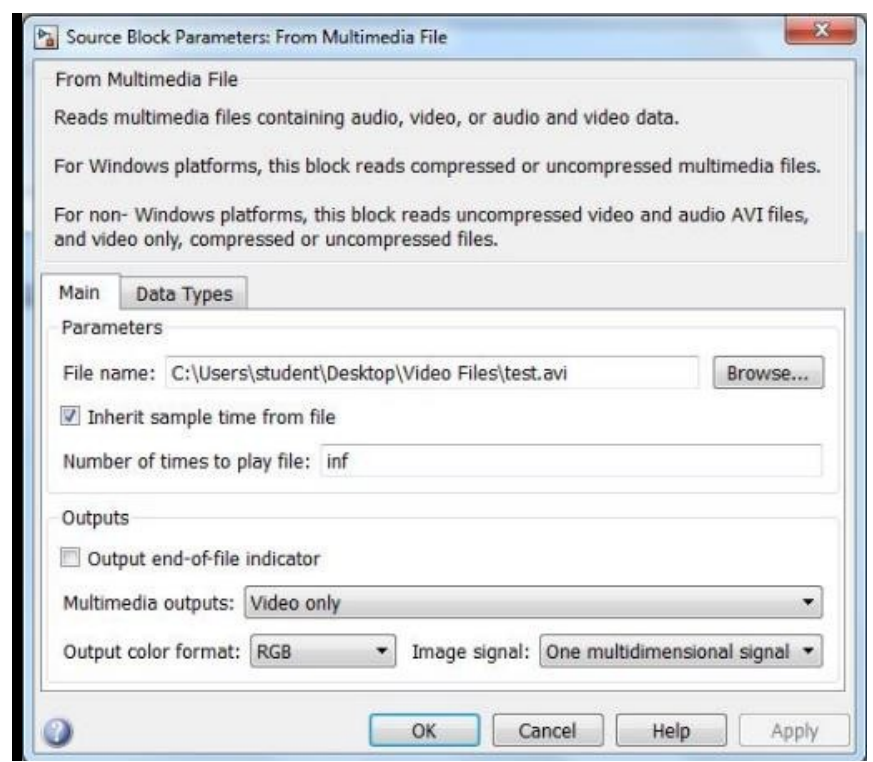

Figure $K 21$ : This is the dialog box that should appear when loading your video file.

19. Hit the "play" button to begin the video analysis. A video viewer should appear that presents a visualization of the part of the analysis process (Figure $K 22$ ).

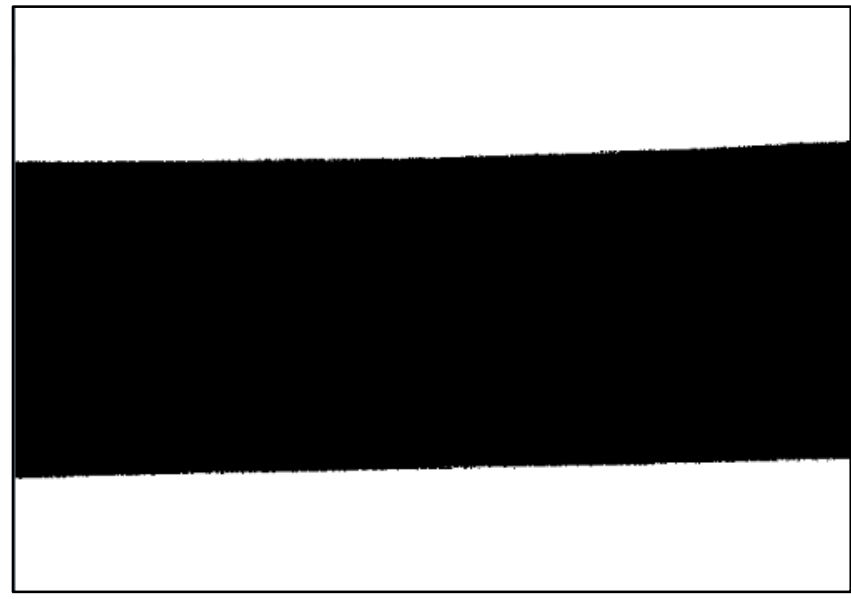

Figure $K$ 22: This is a visualization of how the binary format of the video. The program counts the number of black pixels (representing the scaffold) to monitor diameter change.

20. As seen in Figure $K 23$, there are three output boxes to monitor. MAXIMUM will display the maximum diameter that is achieved (when maximum pressure is applied). CURRENT will display the current diameter that is being counted. \% DIAMETER CHANGE will display maximum percent change in diameter. Familiarize yourself with these output boxes. 


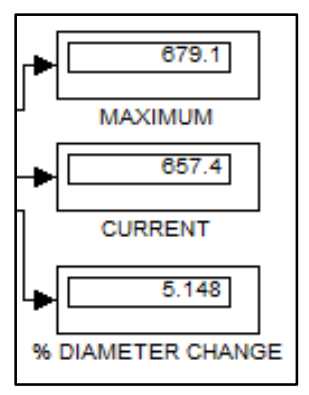

Figure $K$ 23: These output windows need to be monitored in order to know when to end the simulation.

21. While the simulation is running, monitor the T-value and let the program run until it exceeds the T-value recalled from step 15 . Verify that the CURRENT value drops below the MAXIMUM value after this time and hit the "stop" button to end the program. Record the \% Diameter Change.

22. Open your excel file that you saved from DAQ central.

23. Record the maximum voltage value that was achieved. In an empty cell, type "=max(" and then select column B to select the whole array of voltage values (Figure $K 24)$. Delete the contents from row 1.

\begin{tabular}{|c|c|c|c|c|c|}
\hline$\Delta$ & A & B & C & D & $\mathrm{E}$ \\
\hline 1 & Start Time & 6/7/2012 16:09 & & & \\
\hline 2 & Elapsed Time & AN1D & & & \\
\hline 3 & seconds & V & & & \\
\hline 4 & 0 & 0.46461449 & $=\max (\mathrm{B}$ & & \\
\hline 5 & 0.004 & 0.464711812 & \multicolumn{3}{|c|}{ MAX(number1, [number2], ...) } \\
\hline 6 & 0.008 & 0.465158067 & & & \\
\hline 7 & 0.012 & 0.459985774 & & & \\
\hline 0 & م16مـمـ & م-61200522 & & & \\
\hline
\end{tabular}

Figure $K$ 24: Finding the maximum voltage.

24. In the same way, find the minimum voltage value, then calculate the difference between these two values.

25. Convert this change in voltage to a change in PSI by multiplying by 10 (a number provided by OMEGA). Convert this change in PSI to a change in $\mathrm{mmHg}$ (51.7149326 $\mathrm{mmHg} / \mathrm{psi}$.

26. Type the value from the "\% DIAMETER CHANGE" output window in MATLAB into excel. Divide this value by the change in pressure, and multiply by 100 . Units are $\%$ compliance/100 $\mathrm{mmHg}$.

27. Calculate the elastic modulus value from the calculated percent compliance.

28. Make sure to save a copy of this data onto a flash drive and to delete the files from the laptop once you have done so. 


\section{APPENDIX L: SCAFFOLD CONDITIONING PROTOCOL}

This protocol was taken from Sarah Ur's senior project. For the purpose of this thesis, the protocol was modified for a non-sterile setup. Modifications are shown in red.

\section{Prep 1 week prior}

1. Gas sterilize biochambers (with extra piece of tubing to allow for flexibility of PLGA), lids, and 2 port reservoirs

2. Determine target number of cells and passage schedule, then thaw cells

3. Cut grafts, mount on fittings, and suture

4. Autoclave flasks, stop caps, and forceps

Prep the day before when needed

5. Make media:

a. Bioreactor media

b. Conditioning media

c. HUVEC

d. HUVSMC

6. Follow cell tracker protocol to stain cells with cell tracker red, green or violet. Do not use green on ECs.

\section{Set-up day: BVM conditioning}

1. Place 8 roller pump in incubator

2. Sterilize grafts (using 70\%)

a. With forceps place graft in $15 \mathrm{ml}$ conical with $70 \% \mathrm{EtOH}(\sim 9 \mathrm{ml})$ for 30 mins

b. Fill two troughs, one with PBS one with conditioning media. (Fill one trough with conditioning media).

e. Remove grafts from conical with sterile forceps and gloves.

d. Using syringe and stop cap flush luminally (no stop cap) and transmurally (with stop cap at distal end) with PBS conditioning media.

e. Perform same flush procedure with Conditioning Media

3. During 30 minute sterilization:

a. Open sterilized chambers in hood.

b. Fill chamber with bioreactor media (Be sure tubes are closed!)

4. Insert sterile grafts into bioreactors and place lid on chamber. ADD STOP CAP TO SODDING INLET.

5. Prime 2-port reservoirs with conditioning media

6. Attach primed biochamber to 2-port reservoir and condition graft for 6 hours- overnight (14-16 hours)

a. Flow through lumen first to remove air, then clamp lumen and condition transmurally on $150 \mathrm{rpm}$ setting

7. Leave primed biochambers in large incubator until ready for sodding step 


\section{APPENDIX M: CHAPTER III FIBER DIAMETER ANALYSIS WITHOUT OUTLIERS}

6 fiber diameter measurements were outliers and not included in this analysis.

Table $M I$ below shows the mean fiber diameters and standard deviations for the scaffolds excluding outliers. Scaffolds $6,12,18,20$, and 23 were affected by this. The means were slightly reduced, but the standard deviations were more noticeably reduced when outliers were excluded. The ranges of fiber diameters and variability were therefore reduced.

Table M I: Mean fiber diameters and standard deviations of the 20 scaffolds with no outliers.

\begin{tabular}{|c|c|c|}
\hline Spin & $\begin{array}{c}\text { Mean Fiber } \\
\text { Diameter }(\boldsymbol{\mu m})\end{array}$ & $\begin{array}{c}\text { Standard } \\
\text { Deviation }(\boldsymbol{\mu m})\end{array}$ \\
\hline $\mathbf{1}$ & 1.71 & 0.37 \\
\hline $\mathbf{2}$ & 2.79 & 0.51 \\
\hline $\mathbf{3}$ & 2.43 & 0.51 \\
\hline $\mathbf{4}$ & 2.18 & 0.46 \\
\hline $\mathbf{5}$ & 1.95 & 0.64 \\
\hline $\mathbf{6}$ & 2.07 & 0.45 \\
\hline $\mathbf{7}$ & 2.13 & 0.51 \\
\hline $\mathbf{8}$ & 2.07 & 0.53 \\
\hline $\mathbf{9}$ & 2.19 & 0.41 \\
\hline $\mathbf{1 0}$ & 2.58 & 0.63 \\
\hline $\mathbf{1 1}$ & 2.38 & 0.55 \\
\hline $\mathbf{1 2}$ & 2.25 & 0.59 \\
\hline $\mathbf{1 3}$ & 2.22 & 0.42 \\
\hline $\mathbf{1 5}$ & 2.41 & 0.72 \\
\hline $\mathbf{1 7}$ & 2.05 & 0.47 \\
\hline $\mathbf{1 8}$ & 2.21 & 0.55 \\
\hline $\mathbf{1 9}$ & 2.13 & 0.54 \\
\hline $\mathbf{2 0}$ & 2.40 & 0.79 \\
\hline $\mathbf{2 1}$ & 2.12 & 0.59 \\
\hline $\mathbf{2 3}$ & 1.85 & 0.45 \\
\hline $\mathbf{0 v e r a l l}$ & 2.21 & 0.59 \\
\hline & & \\
\hline
\end{tabular}

The data was transformed by taking the square root of the fiber diameters in order to obtain a Normal distribution of the data. These analyses are only accurate if the data are normally distributed. The results of the REML variance components analysis are shown in Table M II. The JMP output from this analysis is shown in Figure M 1 on the following page. The variability between scaffolds increased slightly from $14.211 \%$ to $15.688 \%$ not including outliers. Thus, although the distinctly large fibers were eliminated from this data set, there was still variability between scaffolds. Not including the large fibers actually increased the scaffold-to-scaffold variability. 
Table M II: Results of the REML variance components analysis for random effects with no outliers.

\begin{tabular}{|l|c|c|c|}
\hline & \multicolumn{3}{|c|}{ Variability (\%) } \\
\hline Protocol & Within Scaffold & Between Images & Between Scaffolds \\
\hline Standard & 83.580 & 0.732 & 15.688 \\
\hline
\end{tabular}

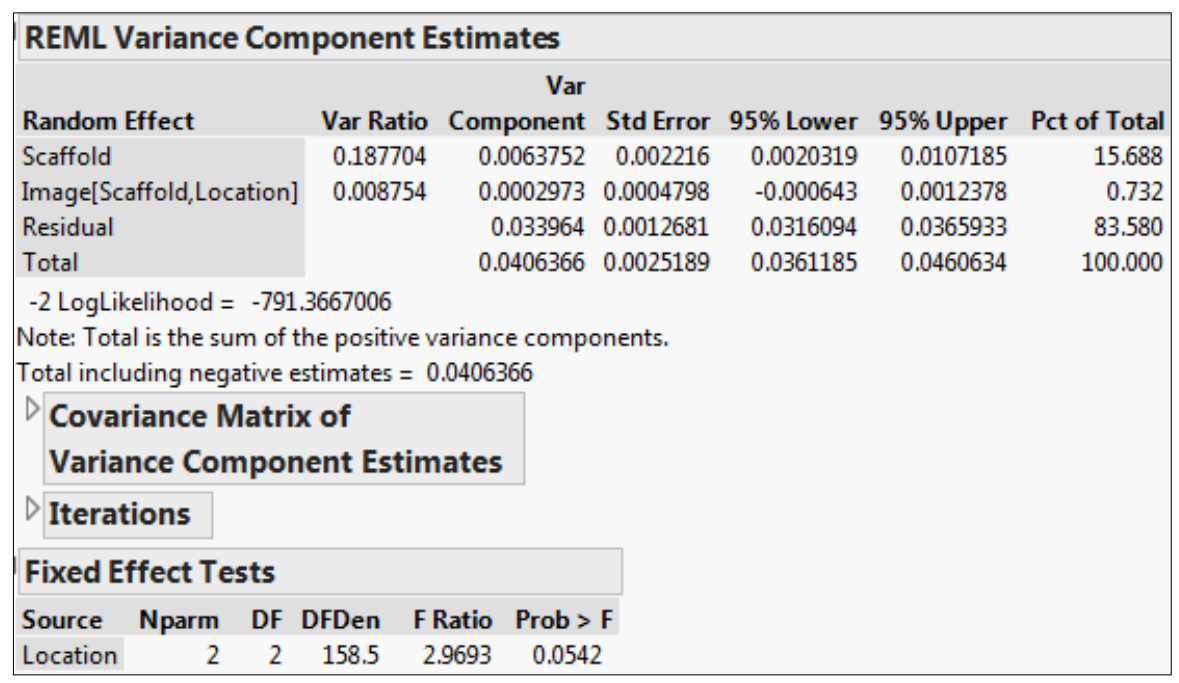

Figure M 1: JMP output of the REML variance components analysis for fiber diameter excluding outliers.

The ANOVA results are shown in Figure $M 2$ below. The p-value was $<0.0001$, so the mean fiber diameter was inconsistent among scaffolds. However, the F Ratio in this analysis was 16.0836 while the F Ratio including outliers was 14.4277. A higher F Ratio indicates more significant difference between means. This corresponds to the results from the variance components analysis above: there was more inconsistency among scaffolds when outliers were not included. 


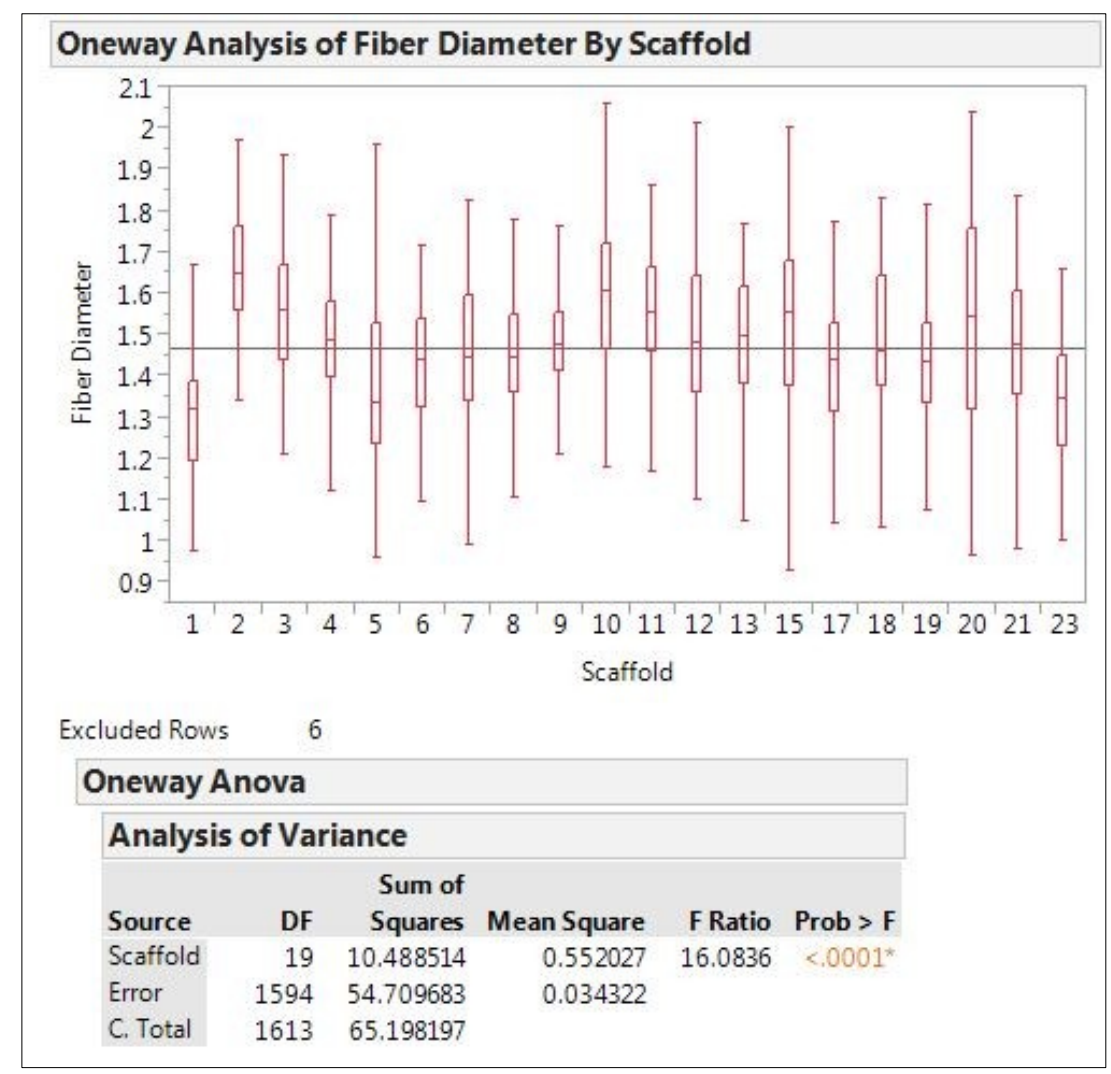

Figure M 2: Boxplot and JMP output of ANOVA for fiber diameter with no outliers.

Finally, Figure M 3 and Figure M 4 below show the results for Tukey's HSD post-hoc test. The graph in Figure M 3 shows the overlapping circles that correspond to similar means. There was less overlap in this analysis compared to the graph obtained in Chapter 3 including outliers; there was therefore more significantly different mean fiber diameters across scaffolds when outliers were not included. Additionally, the Connecting Letters Report shown in Figure M 4 also shows less overlap. Additionally, more letters were used to encompass the scaffolds (A-J), indicating that there were more significantly different groups of scaffolds in this analysis. 


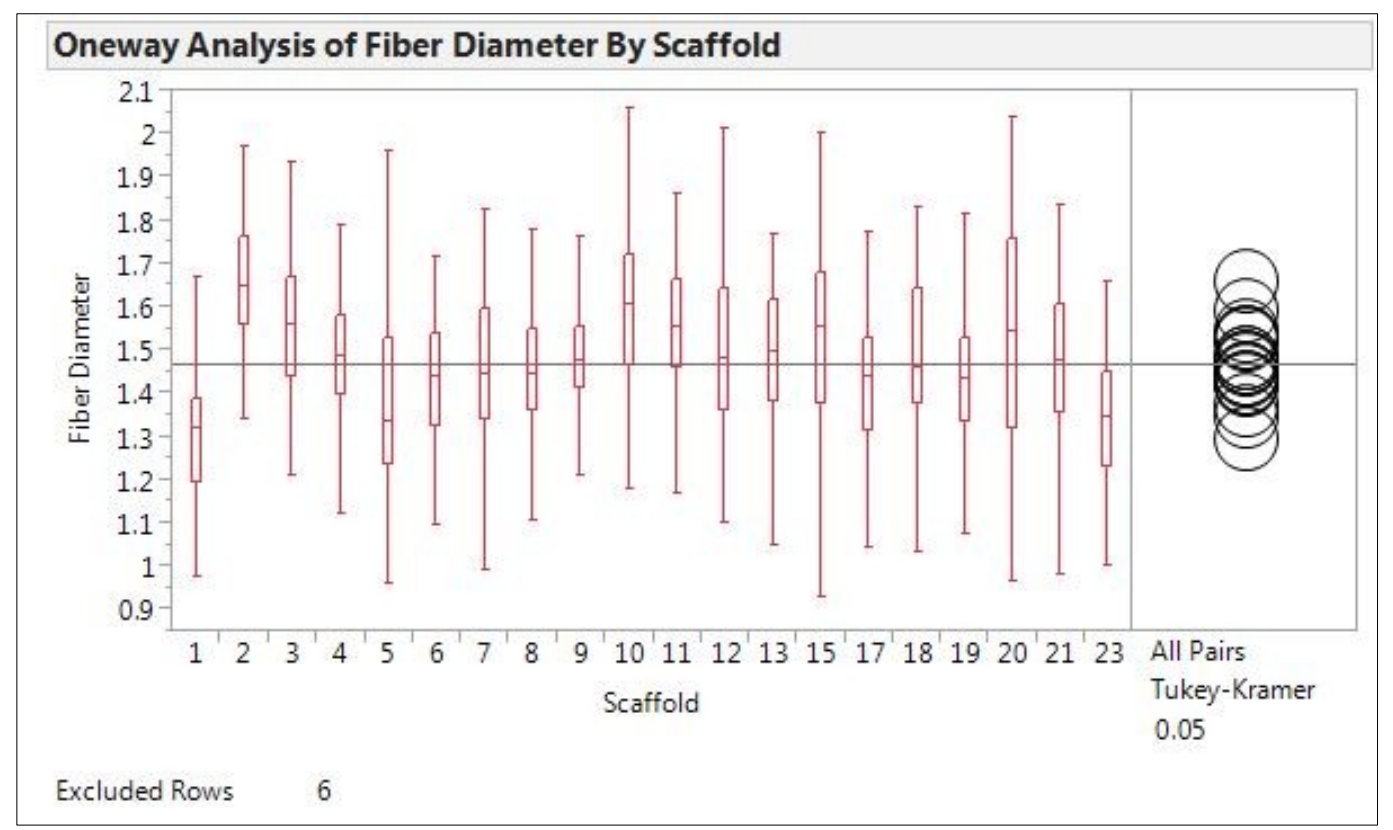

Figure M 3: Graphical results of Tukey's HSD post-hoc test for fiber diameter with no outliers.

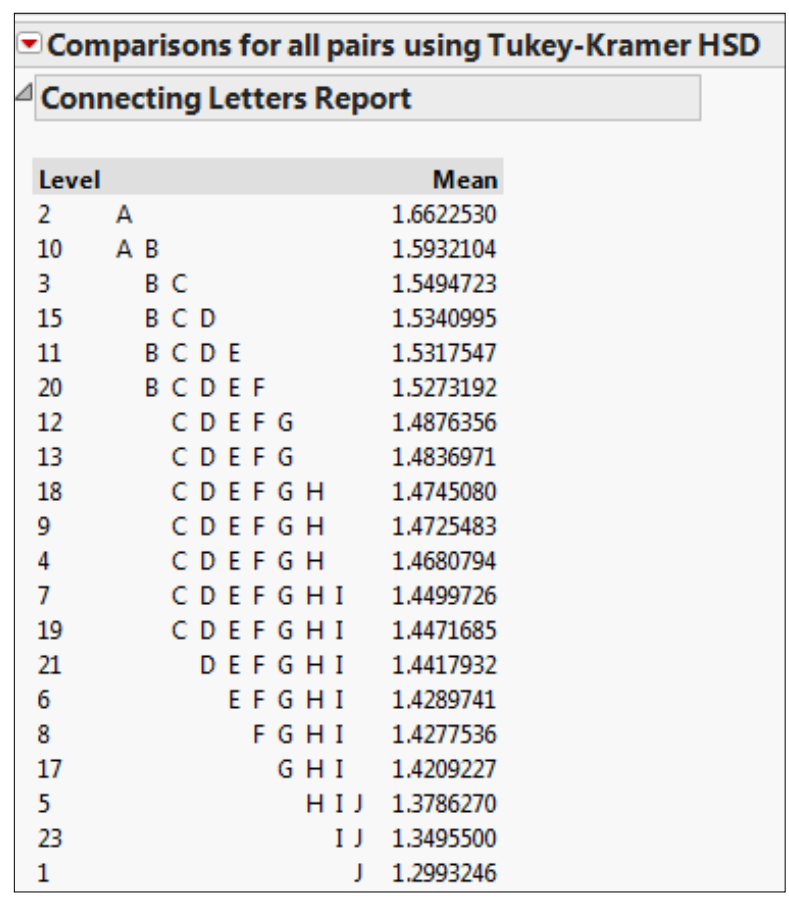

Figure M 4: Connecting Letters Report from Tukey's HSD test for fiber diameter. Levels not connected by the same letter are significantly different. 


\section{APPENDIX N: CHAPTER III SEM AND FIBER DIAMETER RESULTS}

\section{Spin 1}

Process Observations: The initial bead fell as the voltage was first applied and was replaced by the Taylor cone. Initially, the cone was stable and maintained a good shape. However, after about $0.20 \mathrm{~mL}$ of solution was ejected, it started to elongate slowly. It fell and was replaced by another cone. This occurred repeatedly for the rest of the spin.

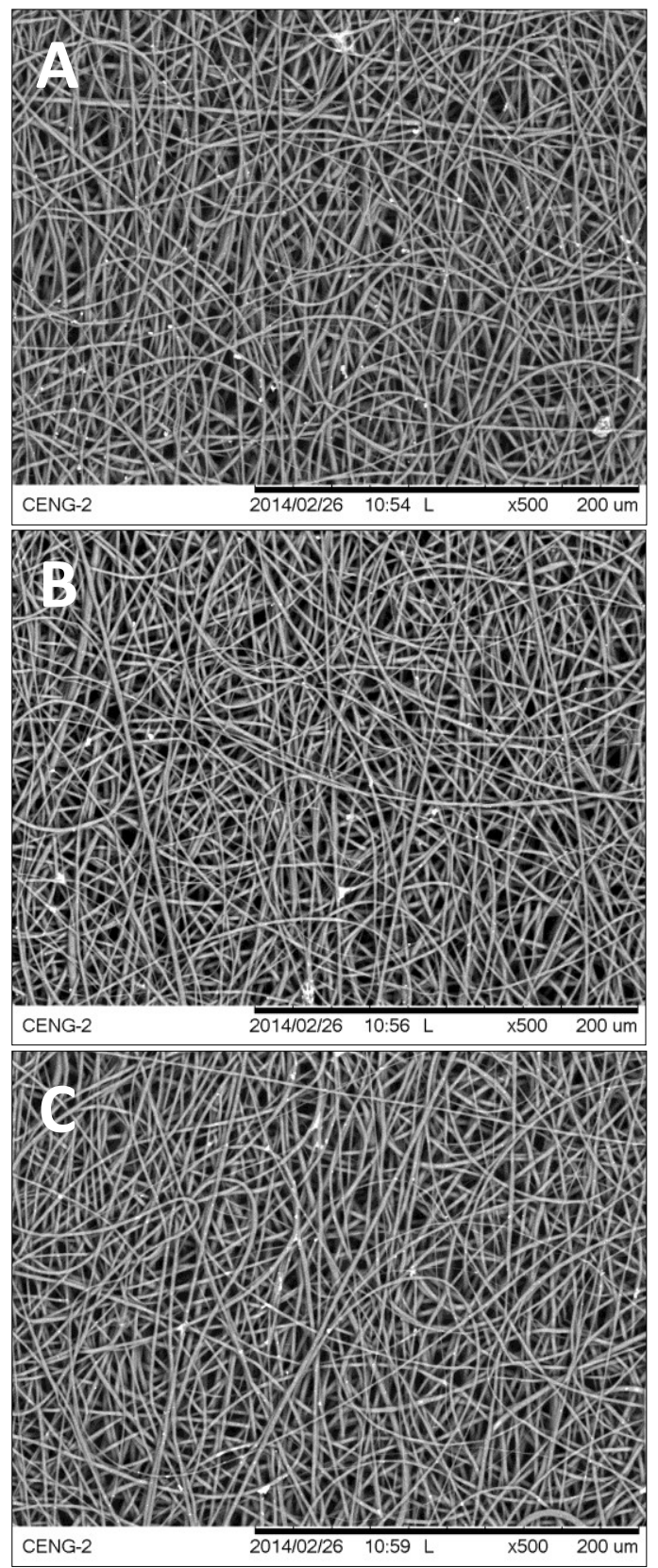

\begin{tabular}{|c|}
\hline Fiber Diameter $(\boldsymbol{\mu m})$ \\
\hline 1.9359 \\
\hline 1.6005 \\
\hline 1.8275 \\
\hline 1.6133 \\
\hline 1.887 \\
\hline 2.1372 \\
\hline 1.5511 \\
\hline 1.8474 \\
\hline 1.4627 \\
\hline
\end{tabular}

\begin{tabular}{|c|}
\hline Fiber Diameter $(\boldsymbol{\mu m})$ \\
\hline 1.7401 \\
\hline 1.3794 \\
\hline 2.4639 \\
\hline 1.4627 \\
\hline 2.1579 \\
\hline 1.4803 \\
\hline 1.8252 \\
\hline 1.2906 \\
\hline 1.3552 \\
\hline
\end{tabular}

\begin{tabular}{|c|}
\hline Fiber Diameter $(\boldsymbol{\mu m})$ \\
\hline 1.3304 \\
\hline 1.8394 \\
\hline 1.7146 \\
\hline 1.253 \\
\hline 2.2054 \\
\hline 1.6528 \\
\hline 1.2791 \\
\hline 2.1372 \\
\hline 2.065 \\
\hline
\end{tabular}

Figure $N$ 1: SEM images of Spin 1 Proximal location at 500x magnification; $(A)$ Image 1; (B) Image 2; (C) Image 3. 


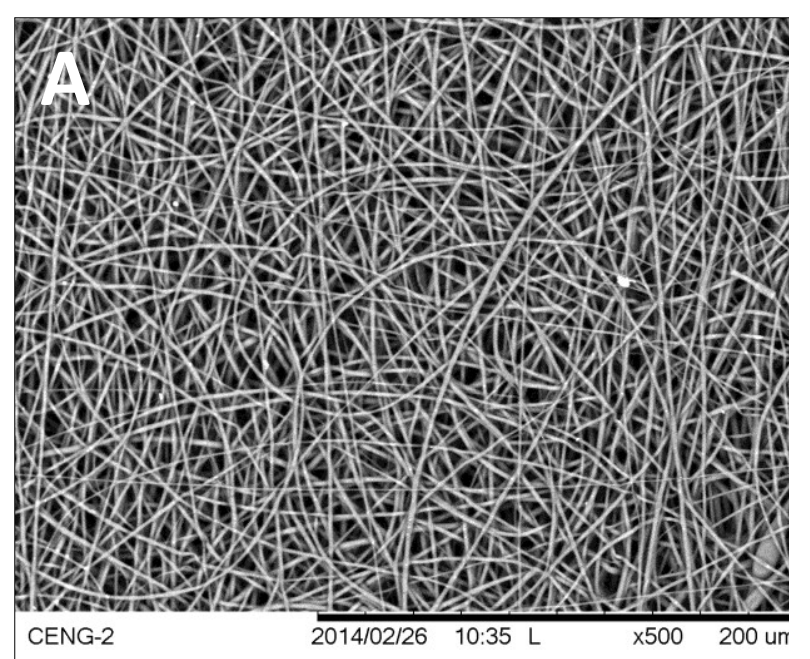

\begin{tabular}{|c|}
\hline Fiber Diameter $(\boldsymbol{\mu m})$ \\
\hline 1.887 \\
\hline 2.0579 \\
\hline 1.5027 \\
\hline 1.8479 \\
\hline 1.1776 \\
\hline 2.0722 \\
\hline 1.1417 \\
\hline 1.808 \\
\hline 1.8172 \\
\hline
\end{tabular}

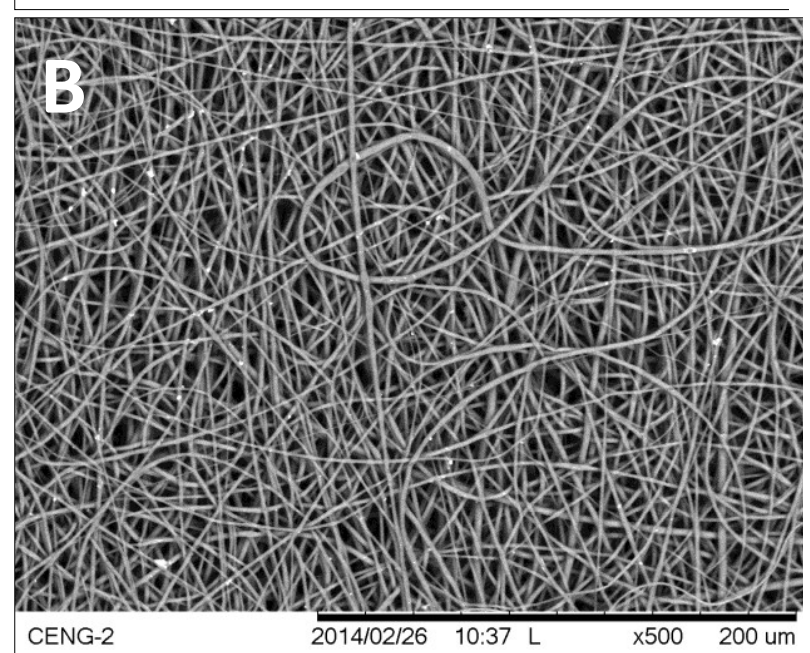

\begin{tabular}{|c|}
\hline Fiber Diameter $(\boldsymbol{\mu m})$ \\
\hline 0.9593 \\
\hline 1.8232 \\
\hline 2.3106 \\
\hline 1.4094 \\
\hline 2.3798 \\
\hline 1.8759 \\
\hline 1.443 \\
\hline 1.2906 \\
\hline 1.8 \\
\hline
\end{tabular}

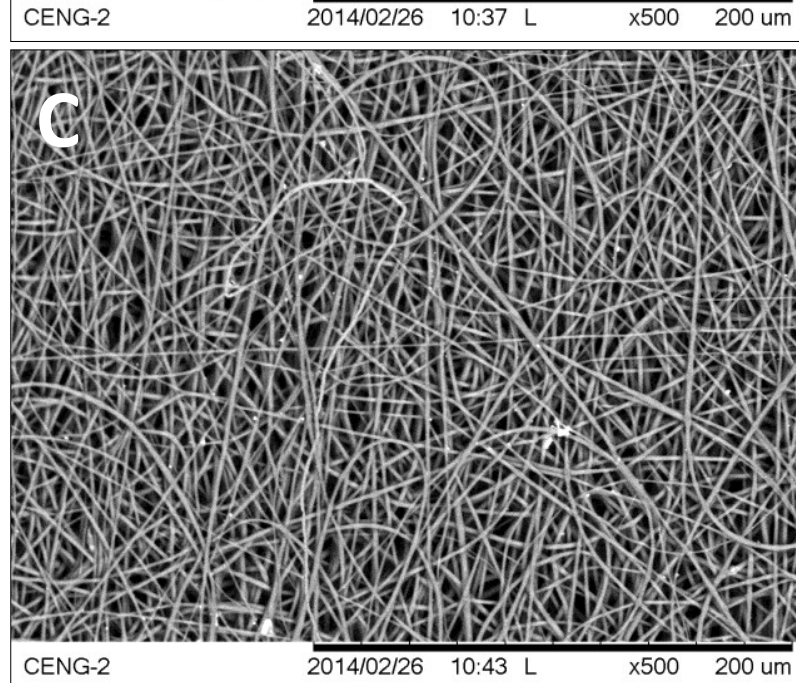

\begin{tabular}{|c|}
\hline Fiber Diameter $(\boldsymbol{\mu m})$ \\
\hline 1.5595 \\
\hline 0.9504 \\
\hline 1.1027 \\
\hline 1.5393 \\
\hline 1.9338 \\
\hline 1.7376 \\
\hline 1.911 \\
\hline 1.4255 \\
\hline 1.0989 \\
\hline
\end{tabular}

Figure N 2: SEM images of Spin 1 Medial location at 500x magnification; (A) Image 1; (B) Image 2; (C) Image 3. 


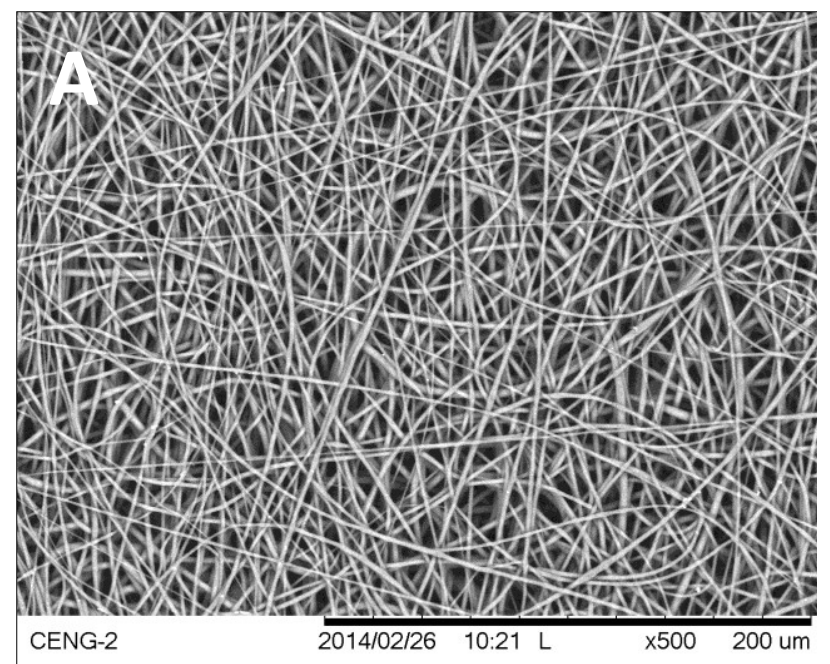

\begin{tabular}{|c|}
\hline Fiber Diameter $(\boldsymbol{\mu m})$ \\
\hline 1.4602 \\
\hline 1.2906 \\
\hline 1.5501 \\
\hline 2.101 \\
\hline 2.0098 \\
\hline 1.8264 \\
\hline 1.937 \\
\hline 1.3856 \\
\hline 1.7376 \\
\hline
\end{tabular}

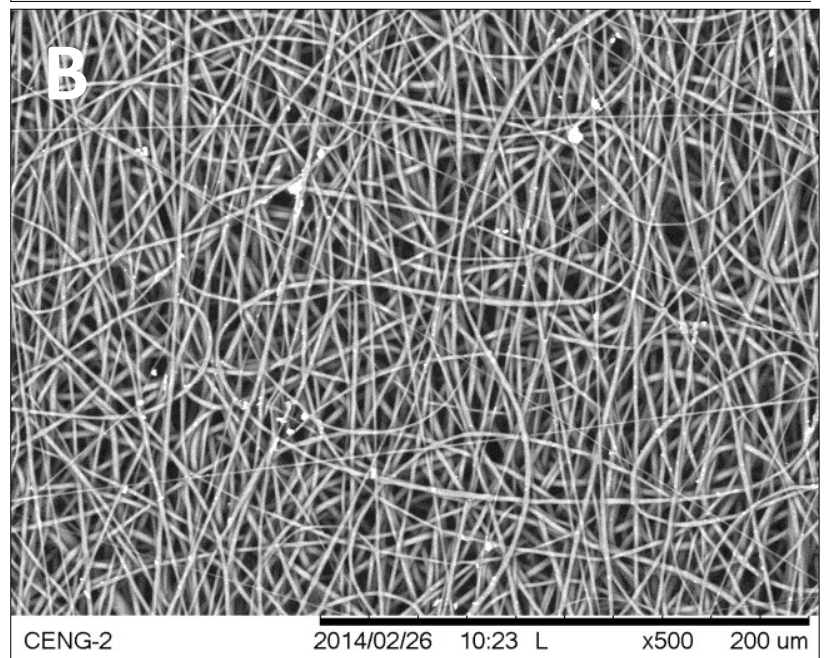

\begin{tabular}{|c|}
\hline Fiber Diameter $(\boldsymbol{\mu m})$ \\
\hline 2.1538 \\
\hline 2.5134 \\
\hline 2.0046 \\
\hline 2.4573 \\
\hline 1.7976 \\
\hline 2.7801 \\
\hline 1.7849 \\
\hline 1.3241 \\
\hline 1.5714 \\
\hline
\end{tabular}

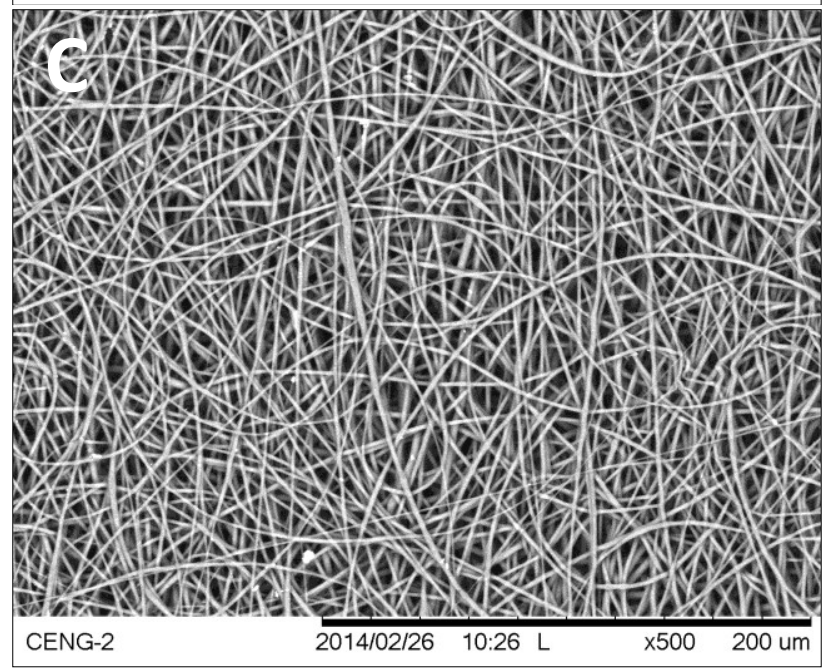

\begin{tabular}{|c|}
\hline Fiber Diameter $(\boldsymbol{\mu m})$ \\
\hline 1.8355 \\
\hline 1.7614 \\
\hline 1.8252 \\
\hline 1.2728 \\
\hline 1.6172 \\
\hline 1.4255 \\
\hline 1.2329 \\
\hline 1.5298 \\
\hline 1.8479 \\
\hline
\end{tabular}

Figure $N$ 3: SEM images of Spin 1 Distal location at 500x magnification; $(A)$ Image 1; (B) Image 2; (C) Image 3. 


\section{Spin 2}

Process Observations: The initial bead fell off of the needle when the voltage was applied. The Taylor cone formed in its place. It maintained a good shape, but eventually began to elongate slowly and fall repeatedly. The elongation lasted for about $0.03 \mathrm{~mL}$ of ejected solution.

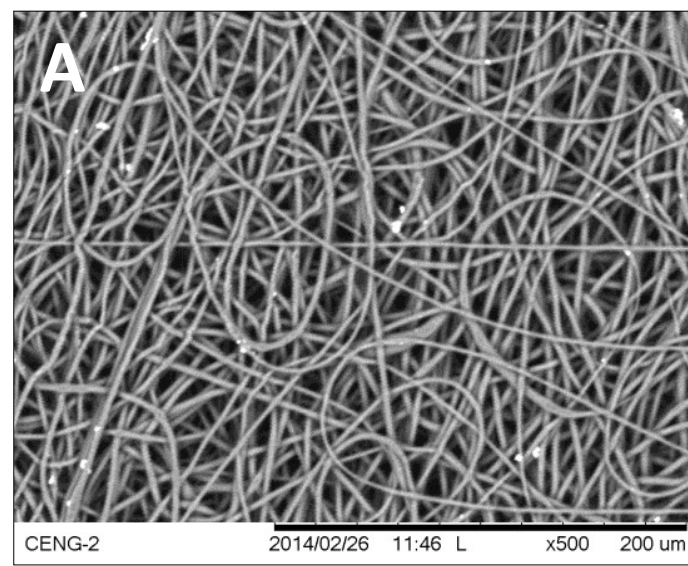

\begin{tabular}{|c|}
\hline Fiber Diameter $(\boldsymbol{\mu m})$ \\
\hline 2.6607 \\
\hline 2.7348 \\
\hline 2.5813 \\
\hline 2.7134 \\
\hline 1.5051 \\
\hline 1.7976 \\
\hline 2.4657 \\
\hline 2.8511 \\
\hline 2.9797 \\
\hline
\end{tabular}

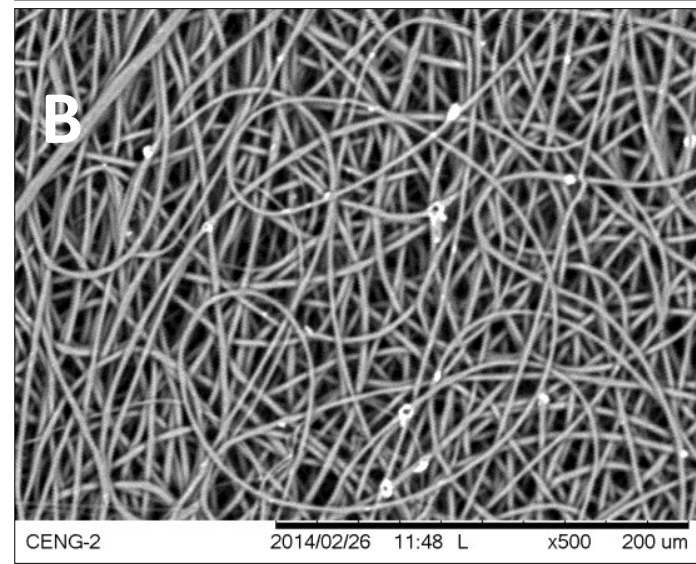

\begin{tabular}{|c|}
\hline Fiber Diameter $(\boldsymbol{\mu m})$ \\
\hline 2.5357 \\
\hline 3.6958 \\
\hline 3.013 \\
\hline 2.3798 \\
\hline 3.4631 \\
\hline 2.0811 \\
\hline 3.2266 \\
\hline 2.4111 \\
\hline 3.1402 \\
\hline
\end{tabular}

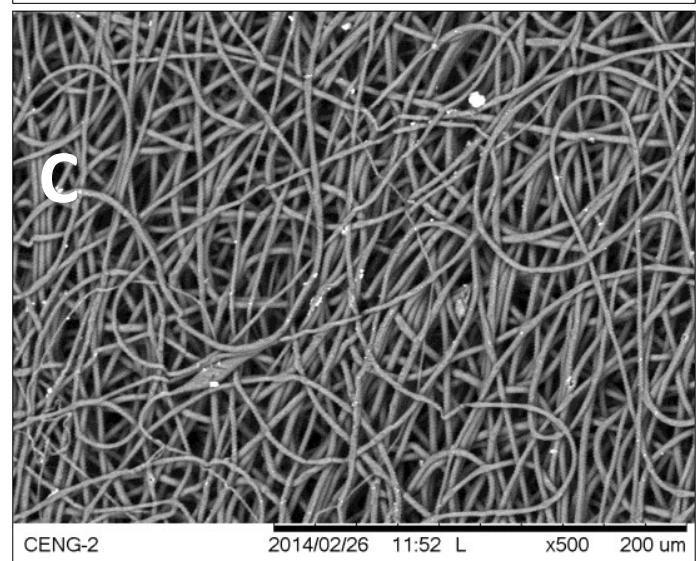

\begin{tabular}{|c|}
\hline Fiber Diameter $(\boldsymbol{\mu m})$ \\
\hline 2.587 \\
\hline 2.3267 \\
\hline 2.148 \\
\hline 2.3088 \\
\hline 2.8569 \\
\hline 3.1083 \\
\hline 2.1979 \\
\hline 2.0851 \\
\hline 2.5845 \\
\hline
\end{tabular}

Figure N 4: SEM images of Spin 2 Proximal location at 500x magnification; (A) Image 1; (B) Image 2; (C) Image 3. 


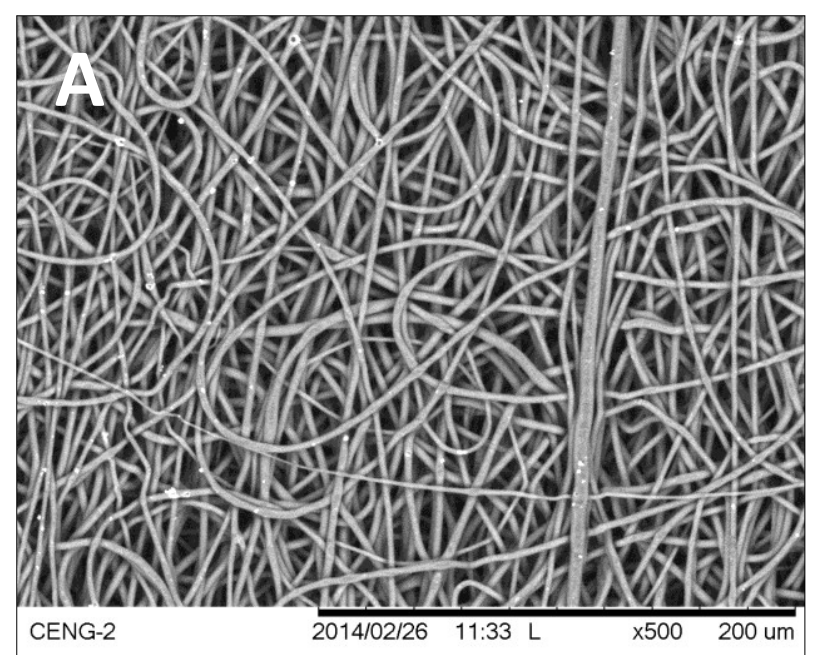

\begin{tabular}{|c|}
\hline Fiber Diameter $(\boldsymbol{\mu m})$ \\
\hline 3.1083 \\
\hline 2.2054 \\
\hline 2.287 \\
\hline 2.9936 \\
\hline 3.024 \\
\hline 2.7591 \\
\hline 2.2092 \\
\hline 3.0975 \\
\hline 3.1745 \\
\hline
\end{tabular}

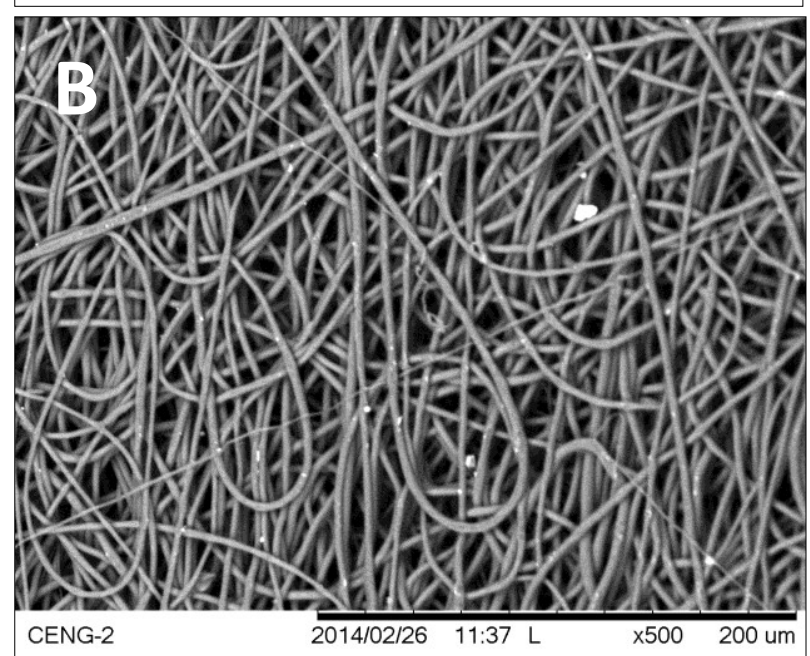

\begin{tabular}{|c|}
\hline Fiber Diameter $(\boldsymbol{\mu m})$ \\
\hline 2.8335 \\
\hline 2.3267 \\
\hline 2.7134 \\
\hline 2.4245 \\
\hline 2.2437 \\
\hline 2.4249 \\
\hline 3.0213 \\
\hline 2.3374 \\
\hline 2.4522 \\
\hline
\end{tabular}

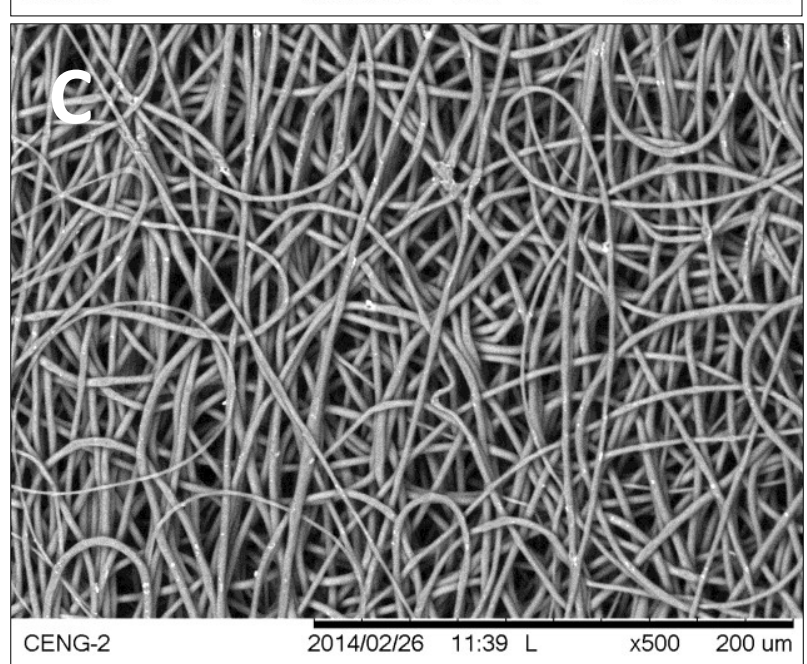

\begin{tabular}{|c|}
\hline Fiber Diameter $(\boldsymbol{\mu m})$ \\
\hline 3.5227 \\
\hline 3.0213 \\
\hline 3.6344 \\
\hline 2.4926 \\
\hline 2.6607 \\
\hline 2.8188 \\
\hline 3.6344 \\
\hline 2.2634 \\
\hline 3.4727 \\
\hline
\end{tabular}

Figure N 5: SEM images of Spin 2 Medial location at 500x magnification; (A) Image 1; (B) Image 2; (C) Image 3. 


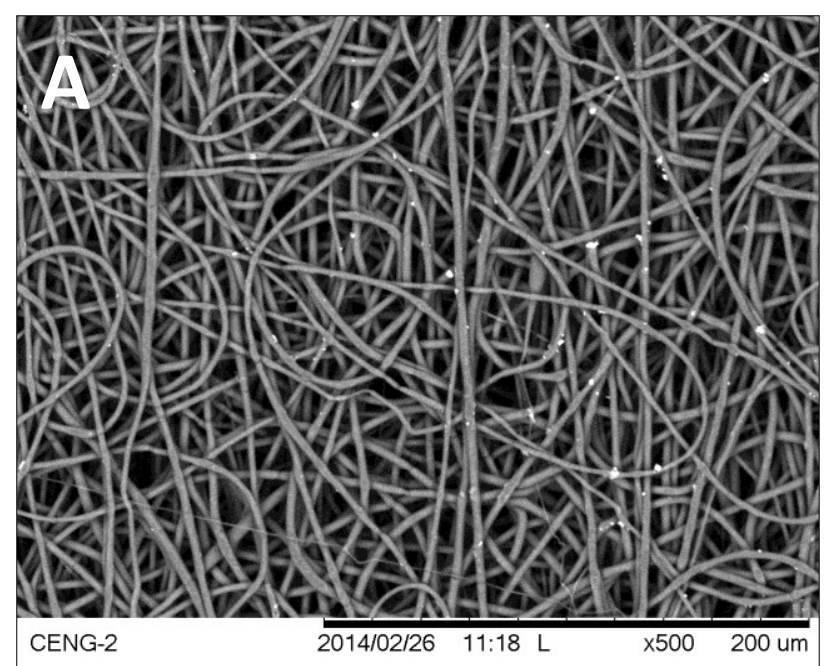

\begin{tabular}{|c|}
\hline Fiber Diameter $(\boldsymbol{\mu m})$ \\
\hline 2.8423 \\
\hline 3.8805 \\
\hline 3.5227 \\
\hline 2.4367 \\
\hline 3.0596 \\
\hline 3.0678 \\
\hline 3.3567 \\
\hline 2.0811 \\
\hline 3.5476 \\
\hline
\end{tabular}

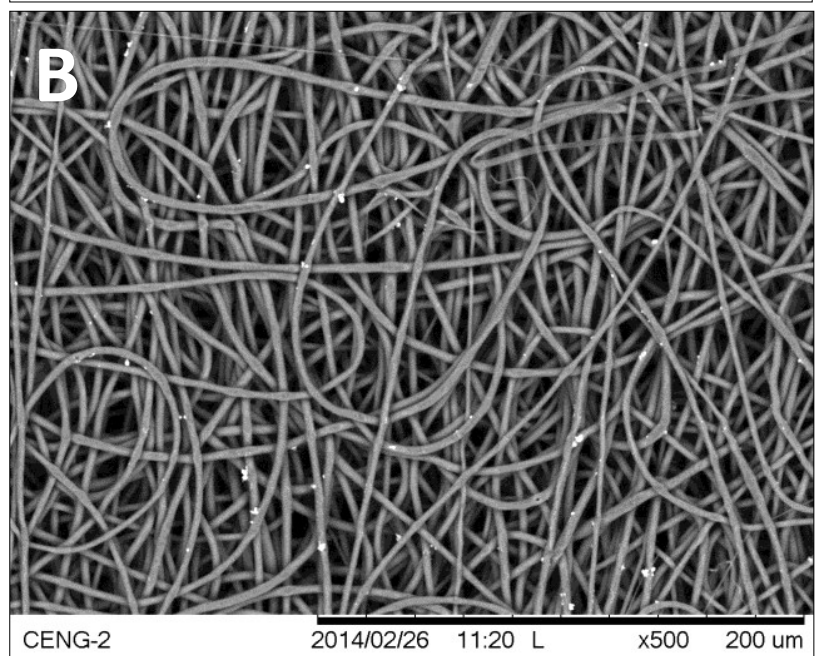

\begin{tabular}{|c|}
\hline Fiber Diameter $(\boldsymbol{\mu m})$ \\
\hline 2.4826 \\
\hline 3.0213 \\
\hline 3.3482 \\
\hline 3.6636 \\
\hline 2.506 \\
\hline 2.4792 \\
\hline 3.2651 \\
\hline 3.6344 \\
\hline 2.5813 \\
\hline
\end{tabular}

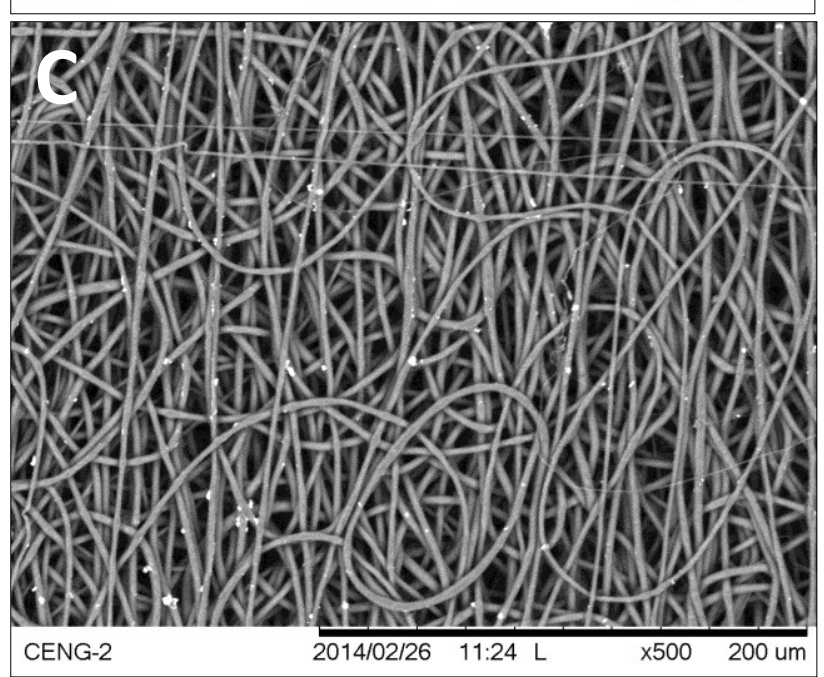

\begin{tabular}{|c|}
\hline Fiber Diameter $(\boldsymbol{\mu m})$ \\
\hline 2.5455 \\
\hline 2.6894 \\
\hline 3.3081 \\
\hline 3.1083 \\
\hline 3.6981 \\
\hline 2.5813 \\
\hline 2.6101 \\
\hline 1.8115 \\
\hline 2.9797 \\
\hline
\end{tabular}

Figure $N$ 6: SEM images of Spin 2 Distal location at 500x magnification; $(A)$ Image 1; (B) Image 2; (C) Image 3. 


\section{Spin 3}

Process Observations: The initial bead fell when the voltage was applied and was immediately replaced by a small, stable Taylor cone. The cone was more stable than previous spins and did not begin elongating until about $0.30 \mathrm{~mL}$ of solution was ejected. It elongated and fell repeatedly throughout the process, but occurred less often and elongated to a much shorter length than previous spins. However, the solution began dripping from the bottom of the cone after $2.50 \mathrm{~mL}$ of solution was ejected.

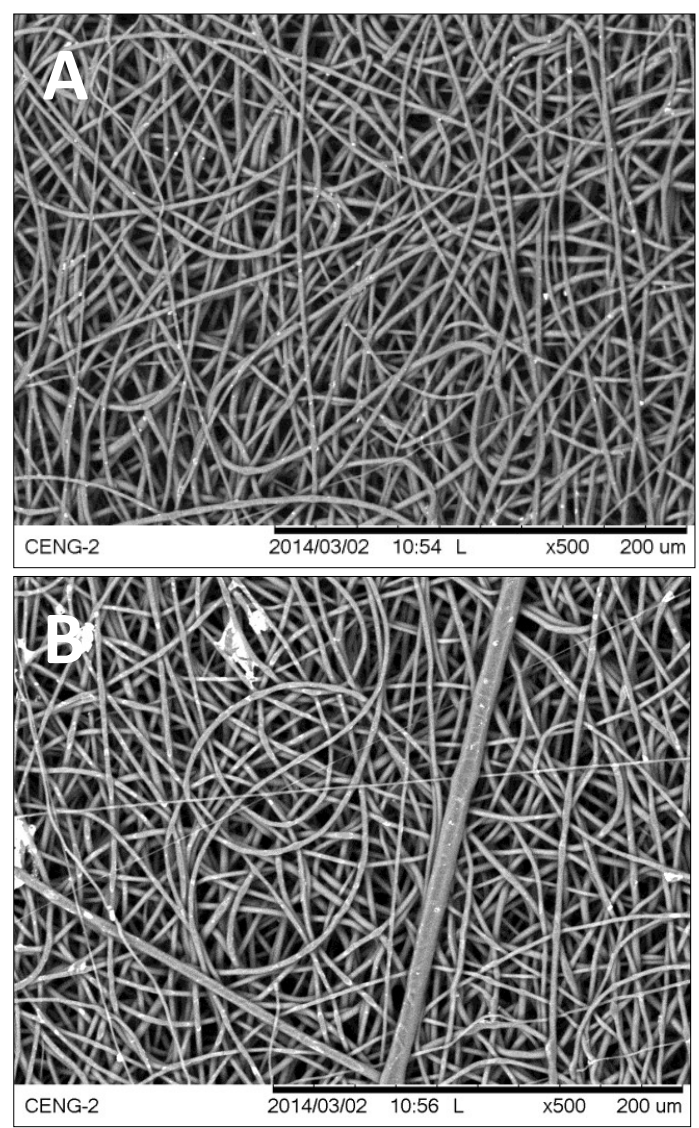

\begin{tabular}{|c|}
\hline Fiber Diameter $(\boldsymbol{\mu m})$ \\
\hline 2.4398 \\
\hline 2.5833 \\
\hline 2.0491 \\
\hline 2.3546 \\
\hline 1.6035 \\
\hline 2.0851 \\
\hline 2.5 \\
\hline 3.1046 \\
\hline 2.6821 \\
\hline
\end{tabular}

\begin{tabular}{|c|}
\hline Fiber Diameter $(\boldsymbol{\mu m})$ \\
\hline 2.3906 \\
\hline 2.8239 \\
\hline 2.5504 \\
\hline 2.2563 \\
\hline 2.7586 \\
\hline 3.7428 \\
\hline 2.6318 \\
\hline 2.4276 \\
\hline 1.9544 \\
\hline
\end{tabular}

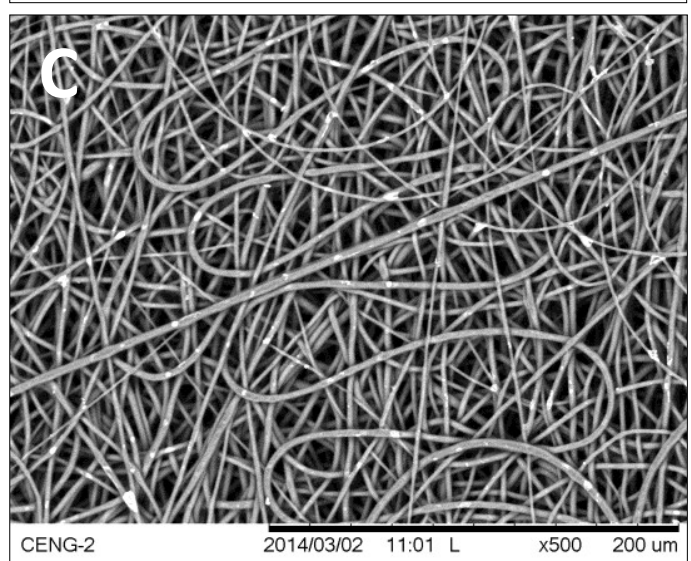

\begin{tabular}{|c|}
\hline Fiber Diameter $(\boldsymbol{\mu m})$ \\
\hline 2.85 \\
\hline 2.8186 \\
\hline 2.6091 \\
\hline 2.5833 \\
\hline 3.1082 \\
\hline 2.6946 \\
\hline 2.3003 \\
\hline 1.8907 \\
\hline 2.5471 \\
\hline
\end{tabular}

Figure $N$ 7: SEM images of Spin 3 Proximal location at 500x magnification; $(A)$ Image 1; (B) Image 2; (C) Image 3. 


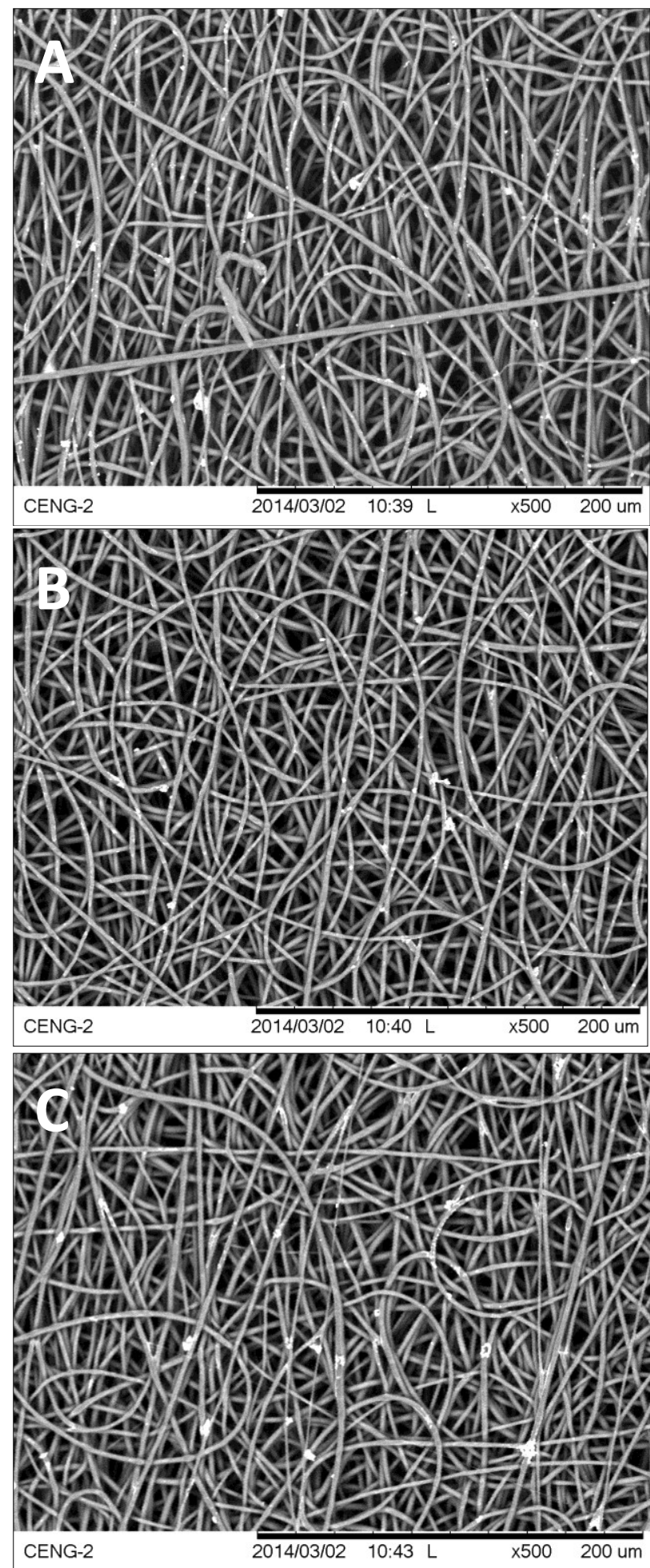

\begin{tabular}{|c|}
\hline Fiber Diameter $(\boldsymbol{\mu m})$ \\
\hline 2.7854 \\
\hline 3.0283 \\
\hline 2.4337 \\
\hline 2.7828 \\
\hline 2.2759 \\
\hline 1.8429 \\
\hline 2.2164 \\
\hline 1.5541 \\
\hline 2.678 \\
\hline
\end{tabular}

\begin{tabular}{|c|}
\hline Fiber Diameter $(\boldsymbol{\mu m})$ \\
\hline 2.5059 \\
\hline 2.0851 \\
\hline 1.8907 \\
\hline 2.4199 \\
\hline 1.9658 \\
\hline 3.0283 \\
\hline 2.0491 \\
\hline 2.4276 \\
\hline 1.9658 \\
\hline
\end{tabular}

\begin{tabular}{|c|}
\hline Fiber Diameter $(\boldsymbol{\mu m})$ \\
\hline 2.7674 \\
\hline 2.7854 \\
\hline 2.4045 \\
\hline 2.3312 \\
\hline 3.4869 \\
\hline 2.5133 \\
\hline 1.5541 \\
\hline 1.6379 \\
\hline 1.4655 \\
\hline
\end{tabular}

Figure $N$ 8: SEM images of Spin 3 Medial location at 500x magnification; $(A)$ Image 1; (B) Image 2; (C) Image 3. 


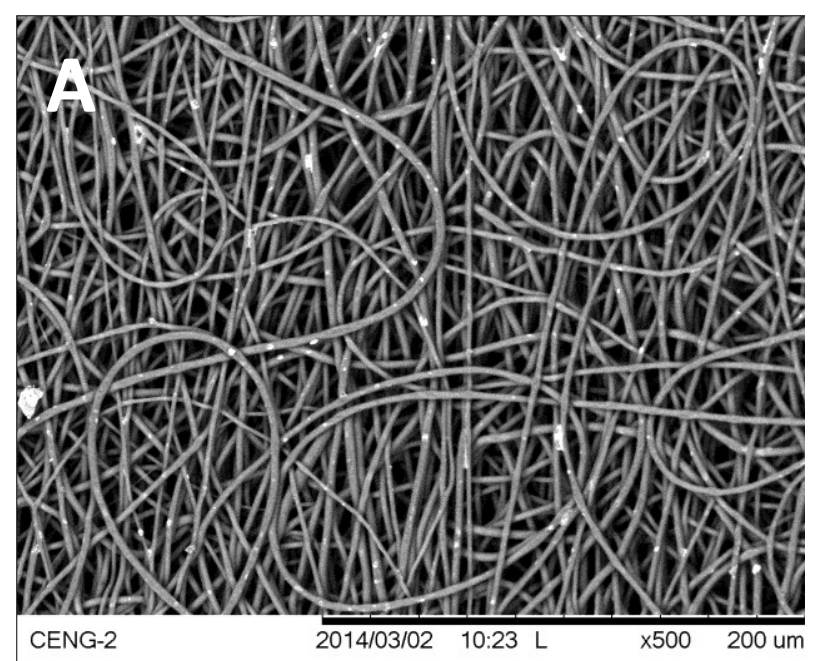

\begin{tabular}{|c|}
\hline Fiber Diameter $(\boldsymbol{\mu m})$ \\
\hline 2.3292 \\
\hline 2.2677 \\
\hline 1.647 \\
\hline 3.0806 \\
\hline 1.6937 \\
\hline 3.3896 \\
\hline 3.1082 \\
\hline 3.294 \\
\hline 2.8773 \\
\hline
\end{tabular}

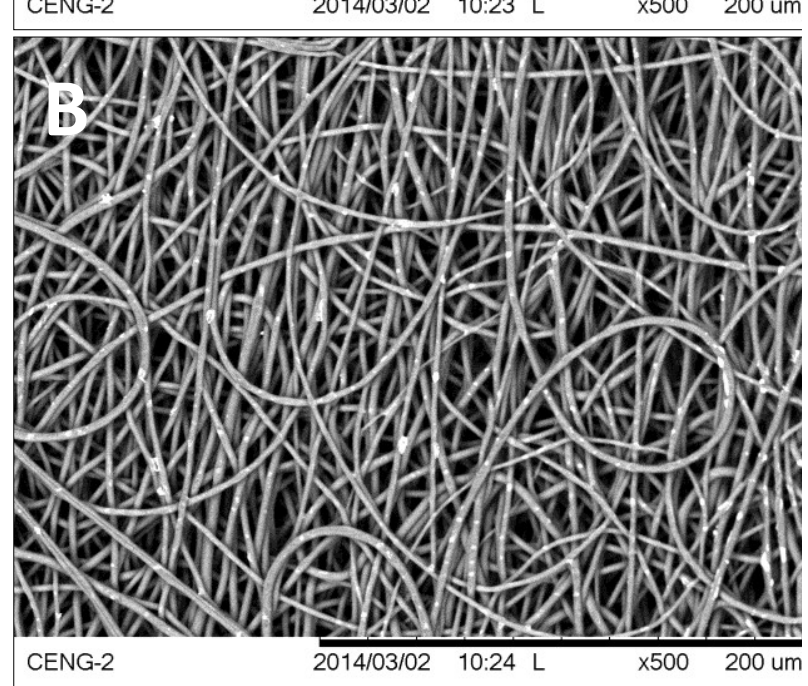

\begin{tabular}{|c|}
\hline Fiber Diameter $(\boldsymbol{\mu m})$ \\
\hline 2.7921 \\
\hline 3.1082 \\
\hline 2.243 \\
\hline 2.452 \\
\hline 2.5237 \\
\hline 1.5849 \\
\hline 2.1292 \\
\hline 1.9141 \\
\hline 2.8915 \\
\hline
\end{tabular}

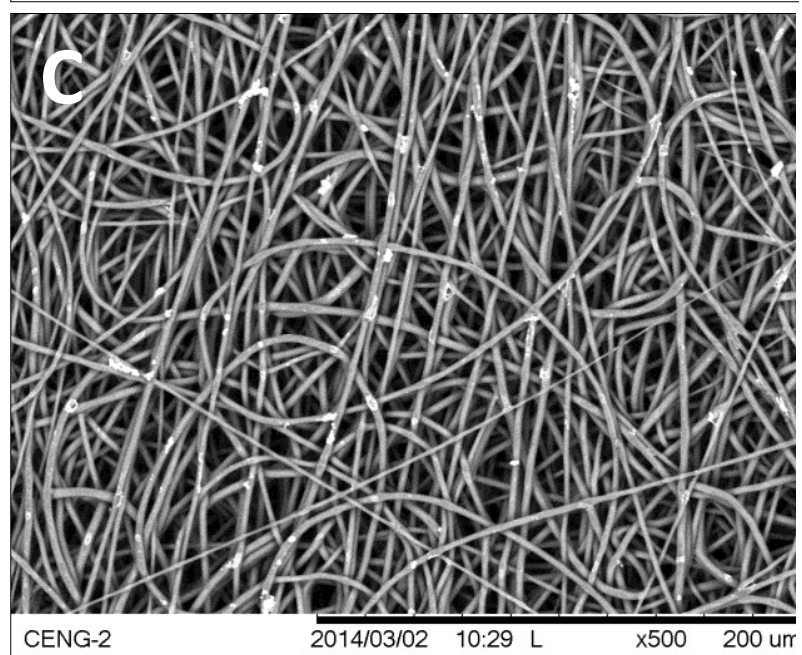

\begin{tabular}{|c|}
\hline Fiber Diameter $(\boldsymbol{\mu m})$ \\
\hline 2.2164 \\
\hline 2.1706 \\
\hline 1.6379 \\
\hline 2.8566 \\
\hline 2.8812 \\
\hline 1.6265 \\
\hline 2.1326 \\
\hline 3.0479 \\
\hline 1.5541 \\
\hline
\end{tabular}

Figure N 9: SEM images of Spin 3 Distal location at 500x magnification; $(A)$ Image 1; (B) Image 2; (C) Image 3. 


\section{Spin 4}

Process Observations: The Taylor cone formed immediately after the voltage was applied. Initially, the cone was small, stable, and exhibited an ideal shape. During the process, it began to slightly elongate and fall, which then occurred repeatedly. The elongation lasted for about $0.03 \mathrm{~mL}$ and the solution began dripping from the bottom of the cone at about $2.00 \mathrm{~mL}$.

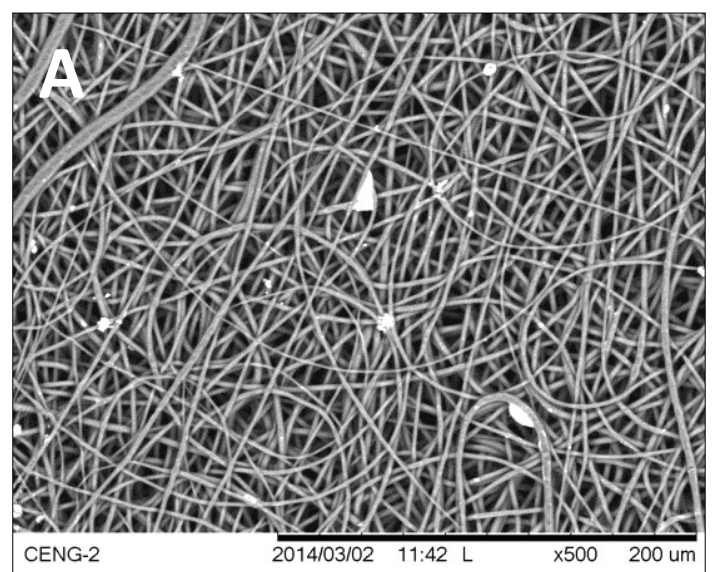

\begin{tabular}{|c|}
\hline Fiber Diameter $(\boldsymbol{\mu m})$ \\
\hline 2.5354 \\
\hline 2.1979 \\
\hline 1.2552 \\
\hline 1.39 \\
\hline 2.2776 \\
\hline 1.7688 \\
\hline 2.678 \\
\hline 2.5934 \\
\hline 1.6738 \\
\hline
\end{tabular}

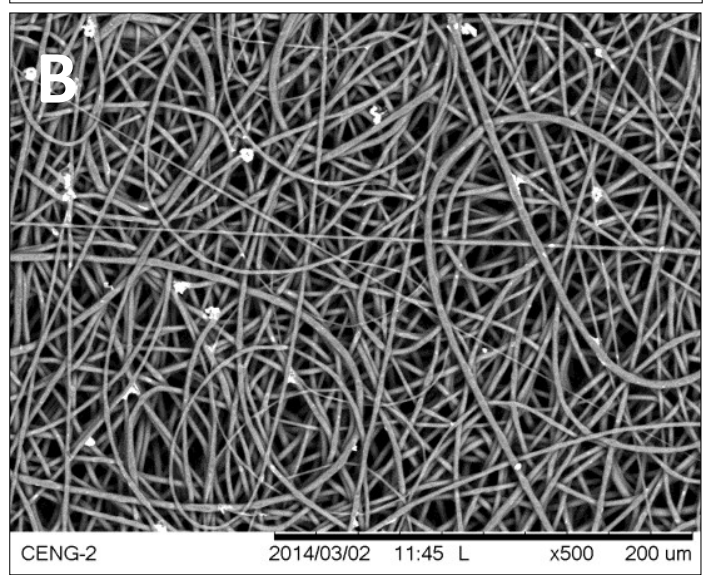

\begin{tabular}{|c|}
\hline Fiber Diameter $(\boldsymbol{\mu m})$ \\
\hline 2.0125 \\
\hline 2.5934 \\
\hline 3.2001 \\
\hline 2.2628 \\
\hline 1.1339 \\
\hline 1.2191 \\
\hline 2.4153 \\
\hline 2.1826 \\
\hline 2.5 \\
\hline
\end{tabular}

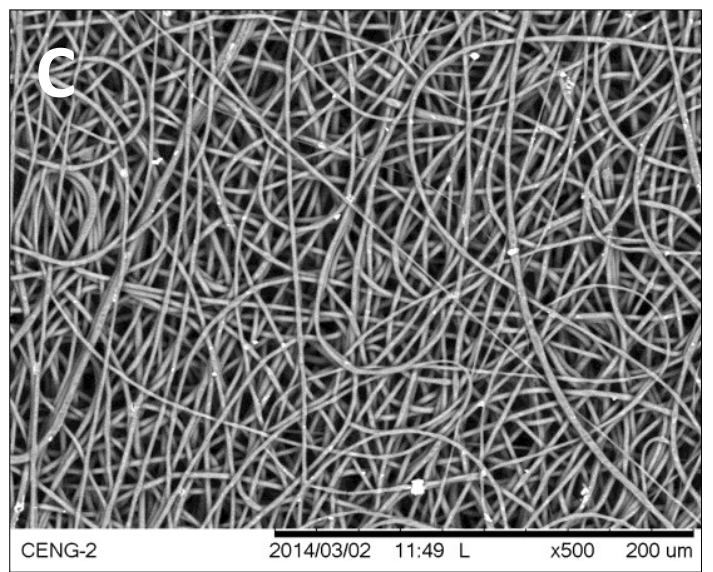

\begin{tabular}{|c|}
\hline Fiber Diameter $(\boldsymbol{\mu m})$ \\
\hline 2.5 \\
\hline 2.1979 \\
\hline 2.8915 \\
\hline 2.0125 \\
\hline 2.2414 \\
\hline 2.2029 \\
\hline 1.5517 \\
\hline 2.5457 \\
\hline 1.9846 \\
\hline
\end{tabular}

Figure N 10: SEM images of Spin 4 Proximal location at 500x magnification; (A) Image 1; (B) Image 2; (C) Image 3. 


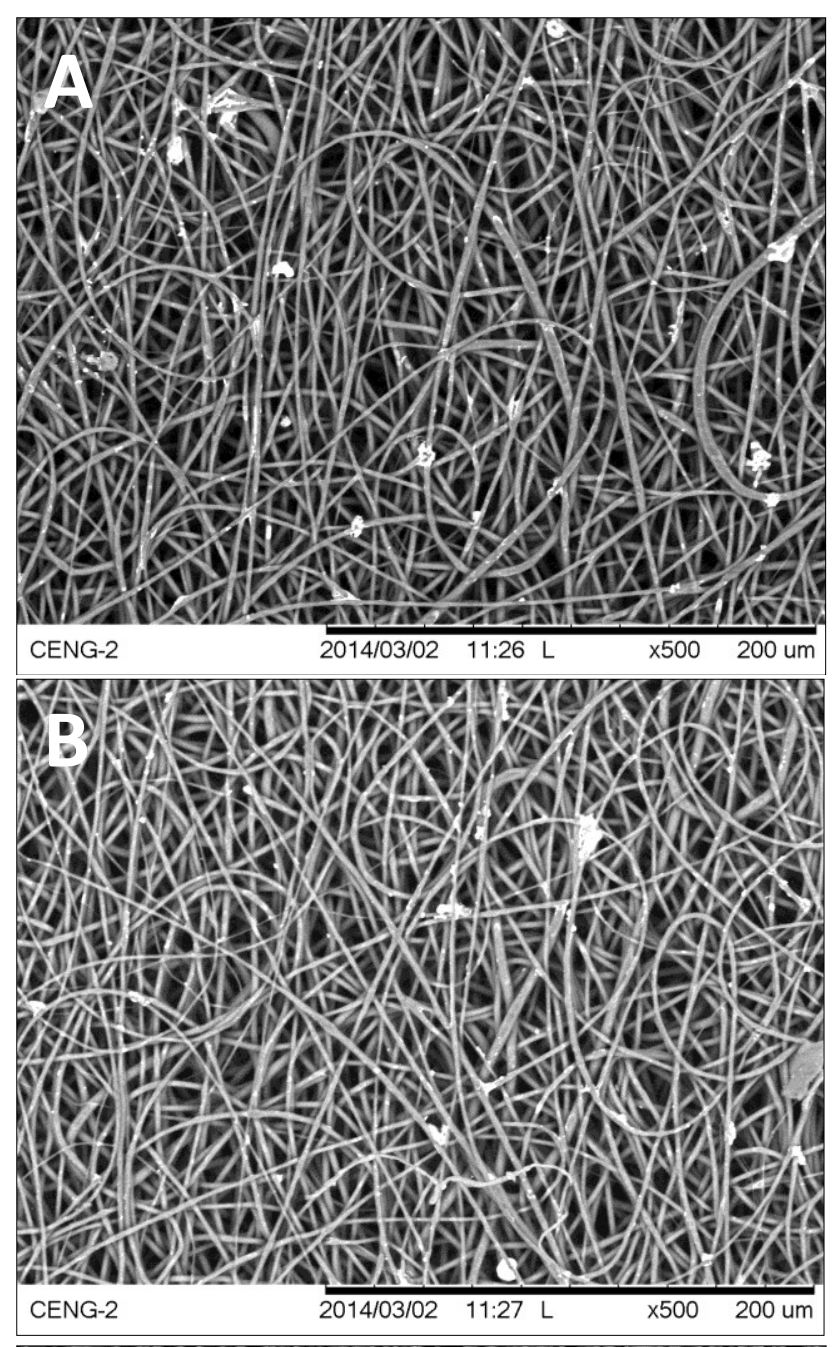

\begin{tabular}{|c|}
\hline Fiber Diameter $(\boldsymbol{\mu m})$ \\
\hline 2.6932 \\
\hline 3.0613 \\
\hline 2.4383 \\
\hline 2.0291 \\
\hline 2.2954 \\
\hline 1.2343 \\
\hline 1.1566 \\
\hline 1.9996 \\
\hline 1.9141 \\
\hline
\end{tabular}

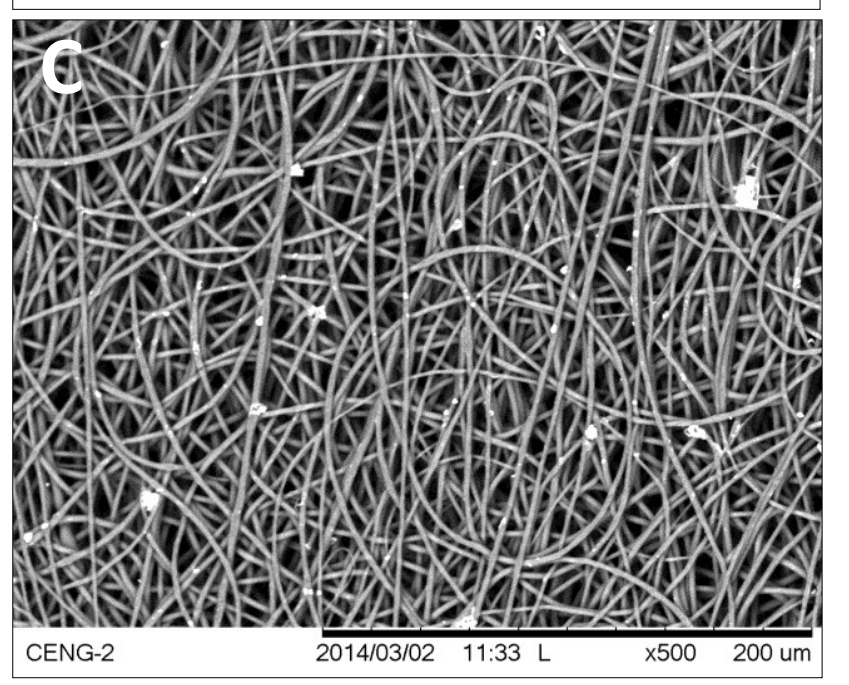

\begin{tabular}{|c|}
\hline Fiber Diameter $(\boldsymbol{\mu m})$ \\
\hline 1.7583 \\
\hline 2.0851 \\
\hline 2.6091 \\
\hline 2.1292 \\
\hline 2.7278 \\
\hline 1.7772 \\
\hline 2.0074 \\
\hline 2.353 \\
\hline 2.1826 \\
\hline
\end{tabular}

\begin{tabular}{|c|}
\hline Fiber Diameter $(\boldsymbol{\mu m})$ \\
\hline 1.9449 \\
\hline 2.3597 \\
\hline 1.9738 \\
\hline 2.2628 \\
\hline 1.9696 \\
\hline 2.7397 \\
\hline 1.4731 \\
\hline 2.3419 \\
\hline 1.2191 \\
\hline
\end{tabular}

Figure N 11: SEM images of Spin 4 Medial location at 500x magnification; (A) Image 1; (B) Image 2; (C) Image 3. 


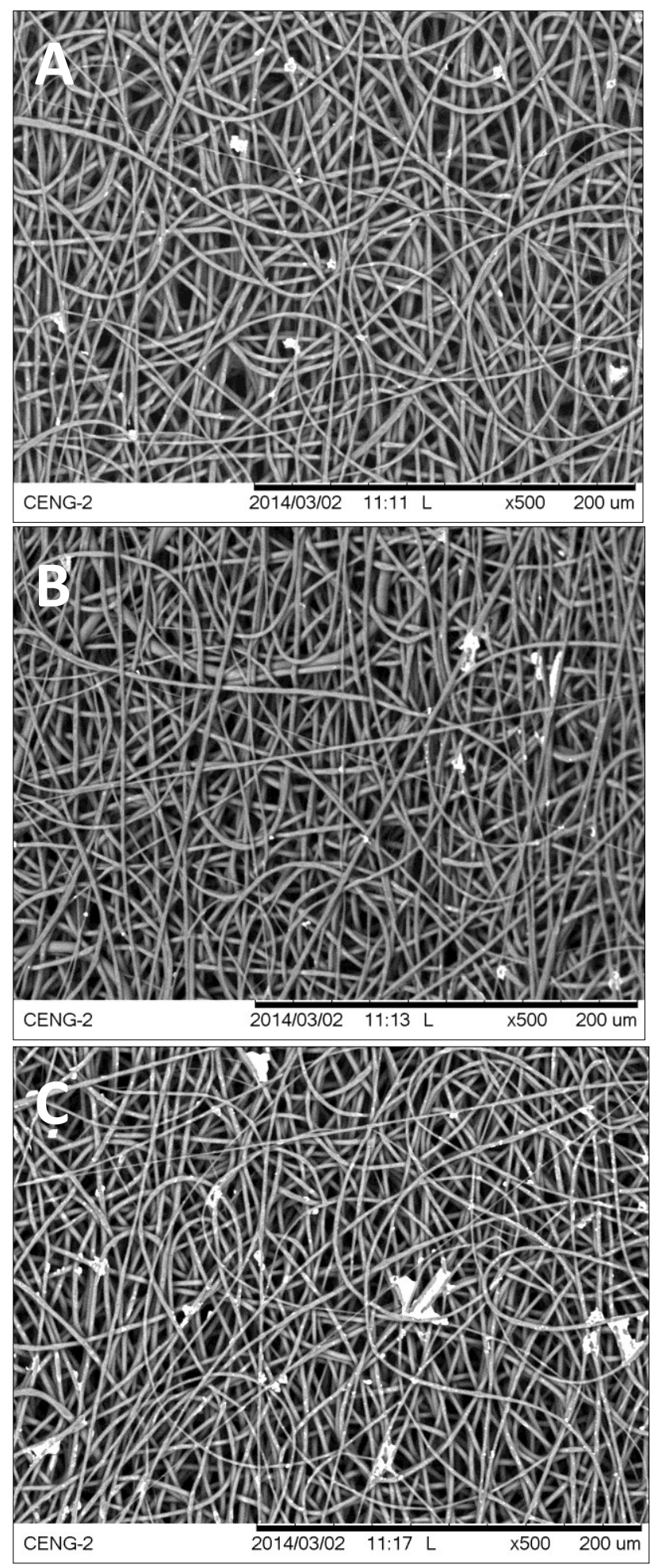

\begin{tabular}{|c|}
\hline Fiber Diameter $(\boldsymbol{\mu m})$ \\
\hline 1.9568 \\
\hline 2.1809 \\
\hline 2.2414 \\
\hline 2.2563 \\
\hline 2.3906 \\
\hline 1.6357 \\
\hline 2.8902 \\
\hline 2.1706 \\
\hline 2.76 \\
\hline
\end{tabular}

\begin{tabular}{|c|}
\hline Fiber Diameter $(\boldsymbol{\mu m})$ \\
\hline 2.334 \\
\hline 2.1552 \\
\hline 2.1706 \\
\hline 2.2134 \\
\hline 2.9323 \\
\hline 2.8708 \\
\hline 2.3419 \\
\hline 2.243 \\
\hline 1.8103 \\
\hline
\end{tabular}

\begin{tabular}{|c|}
\hline Fiber Diameter $(\boldsymbol{\mu m})$ \\
\hline 2.9059 \\
\hline 2.353 \\
\hline 2.5059 \\
\hline 2.6932 \\
\hline 2.3906 \\
\hline 1.8907 \\
\hline 1.7112 \\
\hline 2.0761 \\
\hline 1.8449 \\
\hline
\end{tabular}

Figure $N$ 12: SEM images of Spin 4 Distal location at 500x magnification; (A) Image 1; (B) Image 2; (C) Image 3. 


\section{Spin 5}

Process Observations: The initial Taylor cone formed immediately after the bead of solution fell when the voltage was applied. The cone was small and stable with an ideal shape. After about $0.35 \mathrm{~mL}$ of solution was ejected, it began to elongate slowly and fall repeatedly.

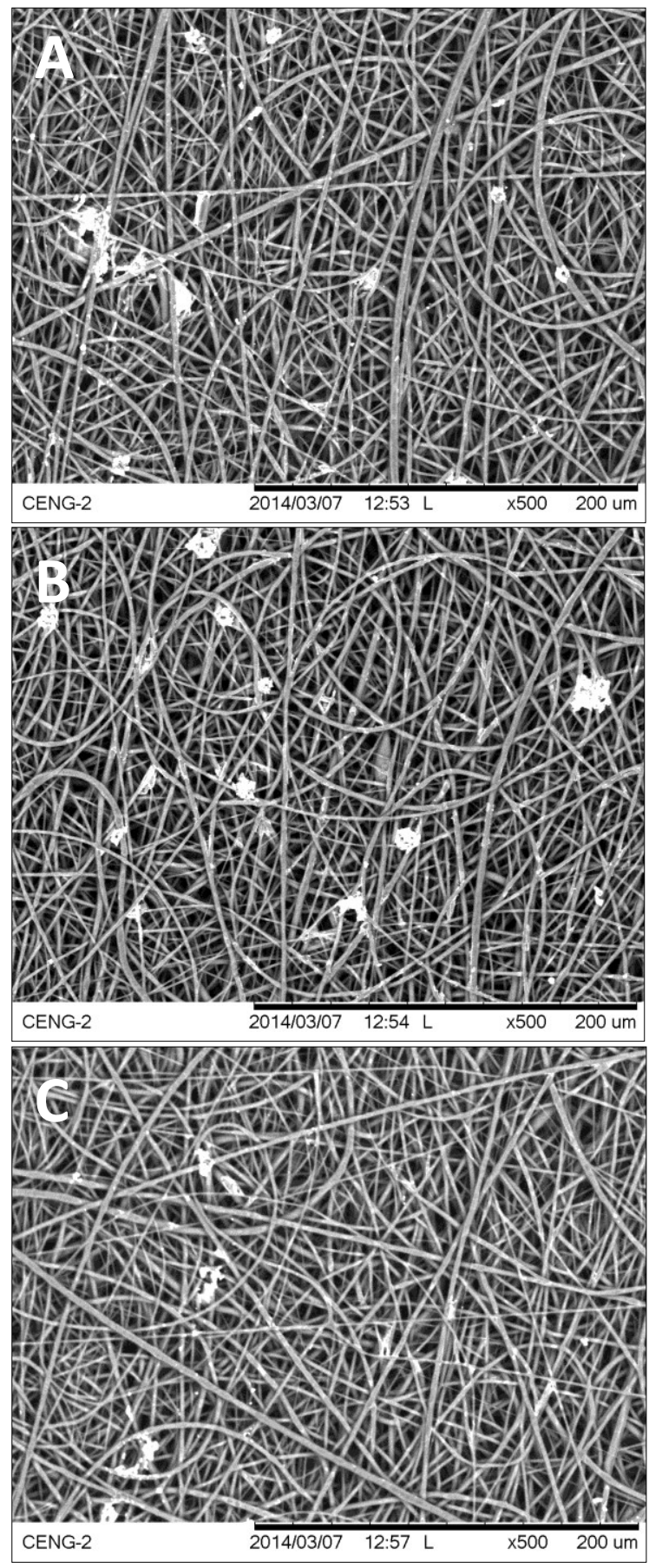

\begin{tabular}{|c|}
\hline Fiber Diameter $(\boldsymbol{\mu m})$ \\
\hline 1.508 \\
\hline 1.6649 \\
\hline 2.0564 \\
\hline 2.5934 \\
\hline 2.5237 \\
\hline 0.9164 \\
\hline 1.861 \\
\hline 1.6379 \\
\hline 1.9544 \\
\hline
\end{tabular}

\begin{tabular}{|c|}
\hline Fiber Diameter $(\boldsymbol{\mu m})$ \\
\hline 3.032 \\
\hline 1.7327 \\
\hline 3.5387 \\
\hline 1.5896 \\
\hline 1.5541 \\
\hline 1.3927 \\
\hline 3.4005 \\
\hline 1.6738 \\
\hline 1.4451 \\
\hline
\end{tabular}

\begin{tabular}{|c|}
\hline Fiber Diameter $(\boldsymbol{\mu m})$ \\
\hline 2.0491 \\
\hline 1.6981 \\
\hline 2.3292 \\
\hline 1.171 \\
\hline 1.9276 \\
\hline 3.8292 \\
\hline 2.4686 \\
\hline 2.0346 \\
\hline 1.5517 \\
\hline
\end{tabular}

Figure N 13: SEM images of Spin 5 Proximal location at 500x magnification; (A) Image 1; (B) Image 2; (C) Image 3. 


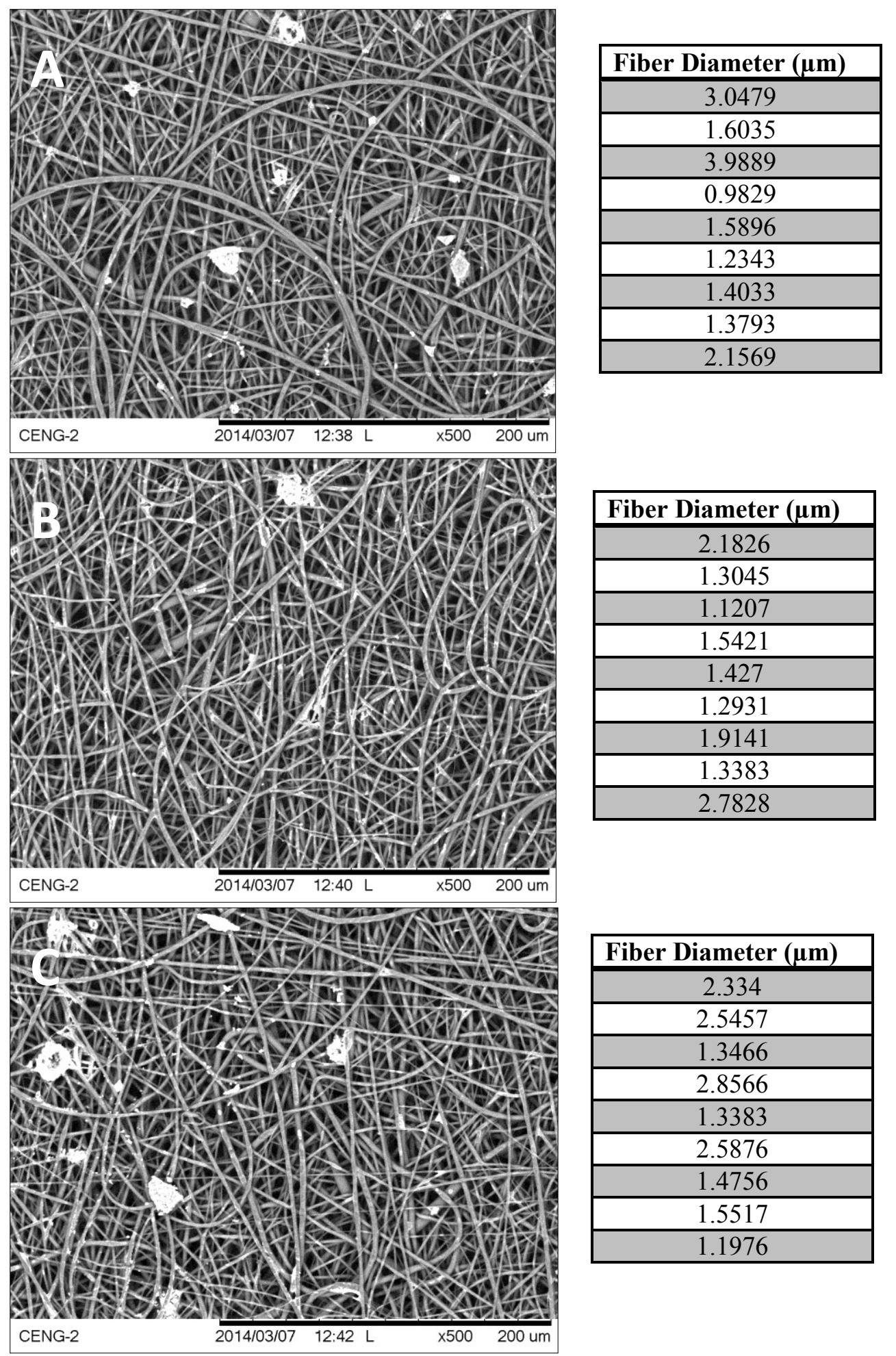

Figure N 14: SEM images of Spin 5 Medial location at 500x magnification; (A) Image 1; (B) Image 2; (C) Image 3. 


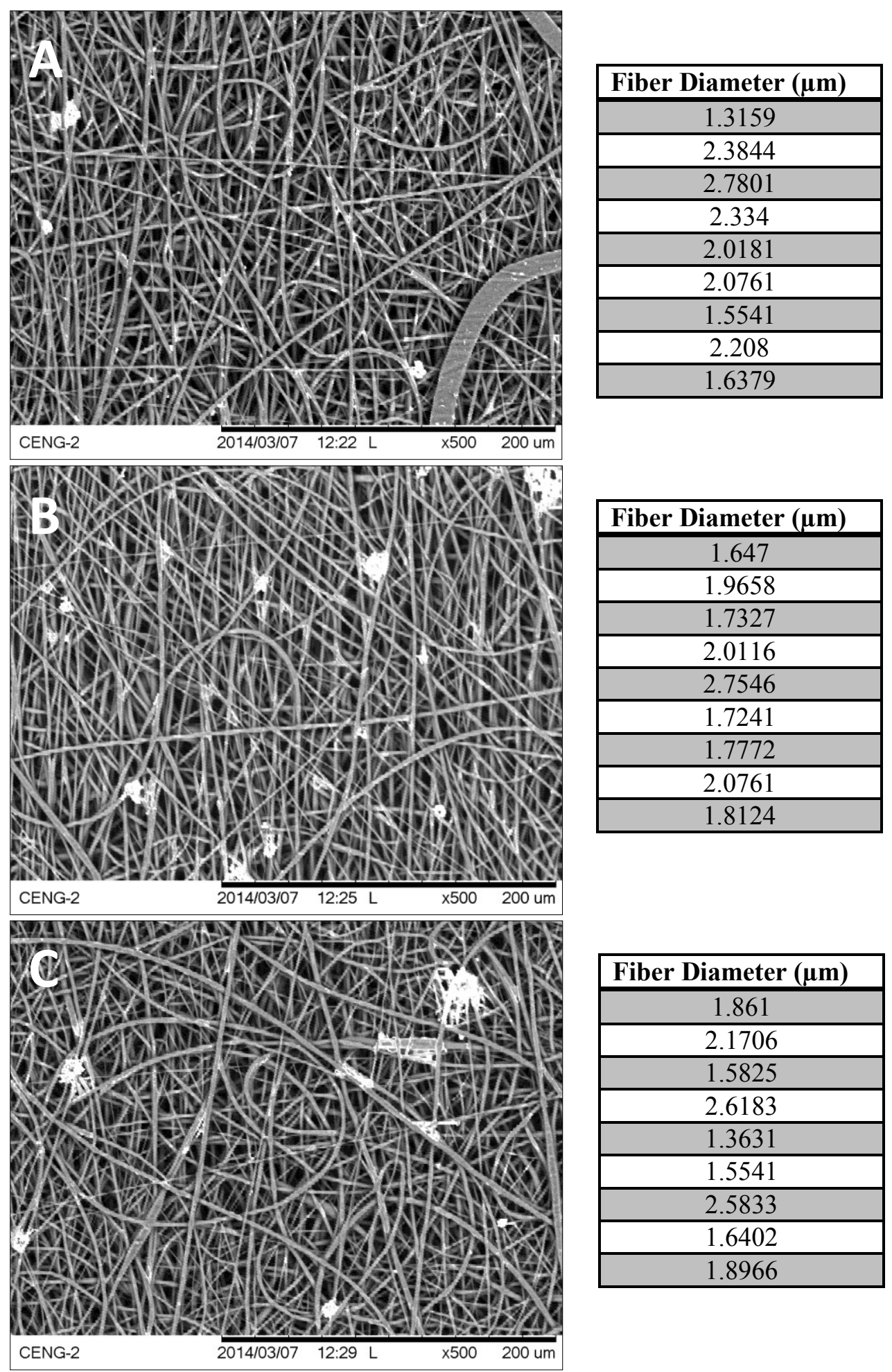

Figure N 15: SEM images of Spin 5 Distal location at 500x magnification; (A) Image 1; (B) Image 2; (C) Image 3. 


\section{Spin 6}

Process Observations: The initial bead fell as the voltage was applied, but the Taylor cone was not immediately visible. It formed inside of the needle tip, but eventually elongated and formed a small, well-shaped cone. The cone elongated downward and fell repeatedly throughout the spin.

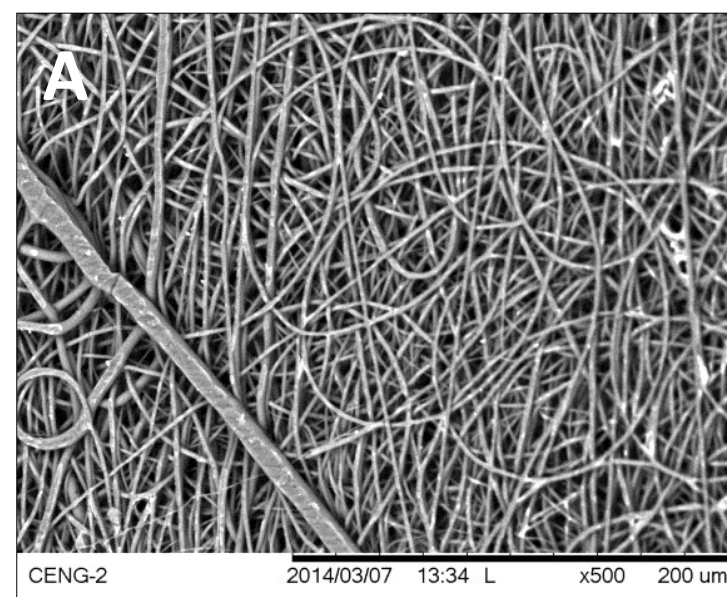

\begin{tabular}{|c|}
\hline Fiber Diameter $(\boldsymbol{\mu m})$ \\
\hline 2.1344 \\
\hline 5.265 \\
\hline 1.3131 \\
\hline 1.9996 \\
\hline 1.5349 \\
\hline 2.2563 \\
\hline 1.4139 \\
\hline 2.4413 \\
\hline 2.1569 \\
\hline
\end{tabular}

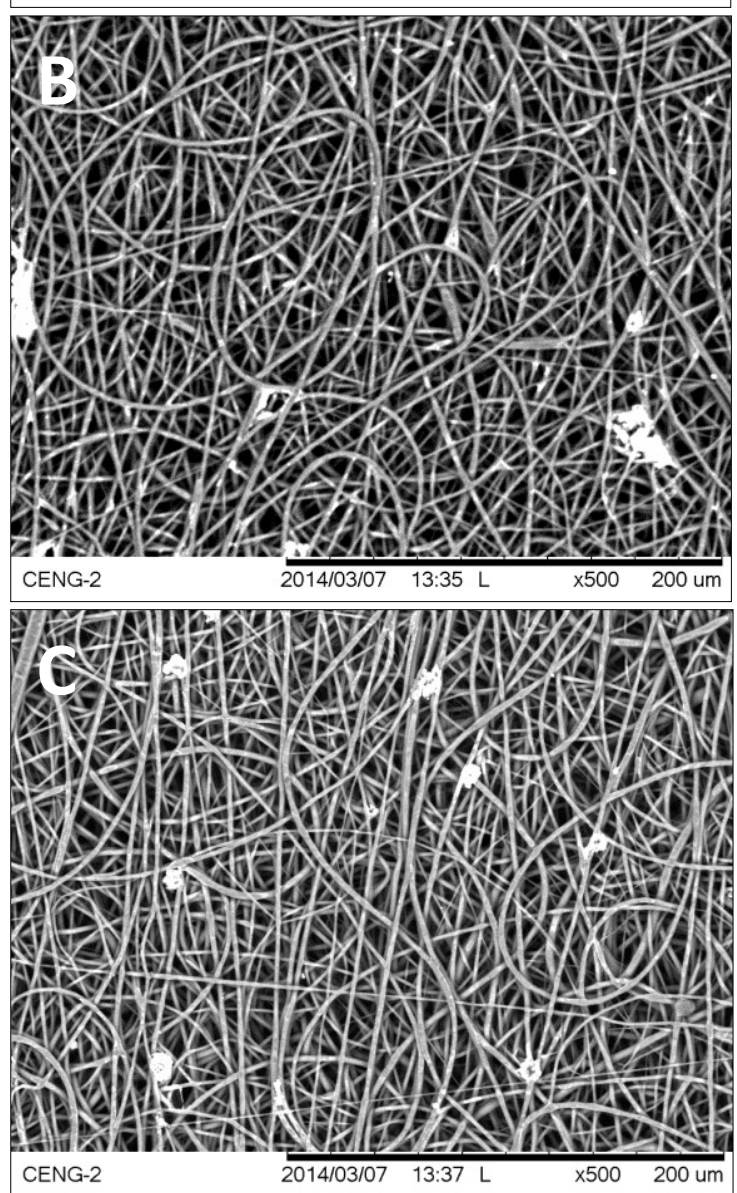

\begin{tabular}{|c|}
\hline Fiber Diameter $(\boldsymbol{\mu m})$ \\
\hline 1.7772 \\
\hline 1.5784 \\
\hline 2.4199 \\
\hline 2.8478 \\
\hline 2.2677 \\
\hline 2.4276 \\
\hline 1.8267 \\
\hline 2.2021 \\
\hline 1.6402 \\
\hline
\end{tabular}

\begin{tabular}{|c|}
\hline Fiber Diameter $(\boldsymbol{\mu m})$ \\
\hline 2.1552 \\
\hline 1.7177 \\
\hline 1.7938 \\
\hline 2.3719 \\
\hline 1.9141 \\
\hline 2.3419 \\
\hline 1.7588 \\
\hline 0.9829 \\
\hline 1.8287 \\
\hline
\end{tabular}

Figure $N$ 16: SEM images of Spin 6 Proximal location at 500x magnification; $(A)$ Image 1; (B) Image 2; (C) Image 3. 


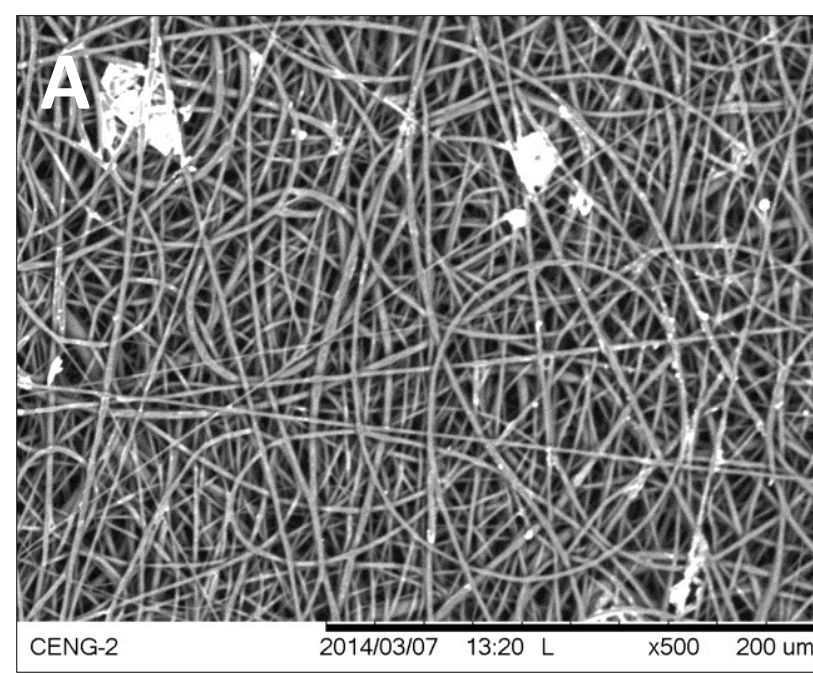

\begin{tabular}{|c|}
\hline Fiber Diameter $(\boldsymbol{\mu m})$ \\
\hline 2.7278 \\
\hline 1.9568 \\
\hline 2.5 \\
\hline 2.0851 \\
\hline 2.4505 \\
\hline 1.8907 \\
\hline 2.2247 \\
\hline 2.6738 \\
\hline 1.9902 \\
\hline
\end{tabular}

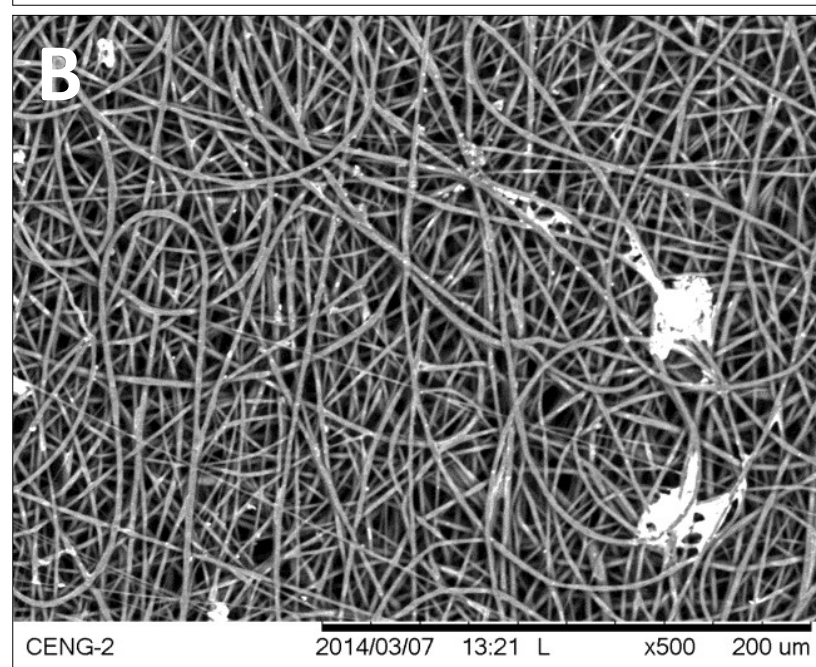

\begin{tabular}{|c|}
\hline Fiber Diameter $(\boldsymbol{\mu m})$ \\
\hline 1.9696 \\
\hline 2.4383 \\
\hline 1.6981 \\
\hline 1.9996 \\
\hline 1.8185 \\
\hline 2.3292 \\
\hline 1.7583 \\
\hline 2.1326 \\
\hline 1.9141 \\
\hline
\end{tabular}

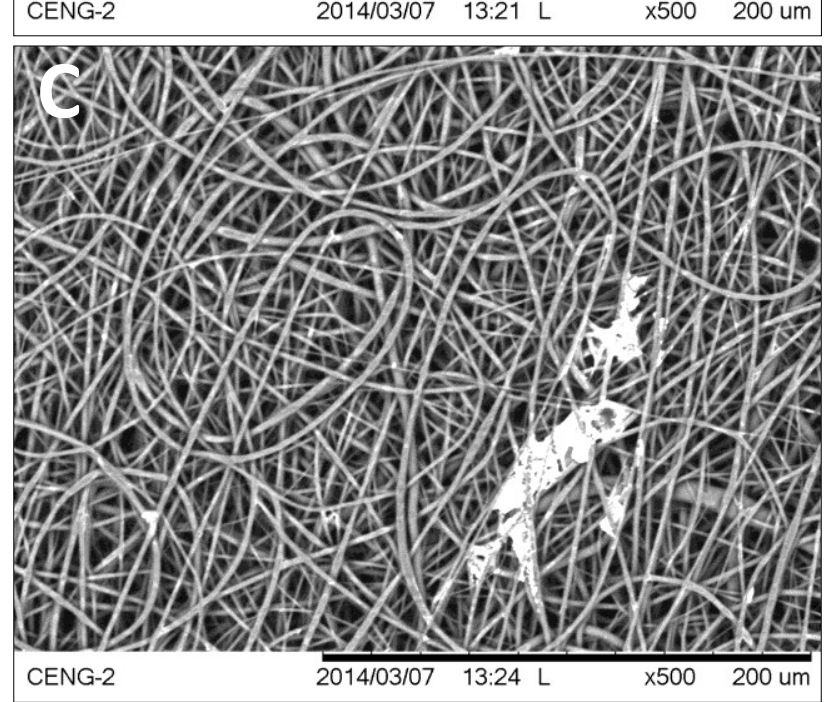

\begin{tabular}{|c|}
\hline Fiber Diameter $(\boldsymbol{\mu m})$ \\
\hline 2.5 \\
\hline 1.296 \\
\hline 2.764 \\
\hline 1.6105 \\
\hline 1.6379 \\
\hline 3.7656 \\
\hline 2.4276 \\
\hline 1.7349 \\
\hline 2.5529 \\
\hline
\end{tabular}

Figure N 17: SEM images of Spin 6 Medial location at 500x magnification; (A) Image 1; (B) Image 2; (C) Image 3. 


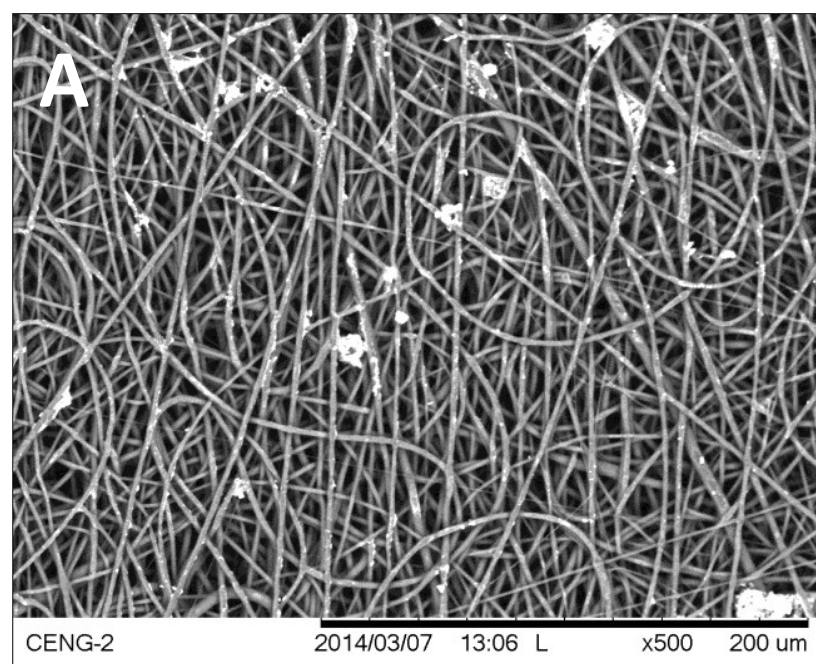

\begin{tabular}{|c|}
\hline Fiber Diameter $(\boldsymbol{\mu m})$ \\
\hline 2.6062 \\
\hline 2.0491 \\
\hline 1.7583 \\
\hline 2.2954 \\
\hline 2.0851 \\
\hline 2.2563 \\
\hline 2.3212 \\
\hline 2.2247 \\
\hline 2.4138 \\
\hline
\end{tabular}

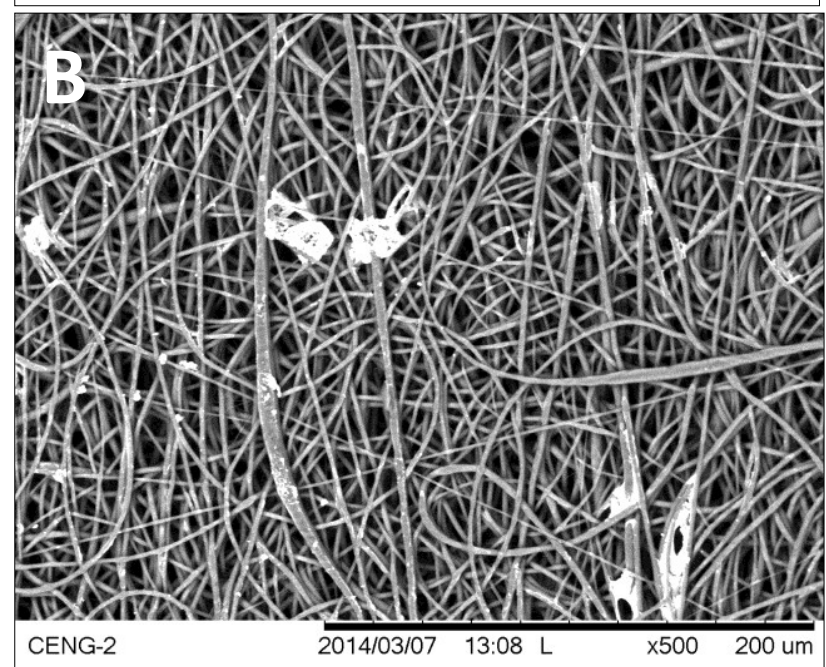

\begin{tabular}{|c|}
\hline Fiber Diameter $(\boldsymbol{\mu m})$ \\
\hline 1.8185 \\
\hline 2.248 \\
\hline 2.1292 \\
\hline 1.6649 \\
\hline 2.0491 \\
\hline 2.9361 \\
\hline 2.5133 \\
\hline 2.248 \\
\hline 2.0761 \\
\hline
\end{tabular}

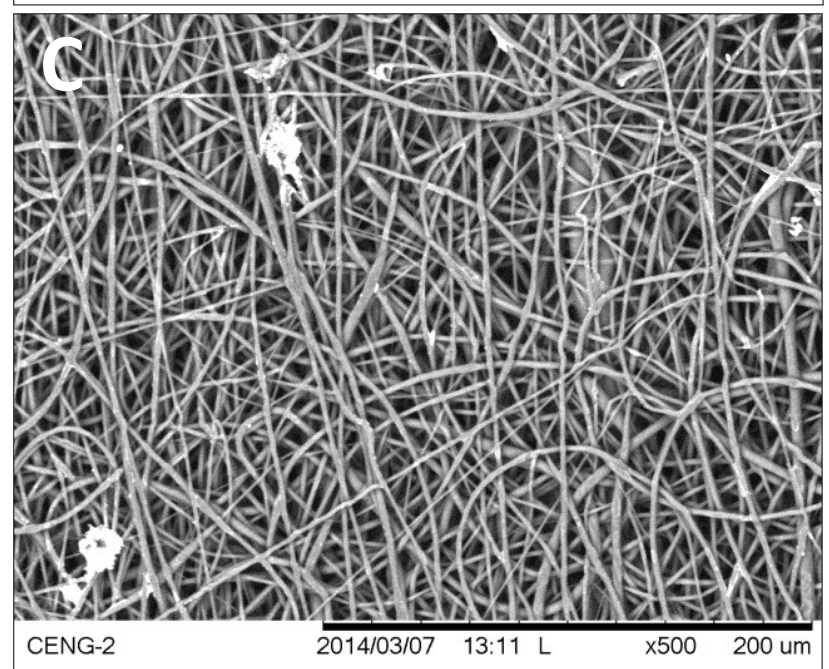

\begin{tabular}{|c|}
\hline Fiber Diameter $(\boldsymbol{\mu m})$ \\
\hline 2.0346 \\
\hline 1.5276 \\
\hline 2.0726 \\
\hline 1.7112 \\
\hline 2.5919 \\
\hline 1.508 \\
\hline 1.3383 \\
\hline 1.1976 \\
\hline 1.6582 \\
\hline
\end{tabular}

Figure $N$ 18: SEM images of Spin 6 Distal location at 500x magnification; $(A)$ Image 1; (B) Image 2; (C) Image 3. 


\section{Spin 7}

Process Observations: The Taylor cone formed immediately after the initial bead fell. It was small and steady with an ideal shape. Around $0.25 \mathrm{~mL}$ of solution was ejected, it began slowly elongating for about $0.03 \mathrm{~mL}$ and falling repeatedly.

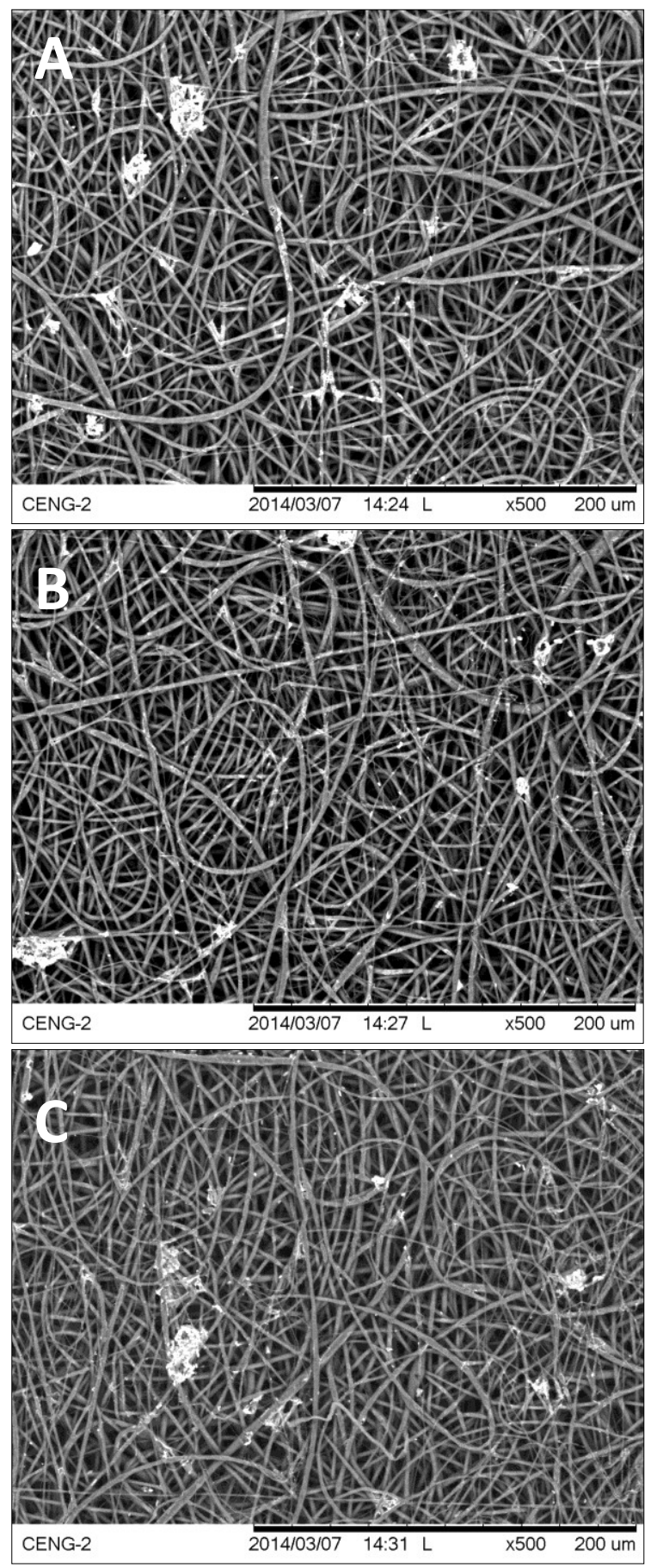

\begin{tabular}{|c|}
\hline Fiber Diameter $(\boldsymbol{\mu m})$ \\
\hline 1.8322 \\
\hline 1.8138 \\
\hline 2.9569 \\
\hline 0.9772 \\
\hline 1.8138 \\
\hline 1.6411 \\
\hline 0.868 \\
\hline 2.6269 \\
\hline 1.9941 \\
\hline
\end{tabular}

\begin{tabular}{|c|}
\hline Fiber Diameter $(\boldsymbol{\mu m})$ \\
\hline 2.0801 \\
\hline 2.5107 \\
\hline 1.721 \\
\hline 2.1728 \\
\hline 1.3212 \\
\hline 1.7035 \\
\hline 2.4733 \\
\hline 1.9734 \\
\hline 1.9313 \\
\hline
\end{tabular}

\begin{tabular}{|c|}
\hline Fiber Diameter $(\boldsymbol{\mu m})$ \\
\hline 1.822 \\
\hline 2.6425 \\
\hline 2.4921 \\
\hline 1.7806 \\
\hline 2.3496 \\
\hline 2.2672 \\
\hline 1.8159 \\
\hline 2.7814 \\
\hline 1.3156 \\
\hline
\end{tabular}

Figure $N$ 19: SEM images of Spin 7 Proximal location at 500x magnification; (A) Image 1; (B) Image 2; (C) Image 3. 


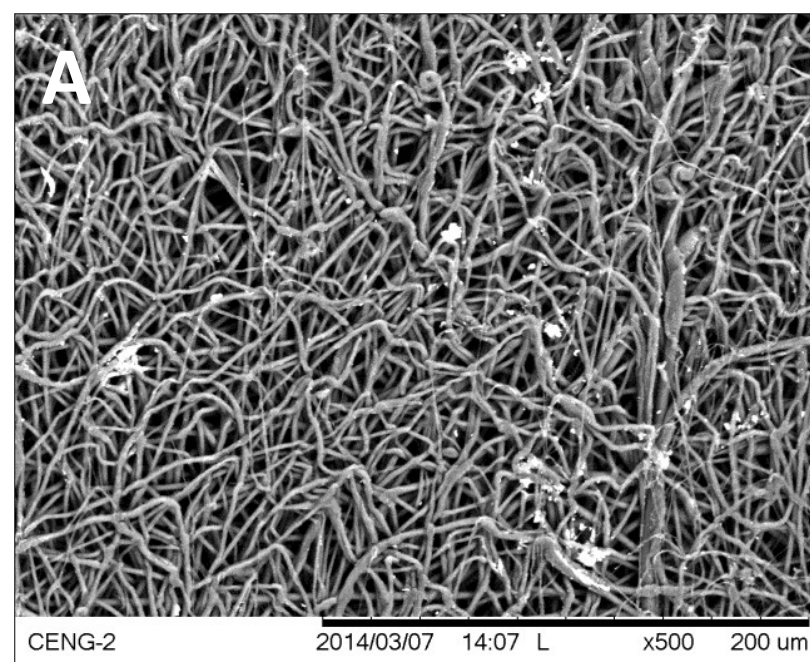

\begin{tabular}{|c|}
\hline Fiber Diameter $(\boldsymbol{\mu m})$ \\
\hline 2.4014 \\
\hline 1.5926 \\
\hline 1.4709 \\
\hline 1.9884 \\
\hline 2.0164 \\
\hline 1.8464 \\
\hline 2.6583 \\
\hline 2.9983 \\
\hline 2.0801 \\
\hline
\end{tabular}

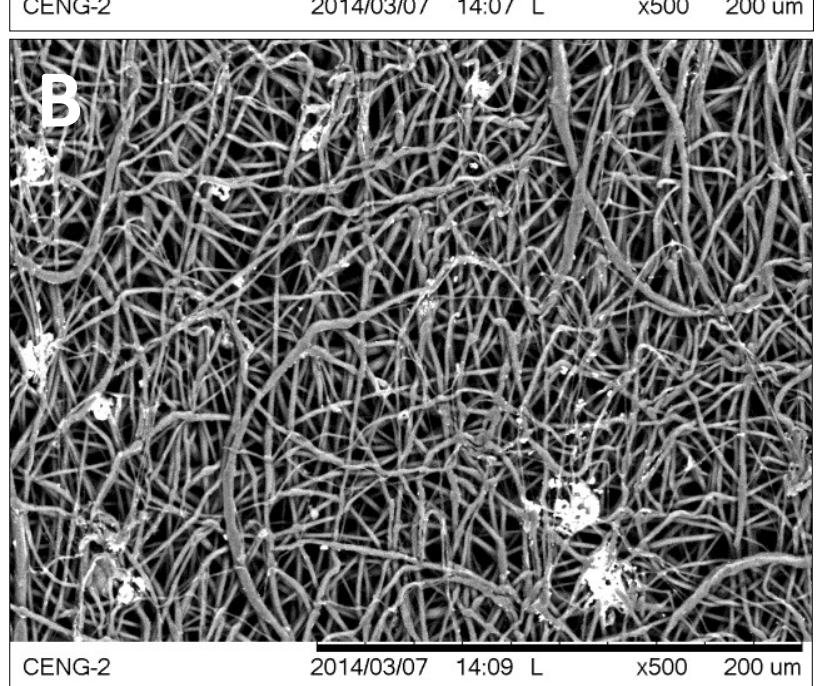

\begin{tabular}{|c|}
\hline Fiber Diameter $(\boldsymbol{\mu m})$ \\
\hline 2.0765 \\
\hline 1.9776 \\
\hline 2.0164 \\
\hline 2.0873 \\
\hline 2.7653 \\
\hline 2.7453 \\
\hline 3.3363 \\
\hline 1.6516 \\
\hline 1.7617 \\
\hline
\end{tabular}

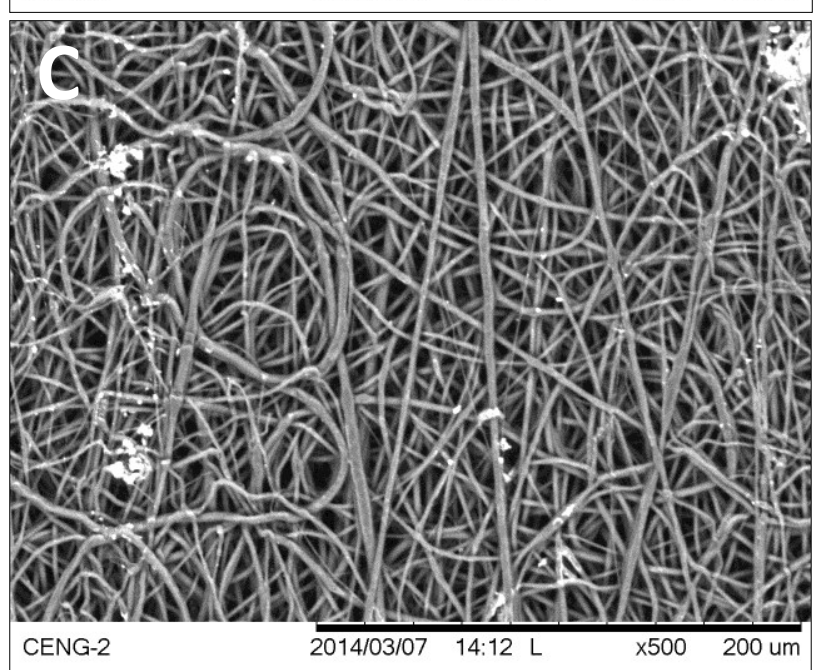

\begin{tabular}{|c|}
\hline Fiber Diameter $(\boldsymbol{\mu m})$ \\
\hline 2.5767 \\
\hline 1.8138 \\
\hline 1.9819 \\
\hline 2.7693 \\
\hline 2.7696 \\
\hline 1.6969 \\
\hline 2.4914 \\
\hline 2.9544 \\
\hline 2.5926 \\
\hline
\end{tabular}

Figure N 20: SEM images of Spin 7 Medial location at 500x magnification; (A) Image 1; (B) Image 2; (C) Image 3. 


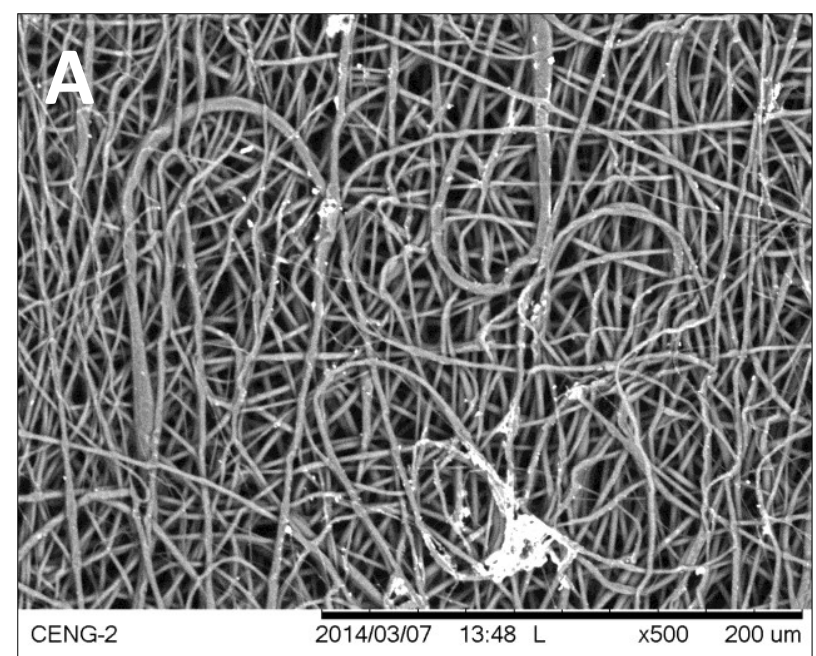

\begin{tabular}{|c|}
\hline Fiber Diameter $(\boldsymbol{\mu m})$ \\
\hline 2.2206 \\
\hline 1.6614 \\
\hline 3.0756 \\
\hline 2.3952 \\
\hline 1.677 \\
\hline 1.9178 \\
\hline 2.5969 \\
\hline 1.4166 \\
\hline 2.2672 \\
\hline
\end{tabular}

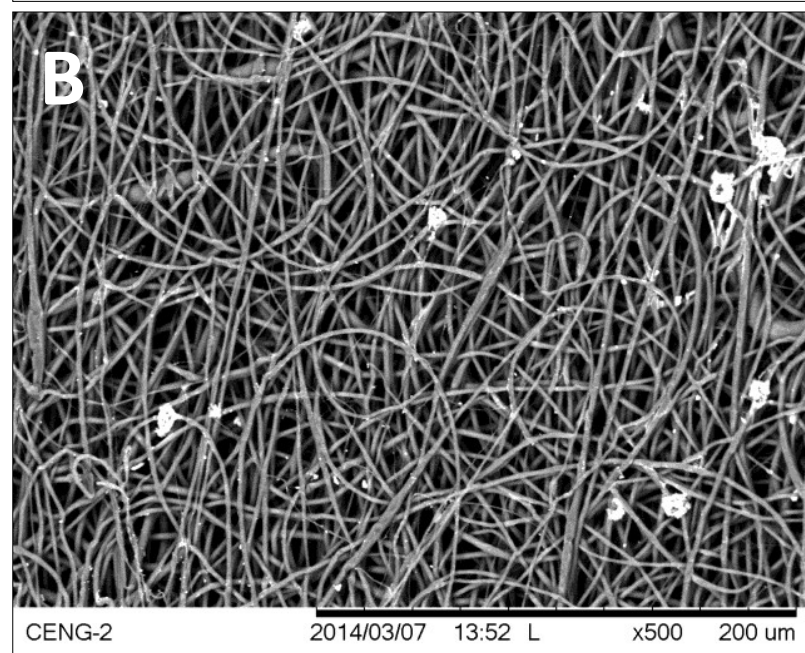

\begin{tabular}{|c|}
\hline Fiber Diameter $(\boldsymbol{\mu m})$ \\
\hline 2.3889 \\
\hline 2.033 \\
\hline 2.6789 \\
\hline 1.9715 \\
\hline 1.5547 \\
\hline 2.1662 \\
\hline 2.0909 \\
\hline 1.7274 \\
\hline 2.1454 \\
\hline
\end{tabular}

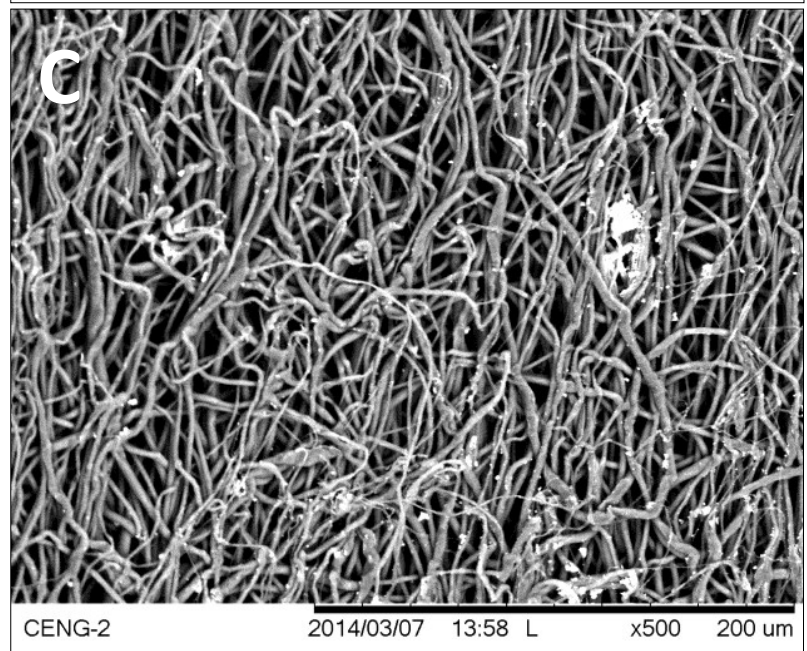

\begin{tabular}{|c|}
\hline Fiber Diameter $(\boldsymbol{\mu m})$ \\
\hline 2.2921 \\
\hline 1.3184 \\
\hline 1.8045 \\
\hline 2.6141 \\
\hline 1.3492 \\
\hline 2.3496 \\
\hline 2.4384 \\
\hline 3.028 \\
\hline 2.8712 \\
\hline
\end{tabular}

Figure $N$ 21: SEM images of Spin 7 Distal location at 500x magnification; $(A)$ Image 1; (B) Image 2; (C) Image 3. 


\section{Spin 8}

Process Observations: The Taylor cone formed immediately after the initial bead of solution fell. The cone was small, extremely stable, and had an ideal shape. It maintained its stability for a longer amount of time than previous spins; it began to elongate after about $0.50 \mathrm{~mL}$ of solution was ejected and elongated for approximately $0.06 \mathrm{~mL}$.

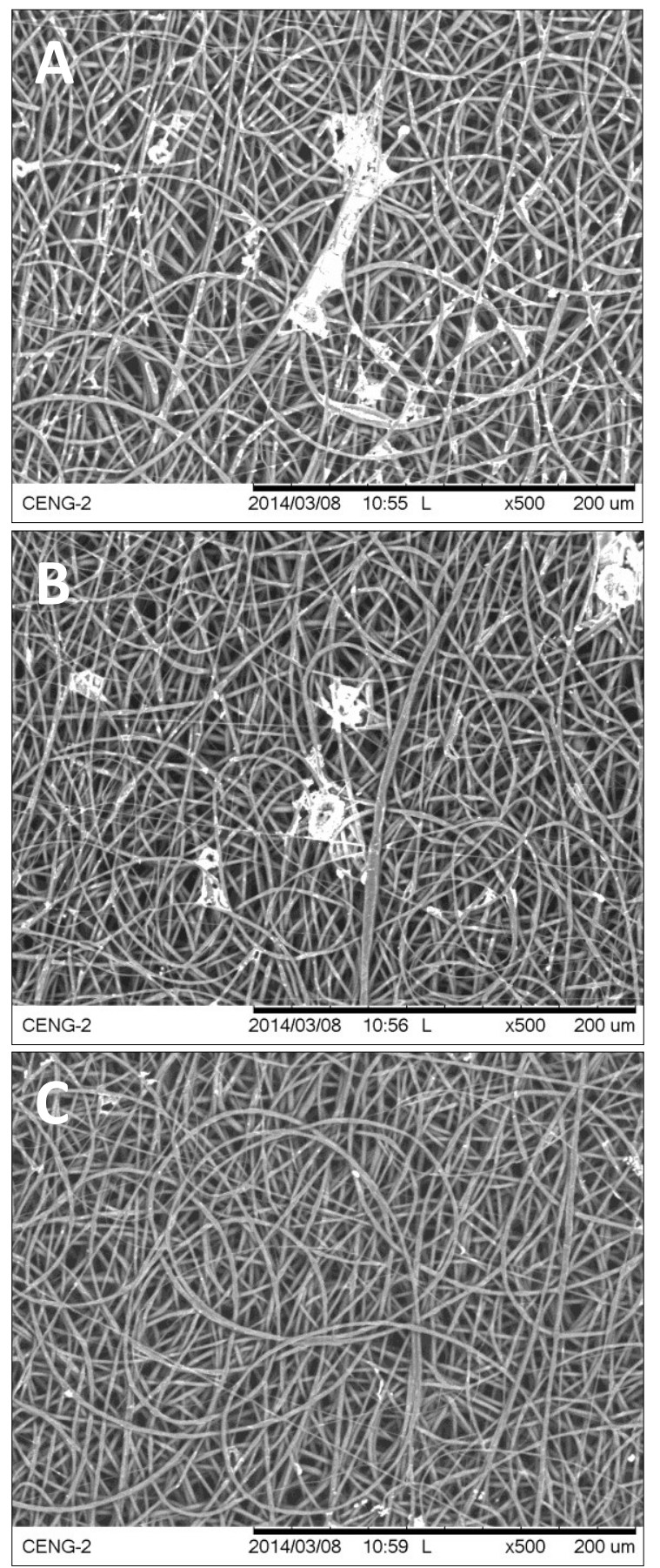

\begin{tabular}{|c|}
\hline Fiber Diameter $(\boldsymbol{\mu m})$ \\
\hline 2.0909 \\
\hline 2.3591 \\
\hline 1.9313 \\
\hline 2.1086 \\
\hline 2.3176 \\
\hline 2.1748 \\
\hline 2.1748 \\
\hline 2.3889 \\
\hline 2.9481 \\
\hline
\end{tabular}

\begin{tabular}{|c|}
\hline Fiber Diameter $(\boldsymbol{\mu m})$ \\
\hline 1.3184 \\
\hline 1.284 \\
\hline 2.1367 \\
\hline 1.6501 \\
\hline 2.2672 \\
\hline 1.9544 \\
\hline 2.0385 \\
\hline 2.3998 \\
\hline 1.721 \\
\hline
\end{tabular}

\begin{tabular}{|c|}
\hline Fiber Diameter $(\boldsymbol{\mu m})$ \\
\hline 0.9343 \\
\hline 2.009 \\
\hline 2.0801 \\
\hline 2.2021 \\
\hline 1.5856 \\
\hline 2.2868 \\
\hline 2.1332 \\
\hline 1.6681 \\
\hline 2.862 \\
\hline
\end{tabular}

Figure $N$ 22: SEM images of Spin 8 Proximal location at 500x magnification; (A) Image 1; (B) Image 2; (C) Image 3. 


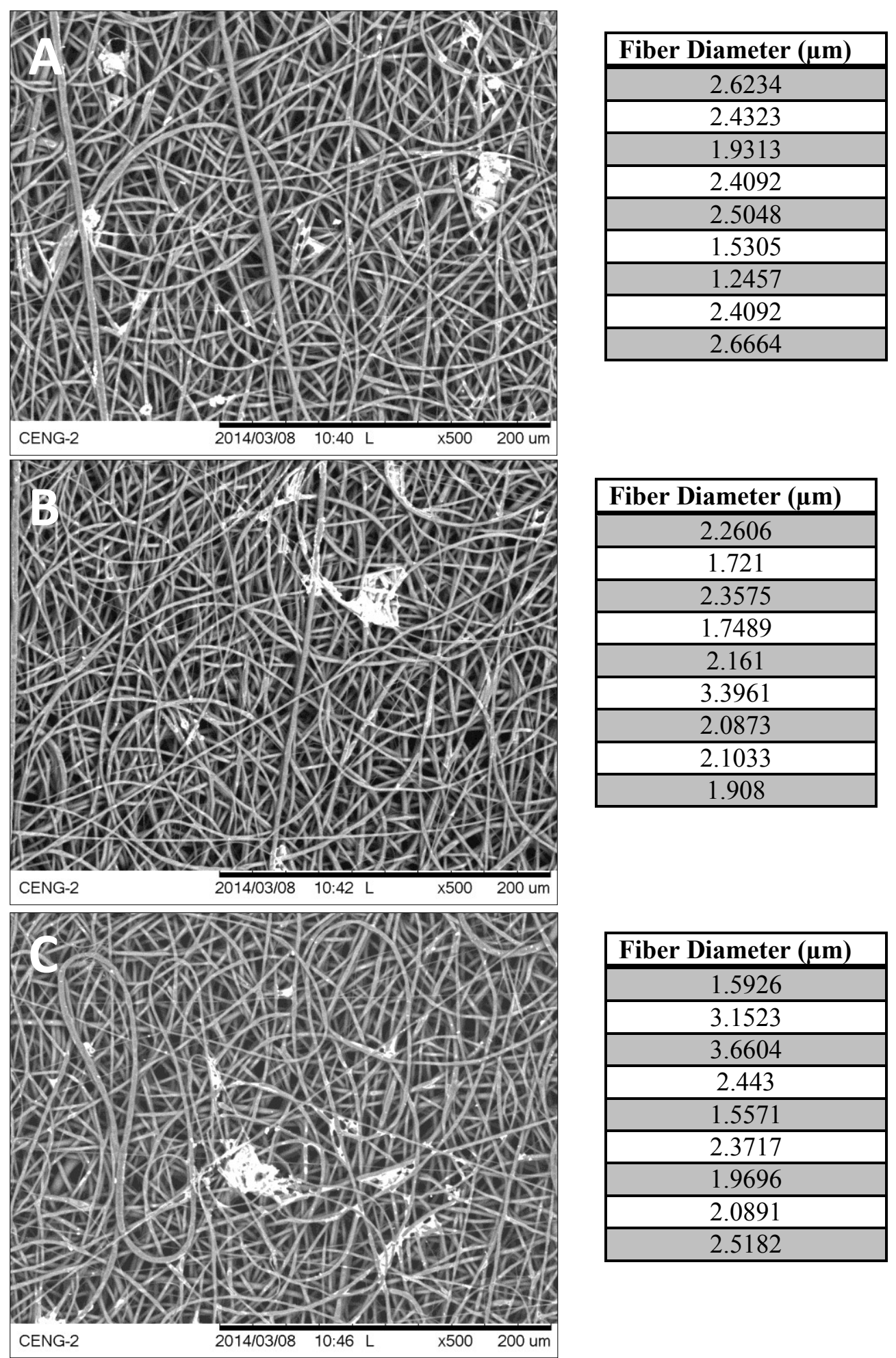

Figure N 23: SEM images of Spin 8 Medial location at 500x magnification; (A) Image 1; (B) Image 2; (C) Image 3. 


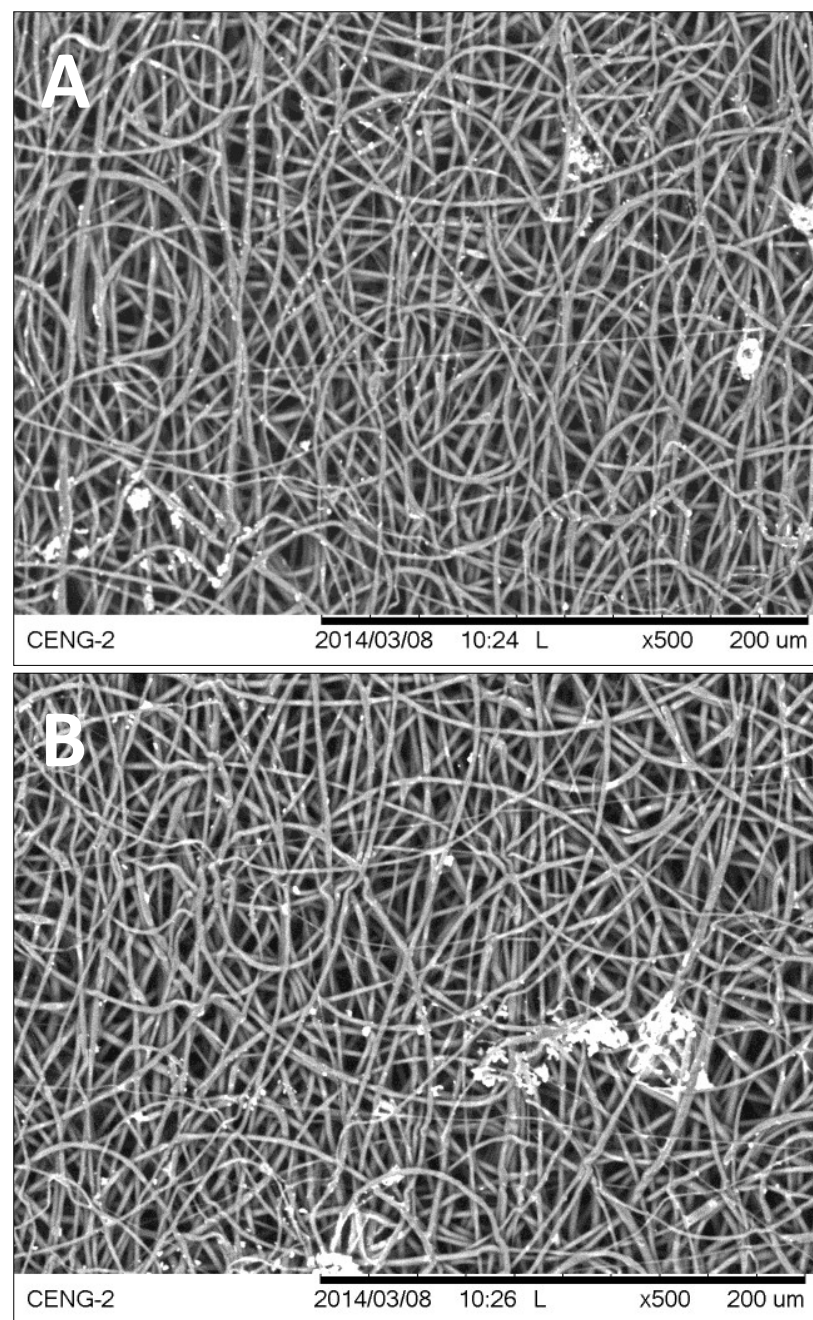

\begin{tabular}{|c|}
\hline Fiber Diameter $(\boldsymbol{\mu m})$ \\
\hline 1.9022 \\
\hline 2.3889 \\
\hline 1.8864 \\
\hline 1.8138 \\
\hline 2.0164 \\
\hline 1.1228 \\
\hline 2.4733 \\
\hline 1.9022 \\
\hline 1.9178 \\
\hline
\end{tabular}

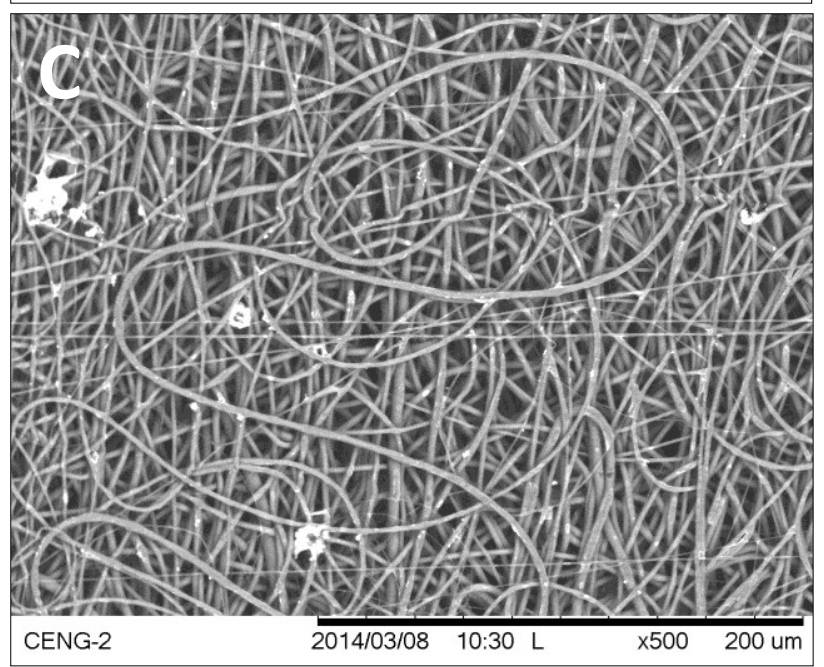

\begin{tabular}{|c|}
\hline Fiber Diameter $(\boldsymbol{\mu m})$ \\
\hline 2.0765 \\
\hline 2.4869 \\
\hline 1.9884 \\
\hline 2.443 \\
\hline 0.8194 \\
\hline 1.9884 \\
\hline 1.677 \\
\hline 1.2215 \\
\hline 0.8194 \\
\hline
\end{tabular}

\begin{tabular}{|c|}
\hline Fiber Diameter $(\boldsymbol{\mu m})$ \\
\hline 2.1987 \\
\hline 2.0164 \\
\hline 2.6678 \\
\hline 2.9417 \\
\hline 1.9582 \\
\hline 2.229 \\
\hline 0.9501 \\
\hline 1.9884 \\
\hline 2.2523 \\
\hline
\end{tabular}

Figure N 24: SEM images of Spin 8 Distal location at 500x magnification; $(A)$ Image 1; (B) Image 2; (C) Image 3. 


\section{Spin 9}

Process Observations: The initial cone was small, stable, and well-shaped. It began elongating and falling repeatedly during the spin. The elongation lasted for about 0.03 $\mathrm{mL}$.

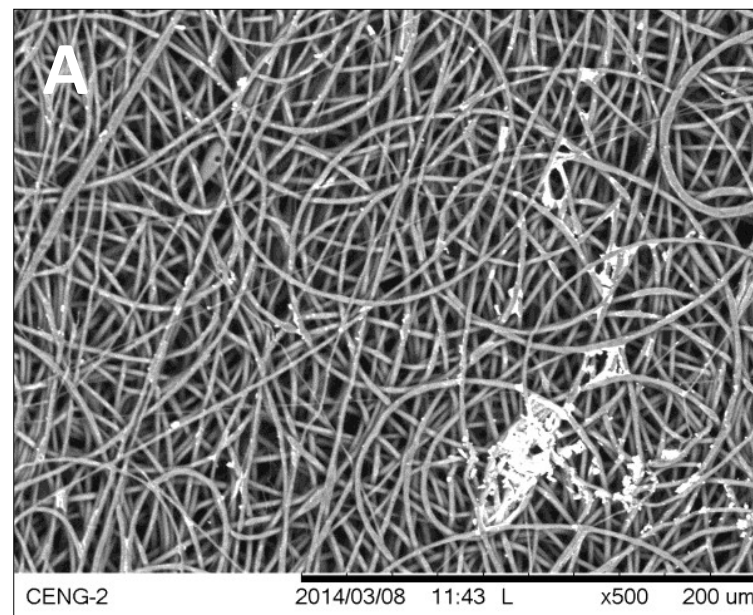

\begin{tabular}{|c|}
\hline Fiber Diameter $(\boldsymbol{\mu m})$ \\
\hline 2.0238 \\
\hline 1.4683 \\
\hline 2.2721 \\
\hline 2.6326 \\
\hline 1.7144 \\
\hline 2.2672 \\
\hline 2.443 \\
\hline 2.4184 \\
\hline 1.6969 \\
\hline
\end{tabular}

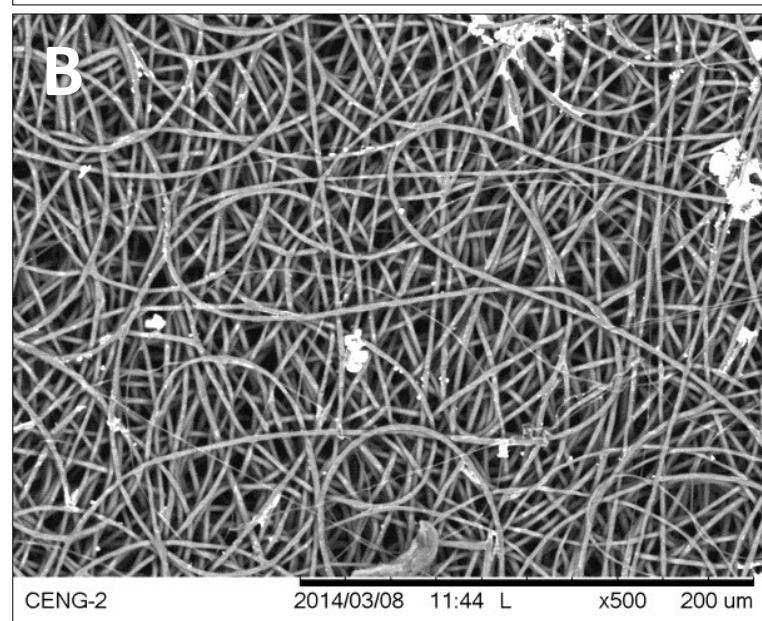

\begin{tabular}{|c|}
\hline Fiber Diameter $(\boldsymbol{\mu m})$ \\
\hline 2.1416 \\
\hline 2.1593 \\
\hline 2.0747 \\
\hline 2.0164 \\
\hline 2.2457 \\
\hline 2.0981 \\
\hline 2.0873 \\
\hline 2.4616 \\
\hline 2.009 \\
\hline
\end{tabular}

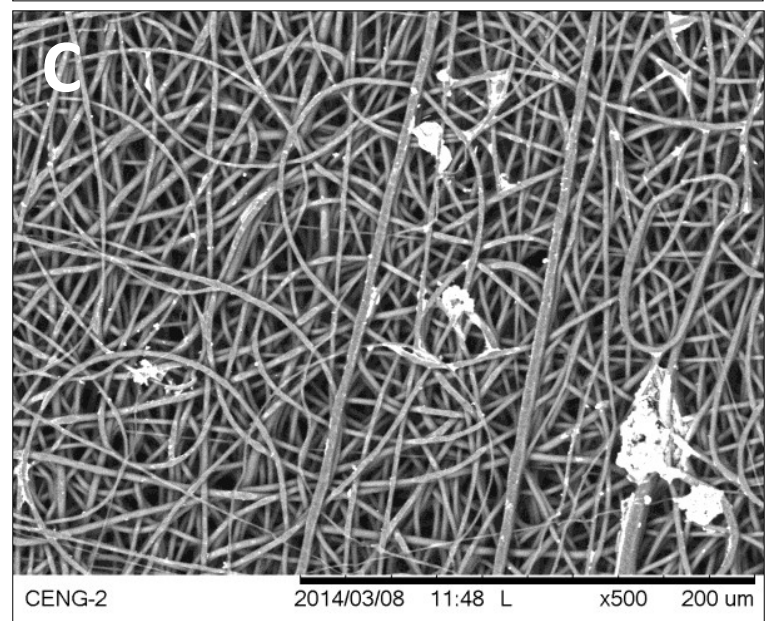

\begin{tabular}{|c|}
\hline Fiber Diameter $(\boldsymbol{\mu m})$ \\
\hline 1.7806 \\
\hline 1.8651 \\
\hline 2.7674 \\
\hline 2.7484 \\
\hline 2.7215 \\
\hline 2.3464 \\
\hline 2.3356 \\
\hline 2.2921 \\
\hline 1.1748 \\
\hline
\end{tabular}

Figure $N$ 25: SEM images of Spin 9 Proximal location at 500x magnification; (A) Image 1; (B) Image 2; (C) Image 3. 


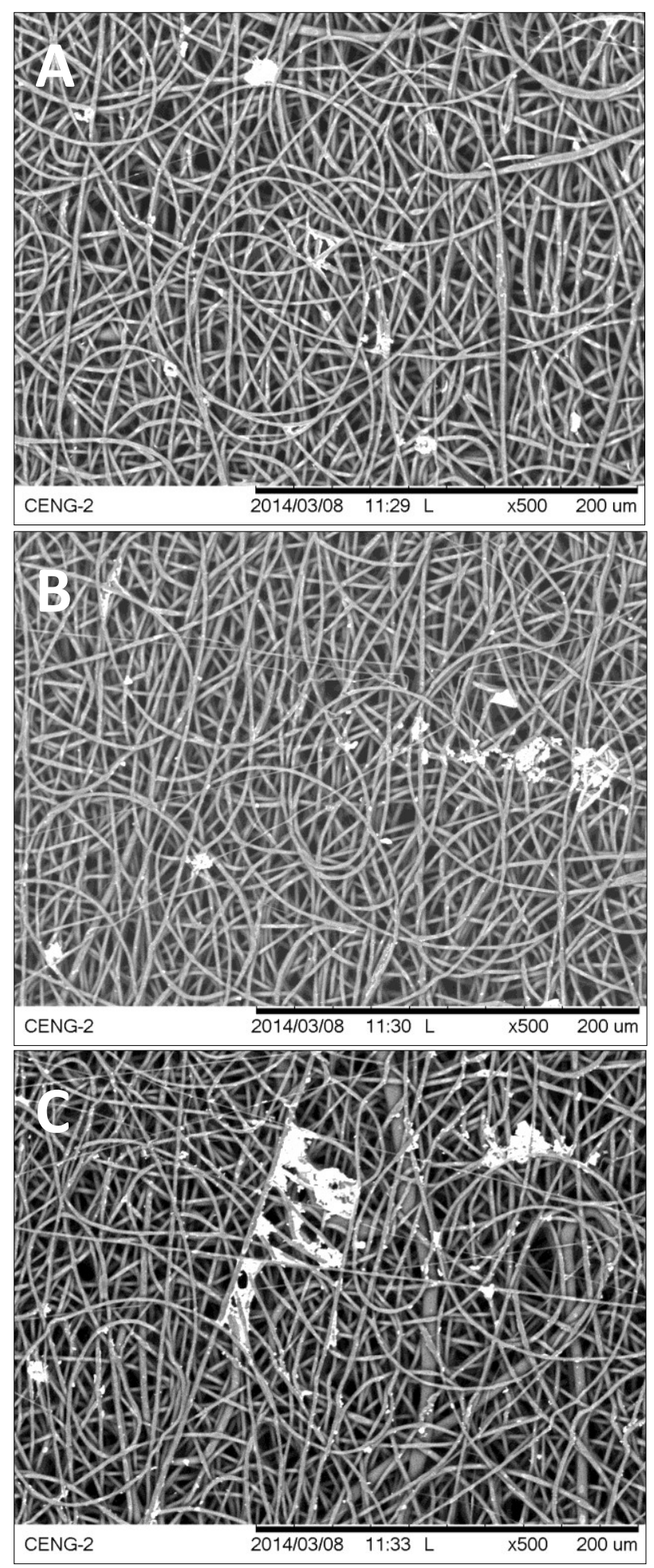

\begin{tabular}{|c|}
\hline Fiber Diameter $(\boldsymbol{\mu m})$ \\
\hline 2.1091 \\
\hline 2.5156 \\
\hline 2.0739 \\
\hline 2.2063 \\
\hline 2.1385 \\
\hline 2.2337 \\
\hline 3.0246 \\
\hline 3.5363 \\
\hline 2.4693 \\
\hline
\end{tabular}

\begin{tabular}{|c|}
\hline Fiber Diameter $(\boldsymbol{\mu m})$ \\
\hline 2.5264 \\
\hline 1.9702 \\
\hline 1.9445 \\
\hline 1.9744 \\
\hline 2.4184 \\
\hline 2.733 \\
\hline 0.8811 \\
\hline 2.3764 \\
\hline 1.9829 \\
\hline
\end{tabular}

\begin{tabular}{|c|}
\hline Fiber Diameter $(\boldsymbol{\mu m})$ \\
\hline 2.4929 \\
\hline 2.5883 \\
\hline 2.1377 \\
\hline 1.5452 \\
\hline 2.282 \\
\hline 0.9848 \\
\hline 2.271 \\
\hline 2.1091 \\
\hline 1.8322 \\
\hline
\end{tabular}

Figure N 26: SEM images of Spin 9 Medial location at 500x magnification; $(A)$ Image 1; $(B)$ Image 2; $(C)$ Image 3. 


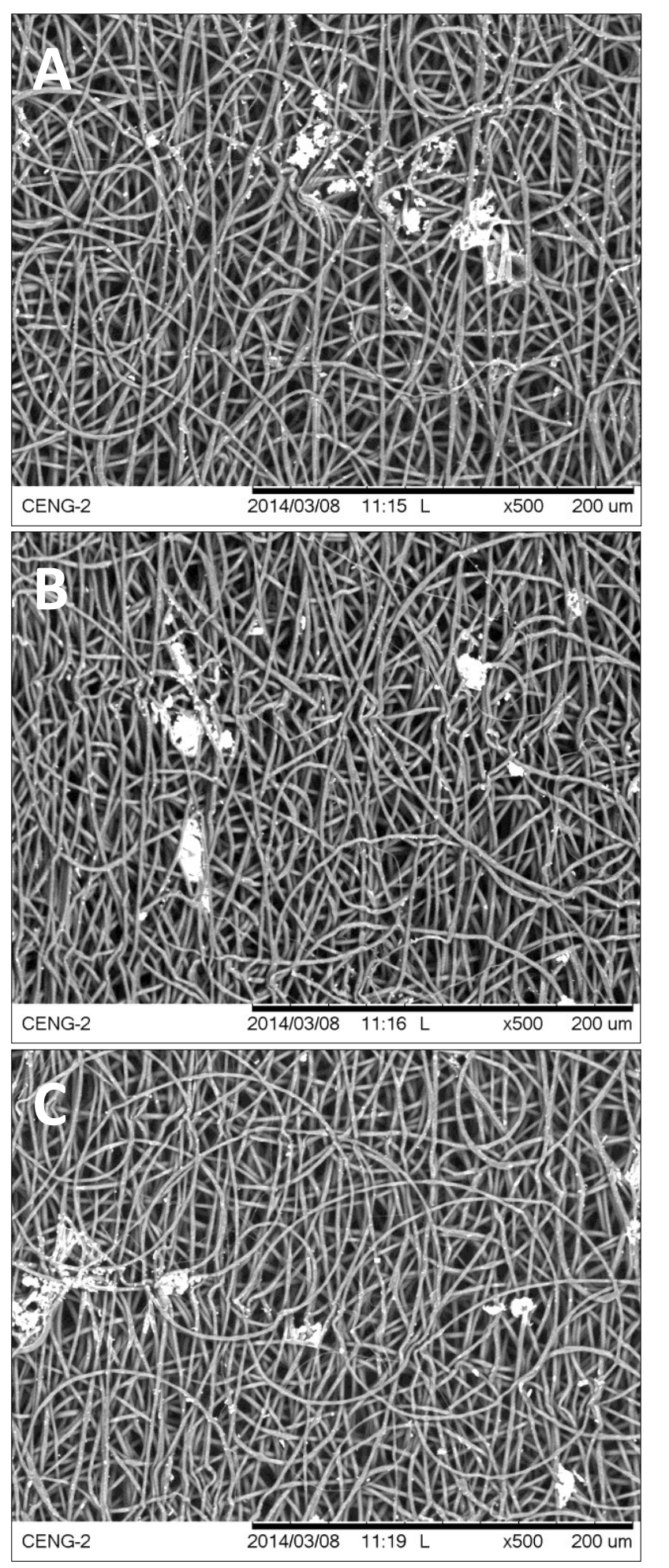

\begin{tabular}{|c|}
\hline Fiber Diameter $(\boldsymbol{\mu m})$ \\
\hline 2.1728 \\
\hline 2.3003 \\
\hline 2.7908 \\
\hline 1.9052 \\
\hline 2.2034 \\
\hline 2.2365 \\
\hline 2.1416 \\
\hline 3.1094 \\
\hline 2.0092 \\
\hline
\end{tabular}

\begin{tabular}{|c|}
\hline Fiber Diameter $(\boldsymbol{\mu m})$ \\
\hline 2.3757 \\
\hline 2.0092 \\
\hline 2.1387 \\
\hline 2.341 \\
\hline 2.0289 \\
\hline 2.2309 \\
\hline 2.2682 \\
\hline 2.4394 \\
\hline 2.552 \\
\hline
\end{tabular}

\begin{tabular}{|c|}
\hline Fiber Diameter $(\boldsymbol{\mu m})$ \\
\hline 1.9412 \\
\hline 2.1387 \\
\hline 2.678 \\
\hline 2.1573 \\
\hline 1.7788 \\
\hline 1.855 \\
\hline 1.7917 \\
\hline 2.2756 \\
\hline 1.855 \\
\hline
\end{tabular}

Figure N 27: SEM images of Spin 9 Distal location at 500x magnification; (A) Image 1; (B) Image 2; (C) Image 3. 


\section{Spin 10}

Process Observations: The initial cone was small, steady, and had an ideal shape. It began to elongate every $0.02 \mathrm{~mL}$ and fall repeatedly for the remainder of the spin.

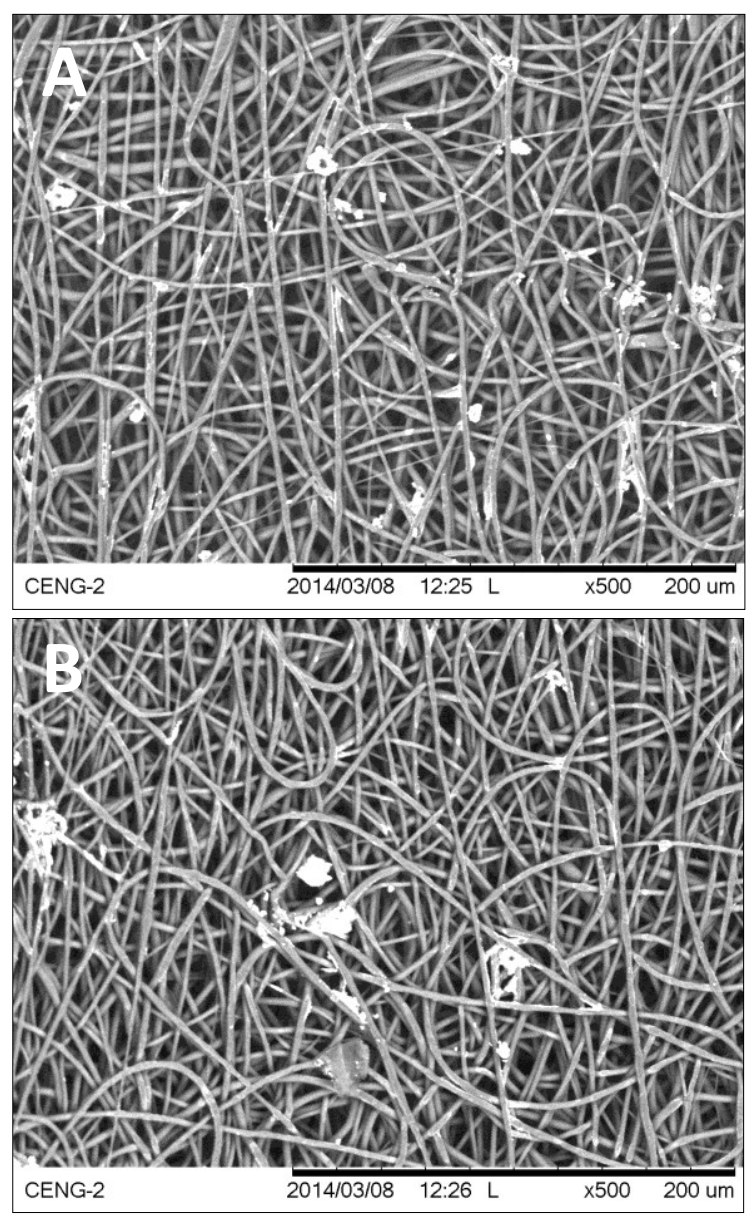

\begin{tabular}{|c|}
\hline Fiber Diameter $(\boldsymbol{\mu m})$ \\
\hline 2.0747 \\
\hline 3.3696 \\
\hline 2.6112 \\
\hline 3.4284 \\
\hline 2.745 \\
\hline 1.4251 \\
\hline 1.3863 \\
\hline 1.3863 \\
\hline 2.7192 \\
\hline
\end{tabular}

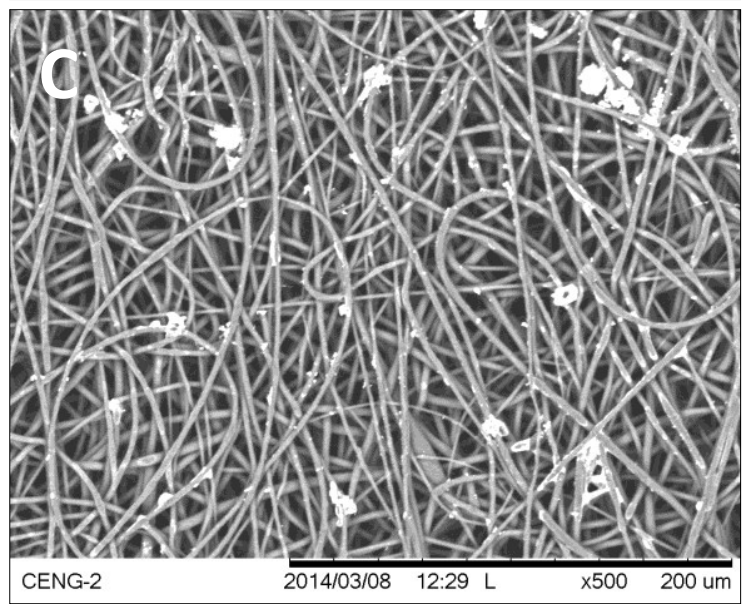

\begin{tabular}{|c|}
\hline Fiber Diameter $(\boldsymbol{\mu m})$ \\
\hline 3.6369 \\
\hline 2.7854 \\
\hline 3.2867 \\
\hline 2.1416 \\
\hline 1.9477 \\
\hline 3.6021 \\
\hline 2.3321 \\
\hline 2.6997 \\
\hline 1.7274 \\
\hline
\end{tabular}

Figure N 28: SEM images of Spin 10 Proximal location at 500x magnification; (A) Image 1; (B) Image 2; (C) Image 3. 


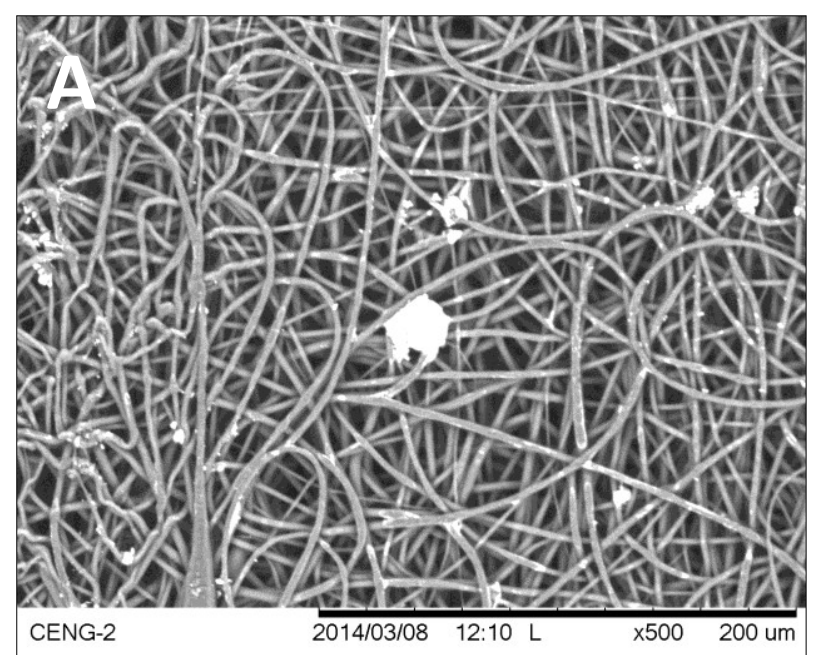

\begin{tabular}{|c|}
\hline Fiber Diameter $(\boldsymbol{\mu m})$ \\
\hline 2.7108 \\
\hline 2.7639 \\
\hline 2.5984 \\
\hline 2.1593 \\
\hline 2.295 \\
\hline 2.7272 \\
\hline 1.1262 \\
\hline 3.0218 \\
\hline 2.5912 \\
\hline
\end{tabular}

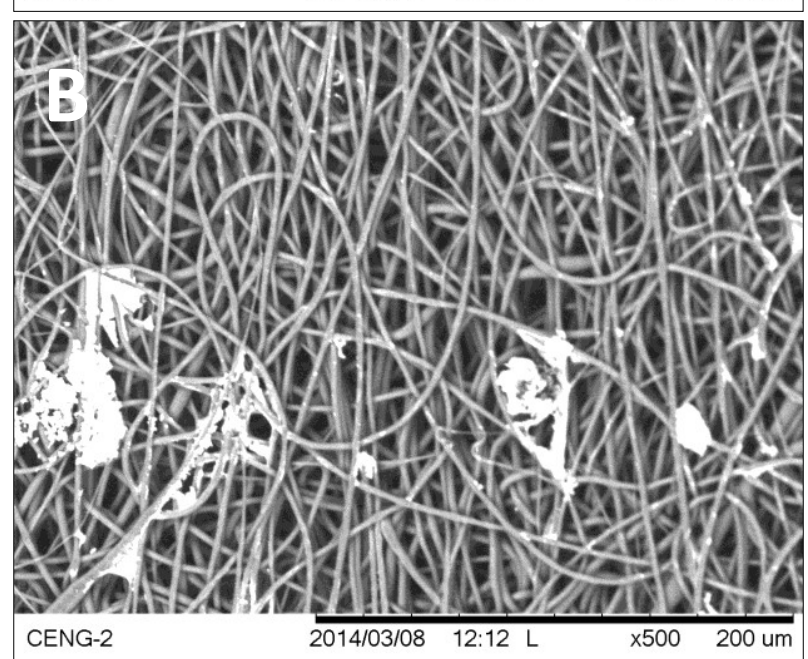

\begin{tabular}{|c|}
\hline Fiber Diameter $(\boldsymbol{\mu m})$ \\
\hline 3.1297 \\
\hline 2.3384 \\
\hline 2.3337 \\
\hline 3.144 \\
\hline 2.5944 \\
\hline 3.3597 \\
\hline 3.5863 \\
\hline 2.862 \\
\hline 2.6608 \\
\hline
\end{tabular}

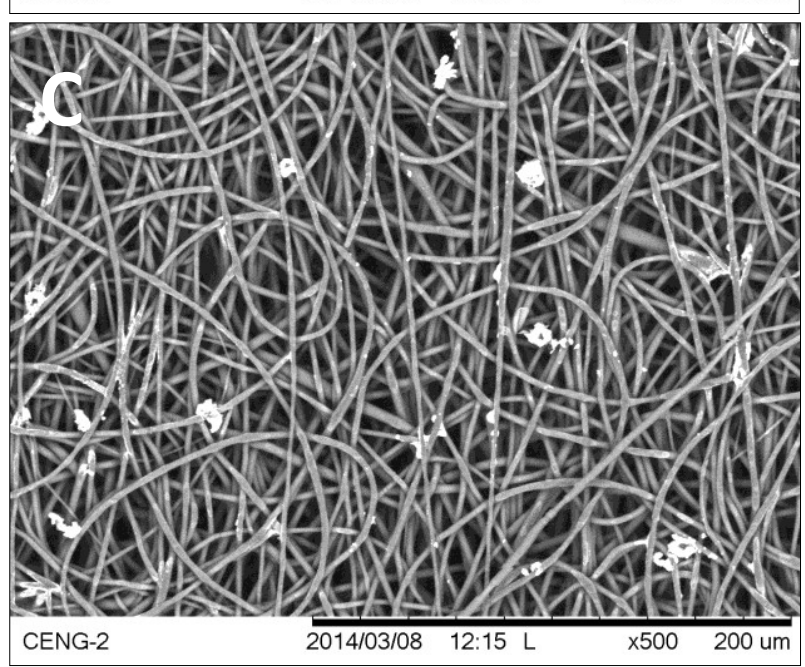

\begin{tabular}{|c|}
\hline Fiber Diameter $(\boldsymbol{\mu m})$ \\
\hline 3.2833 \\
\hline 2.42 \\
\hline 1.7806 \\
\hline 2.1593 \\
\hline 2.4323 \\
\hline 3.0169 \\
\hline 2.2868 \\
\hline 2.4014 \\
\hline 2.5798 \\
\hline
\end{tabular}

Figure $N$ 29: SEM images of Spin 10 Medial location at 500x magnification; $(A)$ Image 1; $(B)$ Image 2; $(C)$ Image 3. 


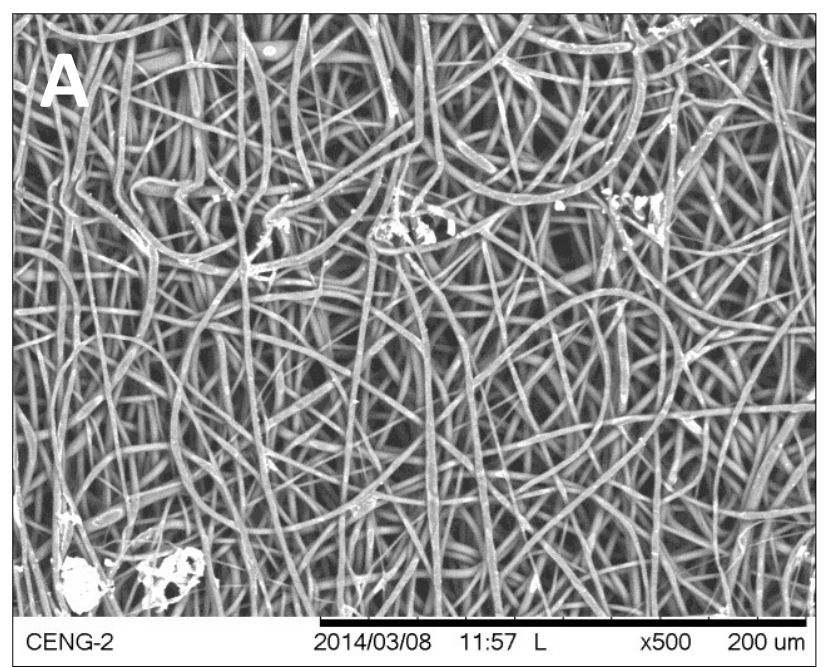

\begin{tabular}{|c|}
\hline Fiber Diameter $(\boldsymbol{\mu m})$ \\
\hline 1.5571 \\
\hline 2.6817 \\
\hline 2.1033 \\
\hline 3.8014 \\
\hline 2.2606 \\
\hline 2.5182 \\
\hline 2.4733 \\
\hline 2.5156 \\
\hline 1.7785 \\
\hline
\end{tabular}

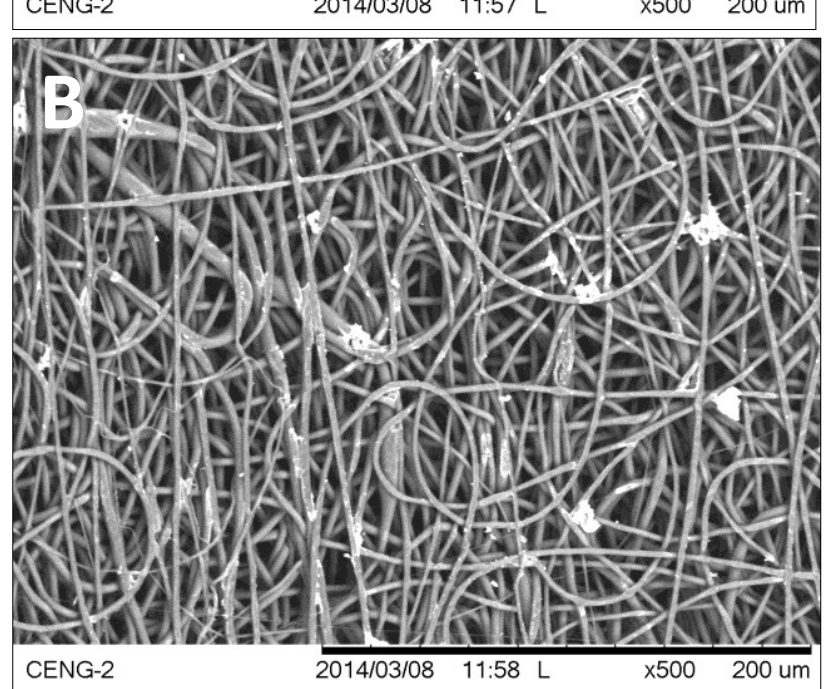

\begin{tabular}{|c|}
\hline Fiber Diameter $(\boldsymbol{\mu m})$ \\
\hline 2.1332 \\
\hline 2.1748 \\
\hline 2.1367 \\
\hline 3.4689 \\
\hline 3.4038 \\
\hline 2.4567 \\
\hline 2.8763 \\
\hline 2.7053 \\
\hline 2.5285 \\
\hline
\end{tabular}

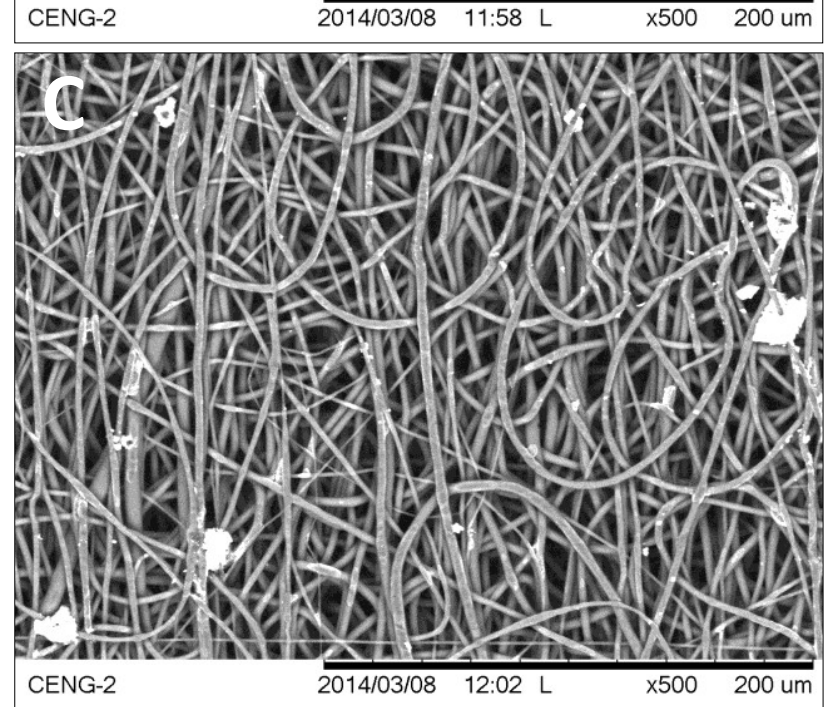

\begin{tabular}{|c|}
\hline Fiber Diameter $(\boldsymbol{\mu m})$ \\
\hline 2.3337 \\
\hline 1.941 \\
\hline 2.8957 \\
\hline 3.9477 \\
\hline 4.2402 \\
\hline 3.4764 \\
\hline 2.4316 \\
\hline 3.1106 \\
\hline 2.0454 \\
\hline
\end{tabular}

Figure N 30: SEM images of Spin 10 Distal location at 500x magnification; $(A)$ Image 1; (B) Image 2; (C) Image 3. 


\section{Spin 11}

Process Observations: The cone formed immediately after the bead fell off of the needle. It was stable and maintained an ideal shape initially, but began to elongate and fall repeatedly. Around $2.00 \mathrm{~mL}$, the cone appeared to be pulsing, but the jet of solution remained thin at its extrusion point from the cone.

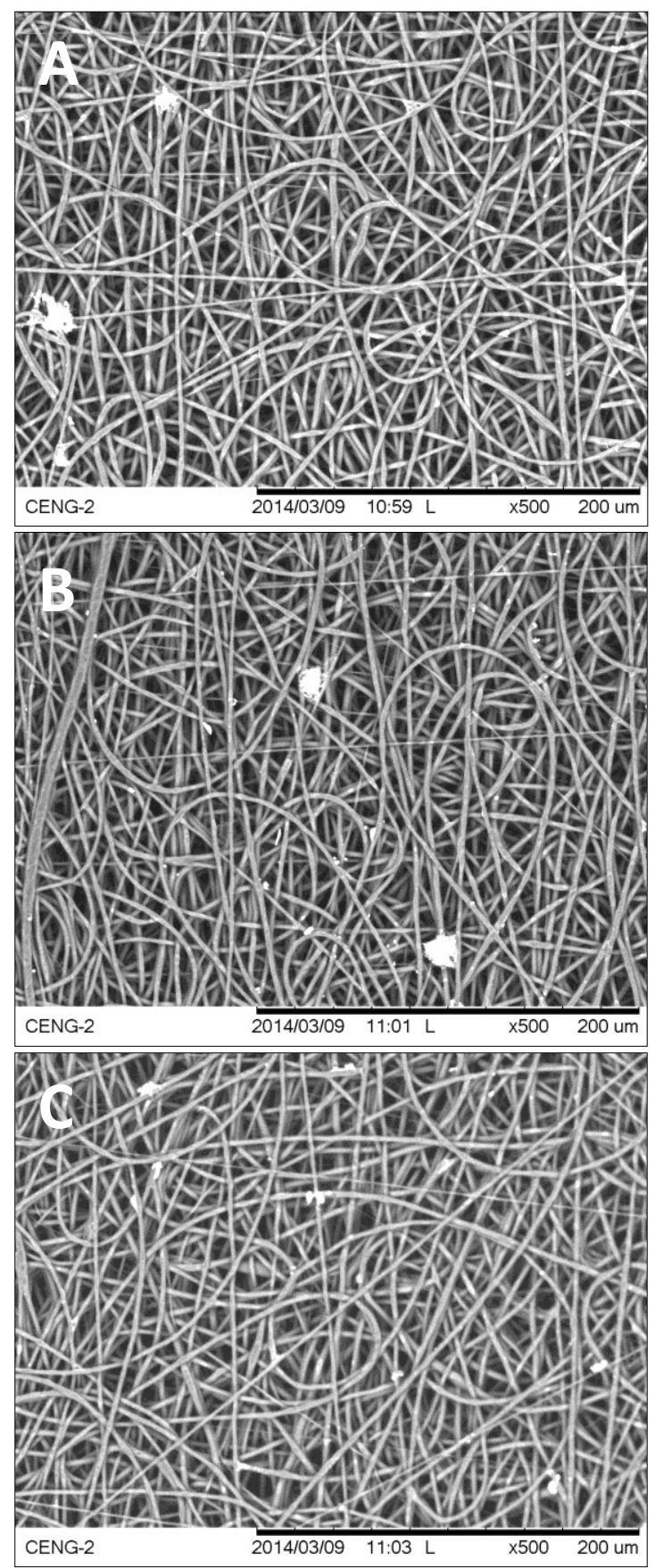

\begin{tabular}{|c|}
\hline Fiber Diameter $(\boldsymbol{\mu m})$ \\
\hline 2.1413 \\
\hline 2.4686 \\
\hline 2.5237 \\
\hline 2.2134 \\
\hline 2.76 \\
\hline 2.926 \\
\hline 2.2639 \\
\hline 2.1552 \\
\hline 3.277 \\
\hline
\end{tabular}

\begin{tabular}{|c|}
\hline Fiber Diameter $(\boldsymbol{\mu m})$ \\
\hline 1.39 \\
\hline 3.1088 \\
\hline 1.5541 \\
\hline 2.9987 \\
\hline 1.7883 \\
\hline 1.8287 \\
\hline 3.4583 \\
\hline 2.353 \\
\hline 2.4138 \\
\hline
\end{tabular}

\begin{tabular}{|c|}
\hline Fiber Diameter $(\boldsymbol{\mu m})$ \\
\hline 2.4027 \\
\hline 2.1552 \\
\hline 2.4701 \\
\hline 3.4526 \\
\hline 2.8448 \\
\hline 3.1908 \\
\hline 3.0498 \\
\hline 2.4062 \\
\hline 2.8461 \\
\hline
\end{tabular}

Figure N 31: SEM images of Spin 11 Proximal location at 500x magnification; (A) Image 1; (B) Image 2; (C) Image 3. 


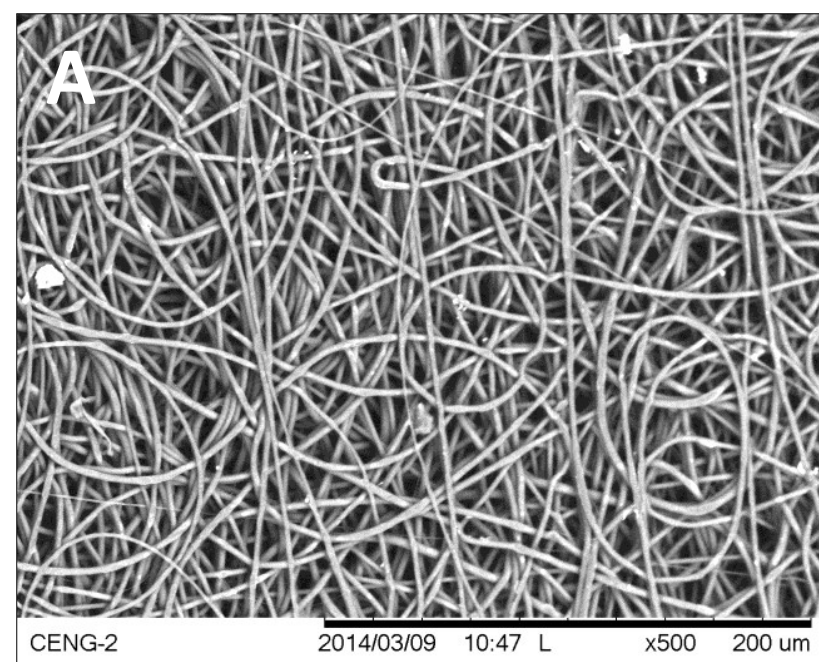

\begin{tabular}{|c|}
\hline Fiber Diameter $(\boldsymbol{\mu m})$ \\
\hline 1.9544 \\
\hline 2.0125 \\
\hline 2.76 \\
\hline 2.2134 \\
\hline 2.4276 \\
\hline 2.4157 \\
\hline 2.6063 \\
\hline 2.2759 \\
\hline 2.5631 \\
\hline
\end{tabular}

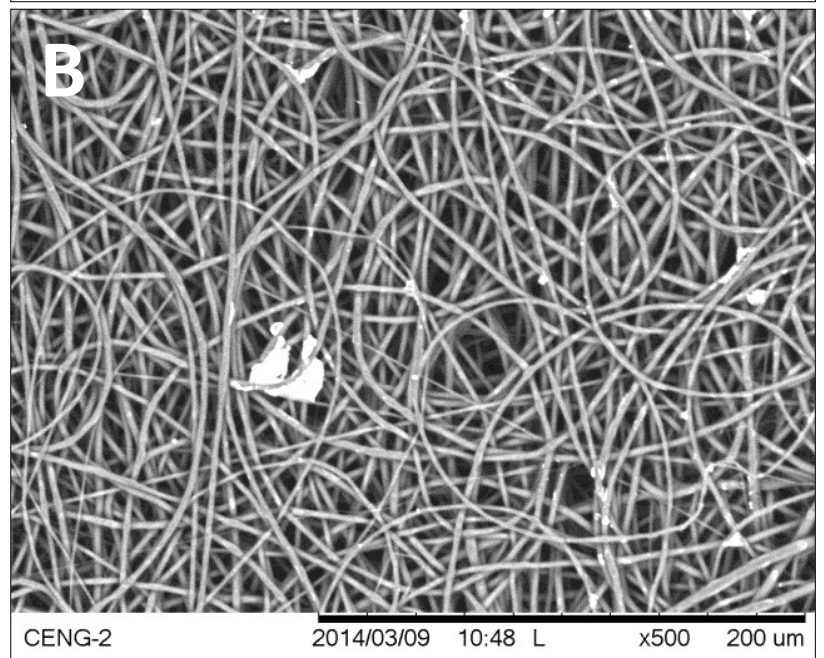

\begin{tabular}{|c|}
\hline Fiber Diameter $(\boldsymbol{\mu m})$ \\
\hline 2.9608 \\
\hline 1.3631 \\
\hline 2.8146 \\
\hline 2.1522 \\
\hline 2.1552 \\
\hline 2.1954 \\
\hline 3.1237 \\
\hline 2.5015 \\
\hline 2.4276 \\
\hline
\end{tabular}

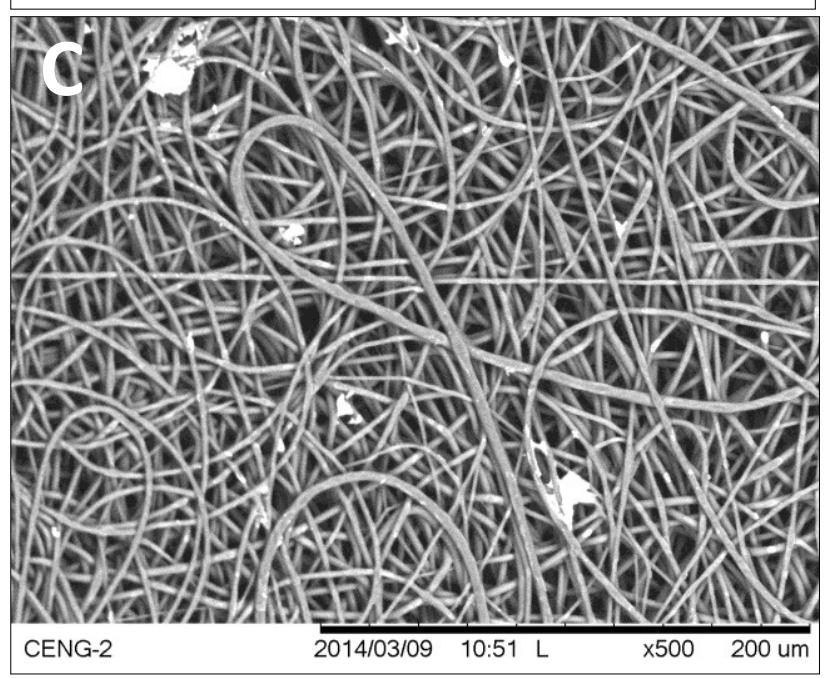

\begin{tabular}{|c|}
\hline Fiber Diameter $(\boldsymbol{\mu m})$ \\
\hline 2.4535 \\
\hline 2.7438 \\
\hline 2.2759 \\
\hline 2.8966 \\
\hline 2.1292 \\
\hline 1.7241 \\
\hline 2.6849 \\
\hline 2.0125 \\
\hline 2.7505 \\
\hline
\end{tabular}

Figure N 32: SEM images of Spin 11 Medial location at 500x magnification; $(A)$ Image 1; (B) Image 2; (C) Image 3. 

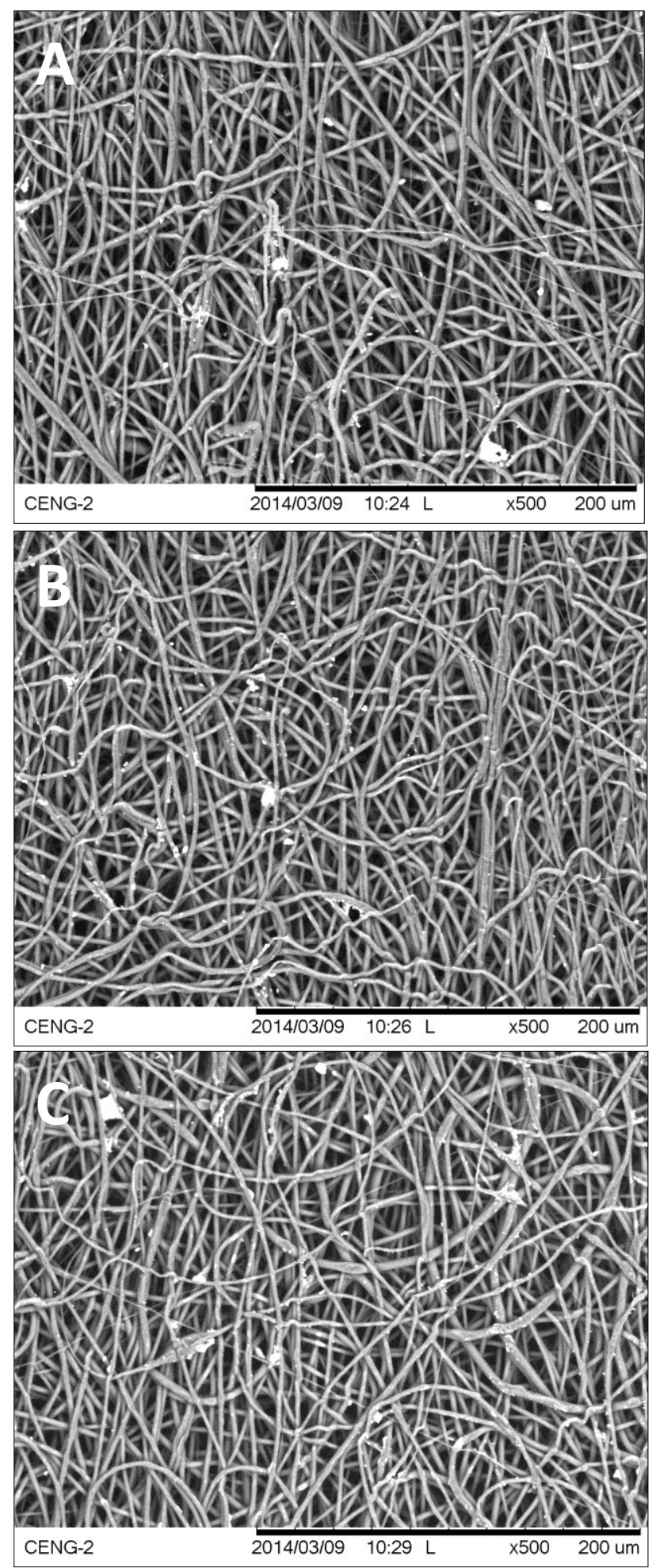

\begin{tabular}{|c|}
\hline Fiber Diameter $(\boldsymbol{\mu m})$ \\
\hline 2.5356 \\
\hline 2.1621 \\
\hline 2.0346 \\
\hline 2.8915 \\
\hline 0.9829 \\
\hline 2.7001 \\
\hline 2.5354 \\
\hline 0.989 \\
\hline 2.6091 \\
\hline
\end{tabular}

\begin{tabular}{|c|}
\hline Fiber Diameter $(\boldsymbol{\mu m})$ \\
\hline 1.0523 \\
\hline 2.76 \\
\hline 2.5471 \\
\hline 2.1552 \\
\hline 1.9449 \\
\hline 2.7261 \\
\hline 1.3383 \\
\hline 2.5934 \\
\hline 1.4451 \\
\hline
\end{tabular}

\begin{tabular}{|c|}
\hline Fiber Diameter $(\boldsymbol{\mu m})$ \\
\hline 2.0761 \\
\hline 2.1552 \\
\hline 2.2219 \\
\hline 1.9696 \\
\hline 1.7564 \\
\hline 3.2082 \\
\hline 3.1698 \\
\hline 2.9726 \\
\hline 2.2932 \\
\hline
\end{tabular}

Figure $N$ 33: SEM images of Spin 11 Distal location at 500x magnification; (A) Image 1; (B) Image 2; (C) Image 3. 


\section{Spin 12}

Process Observations: The solution had to be stopped before the initial bead formed (after $0.07 \mathrm{~mL}$ was displayed on the syringe pump) because dried solution was blocking the tip of the needle; solution from the previous spin was covering the needle hole in the wall of the plastic housing for the electrospinner. After restarting, the bead formed, but the solution was not attracted to the mandrel when the voltage was applied. The needle was replaced and the mandrel was regrounded before starting the pump again. The Taylor cone formed immediately and maintained a good shape until it started to elongate after $0.20 \mathrm{~mL}$ of solution was ejected. It elongated and fell repeatedly for the remainder of the spin.

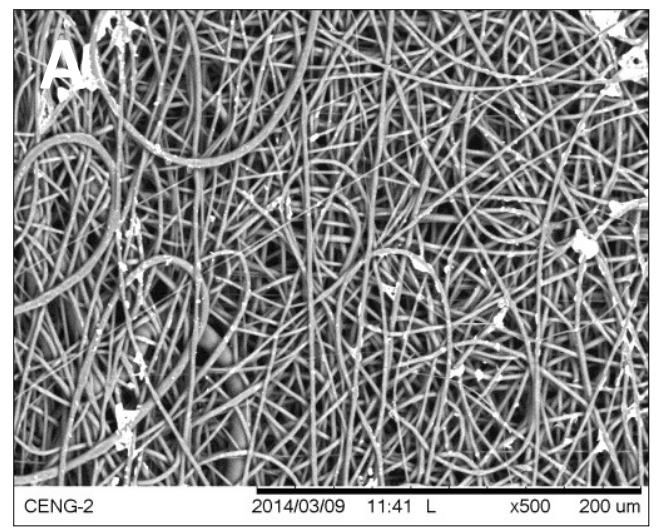

\begin{tabular}{|c|}
\hline Fiber Diameter $(\boldsymbol{\mu m})$ \\
\hline 2.678 \\
\hline 2.1326 \\
\hline 4.5125 \\
\hline 2.3906 \\
\hline 2.9626 \\
\hline 2.764 \\
\hline 1.4658 \\
\hline 2.3419 \\
\hline 2.1552 \\
\hline
\end{tabular}

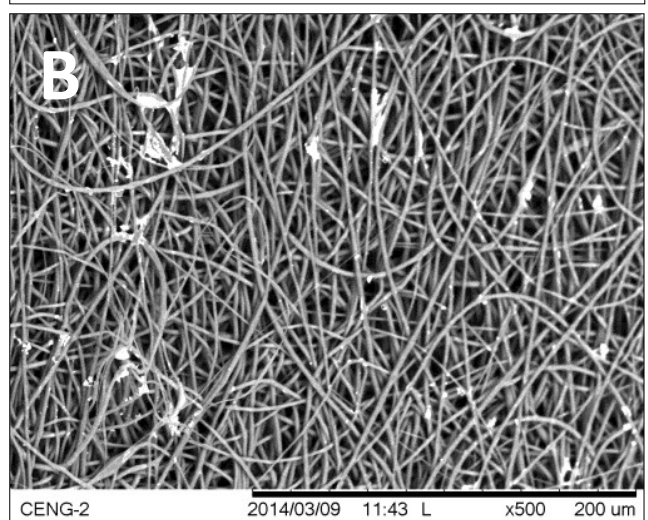

\begin{tabular}{|c|}
\hline Fiber Diameter $(\boldsymbol{\mu m})$ \\
\hline 1.2069 \\
\hline 1.8509 \\
\hline 1.4218 \\
\hline 1.9449 \\
\hline 2.208 \\
\hline 2.3212 \\
\hline 2.0726 \\
\hline 2.1552 \\
\hline 1.4655 \\
\hline
\end{tabular}

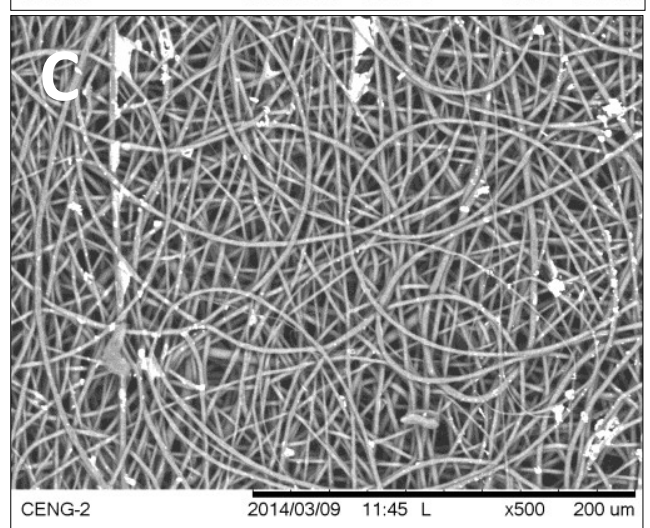

\begin{tabular}{|c|}
\hline Fiber Diameter $(\boldsymbol{\mu m})$ \\
\hline 1.8649 \\
\hline 2.9487 \\
\hline 3.4483 \\
\hline 1.9996 \\
\hline 1.6402 \\
\hline 1.4655 \\
\hline 2.248 \\
\hline 4.0526 \\
\hline 2.2563 \\
\hline
\end{tabular}

Figure $N$ 34: SEM images of Spin 12 Proximal location at 500x magnification; $(A)$ Image 1; (B) Image 2; (C) Image 3. 


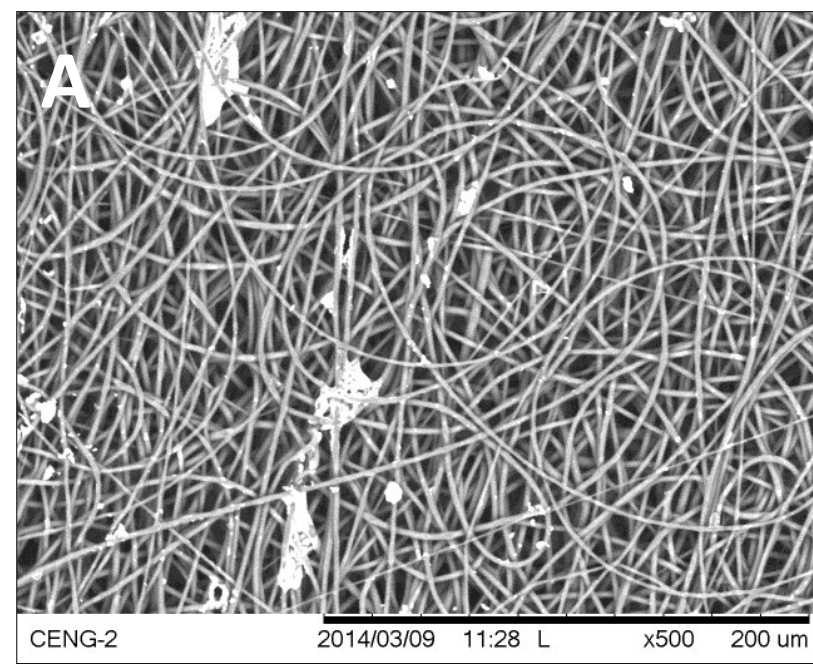

\begin{tabular}{|c|}
\hline Fiber Diameter $(\boldsymbol{\mu m})$ \\
\hline 1.8185 \\
\hline 2.707 \\
\hline 2.5862 \\
\hline 2.248 \\
\hline 2.8067 \\
\hline 2.274 \\
\hline 2.0292 \\
\hline 1.4451 \\
\hline 3.3751 \\
\hline
\end{tabular}

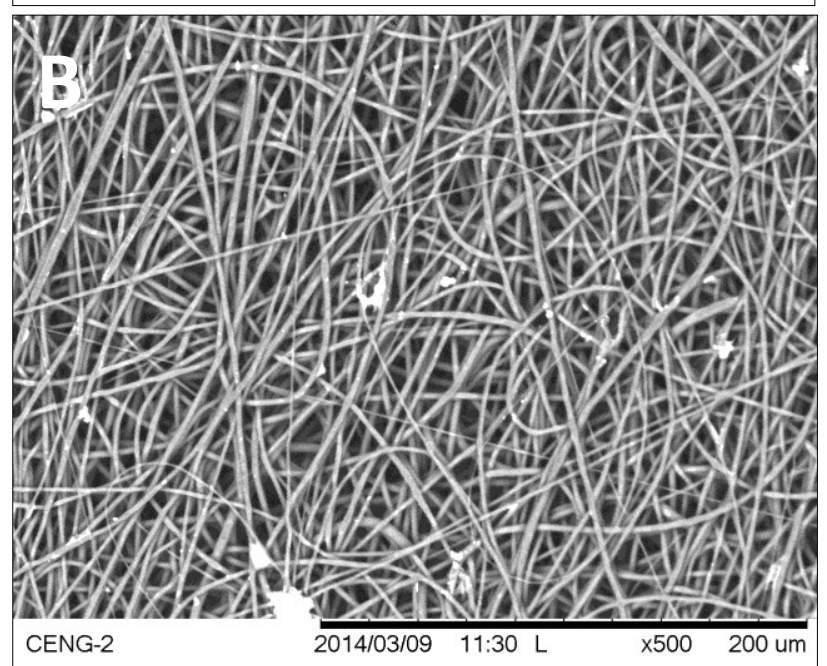

\begin{tabular}{|c|}
\hline Fiber Diameter $(\boldsymbol{\mu m})$ \\
\hline 2.0181 \\
\hline 1.5259 \\
\hline 1.7772 \\
\hline 2.4577 \\
\hline 1.6773 \\
\hline 1.656 \\
\hline 2.1826 \\
\hline 2.3844 \\
\hline 2.248 \\
\hline
\end{tabular}

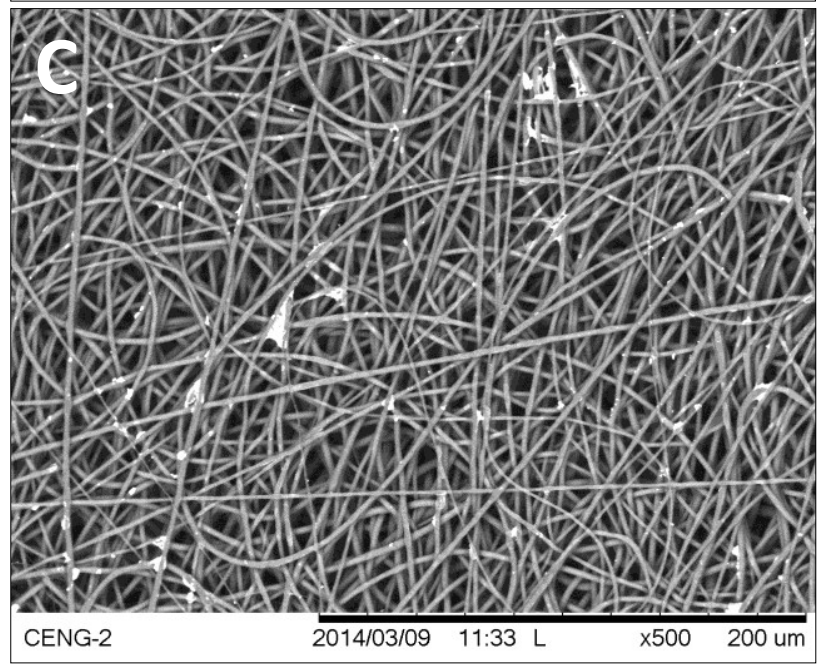

\begin{tabular}{|c|}
\hline Fiber Diameter $(\boldsymbol{\mu m})$ \\
\hline 1.8661 \\
\hline 1.944 \\
\hline 2.1326 \\
\hline 2.0833 \\
\hline 1.7754 \\
\hline 2.3419 \\
\hline 2.3292 \\
\hline 1.2834 \\
\hline 1.4356 \\
\hline
\end{tabular}

Figure N 35: SEM images of Spin 12 Medial location at 500x magnification; (A) Image 1; (B) Image 2; (C) Image 3. 


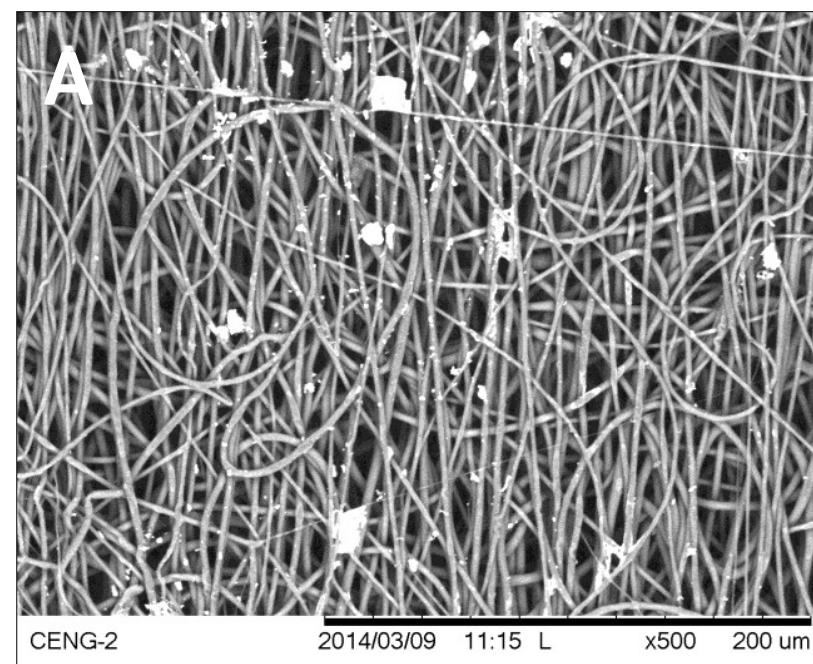

\begin{tabular}{|c|}
\hline Fiber Diameter $(\boldsymbol{\mu m})$ \\
\hline 2.8657 \\
\hline 2.9361 \\
\hline 2.8951 \\
\hline 2.4603 \\
\hline 2.7801 \\
\hline 3.0271 \\
\hline 2.7056 \\
\hline 2.0125 \\
\hline 1.681 \\
\hline
\end{tabular}

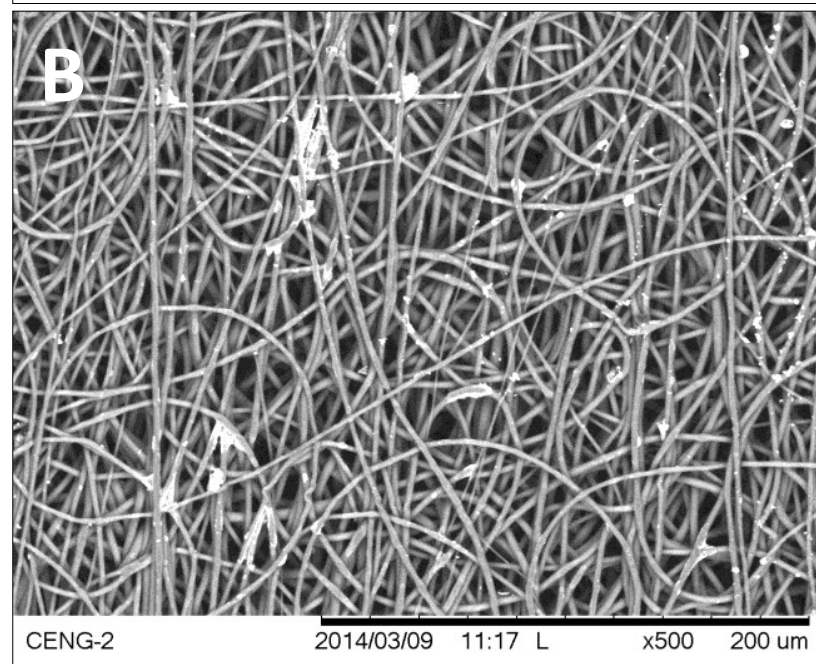

\begin{tabular}{|c|}
\hline Fiber Diameter $(\boldsymbol{\mu m})$ \\
\hline 2.2414 \\
\hline 2.5 \\
\hline 2.8461 \\
\hline 2.1336 \\
\hline 2.6932 \\
\hline 2.836 \\
\hline 2.0043 \\
\hline 2.3672 \\
\hline 3.445 \\
\hline
\end{tabular}

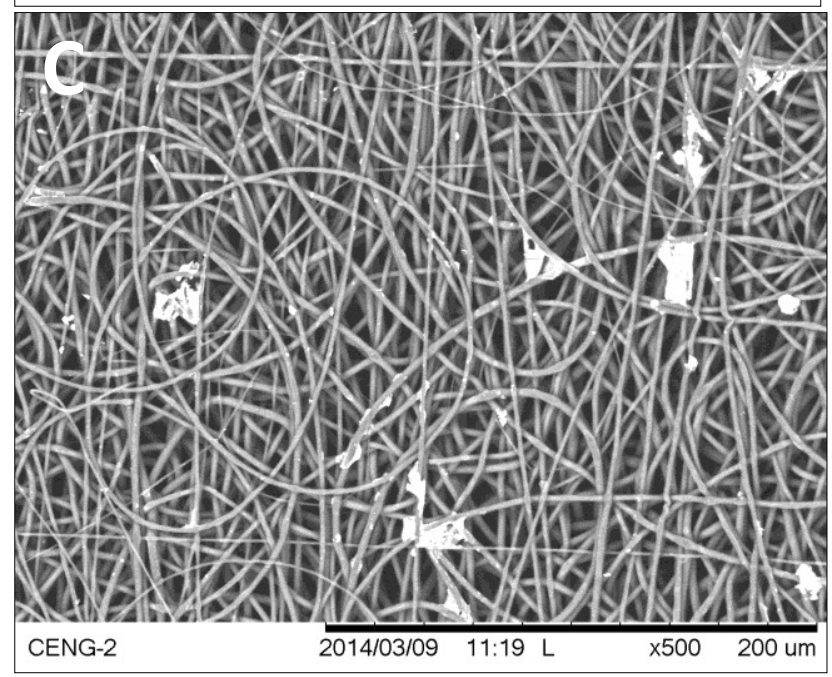

\begin{tabular}{|c|}
\hline Fiber Diameter $(\boldsymbol{\mu m})$ \\
\hline 2.1676 \\
\hline 2.0548 \\
\hline 1.2931 \\
\hline 1.4195 \\
\hline 1.8103 \\
\hline 2.7186 \\
\hline 1.945 \\
\hline 2.3003 \\
\hline 4.0186 \\
\hline
\end{tabular}

Figure N 36: SEM images of Spin 12 Distal location at 500x magnification; $(A)$ Image 1; (B) Image 2; (C) Image 3. 


\section{Spin 13}

Process Observations: The Taylor cone initially had trouble forming; it was repeatedly pulled off before completely forming. A stable cone finally formed after about $0.40 \mathrm{~mL}$. It then slowly elongated and fell repeatedly every $0.03 \mathrm{~mL}$.

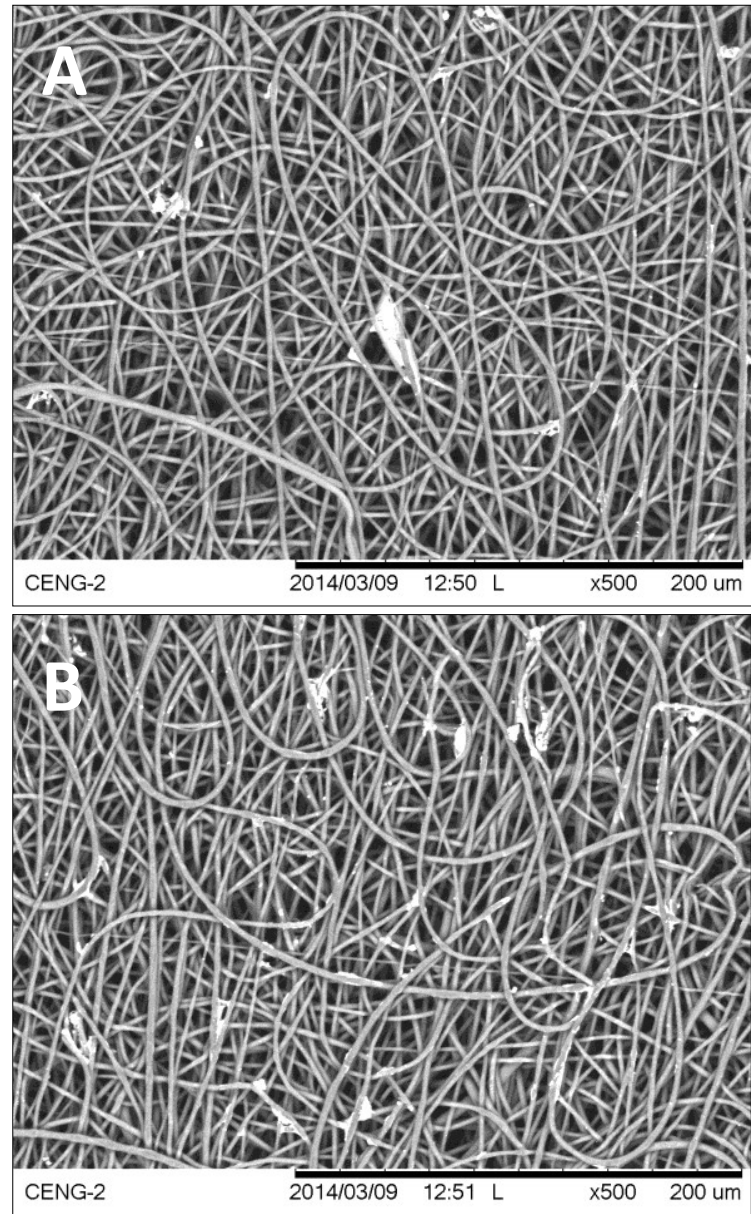

\begin{tabular}{|c|}
\hline Fiber Diameter $(\boldsymbol{\mu m})$ \\
\hline 1.9141 \\
\hline 2.6414 \\
\hline 2.1208 \\
\hline 1.681 \\
\hline 2.7056 \\
\hline 1.7179 \\
\hline 2.3132 \\
\hline 1.9996 \\
\hline 1.5423 \\
\hline
\end{tabular}

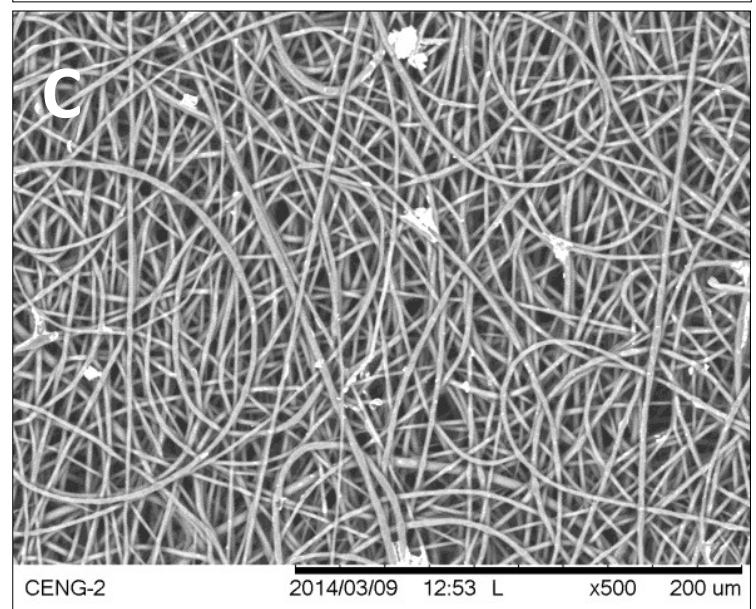

\begin{tabular}{|c|}
\hline Fiber Diameter $(\boldsymbol{\mu m})$ \\
\hline 1.9506 \\
\hline 2.5178 \\
\hline 2.7761 \\
\hline 1.7409 \\
\hline 2.0851 \\
\hline 2.6382 \\
\hline 1.9846 \\
\hline 2.6947 \\
\hline 2.0868 \\
\hline
\end{tabular}

Figure $N$ 37: SEM images of Spin 13 Proximal location at 500x magnification; (A) Image 1; (B) Image 2; (C) Image 3. 


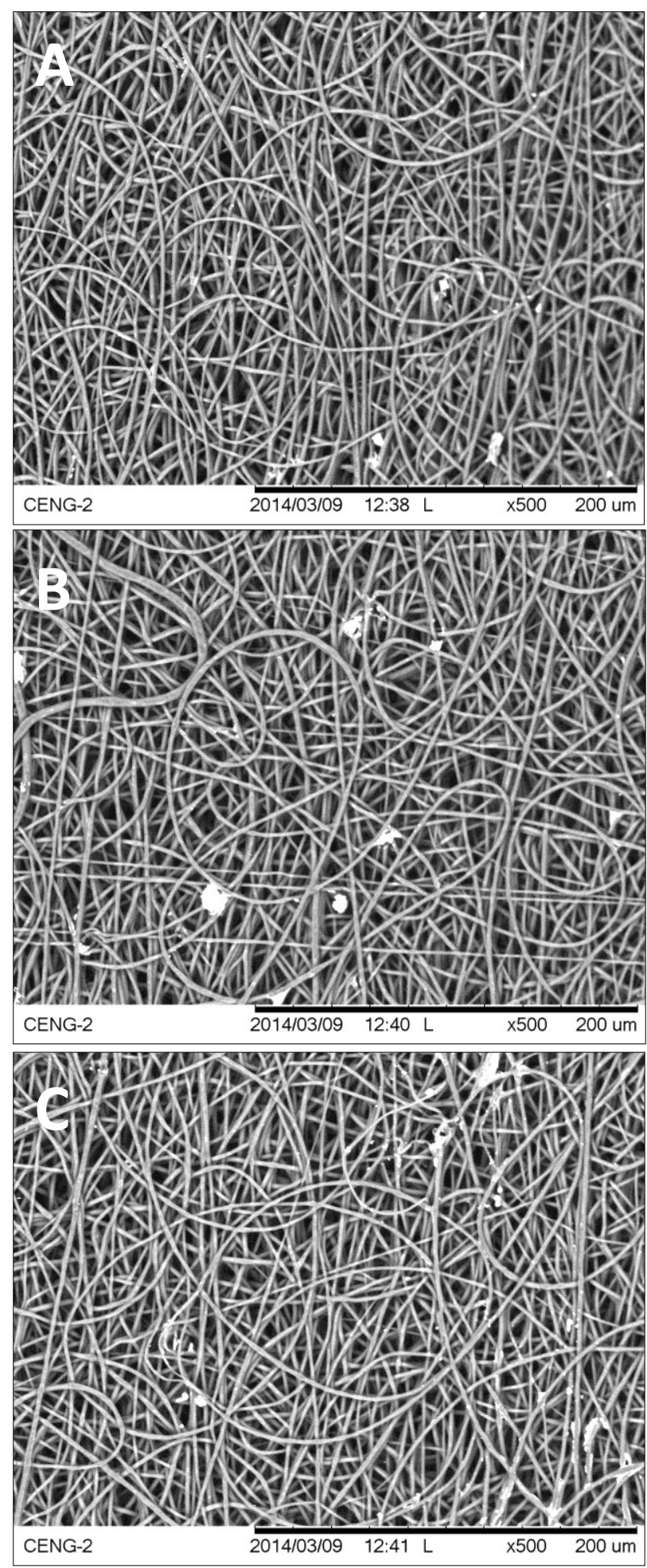

\begin{tabular}{|c|}
\hline Fiber Diameter $(\boldsymbol{\mu m})$ \\
\hline 2.248 \\
\hline 3.1202 \\
\hline 2.8806 \\
\hline 2.2381 \\
\hline 1.7564 \\
\hline 1.9027 \\
\hline 2.7921 \\
\hline 2.0251 \\
\hline 2.3419 \\
\hline
\end{tabular}

\begin{tabular}{|c|}
\hline Fiber Diameter $(\boldsymbol{\mu m})$ \\
\hline 2.4475 \\
\hline 1.6164 \\
\hline 1.6582 \\
\hline 2.1492 \\
\hline 2.1992 \\
\hline 2.6849 \\
\hline 2.7546 \\
\hline 2.2629 \\
\hline 2.3003 \\
\hline
\end{tabular}

\begin{tabular}{|c|}
\hline Fiber Diameter $(\boldsymbol{\mu m})$ \\
\hline 2.4014 \\
\hline 2.0851 \\
\hline 2.0199 \\
\hline 1.4587 \\
\hline 1.9044 \\
\hline 2.3535 \\
\hline 1.6823 \\
\hline 2.1336 \\
\hline 2.7438 \\
\hline
\end{tabular}

Figure $N$ 38: SEM images of Spin 13 Medial location at 500x magnification; (A) Image 1; (B) Image 2; (C) Image 3. 


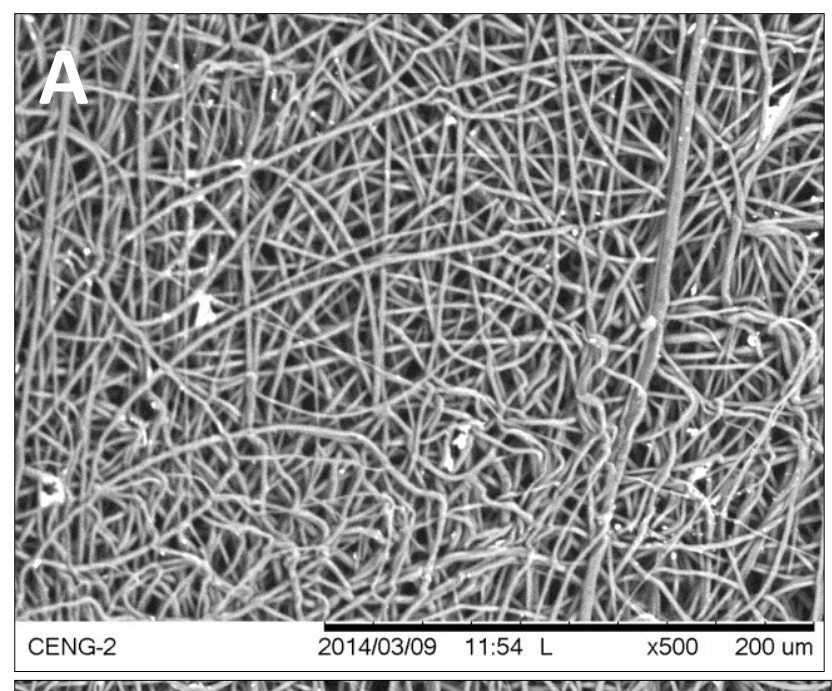

\begin{tabular}{|c|}
\hline Fiber Diameter $(\boldsymbol{\mu m})$ \\
\hline 1.8985 \\
\hline 2.7446 \\
\hline 2.707 \\
\hline 1.8795 \\
\hline 2.4705 \\
\hline 1.5896 \\
\hline 2.3196 \\
\hline 2.0851 \\
\hline 1.2027 \\
\hline
\end{tabular}

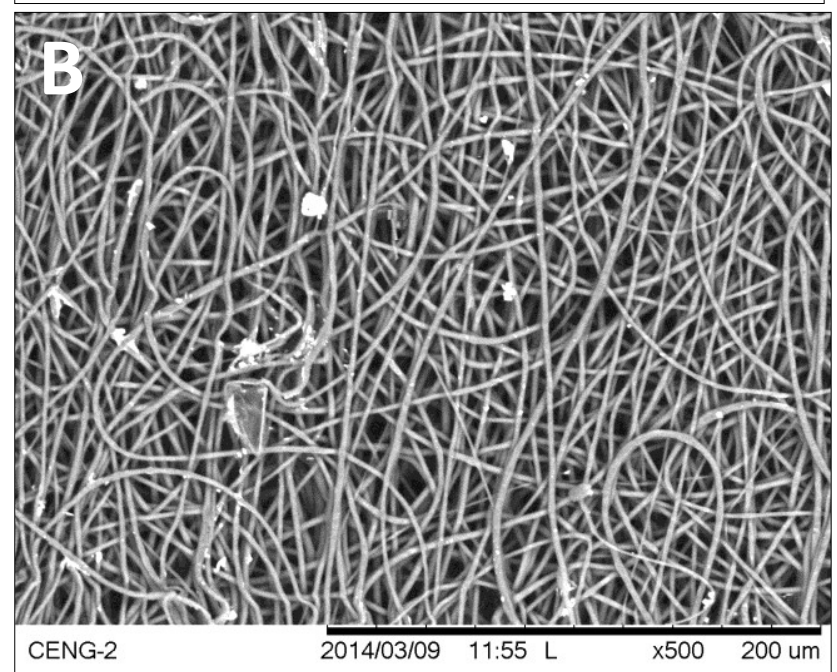

\begin{tabular}{|c|}
\hline Fiber Diameter $(\boldsymbol{\mu m})$ \\
\hline 2.6219 \\
\hline 2.7186 \\
\hline 2.2115 \\
\hline 2.7974 \\
\hline 2.5718 \\
\hline 1.9449 \\
\hline 2.3428 \\
\hline 2.6791 \\
\hline 1.6035 \\
\hline
\end{tabular}

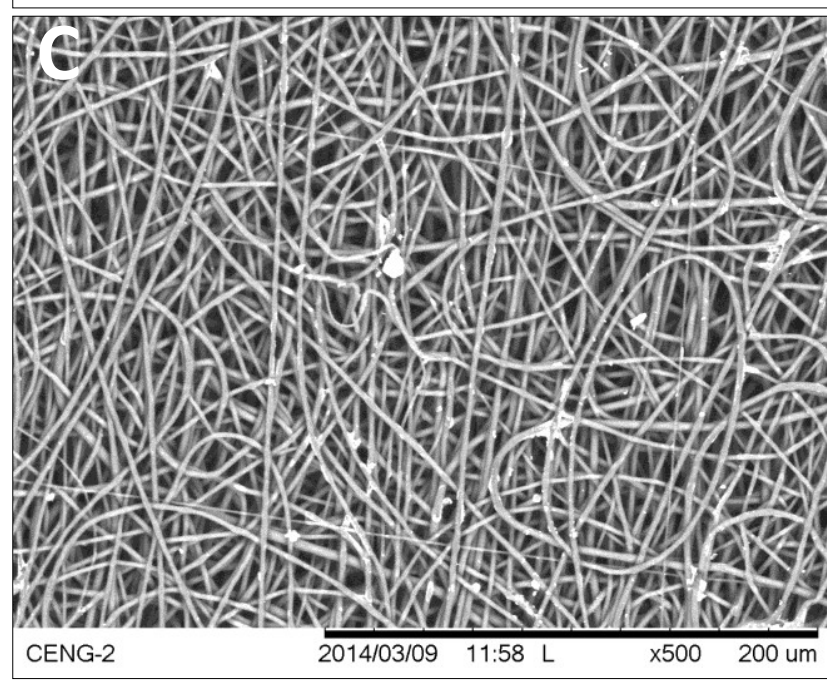

\begin{tabular}{|c|}
\hline Fiber Diameter $(\boldsymbol{\mu m})$ \\
\hline 2.4291 \\
\hline 2.3773 \\
\hline 1.9027 \\
\hline 2.2954 \\
\hline 2.707 \\
\hline 2.2563 \\
\hline 2.3968 \\
\hline 1.0991 \\
\hline 2.7832 \\
\hline
\end{tabular}

Figure $N$ 39: SEM images of Spin 13 Distal location at 500x magnification; $(A)$ Image 1; (B) Image 2; (C) Image 3. 


\section{Spin 15}

Process Observations: A stable, ideal-shaped Taylor cone formed immediately after the voltage was applied. It maintained its stability longer than usual, but began to elongate after $0.50 \mathrm{~mL}$ of solution was ejected. It elongated and fell less often than usual, occurring every $0.08 \mathrm{~mL}$.

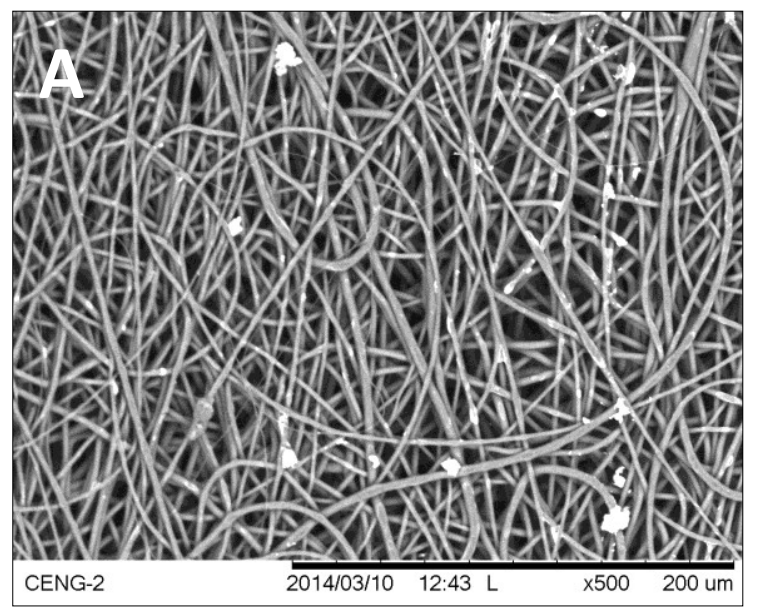

\begin{tabular}{|c|}
\hline Fiber Diameter $(\boldsymbol{\mu m})$ \\
\hline 2.1645 \\
\hline 1.8725 \\
\hline 1.8929 \\
\hline 2.3673 \\
\hline 4.0109 \\
\hline 3.8671 \\
\hline 2.6205 \\
\hline 2.0005 \\
\hline 2.5917 \\
\hline
\end{tabular}

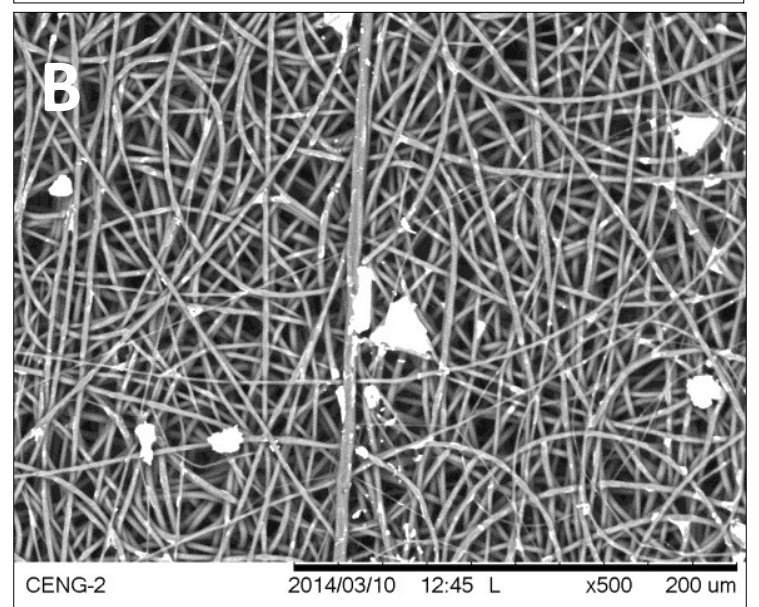

\begin{tabular}{|c|}
\hline Fiber Diameter $(\boldsymbol{\mu m})$ \\
\hline 2.3969 \\
\hline 1.9658 \\
\hline 0.9871 \\
\hline 1.9456 \\
\hline 2.6205 \\
\hline 0.985 \\
\hline 3.0576 \\
\hline 1.8929 \\
\hline 2.7868 \\
\hline
\end{tabular}

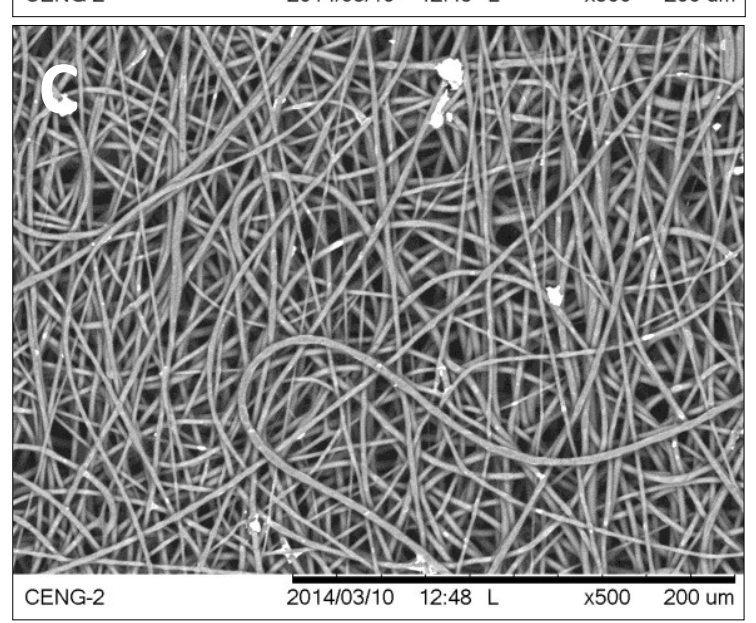

\begin{tabular}{|c|}
\hline Fiber Diameter $(\boldsymbol{\mu m})$ \\
\hline 2.5408 \\
\hline 2.8838 \\
\hline 2.101 \\
\hline 2.3626 \\
\hline 1.8252 \\
\hline 1.8614 \\
\hline 1.6753 \\
\hline 2.9388 \\
\hline 2.9239 \\
\hline
\end{tabular}

Figure N 40: SEM images of Spin 15 Proximal location at 500x magnification; (A) Image 1; (B) Image 2; (C) Image 3. 


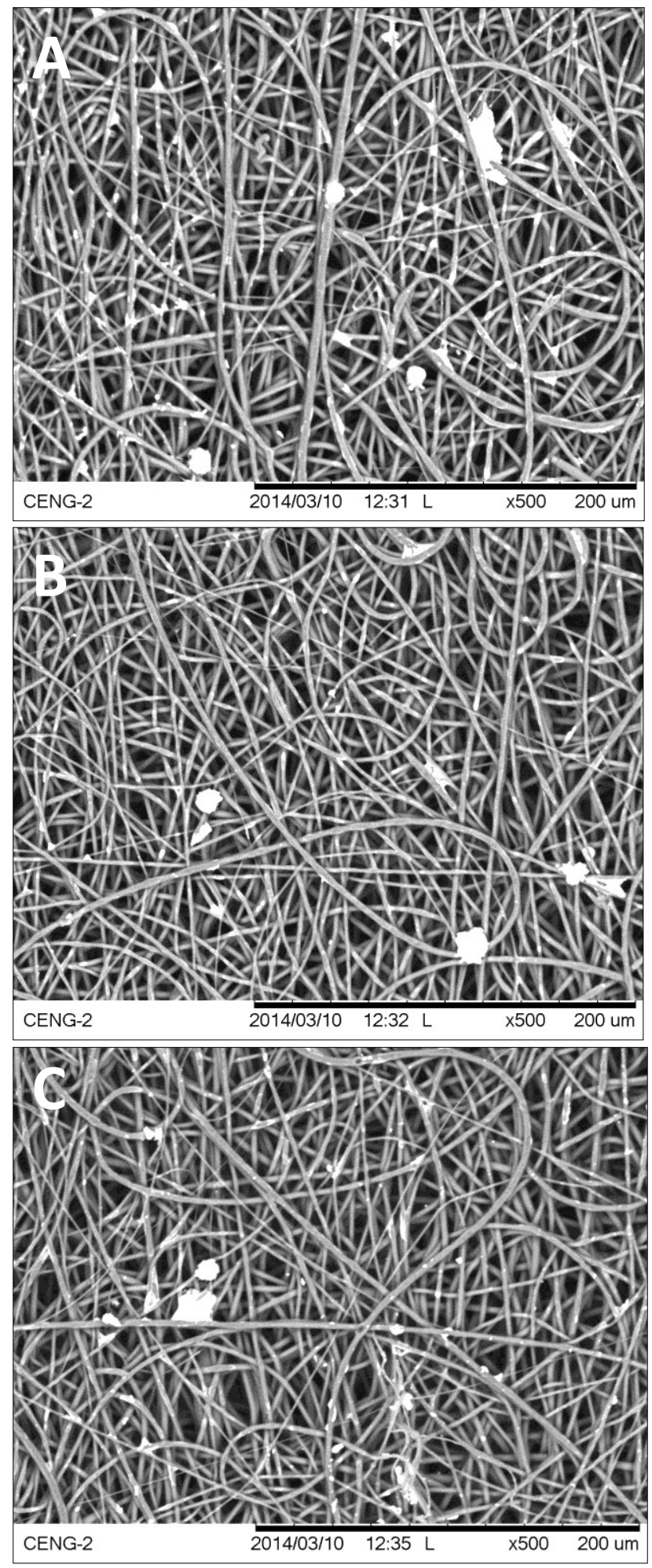

\begin{tabular}{|c|}
\hline Fiber Diameter $(\boldsymbol{\mu m})$ \\
\hline 1.7035 \\
\hline 2.9388 \\
\hline 2.8446 \\
\hline 3.8872 \\
\hline 1.2791 \\
\hline 1.7738 \\
\hline 2.7719 \\
\hline 2.4428 \\
\hline 3.5277 \\
\hline
\end{tabular}

\begin{tabular}{|c|}
\hline Fiber Diameter $(\boldsymbol{\mu m})$ \\
\hline 2.3626 \\
\hline 2.2371 \\
\hline 2.5813 \\
\hline 2.1941 \\
\hline 2.4967 \\
\hline 2.423 \\
\hline 2.6205 \\
\hline 2.1937 \\
\hline 2.4153 \\
\hline
\end{tabular}

\begin{tabular}{|c|}
\hline Fiber Diameter $(\boldsymbol{\mu m})$ \\
\hline 2.3736 \\
\hline 2.423 \\
\hline 2.2321 \\
\hline 2.4107 \\
\hline 1.5392 \\
\hline 3.1882 \\
\hline 2.9838 \\
\hline 2.5306 \\
\hline 2.1836 \\
\hline
\end{tabular}

Figure N 41: SEM images of Spin 15 Medial location at 500x magnification; $(A)$ Image 1; (B) Image 2; (C) Image 3. 


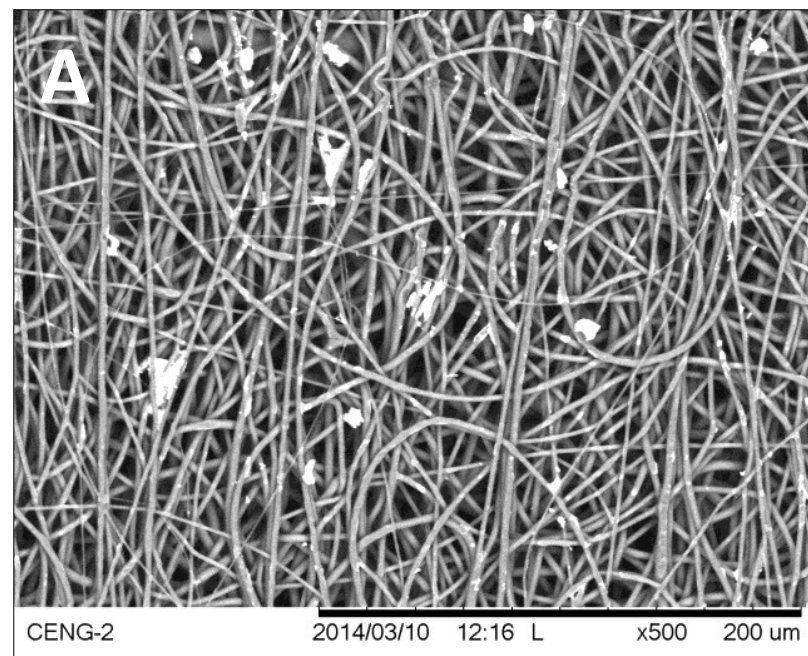

\begin{tabular}{|c|}
\hline Fiber Diameter $(\boldsymbol{\mu m})$ \\
\hline 2.3922 \\
\hline 2.4153 \\
\hline 2.5308 \\
\hline 3.4943 \\
\hline 1.8151 \\
\hline 3.4751 \\
\hline 1.8394 \\
\hline 1.0884 \\
\hline 3.2297 \\
\hline
\end{tabular}

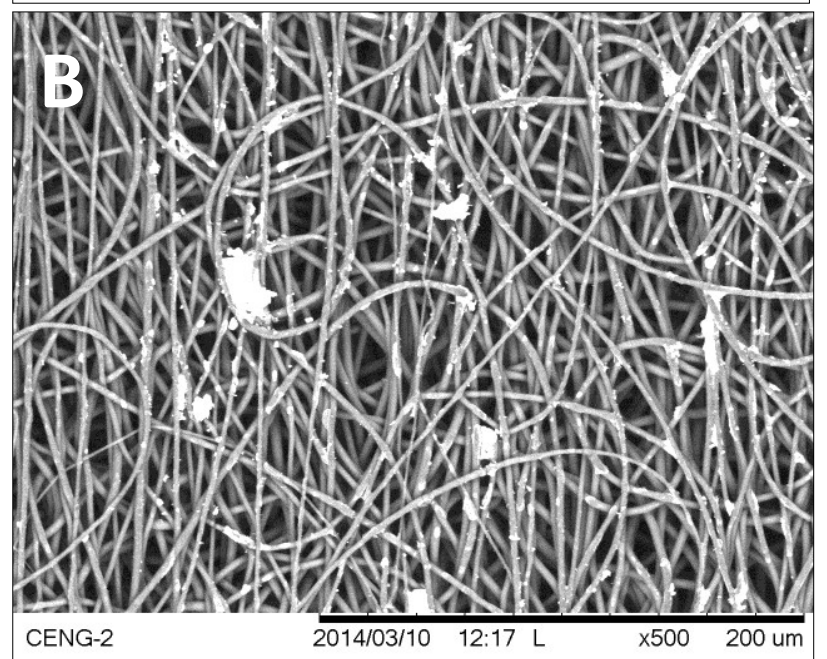

\begin{tabular}{|c|}
\hline Fiber Diameter $(\boldsymbol{\mu m})$ \\
\hline 3.1166 \\
\hline 2.6576 \\
\hline 3.4038 \\
\hline 2.6673 \\
\hline 1.2413 \\
\hline 2.7774 \\
\hline 2.3626 \\
\hline 3.8095 \\
\hline 3.8671 \\
\hline
\end{tabular}

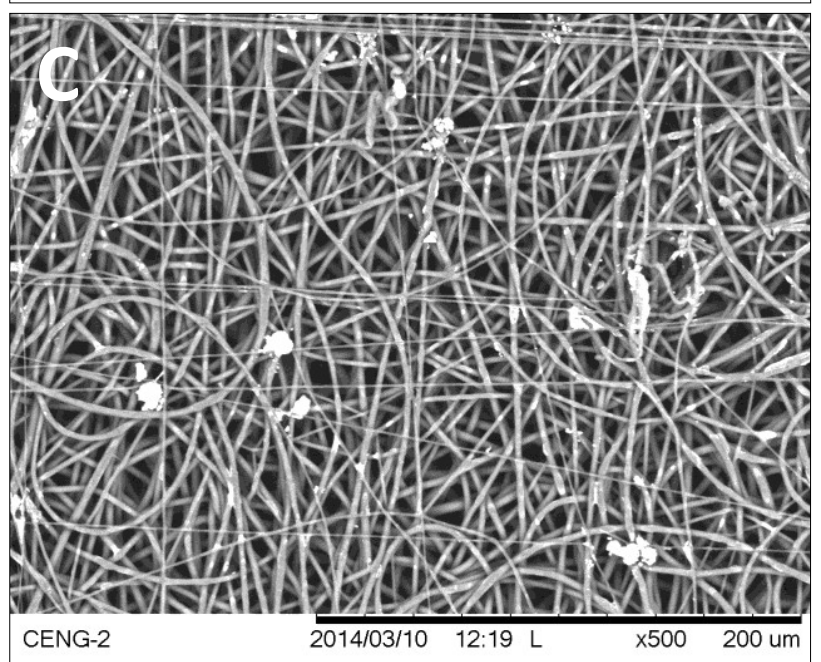

\begin{tabular}{|c|}
\hline Fiber Diameter $(\boldsymbol{\mu m})$ \\
\hline 1.0467 \\
\hline 3.7428 \\
\hline 2.4257 \\
\hline 0.8604 \\
\hline 2.2437 \\
\hline 1.5621 \\
\hline 1.7976 \\
\hline 1.889 \\
\hline 1.7717 \\
\hline
\end{tabular}

Figure $N$ 42: SEM images of Spin 15 Distal location at 500x magnification; (A) Image 1; (B) Image 2; (C) Image 3. 


\section{Spin 17}

Process Observations: The Taylor cone was small, stable, and maintained an ideal shape until it began elongating after $0.30 \mathrm{~mL}$ of solution was ejected. It elongated and fell at a slow rate, occurring every $0.07 \mathrm{~mL}$.

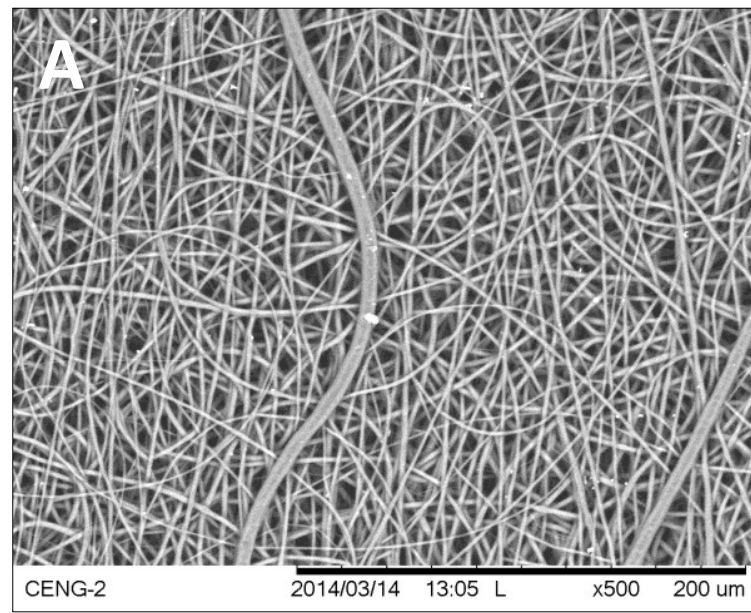

\begin{tabular}{|c|}
\hline Fiber Diameter $(\boldsymbol{\mu m})$ \\
\hline 2.7397 \\
\hline 2.1809 \\
\hline 1.124 \\
\hline 1.7349 \\
\hline 1.7327 \\
\hline 2.0564 \\
\hline 1.5276 \\
\hline 2.1569 \\
\hline 3.138 \\
\hline
\end{tabular}

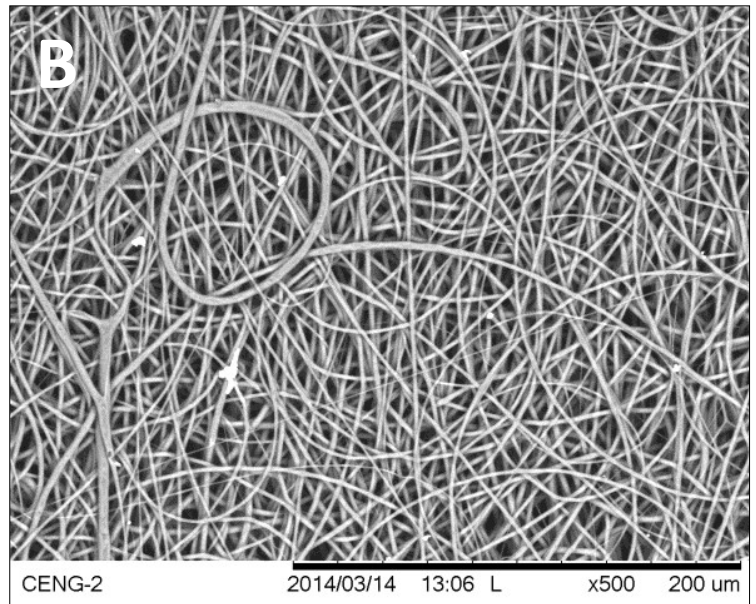

\begin{tabular}{|c|}
\hline Fiber Diameter $(\boldsymbol{\mu m})$ \\
\hline 1.6582 \\
\hline 1.7688 \\
\hline 1.8449 \\
\hline 2.4505 \\
\hline 1.7112 \\
\hline 2.3546 \\
\hline 2.2414 \\
\hline 2.07 \\
\hline 1.2801 \\
\hline
\end{tabular}

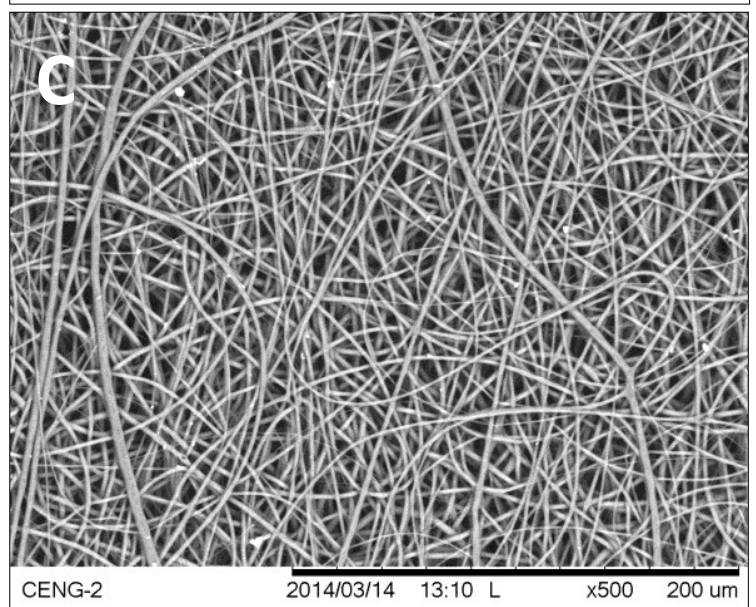

\begin{tabular}{|c|}
\hline Fiber Diameter $(\boldsymbol{\mu m})$ \\
\hline 2.353 \\
\hline 1.5135 \\
\hline 2.0761 \\
\hline 1.5276 \\
\hline 1.9902 \\
\hline 2.0975 \\
\hline 1.6649 \\
\hline 2.0833 \\
\hline 1.3383 \\
\hline
\end{tabular}

Figure N 43: SEM images of Spin 17 Proximal location at 500x magnification; (A) Image 1; (B) Image 2; (C) Image 3. 


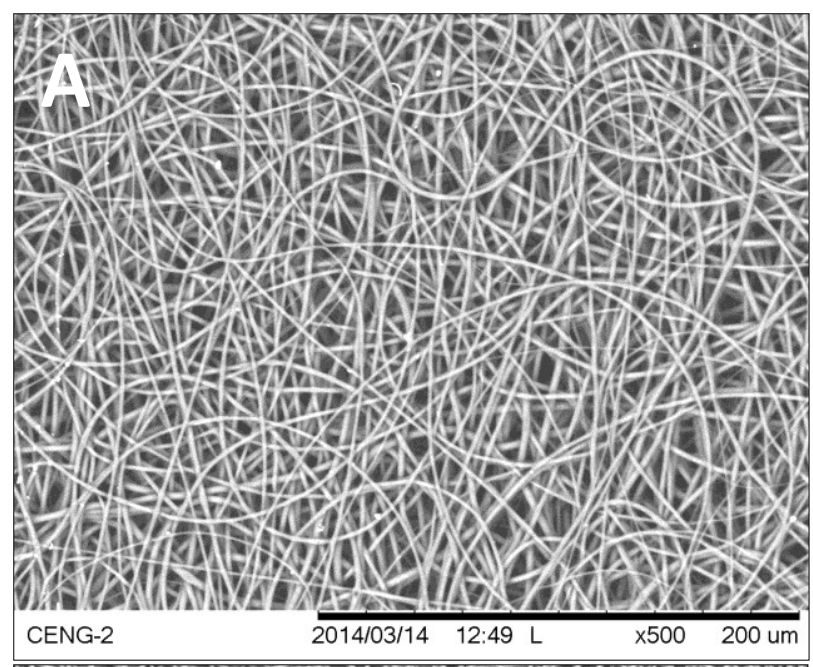

\begin{tabular}{|c|}
\hline Fiber Diameter $(\boldsymbol{\mu m})$ \\
\hline 2.3844 \\
\hline 1.7583 \\
\hline 1.7588 \\
\hline 1.6164 \\
\hline 1.9044 \\
\hline 1.8267 \\
\hline 2.1204 \\
\hline 1.8267 \\
\hline 1.6582 \\
\hline
\end{tabular}

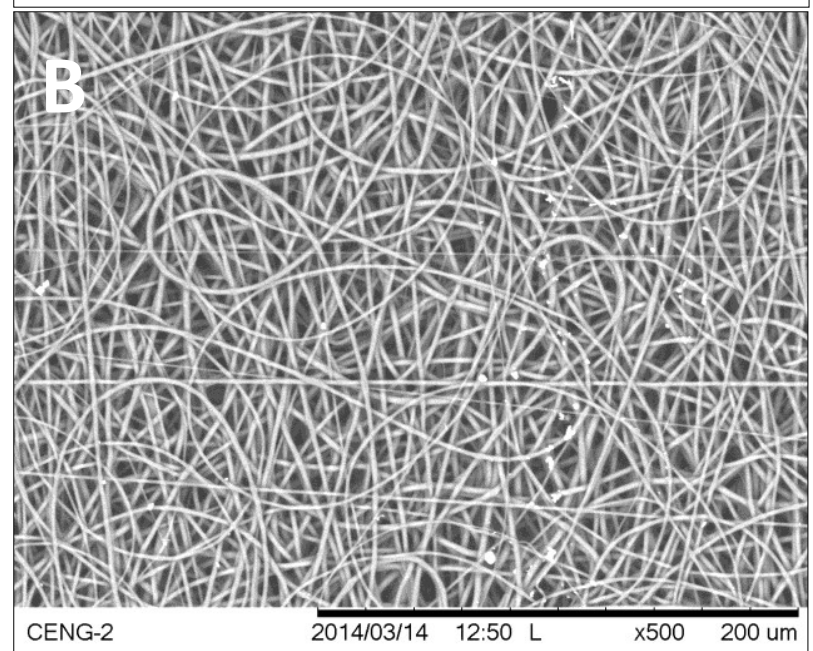

\begin{tabular}{|c|}
\hline Fiber Diameter $(\boldsymbol{\mu m})$ \\
\hline 0.9522 \\
\hline 1.9276 \\
\hline 2.2414 \\
\hline 2.1292 \\
\hline 2.243 \\
\hline 2.5059 \\
\hline 2.0833 \\
\hline 1.6981 \\
\hline 1.5995 \\
\hline
\end{tabular}

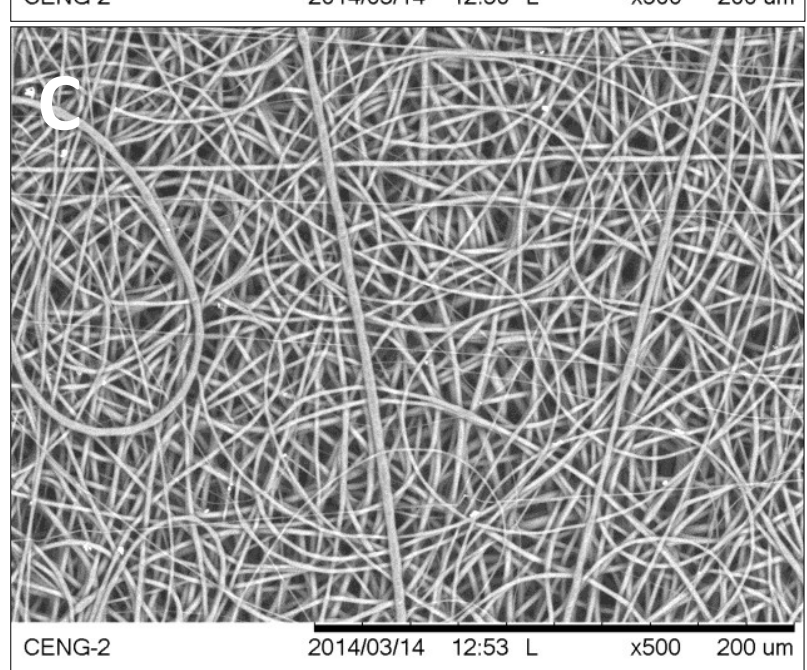

\begin{tabular}{|c|}
\hline Fiber Diameter $(\boldsymbol{\mu m})$ \\
\hline 2.2563 \\
\hline 2.6849 \\
\hline 1.9677 \\
\hline 1.6357 \\
\hline 2.8719 \\
\hline 2.0054 \\
\hline 1.656 \\
\hline 1.8287 \\
\hline 2.0346 \\
\hline
\end{tabular}

Figure $N$ 44: SEM images of Spin 17 Medial location at 500x magnification; (A) Image 1; (B) Image 2; (C) Image 3. 


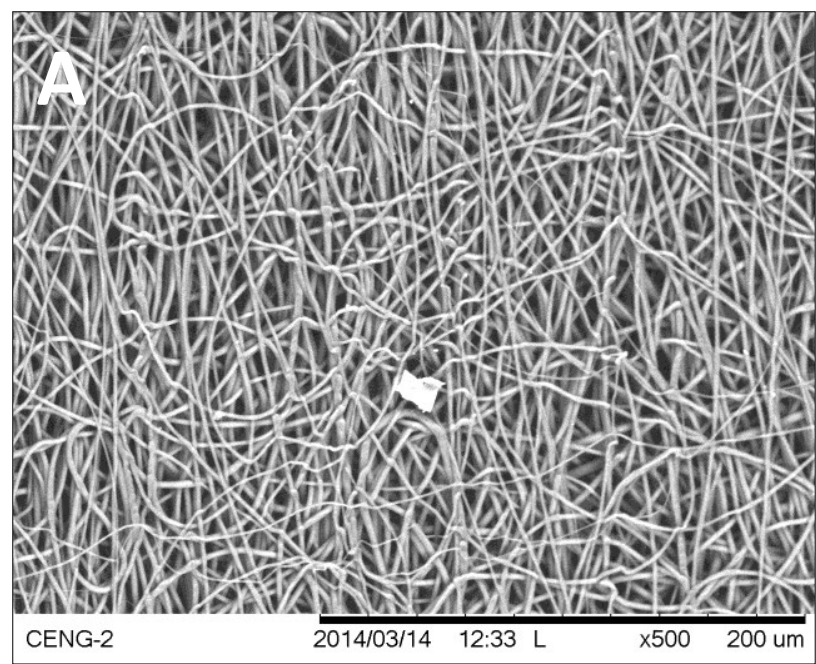

\begin{tabular}{|c|}
\hline Fiber Diameter $(\boldsymbol{\mu m})$ \\
\hline 2.6091 \\
\hline 2.5059 \\
\hline 2.1826 \\
\hline 2.5249 \\
\hline 2.0708 \\
\hline 2.2677 \\
\hline 2.4276 \\
\hline 2.6183 \\
\hline 1.8509 \\
\hline
\end{tabular}

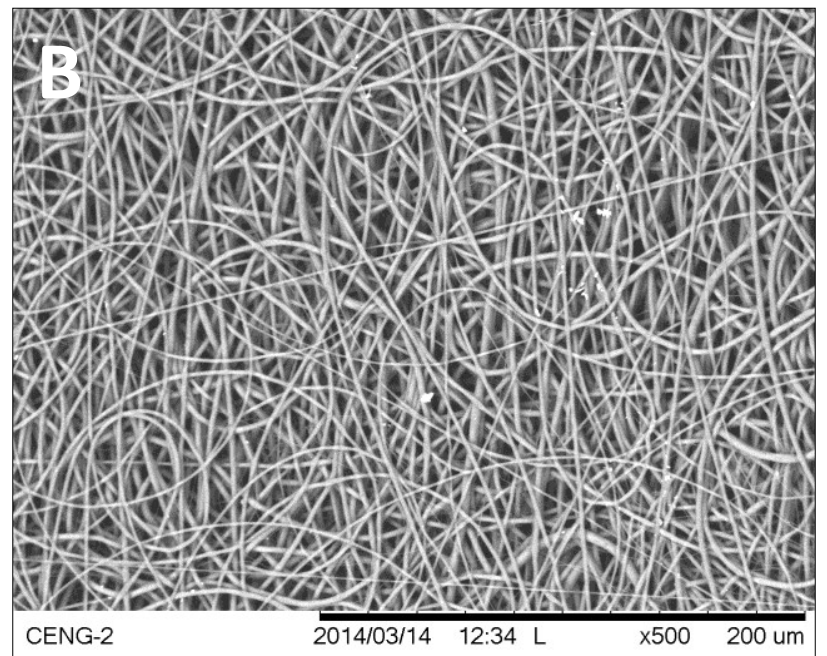

\begin{tabular}{|c|}
\hline Fiber Diameter $(\boldsymbol{\mu m})$ \\
\hline 1.0904 \\
\hline 2.6219 \\
\hline 2.105 \\
\hline 2.07 \\
\hline 1.4139 \\
\hline 1.9223 \\
\hline 2.7009 \\
\hline 1.8034 \\
\hline 2.3428 \\
\hline
\end{tabular}

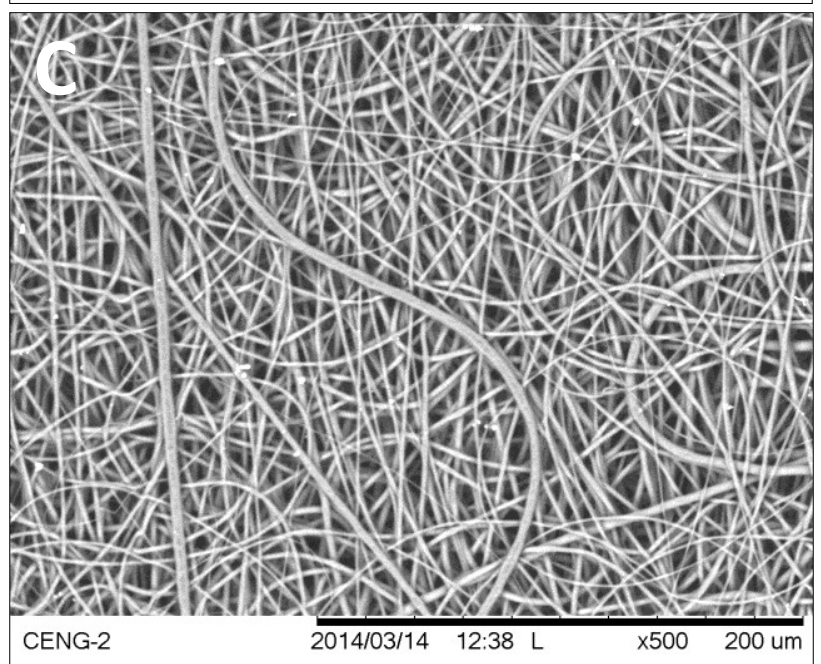

\begin{tabular}{|c|}
\hline Fiber Diameter $(\boldsymbol{\mu m})$ \\
\hline 3.8831 \\
\hline 2.3212 \\
\hline 2.7586 \\
\hline 2.5354 \\
\hline 2.0761 \\
\hline 1.6265 \\
\hline 2.3212 \\
\hline 1.5276 \\
\hline 1.9506 \\
\hline
\end{tabular}

Figure N 45: SEM images of Spin 17 Distal location at 500x magnification; $(A)$ Image 1; (B) Image 2; (C) Image 3. 


\section{Spin 18}

Process Observations: The cone was stable and well-shaped initially, but split into two tips; one larger cone pointed at a downward angle while a smaller cone pointed upward. This cone fell and formed a stable cone again. It then elongated and fell repeatedly for the remainder of the spin. The solution began to pulse around $2.00 \mathrm{~mL}$.

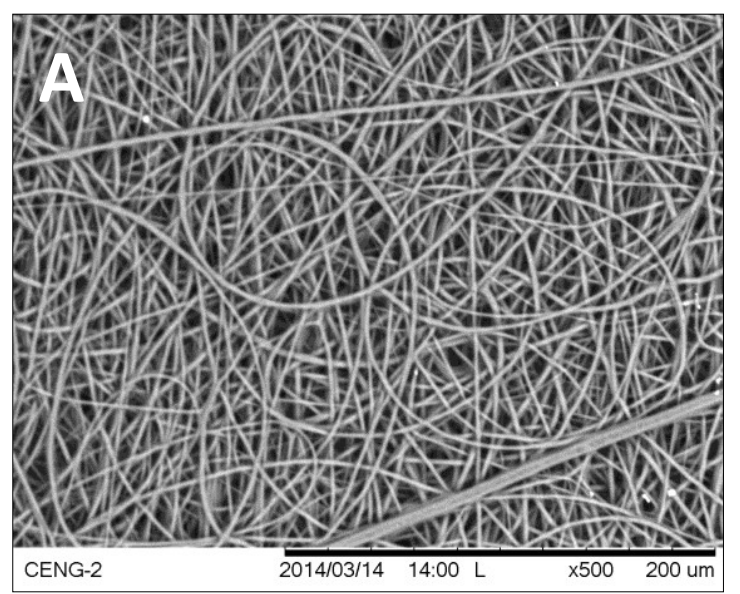

\begin{tabular}{|c|}
\hline Fiber Diameter $(\boldsymbol{\mu m})$ \\
\hline 2.1204 \\
\hline 2.073 \\
\hline 2.484 \\
\hline 1.681 \\
\hline 2.808 \\
\hline 2.3738 \\
\hline 2.7643 \\
\hline 1.6197 \\
\hline 2.8461 \\
\hline
\end{tabular}

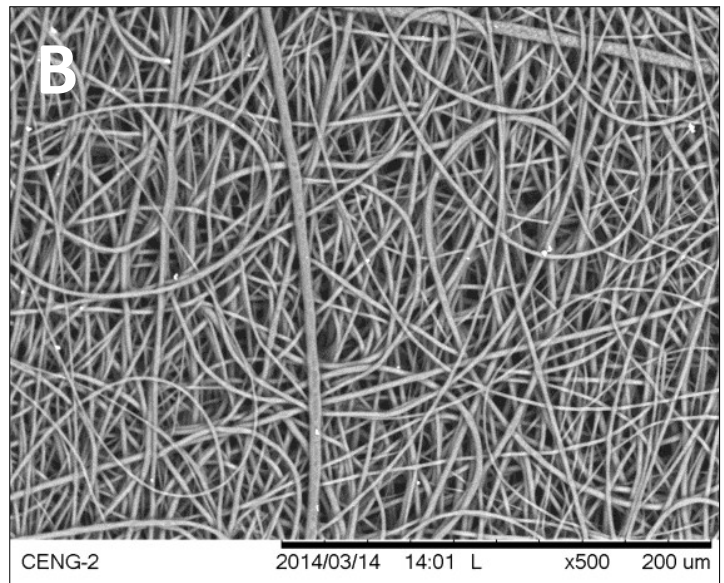

\begin{tabular}{|c|}
\hline Fiber Diameter $(\boldsymbol{\mu m})$ \\
\hline 2.5108 \\
\hline 2.5934 \\
\hline 1.656 \\
\hline 1.9141 \\
\hline 1.8907 \\
\hline 2.6374 \\
\hline 2.1292 \\
\hline 1.7772 \\
\hline 1.4832 \\
\hline
\end{tabular}

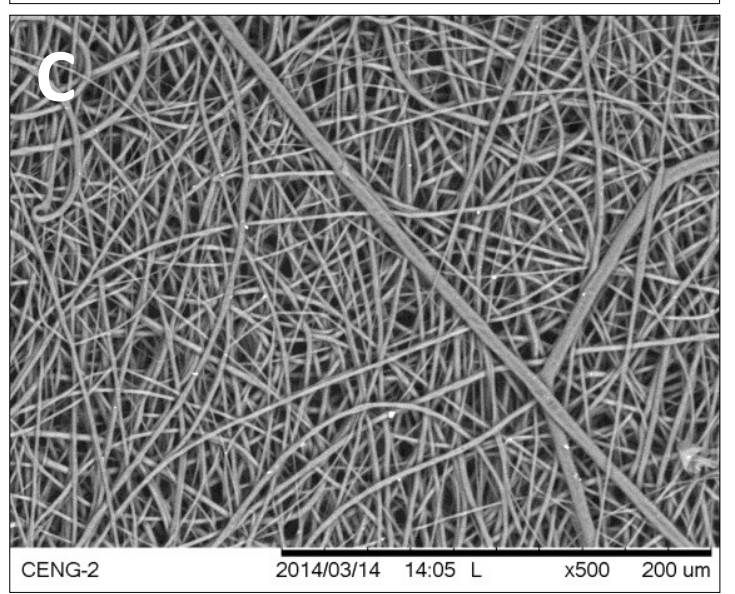

\begin{tabular}{|c|}
\hline Fiber Diameter $(\boldsymbol{\mu m})$ \\
\hline 2.0051 \\
\hline 1.0904 \\
\hline 3.138 \\
\hline 3.1082 \\
\hline 2.9323 \\
\hline 1.4681 \\
\hline 1.6981 \\
\hline 3.0951 \\
\hline 1.9044 \\
\hline
\end{tabular}

Figure $N$ 46: SEM images of Spin 18 Proximal location at 500x magnification; $(A)$ Image 1; (B) Image 2; (C) Image 3. 


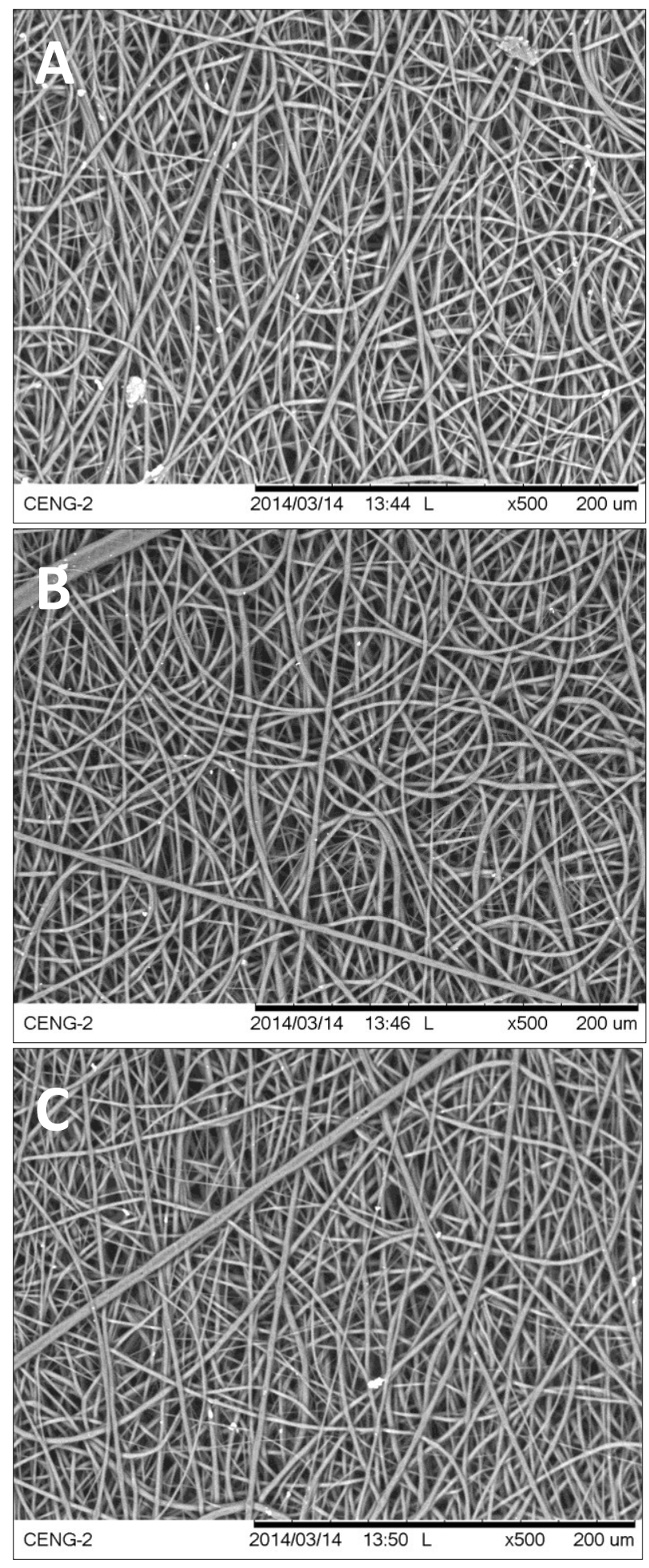

\begin{tabular}{|c|}
\hline Fiber Diameter $(\boldsymbol{\mu m})$ \\
\hline 2.76 \\
\hline 3.1332 \\
\hline 2.7056 \\
\hline 2.5059 \\
\hline 0.9285 \\
\hline 2.9462 \\
\hline 2.3546 \\
\hline 1.9083 \\
\hline 1.9276 \\
\hline
\end{tabular}

\begin{tabular}{|c|}
\hline Fiber Diameter $(\boldsymbol{\mu m})$ \\
\hline 2.248 \\
\hline 2.5059 \\
\hline 1.8985 \\
\hline 1.5541 \\
\hline 1.8124 \\
\hline 1.5349 \\
\hline 2.3844 \\
\hline 3.3477 \\
\hline 2.2906 \\
\hline
\end{tabular}

\begin{tabular}{|c|}
\hline Fiber Diameter $(\boldsymbol{\mu m})$ \\
\hline 1.7434 \\
\hline 6.0252 \\
\hline 2.9361 \\
\hline 2.0851 \\
\hline 2.4276 \\
\hline 1.9828 \\
\hline 1.7434 \\
\hline 2.6932 \\
\hline 2.0761 \\
\hline
\end{tabular}

Figure N 47: SEM images of Spin 18 Medial location at 500x magnification; (A) Image 1, (B) Image 2, (C) Image 3. 


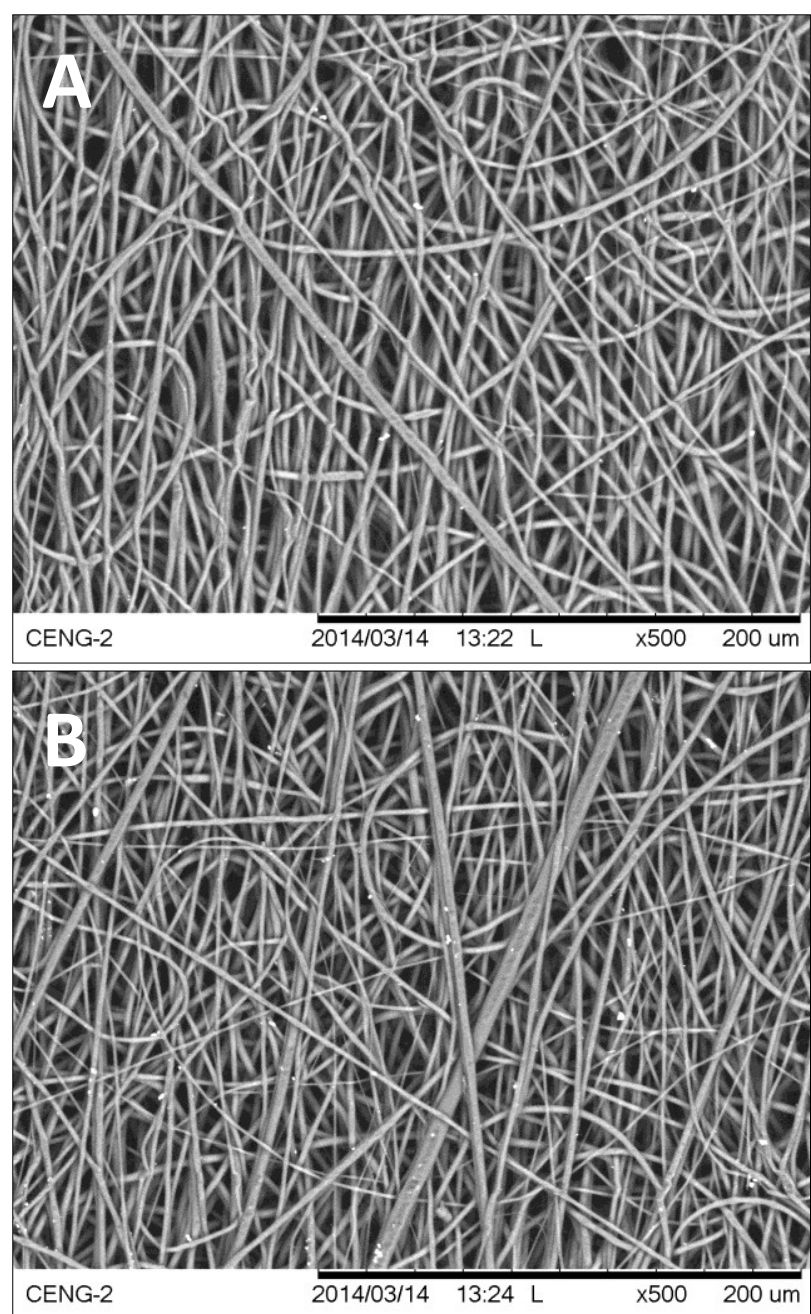

\begin{tabular}{|c|}
\hline Fiber Diameter $(\boldsymbol{\mu m})$ \\
\hline 1.1207 \\
\hline 2.4045 \\
\hline 2.069 \\
\hline 2.6932 \\
\hline 2.6987 \\
\hline 2.9925 \\
\hline 2.208 \\
\hline 2.5 \\
\hline 2.9323 \\
\hline
\end{tabular}

\begin{tabular}{|c|}
\hline Fiber Diameter $(\boldsymbol{\mu m})$ \\
\hline 2.3164 \\
\hline 1.9141 \\
\hline 1.7456 \\
\hline 2.8657 \\
\hline 1.4451 \\
\hline 7.3655 \\
\hline 2.9424 \\
\hline 1.9828 \\
\hline 1.4681 \\
\hline
\end{tabular}

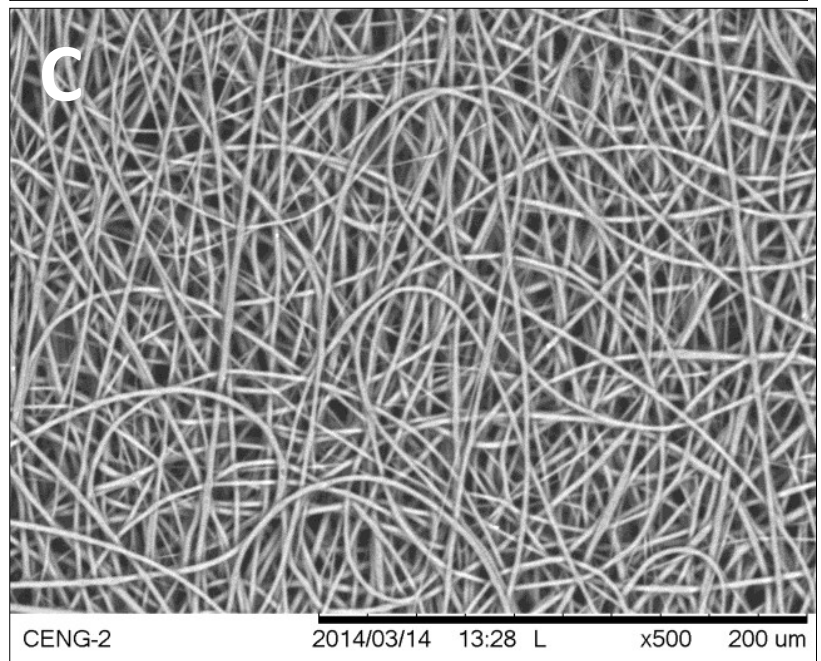

\begin{tabular}{|c|}
\hline Fiber Diameter $(\boldsymbol{\mu m})$ \\
\hline 1.9658 \\
\hline 1.8926 \\
\hline 1.8985 \\
\hline 1.9373 \\
\hline 2.5133 \\
\hline 2.2759 \\
\hline 2.5602 \\
\hline 1.0663 \\
\hline 1.9658 \\
\hline
\end{tabular}

Figure N 48:SEM images of Spin 18 Distal location at 500x

magnification; (A) Image 1, (B) Image 2, (C) Image 3. 


\section{Spin 19}

Process Observations: The Taylor cone was stable and well-shaped initially. It began to elongate for about $0.03 \mathrm{~mL}$ and fell repeatedly for the entire spin. The solution started pulsing at the cone around $2.50 \mathrm{~mL}$.

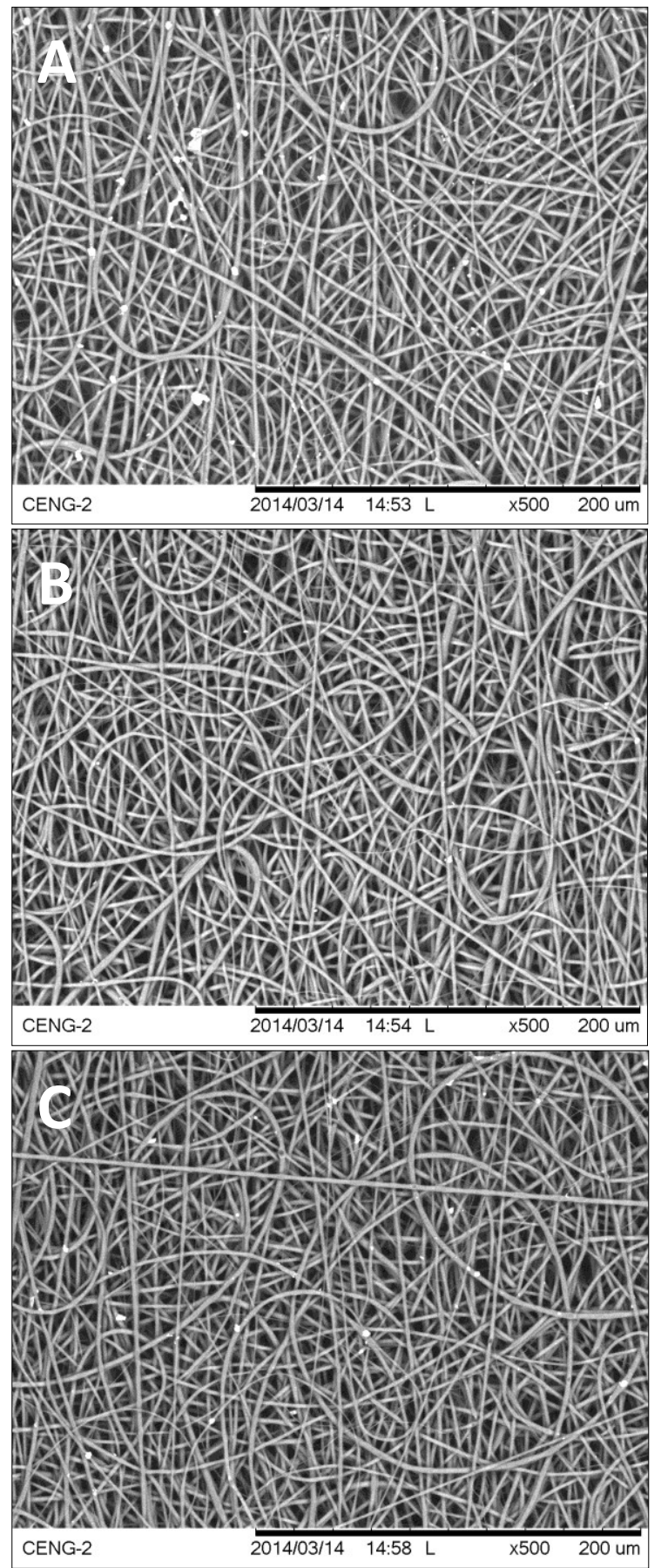

\begin{tabular}{|c|}
\hline Fiber Diameter $(\boldsymbol{\mu m})$ \\
\hline 1.5613 \\
\hline 1.7456 \\
\hline 3.294 \\
\hline 1.9141 \\
\hline 2.5631 \\
\hline 2.0993 \\
\hline 1.7772 \\
\hline 1.8287 \\
\hline 2.2414 \\
\hline
\end{tabular}

\begin{tabular}{|c|}
\hline Fiber Diameter $(\boldsymbol{\mu m})$ \\
\hline 2.2776 \\
\hline 1.7434 \\
\hline 2.7492 \\
\hline 1.5613 \\
\hline 1.6357 \\
\hline 1.4655 \\
\hline 1.7772 \\
\hline 1.9846 \\
\hline 1.647 \\
\hline
\end{tabular}

Figure N 49: SEM images of Spin 19 Proximal location at 500x magnification; (A) Image 1; (B) Image 2; (C) Image 3. 


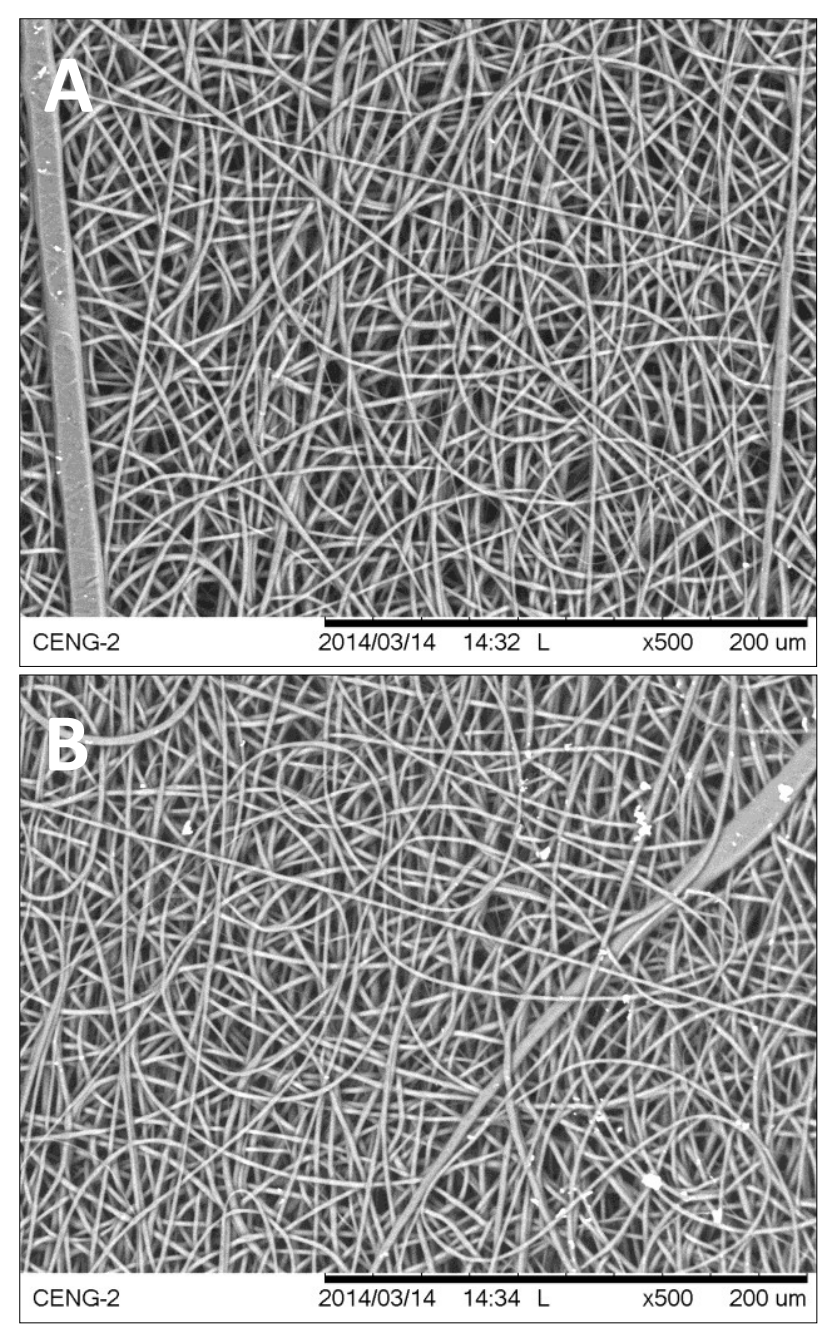

\begin{tabular}{|c|}
\hline Fiber Diameter $(\boldsymbol{\mu m})$ \\
\hline 1.8907 \\
\hline 2.1344 \\
\hline 2.0761 \\
\hline 2.5354 \\
\hline 2.0491 \\
\hline 2.5718 \\
\hline 1.857 \\
\hline 1.8429 \\
\hline 2.2628 \\
\hline
\end{tabular}

\begin{tabular}{|c|}
\hline Fiber Diameter $(\boldsymbol{\mu m})$ \\
\hline 1.7751 \\
\hline 1.4756 \\
\hline 2.5237 \\
\hline 1.7456 \\
\hline 1.857 \\
\hline 4.0112 \\
\hline 2.5833 \\
\hline 2.1552 \\
\hline 2.208 \\
\hline
\end{tabular}

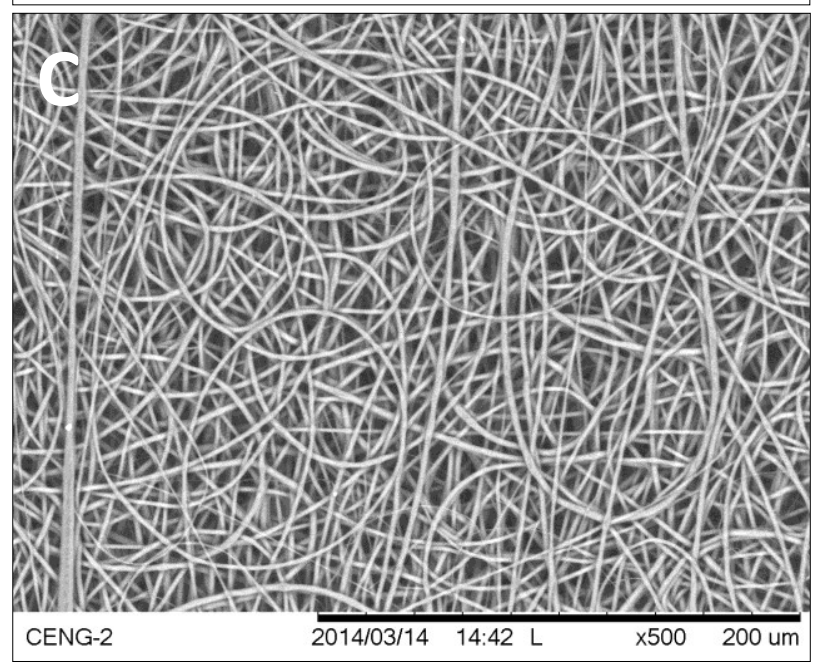

\begin{tabular}{|c|}
\hline Fiber Diameter $(\boldsymbol{\mu m})$ \\
\hline 2.8657 \\
\hline 1.9044 \\
\hline 2.3781 \\
\hline 1.9373 \\
\hline 2.0851 \\
\hline 2.76 \\
\hline 2.5237 \\
\hline 2.2381 \\
\hline 2.2628 \\
\hline
\end{tabular}

Figure N 50: SEM images of Spin 19 Medial location at 500x magnification; (A) Image 1; (B) Image 2; (C) Image 3. 


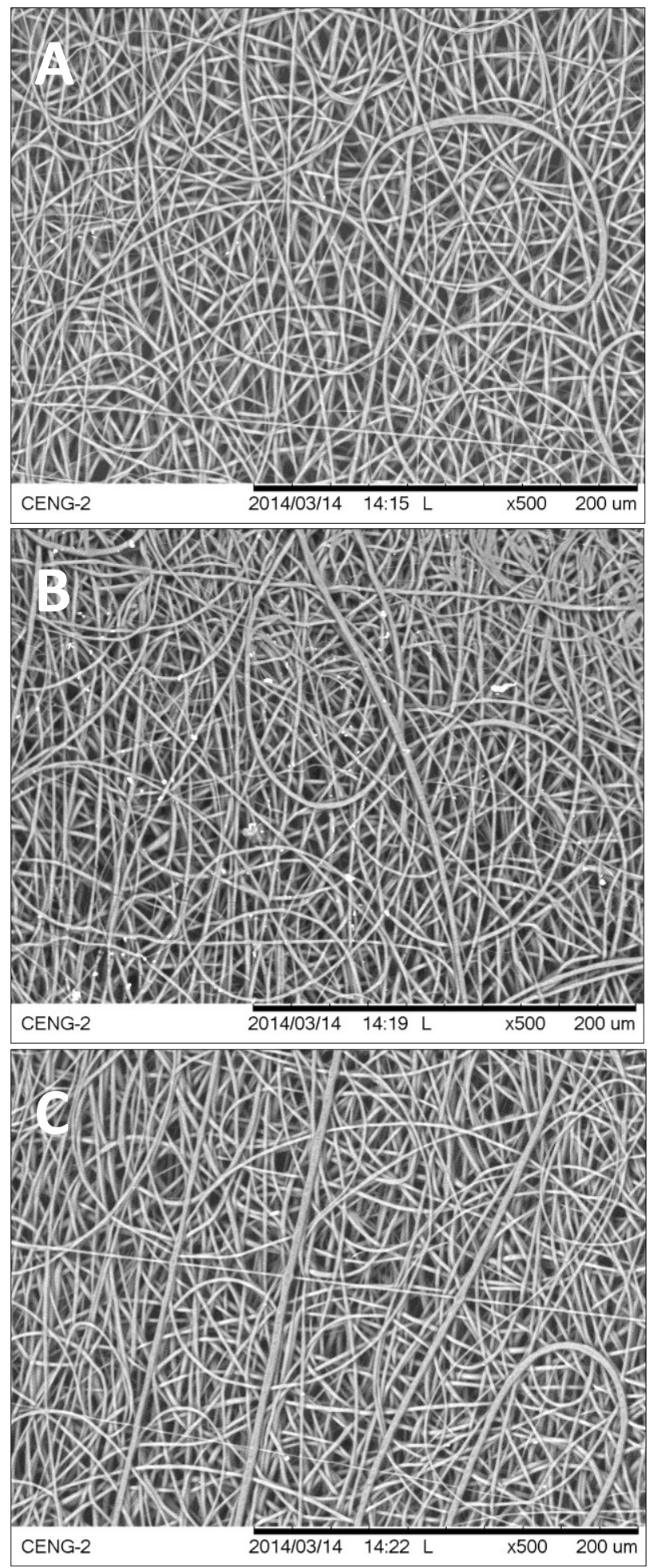

\begin{tabular}{|c|}
\hline Fiber Diameter $(\boldsymbol{\mu m})$ \\
\hline 2.0346 \\
\hline 1.6981 \\
\hline 2.76 \\
\hline 2.2414 \\
\hline 1.5541 \\
\hline 2.1979 \\
\hline 2.2563 \\
\hline 1.6937 \\
\hline 2.3451 \\
\hline
\end{tabular}

\begin{tabular}{|c|}
\hline Fiber Diameter $(\boldsymbol{\mu m})$ \\
\hline 1.9996 \\
\hline 1.8287 \\
\hline 1.6649 \\
\hline 1.8185 \\
\hline 1.5731 \\
\hline 2.0851 \\
\hline 2.0833 \\
\hline 2.069 \\
\hline 2.6557 \\
\hline
\end{tabular}

\begin{tabular}{|c|}
\hline Fiber Diameter $(\boldsymbol{\mu m})$ \\
\hline 3.2462 \\
\hline 1.8287 \\
\hline 3.331 \\
\hline 4.3112 \\
\hline 1.4756 \\
\hline 1.1501 \\
\hline 1.9083 \\
\hline 2.1621 \\
\hline 2.2381 \\
\hline
\end{tabular}

Figure N 51: SEM images of Spin 19 Distal location at 500x magnification; $(A)$ Image 1; $(B)$ Image 2; $(C)$ Image 3. 


\section{Spin 20}

Process Observations: Solution from the previous spin got onto the tip of the needle through the hole in the plastic housing. The bead formed sooner than usual, but when the voltage was applied the bead stretched out into branches. The bead was actually the solution from the previous spin, so the solution had not yet actually traveled through the needle. Solution from the syringe finally appeared and pushed the branched cone off after $0.50 \mathrm{~mL}$ of solution was ejected. A well-shaped cone formed, but elongated every 0.02 $\mathrm{mL}$ and fell repeatedly. The solution started pulsing around $2.00 \mathrm{~mL}$.

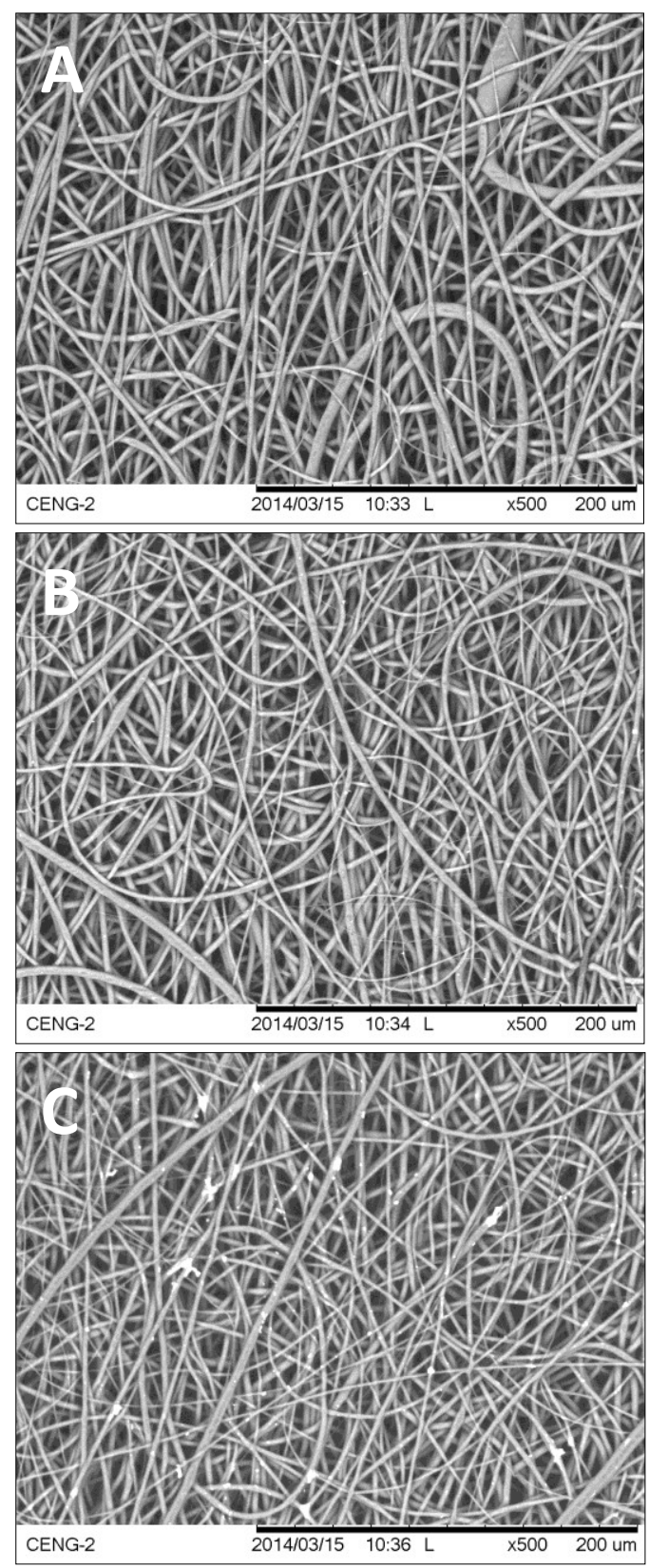

\begin{tabular}{|c|}
\hline Fiber Diameter $(\boldsymbol{\mu m})$ \\
\hline 3.6207 \\
\hline 1.8185 \\
\hline 1.7938 \\
\hline 2.8773 \\
\hline 2.5833 \\
\hline 6.3875 \\
\hline 1.1976 \\
\hline 3.372 \\
\hline 1.3187 \\
\hline
\end{tabular}

\begin{tabular}{|c|}
\hline Fiber Diameter $(\boldsymbol{\mu m})$ \\
\hline 0.9325 \\
\hline 2.0761 \\
\hline 2.3546 \\
\hline 1.7772 \\
\hline 2.0051 \\
\hline 3.445 \\
\hline 2.1758 \\
\hline 1.9902 \\
\hline 3.2405 \\
\hline
\end{tabular}

\begin{tabular}{|c|}
\hline Fiber Diameter $(\boldsymbol{\mu m})$ \\
\hline 2.5602 \\
\hline 1.6265 \\
\hline 2.2563 \\
\hline 2.9424 \\
\hline 3.2996 \\
\hline 3.199 \\
\hline 2.8278 \\
\hline 1.4033 \\
\hline 2.8461 \\
\hline
\end{tabular}

Figure N 52: SEM images of Spin 20 Proximal location at 500x magnification; (A) Image 1; (B) Image 2; (C) Image 3. 


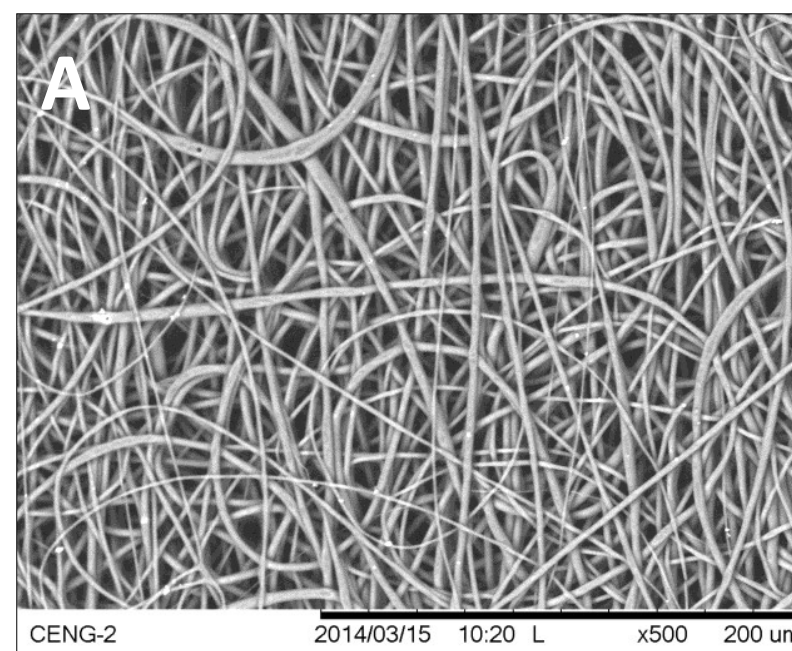

\begin{tabular}{|c|}
\hline Fiber Diameter $(\boldsymbol{\mu m})$ \\
\hline 1.4218 \\
\hline 3.1792 \\
\hline 2.069 \\
\hline 3.1082 \\
\hline 3.0369 \\
\hline 1.9141 \\
\hline 3.3874 \\
\hline 3.2279 \\
\hline 1.5896 \\
\hline
\end{tabular}

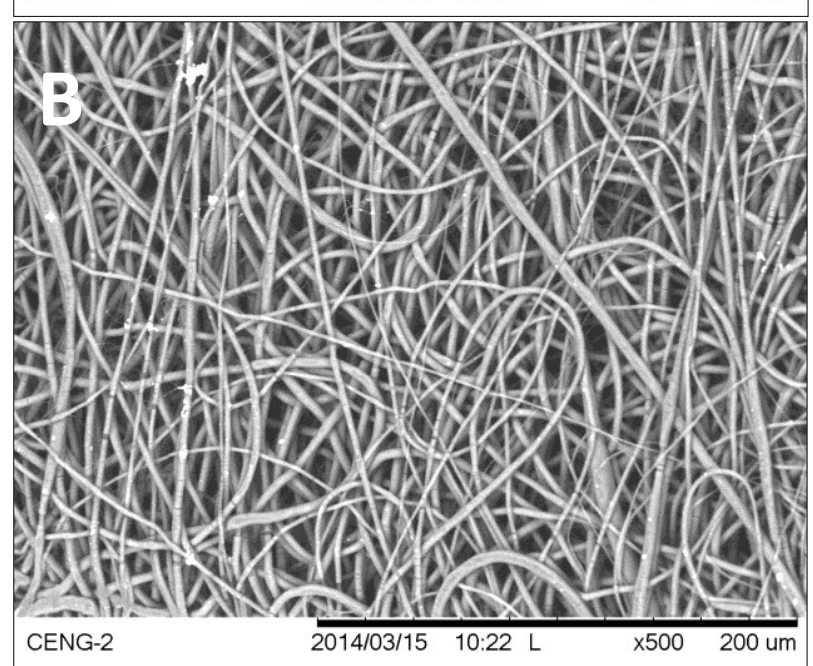

\begin{tabular}{|c|}
\hline Fiber Diameter $(\boldsymbol{\mu m})$ \\
\hline 3.2861 \\
\hline 1.4681 \\
\hline 2.2414 \\
\hline 2.6219 \\
\hline 3.1463 \\
\hline 1.5849 \\
\hline 1.3494 \\
\hline 1.7434 \\
\hline 3.1154 \\
\hline
\end{tabular}

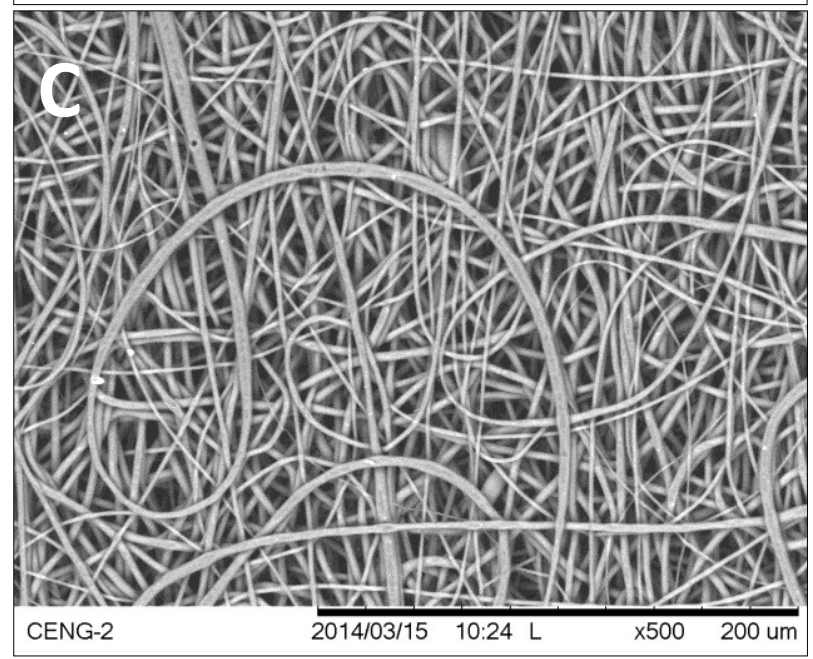

\begin{tabular}{|c|}
\hline Fiber Diameter $(\boldsymbol{\mu m})$ \\
\hline 1.2931 \\
\hline 2.3781 \\
\hline 1.7772 \\
\hline 2.5059 \\
\hline 2.3419 \\
\hline 2.764 \\
\hline 2.7801 \\
\hline 1.9141 \\
\hline 2.9424 \\
\hline
\end{tabular}

Figure N 53: SEM images of Spin 20 Medial location at 500x magnification; (A) Image 1; (B) Image 2; (C) Image 3. 


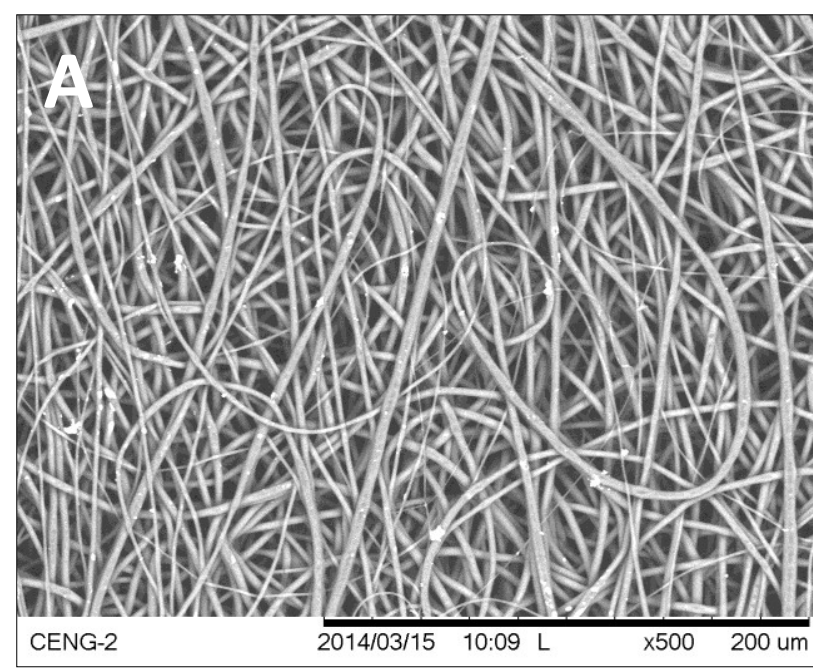

\begin{tabular}{|c|}
\hline Fiber Diameter $(\boldsymbol{\mu m})$ \\
\hline 2.8067 \\
\hline 1.6582 \\
\hline 1.8103 \\
\hline 2.8966 \\
\hline 3.1034 \\
\hline 1.4832 \\
\hline 2.9487 \\
\hline 2.9626 \\
\hline 1.4218 \\
\hline
\end{tabular}

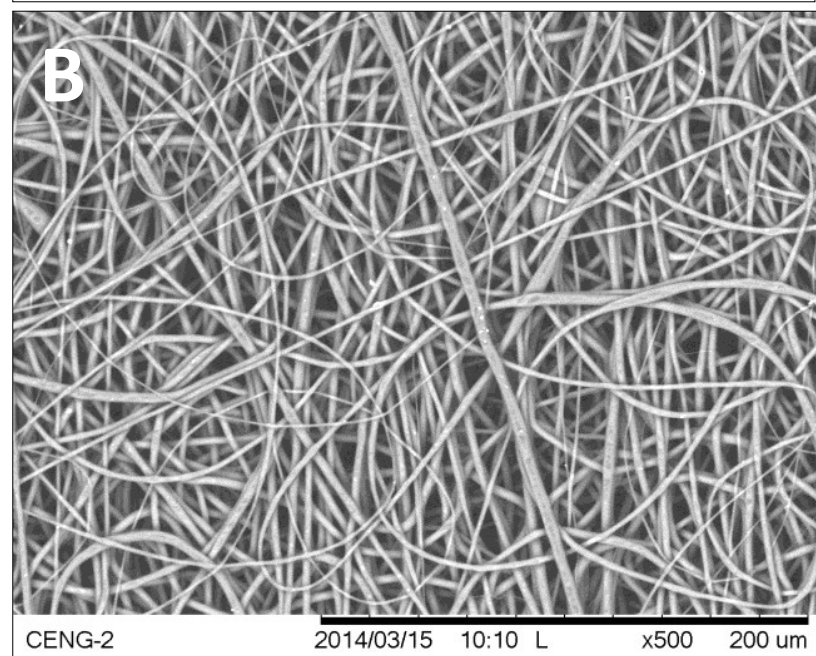

\begin{tabular}{|c|}
\hline Fiber Diameter $(\boldsymbol{\mu m})$ \\
\hline 3.7589 \\
\hline 2.4686 \\
\hline 4.11 \\
\hline 2.7278 \\
\hline 2.3844 \\
\hline 1.296 \\
\hline 2.5991 \\
\hline 3.8447 \\
\hline 1.656 \\
\hline
\end{tabular}

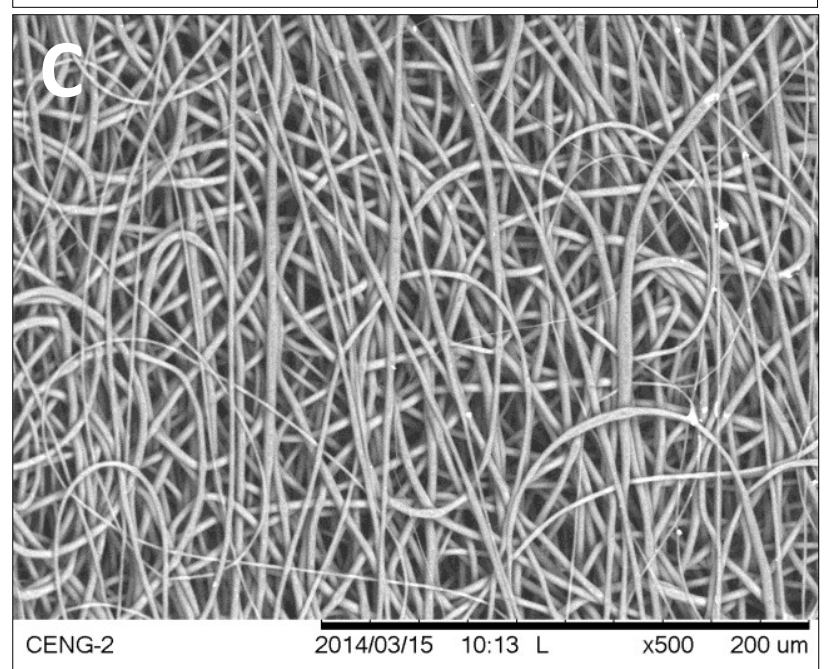

\begin{tabular}{|c|}
\hline Fiber Diameter $(\boldsymbol{\mu m})$ \\
\hline 1.7434 \\
\hline 1.4681 \\
\hline 2.3292 \\
\hline 4.1612 \\
\hline 3.1463 \\
\hline 0.9638 \\
\hline 2.2824 \\
\hline 3.1616 \\
\hline 1.1207 \\
\hline
\end{tabular}

Figure N 54: SEM images of Spin 20 Distal location at 500x magnification; $(A)$ Image 1; $(B)$ Image 2; (C) Image 3. 


\section{Spin 21}

Process Observations: The initial cone was small and had an ideal shape, but did not form immediately. After stabilizing, it began elongating and falling after $0.20 \mathrm{~mL}$ of solution was ejected. It elongated every $0.02 \mathrm{~mL}$.

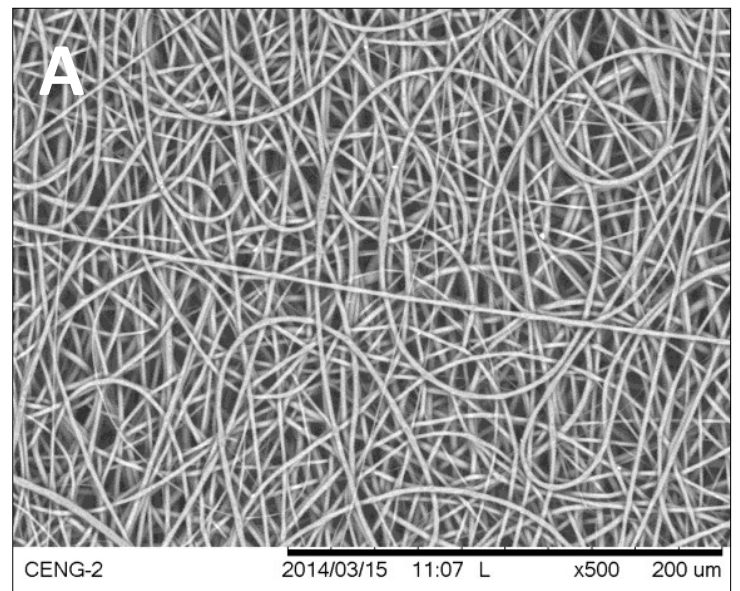

\begin{tabular}{|c|}
\hline Fiber Diameter $(\boldsymbol{\mu m})$ \\
\hline 1.3793 \\
\hline 1.9902 \\
\hline 2.5934 \\
\hline 2.0761 \\
\hline 1.2343 \\
\hline 1.9658 \\
\hline 1.9373 \\
\hline 1.8907 \\
\hline 2.0291 \\
\hline
\end{tabular}

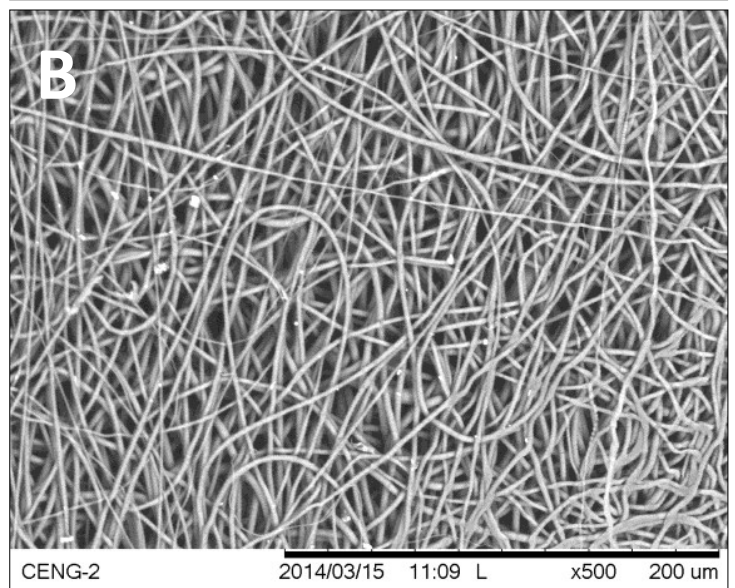

\begin{tabular}{|c|}
\hline Fiber Diameter $(\boldsymbol{\mu m})$ \\
\hline 1.5613 \\
\hline 1.4756 \\
\hline 1.9373 \\
\hline 2.2776 \\
\hline 3.1463 \\
\hline 1.9141 \\
\hline 2.6738 \\
\hline 2.208 \\
\hline 2.243 \\
\hline
\end{tabular}

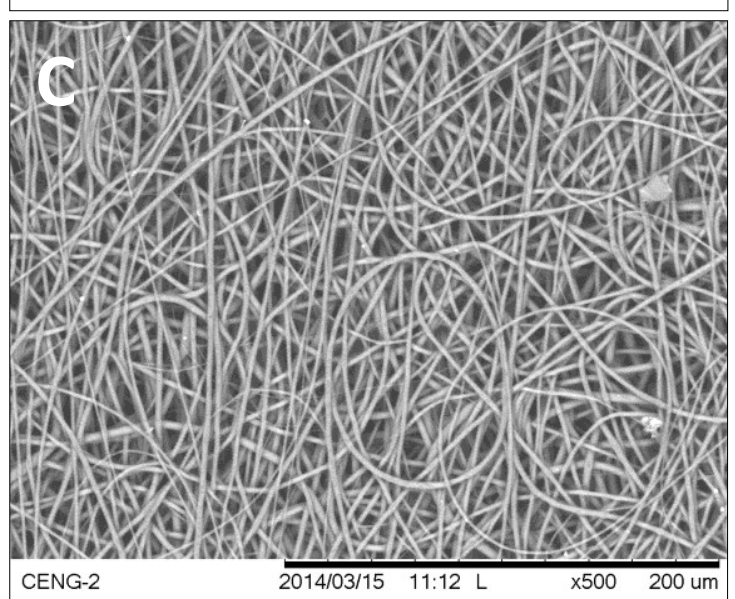

\begin{tabular}{|c|}
\hline Fiber Diameter $(\boldsymbol{\mu m})$ \\
\hline 3.1082 \\
\hline 1.2252 \\
\hline 2.2824 \\
\hline 2.069 \\
\hline 2.5457 \\
\hline 2.3212 \\
\hline 1.1976 \\
\hline 2.6388 \\
\hline 1.9141 \\
\hline
\end{tabular}

Figure $N$ 55: SEM images of Spin 21 Proximal location at 500x magnification; (A) Image 1; (B) Image 2; (C) Image 3. 


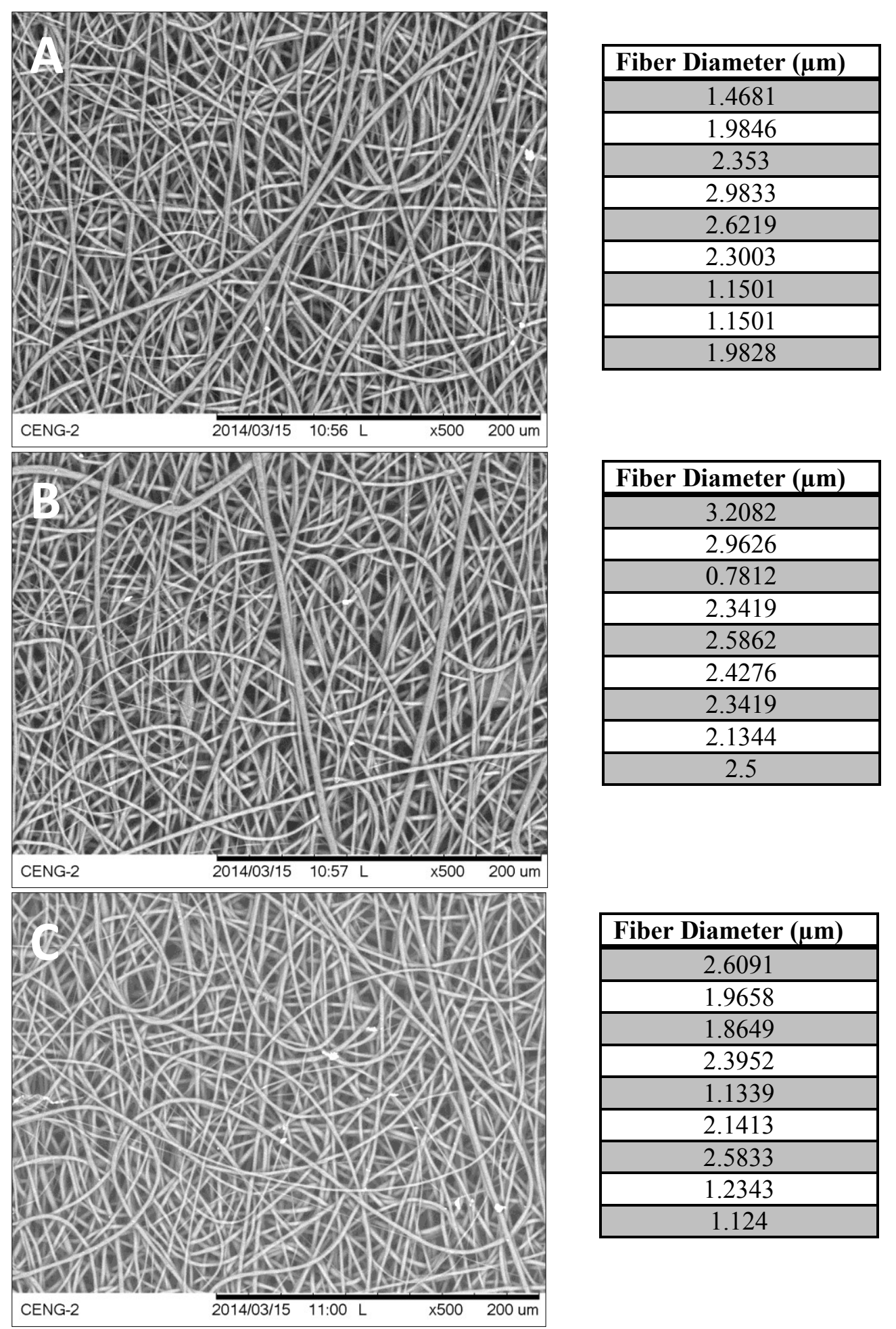

Figure N 56: SEM images of Spin 21 Medial location at 500x magnification; (A) Image 1; (B) Image 2; (C) Image 3. 


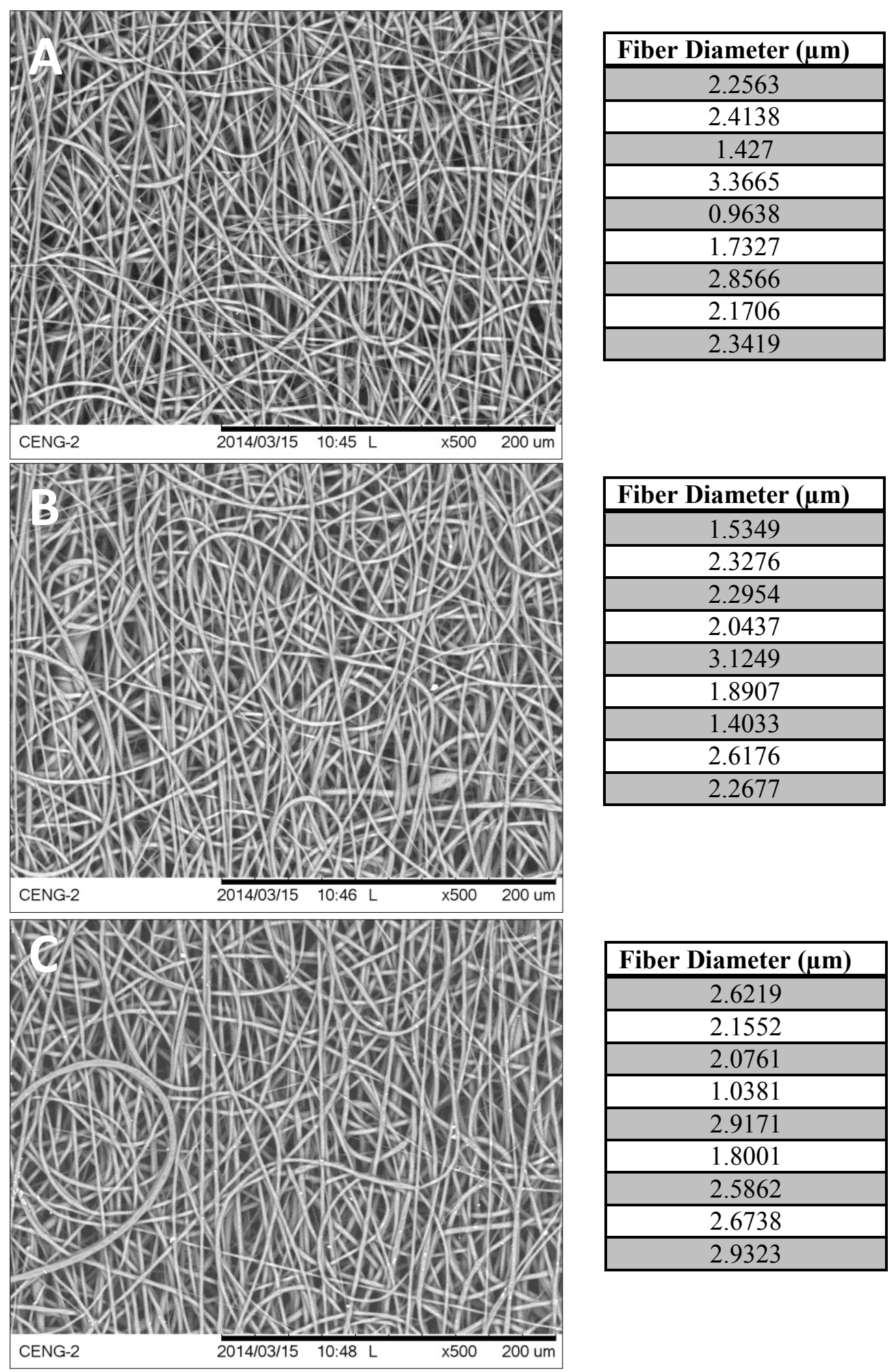

Figure $N$ 57: SEM images of Spin 21 Distal location at 500x magnification; (A) Image 1; (B) Image 2; (C) Image 3. 


\section{Spin 23}

Process Observations: The Taylor cone formed immediately after the initial bead fell. The cone was stable for about $0.10 \mathrm{~mL}$ and then elongated every $0.03 \mathrm{~mL}$ and fell repeatedly.

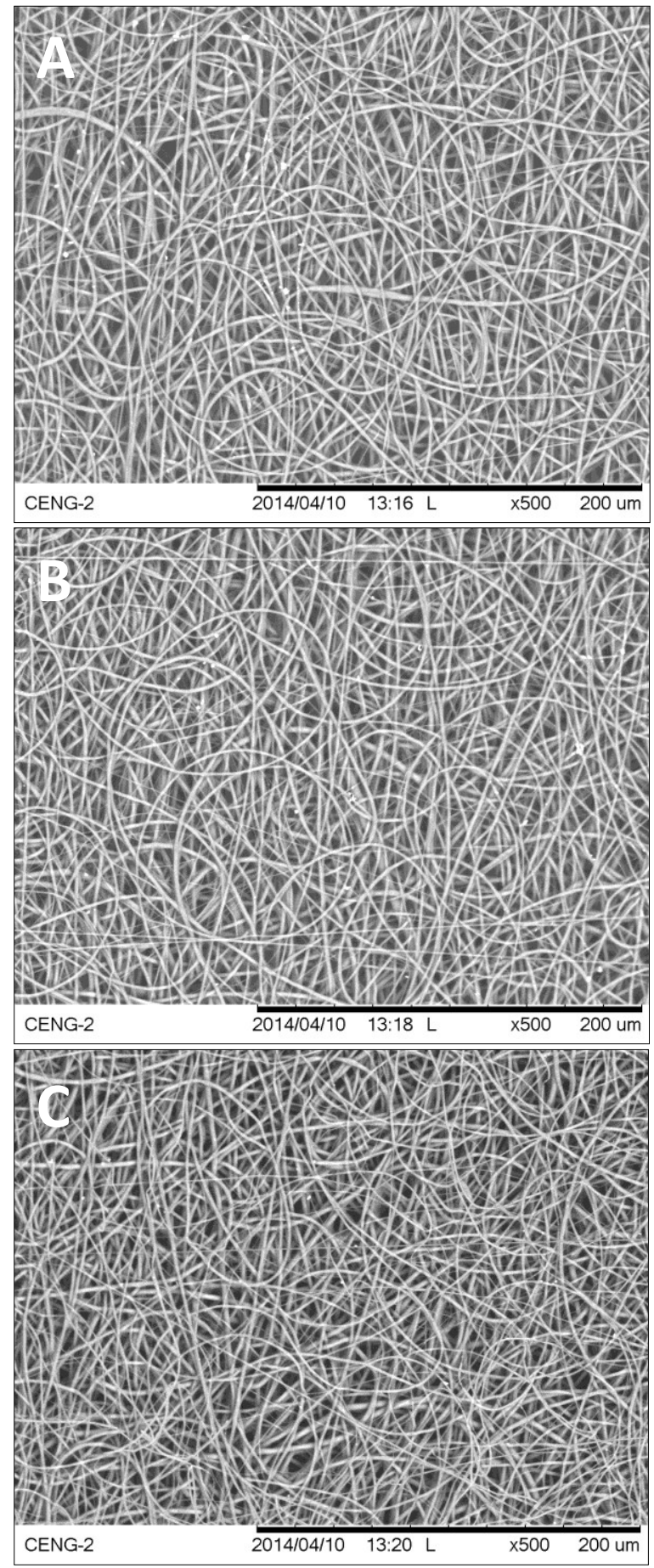

\begin{tabular}{|c|}
\hline Fiber Diameter $(\boldsymbol{\mu m})$ \\
\hline 1.4853 \\
\hline 1.9007 \\
\hline 1.8089 \\
\hline 1.3604 \\
\hline 2.423 \\
\hline 1.3874 \\
\hline 2.3088 \\
\hline 1.4728 \\
\hline 2.6715 \\
\hline
\end{tabular}

\begin{tabular}{|c|}
\hline Fiber Diameter $(\boldsymbol{\mu m})$ \\
\hline 1.4728 \\
\hline 2.0398 \\
\hline 1.6617 \\
\hline 1.8929 \\
\hline 1.9104 \\
\hline 2.3295 \\
\hline 1.9336 \\
\hline 1.6234 \\
\hline 1.3901 \\
\hline
\end{tabular}

Figure N 58: SEM images of Spin 23 Proximal location at 500x magnification; (A) Image 1; (B) Image 2; (C) Image 3. 


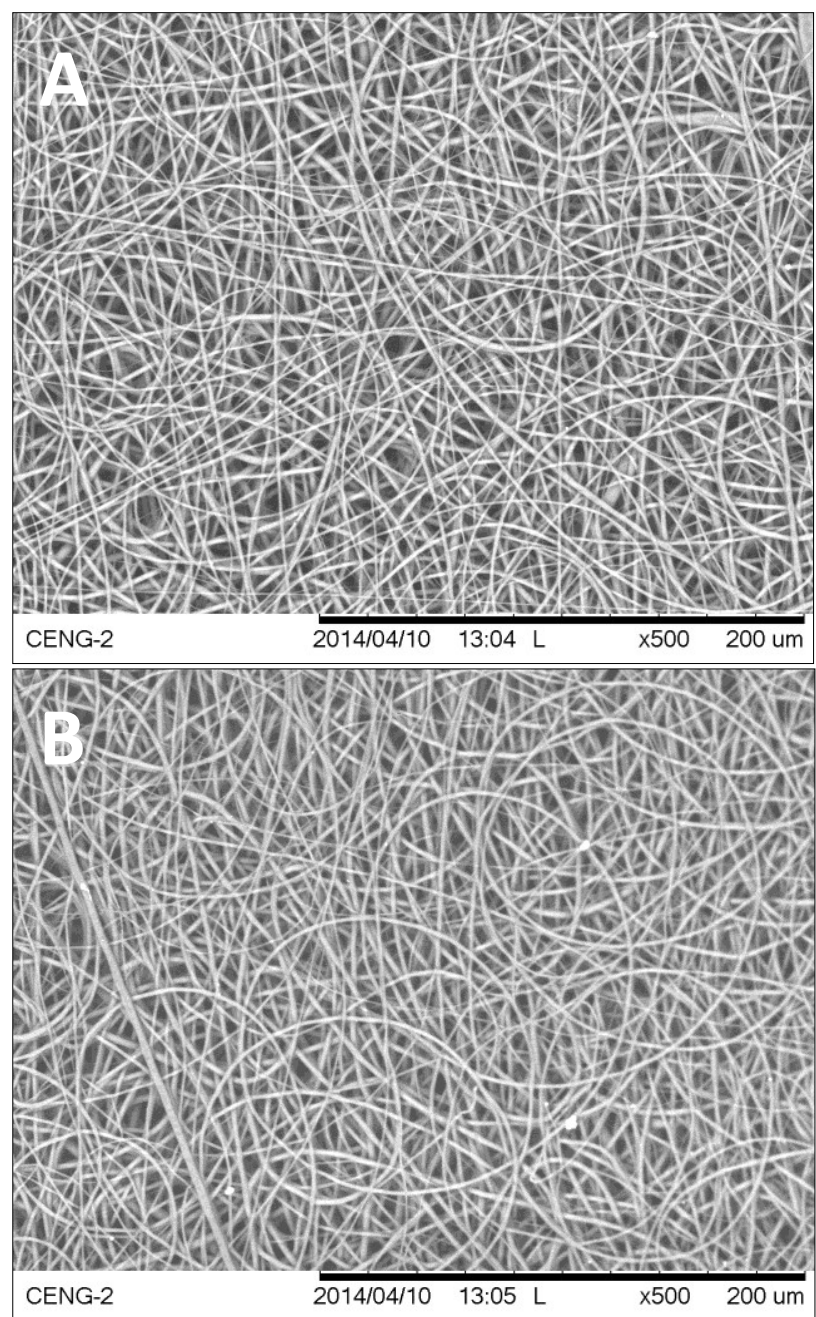

\begin{tabular}{|c|}
\hline Fiber Diameter $(\boldsymbol{\mu m})$ \\
\hline 0.7402 \\
\hline 1.2791 \\
\hline 1.887 \\
\hline 1.6905 \\
\hline 1.5819 \\
\hline 1.5051 \\
\hline 1.5701 \\
\hline 1.5222 \\
\hline 1.5488 \\
\hline
\end{tabular}

\begin{tabular}{|c|}
\hline Fiber Diameter $(\boldsymbol{\mu m})$ \\
\hline 2.5011 \\
\hline 1.8151 \\
\hline 1.2704 \\
\hline 1.5247 \\
\hline 2.4458 \\
\hline 2.1784 \\
\hline 1.5027 \\
\hline 1.723 \\
\hline 1.9507 \\
\hline
\end{tabular}

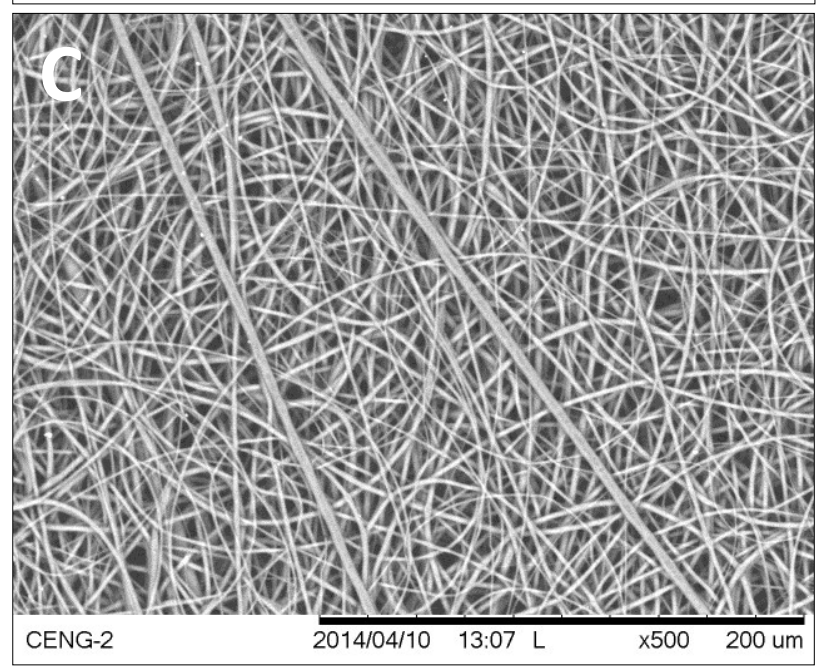

\begin{tabular}{|c|}
\hline Fiber Diameter $(\boldsymbol{\mu m})$ \\
\hline 4.4915 \\
\hline 1.6528 \\
\hline 2.7547 \\
\hline 1.4112 \\
\hline 1.4803 \\
\hline 1.7966 \\
\hline 1.7549 \\
\hline 2.7547 \\
\hline 2.016 \\
\hline
\end{tabular}

Figure N 59: SEM images of Spin 23 Medial location at 500x magnification; $(A)$ Image 1; $(B)$ Image 2; (C) Image 3. 


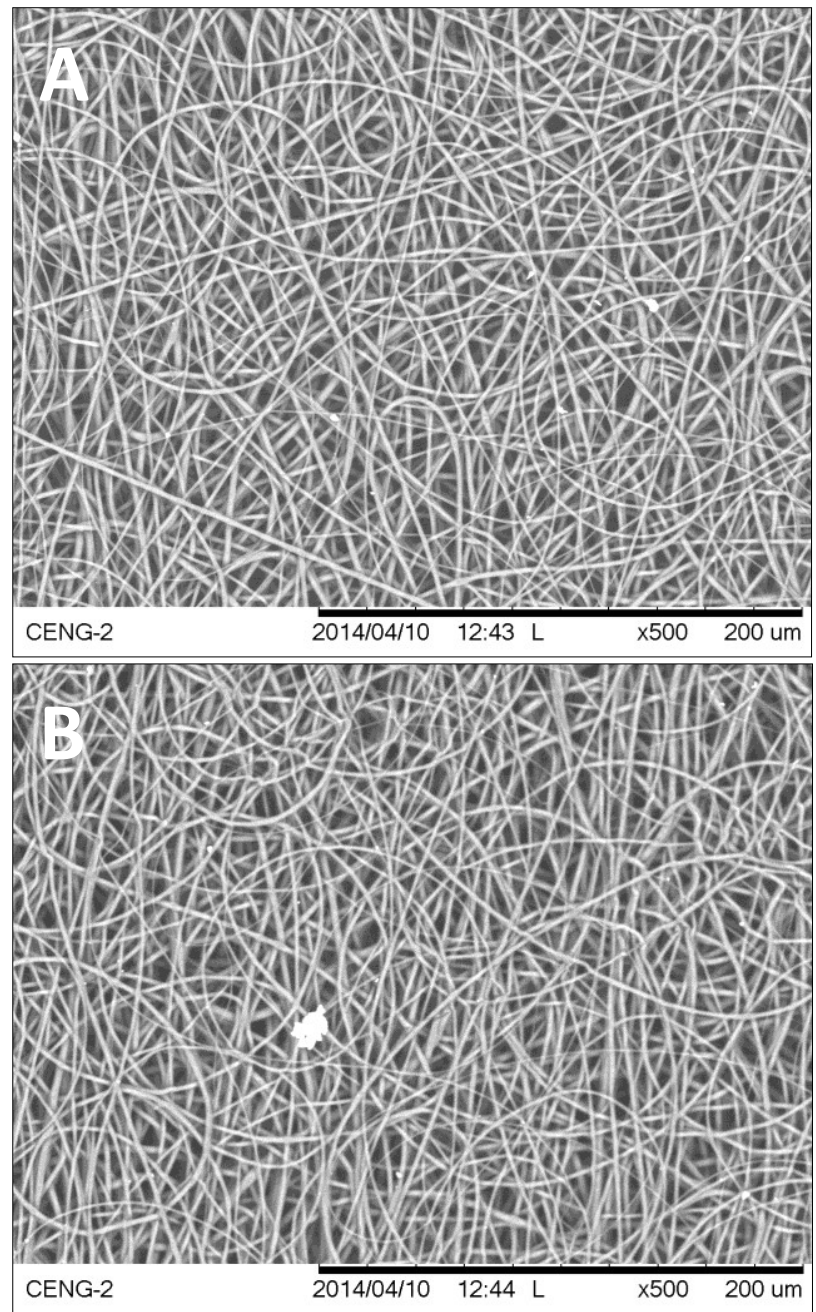

\begin{tabular}{|c|}
\hline Fiber Diameter $(\boldsymbol{\mu m})$ \\
\hline 1.8069 \\
\hline 1.432 \\
\hline 1.8089 \\
\hline 2.0013 \\
\hline 2.6673 \\
\hline 1.1185 \\
\hline 1.9864 \\
\hline 1.5701 \\
\hline 1.7316 \\
\hline
\end{tabular}

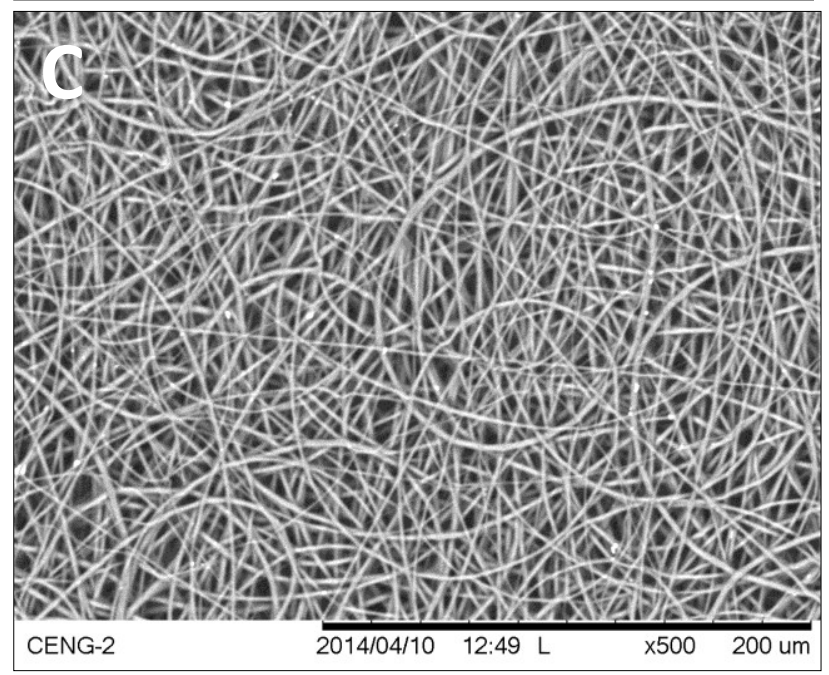

\begin{tabular}{|c|}
\hline Fiber Diameter $(\boldsymbol{\mu m})$ \\
\hline 2.0068 \\
\hline 2.5827 \\
\hline 2.3168 \\
\hline 1.0034 \\
\hline 2.1006 \\
\hline 2.2371 \\
\hline 3.1847 \\
\hline 2.0686 \\
\hline 2.1579 \\
\hline
\end{tabular}

\begin{tabular}{|c|}
\hline Fiber Diameter $(\boldsymbol{\mu m})$ \\
\hline 2.1511 \\
\hline 2.3485 \\
\hline 2.0722 \\
\hline 1.8232 \\
\hline 1.8414 \\
\hline 2.2371 \\
\hline 1.7079 \\
\hline 2.6169 \\
\hline 2.0252 \\
\hline
\end{tabular}

Figure N 60: SEM images of Spin 23 Distal location at 500x magnification; (A) Image 1; (B) Image 2; (C) Image 3. 


\section{APPENDIX O: CHAPTER III FIBER DIAMETER JMP SOFTWARE STATISTICAL ANALYSIS}

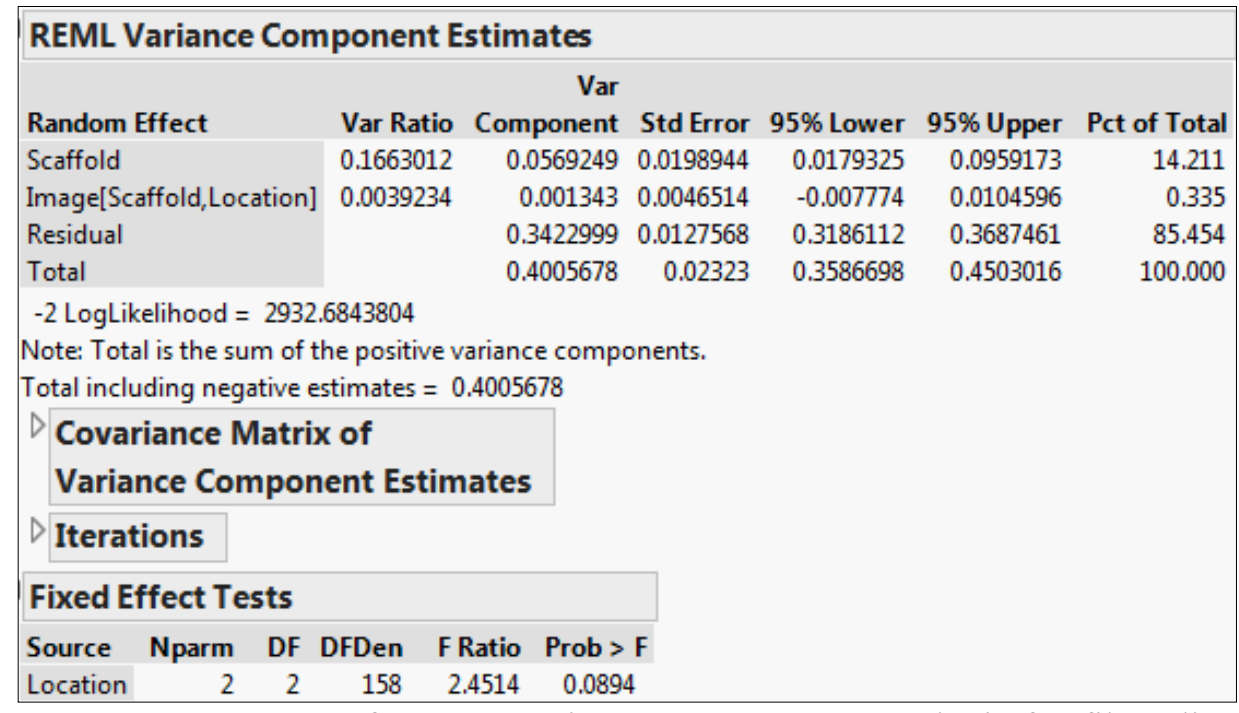

Figure O 1: JMP output of REML variance components analysis for fiber diameter.

\begin{tabular}{|c|c|c|c|c|c|}
\hline \multicolumn{6}{|c|}{ Oneway Anova } \\
\hline \multicolumn{6}{|c|}{$D$ Summary of Fit } \\
\hline \multicolumn{6}{|c|}{$\triangle$ Analysis of Variance } \\
\hline Source & DF & $\begin{array}{l}\text { Sum of } \\
\text { Squares }\end{array}$ & Mean Square & F Ratio & Prob $>F$ \\
\hline Scaffold & 19 & 94.34076 & 4.96530 & 14.4277 & $<.0001^{*}$ \\
\hline Error & 1600 & 550.64246 & 0.34415 & & \\
\hline C. Total & 1619 & 644.98322 & & & \\
\hline
\end{tabular}

Figure O 2: JMP output of ANOVA for fiber diameter. 


\section{APPENDIX P: CHAPTER III RAW COMPLIANCE DATA}

Table P I: Raw data obtained during dry compliance testing, including maximum and minimum voltage (Max V and Min V), change in voltage (Change in V), change in pressure (Change in $\mathrm{mmHg})$, change in diameter (\% Diameter Change), and compliance $(\% / 100 \mathrm{mmHg})$.

\begin{tabular}{|c|c|c|c|c|c|c|c|}
\hline Scaffold & Location & $\operatorname{Max} V$ & Min V & $\begin{array}{c}\text { Change } \\
\text { in } V\end{array}$ & $\begin{array}{c}\text { Change in } \\
\text { mmHg }\end{array}$ & $\begin{array}{c}\text { \% Diameter } \\
\text { Change }\end{array}$ & $\begin{array}{c}\text { Compliance } \\
(\% / 100 \mathrm{mmHg})\end{array}$ \\
\hline 1 & Proximal & 4.554 & 0.6269 & 3.92716 & 610.9031 & 0.2385 & 0.039041 \\
\hline 1 & Proximal & 4.9106 & 1.3154 & 3.59514 & 559.2547 & 0.3235 & 0.057845 \\
\hline 1 & Proximal & 4.4748 & 1.2961 & 3.17868 & 494.4714 & 0.2586 & 0.052298 \\
\hline 1 & Medial & 4.796 & 1.2519 & 3.54413 & 551.3202 & 0.1704 & 0.030908 \\
\hline 1 & Medial & 4.7623 & 1.3504 & 3.41188 & 530.7472 & 0.1398 & 0.02634 \\
\hline 1 & Medial & 4.847 & 1.391 & 3.45594 & 537.6012 & 0.3209 & 0.059691 \\
\hline 1 & Distal & 4.526 & 0.1088 & 4.41717 & 687.1279 & 0.2583 & 0.037591 \\
\hline 1 & Distal & 4.4486 & 1.4344 & 3.01423 & 468.889 & 0.326 & 0.069526 \\
\hline 1 & Distal & 4.417 & 1.6538 & 2.76312 & 429.8276 & 0.8597 & 0.20001 \\
\hline 2 & Proximal & 4.4619 & 0.554 & 3.9079 & 607.9073 & 0.3052 & 0.050205 \\
\hline 2 & Proximal & 4.4634 & 0.6437 & 3.81971 & 594.1882 & 0.2421 & 0.040745 \\
\hline 2 & Proximal & 5.0963 & 1.5285 & 3.56782 & 555.005 & 0.3323 & 0.059873 \\
\hline 2 & Medial & 4.8681 & 0.6388 & 4.22937 & 657.9151 & 0.208 & 0.031615 \\
\hline 2 & Medial & 4.7045 & 1.3092 & 3.39529 & 528.1657 & 0.7036 & 0.133216 \\
\hline 2 & Medial & 4.6329 & 0.8577 & 3.77524 & 587.2715 & 0.1305 & 0.022221 \\
\hline 2 & Distal & 4.5573 & 0.4626 & 4.0947 & 636.9662 & 0.1965 & 0.030849 \\
\hline 2 & Distal & 4.6217 & 0.7194 & 3.90234 & 607.0414 & 0.7605 & 0.12528 \\
\hline 2 & Distal & 4.742 & 0.8326 & 3.90937 & 608.1359 & 0.1931 & 0.031753 \\
\hline 3 & Proximal & 4.6745 & 0.6943 & 3.9802 & 619.1547 & 1.117 & 0.180407 \\
\hline 3 & Proximal & 4.7511 & 0.7118 & 4.03923 & 628.3361 & 1.201 & 0.19114 \\
\hline 3 & Proximal & 4.5877 & 0.704 & 3.88362 & 604.1299 & 1.123 & 0.185887 \\
\hline 3 & Medial & 4.651 & 0.6821 & 3.96894 & 617.403 & 0.8085 & 0.130952 \\
\hline 3 & Medial & 4.5111 & 0.7191 & 3.79205 & 589.8861 & 1.339 & 0.226993 \\
\hline 3 & Medial & 4.5899 & 0.7196 & 3.87029 & 602.057 & 0.9172 & 0.152344 \\
\hline 3 & Distal & 4.599 & 0.6909 & 3.90808 & 607.9354 & 0.494 & 0.081259 \\
\hline 3 & Distal & 4.6785 & 0.3738 & 4.30476 & 669.6425 & 1.092 & 0.163072 \\
\hline 3 & Distal & 4.4516 & 0.6498 & 3.80174 & 591.393 & 0.472 & 0.079812 \\
\hline 4 & Proximal & 4.6943 & 0.7194 & 3.97484 & 618.3205 & 1.353 & 0.218819 \\
\hline 4 & Proximal & 4.7733 & 0.729 & 4.04431 & 629.127 & 0.3064 & 0.048702 \\
\hline 4 & Proximal & 4.5228 & 0.7446 & 3.77826 & 587.7412 & 0.6213 & 0.10571 \\
\hline 4 & Medial & 4.5921 & 0.7188 & 3.87332 & 602.5285 & 0.9608 & 0.159461 \\
\hline 4 & Medial & 4.9408 & 0.71 & 4.23077 & 658.1326 & 1.274 & 0.193578 \\
\hline 4 & Medial & 4.721 & 0.7028 & 4.01821 & 625.0664 & 1.205 & 0.19278 \\
\hline
\end{tabular}




\begin{tabular}{|c|c|c|c|c|c|c|c|}
\hline 4 & Distal & 4.5401 & 0.6983 & 3.84174 & 597.6149 & 0.7938 & 0.132828 \\
\hline 4 & Distal & 4.5134 & 0.6883 & 3.82518 & 595.0386 & 0.8021 & 0.134798 \\
\hline 4 & Distal & 4.7015 & 0.7068 & 3.99473 & 621.4137 & 1.275 & 0.205177 \\
\hline 5 & Proximal & 4.9097 & 0.8299 & 4.07975 & 634.6391 & 0.9749 & 0.153615 \\
\hline 5 & Proximal & 4.6425 & 0.8708 & 3.77172 & 586.7235 & 0.7642 & 0.130249 \\
\hline 5 & Proximal & 4.4504 & 0.9162 & 3.53421 & 549.776 & 0.5782 & 0.10517 \\
\hline 5 & Medial & 4.7607 & 0.7208 & 4.03989 & 628.4398 & 0.2882 & 0.04586 \\
\hline 5 & Medial & 4.665 & 0.7614 & 3.90367 & 607.249 & 0.3633 & 0.059827 \\
\hline 5 & Medial & 4.8331 & 0.837 & 3.99614 & 621.6331 & 0.2971 & 0.047793 \\
\hline 5 & Distal & 4.8524 & 0.5743 & 4.27811 & 665.4962 & 0.7563 & 0.113645 \\
\hline 5 & Distal & 4.6435 & 0.6102 & 4.03323 & 627.4037 & 0.5709 & 0.090994 \\
\hline 5 & Distal & 4.6357 & 0.6356 & 4.00015 & 622.2578 & 0.7347 & 0.11807 \\
\hline 6 & Proximal & 4.6727 & 1.2233 & 3.44934 & 536.5743 & 0.1454 & 0.027098 \\
\hline 6 & Proximal & 4.8282 & 1.2537 & 3.57452 & 556.0466 & 0.2334 & 0.041975 \\
\hline 6 & Proximal & 4.9677 & 1.2685 & 3.69918 & 575.4396 & 0.1131 & 0.019655 \\
\hline 6 & Medial & 4.6333 & 1.0619 & 3.57138 & 555.5589 & 0.7475 & 0.134549 \\
\hline 6 & Medial & 4.5839 & 1.1224 & 3.46146 & 538.4589 & 0.2335 & 0.043364 \\
\hline 6 & Medial & 4.5104 & 1.1526 & 3.35784 & 522.3405 & 0.1856 & 0.035532 \\
\hline 6 & Distal & 4.6001 & 0.8809 & 3.71918 & 578.5505 & 0.2453 & 0.042399 \\
\hline 6 & Distal & 4.6732 & 0.9878 & 3.68546 & 573.305 & 0.2607 & 0.045473 \\
\hline 6 & Distal & 4.6427 & 1.0671 & 3.57559 & 556.2139 & 0.2888 & 0.051922 \\
\hline 7 & Proximal & 4.6468 & 1.3738 & 3.27294 & 509.1339 & 1.206 & 0.236873 \\
\hline 7 & Proximal & 4.8998 & 1.4328 & 3.46704 & 539.3281 & 1.296 & 0.240299 \\
\hline 7 & Proximal & 4.8114 & 1.4711 & 3.34026 & 519.6065 & 0.7157 & 0.137739 \\
\hline 7 & Medial & 4.9778 & 1.2977 & 3.68008 & 572.4679 & 0.5777 & 0.100914 \\
\hline 7 & Medial & 5.0033 & 1.3308 & 3.67256 & 571.2977 & 0.5337 & 0.093419 \\
\hline 7 & Medial & 4.5566 & 1.3498 & 3.20674 & 498.8355 & 0.4992 & 0.100073 \\
\hline 7 & Distal & 4.8943 & 1.2562 & 3.63802 & 565.9255 & 0.8341 & 0.147387 \\
\hline 7 & Distal & 5.1073 & 1.3237 & 3.78355 & 588.5631 & 0.5975 & 0.101518 \\
\hline 7 & Distal & 5.0978 & 1.317 & 3.7808 & 588.1355 & 0.5858 & 0.099603 \\
\hline 8 & Proximal & 5.1944 & 1.1037 & 4.0907 & 636.3425 & 0.6376 & 0.100198 \\
\hline 8 & Proximal & 4.9025 & 1.1447 & 3.75778 & 584.5549 & 0.6411 & 0.109673 \\
\hline 8 & Proximal & 4.4479 & 1.1997 & 3.24819 & 505.2834 & 0.5206 & 0.103031 \\
\hline 8 & Medial & 4.7197 & 0.9474 & 3.77227 & 586.8084 & 0.5543 & 0.09446 \\
\hline 8 & Medial & 4.4423 & 1.0487 & 3.39351 & 527.8892 & 0.8095 & 0.153347 \\
\hline 8 & Medial & 4.4168 & 1.1 & 3.31681 & 515.9587 & 0.6671 & 0.129293 \\
\hline 8 & Distal & 4.4568 & 0.5707 & 3.88607 & 604.5117 & 0.4147 & 0.068601 \\
\hline 8 & Distal & 4.8184 & 0.8665 & 3.952 & 614.7669 & 0.3772 & 0.061357 \\
\hline 8 & Distal & 4.579 & 0.9491 & 3.62986 & 564.6553 & 0.472 & 0.083591 \\
\hline 9 & Proximal & 4.9036 & 1.359 & 3.54456 & 551.386 & 0.5437 & 0.098606 \\
\hline
\end{tabular}




\begin{tabular}{|c|c|c|c|c|c|c|c|}
\hline 9 & Proximal & 4.6566 & 1.4135 & 3.24301 & 504.4773 & 0.3233 & 0.064086 \\
\hline 9 & Proximal & 4.8358 & 1.4168 & 3.41906 & 531.8645 & 0.3177 & 0.059733 \\
\hline 9 & Medial & 4.8347 & 1.3025 & 3.53214 & 549.4544 & 0.6543 & 0.119082 \\
\hline 9 & Medial & 4.4877 & 1.3426 & 3.14507 & 489.2424 & 1.015 & 0.207464 \\
\hline 9 & Medial & 4.6448 & 1.3428 & 3.30208 & 513.6661 & 1.109 & 0.215899 \\
\hline 9 & Distal & 4.5701 & 1.2908 & 3.27929 & 510.122 & 0.8056 & 0.157923 \\
\hline 9 & Distal & 4.9443 & 1.2778 & 3.66651 & 570.3565 & 0.9198 & 0.161268 \\
\hline 9 & Distal & 4.5912 & 1.3171 & 3.27407 & 509.3089 & 1.068 & 0.209696 \\
\hline 10 & Proximal & 4.6882 & 1.5033 & 3.18488 & 495.4355 & 0.3544 & 0.071533 \\
\hline 10 & Proximal & 4.3263 & 1.7059 & 2.62046 & 407.6343 & 0.3543 & 0.086916 \\
\hline 10 & Proximal & 4.6714 & 1.5937 & 3.07769 & 478.7609 & 0.3628 & 0.075779 \\
\hline 10 & Medial & 4.7942 & 1.4363 & 3.3579 & 522.3493 & 0.5183 & 0.099225 \\
\hline 10 & Medial & 4.8843 & 1.4842 & 3.40017 & 528.9256 & 0.3504 & 0.066247 \\
\hline 10 & Medial & 4.3656 & 0.9521 & 3.41349 & 530.9968 & 0.4769 & 0.089812 \\
\hline 10 & Distal & 5.2646 & 1.4144 & 3.85015 & 598.9231 & 0.6371 & 0.106374 \\
\hline 10 & Distal & 4.5851 & 0.8346 & 3.7505 & 583.422 & 0.242 & 0.041479 \\
\hline 10 & Distal & 4.6969 & 1.4401 & 3.25675 & 506.6149 & 0.2358 & 0.046544 \\
\hline 11 & Proximal & 4.5947 & 1.1668 & 3.42789 & 533.2377 & 0.3422 & 0.064174 \\
\hline 11 & Proximal & 4.9159 & 0.6816 & 4.23427 & 658.6773 & 0.5384 & 0.08174 \\
\hline 11 & Proximal & 4.7192 & 1.2446 & 3.47456 & 540.4976 & 0.3322 & 0.061462 \\
\hline 11 & Medial & 4.8226 & 1.0982 & 3.72441 & 579.3644 & 0.6069 & 0.104753 \\
\hline 11 & Medial & 4.5706 & 1.1299 & 3.44062 & 535.218 & 0.2369 & 0.044262 \\
\hline 11 & Medial & 4.5362 & 1.1544 & 3.38184 & 526.0739 & 0.2276 & 0.043264 \\
\hline 11 & Distal & 4.4202 & 0.9817 & 3.43851 & 534.8898 & 0.2873 & 0.053712 \\
\hline 11 & Distal & 4.5147 & 1.0491 & 3.46563 & 539.1077 & 0.3825 & 0.070951 \\
\hline 11 & Distal & 4.4645 & 1.0706 & 3.39392 & 527.9538 & 0.9343 & 0.176966 \\
\hline 12 & Proximal & 5.295 & 0.6666 & 4.62842 & 719.9904 & 0.9038 & 0.125529 \\
\hline 12 & Proximal & 4.7277 & 1.2884 & 3.43929 & 535.0116 & 0.6761 & 0.126371 \\
\hline 12 & Proximal & 4.6976 & 1.3015 & 3.39606 & 528.2857 & 0.5173 & 0.09792 \\
\hline 12 & Medial & 4.5239 & 1.2336 & 3.2903 & 511.8342 & 0.6569 & 0.128342 \\
\hline 12 & Medial & 4.3923 & 0.6837 & 3.70869 & 576.9185 & 0.4687 & 0.081242 \\
\hline 12 & Medial & 4.4105 & 0.7508 & 3.65968 & 569.2946 & 1.046 & 0.183736 \\
\hline 12 & Distal & 4.9904 & 0.6453 & 4.3451 & 675.9175 & 1.273 & 0.188337 \\
\hline 12 & Distal & 4.585 & 1.2241 & 3.3609 & 522.8172 & 0.6494 & 0.124212 \\
\hline 12 & Distal & 4.8687 & 1.2399 & 3.6288 & 564.491 & 1.257 & 0.222678 \\
\hline 13 & Proximal & 4.6545 & 0.836 & 3.81853 & 594.0044 & 1.224 & 0.206059 \\
\hline 13 & Proximal & 4.6905 & 1.5201 & 3.17035 & 493.1749 & 1.461 & 0.296244 \\
\hline 13 & Proximal & 4.66 & 0.99 & 3.66994 & 570.8897 & 1.195 & 0.209322 \\
\hline 13 & Medial & 4.5289 & 0.7397 & 3.78923 & 589.4467 & 0.369 & 0.062601 \\
\hline 13 & Medial & 4.4638 & 1.4274 & 3.0364 & 472.3382 & 0.2698 & 0.05712 \\
\hline
\end{tabular}




\begin{tabular}{|c|c|c|c|c|c|c|c|}
\hline 13 & Medial & 4.5743 & 0.8898 & 3.68445 & 573.1473 & 0.866 & 0.151096 \\
\hline 13 & Distal & 4.8753 & 0.753 & 4.12236 & 641.2683 & 1.183 & 0.184478 \\
\hline 13 & Distal & 4.6127 & 1.3744 & 3.23835 & 503.7528 & 1.217 & 0.241587 \\
\hline 13 & Distal & 4.5243 & 1.406 & 3.11827 & 485.0729 & 1.293 & 0.266558 \\
\hline 15 & Proximal & 4.6592 & 1.1188 & 3.54036 & 550.7339 & 0.7245 & 0.131552 \\
\hline 15 & Proximal & 4.5973 & 1.1999 & 3.39737 & 528.4892 & 0.5867 & 0.111015 \\
\hline 15 & Proximal & 4.5575 & 1.2371 & 3.32043 & 516.5215 & 1.067 & 0.206574 \\
\hline 15 & Medial & 5.1405 & 1.0149 & 4.12565 & 641.7793 & 0.6718 & 0.104678 \\
\hline 15 & Medial & 5.0294 & 1.0558 & 3.97364 & 618.1333 & 0.3594 & 0.058143 \\
\hline 15 & Medial & 4.4913 & 1.0771 & 3.41419 & 531.1068 & 0.2355 & 0.044341 \\
\hline 15 & Distal & 4.8512 & 0.8045 & 4.04667 & 629.494 & 0.6309 & 0.100223 \\
\hline 15 & Distal & 4.812 & 0.8927 & 3.9192 & 609.6657 & 0.8688 & 0.142504 \\
\hline 15 & Distal & 4.5452 & 0.9911 & 3.55413 & 552.8755 & 0.4273 & 0.077287 \\
\hline 17 & Proximal & 4.4386 & 0.6513 & 3.78725 & 589.1391 & 0.4553 & 0.077282 \\
\hline 17 & Proximal & 4.3768 & 0.709 & 3.6678 & 570.5574 & 0.4286 & 0.07512 \\
\hline 17 & Proximal & 4.6233 & 0.7584 & 3.86492 & 601.2213 & 0.4645 & 0.077259 \\
\hline 17 & Medial & 4.4622 & 0.5449 & 3.9173 & 609.3692 & 0.6894 & 0.113133 \\
\hline 17 & Medial & 4.7117 & 0.6047 & 4.10692 & 638.867 & 0.6533 & 0.102259 \\
\hline 17 & Medial & 4.4733 & 0.6489 & 3.82442 & 594.9212 & 0.487 & 0.08186 \\
\hline 17 & Distal & 4.5614 & 0.4553 & 4.10612 & 638.7422 & 1.794 & 0.280864 \\
\hline 17 & Distal & 4.2867 & 0.3925 & 3.89411 & 605.762 & 0.3831 & 0.063243 \\
\hline 17 & Distal & 4.3622 & 0.5344 & 3.82786 & 595.4563 & 0.3572 & 0.059988 \\
\hline 18 & Proximal & 4.5768 & 0.9161 & 3.66064 & 569.4441 & 1.246 & 0.21881 \\
\hline 18 & Proximal & 4.8171 & 0.9568 & 3.8603 & 600.5024 & 1.321 & 0.219982 \\
\hline 18 & Proximal & 4.5459 & 1.0011 & 3.54487 & 551.4347 & 1.094 & 0.198392 \\
\hline 18 & Medial & 4.7529 & 0.8528 & 3.90014 & 606.6995 & 0.5815 & 0.095846 \\
\hline 18 & Medial & 4.5023 & 0.8858 & 3.61648 & 562.5735 & 0.465 & 0.082656 \\
\hline 18 & Medial & 4.5383 & 0.917 & 3.62133 & 563.3282 & 1.185 & 0.210357 \\
\hline 18 & Distal & 4.3603 & 0.8062 & 3.55412 & 552.8741 & 0.5785 & 0.104635 \\
\hline$\overline{18}$ & Distal & 4.5751 & 0.8233 & 3.75186 & 583.6344 & 0.6243 & 0.106968 \\
\hline 18 & Distal & 4.6864 & 0.8557 & 3.8307 & 595.8975 & 0.6766 & 0.113543 \\
\hline 19 & Proximal & 4.8913 & 1.0981 & 3.79317 & 590.06 & 0.6534 & 0.110734 \\
\hline 19 & Proximal & 4.6776 & 1.1444 & 3.53318 & 549.6158 & 1.226 & 0.223065 \\
\hline 19 & Proximal & 4.4299 & 1.161 & 3.26892 & 508.5087 & 1.246 & 0.24503 \\
\hline 19 & Medial & 4.865 & 1.0509 & 3.81406 & 593.3102 & 0.7765 & 0.130876 \\
\hline 19 & Medial & 4.3283 & 1.0898 & 3.2385 & 503.7761 & 0.9003 & 0.17871 \\
\hline 19 & Medial & 4.4337 & 1.0938 & 3.33987 & 519.5449 & 0.4528 & 0.087153 \\
\hline 19 & Distal & 4.8885 & 0.4461 & 4.44245 & 691.0608 & 0.7672 & 0.111018 \\
\hline 19 & Distal & 4.6635 & 1.0436 & 3.61995 & 563.1133 & 1.19 & 0.211325 \\
\hline 19 & Distal & 4.2614 & 1.0772 & 3.18421 & 495.3317 & 0.6487 & 0.130963 \\
\hline
\end{tabular}




\begin{tabular}{|c|c|c|c|c|c|c|c|}
\hline $\mathbf{2 0}$ & Proximal & 5.0356 & 0.8278 & 4.20781 & 654.5609 & 0.7321 & 0.111846 \\
\hline $\mathbf{2 0}$ & Proximal & 4.2787 & 0.847 & 3.43169 & 533.8282 & 0.2803 & 0.052508 \\
\hline $\mathbf{2 0}$ & Proximal & 4.6368 & 0.8635 & 3.77333 & 586.9742 & 0.7441 & 0.126769 \\
\hline $\mathbf{2 0}$ & Medial & 4.6982 & 0.7579 & 3.94027 & 612.9421 & 0.4828 & 0.078768 \\
\hline $\mathbf{2 0}$ & Medial & 4.5646 & 0.7726 & 3.79202 & 589.881 & 0.1123 & 0.019038 \\
\hline $\mathbf{2 0}$ & Medial & 4.4245 & 0.7974 & 3.62711 & 564.2285 & 0.1465 & 0.025965 \\
\hline $\mathbf{2 0}$ & Distal & 4.7473 & 0.6872 & 4.06011 & 631.5851 & 0.8247 & 0.130576 \\
\hline $\mathbf{2 0}$ & Distal & 4.4785 & 0.7223 & 3.75621 & 584.3108 & 0.2044 & 0.034981 \\
\hline $\mathbf{2 0}$ & Distal & 4.6246 & 0.7494 & 3.87517 & 602.8154 & 0.4517 & 0.074932 \\
\hline $\mathbf{2 1}$ & Proximal & 4.3482 & 1.0307 & 3.31747 & 516.0603 & 0.5433 & 0.105278 \\
\hline $\mathbf{2 1}$ & Proximal & 4.3053 & 1.074 & 3.23128 & 502.6532 & 0.2254 & 0.044842 \\
\hline $\mathbf{2 1}$ & Proximal & 5.1051 & 1.1067 & 3.99842 & 621.989 & 0.2045 & 0.032878 \\
\hline $\mathbf{2 1}$ & Medial & 4.7862 & 0.9835 & 3.80268 & 591.5389 & 0.364 & 0.061534 \\
\hline $\mathbf{2 1}$ & Medial & 4.6152 & 0.9947 & 3.62041 & 563.1861 & 0.9173 & 0.162877 \\
\hline $\mathbf{2 1}$ & Medial & 4.3727 & 1.0204 & 3.3523 & 521.4794 & 0.2764 & 0.053003 \\
\hline $\mathbf{2 1}$ & Distal & 4.3547 & 0.8935 & 3.46118 & 538.4154 & 0.3948 & 0.073326 \\
\hline $\mathbf{2 1}$ & Distal & 4.2967 & 0.924 & 3.37264 & 524.6424 & 0.2313 & 0.044087 \\
\hline $\mathbf{2 1}$ & Distal & 4.814 & 0.9607 & 3.85335 & 599.4213 & 0.8717 & 0.145424 \\
\hline $\mathbf{2 3}$ & Proximal & 2.0136 & 0.2988 & 1.71472 & 886.768 & 1.363 & 0.153704 \\
\hline $\mathbf{2 3}$ & Proximal & 2.1448 & 0.1608 & 1.984 & 1026.022 & 0.8478 & 0.08263 \\
\hline $\mathbf{2 3}$ & Proximal & 2.1772 & 0.1139 & 2.06332 & 1067.047 & 1.023 & 0.095872 \\
\hline $\mathbf{2 3}$ & Medial & 1.9262 & 0.2356 & 1.69057 & 874.2763 & 1.126 & 0.128792 \\
\hline $\mathbf{2 3}$ & Medial & 1.9069 & 0.2179 & 1.68895 & 873.4391 & 1.08 & 0.123649 \\
\hline $\mathbf{2 3}$ & Medial & 2.0465 & 0.2518 & 1.7947 & 928.1256 & 1.33 & 0.1433 \\
\hline $\mathbf{2 3}$ & Distal & 2.0889 & 0.3572 & 1.73173 & 895.5646 & 1.132 & 0.126401 \\
\hline $\mathbf{2 3}$ & Distal & 1.9335 & 0.3527 & 1.58073 & 817.476 & 1.103 & 0.134928 \\
\hline $\mathbf{2 3}$ & Distal & 1.998 & 0.3945 & 1.6035 & 829.2471 & 1.261 & 0.152066 \\
\hline
\end{tabular}


Table P II: Raw data obtained during dry compliance testing, including maximum and minimum voltage (Max V and Min V), change in voltage (Change in V), change in pressure (Change in $\mathrm{mmHg}$ ), change in diameter (\% Diameter Change), and compliance $(\% / 100 \mathrm{mmHg})$.

\begin{tabular}{|c|c|c|c|c|c|c|c|}
\hline Scaffold & Location & Max V & Min V & $\begin{array}{l}\text { Change } \\
\text { in V }\end{array}$ & $\begin{array}{c}\text { Change in } \\
\text { mmHg }\end{array}$ & $\begin{array}{l}\text { \% Diameter } \\
\text { Change }\end{array}$ & $\begin{array}{c}\text { Compliance } \\
(\% / 100 \mathrm{mmHg})\end{array}$ \\
\hline 1 & Proximal & 4.9032 & 0.535 & 4.36815 & 679.5033 & 1.494 & 0.219866 \\
\hline 1 & Proximal & 4.7899 & 0.6907 & 4.09912 & 637.65332 & 1.454 & 0.228024 \\
\hline 1 & Proximal & 4.6654 & 0.8623 & 3.80316 & 591.61458 & 0.4578 & 0.077381 \\
\hline 1 & Medial & - & - & - & - & - & - \\
\hline 1 & Medial & - & - & - & - & - & - \\
\hline 1 & Medial & - & - & - & - & - & - \\
\hline 1 & Distal & 4.5568 & 0.8823 & 3.67442 & 571.58761 & 1.228 & 0.21484 \\
\hline 1 & Distal & 4.892 & 0.9135 & 3.97849 & 618.88807 & 0.8831 & 0.142691 \\
\hline 1 & Distal & 4.9248 & 0.9255 & 3.99931 & 622.1275 & 0.989 & 0.158971 \\
\hline 2 & Proximal & 4.5727 & 0.8452 & 3.72745 & 579.8359 & 1.076 & 0.18557 \\
\hline 2 & Proximal & 4.7774 & 0.9749 & 3.80252 & 591.51451 & 1.509 & 0.255108 \\
\hline 2 & Proximal & 4.7148 & 1.0161 & 3.69873 & 575.36835 & 1.007 & 0.175018 \\
\hline 2 & Medial & 4.6973 & 0.9857 & 3.71165 & 577.37891 & 0.5919 & 0.102515 \\
\hline 2 & Medial & 4.947 & 1.0376 & 3.90944 & 608.14661 & 0.3549 & 0.058358 \\
\hline 2 & Medial & 4.9197 & 1.0518 & 3.86794 & 601.69066 & 1.723 & 0.28636 \\
\hline 2 & Distal & 5.0793 & 1.0017 & 4.07759 & 634.30423 & 1.163 & 0.183351 \\
\hline 2 & Distal & 4.886 & 1.0885 & 3.79755 & 590.74131 & 0.8856 & 0.149913 \\
\hline 2 & Distal & 4.6559 & 1.1037 & 3.55224 & 552.58124 & 1.604 & 0.290274 \\
\hline 3 & Proximal & 4.6948 & 0.5464 & 4.14846 & 645.32891 & 1.673 & 0.259248 \\
\hline 3 & Proximal & 4.6 & 0.636 & 3.964 & 616.63344 & 1.427 & 0.231418 \\
\hline 3 & Proximal & 4.716 & 0.6583 & 4.0577 & 631.21029 & 1.485 & 0.235262 \\
\hline 3 & Medial & 4.7553 & 0.7236 & 4.03167 & 627.16 & 1.471 & 0.234549 \\
\hline 3 & Medial & 4.7333 & 0.7351 & 3.99825 & 621.96208 & 1.062 & 0.17075 \\
\hline 3 & Medial & 4.7633 & 0.7216 & 4.0417 & 628.72118 & 1.107 & 0.176072 \\
\hline 3 & Distal & 4.9267 & 0.7243 & 4.20231 & 653.70496 & 1.132 & 0.173167 \\
\hline 3 & Distal & 4.8466 & 0.7324 & 4.11418 & 639.99584 & 2.813 & 0.439534 \\
\hline 3 & Distal & 4.9766 & 0.7575 & 4.21907 & 656.31297 & 0.8205 & 0.125017 \\
\hline 4 & Proximal & 4.5335 & 0.7658 & 3.76771 & 586.09874 & 1.492 & 0.254565 \\
\hline 4 & Proximal & 4.4441 & 0.806 & 3.6381 & 565.93809 & 2.648 & 0.467896 \\
\hline 4 & Proximal & 4.4659 & 0.8621 & 3.60379 & 560.60022 & 1.138 & 0.202997 \\
\hline 4 & Medial & 4.4652 & 0.8696 & 3.59561 & 559.32853 & 1.174 & 0.209895 \\
\hline 4 & Medial & 4.5838 & 0.935 & 3.64885 & 567.60968 & 0.558 & 0.098307 \\
\hline 4 & Medial & 4.6903 & 0.9876 & 3.70272 & 575.9898 & 0.5318 & 0.092328 \\
\hline 4 & Distal & 4.6866 & 1.012 & 3.67465 & 571.62268 & 1.383 & 0.241943 \\
\hline 4 & Distal & 4.5776 & 1.0152 & 3.56234 & 554.15239 & 1.402 & 0.252999 \\
\hline
\end{tabular}




\begin{tabular}{|c|c|c|c|c|c|c|c|}
\hline 4 & Distal & 4.6168 & 1.0484 & 3.5684 & 555.09545 & 1.371 & 0.246985 \\
\hline 5 & Proximal & 4.8324 & 0.5014 & 4.33098 & 673.72086 & 1.53 & 0.227097 \\
\hline 5 & Proximal & 4.4507 & 1.1525 & 3.29824 & 513.06971 & 1.314 & 0.256106 \\
\hline 5 & Proximal & 4.5249 & 1.1737 & 3.35116 & 521.30139 & 0.7867 & 0.150911 \\
\hline 5 & Medial & 4.4168 & 1.181 & 3.23577 & 503.35218 & 0.8364 & 0.166166 \\
\hline 5 & Medial & 4.4726 & 1.2529 & 3.21965 & 500.84387 & 0.8175 & 0.163225 \\
\hline 5 & Medial & 4.7215 & 1.2803 & 3.44122 & 535.31181 & 0.7558 & 0.141189 \\
\hline 5 & Distal & 5.0743 & 1.3384 & 3.73597 & 581.16187 & 1.257 & 0.216291 \\
\hline 5 & Distal & 4.3609 & 1.3665 & 2.99441 & 465.80543 & 1.232 & 0.264488 \\
\hline 5 & Distal & 4.7246 & 1.386 & 3.3387 & 519.36246 & 1.18 & 0.227202 \\
\hline 6 & Proximal & 4.7177 & 1.3688 & 3.34884 & 520.94063 & 1.54 & 0.295619 \\
\hline 6 & Proximal & 4.9616 & 1.4675 & 3.49417 & 543.54866 & 2.42 & 0.445222 \\
\hline 6 & Proximal & 4.4884 & 0.7571 & 3.7313 & 580.43593 & 1.56 & 0.268764 \\
\hline 6 & Medial & 4.3582 & 1.4256 & 2.93266 & 456.20015 & 1.247 & 0.273345 \\
\hline 6 & Medial & 4.8021 & 0.7064 & 4.09571 & 637.12234 & 1.199 & 0.18819 \\
\hline 6 & Medial & 4.6401 & 1.5109 & 3.12918 & 486.77104 & 1.18 & 0.242414 \\
\hline 6 & Distal & 4.6579 & 1.5323 & 3.12561 & 486.21532 & 1.945 & 0.400029 \\
\hline 6 & Distal & 4.7518 & 1.5913 & 3.16048 & 491.63922 & 1.289 & 0.262184 \\
\hline 6 & Distal & 4.7357 & 1.6283 & 3.10735 & 483.37505 & 1.692 & 0.350039 \\
\hline 7 & Proximal & 4.7855 & 0.0884 & 4.69708 & 730.67018 & 1.246 & 0.170528 \\
\hline 7 & Proximal & 5.0308 & 0.1047 & 4.92611 & 766.29831 & 1.195 & 0.155944 \\
\hline 7 & Proximal & 4.8599 & 0.7255 & 4.1344 & 643.14111 & 1.382 & 0.214883 \\
\hline 7 & Medial & 4.5662 & 0.6679 & 3.89831 & 606.4152 & 2.774 & 0.457442 \\
\hline 7 & Medial & 5.0709 & 0.686 & 4.38487 & 682.10356 & 3.557 & 0.521475 \\
\hline 7 & Medial & 4.9074 & 0.6924 & 4.215 & 655.67971 & 3.099 & 0.472639 \\
\hline 7 & Distal & 5.0202 & 0.7094 & 4.31082 & 670.58409 & 1.119 & 0.166869 \\
\hline 7 & Distal & 5.1239 & 0.7009 & 4.42301 & 688.03739 & 1.117 & 0.162346 \\
\hline 7 & Distal & 4.7114 & 0.6989 & 4.01247 & 624.17314 & 1.34 & 0.214684 \\
\hline 8 & Proximal & 5.058 & 0.7151 & 4.34287 & 675.57043 & 1.631 & 0.241426 \\
\hline 8 & Proximal & 4.582 & 0.7342 & 3.84786 & 598.56755 & 1.263 & 0.211004 \\
\hline 8 & Proximal & 4.9777 & 0.7406 & 4.23713 & 659.12148 & 0.8586 & 0.130264 \\
\hline 8 & Medial & 4.6684 & 0.7249 & 3.94347 & 613.44054 & 1.373 & 0.22382 \\
\hline 8 & Medial & 5.2204 & 0.7707 & 4.44975 & 692.19699 & 1.484 & 0.21439 \\
\hline 8 & Medial & 5.0306 & 0.7845 & 4.24611 & 660.51909 & 1.401 & 0.212106 \\
\hline 8 & Distal & 5.0754 & 0.769 & 4.30642 & 669.90061 & 1.388 & 0.207195 \\
\hline 8 & Distal & 4.9334 & 0.7965 & 4.13686 & 643.52328 & 1.764 & 0.274116 \\
\hline 8 & Distal & 4.9231 & 0.8181 & 4.10499 & 638.56685 & 1.269 & 0.198726 \\
\hline 9 & Proximal & 4.3892 & 0.4434 & 3.94584 & 613.80866 & 1.351 & 0.220101 \\
\hline 9 & Proximal & 4.4884 & 0.4482 & 4.04023 & 628.49282 & 1.544 & 0.245667 \\
\hline 9 & Proximal & 4.8032 & 0.4556 & 4.34757 & 676.30221 & 1.263 & 0.186751 \\
\hline
\end{tabular}




\begin{tabular}{|c|c|c|c|c|c|c|c|}
\hline 9 & Medial & 4.6932 & 0.4644 & 4.22875 & 657.81877 & 0.8104 & 0.123195 \\
\hline 9 & Medial & 4.5414 & 0.5405 & 4.00099 & 622.38745 & 0.8004 & 0.128602 \\
\hline 9 & Medial & 4.609 & 0.587 & 4.02203 & 625.66121 & 1.3345 & 0.213294 \\
\hline 9 & Distal & - & - & - & - & - & - \\
\hline 9 & Distal & - & - & - & - & - & - \\
\hline 9 & Distal & - & - & - & - & - & - \\
\hline 10 & Proximal & 4.3681 & 0.6035 & 3.76465 & 585.62277 & 0.7956 & 0.135855 \\
\hline 10 & Proximal & 4.8332 & 0.66 & 4.17314 & 649.168 & 0.7803 & 0.1202 \\
\hline 10 & Proximal & 4.5047 & 0.6985 & 3.80619 & 592.085 & 0.7122 & 0.120287 \\
\hline 10 & Medial & 4.7442 & 0.7131 & 4.03103 & 627.06141 & 1.198 & 0.19105 \\
\hline 10 & Medial & 5.2485 & 0.7384 & 4.51016 & 701.59364 & 1.384 & 0.197265 \\
\hline 10 & Medial & 5.0047 & 0.7787 & 4.22597 & 657.3849 & 1.455 & 0.221332 \\
\hline 10 & Distal & 4.8238 & 0.8216 & 4.00213 & 622.56617 & 0.7663 & 0.123087 \\
\hline 10 & Distal & 4.8516 & 0.8811 & 3.97046 & 617.63964 & 1.1154 & 0.180591 \\
\hline 10 & Distal & 4.8183 & 0.9313 & 3.88698 & 604.65314 & 0.8351 & 0.138112 \\
\hline 11 & Proximal & 5.5223 & 0.0694 & 5.45289 & 848.24286 & 1.817 & 0.214208 \\
\hline 11 & Proximal & 4.5738 & 0.605 & 3.96875 & 617.37304 & 1.466 & 0.237458 \\
\hline 11 & Proximal & 5.0066 & 0.6319 & 4.37474 & 680.5276 & 1.296 & 0.19044 \\
\hline 11 & Medial & 4.4913 & 0.5856 & 3.90563 & 607.55434 & 0.884 & 0.145501 \\
\hline 11 & Medial & 5.0438 & 0.656 & 4.38777 & 682.55478 & 1.5654 & 0.229344 \\
\hline 11 & Medial & 4.968 & 0.6934 & 4.27464 & 664.95673 & 1.464 & 0.220165 \\
\hline 11 & Distal & 4.3701 & 0.7004 & 3.66973 & 570.85723 & 1.4476 & 0.253584 \\
\hline 11 & Distal & 4.4612 & 0.7499 & 3.71127 & 577.31946 & 1.3329 & 0.230877 \\
\hline 11 & Distal & 4.5226 & 0.7834 & 3.73918 & 581.66147 & 1.3321 & 0.229016 \\
\hline 12 & Proximal & 4.5687 & 0.7831 & 3.78555 & 588.87512 & 1.5697 & 0.266559 \\
\hline 12 & Proximal & 4.4257 & 0.8393 & 3.58642 & 557.89806 & 0.8385 & 0.150296 \\
\hline 12 & Proximal & 4.5002 & 0.8647 & 3.63556 & 565.54226 & 0.9049 & 0.160006 \\
\hline 12 & Medial & 4.7352 & 0.867 & 3.86822 & 601.73497 & 2.591 & 0.430588 \\
\hline 12 & Medial & 4.6991 & 0.9261 & 3.77297 & 586.91699 & 2.314 & 0.394264 \\
\hline 12 & Medial & 4.6715 & 0.9198 & 3.7517 & 583.60852 & 2.781 & 0.476518 \\
\hline 12 & Distal & 4.89 & 0.999 & 3.89102 & 605.28161 & 2.919 & 0.482255 \\
\hline 12 & Distal & 4.6292 & 0.643 & 3.98619 & 620.08518 & 2.769 & 0.446552 \\
\hline 12 & Distal & 4.9875 & 0.8685 & 4.11901 & 640.74726 & 2.363 & 0.368788 \\
\hline 13 & Proximal & 4.7521 & 0.436 & 4.31615 & 671.41416 & 1.252 & 0.186472 \\
\hline 13 & Proximal & 4.9496 & 0.5607 & 4.3889 & 682.73017 & 1.41 & 0.206524 \\
\hline 13 & Proximal & 4.9122 & 0.5726 & 4.33963 & 675.0664 & 1.263 & 0.187093 \\
\hline 13 & Medial & 4.7116 & 0.566 & 4.14555 & 644.87621 & 1.181 & 0.183136 \\
\hline 13 & Medial & 5.1414 & 0.6915 & 4.4499 & 692.21988 & 1.1479 & 0.165829 \\
\hline 13 & Medial & 4.7118 & 0.7772 & 3.93452 & 612.04736 & 1.305 & 0.213219 \\
\hline 13 & Distal & 4.6139 & 0.8819 & 3.732 & 580.54412 & 1.321 & 0.227545 \\
\hline
\end{tabular}




\begin{tabular}{|c|c|c|c|c|c|c|c|}
\hline 13 & Distal & 4.7017 & 1.2465 & 3.45515 & 537.4782 & 1.3063 & 0.243042 \\
\hline 13 & Distal & 4.8406 & 1.0552 & 3.78541 & 588.85223 & 1.2 & 0.203786 \\
\hline 15 & Proximal & 4.7035 & 0.3441 & 4.35932 & 678.12933 & 1.751 & 0.25821 \\
\hline 15 & Proximal & 4.6295 & 0.763 & 3.86648 & 601.46431 & 1.122 & 0.186545 \\
\hline 15 & Proximal & 4.8752 & 0.7461 & 4.1291 & 642.31621 & 1.685 & 0.262332 \\
\hline 15 & Medial & 4.5878 & 1.1653 & 3.42252 & 532.4025 & 1.343 & 0.252253 \\
\hline 15 & Medial & 4.4599 & 0.712 & 3.74795 & 583.0251 & 1.221 & 0.209425 \\
\hline 15 & Medial & 4.6264 & 0.1888 & 4.43758 & 690.30274 & 0.9803 & 0.14201 \\
\hline 15 & Distal & 4.966 & 0.1992 & 4.76681 & 741.51836 & 1.534 & 0.206873 \\
\hline 15 & Distal & 4.2671 & 1.2009 & 3.06623 & 476.97818 & 1.165 & 0.244246 \\
\hline 15 & Distal & 4.838 & 0.9621 & 3.87599 & 602.94389 & 1.352 & 0.224233 \\
\hline 17 & Proximal & 4.6789 & 0.6217 & 4.0572 & 631.13164 & 1.3322 & 0.211081 \\
\hline 17 & Proximal & 4.2212 & 0.6955 & 3.52565 & 548.44491 & 1.3074 & 0.238383 \\
\hline 17 & Proximal & 4.7019 & 0.648 & 4.05389 & 630.61691 & 1.2033 & 0.190813 \\
\hline 17 & Medial & 4.742 & 0.791 & 3.95098 & 614.60884 & 0.6901 & 0.112283 \\
\hline 17 & Medial & 5.0085 & 0.8024 & 4.20617 & 654.3061 & 1.816 & 0.277546 \\
\hline 17 & Medial & 4.9156 & 0.8599 & 4.05565 & 630.89089 & 1.251 & 0.198291 \\
\hline 17 & Distal & 4.609 & 0.6221 & 3.98686 & 620.18968 & 1.2567 & 0.202632 \\
\hline 17 & Distal & 5.3594 & 0.2808 & 5.07864 & 790.02516 & 1.3935 & 0.176387 \\
\hline 17 & $\overline{\text { Distal }}$ & 4.7046 & 0.918 & 3.7866 & 589.03833 & 1.1764 & 0.199715 \\
\hline 18 & Proximal & 4.7668 & 0.2519 & 4.51491 & 702.33214 & 1.121 & 0.159611 \\
\hline 18 & Proximal & 4.9637 & 0.8971 & 4.06664 & 632.60089 & 0.8041 & 0.12711 \\
\hline 18 & Proximal & 4.3922 & 0.4761 & 3.91614 & 609.189 & 1.153 & 0.189268 \\
\hline 18 & Medial & 4.4666 & 0.6013 & 3.86529 & 601.27894 & 1.269 & 0.21105 \\
\hline 18 & Medial & 4.5539 & 0.5944 & 3.95952 & 615.93666 & 1.227 & 0.199209 \\
\hline 18 & Medial & 4.4586 & 0.6374 & 3.82122 & 594.42309 & 2.411 & 0.405603 \\
\hline 18 & Distal & 5.113 & 0.5582 & 4.55484 & 708.54476 & 0.9624 & 0.135828 \\
\hline 18 & Distal & 4.7696 & 0.5799 & 4.18965 & 651.73612 & 1.02 & 0.156505 \\
\hline 18 & Distal & 4.7535 & 0.5782 & 4.17536 & 649.51287 & 0.837 & 0.128866 \\
\hline 19 & Proximal & 1.8787 & 0.2531 & 1.62566 & 840.70876 & 2.705 & 0.321752 \\
\hline 19 & Proximal & 1.7786 & 0.1236 & 1.655 & 855.88383 & 3.518 & 0.411037 \\
\hline 19 & Proximal & 1.7601 & 0.1103 & 1.64974 & 853.16356 & 3.088 & 0.361947 \\
\hline 19 & Medial & 2.3543 & 0.2975 & 2.05672 & 1063.632 & 2.316 & 0.217744 \\
\hline 19 & Medial & 2.5318 & 0.3119 & 2.21989 & 1148.0143 & 2.6713 & 0.232689 \\
\hline 19 & Medial & 2.272 & 0.2533 & 2.01867 & 1043.9542 & 2.291 & 0.219454 \\
\hline 19 & Distal & 2.0477 & 0.0823 & 1.96535 & 1016.3796 & 2.226 & 0.219013 \\
\hline 19 & Distal & 2.0163 & 0.3293 & 1.68701 & 872.43499 & 3.316 & 0.380086 \\
\hline 19 & Distal & 1.95 & 0.3899 & 1.5601 & 806.80608 & 2.906 & 0.360186 \\
\hline 20 & Proximal & 1.9582 & 0.349 & 1.60914 & 832.16619 & 1.9457 & 0.233811 \\
\hline 20 & Proximal & 2.1078 & 0.3991 & 1.70865 & 883.62909 & 3.028 & 0.342678 \\
\hline
\end{tabular}




\begin{tabular}{|c|c|c|c|c|c|c|c|}
\hline 20 & Proximal & 2.1084 & 0.2349 & 1.87345 & 968.85473 & 2.601 & 0.268461 \\
\hline 20 & Medial & 2.0355 & 0.2732 & 1.7623 & 911.37312 & 2.366 & 0.259608 \\
\hline 20 & Medial & 2.0869 & 0.2228 & 1.86406 & 963.99483 & 2.212 & 0.229462 \\
\hline 20 & Medial & 2.0261 & 0.2613 & 1.76479 & 912.6596 & 2.419 & 0.26505 \\
\hline 20 & Distal & 2.1371 & 0.2355 & 1.90165 & 983.43934 & 2.8256 & 0.287318 \\
\hline 20 & Distal & 2.1232 & 0.2081 & 1.91508 & 990.38363 & 2.7687 & 0.279558 \\
\hline 20 & Distal & 2.1133 & 0.2202 & 1.89303 & 978.97717 & 2.9979 & 0.306228 \\
\hline 21 & Proximal & 1.9877 & 0.3149 & 1.67286 & 865.11875 & 2.181 & 0.252104 \\
\hline 21 & Proximal & 2.0386 & 0.1505 & 1.8881 & 976.42998 & 2.953 & 0.302428 \\
\hline 21 & Proximal & 2.0655 & 0.2415 & 1.82409 & 943.32889 & 2.497 & 0.264701 \\
\hline 21 & Medial & 1.986 & 0.2249 & 1.76116 & 910.78143 & 2.969 & 0.325984 \\
\hline 21 & Medial & 2.0197 & 0.2383 & 1.78137 & 921.23654 & 2.029 & 0.220247 \\
\hline 21 & Medial & 2.2615 & 0.1468 & 2.11465 & 1093.5881 & 2.793 & 0.255398 \\
\hline 21 & Distal & 1.8959 & 0.2386 & 1.6573 & 857.07088 & 2.136 & 0.249221 \\
\hline 21 & Distal & 2.0507 & 0.2263 & 1.82446 & 943.51917 & 2.553 & 0.270583 \\
\hline 21 & Distal & 1.99 & 0.2901 & 1.69994 & 879.12272 & 2.422 & 0.275502 \\
\hline 23 & Proximal & 1.8744 & 0.233 & 1.64145 & 848.87693 & 2.381 & 0.280488 \\
\hline 23 & Proximal & 2.1882 & 0.304 & 1.88423 & 974.42907 & 2.877 & 0.29525 \\
\hline 23 & Proximal & 2.0284 & 0.3374 & 1.69107 & 874.53657 & 2.754 & 0.31491 \\
\hline 23 & Medial & 1.8392 & 0.3249 & 1.51434 & 783.13754 & 2.521 & 0.32191 \\
\hline 23 & Medial & 2.2146 & 0.3365 & 1.87806 & 971.23496 & 3.16 & 0.325359 \\
\hline 23 & Medial & 2.1132 & 0.2668 & 1.84642 & 954.87407 & 3.792 & 0.39712 \\
\hline 23 & Distal & 1.9853 & 0.1451 & 1.84019 & 951.65419 & 2.937 & 0.308621 \\
\hline 23 & Distal & 2.0936 & 0.2856 & 1.80805 & 935.03306 & 2.683 & 0.286942 \\
\hline 23 & Distal & 2.0352 & 0.2865 & 1.74872 & 904.34903 & 2.856 & 0.315807 \\
\hline
\end{tabular}




\section{APPENDIX Q: CHAPTER III COMPLIANCE JMP SOFTWARE STATISTICAL ANALYSIS}

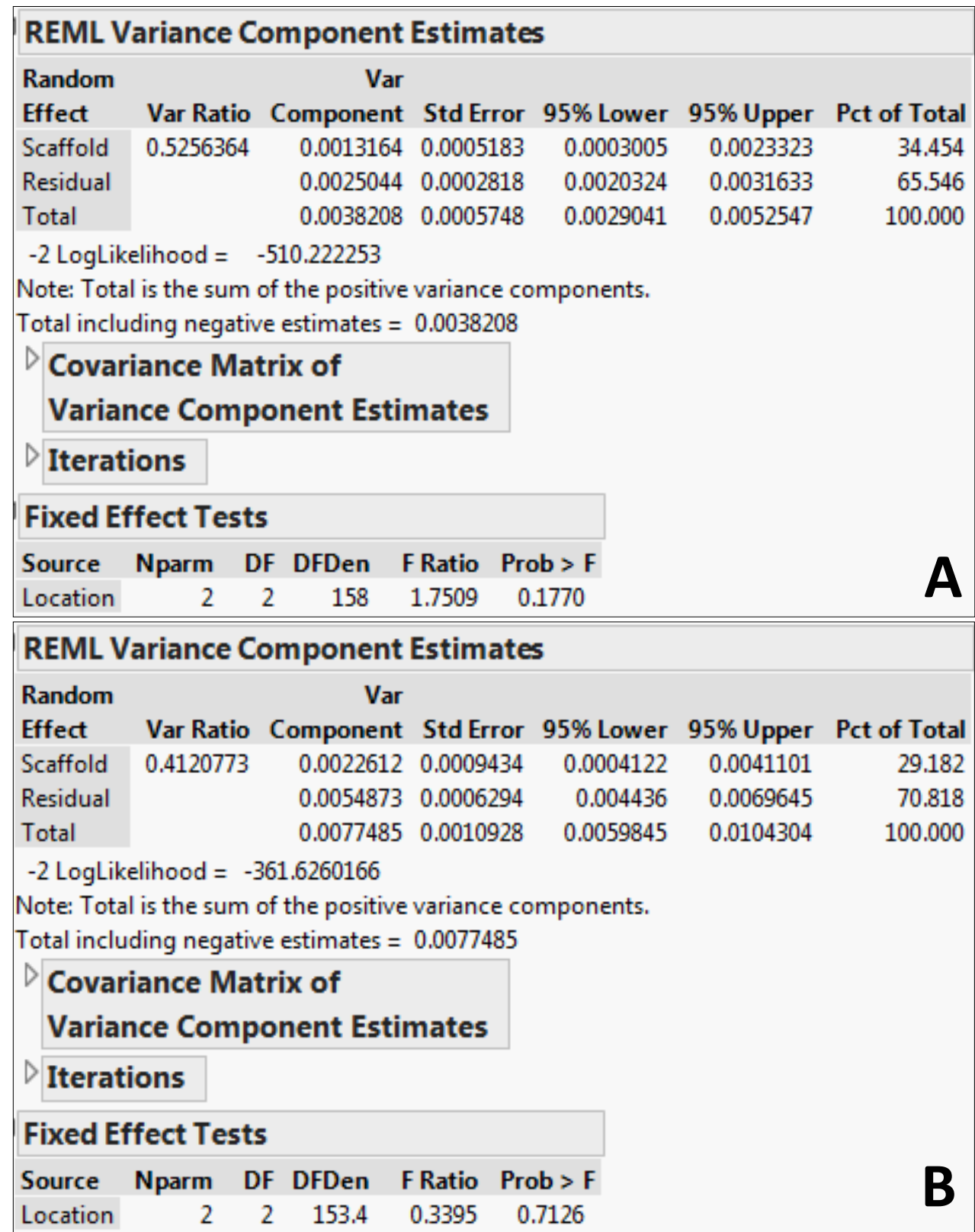

Figure $Q$ 1: JMP output of the REML variance components analysis for $(A)$ dry compliance and $(B)$ wet compliance. 


\begin{tabular}{|c|c|c|c|c|c|}
\hline \multicolumn{6}{|c|}{ Analysis of Variance } \\
\hline Source & DF & $\begin{array}{l}\text { Sum of } \\
\text { Squares }\end{array}$ & Mean Square & F Ratio & Prob $>F$ \\
\hline Scaffold & 19 & 0.27268897 & 0.014352 & 5.6774 & $<.0001^{*}$ \\
\hline Error & 160 & 0.40446578 & 0.002528 & & \\
\hline C. Total & 179 & 0.67715475 & & & \\
\hline \multicolumn{6}{|c|}{ Analysis of Variance } \\
\hline Source & DF & $\begin{array}{r}\text { Sum of } \\
\text { Squares }\end{array}$ & Mean Square & F Ratio & Prob $>$ F \\
\hline Scaffold & 19 & 0.4786903 & 0.025194 & 4.6330 & $<.0001^{*}$ \\
\hline Error & 154 & 0.8374497 & 0.005438 & & \\
\hline C. Total & 173 & 1.3161400 & & & \\
\hline
\end{tabular}

Figure $Q$ 2: JMP output of ANOVA results for $(A)$ dry compliance and $(B)$ wet compliance. 